\title{
Effectiveness of Different Approaches to Disseminating Traveler Information on Travel Time Reliability
}

SHRP 2 RELIABILITY RESEARCH 


\section{TRANSPORTATION RESEARCH BOARD 2014 EXECUTIVE COMMITTEE*}

\section{OFFICERS}

CHAIR: Kirk T. Steudle, Director, Michigan Department of Transportation, Lansing

VICE CHAIR: Daniel Sperling, Professor of Civil Engineering and Environmental Science and Policy; Director, Institute of Transportation Studies,

University of California, Davis

EXECUTIVE DIRECTOR: Robert E. Skinner, Jr., Transportation Research Board

\section{MEMBERS}

Victoria A. Arroyo, Executive Director, Georgetown Climate Center, and Visiting Professor, Georgetown University Law Center, Washington, D.C. Scott E. Bennett, Director, Arkansas State Highway and Transportation Department, Little Rock

Deborah H. Butler, Executive Vice President, Planning, and CIO, Norfolk Southern Corporation, Norfolk, Virginia (Past Chair, 2013)

James M. Crites, Executive Vice President of Operations, Dallas-Fort Worth International Airport, Texas

Malcolm Dougherty, Director, California Department of Transportation, Sacramento

A. Stewart Fotheringham, Professor and Director, Centre for Geoinformatics, School of Geography and Geosciences, University of St. Andrews, Fife, United Kingdom

John S. Halikowski, Director, Arizona Department of Transportation, Phoenix

Michael W. Hancock, Secretary, Kentucky Transportation Cabinet, Frankfort

Susan Hanson, Distinguished University Professor Emerita, School of Geography, Clark University, Worcester, Massachusetts

Steve Heminger, Executive Director, Metropolitan Transportation Commission, Oakland, California

Chris T. Hendrickson, Duquesne Light Professor of Engineering, Carnegie Mellon University, Pittsburgh, Pennsylvania

Jeffrey D. Holt, Managing Director, Bank of Montreal Capital Markets, and Chairman, Utah Transportation Commission, Huntsville, Utah

Gary P. LaGrange, President and CEO, Port of New Orleans, Louisiana

Michael P. Lewis, Director, Rhode Island Department of Transportation, Providence

Joan McDonald, Commissioner, New York State Department of Transportation, Albany

Abbas Mohaddes, President and CEO, Iteris, Inc., Santa Ana, California

Donald A. Osterberg, Senior Vice President, Safety and Security, Schneider National, Inc., Green Bay, Wisconsin

Steven W. Palmer, Vice President of Transportation, Lowe's Companies, Inc., Mooresville, North Carolina

Sandra Rosenbloom, Professor, University of Texas, Austin (Past Chair, 2012)

Henry G. (Gerry) Schwartz, Jr., Chairman (retired), Jacobs/Sverdrup Civil, Inc., St. Louis, Missouri

Kumares C. Sinha, Olson Distinguished Professor of Civil Engineering, Purdue University, West Lafayette, Indiana

Gary C. Thomas, President and Executive Director, Dallas Area Rapid Transit, Dallas, Texas

Paul Trombino III, Director, Iowa Department of Transportation, Ames

Phillip A. Washington, General Manager, Regional Transportation District, Denver, Colorado

\section{EX OFFICIO MEMBERS}

Thomas P. Bostick, (Lt. General, U.S. Army), Chief of Engineers and Commanding General, U.S. Army Corps of Engineers, Washington, D.C. Alison J. Conway, Assistant Professor, Department of Civil Engineering, City College of New York, New York, and Chair, TRB Young Members Council Anne S. Ferro, Administrator, Federal Motor Carrier Safety Administration, U.S. Department of Transportation

David J. Friedman, Acting Administrator, National Highway Traffic Safety Administration, U.S. Department of Transportation

LeRoy Gishi, Chief, Division of Transportation, Bureau of Indian Affairs, U.S. Department of the Interior, Washington, D.C.

John T. Gray II, Senior Vice President, Policy and Economics, Association of American Railroads, Washington, D.C.

Michael P. Huerta, Administrator, Federal Aviation Administration, U.S. Department of Transportation

Paul N. Jaenichen, Sr., Acting Administrator, Maritime Administration, U.S. Department of Transportation

Therese W. McMillan, Acting Administrator, Federal Transit Administration

Michael P. Melaniphy, President and CEO, American Public Transportation Association, Washington, D.C.

Gregory Nadeau, Acting Administrator, Federal Highway Administration, U.S. Department of Transportation

Cynthia L. Quarterman, Administrator, Pipeline and Hazardous Materials Safety Administration, U.S. Department of Transportation

Peter M. Rogoff, Under Secretary for Policy, U.S. Department of Transportation

Craig A. Rutland, U.S. Air Force Pavement Engineer, Air Force Civil Engineer Center, Tyndall Air Force Base, Florida

Joseph C. Szabo, Administrator, Federal Railroad Administration, U.S. Department of Transportation

Barry R. Wallerstein, Executive Officer, South Coast Air Quality Management District, Diamond Bar, California

Gregory D. Winfree, Assistant Secretary for Research and Technology, Office of the Secretary, U.S. Department of Transportation

Frederick G. (Bud) Wright, Executive Director, American Association of State Highway and Transportation Officials, Washington, D.C.

Paul F. Zukunft, Adm., U.S. Coast Guard, Commandant, U.S. Coast Guard, U.S. Department of Homeland Security.

\footnotetext{
* Membership as of July 2014 .
} 


\title{
Effectiveness of Different Approaches to Disseminating Traveler Information on Travel Time Reliability
}

\author{
Beverly Kuhn, Laura Higgins, Alicia Nelson, Melisa Finley, and Gerald Ullman \\ Texas A\&M Transportation Institute \\ College Station, Texas \\ SUSAN CHRYSLER \\ University of Iowa \\ Iowa City, Iowa \\ KarL Wunderlich and Vaishali ShaH \\ Noblis, Inc. \\ Washington, D.C. \\ ConRad Dudek \\ Dudek \& Associates \\ Bryan, Texas
}




\section{Subject Areas}

Highways

Operations and Traffic Management

Passenger Transportation

Public Transportation

Safety and Human Factors 


\section{The Second Strategic Highway Research Program}

America's highway system is critical to meeting the mobility and economic needs of local communities, regions, and the nation. Developments in research and technology_such as advanced materials, communications technology, new data collection technologies, and human factors science-offer a new opportunity to improve the safety and reliability of this important national resource. Breakthrough resolution of significant transportation problems, however, requires concentrated resources over a short time frame. Reflecting this need, the second Strategic Highway Research Program (SHRP 2) has an intense, large-scale focus, integrates multiple fields of research and technology, and is fundamentally different from the broad, mission-oriented, discipline-based research programs that have been the mainstay of the highway research industry for half a century.

The need for SHRP 2 was identified in TRB Special Report 260: Strategic Highway Research: Saving Lives, Reducing Congestion, Improving Quality of Life, published in 2001 and based on a study sponsored by Congress through the Transportation Equity Act for the 21st Century (TEA-21). SHRP 2, modeled after the first Strategic Highway Research Program, is a focused, timeconstrained, management-driven program designed to complement existing highway research programs. SHRP 2 focuses on applied research in four areas: Safety, to prevent or reduce the severity of highway crashes by understanding driver behavior; Renewal, to address the aging infrastructure through rapid design and construction methods that cause minimal disruptions and produce lasting facilities; Reliability, to reduce congestion through incident reduction, management, response, and mitigation; and Capacity, to integrate mobility, economic, environmental, and community needs in the planning and designing of new transportation capacity.

SHRP 2 was authorized in August 2005 as part of the Safe, Accountable, Flexible, Efficient Transportation Equity Act: A Legacy for Users (SAFETEA-LU). The program is managed by the Transportation Research Board (TRB) on behalf of the National Research Council (NRC). SHRP 2 is conducted under a memorandum of understanding among the American Association of State Highway and Transportation Officials (AASHTO), the Federal Highway Administration (FHWA), and the National Academy of Sciences, parent organization of TRB and NRC. The program provides for competitive, merit-based selection of research contractors; independent research project oversight; and dissemination of research results.
SHRP 2 Report S2-L14-RW-1

ISBN: 978-0-309-27305-3

(c) 2014 National Academy of Sciences. All rights reserved.

\section{Copyright Information}

Authors herein are responsible for the authenticity of their materials and for obtaining written permissions from publishers or persons who own the copyright to any previously published or copyrighted material used herein.

The second Strategic Highway Research Program grants permission to reproduce material in this publication for classroom and not-for-profit purposes. Permission is given with the understanding that none of the material will be used to imply TRB, AASHTO, or FHWA endorsement of a particular product, method, or practice. It is expected that those reproducing material in this document for educational and not-for-profit purposes will give appropriate acknowledgment of the source of any reprinted or reproduced material. For other uses of the material, request permission from SHRP 2.

Note: SHRP 2 report numbers convey the program, focus area, project number, and publication format. Report numbers ending in " $w$ " are published as web documents only.

\section{Notice}

The project that is the subject of this report was a part of the second Strategic Highway Research Program, conducted by the Transportation Research Board with the approval of the Governing Board of the National Research Council.

The members of the technical committee selected to monitor this project and review this report were chosen for their special competencies and with regard for appropriate balance. The report was reviewed by the technical committee and accepted for publication according to procedures established and overseen by the Transportation Research Board and approved by the Governing Board of the National Research Council.

The opinions and conclusions expressed or implied in this report are those of the researchers who performed the research and are not necessarily those of the Transportation Research Board, the National Research Council, or the program sponsors.

The Transportation Research Board of the National Academies, the National Research Council, and the sponsors of the second Strategic Highway Research Program do not endorse products or manufacturers. Trade or manufacturers' names appear herein solely because they are considered essential to the object of the report.

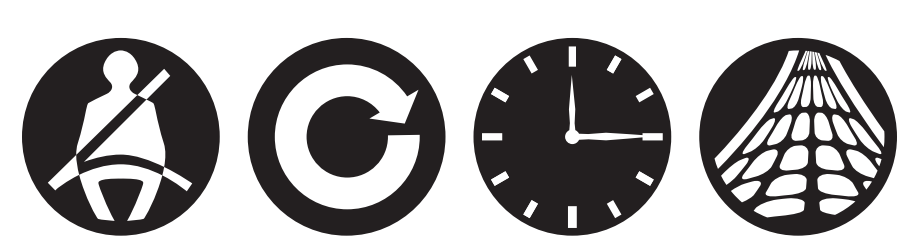

\section{SHRP 2 Reports}

Available by subscription and through the TRB online bookstore: www.TRB.org/bookstore

Contact the TRB Business Office: 202-334-3213

More information about SHRP 2: www.TRB.org/SHRP2 


\section{THE NATIONAL ACADEMIES Advisers to the Nation on Science, Engineering, and Medicine}

The National Academy of Sciences is a private, nonprofit, self-perpetuating society of distinguished scholars engaged in scientific and engineering research, dedicated to the furtherance of science and technology and to their use for the general welfare. On the authority of the charter granted to it by Congress in 1863, the Academy has a mandate that requires it to advise the federal government on scientific and technical matters. Dr. Ralph J. Cicerone is president of the National Academy of Sciences.

The National Academy of Engineering was established in 1964, under the charter of the National Academy of Sciences, as a parallel organization of outstanding engineers. It is autonomous in its administration and in the selection of its members, sharing with the National Academy of Sciences the responsibility for advising the federal government. The National Academy of Engineering also sponsors engineering programs aimed at meeting national needs, encourages education and research, and recognizes the superior achievements of engineers. Dr. C. D. (Dan) Mote, Jr., is president of the National Academy of Engineering.

The Institute of Medicine was established in 1970 by the National Academy of Sciences to secure the services of eminent members of appropriate professions in the examination of policy matters pertaining to the health of the public. The Institute acts under the responsibility given to the National Academy of Sciences by its congressional charter to be an adviser to the federal government and, on its own initiative, to identify issues of medical care, research, and education. Dr. Victor J. Dzau is president of the Institute of Medicine.

The National Research Council was organized by the National Academy of Sciences in 1916 to associate the broad community of science and technology with the Academy's purposes of furthering knowledge and advising the federal government. Functioning in accordance with general policies determined by the Academy, the Council has become the principal operating agency of both the National Academy of Sciences and the National Academy of Engineering in providing services to the government, the public, and the scientific and engineering communities. The Council is administered jointly by both Academies and the Institute of Medicine. Dr. Ralph J. Cicerone and Dr. C. D. (Dan) Mote, Jr., are chair and vice chair, respectively, of the National Research Council.

The Transportation Research Board is one of six major divisions of the National Research Council. The mission of the Transportation Research Board is to provide leadership in transportation innovation and progress through research and information exchange, conducted within a setting that is objective, interdisciplinary, and multimodal. The Board's varied activities annually engage about 7,000 engineers, scientists, and other transportation researchers and practitioners from the public and private sectors and academia, all of whom contribute their expertise in the public interest. The program is supported by state transportation departments, federal agencies including the component administrations of the U.S. Department of Transportation, and other organizations and individuals interested in the development of transportation. Www.TRB.org

wWw.national-academies.org 


\section{SHRP 2 STAFF}

Ann M. Brach, Director

Stephen J. Andrle, Deputy Director

Neil J. Pedersen, Deputy Director, Implementation and Communications

Cynthia Allen, Editor

Kenneth Campbell, Chief Program Officer, Safety

JoAnn Coleman, Senior Program Assistant, Capacity and Reliability

Eduardo Cusicanqui, Financial Officer

Richard Deering, Special Consultant, Safety Data Phase 1 Planning

Shantia Douglas, Senior Financial Assistant

Charles Fay, Senior Program Officer, Safety

Carol Ford, Senior Program Assistant, Renewal and Safety

Jo Allen Gause, Senior Program Officer, Capacity

James Hedlund, Special Consultant, Safety Coordination

Alyssa Hernandez, Reports Coordinator

Ralph Hessian, Special Consultant, Capacity and Reliability

Andy Horosko, Special Consultant, Safety Field Data Collection

William Hyman, Senior Program Officer, Reliability

Linda Mason, Communications Officer

Reena Mathews, Senior Program Officer, Capacity and Reliability

Matthew Miller, Program Officer, Capacity and Reliability

Michael Miller, Senior Program Assistant, Capacity and Reliability

David Plazak, Senior Program Officer, Capacity and Reliability

Rachel Taylor, Senior Editorial Assistant

Dean Trackman, Managing Editor

Connie Woldu, Administrative Coordinator

\section{ACKNOWLEDGMENTS}

This work was sponsored by the Federal Highway Administration in cooperation with the American Association of State Highway and Transportation Officials. It was conducted in the second Strategic Highway Research Program (SHRP 2), which is administered by the Transportation Research Board of the National Academies. The project was managed by Abdelmename Hedhli, Visiting Professional for SHRP 2 Reliability.

The research reported was performed by Texas A\&M Transportation Institute, supported by Noblis, Inc., and Dudek \& Associates. Beverly Kuhn, Texas A\&M Transportation Institute, was the principal investigator along with Susan Chrysler, University of Iowa, when she was with Texas A\&M Transportation Institute. The other authors of the report are Laura Higgins, Alicia Nelson, Melisa Finley, and Gerald Ullman of Texas A\&M Transportation Institute; Karl Wunderlich and Vaishali Shah of Noblis, Inc.,; and Conrad Dudek of Dudek \& Associates. The authors acknowledge the contributions to this research from Katie Connell, Sarah Hammond, Brenda Manak, Kristine Miller, Lisa Minjares, Lisa Patke, Roma Stevens, Sandra Stone, Luann Theiss, Nada Trout, Brooke Ullman, and Christine Yager of Texas A\&M Transportation Institute and Jim Larkin of Noblis, Inc. 


\section{F OR E W O R D}

Abdelmename Hedhli, SHRP 2 Visiting Professional, Reliability

Travel time reliability can be defined as consistency of travel time over time. The primary goal of SHRP 2 Reliability research is to improve the reliability of highway travel times by mitigating the effects of events that cause travel times to fluctuate unpredictably. Seven sources of unreliable travel times are now generally accepted: traffic incidents, work zones, demand fluctuations, special events, traffic control devices, weather, and inadequate base capacity.

A key component to addressing the reliability issue related to urban mobility is conveying this reliability-related information to system users so that they can make informed decisions about their travel. The goal of the SHRP 2 L14 project, Effectiveness of Different Approaches to Disseminating Traveler Information on Travel Time Reliability, is to examine what combination of words, numbers, and other features of user information messages, along with communications methods and technology platforms, best communicate information about travel time and reliability to travelers so they can make optimal travel choices from their point of view, such as whether to take a trip, departure time, mode choice, and route choice.

This final report documents the research conducted as part the SHRP 2 L14 project. One of the main work products from the L14 project is the Lexicon for Conveying Travel Time Reliability Information, which offers recommendations to system operators on appropriate ways to provide travel time reliability information to travelers so that the information is most likely to be understood and used by travelers to influence their travel choices. The research team identified several key elements of a lexicon entry that were deemed necessary to completely present each term and how it might be used within the transportation community. One purpose of this final report is to describe the process that led to the development of the lexicon.

The research began with a literature review to document existing practices and lessons learned regarding the communication of both travel and non-travel-related reliability information. The team then focused on conducting expert interviews and a technology and innovation scan to further examine the state of the practice in communicating information

to travelers. The team also conducted a series of human factors experiments, including focus groups and surveys, to assess travelers' comprehension and preferences for various reliability-related words and phrases.

Finally, two laboratory experiments developed a utility function for travel time reliability information by observing participants' use of reliability information during simulated commute trips and soliciting their opinions about the monetary value of that information. The lexicon was developed as the final task of this project. 


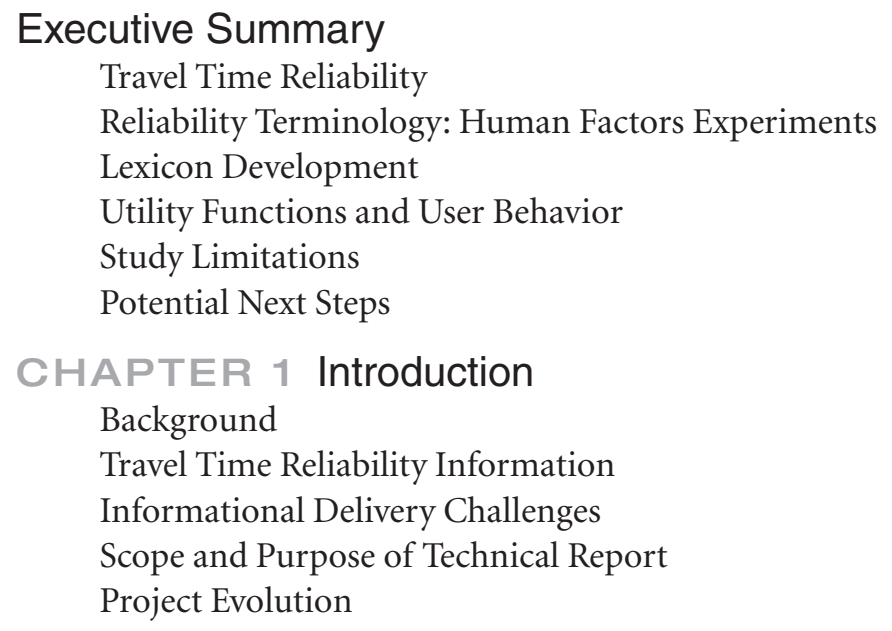

CHAPTER 4 Technology and Innovation Scan Introduction and Context Underlying Technology Trends: Better Data to Come Innovative Media for Traveler Information Users Traveler Information Market Participants Outlook for Traveler Reliability Information

\section{CHAPTER 6 Focus Groups}

Script Development

Participants

Results Summary

Lexicon Information 
49

49

49

50

71

71

73

83

84

85

86

86

87

90

97

102

103

105

105

106

111

114

125

127

130

132

132

133

137

138

138

146

146

147

147

148

151

155

156

167

172

182 Appendix D. Computer Survey Questions and Results Tables

CHAPTER 7 Usability Surveys

Study Locations

Study Participants

Computer Survey

\section{CHAPTER 8 Open-Ended Survey}

Research Method

Results

Conclusions

Recommendations

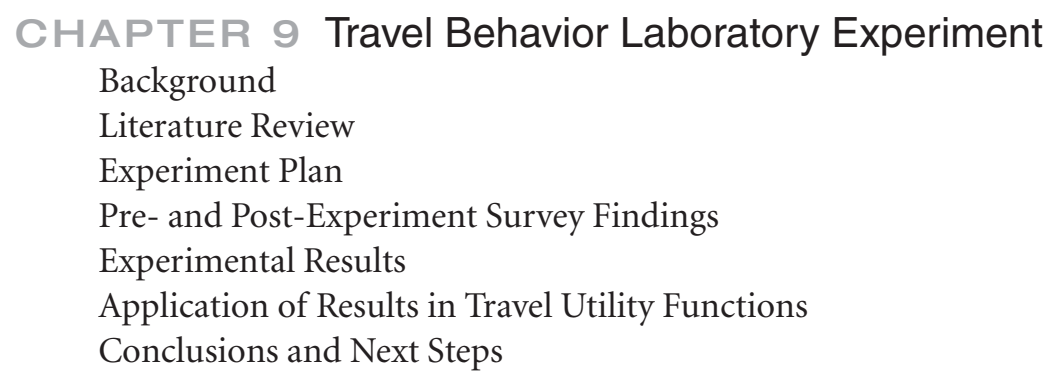

\section{CHAPTER 10 Enhanced Laboratory Experiment}

Experiment Scope and Hypotheses

Experimental Plan

Pre-Experiment Survey Findings

Experiment 1 Findings: Effectiveness and Valuation of Reliability Terms for Unfamiliar Trips

Experiment 2 Findings: Reliability Data Expedites Learning Curve for Trip Familiarity

Post-Experiment Survey Findings

Conclusions and Looking Forward

\section{CHAPTER 11 Lexicon Development}

Frequently Used Terms

Terminology Assessment

Lexicon Format

Limitations of Lexicon Information

Travel Time Reliability Lexicon

\section{CHAPTER 12 Final Remarks}

Study Limitations

Key Findings on Travel Time Reliability Terminology

Key Study Observations on User Behavior

Potential Next Steps

\section{References}

Glossary

Appendix A. General Literature Review

67 Appendix B. Telephone Interview Guide for Expert Interviews

72 Appendix C. Detailed Focus Group Summary 
211 Appendix E. Open-Ended Survey Materials

218 Appendix F. Travel Behavior Laboratory Experiment Documentation

228 Appendix G. Supplemental Figures from Travel Behavior Laboratory Experiment Pre-Survey

232 Appendix H. Enhanced Laboratory Experiment Documentation

255 Appendix I. Supplemental Figures from Enhanced Laboratory Experiment Results 


\section{Executive Summary}

The second Strategic Highway Research Program (SHRP 2) Reliability program aims to improve trip time reliability by reducing the frequency and effects of events that cause travel times to fluctuate unpredictably. Congestion caused by unreliable, or nonrecurring, events is roughly as extensive as congestion caused by routine bottlenecks (Cambridge Systematics, Inc. 2003). Nonrecurring events such as crashes, work zones, special events, and weather disrupt normal traffic flow by causing reduced speeds, lane closures, and erratic driving maneuvers. The goals of the SHRP 2 Reliability program focus on travel time variation-the characteristic of the transportation system that can cause the driver's current trip to take much longer than normally expected. For example, a driver must allow an hour to make a trip that normally takes 30 minutes. This transportation system characteristic is important for travelers and shippers and is a component of the congestion problem in which transportation agencies can make significant and measurable gains even as travel demand grows. Reducing delays related to reliability has the added benefit of reducing primary and secondary crashes, vehicle emissions, and fuel use, as well as other benefits.

Travel time reliability information includes cumulative data about traffic speeds and trip times that take into account historical variations from day to day and enable individuals to understand the level of variation in traffic. Unlike real-time travel time information, which provides a current snapshot of trip conditions and travel time, reliability information can be used to plan and budget in advance for a trip.

A key component in addressing the reliability issue related to urban mobility is conveying reliability-related information to system users so they can make informed decisions about their travel. The challenge for transportation professionals lies in selecting the best means of conveying that information so it is usable and effective. The goal of this research project was to examine what combination of words, numbers, and other features of user information messages, along with communications methods and technology platforms, best communicates information about travel time and reliability to travelers so that they can make optimal travel choices from their own point of view. Such choices include whether to take a trip, departure time, mode choice, and route choice.

\section{Travel Time Reliability}

Past research related to travel time reliability has, for the most part, examined how people use their own experience to judge travel time reliability with regard to route choice or time of departure. Researchers have not specifically examined when people prefer to have this information or how they use it. Many stated preference surveys allow users unlimited time to consider all the possibilities. In an unpressured situation, the message content and display are not as critical as 


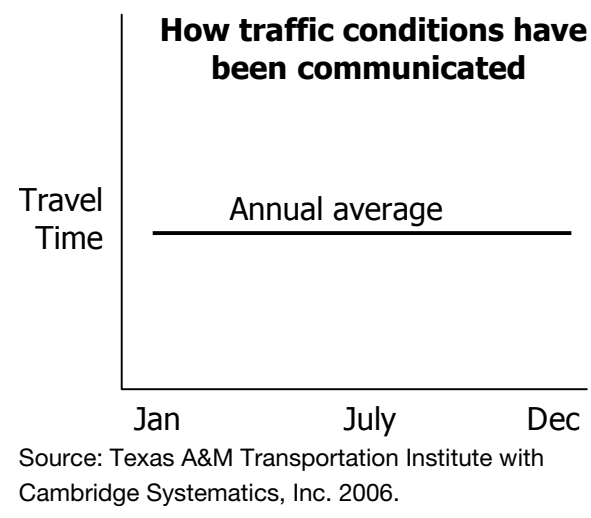

Figure ES.1. Average travel time used by professionals.

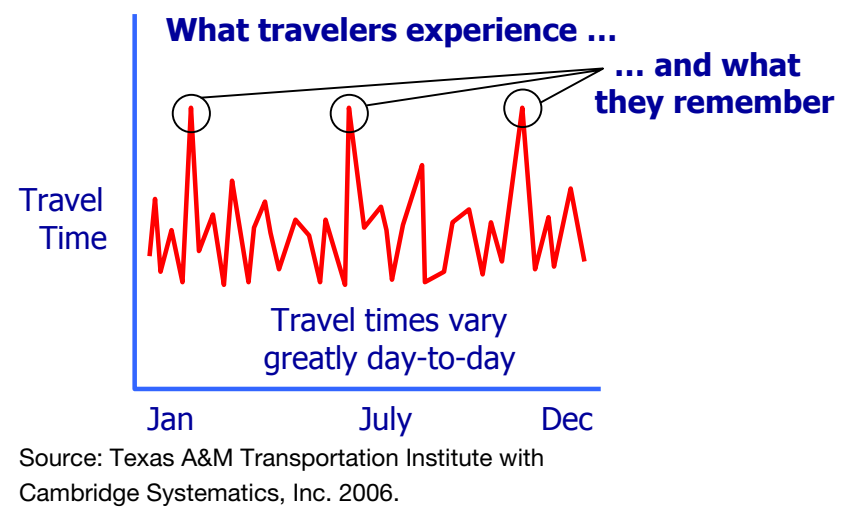

Figure ES.2. Travelers' travel time experiences.

they would be in a time-pressured situation immediately before departure or actually en route in the vehicle. Thus, message content and display-that is, the optimal display, sequence of inputs required, and display of search results-were the main focus of this project.

Transportation professionals most commonly discuss travel time reliability in terms of historical average travel times calculated over periods of a year or longer, as shown in Figure ES.1. A typical definition for travel time reliability is the following:

The consistency or dependability in travel times, as measured from day to day or across different times of the day.

However, most travelers do not experience the same average travel time each day. Travelers experience and remember something much different from the average throughout a year of commutes (Figure ES.2). Their travel times may vary greatly from day to day, and they remember the few bad days when they suffered through unexpectedly longer travel times. Research has shown that travel time reliability information can provide transportation system users with a more complete picture of the expected travel time along a particular route. The challenge is how to communicate that reliability information effectively to road and transit system users so that they understand it clearly.

To date, the primary travel time information conveyed to travelers, either pre-trip or en route, is real-time information. Real-time travel time messages have been in use in the United States for well over a decade, ever since traffic monitoring and integration systems became reliable. The most commonly used media for real-time messages are dynamic message signs (DMSs) and transportation agency websites. However, the widespread use of cell phones and other mobile devices is prompting a growing number of transportation agencies and providers to offer real-time updates on transportation conditions and options via e-mails, text messages, and Twitter feeds.

Real-time travel time estimates are most often provided for a particular roadway segment or a particular transit route on the basis of recent travel speeds or conditions. Some agencies also provide travel time comparisons among two or more routes or roadways to help travelers make decisions about the route or transportation mode to take. Most recent and most rare are the information sources that advise travelers about travel time reliability-the likelihood that the estimated travel time for a particular trip or trip segment will be dependable.

The concept of travel time reliability is complex, and travel time information may affect system users, system operators, and service providers in myriad ways. Several potential issues identified by the project team are addressed in detail in the following sections. These issues can be refined through additional investigation. 


\section{Reliability Terminology: Human Factors Experiments}

This project developed the Lexicon for Conveying Travel Time Reliability Informations to provide information on appropriate ways to introduce and provide travel time reliability information to travelers so that such information will most likely be understood and used by the travelers to influence their travel choices, while not presenting a safety hazard in the process. The lexicon was developed on the basis of an increasingly detailed series of human factors experiments and the development of a utility function, with input from a literature review, expert interviews, and a technology and innovation scan. All of those resources provided important information and insight into how individuals comprehend and interpret travel time reliability information, how they use that information to make trip decisions, and how reliability terms can be phrased to reach the highest percentage of travelers so their travel decisions yield some benefit to them.

\section{Focus Groups}

The first human factors study undertaken for this project was a series of focus groups in cities across the United States. Participants discussed recurring and nonrecurring conditions that affect travel times on roadways, a hypothetical traveler information system that would provide information on travel time reliability, and words and phrases that participants used and preferred to describe travel time and reliability concepts.

Focus group participants used words such as possibly, probably, chance, or likely to describe variability at a certain time of day. Generally, they preferred to precede those words with descriptors, such as "X\% chance" or "highly likely," to make the term more specific. When speaking about the additional time needed for a trip to ensure on-time arrival, participants' terminology varied greatly, and no real consensus emerged. For example, terms like additional time, traffic time, leeway, driving time, just-in-case time, fluff time, and additional drive time all came up as possible descriptors. Unprompted, participants used the words or phrases cushion, allow an additional $X$ minutes for variables, tack on extra, and extra time during discussion. Several terms specifically addressed reliability information, including reliability factors, plus or minus $10 \%$, and 9 out of 10 days. Terms that addressed a range of time were approximately, usually, window of time instead of exact time, give or take, variation, cushion, buffer, time frame that's certain, average, and depends.

\section{Surveys}

A computer-based survey tested participants' comprehension of and preference for terms used to present reliability information and related trip planning concepts. An open-ended survey was later added to the computer-based survey to further test user comprehension of selected terms for crucial reliability concepts such as average trip time, buffer time, and 95th percentile trip time.

The results of both surveys indicated a disconnect between the technical terms used by professionals and the layperson's understanding of and preference for those terms, a finding that was also evident in the focus group comments. Results related to some of the specific concepts and terms tested in the two surveys are as follows.

\section{Average Travel Time}

Only $18 \%$ of participants in the computer survey understood average to mean "about half the time (10 days out of 20)"; the largest group (about $38 \%$ of participants) interpreted average to 
mean "most of the time (15 days out of 20)." Other terms tested for this concept (typical, historical, estimated) were also more often interpreted as meaning "most of the time" than "about half the time." Of the four terms, estimated travel time was preferred by the highest number of participants; typical and historical were the least preferred.

The open-ended survey tested the terms average, estimated, typical, and expected for conveying a normal or "average" trip time. None of the four terms stood out as being significantly better or worse for conveying this travel time concept, and no clear trends in participant preference emerged.

On the basis of the results from the focus groups and the two surveys, the research team selected estimated travel time as the preferred term for conveying the "average travel time" concept, followed by average travel time and expected travel time.

\section{Buffer Time}

The large majority (90\%) of participants in the computer survey had a desire for information about whether a planned trip had a chance of taking longer than average. When asked to select a term to describe that additional time, only $10 \%$ selected buffer time, which is the term used by professionals in the travel time reliability field. The largest percentage $(33 \%)$ of participants preferred extra time to describe this concept.

The open-ended survey tested the terms added time, extra time, cushion time, and recommended cushion time for this concept. Of those terms, recommended cushion time was marginally more likely to encourage participants to calculate the total travel time that the researchers intended (adding a given buffer time to a given average trip time). The term extra time was somewhat more likely to result in a participant selecting a total trip time that was slightly longer than the researchers intended. Participants expressed no clear preference among the four terms.

Given the results of the focus groups and the two surveys, the research team selected extra time as the preferred term for conveying the buffer time concept, followed by added time, then recommended cushion.

\section{5th Percentile Travel Time}

Only $37 \%$ of participants in the computer survey understood 95 th percentile to mean "nearly all the time (19 days out of 20)."

The open-ended survey tested the terms 95th percentile travel time, majority of the time, most of the time, and travel time for planning. Of these terms, majority of the time encouraged the greatest percentage of participants to select a trip time that most closely matched a 95th percentile travel time. Most of the time encouraged participants to select a trip time slightly longer than the 95th percentile trip time, but participants who saw this term were also the most confident that they would arrive at their destination on time. Participants who saw the term 95th percentile travel time were the least confident about arriving on time.

Based on the focus groups and the two surveys, the research team selected majority of the time as the preferred term for this concept, followed by most of the time, travel time for planning, and 95th percentile trip time (the latter to be used only with an accompanying description).

\section{Lexicon Development}

The research team developed a structure for the lexicon that organized various data elements for each term in a way that could be applied to both reliability terms at a concept level and user interface phrases and terms. The data elements include a definition, the usage of the term, a ranking of messages or terms to be used in order of preference, alternate phrases, and information technology platforms. This structure also provides a convenient checkbox matrix indicating the 
platforms for which each variant of a term is appropriate. Chapter 11 of this report includes the lexicon—as Tables 11.3 through 11.10 - and contains recommendations for terms describing the concepts of 95th percentile trip time, arrival time, average travel time, buffer time, departure time, recommended departure time, recommended route, and reliability.

Terms recommended by the research team in the lexicon were used to convey travel time reliability concepts to participants in the second (enhanced) utility function laboratory study.

\section{Utility Functions and User Behavior}

Findings from the focus groups brought into question the relative value of reliability information that is specific to travelers. The most frequent potential use of travel time reliability (TTR) information seemed to be for pre-trip planning of constrained, unfamiliar trips within a known community or trips within or to an unfamiliar location. For the unconstrained trip scenarios, overwhelmingly, most people would not use it; for weekends, it would be used the least.

In general, focus group participants believed the TTR system would be valuable in some instances but would be immensely more valuable if it contained real-time data in addition to historical data. For example, they felt that a TTR system would be useful when planning a trip to an unfamiliar location given significant time constraints on one or both ends of the trip. However, planning such a trip on the basis of historical information would not be accurate unless the information was updated with current road and traffic conditions.

\section{First Utility Function Laboratory Study}

The objectives of the first utility function laboratory study were (a) to assess the value participants placed on traveler information, and specifically reliability information, within the context of a simulated time-constrained trip; and (b) to determine whether having reliability information helped speed the transition from an unfamiliar commuter to an experienced commuter.

The computer-based laboratory experiment was prepared by Noblis and administered by the Texas A\&M Transportation Institute (TTI) research team in concert with the computer and open-ended surveys. It presented participants with a scenario in which they were commuting in an unfamiliar city with a constrained arrival time (with penalties for both late and early arrival) for two work weeks, or 10 commutes to work. Participants had access to one of three additive levels of travel time information for their planned route and optional route deviations: real-time DMS messages only, real-time DMS plus real-time travel time information, or realtime DMS plus both real-time and historical travel time information. Participants selected from three departure time options and two route modification points to arrive at their destination on time.

The results of this initial laboratory experiment showed that participants who received reliability information arrived on time more frequently than those who received real-time information only. Results concerning participants' perceived value of this information were inconclusive.

\section{Enhanced Utility Function Laboratory Study}

After careful assessment of the aforementioned travel time reliability terms and the results obtained in the various human factors studies and experiments conducted throughout the course of the L14 project, the research team established three key hypotheses related to the use and value of travel time reliability information from the user's perspective. Those hypotheses were tested in the second, enhanced laboratory study.

Two slightly different experiments were developed for the enhanced laboratory study. In both experiments, the reliability information presented to participants did not vary from day 
to day but instead reflected the historical reliability of travel along a route. In addition to differing levels of reliability information, all participants received pre-trip and en route advisory messages via simulated dynamic message signs as well as text-format "radio messages." In both experiments, participants selected a departure time on the basis of pre-trip information, experienced travel time as a function of trip duration, and rated the value of the information simulated from day to day.

Experiment 1 framed the simulation as that of traveling for work to five different cities and commuting for five weekdays from each city's regional headquarters to a morning client meeting that was $40 \mathrm{~min}$ to $60 \mathrm{~min}$ from headquarters. Each participant experienced four of the seven reliability-related data, a specific term each for a week, and during one week there was no reliability-related data.

Experiment 2 framed the simulation as that of traveling to a new city for work and making daily departure time decisions to work for a month ( 4 weeks, 5 days a week). In this version, half of the participants received graphical 20th, average, and 95th percentile arrival time reliability information with a legend identifying data as "good day," "typical day," and "bad day." The other half of the participants did not receive reliability information.

Pre- and post-trip surveys during the commute simulation programs and surveys following the completion of the experiments measured participants' evaluations of the usefulness of the traveler information they received, their confidence in selecting trip times on the basis of that information, their level of stress in completing their trips, and the monetary value they would place on the information.

\section{Hypothesis 1}

Hypothesis 1 states that the provision of accurate reliability information (in an easy-tounderstand format) will result in improved on-time performance and lower generalized travel disutility compared with a control group receiving no reliability information. The results of the first laboratory study and the later enhanced laboratory study strongly supported this hypothesis. Of the seven different forms of delivery of reliability information tested in the experiment, users presented with five of the options demonstrated statistically significant reductions in weekly schedule offset costs compared with the control group receiving no reliability information. Those five options were also the simplest of the forms of reliability information, focusing on average and 95th percentile travel time values, delivered in various forms. Participants receiving those simple forms of reliability information reduced schedule offset costs by $9 \%$ to $21 \%$ compared with the control group.

\section{Hypothesis 2a (First Laboratory Study)}

Hypothesis $2 \mathrm{a}$, tested in the first laboratory study, proposes that while travel outcomes will improve with the provision of reliability information, the perceived value of the reliability information will underestimate the realized benefit in terms of reduced delay, improved on-time reliability, and reduced stress.

Findings from the first laboratory study supported this hypothesis. Measured perceptions of benefit associated with reliability information were not commensurate with the observed improvements in trip outcomes. Changes in the value of information and stress reduction, although generally favorable to reliability information, were not statistically significant.

\section{Hypothesis 2b (Enhanced Laboratory Study)}

Hypothesis $2 b$, tested in the enhanced laboratory study, states that experimental subjects receiving contextual information on underlying variation with numeric indicators reinforced with en route information (travel time reliability signposting) will have improved on-time performance 
compared with both an experimental group that receives reliability information but no contextual information and a control group that receives no reliability information.

Hypothesis $2 \mathrm{~b}$ was not supported by the enhanced laboratory study. The signposting concept was not successful for participants in managing trip outcomes and stress reduction. To some degree, this result was due to the complexity of the presentation. Signposting may still be a valuable concept to pursue for providing reliability information, but work remains to convey the concept in a more accessible manner.

\section{Hypothesis 3}

Hypothesis 3 states that the benefits of travel time reliability information will decline over time as both experimental and control subjects learn and understand the underlying travel time variability. That is, the benefit from reliability information during the first weeks will be larger than during the last weeks.

Findings from the first laboratory study were inconclusive. Participants with access to reliability information did see total early- and late-arrival penalties decline from week 1 to week 2 of the simulated trip-making. Control group participants who did not receive this information were mixed, with one group realizing a reduction in total costs and another seeing a rise in costs. Interestingly, participants' willingness to pay for reliability information declined over time in the simulation experiment, indicating that experience within the simulated trip began to offset reliance on the reliability information provided.

The enhanced laboratory study supported hypothesis 3. Participants using travel time reliability information were as effective in managing trip outcomes (late arrivals, schedule delays, and offset costs) in the first week of exposure to unfamiliar travel time variability patterns as their counterparts without reliability information were after 4 weeks of experience in an unfamiliar system.

\section{Study Limitations}

Note that the studies conducted in this project were laboratory studies, and none of the travel time reliability terms was tested in the field. Only in a field test with specific, detailed, travel behavior data can researchers determine the true effects and benefits of the use of travel time reliability information on behavior and resulting trip performance. Also note that nowhere in the various human factors studies were the specific phrases suggested for display on DMSs tested specifically as being displayed on a DMS and as en route information. The suggested phrases were developed by the research team on the basis of the results discussed for the related terminologies. The team developed the phrases using the general guidance for DMS message development provided in the Manual on Uniform Traffic Control Devices (MUTCD). Note that the formatting of these travel time messages is very different from the standard messages state transportation agencies use on DMSs.

\section{Potential Next Steps}

The concept of travel time reliability is complex, and travel time information may affect system users, system operators, and service providers in myriad ways. The project team identified several potential issues that can be addressed in further detail and refined through additional investigation. These issues include the following:

- Travelers' perceived value of reliability information in the context of more complex trip planning (e.g., a wider range of route and/or mode options);

- Mechanisms and reasons behind the under-valuation of reliability information by users; 
- Changing effects and value of reliability information for travelers on familiar routes;

- Effects of reliability information on travel-related choices such as home and work locations, facility locations for businesses, and others;

- Use of reliability information by the freight industry;

- Effects of reliability information in public transit;

- Traveler responses to increased travel time reliability;

- Further examination of graphical formats for reliability information; and

- Field tests of reliability terminology. 


\section{CHAPTER 1 \\ Introduction}

The second Strategic Highway Research Program (SHRP 2) Reliability program aims to improve trip time reliability by reducing the frequency and effects of events that cause travel times to fluctuate unpredictably. Congestion caused by unreliable, or nonrecurring, events is roughly as extensive as congestion caused by routine bottlenecks (Cambridge Systematics, Inc. 2003). Nonrecurring events such as crashes, work zones, special events, and weather disrupt normal traffic flow by causing reduced speeds, lane closures, and erratic driving maneuvers. The goals of the SHRP 2 Reliability program focus on travel time variation - the characteristic of the transportation system which means the driver's current trip will take much longer than normally expected (see Figure 1.1). For example, a driver must allow an hour to make a trip that normally takes 30 minutes. This transportation system characteristic is important for travelers and shippers and is a component of the congestion problem in which transportation agencies can make significant and measurable gains even as travel demand grows. Reducing delays related to reliability has the added benefit of reducing primary and secondary crashes, vehicle emissions, and fuel use, and yields other benefits.

\section{Background}

The highway system in the United States is a critical component of American life. The Federal Highway Administration (FHWA) works to ensure that the system provides extensive and flexible personal mobility to American citizens and efficient freight movement to support the domestic economy (FHWA 2003). Both personal mobility and efficient freight movement are affected by transportation investment and location decisions made by governmental entities across the country in their planning processes. However, an increase in travel by users, congestion, and environmental and financial constraints interfere with the system's ability to provide these services. For example, the growth in vehicle miles traveled (VMT) continues to outpace lane mile growth across the country. Between 1993 and 2000, VMT increased by $2.7 \%$ annually, while the number of lane miles in the United States grew by only $0.2 \%$ annually during the same time period (FHWA 2003). This growth in travel strains a transportation system that is already overburdened.

Congestion interferes with daily life, so any method to alleviate it and improve trip reliability can help reduce its effect on productivity. Another reality facing transportation agencies today is the need to function within environmental constraints. Agencies must consider the environment in the planning of transportation projects, minimize the negative effects of construction, and work to reduce transportation-related pollution in the process. They must demonstrate environmental stewardship and improve the environmental quality of their transportation decision making (FHWA 2002).

Financial constraints are another burden on transportation agencies. As public resources become more and more scarce, state and local governments are challenged to meet growing transportation needs with inadequate funding. An emerging trend in transportation spending is the reality that state and local governments are devoting a larger share of their capital spending to preserving and operating their existing transportation infrastructure, thereby leaving less money available for new roads and bridges and system enhancements (FHWA 2002). Despite these constraints, every effort to better manage the transportation infrastructure helps address the goals of the SHRP 2 Reliability program to enhance the quality of life for all system users by improving the reliability of the daily trip.

\section{Travel Time Reliability Information}

Travel time reliability information includes cumulative data about traffic speeds and trip times that take into account historical variations from day to day and enable individuals to understand the level of variation in traffic. 


\begin{tabular}{|l|}
\hline \multicolumn{2}{|c|}{ The Seven Factors } \\
\hline Incidents \\
\hline Weather \\
\hline Work Zones \\
\hline Fluctuation in Demand \\
\hline Special Events \\
\hline Traffic Control Devices \\
\hline Inadequate Base Capacity \\
\hline
\end{tabular}

Source: SHRP 2 Reliability program.

\section{Figure 1.1. Seven factors contributing to non- recurring congestion.}

Unlike real-time travel time information, which provides a current snapshot of trip conditions and travel time, reliability information can be used to plan and budget in advance for a trip.

A key component to addressing the reliability issue related to urban mobility is conveying reliability-related information to system users so they can make informed decisions about their travel. The challenge for transportation professionals lies in selecting the best means of conveying that information so it is usable and effective. The goal of this research project was to examine what combination of words, numbers, and other features of user information messages, along with communications methods and technology platforms, best communicates information about travel time and reliability to travelers so that they can make optimal travel choices from their own point of view. Such choices include whether or not to take a trip, departure time, mode choice, and route choice.

For the most part, past research related to travel time reliability has examined how people use their own experience to judge travel time reliability with regard to route choice or time of departure. Researchers have not specifically examined when people prefer to have this information or how they use it. Many stated preference surveys allow users unlimited time to think about all of the possibilities. In an unpressured situation, the message content and display are not as critical as they would be in a time-pressured situation immediately before departure or actually en route in the vehicle. Thus, message content and display-that is, the optimal display, sequence of inputs required, and display of search resultswere the main focus of this project. Logically, the trip-making process includes three points at which users would want to access travel time reliability information: trip planning for habitual trips when new to an area; pre-trip planning immediately before departure; and planning en route before a route or mode choice point. These points are discussed in the following sections.

\section{Trip Planning for Habitual Trips When New to an Area}

When people move to a new area or start a new job, they must find the best mode, time of departure, and route for their commute. This can be accomplished by talking with neighbors and colleagues, trying different times if their work schedule allows, and trying different routes. Once the decision is made, the trip becomes routine. Users may find travel time reliability information helpful at this point to make direct comparisons across modes, routes, and times. System users would most likely seek out this information through an Internet source outside the vehicle under no particular time pressure. The user may desire maps and tables as outputs and wish to input two distinct scenarios and compare the results. In essence, the user would use the travel time reliability information in a series of what-if scenarios and weigh the potential travel time savings against the volatility in that travel time. This type of use may require a rich user interface with many input options, including specific origin-destination pairs. An analogous situation would be using a service like MapQuest to get door-to-door driving directions with specific addresses.

\section{Pre-Trip Planning Immediately Before Departure}

Many users may want to check traffic or check how transit is running just before departure. They may do this by visiting a traffic management center (TMC) website, consulting a smartphone or navigation system that includes real-time traffic information (e.g., a global positioning system, or GPS), or listening to a traffic advisory radio broadcast. This information is typically sought immediately before beginning the trip (i.e., not while driving). Users may be able to delay their departure, choose a known alternate route, or choose to take the bus rather than the train. For these purposes, users may want a subscription system in which they have entered their origin-destination or typical route information once, and the system is able to show them the travel time information specifically for their route. The display can be simple text or a color-coded travel time system map common on many TMC websites. These users are not necessarily looking to find the best route. More likely, they want to change their mode or departure time to avoid congested conditions and incidents.

\section{Planning En Route Before a Route or Mode Choice Point}

Some users may use these same sources-TMC website, smartphone, GPS, or radio report-to seek information en route before a major interchange or key decision point along their route. Because travel time reliability shifts throughout the day and with incidents, users may want to know reliability associated with current conditions. For instance, route A may 
be the shortest mileage and trip time under level of service A; but when conditions deteriorate because of traffic volume or an incident, reliability suffers and route B (though longer in distance) has a more reliable trip time. For these users, en route information becomes useful because people cannot remember a whole set of values - such as when travel time is $20 \mathrm{~min}$ on route $\mathrm{A}$, the variability is $\pm 5 \mathrm{~min}$; but when travel time is $40 \mathrm{~min}$, the variability is $\pm 15 \mathrm{~min}$. Likewise, with mode shifts, users may use travel time reliability information to prompt the decision to divert to a park-and-ride lot and take transit. These users may not want to risk being caught in traffic for a long period of time and prefer to ride the bus or train during congested conditions.

\section{Informational Delivery Challenges}

Travel time reliability information delivered en route must take a different form because of the risks of distracted driving. Displays that have been designed and tested with users sitting in front of a computer screen with their full attention devoted to the task will not fare well in a moving vehicle. The safety concerns of requiring long eyes-off-the-road glances to displays are considerable. Although designing in-vehicle and portable device displays was beyond the scope of this project, determining the key elements that should be present was part of the scope. This information can be used by automotive suppliers, and smartphone manufacturers can include this information in systems that are already used to display travel time in-vehicle. Some systems use auditory messages as another way of presenting this information in-vehicle.

Users' diverse needs for reliability information, the times at which users may want that information, and the broad range of communications media and information formats already in the marketplace and on the horizon present a challenge for the transportation profession set on conveying travel time reliability. Consequently, this project sought to answer the critical questions of what, when, and how to deliver travel time reliability information.

\section{Scope and Purpose of Technical Report}

This report documents the research activities undertaken throughout the duration of the SHRP 2 L14 research project. The key product of the research is a lexicon that was developed to provide information on appropriate ways to introduce travel time reliability information to travelers. This information should be able to be understood and used by travelers to influence their travel choices and not present any safety hazards in the process. This report provides detailed results of all of the tasks included in the study, as illustrated in Figure 1.2.

\section{Project Evolution}

Travel time reliability information includes static data about traffic speeds or trip times that capture historical variations from day to day and enable individuals to understand the level of variation in traffic. Unlike real-time travel time information, which provides a current snapshot of trip conditions and travel time, reliability information can be used to plan and budget in advance for a trip. The research undertaken as a part of the L14 project was the first to examine road user understanding of travel time reliability information in the United States. The approach was to apply a series of successively more focused

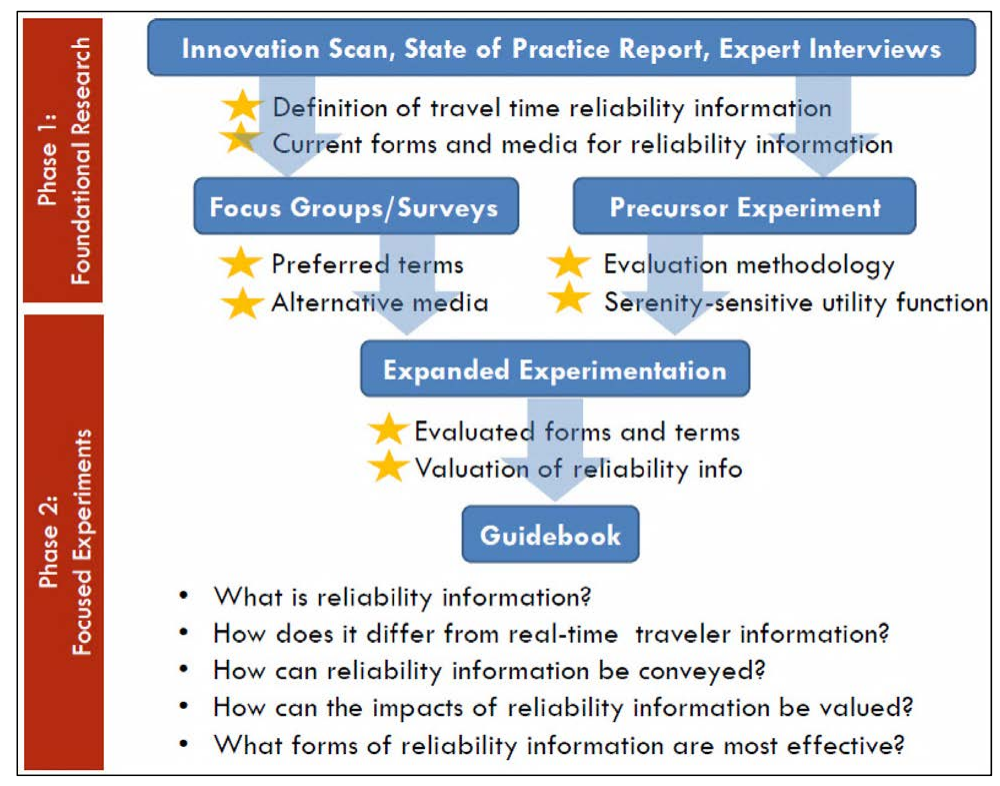

Figure 1.2. SHRP 2 L14 research project activities. 
methodologies, thus allowing researchers to cast a wide net initially and gather the broadest understanding of users' preconceived notions of travel time reliability. Developing and tailoring traveler information systems on the basis of users' inherent understanding of trip reliability provides the best chance for a high level of user acceptance of the ultimate traveler information system.
The results of the three Phase 1 and five Phase 2 research activities contributed to the travel time reliability lexicon (Task 13) and guidebook and deployment advisory (Task 14) which are the primary products of this research project. These activities also informed the plans for the development of utility functions (Task 12). The overall project concept is illustrated in Figure 1.3.

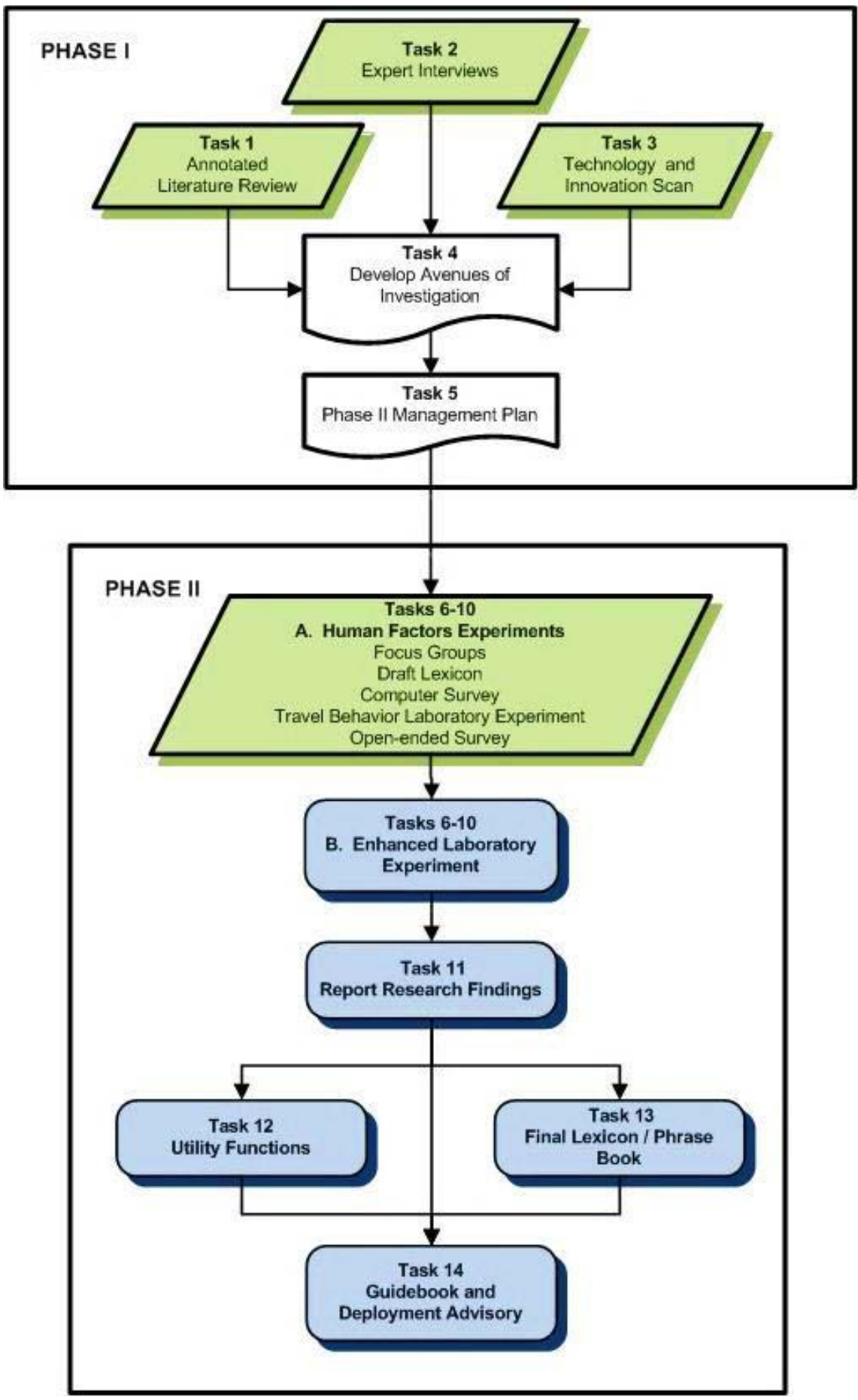

Figure 1.3. SHRP 2 L14 project overview. 
A few shifts in focus emerged after the proposal stage as a result of Phase 1, so some of the specific activities undertaken in Phase 2 were modified. Those modifications were reflected in the Phase 2 Management Plan and are summarized as follows:

- Increase focus on market demand for information. In the original proposal, the research team hypothesized that travelers would intrinsically value reliability information for trip planning purposes. During the Phase 1 tasks, many people - both transportation officials and general usersdid not show particular interest in providing or receiving this information. Phase 2 sought to determine more definitively if a market for this type of information exists, what value users place on it, and how and when it might be used.

- Eliminate the field operational test. In the proposal, the team planned a field operational test of the lexicon to gather real-world information on how travelers used the information conveyed and what were the results for their travel. Initially, the team considered deploying the test using smartphones or some other in-vehicle device. With the United States Department of Transportation's (U.S. DOT's) increasing emphasis on distracted driving, this approach was no longer prudent. Instead, the team proposed a web-based system, intended to be accessed from a home computer exclusively. Participants would be recruited on the basis of access to a personal computer and would be instructed not to access the system from a portable mobile device. After further consideration of the challenges associated with conducting a field operational test and the limited control that the project team would have over the design of the web-based system, the team proposed eliminating the field operational test. The team and the SHRP 2 L14 technical expert task group (TETG) agreed that the resources dedicated to the field operational test would be better used on other activities.

- Eliminate simulator testing. Because the project team decided to focus on a personal computer-based system as the testing platform, that eliminated need to test the system while driving. Therefore, the simulator study was also eliminated from the research plan. With the elimination of both the driving simulator study and the field operational test, the research team was able to use project resources to add two additional human factors activities; thus, the larger usability study also included an open-ended survey and an initial travel behavior laboratory experiment and was followed by an enhanced travel behavior laboratory experiment.

The project team developed a diagram to summarize all of the research activities to make clear how the activities interrelated and contributed to one another (see Figure 1.4). The remaining chapters in this report provide detailed information on all of the project tasks and the specific results that were used to generate the final products. 


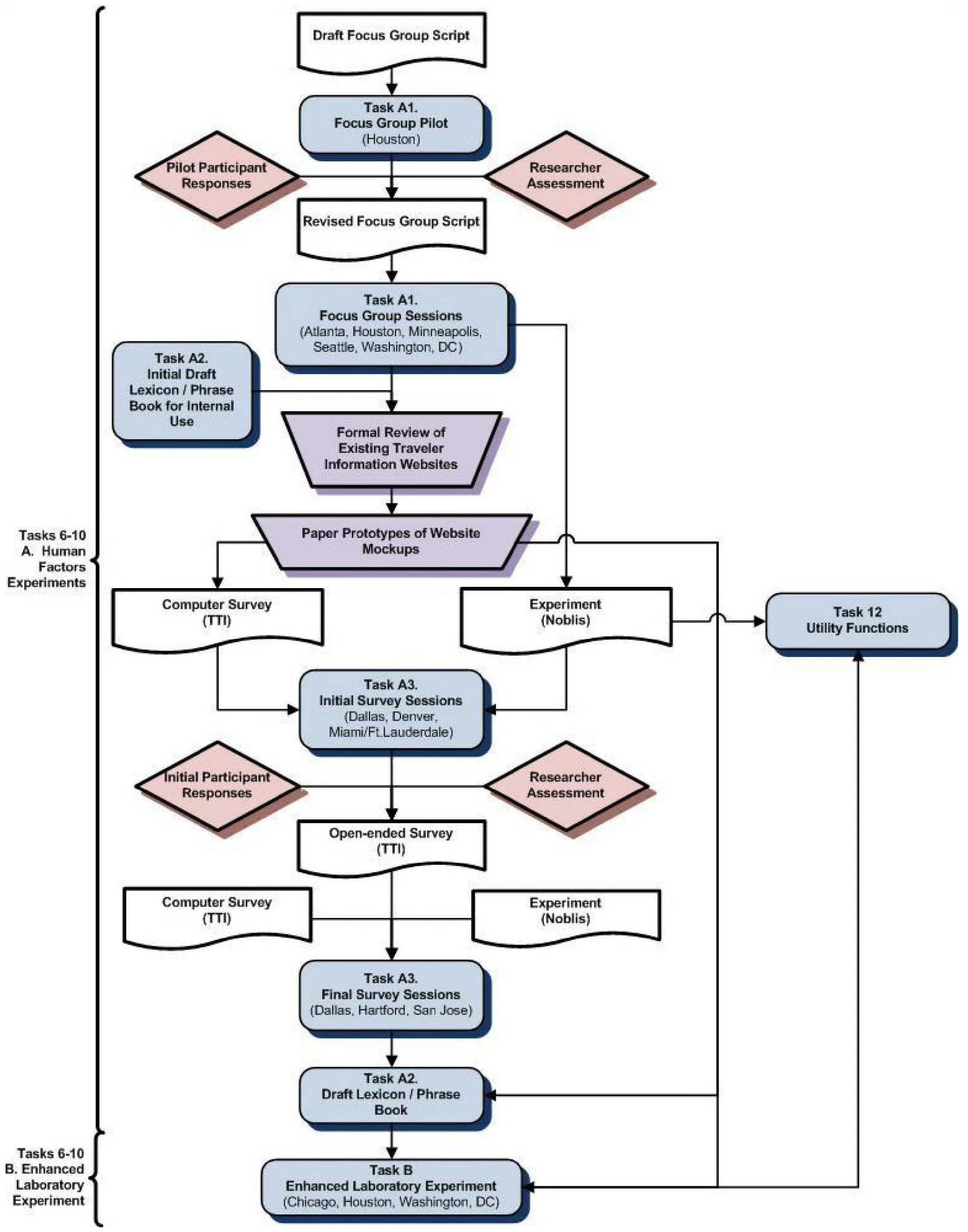

Figure 1.4. SHRP 2 L14 project task interrelationships. 


\section{CHAPTER 2 \\ Literature Review}

In the many cities where congestion on the transportation system is commonplace, drivers are accustomed to congestion and expect and plan for some increase in travel time, particularly during peak driving times. Many system users either adjust their schedules to avoid peak hours or budget extra time to allow for unexpected traffic congestion or incidents. However, problems arise when travel times are much higher than anticipated. Most travelers are less tolerant of unexpected travel time increases because those longer travel times cause travelers to be late for work or important meetings, to miss appointments, or to incur extra child-care fees. Moreover, shippers that face unexpected delays may lose money, experience disruptions in just-in-time delivery and manufacturing processes, and lose their competitive edge (Texas A\&M Transportation Institute with Cambridge Systematics, Inc. 2006).

Transportation professionals most commonly discuss travel time reliability in terms of historical average travel times calculated over periods of a year or longer, as illustrated in Figure 2.1. A typical definition for travel time reliability is the following:

The consistency or dependability in travel times, as measured from day to day or across different times of the day.

However, most travelers do not experience the same average travel time each day. As shown in Figure 2.2, travelers experience and remember something much different than the average throughout a year of commutes. Their travel times vary greatly from day to day, and they remember the few bad days they suffered through unexpectedly longer travel times. Research within the profession has shown that travel time reliability information can provide transportation system users with a more complete picture of the expected travel time along a particular route. The challenge is how to communicate that reliability information effectively to road and transit system users so that they understand it clearly.
Another example illustrating travel time reliability is shown in Figure 2.3, which presents travel time data from a major commuter route in Seattle, Washington. Without congestion along the route, travel times are about 12 min (e.g., see President's Day in the figure). On all other weekdays, the average travel time is $18 \mathrm{~min}$. However, when traffic incidents and weather combine to cause unexpected congestion, travel times may be $25 \mathrm{~min}$ or more, or 39\%, longer than usual. Commuters who travel this route must plan for this variability if they want to arrive on time. If they plan their commute on the basis of the average travel time, they will be late half the time and early the other half of the time. In other words, commuters have to build in a time cushion, or buffer, to their trip planning to account for the variability. If they build in a buffer, they will arrive early some days. That is not necessarily a bad thing, but the extra time is carved out of their day-time they could be using on pursuits other than commuting.

\section{Travel Time Reliability Metrics}

The measurement of travel time reliability is an emerging practice. However, a few measures appear to have technical merit and are thought to be easily understood by nontechni$\mathrm{cal}$ audiences. Most of these measures compare high travel time days with average travel time days. Four recommended measures are as follows (Texas A\&M Transportation Institute with Cambridge Systematics, Inc. 2006):

- 90th or 95th percentile travel time;

- Buffer index;

- Planning time index; and

- Frequency the congestion exceeds some expected threshold.

The 90th or 95th percentile travel time is a time identified for a specific travel route that indicates how long the delay will be on the heaviest travel days (Texas A\&M Transportation Institute with Cambridge Systematics, Inc. 2006). These travel 


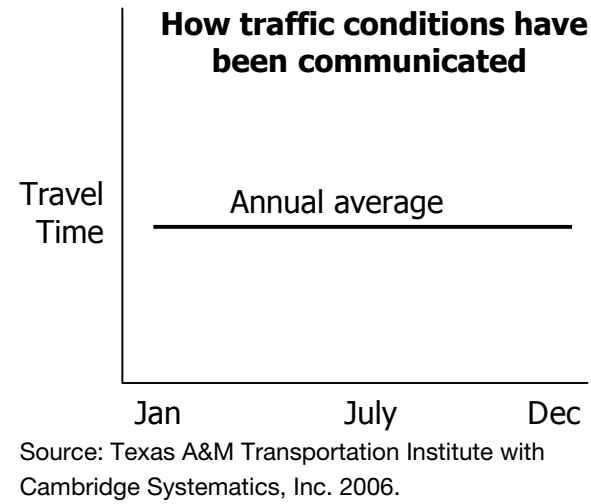

Figure 2.1. Average travel time used by professionals.

times are reported in minutes and seconds and are thought to be easily understood by commuters familiar with their trips. Therefore, this measure is ideally suited for traveler information. It has the disadvantage of not being easily compared across trips, because most trips have different lengths. Nor can this measure be used to easily combine route or trip travel times into a subarea or citywide average. Other reliability indices presented below do enable comparisons or combinations of routes or trips with different lengths.

The buffer index represents the extra time cushion (or buffer) that most travelers add to their average travel time when planning trips to account for unforeseen delays and to ensure

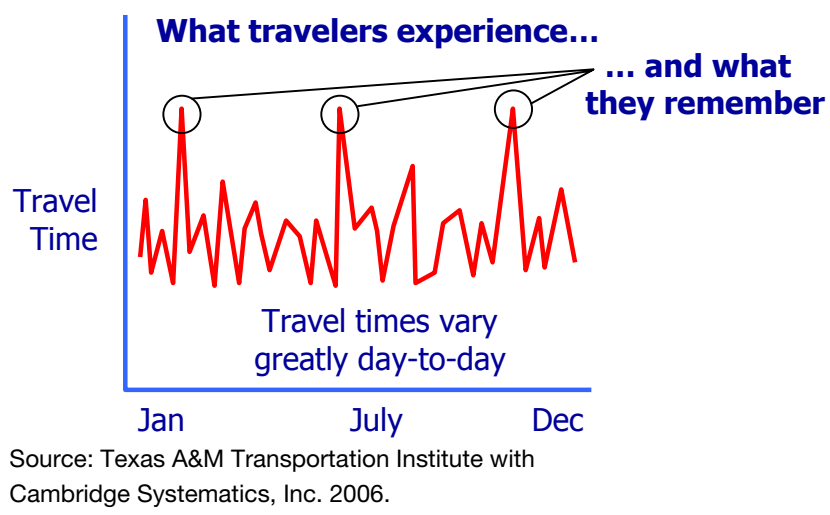

Figure 2.2. Traveler travel time experiences.

on-time arrival (Texas A\&M Transportation Institute with Cambridge Systematics, Inc. 2006). The buffer index is expressed as a percentage, and its value increases as reliability worsens. For example, a buffer index of $40 \%$ means that for a 20 -min average travel time, a traveler should budget an additional $8 \mathrm{~min}$ ( $20 \mathrm{~min} \times 40 \%=8 \mathrm{~min})$ to ensure on-time arrival most of the time. In this example, the eight extra minutes is called the buffer time. The buffer index is computed as the difference between the 95th percentile travel time and average travel time, divided by the average travel time.

The planning time index represents the total travel time that a traveler should expect or plan on when an adequate

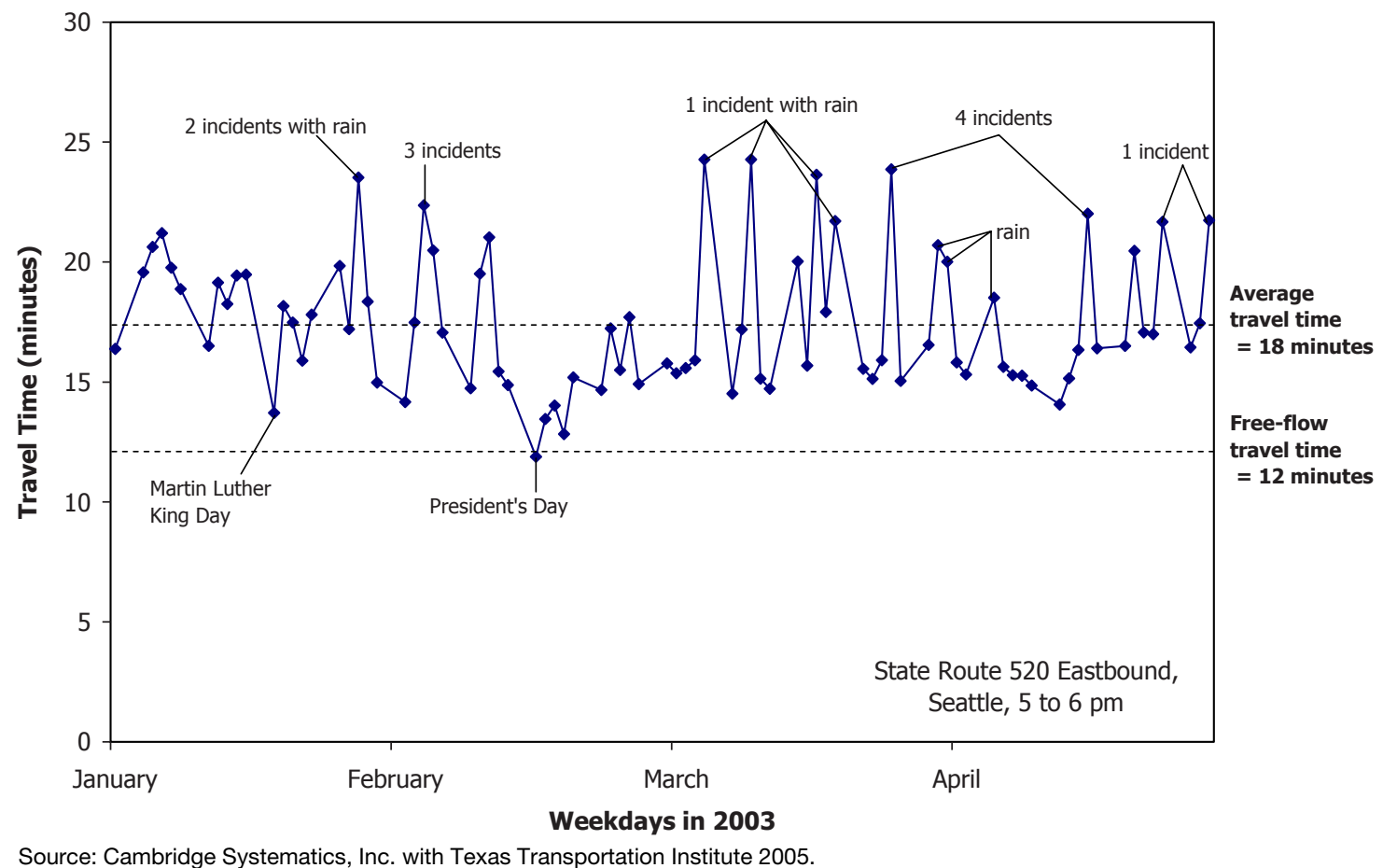

Figure 2.3. Example of commuters planned trips based on the worst days, not an average day. 
buffer time is included (Texas A\&M Transportation Institute with Cambridge Systematics, Inc. 2006). The planning time index differs from the buffer index in that it includes typical delay as well as unexpected delay. Thus, the planning time index compares near-worst-case travel time with a travel time in light or free-flow traffic. For example, a planning time index of 1.60 means that for a 15-min trip in light traffic, the total time that should be planned for the trip is $24 \mathrm{~min}$ ( $15 \mathrm{~min} \times$ $1.60=24 \mathrm{~min}$ ). The planning time index is useful because it can be directly compared with the travel time index (a measure of average congestion) on similar numeric scales. The planning time index is computed as the 95th percentile travel time divided by the free-flow travel time.

From a data perspective, continuous travel time data are the only way to establish reliability patterns empirically. Although predictive methods - such as the ones developed by the project team for the SHRP 2 L03 final report, Analytic Procedures for Determining the Impacts of Reliability Mitigation Strategiesmay be used in a reliability monitoring system when the data are unavailable, only continuously collected travel time data can produce the actual travel time distribution from which all reliability metrics are derived. For example, the reliability metrics being used in the SHRP 2 L03 project, as shown in Table 2.1, are all derivatives of the travel time distribution.

At present, agreement is lacking within the professional field on the terms to be used and what the mathematical calculations of each term should be. If the professionals cannot reach consensus on the technical terms, then the general public certainly will not do so. The purpose of the L14 project was to discover what terms the layperson would use to refer to travel time reliability concepts and to encourage the use of those terms in communications with transportation system users.

\section{Importance of Travel Time Reliability}

Travel time reliability is significant to many transportation system users, whether they are vehicle drivers, transit riders, freight shippers, or even air travelers. Good and consistent system reliability is a valuable service that can be provided on privately operated and publicly operated highways alike. Because reliability is so important for transportation system users, transportation planners, operators, and decision makers should consider travel time reliability a key performance measure.

\section{Travel Time Reliability and Highway Travel}

Travel time reliability is valuable to traffic professionals because it better quantifies the benefits of traffic management and operation activities than simple averages. For example, consider a typical before-and-after study that attempts to quantify the benefits of an incident management or ramp metering program. The improvement in average travel time may appear modest, as shown on the left side of Figure 2.4. However, reliability measures will show a much greater improvement-as illustrated on the right side of Figure 2.4-because they show the effect of improving the worst few days of unexpected delay.

For drivers, travel time reliability information can be valuable when they are selecting a route. For example, the value of travel time reliability was assessed through a mail survey, trip diaries, and loop-detector data by Lam and Small (2001) soon after the first high-occupancy/toll (HOT) lane opened on State Route 91 in Riverside, California. The researchers found that, for women in this study, the value of travel time reliability was actually higher than simple travel time information. For men, the value of travel time was roughly $50 \%$

Table 2.1. Recommended Reliability Performance Metrics from the SHRP 2 L03 Report, Analytic Procedures for Determining the Impacts of Reliability Mitigation Strategies

\begin{tabular}{|l|l|l|}
\hline Reliability Performance Metric & Definition & Unit \\
\hline Buffer index (BI), mean-based & $\begin{array}{c}\text { The difference between the 95th percentile travel time and the average travel time, } \\
\text { normalized by the average travel time }\end{array}$ & Percent \\
\hline Buffer index, median-based & $\begin{array}{c}\text { The difference between the 95th percentile travel time and the median travel time, } \\
\text { normalized by the median travel time }\end{array}$ & Percent \\
\hline Failure or on-time measures, median-based & $\begin{array}{l}\text { Percentage of trips with travel times less than } 1.1 \times \text { median travel time and/or } \\
1.25 \times \text { median travel time }\end{array}$ & Percent \\
\hline Failure or on-time measures, speed-based ${ }^{2}$ & Percentage of trips with travel times less than 50, 45, and/or 30 mph & Percent \\
\hline Misery index (modified) & The average of the top 5\% worst travel times divided by the free-flow travel time & None \\
\hline Planning time indices & 95th, 90th, and 80th percentile travel times divided by the free-flow travel time & None \\
\hline Skew statistic & $\begin{array}{c}\text { The ratio of (90th percentile travel time minus the median) to (the median minus } \\
\text { the 10th percentile) }\end{array}$ & None \\
\hline
\end{tabular}

Source: Cambridge Systematics, Inc. (2007).

a Speed is the space-mean speed over the study section. 


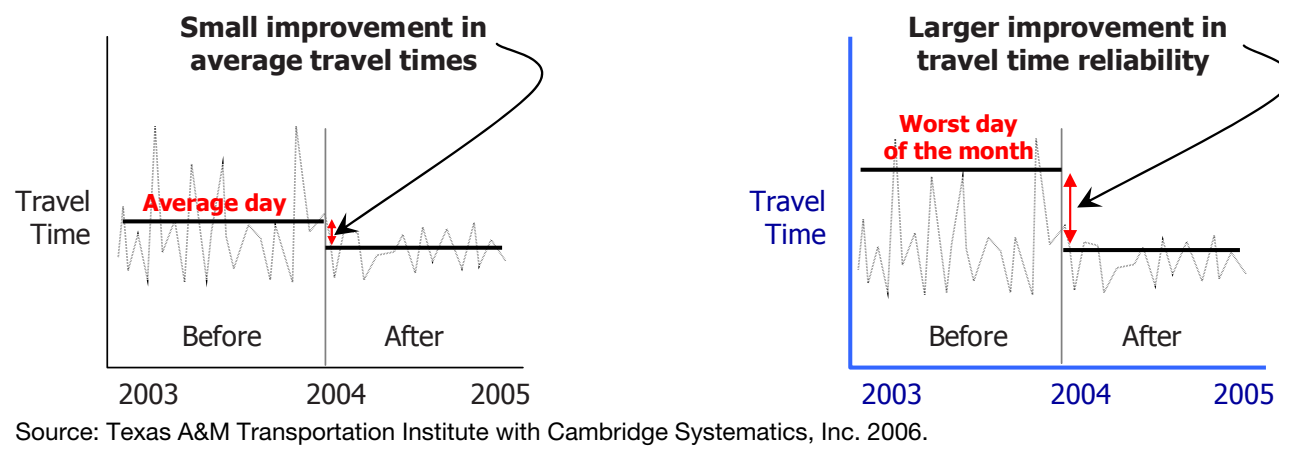

Figure 2.4. Reliability measures capture the benefits of traffic management.

higher than the value of reliability information. The reasons for this difference were not clear from the data collected, though some have interpreted the data to indicate that women have more time-critical commitments related to child-care trips. For this study, the researchers defined travel time as the 90th percentile travel time minus the median travel time. The authors discuss further how the transponder usage records of participants showed that few drivers habitually used the HOT lane. Rather, people made the decision whether to pay for the HOT lane on a daily basis depending on trip purpose and traffic conditions. In applications such as HOT lanes, travel time reliability information may be most useful en route to help drivers make the purchase decision to use the HOT lanes. The influence of pre-trip and en route travel information on route decisions has been demonstrated in other studies: An evaluation of the Washington State DOT's 511 travel information system in 2005 found that $21 \%$ of respondents changed their original travel plans on the basis of information they got from the 511 system (PRR, Inc. 2005). Drivers on an Orlando, Florida, toll road who stated that they used information from the state's 511 service or from DMSs (which displayed estimated delay times for the road) were more likely to change their route in response to unexpected congestion.

A review of research on travel time and travel time reliability conducted by the Center of Urban Transportation Research (University of South Florida) includes the finding that most travelers value trip time reliability at least as much as actual trip time. In fact, when travelers' arrival and departure times were inflexible because of the nature of the trip, the value of reliability was as much as three times that of trip time (Concas and Kolpakov 2009).

\section{Travel Time Reliability and Transit}

Studies of transit ridership have shown that trip time reliability (including the reliability of a rider's wait time at transit stops) is more important to retaining riders than the trip and waiting times themselves. Wait-time reliability is particularly important, as transit riders tend to perceive time spent waiting for a transit vehicle as being longer than an equivalent amount of time spent riding in the vehicle. Real-time information that allows transit riders to schedule their own arrival at a transit stop and/or to monitor the wait time remaining until the vehicle's arrival increases rider confidence in the service (Perk et al. 2008). Transit passengers surveyed in two cities ranked knowledge of when their bus would arrive and knowledge that it would arrive on time as the two most important factors affecting their decision to ride transit (Peng et al. 2002).

\section{Travel Time Reliability and Freight}

In terms of economic value, reliability is probably more important to freight carriers and shippers than to personal travelers. With the rise in just-in-time deliveries (largely as a replacement for extensive warehousing), providing dependable (reliable) service has become extremely valuable, while failure to provide dependable service can increase costs considerably (Cambridge Systematics, Inc. 2007). For example, improvements in transportation reliability play an important role in reducing inventory in the chemical supply chain for freight shippers. Because of the many nodes in the supply chain, upwards of one-third of all chemical inventory is in transit at any point in time. Inventory managers keep safety or buffer supplies to cushion against variability of inbound arrivals, and the amount of safety supplies increases with the degree of unreliability and the number of stocking locations (Cambridge Systematics, Inc. 2007). However, the capacity to receive chemical supplies is limited by the size of the liquid storage silos. Balancing capacity with demand is a challenge. As transportation reliability decreases, wait time, dead freight, and cost increase (Cambridge Systematics, Inc. 2006).

\section{Travel Time Information: State of the Practice}

Real-time travel time messages have been in use in the United States for well over a decade, ever since traffic monitoring and integration systems became reliable. The most commonly 
used media for these messages are DMSs and transportation agency websites; but the widespread use of cell phones and other mobile devices is prompting a growing number of transportation agencies and providers to offer real-time updates on transportation conditions and options via e-mails, text messages, and Twitter feeds.

Real-time travel time estimates are most often provided for a particular roadway segment or a particular transit route on the basis of recent travel speeds or conditions. Some agencies also provide travel time comparisons among two or more routes or roadways to help travelers make decisions about the route or transportation mode to take. Recent and rare sources are the information sources that advise travelers about travel time reliability - that is, about the likelihood that the estimated travel time for a particular trip or trip segment can be relied on dependably. The following subsections provide a concise summary of the state of the practice regarding travel time information. A more detailed literature review of this information is provided in Appendix A.

\section{Real-Time Travel Time Information on Dynamic Message Signs}

Changeable message signs (CMSs), also known as dynamic or variable message signs (DMSs or VMSs), can be used to provide several types of travel time information to drivers:

- Travel time information between specified locations;

- Comparative travel times for alternate routes (e.g., "Airport via Route $1-20$ min, I-94-35 min");

- Time saved by taking an alternate route (e.g., "Accident at exit 12; use Route 46-save 20 min");

- Delay on the freeway; and

- Delay avoided by taking the alternate route (Dudek and Huchingson 1991; Dudek 2004).

Displaying travel times on CMSs is not a universal practice. A 2008 survey of 100 traffic management centers in 40 states found that only $30 \%$ displayed travel times on some of the CMSs in their jurisdictions during peak traffic periods, and only $23 \%$ displayed travel times during off-peak periods. Reasons for not displaying travel times on CMSs include $(a)$ the CMS not being located where travel time messages would be useful and $(b)$ a lack of communications infrastructure and software to maintain up-to-date information and messages. The primary lessons learned by the TMCs were that travel time information must be accurate and that displaying accurate travel times during rapidly deteriorating traffic conditions (e.g., transition between off-peak and peak periods, occurrence of incidents) is difficult (Dudek 2008).

To be effective, a CMS must communicate a meaningful message that can be read and understood by motorists within a very short time period, constrained by the available sight distance and design features of the CMS (Dudek 2001, 2004, 2006). Some transportation agencies use signs that show one part of the message in static form (e.g., locations) and the travel times in changeable form. These types of signs are placed upstream of major diversion points which have no need for fully changeable message capability. Although these signs likely contain more units of information than can be fully read by unfamiliar drivers, drivers who are familiar with the area learn the static messages and concentrate only on the changeable travel times. One study of this sign type found that drivers rated the combined static/changeable format as easier to process than information displayed on a traditional CMS (Lerner et al. 2009).

The credibility of the message, in this case of the travel time provided, is also a concern. Real-time travel times posted on CMSs are actually historical travel times based on the past several minutes or hours of travel speeds that have been recorded; thus they may not reflect recent changes in traffic speeds. To alleviate the potential credibility problems that can result from a large difference between posted travel time and the travel time that drivers experience, some TMCs display a time stamp to indicate when travel speeds were last calculated; others display a range of estimated travel time (Houston TranStar 2012; San Antonio TransGuide 2012).

\section{Reliability Information on DMSs}

The travel time ranges used by some TMCs in real-time travel time messages can be one way of including some travel time reliability information along with real-time travel time information, though studies have found that drivers tend to prefer single time values to ranges and to accept that the actual travel time may vary from the single value posted (Ban et al. 2009; Phoenix Tightens Travel Time Estimates 2008). The French Ministry of Transportation in cooperation with the City of Paris experimented with another format: a travel time message that included slanted up and down arrows to indicate to drivers whether travel times were increasing or decreasing from the posted estimate (A. Hedhli, interview by S. Chrysler, May 26, 2010). Another study of travel time messages using trend arrows found that drivers took longer to process the information on CMSs when trend arrows were added and that they were more confident in the travel time value provided without a trend arrow (A. Hedhli, interview by S. Chrysler, May 26, 2010).

The Long Island Expressway uses the term average travel time on its combination static/CMS signs showing travel times to multiple destinations along a single route. That term is a slight departure from the Manual on Uniform Traffic Control Devices (MUTCD), which shows signs with the term estimated travel time. Both terms are associated with travel time reliability, but in the Long Island Expressway case, the travel times 
displayed on the changeable portions of the signs are based on recent sensor data rather than longer-term historical data (FHWA 2004).

Although symbols and pictograms on roadway signs have not been tested for use with travel time or reliability concepts, the results of research on their use on other types of road signs and CMSs indicate mixed results for driver comprehension (Luoma and Rama 2001; Arbaiza and Lucas 2010; Knoblauch et al. 1995). Another category of graphical sign is a graphical route information panel (GRIP). GRIPs display part of a road network using color coding to display information such as the level of traffic congestion on various roadway segments, similar to the color-coded traffic maps that appear on many TMC traveler information websites. Studies of GRIPs have identified several advantages over word messages, including the ability to convey more information about multiple roadways and to communicate with foreign travelers; a disadvantage is that drivers who have difficulty understanding maps may also have difficulty understanding GRIPs (Schouten et al. 1998; Alkim et al. 2000; Techie-Menson 2001). GRIPs that contain travel time information along with the graphical traffic status information are in use in other parts of the world (not yet in the United States); their effectiveness has not yet been determined (Lerner et al. 2004; Task Group 09 2009).

\section{Reliability Information via Websites and Mobile Devices}

The types of travel time information offered on travel information websites vary, with real-time information much more commonly available than historical and reliability information. Several websites operated by state or local TMCs reproduce the travel time messages displayed on DMSs; users can select a DMS location from a map of area freeways to check real-time travel times and conditions for a particular section of roadway. Color-coded freeway maps displaying either traffic speeds or congestion levels are also common. Other features available on some TMC maps show incidents and weatherrelated hazards such as snow and ice or flooding.

At the time of the literature review, only a handful of travel websites offered reliability information:

- The Wisconsin DOT website provides a table of current and normal travel times for highways in the Milwaukee area, with travel times that are $20 \%$ or more above normal shown in bold print (Kothuri et al. 2007).
- Washington State DOT's website includes a similar table that also displays travel times for high-occupancy-vehicle (HOV) lanes, where applicable, and displays the 95th percentile travel time for an input roadway segment (Seattle Area Travel Times 2012).

- The table of travel times on the Gary-Chicago-Milwaukee travel information website includes links from each average travel time estimate to a graph displaying detailed historical travel time data for the corresponding roadway segment (Travel Midwest Stats 2012).

- Rutgers University provides travel time reliability information for public transit routes. While most transit providers generate reliability information for their own use, few pass the information on to riders (Rutgers Department of Transportation 2012).

A growing number of TMCs and transit providers are communicating travel time information and traffic alerts via e-mail, Twitter, or text message.

\section{Communicating Reliability Information in Nontransportation Fields}

Cognitive science has shown that most people are not good at understanding statistical concepts and applying them to everyday situations such as medical diagnoses, gambling odds, or travel time probabilities (Gal 2002). Qualitative terms for conveying statistical concepts (e.g., probably, most likely, rarely) can be interpreted in different ways, so selecting a term that has a consistent enough definition to communicate the desired message about probability to the public can be difficult (Teigen 1988; Wallsten et al. 1993; Biehl and Halpern-Felsher 2001).

Medicine and weather forecasting are two fields that deal with probabilities and statistical concepts and must find ways of effectively communicating those concepts to the public. Graphical depictions of probabilities have been shown to improve comprehension among study participants, both for the probability of rain in a weather forecast and for the probability that a course of medical treatment will be effective (Schwartz 2009; BBC News 2007; Price et al. 2007). Paling (2003) recommended using numbers to supplement descriptive terms, expressing probabilities as frequencies (e.g., 19 out of 20) instead of percentages, and using a consistent denominator or scale (e.g., expressing two hypothetical probabilities as " 2 out of 10 " and " 9 out of 10 ," rather than expressing those same probabilities as " 1 out of 5 " and " 9 out of 10 ") to communicate probabilities to medical patients. 


\section{CHAPTER 3 Expert Interviews}

The Texas A\&M Transportation Institute (TTI) research team conducted nine telephone interviews in February 2010. The purpose of the interviews was to gather information regarding the state of the practice for disseminating travel time reliability information. TTI researchers conducted interviews with experts in the public and private sectors. As part of the interview, the research team asked questions related to the definition, computation, format, and users of travel time reliability information. The team also asked about current applications and future plans for the dissemination of travel time reliability information. Finally, the team identified lessons learned and pitfalls related to providing travel time reliability information to the public. This chapter summarizes the information learned from the interviews.

\section{Design of Telephone Interview}

The guide for the telephone interviews is presented in Appendix B. TTI researchers developed the guide on the basis of the results from the Task 1 annotated literature review and their expert knowledge of the subject matter. The interview guide has a section for recording participant information, a script for introducing the interviewer and subject matter to the participant, and questions with space for freeform responses. Under most questions, additional instructions and potential responses-or both-are provided for the interviewer.

\section{Telephone Interview Participants}

The TTI research team interviewed experts in the public and private sectors to gather information on the state of the practice for disseminating travel time reliability information. The interview participants represented the following agencies and private companies:

- Maryland State Highway Agency (Maryland SHA);

- Metropolitan Transportation Commission in Oakland, California;

- Minnesota Department of Transportation (Minnesota DOT);

- Utah Department of Transportation (Utah DOT);

- Virginia Department of Rail and Public Transportation;

- Washington State Department of Transportation (Washington State DOT);

- INRIX;

- TeleCommunications Systems (formerly Networks in Motion Inc.); and

- Ryder Logistics.

\section{Telephone Interview Results}

The following sections provide a concise summary of the results of the telephone interviews conducted by the project team. The results are organized by the individual questions discussed during the interviews.

\section{What Is Travel Time Reliability?}

First, TTI researchers asked participants to define travel time reliability. Participants provided the following definitions (in no particular order):

- Measure that allows you to predict trip time on a day-today basis;

- How trip time fluctuates from normal;

- Assessment of riskiness of a specific route;

- Statistical analysis of historical time pattern; 
- Planning time index (i.e., 95th percentile\% travel time index);

- Estimated travel time, which needs to be at least $95 \%$ accurate to gain public trust;

- Travel time to use if you want to be late to work once a month;

- Buffer time or best time to leave;

- Travel time that you can feel comfortable with;

- One mile per minute average over the course of a full day;

- Goal of the agency looking for an efficient system; and

- System performance monitoring.

Most of these definitions deal with providing travel time that is based on certain reliability criteria (e.g., 95\% reliable, late once a month, best time to leave). Only a few describe travel time reliability as a separate measure (e.g., how trip time fluctuates or the riskiness of a route). Most participants also thought of travel time reliability as a historical data projection (e.g., using 6 months of data to estimate the 95th percentile travel time), not a real-time measure (e.g., an estimate that can change depending on current conditions). However, two public-sector participants and one private-sector participant stated that travel time reliability needs to be a real-time measure because it is affected by nonrecurring events (e.g., incidents), and it needs to be accurate to gain the public's trust. Several participants also noted that travel time reliability can be computed from historical data and updated with real-time data when a nonrecurring event arises.

\section{Does Travel Time Reliability Information Have Value to the Public?}

The overwhelming answer was yes. Most of the agencies interviewed currently provide travel time estimates, but estimates can change drastically between the pre-trip and en route stages if a nonrecurring event (e.g., incident) happens. Travel time reliability gives the public an idea of the riskiness of the route (what the delay could be if a nonrecurring event occurs) or the buffer time needed to get to a destination on time. Thus, the public can use travel time reliability to make decisions regarding departure time, mode choice, and route choice. However, many of the participants noted that the public does not use the term reliability and may not understand statistical probability (i.e., 95th percentile travel time index). Participants also noted that the value of travel time reliability information to the public depends on the accuracy of the data and the public's understanding of the information.

Most participants identified local commuters who are familiar with the road network as the primary audience. However, participants expressed concern that travel time reliability information may be difficult for most commuters to understand.

\section{Does Travel Time Reliability Information Have Value to Transportation Operations Staff and Public Officials?}

All of the participants thought that travel time reliability information was of value to transportation operations staff and public officials. Within an agency, travel time reliability can be used to assess the performance of the transportation system and help agencies determine where improvements are needed. In addition, travel time reliability is a tool that can be used to manage transportation networks and justify programs such as incident management programs and freeway service patrols. One of the private-sector companies interviewed uses travel time reliability information to ensure minimum hours of travel, miles traveled, fuel usage, wear and tear on vehicles, and emissions - all of which have financial effects. Two public-sector participants felt that transportation operations staff do not fully understand the effects of nonrecurring congestion on the reliability and performance of the transportation system because the operators normally focus on responding to current traffic conditions. These two public-sector participants thought that travel time reliability information could be used to demonstrate the effects of nonrecurring congestion on system performance.

\section{What Are the Potential Effects of Providing Effective Travel Time Reliability Information?}

Researchers asked the question-What are the potential effects of providing effective travel time reliability information?-in relation to four measures: customer satisfaction, travel choices, system reliability and performance, and management and public officials. Participants felt that the effect of providing travel time reliability on customer satisfaction depends largely on the accuracy of the information and whether or not the public understands it. Participants thought that the effect on travel choices was less clear since only a small portion of commuters are likely to use travel time reliability information for trip planning; anecdotal evidence shows that users are more likely to change their schedule than their mode or route. Participants expressed hope that travel time reliability information will improve system performance; however, quantifying its effects would be difficult. (The effect of providing travel time reliability on management and public officials was discussed in the preceding section.)

\section{Do You Currently Disseminate Travel Time Reliability Information?}

Almost half of the agencies and companies contacted (four of the nine) are currently disseminating travel time reliability information. However, none are specifically measuring the 
effectiveness of conveying that information to users. One private-sector company provides its customers (mainly DOTs and commercial companies) with data from which travel time reliability can be computed. In addition, one private-sector company uses travel time reliability internally to compute estimated time of arrival (ETA) and to determine reasons for late deliveries. The following sections contain more detailed discussions of the two public agencies and two private companies that currently disseminate travel time reliability information.

\section{Metropolitan Transportation Commission in Oakland, California}

In the San Francisco Bay Area, the 511 phone system and 511 website (http://www.511.org/) provide travel time reliability as a comparison between the typical and current travel times for a particular roadway segment. Typical is the term used for the historical average driving time and speed between a starting and ending point for a particular day of the week and time of day. An averaging scheme that gives more weight to current data is used so that the typical values are representative of current, seasonal traffic patterns.

Users can see information in text or graphical form. The text version allows users to choose an origin-destination pair from drop-down menus, then the current and typical travel times are displayed for several routes. The graphical version is accessed on the traffic map for the region. Users choose an origin and destination by clicking the desired nodes on the map, then the current driving time is computed and displayed.

If current travel conditions are unknown, the following message is provided: "Do not have a current driving time. Typical trip is XX minutes." For routes with lane closures, the following message is provided: "Cannot give travel time because a portion of the route is closed." Similar information is provided via the phone system; however, it is only provided for the specifically requested route or location.

An alert feature can also be personalized by users. The alert sends trip information to a mobile device or e-mail about a specific trip the user has established. Like the travel time tools on the website, the alert contains the current trip time and the typical trip time as a reference. Users can also receive a severity alert when the current travel time exceeds a threshold established by the user (e.g., 10 min longer than the typical time).

\section{Washington State DOT}

The Washington State DOT website provides travelers the option of planning their commute using $95 \%$ reliable travel times. The commute calculator uses weekday travel time data from 6:00 a.m. to 7:00 p.m. to provide a reasonable approximation of the worst-case travel time scenario. Commuters who allow for the calculated travel time can expect to arrive at their destination on time 19 out of 20 working days a month (i.e., $95 \%$ of trips). Users choose an origin and destination from a predefined list of locations, as well as the time they need to arrive. The calculator then shows the user how long the trip will take and a recommended time to leave to arrive at the destination on time. In 2008, Washington State DOT introduced a new analysis of reliability to complement the traditional measures. The new analysis includes three travel times: 50th percentile (median), 80th percentile (how much time to leave to be late 1 day per week), and 90th percentile (how much time to leave to be late 2 days per month).

\section{INRIX}

INRIX is a private-sector company that collects travel time information on roadways across the United States using probe vehicles enabled with a global positioning system (GPS). INRIX then aggregates the data and provides historical or real-time information, or both, to DOTs and commercial companies (e.g., Garmin, Ford). Those entities in turn use the data to compute various statistics for performance measurement and to provide traffic conditions to the public. The historical information provided by INRIX includes the following reliability statistics: percentile speeds (e.g., 10th, 15th, 25th, 50th, and 85th) and failure rates at $30 \mathrm{mph}, 50 \mathrm{mph}$, and $60 \mathrm{mph}$. While INRIX's primary customers are DOTs and commercial companies, INRIX does have an iPhone application called INRIX Traffic whose end user is the public. A free version of the application has a color-coded map to compare current and future departure times. The paid version, called INRIX Traffic Pro, lets users drive and record favorite routes, as well as save frequent destinations, so the user can obtain fastest-route and when-to-leave information for those user-defined routes and locations.

\section{TeleCommunications Systems}

TeleCommunications Systems (formerly Networks in Motion Inc.) is a private-sector company similar to INRIX; however, its primary customer is the public. The navigation software uses GPS navigation on a cell phone, allows the user to enter a destination, and then selects a route on the basis of current traffic conditions. If an incident occurs once the user is en route, TeleCommunications Systems sends an incident alert that offers to re-route the user around the incident area. The company uses data from the public sector and proprietary algorithms to improve the data. It also calculates ETAs within $\pm 5 \%$. Over the years, TeleCommunications Systems has found that the color-coded information needs to be extremely accurate $(95 \%)$ or users will not trust the information provided. 
In addition to working with the public, TeleCommunications Systems also works directly with DOTs. On the basis of its review of raw sensor data, TeleCommunications Systems has had occasion to report problems with the DOTs' sensor networks and has worked with DOTs to prioritize the repair and deployment of future infrastructure.

\section{What Are the Challenges to Disseminating Travel Time Reliability Information?}

For the four public agencies that do not currently disseminate travel time reliability information (Maryland SHA, Minnesota DOT, Utah DOT, and the Virginia Department of Rail and Public Transportation), participants cited the following as their main challenges:

- Inability to quickly collect and analyze data,

- Limited staffing, and

- Determining a format that users will accept and understand.

These public agencies do not currently have a quick method (e.g., algorithms, software) to collect data and calculate travel time reliability information. In addition, because of workforce reductions, public agency staff do not have time to develop these methods internally. They would prefer that a format depicting travel time reliability information be developed and standardized before they implement it. That format must be understood and considered valuable by the traveling public for travelers to process and use travel time reliability information effectively.

Other participants cited additional challenges, including the need for quality data and the ability to implement the system in multijurisdictional areas.

Obviously, the quality of the data will affect customer satisfaction and trust of the data provided. Several participants expressed concern regarding potential political effects from disseminating travel time reliability information (e.g., what if one agency's reliability is not as good as another's). Also, agencies in the same area may have different sources, levels, and accuracy of information, so users might have trouble obtaining information on an entire region.

\section{What Are the Short-Term and Long-Term Goals Regarding the Dissemination of Travel Time Reliability Information?}

Among the agencies that do not currently disseminate travel time reliability information, most have begun internal discussions on providing that information to the public and would like actual implementation to occur within years. They mentioned various potential media, including the Internet, 511 systems, and cell phone applications. Among the agencies that are currently providing travel time reliability information, most of them want to improve data quality, expand coverage, provide customizable reports, use new media (e.g., cell phone applications), and provide new travel time reliability statistics (e.g., 50th percentile, 80th percentile). 


\section{онАВтеп 4 \\ Technology and Innovation Scan}

\section{Introduction and Context}

Today, travelers can acquire traveler information through a number of media, ranging from traditional roadside signage and broadcast media to newer electronic wireless and webbased media. The resulting mix of traffic and transportation system status data has greatly increased information accessibility and permitted an ever-greater degree of traveler-specific customization.

The array of organizations providing traveler information continues to broaden beyond traditional public agencies and news organizations. Many private corporations, data integrators, and social networking applications now offer access to traveler information. The methods of measuring travel conditions also have evolved beyond traditional technologies, such as loop detectors and infrared sensors, to video imaging, cell phone tracking, Bluetooth monitoring, GPS traces, and traveler-to-traveler information sharing. The methods for integrating multiple data are becoming more sophisticated and complex as well, blending multiple sources of data, including real-time and historical data - both quantitative and qualitative. Furthermore, the means of transmitting information are increasing in sophistication beyond dynamic message signs and web content toward in-vehicle devices with innovative voicebased transactions and head-up displays (HUDs). These concurrent trends in technology and innovation will provide the potential for traveler information granularity, coverage, and accuracy far exceeding what has historically been available and consequently will offer the opportunity for delivering trip reliability information in a variety of forms, both traditional and newly conceived.

Trip reliability information for travelers can be interpreted through two distinct lenses: (1) information on the historical trip time variability of a specific trip and (2) the reliability of traveler information (e.g., How reliable are the messages "expect delays" or "20 minutes to downtown"?). Reliability information on historical trip time variability can be used to help determine an appropriate departure time and route on the basis of the traveler's willingness to accept the risk of late arrivals. For example, a traveler may budget $75 \mathrm{~min}$ for a trip to the airport because he or she has been informed that, historically, the average travel time to the airport on a rainy Friday afternoon is $45 \mathrm{~min}$, but the 95 th percentile travel time is $70 \mathrm{~min}$. Trip reliability information can be delivered to the traveler through many metrics. As an example, a traveler who is driving to the airport may be informed en route that the travel time is between $40 \mathrm{~min}$ and $50 \mathrm{~min}$ with a $10 \%$ probability that the trip will take more than 50 min given current traffic conditions.

Chapter 2 provides the definitions of trip reliability metrics; outlines the prevalent formats and types of reliability information disseminated mainly through variable message signs, 511 phone systems, and websites; describes how reliability information is communicated in other fields, such as weather forecasts; and highlights recent technological means for transmission of traveler information, including personalized e-mails, texts, and tweets.

This chapter presents an overview of technology and innovation that focuses on trends likely to affect the provision of traveler information over the next 5 years. The chapter is organized into three exploratory sections, each ending with a synopsis of findings. Following the three exploratory sections is a section discussing the outlook for traveler information. The first section identifies the underlying technology trends that will drive the availability of better reliability data. The second section presents innovative media for disseminating traveler information that have come center stage in the past few years. These include portable navigation devices and applications on personal digital assistants (PDAs), smartphones, and mobile phones. The second section also looks at media on the cusp of center stage (in-vehicle agents) and future technologies such as integrated HUDs. Examples of specific applications for these media are highlighted. The use of social media to reach travelers as well as the phenomena of crowdsourcing 
as it pertains to the provision of trip reliability information are explored in this section as well. The third section identifies the market players in provision of traveler information.

Following the three exploratory sections, the fourth section presents the outlook for traveler information, specifically reliability data. This section addresses a number of foundational considerations, two of which are driving safety and the varying needs for driver information.

Driver information overload and distraction is a critical issue gaining significant attention under the Obama Administration. In September 2009, the U.S. DOT held a Distracted Driving Summit; and the discussions at that meeting related to distracted driving legislation will likely have direct implications for the means and content of delivering reliability information (NHTSA 2012). Furthermore, in January 2010, the U.S. DOT banned truck and bus drivers from sending text messages on handheld devices while operating commercial vehicles. This issue is discussed further in the final section of this chapter.

The most basic considerations for trip reliability information relate to the points during a trip at which reliability information should be provided, the content of the reliability information provided, and how content differs as a trip progresses from planning to origin to ultimate destination. Another consideration is how reliability information needs differ among travelers: those with familiarity and experience with a recurrent trip compared with those who make a trip without the benefit of day-to-day experience of its reliability. Likewise, how might transmission media and message content differ according to the needs of different driver types and trip purposes (e.g., older drivers versus newer drivers, commercial vehicle operators versus carpool organizers)? Furthermore, what innovations can help providers efficiently meet these varying needs? These considerations are also discussed in the final section of this chapter.

The chapter concludes with a discussion of likely trends in traveler information on trip reliability in light of factors such as market-player alignment, better underlying technologies, and innovative media for displaying and acquiring information.

\section{Underlying Technology Trends: Better Data to Come}

Major freeways in many metropolitan areas are fitted with in-road or roadside sensors (e.g., inductive loop, radar, and video image detectors) to help monitor performance of the road system, to better manage real-time operations, and to inform travelers of traffic conditions. According to a survey of 78 to 108 metropolitan areas from the years 1999 through 2007, the percentage of freeway centerline miles under electronic surveillance has increased in line with a pessimistic projection over the last decade, nearing $41 \%$ deployment
(RITA 2012a). Given the costs associated with deployment and maintenance of roadside sensors, adding more sensors may be increasingly difficult for public-sector agencies with mature traffic management and traveler information systems to justify.

The sensors measure traffic volume and occupancy and can measure or estimate spot speed. From spot speeds, travel time is estimated through extrapolation methods, statistical methods, or methods based on traffic-flow theory. The sensor provides performance information for a single location, though that location may not accurately represent the performance of the rest of the roadway segment associated with that location. Also, information is only available where sensors are present, which is mainly on freeways. Sensor technologies deployed for freeway systems are less effective on interrupted flow facilities like signalized arterials. Consequently, travel time reliability estimation is limited to measurement of roadway variability at points rather than along a stretch of roadway. The fundamental limitation of point sensors is that travel reliability must be inferred rather than directly observed since the sensors report on roadway segments, not trip makers.

As a whole, probe vehicle technologies differ in concept from spot speed sensors. Probe vehicle technologies enable direct measurement of travel time and space-mean speed, but for now, only a subset of all vehicles is monitored. The most prevalent probe vehicle technology in use over the past two decades is toll tags. Another probe vehicle technology, license plate matching, is able to monitor the majority of vehicles but at significantly higher costs. In the past decade, three key technology trends have enabled new means of collecting data that promise varying degrees of success in garnering far greater geographic coverage and granularity of data that can support estimation of trip reliability. These trends include the proliferation of cell phone usage, Bluetooth usage, and GPSenabled devices. The application of these technologies for travel time and reliability information is discussed in detail after a brief discussion of toll tag reader and license plate matching technologies.

\section{Toll Tag Readers}

Toll tag systems collect and record the time at which individual tag-equipped vehicles pass particular toll reader locations for the purpose of billing. By comparing the time at which a vehicle passes consecutive readers, the travel time can be measured for each toll tag vehicle. Given that ground infrastructure, communications, and necessary data sets are already present for toll billing purposes, agencies would incur a far lower cost in using the data to generate traveler information data on travel time and reliability. For closed toll systems, travel time data are highly accurate given only one path for travel and capture of entry and exit points. 
As of the mid-1990s, agencies have placed toll readers not for the purpose of billing but rather to acquire travel time estimates along facilities where significant numbers of vehicles have toll tags. The Houston region has been very successful in using nonrevenue toll tag readers on nontoll highways to acquire and transmit traveler information. As of 2010, more than 857 directional miles were being monitored using toll tag readers (Houston TranStar 2010). Deployments of toll readers were attempted in San Antonio (Hicks and Carter 2000), but the level of market penetration proved insufficient for travel time and speed measurements through all times of day. During a similar deployment in Orlando, a number of issues regarding the actual path of vehicle travel along arterial roads, the possibility of vehicle stops along the path, and low percentages of traffic having toll tags during off-peak hours, among other issues, made the strategy unsuccessful (RITA 2012b). Consequently, estimating travel times on facilities beyond toll routes and highways with high concentrations of vehicles with toll tags has proven a challenge.

\section{License Plate Matching}

Another traditional probe vehicle technique that is able to monitor a far larger sample of the total traffic between two points, albeit at a higher cost, is license plate matching. Matching can be done either manually or through the use of portable computers, manual transcription of video, or video and character recognition. Only the last of the four methods is amenable to real-time monitoring; but it raises significant privacy issues and is cost-effective for only specialized studies.

\section{Cell Phone Triangulation Using Towers}

To route calls to a cell phone, antenna towers listen for a signal sent from the phone and negotiate which tower can best communicate with the phone. As the phone changes location, the antenna towers monitor the signal and the phone is transferred to an adjacent tower as appropriate. By comparing the relative signal strength from multiple antenna towers, the general location of a phone can be roughly determined whether or not the phone is in use. This triangulation process is used to anonymously track the movement of cell phones and extrapolate such movements to measure travel times and roadway speeds. The triangulation technology has a location accuracy range of $50 \mathrm{~m}$ to $150 \mathrm{~m}$.

The key strengths for use of cell phone triangulation to obtain traffic data include a low deployment cost, the potential for large sample size without recruitment or vehicle instrumentation, and the potential for far greater geographic and temporal coverage. A key challenge to this technology is the inherent inaccuracy of position, direction, and speed identification. This inaccuracy makes distinctions among walking and standing pedestrians, stop-and-go roadway traffic, and bus or transit services difficult to ascertain. Likewise, assignment of observations across multideck or closely parallel roads is challenging. Travel time providers using cell phones as traffic probes also depend on the willingness of wireless cell phone companies to provide the anonymous positional data of cell phone users to compute travel time estimations on roadways.

Key providers of cell tower triangulation systems include AirSage, CellInt, Delcan/ITIS, IntelliOne, Globis Data, and TrafficCast. Other players such as TomTom and INRIX are joining in the use of cell phone tracking techniques in Europe. According to analyses of deployments by providers, the accuracy of travel time and travel speed data is relatively comparable for long road segments during free-flow conditions, and the technology is able to detect the occurrence of congestion. However, for shorter road segments and during moderate to lower speeds, this technology has not yet proved successful compared with traditional spot estimates. Following are snapshots of the first few entrants into this technology.

\section{AirSage}

AirSage collects and analyzes real-time cell phone signals that produce more than 15 billion anonymous locations every day (AirSage 2012). A number of evaluations have been conducted on AirSage's system, including tests in Salt Lake City, Utah; Minneapolis, Minnesota; Milwaukee and Madison, Wisconsin; Atlanta and Macon, Georgia; and Hampton Roads, Virginia (Fontaine and Smith 2007). The most recent evaluation, conducted by GeoStat, reports that for freeways evaluated in Detroit, San Diego, and New York, AirSage correctly detected congestion $84 \%$ to $93 \%$ of the time but did not present analyses on the accuracy of travel time or speed measurements. In Minnesota, speed and travel time were compared with loop-detector data on I-394 East (2.9 miles) and with license plate matching data on County Road 81 (2.2 miles) (Liu et al. 2008). Results suggest significant differences during low-to-moderate speeds. The cell phone tracking system did not correlate with observed trends. In Hampton Roads, Virginia, speed data were not acceptable at speeds below $30 \mathrm{mph}$ and travel time was not reliable (Liu et al. 2008).

\section{Cellint}

CellInt combines its cellular data with GPS probes and other data streams and provides real-time road traffic information in Atlanta, Kansas City, Israel, and the Skåne region of Sweden. With regard to cellular tracking, Cellint uses a pattern matching geo-location approach, which allows the company to correlate each probe vehicle's position with a more exact 
location on a roadway. Reports are unclear as to whether estimates of accuracy are for peak periods or other conditions.

\section{Delcan/ITIS}

Delcan/ITIS provides a system for cell phone tracking that has been implemented in the Baltimore metropolitan region of Maryland for Cingular (now AT\&T) users. Results from a pilot test conducted by the University of Maryland in 2006 suggest that average errors were in the 10-mph range on I-895 and 20-mph range on I-395, with significant quality degradation during peak periods. Another study using Estimotion Ltd, an ITIS product, compared the Estimotion cellular-based data with loop detectors and a small subset of GPS trace runs over a 14-km stretch of freeway in Israel (Bar-Gera 2007). The study reported far greater noise using cellular data than loop detectors, with overall similar speed ranges.

\section{TrafficCast}

TrafficCast, in strategic partnership with China Mobile, began deployment of the world's largest cellular probe system in Shanghai in September 2006. The anonymous mobile phone position and signaling data in China Mobile's GSM Network are collected, analyzed, and converted into travel time and speed information for major highways and surface streets in Shanghai. The system collects cellular data from 10 million subscribers in Shanghai and covers all roads within the Inner Ring Area, including 483 miles of major roads comprising 1,700 roadway segments.

\section{Bluetooth Tracking}

Bluetooth is a telecommunications industry specification that defines the manner in which mobile phones, computers, personal digital assistants, car radios, and other digital devices can be interconnected using short-range wireless communications (Traffax Inc. 2012). The application of this technology that interconnects a mobile phone with a wireless earpiece to permit hands-free operation can be leveraged for use in traffic monitoring. Because each Bluetooth device has a unique media access code, traditional matching algorithms such as those used for license plate or toll tag tracking can be used to estimate travel time along a freeway or arterial.

The use of Bluetooth technology offers advantages in that travel time and space-mean speed are directly measured with greater accuracy for multiple modes of travel (road, transit, and walking) without a concern for personally identifiable information as with toll tags or cell phones. The radius of Bluetooth recognition is approximately $100 \mathrm{~m}$; consequently, the maximum measurement error is less than $6 \%$ given a distance between trackers of $2 \mathrm{mi}$. This system would require the placement of Bluetooth receivers along the routes for which travel time information is desired, much like toll tag receivers.

Bluetooth tracking has been applied as an alternative to floating car or other probe vehicle tests and can supplant such tactics in computing ground truth. Field tests using this technology have been performed by the University of Maryland on I-495 (Center for Advanced Transportation Technology 2008) and by Purdue University with the Indiana DOT on I-465, SR-37, and other locations (Wasson et al. 2008). The Texas A\&M Transportation Institute began tests of Bluetooth tracking as a viable, cost-effective alternative for expansion of coverage of the TranStar system on arterial roadways. Initial results comparing Bluetooth tracking with license plate recognition systems on urban arterials in Houston demonstrated sufficient concentrations of Bluetooth traffic and often superior travel time and speed data generated by Bluetooth tracking (Texas A\&M Transportation Institute 2012). In June 2009, TrafficCast introduced BlueTOAD (Bluetooth Travel-time Origination And Destination), a traffic monitoring technology that traces anonymous Bluetooth signals to derive travel times, road speeds, and vehicle movements (TrafficCast 2012).

One other consideration with Bluetooth-enabled probe approaches is that Bluetooth is not a dedicated transportation wireless protocol; rather, Bluetooth directly serves the purpose of short-range wireless connectivity between mobile devices. Bluetooth will evolve over time, primarily in response to the needs of non-traffic-related applications. At a minimum, that means traffic-related probe technologies will have to evolve along with any changes in Bluetooth. Some risk remains that, over the long term, Bluetooth technology may evolve such that it no longer supports traffic data collection.

\section{GPS-Enabled Devices}

GPS is a satellite-based navigation system made up of a network of 24 active satellites placed into orbit by the U.S. Department of Defense. GPS works in any weather conditions, anywhere in the world, 24 hours a day, and without a subscription fee. GPS receivers use trilateration to calculate the user's exact location on the earth on the basis of radio signals from satellites. Whereas cellular technology enables location accuracy near $100 \mathrm{~m}$, GPS technology enables location accuracy within a few meters, along with highly accurate speed, trip distance, and travel time computations.

What can degrade the GPS signal and thus affect accuracy in downtown settings is the signal multipath effect, which occurs when the signal bounces off tall buildings or rock surfaces before reaching the receiver. The location accuracy also degrades at very low speeds and during vehicle stops, situations in which assigning road use in dense urban networks can be difficult. As is the case with cell phone triangulation, distinguishing 
whether the device transmission is occurring during walking, transit use, or auto use can also be difficult.

Application of GPS technology for fleet management has become common for both public and private fleet operators, ranging from goods delivery vehicles to public bus transit. Many studies have investigated the use of dedicated fleets of vehicles equipped with GPS, including FedEx and UPS trucks, taxi fleets, and transit fleets (Schafer et al. 2002; Bertini and Tantiyanagulchai 2004). These studies demonstrate that GPS-based estimates of travel time and roadway speeds far exceed existing granularity and accuracy and that a market penetration of about $2 \%$ in the vehicle fleet is sufficient for quality estimates of travel time and speed. The location and speed of mobile devices with GPS capability can be tracked.

Nearly 4.1 million mobile phones were active worldwide at the end of 2008 (Tryhorn 2009). Among new features in mobile devices (e.g., smartphones or PDAs), GPS is one of the most common, especially for mid- to high-end mobile devices. Most individual mobile devices have settings that enable or disable GPS; mobile device providers can track and record locations for GPS-enabled devices.

A number of private traveler information providers leverage real-time GPS reports from commercial and consumer vehicles and devices in providing estimates of travel speed and travel time. INRIX blends real-time road sensor data with billions of real-time data points from more than 1 million GPS-enabled commercial and consumer devices in taxis, service vehicles, airport shuttle services, cars, and long-haul trucks (INRIX 2012a). NAVTEQ, a wholly owned subsidiary of Nokia, integrates GPS data from Nokia handsets into NAVTEQ Traffic products in Europe and North America. Google has also begun using GPS-enabled mobile devices to acquire probe data.

The Mobile Millennium project-jointly run by the University of California's California Center for Innovative Transportation, the California Department of Transportation (Caltrans), and Nokia-began development of the first realtime permanent traffic monitoring system using GPS data in mid-2008. The project tracked 100 vehicles traveling with their GPS-enabled Nokia phones (University of California, Berkeley 2012). Researchers planned to continue, with greater attention to arterial coverage, but the project ended in 2010. During that time, a number of crowdsourcing services (e.g., Waze, Google Maps, and Aha Mobile) emerged that use GPS-enabled mobile devices to make available experiences of drivers using their service through either active driver reporting or passive GPS tracing.

\section{Future Technologies}

Connected Vehicles is a suite of technologies and applications that uses wireless communications to provide connectivity between vehicles and among vehicles, wireless devices, and the roadway infrastructure. These technologies are, in essence, similar to toll tag readers but have far richer information and greater emphasis on vehicle safety and driver assistance applications. On-board equipment (OBE) integrated with vehicle electronic systems could be designed to anonymously relay information on speeds and vehicle conditions, such as traction control or antilock braking activation, which are proxies for road surface conditions. The OBE could transmit this data anonymously to roadside equipment (RSE), which in turn could relay the information for use as traffic data. Safety applications are expected to operate through dedicated shortrange communications (DSRCs) that take place over a dedicated $75 \mathrm{MHz}$ spectrum band around $5.9 \mathrm{GHz}$, allocated by the U.S. Federal Communications Commission for vehicle safety applications. Applications with less-stringent latency requirements (e.g., traveler information) may use a broader range of communication links, ranging from DSRC to Wi-Fi and other wide-area media. Many Connected Vehicles technologies are still in the research and development phase, with some innovative safety applications emerging into the market. With regard to travel time and travel time reliability data acquisition, however, they are not expected to significantly affect the market in the near term.

\section{Synopsis of Underlying Technologies}

Traditional in-road and roadside technologies have been around for more than half a century. Although these technologies have documented difficulties with system reliability, geographic availability, and travel time accuracy, they can adequately measure the flow, occupancy, and volume of traffic along major roadways. Those data that are invaluable to the management and operation of roadway infrastructure.

Among newer probe technologies, GPS mobile devices offer the greatest promise given that GPS's data accuracy is far superior to cell phone tower triangulation's. Also, GPS mobile devices require no additional infrastructure as would be needed with Bluetooth tracking. Advantages of advanced probe vehicle techniques include low costs per unit of data, continuous and automated data collection without disruption to traffic, ability to directly measure travel times, and fewer privacy concerns. Furthermore, unlike traditional probe systems such as transit automatic vehicle location (AVL), which have high implementation costs and fixed infrastructure constraints (e.g., locations of receiving antennae), the proliferation of cellular and GPS-enabled mobile devices provides greater opportunities for data accuracy, coverage, and reliability than ever before.

Bluetooth tracking technologies have some notable advantages compared with GPS and cell phone tracking. Foremost, Bluetooth tracking does not entail the recording of any 
personally identifiable data. Thus, use of this technology eliminates privacy concerns associated with GPS and cellular sources of data. Additionally, the data generated through GPS and cellular devices require agreements with private parties, and the ownership of the data typically remains with a private-sector entity. Bluetooth data can be acquired directly by public agencies at relatively lower costs compared with toll tag technologies, and data ownership rights remain with the public agency.

The data to support end-to-end trip reliability will be increasingly available in the near future. The potential availability of advanced probe data also comes with new challenges for developing efficient means of processing, presenting, and archiving data. More complex processes for merging probe data with traditional data will need to be developed to provide information that is both timely and relevant to system managers and travelers. Would-be data integrators will also have to manage relationships between public agencies and commercial mobile phone corporations with regard to access and use of vehicle location and speed data from mobile subscribers. The need for traditional traffic monitoring techniques will remain for the foreseeable future, given public agency needs for data on traffic volumes and occupancy as well as other functions such as intersection management and tolling.

\section{Innovative Media for Traveler Information Users}

Traditionally, travelers have relied on commercial radio, television, and highway advisory radio for traveler information. Information content from these media is generalized, covering locations of accidents and providing qualitative descriptions of localized roadway traffic conditions such as "slow" or "congested." Additionally, content and delivery time from these media are on a schedule determined by the media rather than traveler needs. Changeable message signs, transportation agency websites, and phone services followed supplementing qualitative traffic reports with more precise quantitative data that covers ranges of travel speed, construction and event information, transit options and times to arrival, and travel time estimates along primary and alternate paths. Travelers can acquire data when they need it, but accessibility is limited by when travelers can access the websites. Furthermore, travelers frequently have to perform relatively extensive searches to find the data they need.

Concurrently with improved public-sector information on CMSs and traveler information websites, new consumer electronics emerged in the marketplace. In 2000, the Federal Communications Commission designated 511 as the national travel information telephone number across the country, spurring the growth of roadway traffic information content available by phone. In 2009, the Federal Highway Administration submitted a Notice for Proposed Rulemaking for the RealTime System Management Information Program. The FHWA proposes "general uniformity among the real-time information programs to ensure consistent service to travelers and to other agencies." This notice outlines, among other items, requirements for the timeliness, availability, and accuracy of traffic content (Real-Time System Management Information Program 2010).

In 2001, two satellite radio providers, Sirius Satellite Radio and XM Satellite Radio, began providing music, news, talk, traffic, and weather content in vehicles through paid subscriptions. Subscribers receive the same programming anywhere in the footprint of the service. Satellite radio provides local traffic and weather information continuously in a loop format on a dedicated channel, thus giving it an advantage over commercial radio, which provides travelers localized traffic information among other content. In 2008, the two companies completed a merger and continue to provide hundreds of channels including localized traffic data for over a dozen metropolitan regions.

More recently, public and private websites have begun to offer personalized information through e-mails to travelers depending on routes of interest to the traveler. Private commercial enterprises have brought to market a number of personal navigation devices (PNDs) that enable acquisition of real-time information along with turn-by-turn trip routing. Public agencies, local morning television shows, and commercial radio broadcasters are leveraging newer communication media to deliver traveler information. For example, KIROTV7 in Seattle sends messages (called tweets) regarding traffic on Twitter, a free social networking and microblogging service that enables its users to send and read other users' updates via their text-messaging phones. While most states only distribute information on traffic, a few states, such as Washington, let motorists tweet about traffic conditions. State agencies providing traffic information via Twitter include California, Colorado, Delaware, the District of Columbia, Illinois, Indiana, Kansas, Louisiana, Maine, Maryland, Minnesota, Mississippi, Missouri, Nebraska, New York, North Carolina, Oregon, Tennessee, Texas, Utah, and Virginia (Washington Times 2009). Tweet content varies from state to state; some states use tweets to distribute up-to-the-minute traffic congestion information, and others reserve tweets for emergencies such as hurricane evacuations.

With greater availability of PDAs and smartphones in the past few years, a number of applications have become available for travelers to acquire real-time traffic information along with routing support over their mobile devices. Such applications use information from public agencies, data from private commercial enterprises, and GPS or user input directly from traveling subscribers. 
Another technology that emerged about the same time as traveler information on GPS-enabled mobile devices is that of vehicle telematics systems (or early in-vehicle agent technologies). These systems control operations of relevant invehicle devices on the basis of driver requests or vehicle actions. The vehicle, in essence, has a built-in computerized interface, and the owner can subscribe to various services and applications for use in the vehicle.

Another phenomenon in traveler information that is gaining momentum is crowdsourcing. A crowdsourced network gathers reports directly from drivers themselves, bringing information on even smaller side roads or neighborhood roads to the individuals using the network. Many applications only receive information when the driver actively sends a message or turns on the application; some phones continue to send speed and location data even when the application is not in use. Some crowdsourced media are standalone enterprises, while others intertwine crowdsourced data with public traffic and other private data to provide traveler information.

As of mid-2009, nearly 35 million factory-installed and aftermarket in-dash navigation systems, more than 90 million PNDs, and an estimated 28 million navigation-enabled mobile handsets with GPS were being used worldwide (Berg InSight 2009). Features beyond turn-by-turn guidancesuch as local search, traffic-flow information, speed camera locations, and real-time travel time- - are now available through these media. What is missing by and large is trip reliability information, as well as assessments of the accuracy and completeness of information provided. The following sections highlight the state of the art in PNDs, GPS-enabled mobile devices, vehicle agents, crowdsourced applications, and mobile applications specific to transit.

\section{Personal Navigation Devices}

PNDs first entered the market in the early 1980s, but these devices contained only maps for a small area. The PND market expanded rapidly in the last decade, and highly capable, lowend devices now sell below $\$ 100$ per unit. The newest generation of PNDs offers many more features, such as real-time traffic information, Bluetooth compatibility, touch screen buttons, voice and command control, and spoken direction with text-to-speech. Following the increased competition and trend toward multipurpose GPS-enabled mobile devices, many players have exited the PND market since 2009. Going forward, the PND industry is likely to see further consolidation and additional exits. The four major vendors of PNDs are Garmin, Magellan (MiTAC Corp.), Navigon, and TomTom.

As the PND-smartphone convergence in the navigation market continues, the major PND vendors have begun developing application versions of their standalone GPS devices with additional features, while other PND vendors are pushing to add cellular technology to their portable devices with hopes of maintaining their market position. The next section highlights mobile applications from PND vendors as well as others vying for market share.

\section{Applications for GPS-Enabled Mobile Devices}

Apple, Research in Motion (BlackBerry), HTC, LG, Nokia, Palm, Samsung, and T-Mobile all offer smartphones, which can be broadly defined as multipurpose GPS-enabled mobile communication and computing platforms. The iPhone, BlackBerry, Droid, and others have applications that enable travelers to access traffic information. For example, the free California Traffic Report application for iPhone users was introduced in February 2009, and within the first 10 days, more than 2,600 individuals downloaded the application (Ramsey 2009). The application offers personalized reports with real-time commute times based on data supplied by Caltrans.

PND vendors are also transitioning from marketing singlepurpose navigation devices to providing navigation applications for multipurpose mobile devices. In 2009, Navigon introduced a third update to its iPhone application with textto-speech features and live traffic called the Navigon Mobile Navigator (Ngo 2009). The product is not a free application. The Magellan RoadMate for iPhone inherits many of the features of the RoadMate 1470 PND, including spoken street names, NAVTEQ maps, three-dimensional (3D) landmarks, in-application music control, and a pedestrian mode; but it does not yet offer real-time traveler information.

Other popular traffic applications include GPS Traffic by eMobile, Traffic Vizzion, TrafficGauge, and Google Maps. Traffic Vizzion, which partnered with INRIX in April 2009, offers real-time viewing of traffic cameras across 180 cities and a suite of additional functions for GPS-enabled BlackBerry smartphones (Traffic Vizzion 2012). TrafficGauge offers applications for multiple platforms in nearly a dozen cities with colorcoded maps; it claims that it only uses actual real-time data to guide users around traffic (TrafficGauge, Inc. 2012). Traffic .com's free application for BlackBerry gives the user a detailed view of traffic conditions, including color-coded traffic-flow maps, incident data, mass transit data, and more (Zeis 2009).

In November 2009, Google Maps introduced as a free service the application Google Maps Navigation for mobile devices running the Google-developed open-source Android 2.0 mobile operating system. The application enables both voice guidance and automatic routing and provides traffic information through color-coded maps. Color-coded traffic maps are also accessible for free and are based on crowdsourced GPS data from application users. If this application expands in popularity commensurate with the success of other Google services, the viability of PND devices and other paid traffic applications may be called into question. The business 
model for the consortium of Google, partnering telecommunications companies, and partner device manufacturers is to establish the dominant multipurpose mobile platform. These devices recover costs for Google by ensuring ease of access to Google search engines and other Google-branded services that generate revenue on the basis of large numbers of users performing searches and viewing advertisements.

During the Consumer Electronics Showcase in January 2010, INRIX debuted its Traffic Pro application for the iPhone and iPod (INRIX Traffic Pro 2010). The application appears to be the first to provide real-time, trip-based, travel time prediction capability. The technology merges data on the current traffic conditions, weather forecasts, event information, and roadwork with historical day of week, seasonal, holiday, weather, and accident data to predict travel time at 15-min intervals. Traffic Pro is now available for a few metropolitan regions in the United States. The application answers basic questions for travelers, such as when to leave to get home, what is the best route, when to expect to arrive home, and whether any accidents or events appear along the way. The level of accuracy of the predictive data is unclear. A previous iPhone traffic application, offered by INRIX, covered 126 U.S. cities. That INRIX application, like many others, provided color-coded traffic congestion maps but differed in its forecast function, which let users see traffic predictions up to an hour in advance (Cunningham 2009).

\section{Crowdsourced Applications for Traveler Information}

Crowdsourcing is potentially a win-win strategy for both consumers and businesses; and it has significant implications for potentially transforming traditional roles in traffic condition data collection, aggregation, and provision. Crowdsourcing makes possible new services at reduced costs and can offer participants value from the experience of peers. Crowdsourcing has been driven by the explosive growth of GPS-enabled mobile devices, leaps in software technology, and willingness of consumers to contribute to a shared experience.

A number of companies aim to provide traffic information through the experience of their members. Some use experiential knowledge to share information among members; others actually use GPS data from members to share information among users and improve traveler information estimates beyond the application's mobile users. Crowdsourcing services highlighted below include Aardvark, Aha Mobile, Waze, Google Maps, and INRIX.

\section{Aardvark}

Aardvark, founded in late 2007, launched its mobile social networking search application for the iPhone in September 2009.
The application uses Facebook or an e-mail import to determine a user's social network. Users receive a notification when someone in their network has a question that they may be able to answer. The service may solicit input from networks of those in the user's network and consequently have a far larger pool of experts. A user, for example, may ask what the best route from San Francisco to San Diego might be on a Wednesday afternoon. Responses usually arrive within $5 \mathrm{~min}$. The service extends far beyond traffic information but can easily garner enough responses from members and experts to support information on trip reliability when no institutional data are available.

\section{Aha Mobile}

In September 2009 Aha Mobile launched a new version of its free iPhone application, providing real-time traffic reports using a voice-based, non-map-centric approach with national traffic coverage and a few entertainment and social media features (Rao 2009). Using Aha's application, drivers record and share their personal traffic reports, up to $15 \mathrm{~s}$ in length, on their iPhone to help those around them. The audio messages, called Aha Shouts, are broadcast to nearby drivers. Shout content can be related to traffic incidents (e.g., bottlenecks, speed traps, or accidents) as well as unrelated to traffic (e.g., sharing a karaoke performance). Shouts can be posted to Twitter or Facebook. Posts are automatically populated and include Shout type, the user's current location and speed (for traffic Shouts), and a link to play the Shout. In addition to Facebook and Twitter integration, users of Aha's application can listen to INRIX and Clear Channel traffic, get nearby restaurant information pulled from Yelp, find the closest bathroom along the trip route from SitOrSquat, and identify speed camera locations from Photoenforced.com.

Emphasis in Aha Mobile is on the top markets, including Los Angeles, San Francisco, Washington, D.C., New York, Boston, Chicago, Dallas, Detroit, Houston, Atlanta, Miami, and Seattle. Aha Mobile plans to sell 10-s advertising spots on the application in the future and hopes to integrate its product with automakers and car navigation systems to make the application sustainable; the company also plans to begin moving into other platforms such as the BlackBerry (Roizen 2009). The set of applications, as with other in-vehicle, nondriving activities, can pose a significant safety hazard by distracting motorists from the task of driving.

\section{Waze}

Waze debuted in the U.S. market in May 2009 after success in Israel with more than 80,000 downloads, $90 \%$ geographic coverage, 10,000 daily users, and better than $85 \%$ arrival time accuracy (Martin 2009a). The goal of this free social mobile 
application is to map road networks through its community of members. Members (called wazers) share their road experiences in real time with other local wazers. The U.S. Census Bureau TIGER maps are the foundation of the Waze map network. The U.S. version posts Westwood One (radio) alerts in addition to member alerts. The business plan involves creating a complete road network comparable to products by NAVTEQ or Tele Atlas and then selling that informationwhile providing value to Waze members through real-time traffic information. Rollout is planned first for San Francisco, Boston, and Chicago, with coverage for the rest of the United States within a year. Participation in Waze requires the Android, Apple iOS, Symbian, Windows Mobile, or J2ME operating system.

\section{Google Maps}

Google Maps for Mobile has been around since 2007 and provides the functionality of Google Maps on the web, such as current location, driving directions, satellite views, and phone numbers and addresses for local businesses. In April 2008, Google Maps debuted a feature that allows users to view traffic congestion patterns by time of day and day of week. In 2009, Google debuted Google Maps Navigation (beta version) on most phones, including the Droid, BlackBerry, iPhone, and others. This free application provides turn-byturn GPS navigation with voice guidance. Additionally, business listings, street views, traffic, and transit and walking directions are all accessible.

The traffic data come from various data sources including crowdsourcing from users who have the My Location feature turned on. Location and speed are acquired from GPS data directly from mobile users with GPS-enabled devices, while cell tower triangulation is applied to estimate location and speed for mobile devices without GPS. Google Maps already has 50 million active users across various mobile phones, but the percentage of users who have enabled the My Location feature is unclear (Arrington 2009). Even if a small fraction of users enable My Location, the quantity of data acquired from this crowdsourced media will be significant.

\section{INRIX Traffic}

INRIX Traffic, unlike Google Maps, receives GPS probe data, including speed and location, from every INRIX Traffic application user. INRIX is the world's largest crowdsourced driver network with more than 1.3 million GPS-enabled vehicles, mobile devices, and road sensors. INRIX has been crowdsourcing GPS-enabled vehicle data commercially to deliver traffic information since 2006. The data are combined with billions of real-time speed data points from commercial and consumer vehicles, as well as road sensor information from
DOTs across the country. INRIX delivers traffic content to clients and consumers on terrestrial and satellite radio, on broadcast and cable TV, through wireless applications and services, and via the Internet.

\section{In-Vehicle Systems}

Since 1995, OnStar has offered 24-hour access to advisers, a connection to emergency assistance, and access to OnStar hands-free calling. The introduction of OnStar was important in moving in-vehicle connectivity beyond simpler carphone technologies. General Motors continues to offer and market OnStar across a broad portion of its vehicle fleet. Alternatively, BMW links multiple in-vehicle functions, including navigation, in its iDrive in-vehicle system. Both OnStar and iDrive generally do not support aftermarket media players and mobile devices except through industry standards like Bluetooth.

In 2007, Ford, with development assistance from Microsoft, introduced SYNC on 12 Ford, Lincoln, and Mercury models; SYNC is now available on more than 20 Ford, Lincoln, and Mercury models. SYNC permits drivers to bring virtually any digital media player or mobile phone into their vehicle and operate the devices using voice commands, the vehicle's steering wheel, or radio controls. In 2009, Sirius Travel Link joined the SYNC consortium, offering access to up-to-the-minute information and entertainment content through the vehicle's navigation system. That content includes current gas prices from an estimated 120,000 filling stations, local real-time traffic information for 78 markets, coast-tocoast weather conditions with 5-day forecasts, sports scores, and movie listings. Voice data commands and voice information response limit driver distraction. In May 2009, Ford shipped its millionth SYNC-installed vehicle (Cooney 2009). In January 2010, Ford announced that the SYNC system would be able to speak incoming tweets; however, SYNC does not yet have the functionality for drivers to send tweets (Associated Press 2010).

\section{Mobile Applications for Public Transit}

Many transit agencies provide open data that enable independent programmers to create useful transit tools for riders. The website City-Go-Round has information on 113 applications that use open data from 96 transit agencies (Transit App Gallery 2012). Despite the many applications for public transit, none were multimodal mobile applications that combine both auto and transit-based trips. A scan of mobile applications follows.

In 2010, the Washington Metropolitan Area Transit Authority began releasing transparent data sets so third parties could develop applications for mobile devices to provide train 
arrival and departures information, predict bus arrivals, locate bus routes, and list elevator and escalator outages. In Portland, Oregon, and its suburbs, more than 10 mobile applications provide next-bus arrival time information for TriMet. PDX Bus for the iPhone, also in Portland, offers trip planning functions for bus and train services. Other applications, such as Unibus for the iPhone, provide additional features, including searching for nearby bus stops and routes.

An application without real-time data, called Exit Strategy NYC, offers users the ideal place to stand in the transit train to be in position for an efficient exit according to the layout of the exit station, potentially saving minutes of time in the exit process. Other features of the application include bus maps of all five New York City boroughs and entry points for train stations. The application Google Maps for Mobile also offers bus, train, and ferry transit information for more than 50 cities worldwide. The application provides the combination of walking and transit options.

\section{Synopsis of Innovative Media}

Historically, the value of traffic data has been limited by mediocre quality, incomplete coverage, and limited accessibility. In the near future, travelers will increasingly have continuous access to high-quality traveler information with detailed coverage beyond major freeways. This change has been and will continue to be spurred by a confluence of three trends: established and projected market share for GPS-enabled devices (mobile and in-vehicle), availability of simplified mobile and in-vehicle applications for traveler information, and growth in crowdsourced data from GPS-enabled devices sharing vehicle speed and location information.

ABI Research, a market intelligence company specializing in global connectivity and emerging technology, predicts GPS will become a standard feature on all mobile devices, and "location awareness will be synonymous with smart devices, a point where personal navigation, social spatial knowledge, and location-specific contextual information will be assumed handset capabilities" (Montgomery 2009). Consequently, the quantity of GPS probe data collected through crowdsourcing will likely experience continued growth as well.

Despite these advances, the current media providing traffic information do not have sufficient market share or critical mass as data collectors, so the levels of accuracy, precision, and coverage reliability of traveler information is not uniformly excellent. Roadways with larger numbers of vehicles (typically freeways) tend to have more consistently accurate travel time estimates from mobile technologies, much like roadside sensor deployments. Another concern is that the processes of data integration and quality assurance, by and large, have not been made publically available. Public-sector purchasers of probe data are increasingly linking payment to accuracy requirements. A search on the accuracy of most PNDs and mobile and crowdsourced applications yields forums with comments citing poor navigation, incorrect traffic information content, and product shortcomings (Android Applications 2012). INRIX is one of the few entities with published overviews of its processes for collecting, aggregating, and quality checking its data presented in INRIX Traffic products (Martin 2009b).

Traditional traveler information media such as commercial radio and television are likely to remain and provide value to technology-averse travelers. Furthermore, media such as changeable message signs, public agency websites, and 511 phone services are also likely to continue because they are accessible without the need for GPS-enabled mobile devices. One consideration, however, is that those who use the Internet and 511 services are early technology adopters who have a need for traveler information; those individuals may be more likely to transition to more innovative media, consequently reducing the patronage of public traveler information websites.

The providers of traveler information media that are likely to be the most successful are those that provide increasingly simplified, personalized, multifunctioning interfaces with voice-based communications (e.g., in-vehicle systems). Standalone devices such as the PNDs of the past decade will likely face a period of integration to mobile or in-vehicle platforms or face significant decline.

\section{Traveler Information Market Participants}

Roles among data collectors, data integrators, data providers, and delivery media providers of real-time traffic information are all shifting. In a traditional model of relationships in regional traveler information, a public agency (typically a state DOT or metropolitan planning organization [MPO]) provided resources and leadership. In some cases, those agencies collected the data and managed a related traveler information service. Other agencies elected to turn over one or more functions after data collection (e.g., systems development, management, and operations of traveler information systems) to the private sector. Private-sector partners, in turn, managed the information systems and developed applications to (a) provide real-time data to the traveler on behalf of the public agency and (b) provide real-time data to the public agency for traffic management functions.

While the traditional model of regional traveler relationships may still remain the norm for many urban areas, the private sector is increasingly acting in the roles of data collector and data integrator, as well as traveler information service provider. The private sector is actively looking for innovative 
means of data collection and aggregation, including probe vehicle data and data from multiple private sources, through partnerships to leverage its market share, particularly in the navigation system arena. The shift from public to private sector is driven by the reality that one of the most important enhancements for navigation systems is real-time traffic. Consequently, organizations that provide mobile media and media content strive to increase their first-hand acquisition of the broadest geographic content of traveler information. This shift will increasingly turn the public sector into a potential consumer of private-sector data and introduce the responsibility to adhere to the data privacy needs of the private sector. The following subsections discuss the key players in the traveler information market, focusing on market presence, partnerships, and integration.

\section{INRIX}

INRIX is a traffic information provider for many public agencies' TMCs. Like other entities in this field, INRIX has engaged in a number of data-exchange partnerships with TomTom, Clear Channel Radio's Total Traffic Network, Navigon, and others. Notably, INRIX provides the I-95 Corridor Coalition and its 11 state partners real-time traffic information through data licensing. INRIX also provides data to the Wisconsin DOT and Alabama DOT (under subcontract to Jacobs Carter Burgess) (INRIX 2012b). INRIX has also expanded operations into Europe and multiplied the number of partnerships with traffic providers to continuously increase the coverage, quality, and accuracy of its traffic information and routing engines. In April 2009, INRIX positioned itself to be the exclusive commercial licensor of traffic camera information from Traffic Vizzion, a leading developer of traffic camera data services (INRIX 2009).

\section{Nokia/NAVTEQ}

The trends toward vertical integration and partnerships in the PND arena steepened around 2006 with NAVTEQ's purchase of Traffic.com, a provider of personalized traffic information in more than 50 metropolitan areas in the United States. That acquisition provided NAVTEQ with proprietary traffic content, as well as the technology and expertise to deliver that content to a wide variety of customers across multiple industries including AOL, Microsoft, and Garmin (TSC Staff 2006). In August 2007, TomTom expressed its intention to acquire Tele Atlas, one of the two providers of map content for all of the major navigation device producers. In response to TomTom's move, and to bolster its position in location-based services, Nokia bought the NAVTEQ mapping service in October 2007 (Niccolai 2007). Consequently, Nokia subsidiaries now produce the mobile media, the map content for use in the mobile media, and the traffic information data for use in travelers' mobile media.

\section{TomTom}

In 2006, TomTom was the leading provider of navigation devices, with more than $35 \%$ of the market share of installed and personal navigation devices. In 2007, TomTom also began working with mobile providers to acquire data through cell phone tower triangulation (Farivar 2006). The company now provides HD Traffic and IQ Routes to its devices as well as to third-party applications in Europe. These products are based on a data fusion engine that aggregates probe data (GPS and cellular), incident data, TMC messages, and historical speed data. TomTom's HD Traffic is based on direct agreements with carriers across Europe for the collection of granular real-time traffic and speed profile data allowing widening coverage and rerouting capabilities. TomTom completed acquisition of Tele Atlas in 2008.

TomTom recently announced an exclusive partnership with SFR, a French telecommunications company, to make its realtime traffic service available in France in 2009. This follows similar agreements with Vodafone in The Netherlands, Germany, and the United Kingdom (ABI: Win-Win Partnerships Key to Traffic Data 2008). Given competition from INRIX in European crowdsourcing, TomTom, in April 2009, turned to TrafficCast to provide real-time traffic on the TomTom GO Live 740 PND model (Privat 2009). Consequently, TomTom is a PND vendor with map content (Tele Atlas) that generates crowdsourcing traffic data and purchases traffic information (TrafficCast) to provide value navigation for commercial fleets and individual travelers.

\section{Clear Channel}

Clear Channel Communications Inc. began in the 1970s as owners of AM and FM radio stations and in the late 1980s entered the television market with acquisition of television stations. By the year 2000, through continued acquisitions, Clear Channel owned or programmed a total of 1,100 radio stations worldwide and 700,000 outdoor advertising displays. In January 2007, Clear Channel's Total Traffic Network and INRIX Inc. announced that they were extending their existing partnership to include real-time traffic speed data from INRIX's Smart Dust Network. That network intelligently combines the largest GPS-enabled vehicle probe network in the world with speed information from conventional road sensors and numerous other sources. In November 2009, Clear Channel Radio's Total Traffic Network announced that its real-time traffic service reached more than 125 million users, spanning broadcast, mobile, and in-vehicle devices (ClearChannel 2009). Mazda has joined other leading 
automotive original equipment manufacturers, including BMW, MINI, and Volvo, in offering the service.

\section{Westwood One}

Westwood One began as a provider of content on the radio, including simulcast in the 1970s, and is now one of the largest producers and distributors of radio programming in the United States. In 1999, Westwood One acquired Metro Networks, provider of 1,800 traffic reporters in markets across the United States. This subsidiary, along with Westwood One, unified a number of helicopter aviation companies providing traffic reporting under Global Traffic Network. Then in 2000, Westwood One acquired Smart Routes. Smart Routes, originally funded by government grants to make available traffic reports for local DOTs, provides the product www.smarttraveler.com and specializes in delivering operation support, program management, data collection and fusion, and 511 services for local and regional public agencies. In 2009, Westwood One, through Metro Networks, partnered with Traffic Land and began providing national traffic video along with its existing traffic incident data, speed and flow information, and more than 1,000 traffic reporters and anchors (Westwood One 2009). It also partnered with TrafficCast to provide road speed data. Westwood One provides content to both NAVTEQ and OnStar.

\section{Google}

A relatively recent entrant in the traveler information marketplace, and one with significant resources, is Google. In 2005, Google released Google Maps and within 2 years began supporting multipath driving directions, local business searches, street views, and mobile applications. That same year the company officially launched Google Traffic Info, which includes real-time traffic-flow conditions for 30 major metropolitan areas. Google acquires traffic information from TrafficCast as well as other sources. In August 2009, Google entered the crowdsourcing arena for data acquisition through the Google Maps for Mobile My Location feature. The feature acquires GPS or cell-based probe data for Google to use in its provision of traveler information and improved mapping. In October 2009, Google replaced its primary geospatial data provider, Tele Atlas, with its own data gathered from their Street View cars in the United States. (Blumenthal 2009). At about the same time, Google introduced its free Navigation device on the Droid, completing nearly seamless vertical integration of traveler information provision from data collection to data integration, data provision, and delivery media. The company's dominance will likely mean significant streamlining among data integrators, data providers, and delivery media.

\section{Synopsis of Market Participants}

The navigation market experienced significant contraction and concentration in the past 3 years. For example, MiTAC acquired Navman and Magellan in 2007 and 2008, respectively (Privat 2008). Navigon exited the U.S. market in 2009 (Vochin 2009), as did JVC in 2007 (Martin 2007). Unlike the navigation market, the traveler information business is in the midst of its market concentration and contraction, particularly in light of recent Google announcements on crowdsourcing and navigation. At present, a complex web of partnering relationships are enabling data providers to make available an array of content-from roadway speeds, camera and video images, incident data, real-time and predictive travel times to more unique information such as location-based gas prices and other points of interest. However, as Google continues to leverage crowdsourcing for acquisition of traffic data and to offer free navigation and traveler information services, Google's competitors will have to adapt their business models. The trend toward vertical integration is expected to continue, as the costs and technological barriers to data collection, integration, and provision continue to decline.

With trends toward more accurate crowdsourced data obtained through mobile GPS devices, traveler information providers have the opportunity to create new reliability metrics and reliability services. These providers could be from the public or private sectors, but the private sector is expected to lead the innovation and deployment of new services. This expectation is based on the central role that traveler information is playing in the battle for mobile device market share and the relatively impoverished condition of traditional public-sector providers (e.g., state DOTs and regional MPOs). In either case, public or private, the key to success will be whether providers present reliability services with sufficient clarity and simplicity that customers value the content. Conceivably, reliability services will be able to suggest travel time ranges based on driver and environmental tendencies, such as whether mobile users follow or lead traffic, or how weather contributes to greater variability. The presence of reliability data in the suite of traffic offerings to mobile traffic customers will be an effective litmus test of the inherent value of travel reliability information.

In the United States and in Europe, public agencies have served as the primary source of traveler information, specifically, real-time traffic and trip reliability information. The technologically savvy population and those that most needed traveler information were the first to actively use public sources such as the Internet or 511. This same population is likely to transition to traveler information provided by private mobile devices because the data may likely supersede what is available from public providers in terms of customization, seamlessness, accuracy, and coverage. Even technologically 
averse drivers may have access to advanced in-vehicle telematics, such as SYNC, if they acquire higher-end vehicles that provide more advanced active and passive safety systems. Still, for a significant portion of the population for the foreseeable future, information sources such as variable message signs, radio, and television will hold value. In particular, VMSs have the ability to reach the entire road population. Consequently, the public-sector traveler delivery model will continue but may lose some users to private traveler information delivery agents.

\section{Outlook for Traveler Reliability Information}

Traveler information providers operate on the principles that people want to be as fully informed as possible and that the only impediment is the availability of information itself. Travelers in possession of all the facts are expected to make rational and efficient choices as to the most attractive travel option, and technology is clearly the means for delivering the information. Traditionally, barriers to travelers' use of information included the complexity in accessing information, the absence of information beyond freeways, and uncertainty regarding its accuracy. The proliferation of mobile and invehicle devices offers tremendous opportunities for probe vehicle data collection and provision of traveler information. Thus, the potential for making complex reliability information available among the suite of other data at the fingertips of travelers is real. Although travel reliability data at present are available from only a handful of public-sector information providers and, by and large, even fewer private sources, reliability-related applications will likely be developed and adopted rapidly.

Traveler information services can be identified in both push and pull paradigms. In push paradigm services, the service provider initiates an action to push information to travelers on the basis of user information preference parameters. That is, information is provided to users without direct action on their part to acquire information. Under the pull paradigm, a user initiates a search for information, such as logging onto a computer before a trip to check a congestion map. While both push and pull paradigms are appropriate for the provision of travel reliability information, new push paradigm services are increasingly being developed as the capability to deliver personalized context-specific alerts is refined. For example, at many public and private traveler information sites (e.g., Traffic.com and the San Francisco Bay Area's 511 .org), travelers can set up trip routes for specific times of day and receive alerts via e-mail, phone, or text when traffic for the set routes exceeds the norm.

In the future, more advanced push systems or in-vehicle systems may use reliability information along with situational awareness to filter what information is provided. For example, route reliability might be used to narrow a long set of alternate routes to two options on the basis of the traveler's prespecified reliability preferences. Alternatively, an in-vehicle system might provide reliability information when it recognizes trips on roads or at times of day that are not recurrent routes for the vehicle-and provide only predictive travel time and incident information for regular work commute trips.

The acquisition of roadway data has traditionally been spearheaded by public agencies, with data made available freely to all entities from radio stations to television broadcasters. This pattern is dramatically shifting, and trip-based data are now being collected by private entities with ownership rights to the data. With this shift, public agencies have to pay for trip reliability and other trip-related data, which at some point may be cost prohibitive. Consequently, the provision of tailored traveler information may shift even more to the domain of private entities in the future.

To acquire trip-based data from cellular, GPS-enabled, or satellite devices requires communication from the handset through the phone service provider to the data integrator; the data integrator, in turn, offers traveler information services directly to the traveler or to third-party entities who form bundled services. Thus far, private providers of traveler information have had limited success creating financially viable, standalone, real-time traveler information services across the nation. This connectivity, along with the need for financial viability, has led to significant vertical integration in the industry as navigation device manufacturers merge with realtime data integrators. The trend will be toward greater bundling of traveler information with roadway navigation and other in-vehicle services such as weather, news, voice-based phone and music requests, and other functions. Those functions are likely to be integrated services through in-vehicle agents such as the Ford SYNC, while head-up displays are likely to be reserved for safety applications. As these more tailored services and systems come to the marketplace, both driving safety and user needs will remain key considerations.

\section{Considering Safety}

At the same time that more complex data are being made available to travelers, lawmakers are contending with the growing issue of how technology leads to driver distraction. Several state legislatures have begun passing legislation to limit many invehicle behaviors, such as texting and otherwise communicating on handheld devices. Visual distraction is the primary concern with mobile devices. As of 2012, 6 states had banned the use of handheld cell phones, 39 states had banned text messaging for all drivers, and 32 states and the District of Columbia had banned all cell phone use (handheld and hands-free) by novice drivers (Governors Highway Safety Association 2012). 
In the research community, an FHWA-supported project called the Mobile Millenniums was suspended until issues related to driver distraction were addressed. The project gives travelers a mobile device with traffic information and uses the devices as GPS probes. Given the movement against in-vehicle distraction, providers of complicated map-based products may also find limitations placed on their use.

Four societal factors are converging that potentially make driving a far less safe activity: increasing congestion, greater use of mobile technologies by younger and less-experienced drivers, greater numbers of mobile technologies (gadgets) taking drivers' eyes off the roads, and the ageing of the population marked by declines in cognitive and visual functioning (Eby 2009). Technology can mitigate the negative outcomes of these societal factors in various ways. Workload management systems - for example, Saab Dialogue Manager, Volvo Intelligent Driver Information System, and NHTSA's SAfety VEhicle using adaptive interface technology (SAVE-IT) — can help manage the source of distraction. The number of driving tasks can be reduced through mechanisms like adaptive cruise control and lane-keeping assistance. And the negative outcomes of distraction can be mitigated, for example, by improving the crashworthiness of vehicles and crash-imminent braking systems. In Volvo's case, the Intelligent Driver Information System delays incoming phone calls or other nonessential information if the driving situation requires greater attentiveness, such as during acceleration or lane shifting (Volvo 2006).

The potential for technology-based distractions in the vehicle is a serious and timely issue. In response, providers of mobile applications have begun shifting from visual directions and manual entry to auditory directions and verbal entry. In the same vein, providers of traveler information, including trip reliability information, must take heed when developing new information interfaces and information content.

\section{Considering What Reliability Information Is Needed}

Travelers require information for three main purposes: to identify travel options (e.g., mode, route, timing, and destination), to assess characteristics of alternatives (e.g., the times of different options), and to complete a trip successfully. Trip reliability information will aid in the latter two purposes; demand for specific reliability information will depend on the travel context and user characteristics. In reality most people, most of the time, do not consult travel information because the majority of trips are familiar and local, have minimal day-to-day variability, and are of a nature that does not necessitate a stringent on-time arrival (Peirce and Lappin 2004).

For the trips when travelers are not fully familiar with the route and have less knowledge of day-to-day variability, reliability information will prove valuable. For example, when planning a trip to a new client, a worker might benefit from knowing that the reliability of travel on a major arterial is far greater than the freeways on Friday mornings; and though that route might take a few more minutes, it would reduce the risk of a late arrival. This example demonstrates the value of situational reliability information-reliability for a roadway or trip based on factors such as time of day, day of week, weather conditions, and other considerations (e.g., major sporting events or holiday travel).

Reliability information can be tailored to encompass driver characteristics as well-perhaps offering data on ranges of likely travel time that reflect differences in outcomes for a traveler whose driving style is to go with the flow compared with one who prefers to lead. Reliability data can also be valuable for traveler information systems that provide information based on levels of user tolerance for travel time variability. The system might use reliability data in the system database to provide the route with the greatest likelihood of arriving on time.

Probability is another type of reliability information. For example, a traveler could be informed that taking the arterial route has a $60 \%$ probability of reducing overall trip time given the occurrence of an accident on the freeway. In that case, the $60 \%$ might reflect a comparison of travel time distributions on the two facilities plus some predictive estimates of travel time. The task for the industry and this SHRP 2 L14 project is to identify what pieces of trip reliability data best support travelers' decision-making needs at certain points along the trip process (i.e., trip planning or en route) and given the situational circumstances (e.g., on a freeway during rain), as well as to provide the data in a way that is clear and concise.

\section{Looking to the Future}

This section presents a short summary of observations regarding emerging technology and innovation trends and how they are likely to affect reliability-related services in the near term.

- Conditions are favorable for reliability-related traveler information services to develop.

$\circ$ Data to support end-to-end trip reliability assessment are in place or emerging in most urban markets and major interurban corridors.

o Costs to acquire and assemble travel time data will continue to decline because of increasing market share of GPS-enabled devices and innovations in crowdsourcing.

$\circ$ Options for free or near-free services on GPS-enabled mobile devices are increasing.

- New market players with significant resources (e.g., Google) are entering the market and are looking to differentiate their services. 
O Consumer demands for higher accuracy in travel time provision may reflect a hidden demand for reliability data.

- That said, there is limited signaling from the traveler information market regarding reliability-related applications. Mobile traveler information content is more or less the same as what is available on public traffic websites: map-based speed ranges, trip times, and incident details. The primary focus for mobile traveler information seems directed at increasing geographic coverage and providing more userfriendly applications. The exception to this comes from a few sources that combine reliability-type data to provide real-time predictive travel times, as is the case with INRIX's Traffic and a few other services providing travel time ranges to suggest reliability of the trip information.

- The GPS and cell-phone-based travel time and roadway speed information will be highly valued by travelers. Analysis of these data, combined with other relevant information, will enable travelers to make better trip choices and system operators to make more efficient operations decisions.

- More and more travelers will obtain trip information through private-sector providers as those companies expand ownership of trip-based traveler information data through technologies such as GPS and cell phone tracking.

- Traditional methods of providing traveler information (e.g., VMSs, commercial radio) will remain relevant to the large numbers of people who are not early technology adopters. Variable message signs can reach the entire driving population of a roadway and will continue to be valuable.
In addition, traveler information on commercial radio will continue to be present given its strength in garnering advertising revenue.

- No standard methods or lexicon to deliver reliability information to travelers has emerged in either the United States or Europe. The Institute of Transportation Engineers standard Message Sets for External Traffic Management Center Communications (MS/ETMCC) and the Society of Automotive Engineers standard J2354 Advanced Travel Information Systems (ATIS) define the structure for messages containing traffic data elements and data phrases (RITA 2012c). None of the intelligent transportation system (ITS) standards provide a clear structure for transmitting information relating to reliability, or probability for forecasts of travel time. Furthermore, the development of the traveler information systems described in this report has been largely independent of these standards; and the private sector has preferred to use the data-exchange formats it has developed rather than public-sector standards.

- A longer-term opportunity for trip reliability services could be integrated into emerging intelligent agent technologies. The foundational technologies are already present for increasingly capable in-vehicle agent technologies (i.e., next-generation vehicle telematics) to meet a road user's information and entertainment needs with seamless connectivity. The agent has the potential to serve in increasingly complex roles as the arbiter of what information is made available to the traveler, as well as when and how the information is presented. 


\section{CHAPTER 5}

\section{Developed Avenues of Investigation}

Tasks 1 through 3 of this project examined previous literature, expert opinions, and state-of-the-art technology and future trends concerning the dissemination of travel time reliability information. In Task 4, building on the results of the first three tasks, the project team formulated a focused set of avenues of investigation to be conducted in Phase 2. During this task, the TTI team honed the research effort to ensure that a manageable and realistic experimental design was developed for the remainder of the project. In reality, not enough time or dollars existed to examine every feasible combination of technology platforms, communication channels, message sets, and message formats to arrive at a single solution to the challenge of disseminating travel time reliability information. Thus, Task 4 focused on summarizing the most important gaps that emerged from Tasks 1 through 3 related to the most effective means of communicating reliability information to travelers.

\section{Key Research Issues}

The research team identified several important emerging research issues (which were addressed in Phase 2 of the project). The following items highlight those issues, the answers to which were pursued in the remainder of the project.

\section{Do Travelers Want Reliability Information?}

The central issue is to determine if there is market demand for reliability information. Transportation planners, policymakers, and operations staff clearly find value in reliability data as a performance metric. Whether travelers find the same value is less obvious.

\section{Do Travelers Comprehend the Basic Idea of Reliability as Separate from Real-Time Travel Time Information?}

During Phase 1 of this project, the research team struggled to separate the two concepts—reliability information versus real-time travel time information-in discussions, writing, and planning. In discussions with the experts interviewed, Technical Coordinating Committee members, and even other Reliability program researchers, the team became aware that most people lapse into talking about real-time information when the topic is information dissemination. This is to be expected because most travelers have experience with freeway CMSs that display current conditions. The L14 team continued to correct people about this misunderstanding and stress the role of historical data in travel time reliability information.

\section{Do Travelers Comprehend the Variability Inherent in Reliability Information?}

The literature review showed, across several domains, how poorly people understand the concepts of variability, probability distributions, and uncertainty.

\section{Do Travelers Comprehend the Average Travel Time?}

Some travelers may be making their trip time estimates on a near-worst-case basis, rather than on the average time the trip might take. If a regular commuter always travels in a narrow time window during peak hours, the overall average is not relevant for him or her. Thus, that commuter may have a different concept of typical or average, or both.

\section{What Terms and Displays Can Best Communicate Average, Variability, and Reliability?}

The most easily comprehended system needs to use terms that are generated by laypeople, even if the terms are not technically precise. 


\section{Can Travelers Assign a Dollar Value to Reliability Information, or Incremental Changes in Reliability?}

Researchers need to understand whether travelers might be willing to pay a premium for reliability information. Understanding the value travelers place on reliable trips could provide insight into how agencies might prioritize design and operational changes to improve reliability on critical routes.

\section{Does Reliability Information Have a Role in Real-Time Systems?}

Algorithm designers can certainly use historical travel time information along with real-time data to improve the accuracy of real-time predictions of travel time for a particular trip. From a traveler point of view, however, the role for historical data is unclear. If the real-time system says a trip takes $\mathrm{X}$ minutes, travelers may not care if it typically takes $\mathrm{X}$ to 10 minutes. Real-time data seem to trump historical data, as real-time data are most pertinent to today's trip. Systems may need to be opaque about their use of historical data.

\section{Do Travelers Want or Need Reliability Information En Route, or Is It Mainly a Pre-Trip Planning Tool?}

Assuming travelers can comprehend reliability concepts and a lexicon can be developed, the question remains whether they would use the reliability information en route. The more apparent use is as a pre-trip planning tool to select recurring times of departure, routes, and modes. Thus, travelers may only access a system once when initially planning a commuting route or a particular trip to a new destination.

\section{Does a Traveler's Desire for Reliability Information Change as a Function of Trip Purpose, Route, and Constraint?}

Trips are always made in a context. For constrained trips (e.g., the traveler needs to get to the airport), reliability information may be valuable and desirable for route choice or time of departure selection. However, for unconstrained trips (e.g., grocery shopping), reliability information may not be desired because the traveler incurs no penalty for being late or early.

\section{Can Travelers Safely Use an En Route, In-Vehicle System?}

Given the emerging evidence of driver distraction caused by in-vehicle devices - whether nomadic, such as cell phones and portable navigation systems, or native to the vehicle- traveler safety is an important question. Travelers may use reliability information en route to make route or mode shift decisions. Such decisions may involve a complicated decision-making process that can require repeated queries to the system to examine alternatives. That level of cognitive effort and interaction with a system is surely distracting. Here again, the system may need to make an opaque calculation that results in simple alternative route advice. Since more states are banning all use of manual input devices - especially smartphones - the deployment of these systems must be carefully considered.

The Task 2 technology scan revealed that the future holds more automation and customization in smartphone and navigation system applications. Those tools may mitigate distraction effects. Ultimately, en route use of in-vehicle systems was not tested in the human factor studies because the decision was made to replace the field operational test with the enhanced laboratory experiment.

\section{Do Travelers Want to Actively Seek Reliability Information (Pull) or Have It Sent Automatically (Push)?}

For both pre-trip planning systems and en route systems, some question remains whether reliability information should be pushed or pulled. Reliability information is calculated on a historical basis and changes slowly over time, so the need for a push may not be urgent. Push data, instead, could be limited to real-time information. Users may want a quarterly update or a push message when the reliability index of their selected route changes above some user-provided threshold value.

\section{How Can Multiple Data Sources and Factors Be Displayed for Reliability Information?}

Reliability algorithms use many factors to make travel time predictions, including weather, time of day, day of week, construction, transit system schedules, and so on. Do travelers need to know all of the factors, and do the factors need to be displayed at all? Even if all of the calculations are hidden or only available on secondary informational screens, travelers may develop trust in a system.

\section{Will Travelers Change Their Habitual Time of Departure and Route on the Basis of a Travel Time Reliability Information System?}

Humans are creatures of habit. In many ways, regular commuters have done all of the reliability calculations in their head using their $N=1$ data set. They know that when it rains, their trip usually takes five extra minutes; and if they leave at 7:40 a.m. instead of 7:30 a.m., the traffic is worse. What is not clear is whether travelers will develop enough trust in a system to make them change those regular habits.

As the research project moved forward, the project team attempted to answer these questions within the framework of Phase 2, as illustrated in Table 5.1. 
Table 5.1. Key Research Issues and Tasks

\begin{tabular}{|c|c|c|c|c|c|}
\hline Question & $\begin{array}{l}\text { Tasks 6-10 A1: } \\
\text { Focus Groups }\end{array}$ & $\begin{array}{l}\text { Tasks 6-10 A3: } \\
\text { Computer } \\
\text { Survey }\end{array}$ & $\begin{array}{l}\text { Tasks 6-10 A3: } \\
\text { Travel Behavior } \\
\text { Laboratory } \\
\text { Experiment }\end{array}$ & $\begin{array}{l}\text { Tasks 6-10 A3: } \\
\text { Open-Ended } \\
\text { Survey }\end{array}$ & $\begin{array}{l}\text { Tasks 6-10 B: } \\
\text { Enhanced } \\
\text { Laboratory } \\
\text { Experiment }\end{array}$ \\
\hline Do travelers want reliability information? & $\sqrt{ }$ & $\sqrt{ }$ & $\sqrt{ }$ & na & $\sqrt{ }$ \\
\hline $\begin{array}{l}\text { Do travelers comprehend the basic idea of } \\
\text { reliability, as separate from real-time travel } \\
\text { time information? }\end{array}$ & $\sqrt{ }$ & $\sqrt{ }$ & $\sqrt{ }$ & $\sqrt{ }$ & $\sqrt{ }$ \\
\hline $\begin{array}{l}\text { Do travelers comprehend the variability inherent in } \\
\text { reliability information? }\end{array}$ & $\sqrt{ }$ & $\sqrt{ }$ & $\sqrt{ }$ & $\sqrt{ }$ & $\sqrt{ }$ \\
\hline $\begin{array}{l}\text { Do travelers comprehend the term average travel } \\
\text { time? }\end{array}$ & $\sqrt{ }$ & $\sqrt{ }$ & $\sqrt{ }$ & $\sqrt{ }$ & $\sqrt{ }$ \\
\hline $\begin{array}{l}\text { What terms and displays can best communicate } \\
\text { average, variability, and reliability? }\end{array}$ & na & $\sqrt{ }$ & na & $\sqrt{ }$ & $\sqrt{ }$ \\
\hline $\begin{array}{l}\text { Can travelers assign a dollar value to reliability } \\
\text { information, or incremental changes in reliability? }\end{array}$ & $\sqrt{ }$ & na & $\sqrt{ }$ & na & $\sqrt{ }$ \\
\hline $\begin{array}{l}\text { Does reliability information have a role in real-time } \\
\text { systems? }\end{array}$ & $\sqrt{ }$ & na & $\sqrt{ }$ & na & $\sqrt{ }$ \\
\hline $\begin{array}{l}\text { Do travelers want or need reliability information } \\
\text { en route, or is it mainly a pre-trip planning tool? }\end{array}$ & $\sqrt{ }$ & na & na & na & na \\
\hline $\begin{array}{l}\text { Does a traveler's desire for reliability information } \\
\text { change as a function of trip purpose, route, and } \\
\text { constraint? }\end{array}$ & $\sqrt{ }$ & na & $\sqrt{ }$ & na & $\sqrt{ }$ \\
\hline $\begin{array}{l}\text { Can travelers safely use an en route, in-vehicle } \\
\text { system? }\end{array}$ & na & na & na & na & na \\
\hline $\begin{array}{l}\text { Do travelers want to actively seek reliability informa- } \\
\text { tion (pull) or have it sent automatically (push)? }\end{array}$ & $\sqrt{ }$ & na & na & na & na \\
\hline $\begin{array}{l}\text { How can multiple data sources and factors be } \\
\text { displayed for reliability information? }\end{array}$ & na & na & $\sqrt{ }$ & na & $\sqrt{ }$ \\
\hline $\begin{array}{l}\text { Will travelers change their habitual time of departure } \\
\text { and route on the basis of a travel time reliability } \\
\text { information system? }\end{array}$ & $\sqrt{ }$ & na & $\sqrt{ }$ & na & $\sqrt{ }$ \\
\hline
\end{tabular}

Note: $n a=$ not recommended for this message; $\sqrt{ }=$ suitable for this message. 


\section{онАВтеп Б \\ Focus Groups}

For the most part, human factor research done to date on traveler information systems has been concerned with segment trip time displays based on historical data. Another line of inquiry has focused on driver faith in the accuracy of the times displayed. The research undertaken as a part of the L14 project sought to examine user understanding of reliability information. Researchers employed a series of successively more objective methodologies, using a mix of methods. That approach allowed them to cast a wide net initially to gather the broadest understanding of users' preconceived notions of travel time reliability. Building information systems based on users' inherent understanding provides the best chance for a high level of user acceptance of the ultimate system.

Task A1 was the first of the series of human factor methodologies. The task involved conducting a series of focus groups in various cities across the country using a script developed by the project team. The script was based on the results of the literature review, the expert interviews, and the technology and innovation scan.

\section{Script Development}

The goal of the focus group task was to develop the script, or discussion guide, from which the facilitators would work during the focus group sessions. The following sections highlight the steps the research team took in the development of the discussion guide.

\section{Discussion Guide Development: Phase 1}

The focus group discussion guide went through several iterations and pre-tests with TTI staff unfamiliar with the project. The first draft of the discussion guide was based on the questions posed in the proposal as well as the results from the literature review, the expert interviews, and the technology and innovation scan. The project team used those research questions and related task results to develop the following set of objectives and questions to address:

- What things affect how long it takes to make a trip?

- What are drivers' understandings of travel time?

- What do drivers think of the accuracy of the travel time provided by current systems?

- How does past experience of travel times influence drivers' perceptions/interpretation of drive time?

- When is it the most important to drivers to have an accurate travel time prediction?

- How do drivers currently receive travel time information?

- When do drivers most need travel time information?

- How does reported travel time affect drivers' decisions on whether or not to take a trip, departure time, mode choice, and route choice?

- How does network travel time influence drivers?

- What travel time terminology makes the most sense to drivers?

- What is the best format for conveying travel time?

Researchers quickly realized that this list of questions did not differentiate enough between systems that provided realtime information and those that would provide a predicted travel time or information about reliability based on historical data. Therefore, the objectives were narrowed to focus more directly on the concept of reliability as defined by the transportation profession:

- How do drivers plan their trip using their own knowledge of the network?

- When is it the most important to drivers to have an accurate travel time prediction?

- Do drivers want reliability information, when do they need it, and how would they like to receive it?

- What are drivers' understandings and trust of traveler information that is currently provided, and how accurate do they think it is? 
- What travel time reliability terminology makes the most sense to drivers?

- What are the best method and format for conveying travel time reliability?

\section{Discussion Guide Development: Phase 2}

At this point in the discussion guide development, researchers began to fear that too much discussion of travel time might cause the focus group to run long as well as lead participants astray. Researchers were concerned they would have difficulty getting participants to distinguish between realtime travel time and travel time reliability. Their solution was to get to the heart of the discussion earlier and eliminate some objectives by jumping straight to (a) identifying the need for travel time reliability information, and (b) determining what travel time reliability terminology and sources make the most sense to drivers. Because the statistical concepts underlying reliability may be hard to grasp, the research team pulled from other, more familiar, domains to convey the statistical concepts. Scenarios unrelated to transportation were developed to discuss reliability in hopes of drawing out words and phrases that drivers use to discuss the concept. The scenarios included probability terms used in weather predictions and ranges of delivery dates for packages. Following those examples, the researchers planned to turn the discussion to a travel time reliability example and include various graphic examples to convey reliability.

\section{Discussion Guide Development: Phase 3}

After further consideration, the research team decided to begin the focus groups with non-travel time reliability scenarios and avoid mentioning travel time reliability until halfway through the discussion. The first pilot focus group was conducted with nontransportation TTI employees using this discussion guide. The scenarios discussed were

- Weather prediction;

- Restaurant wait time;

- Online order shipping time; and

- Prime parking spots (based on time of arrival).

The online order shipping time example described a scenario in which 10 people ordered a package on the same day, and the shipper estimated a delivery window of 5 days to 9 days. The graphic shown in Figure 6.1 was used to illustrate the days on which people actually received their packages. The graphic was meant to encourage discussion of probability distribution and was intended to generate words and phrases people

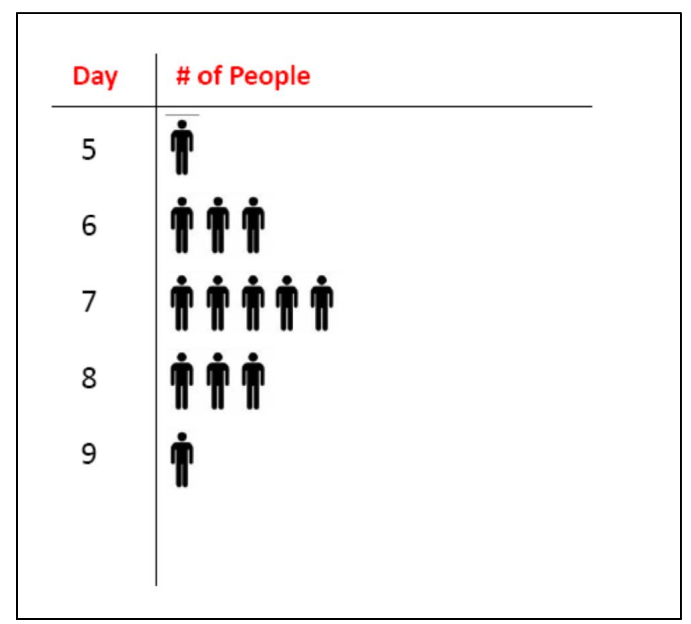

Figure 6.1. Shipping time graphic.

use to describe the reliability of the shipping service. The same distribution was later presented in a traffic context to show a distribution of travel times.

After the initial pilot, additional graphics, such as the one shown in Figure 6.2, were added to the focus group discussion once the concept of travel time reliability had been introduced by the facilitator. Using similar graphics, the research team explored different formats, colors, and symbols to display travel time reliability.

After these script revisions, two official pilot focus groups were conducted in Houston using the target demographics for participants. Although the two groups ran smoothly, the format of starting with non-transportation-related scenarios to discuss terms did not work as researchers planned. Although those everyday examples were well understood, the pilot group participants had great trouble making the leap from the scenarios to a traffic situation. Other feedback from the pilot group indicated that participants were confused by being invited to participate in a focus group about travel information and then being asked about seemingly unrelated topics like weather and shipping. For example, when asked what a " $70 \%$ chance of rain means to you," participants responded that they needed to leave earlier to allow more time on the road, rather than discussing the variability of the weather. Another problem that emerged during these pilots was that the discussion and questions were not drawing out many terms that the team could use to develop a lexicon.

Additionally, participants overwhelmingly disliked the graphics proposed for providing reliability information. Participants thought they contained too much information and were too confusing. This feedback led researchers to think that they were offering very limited options for displaying reliability information and possibly biasing drivers' opinions on the usefulness of the system. 


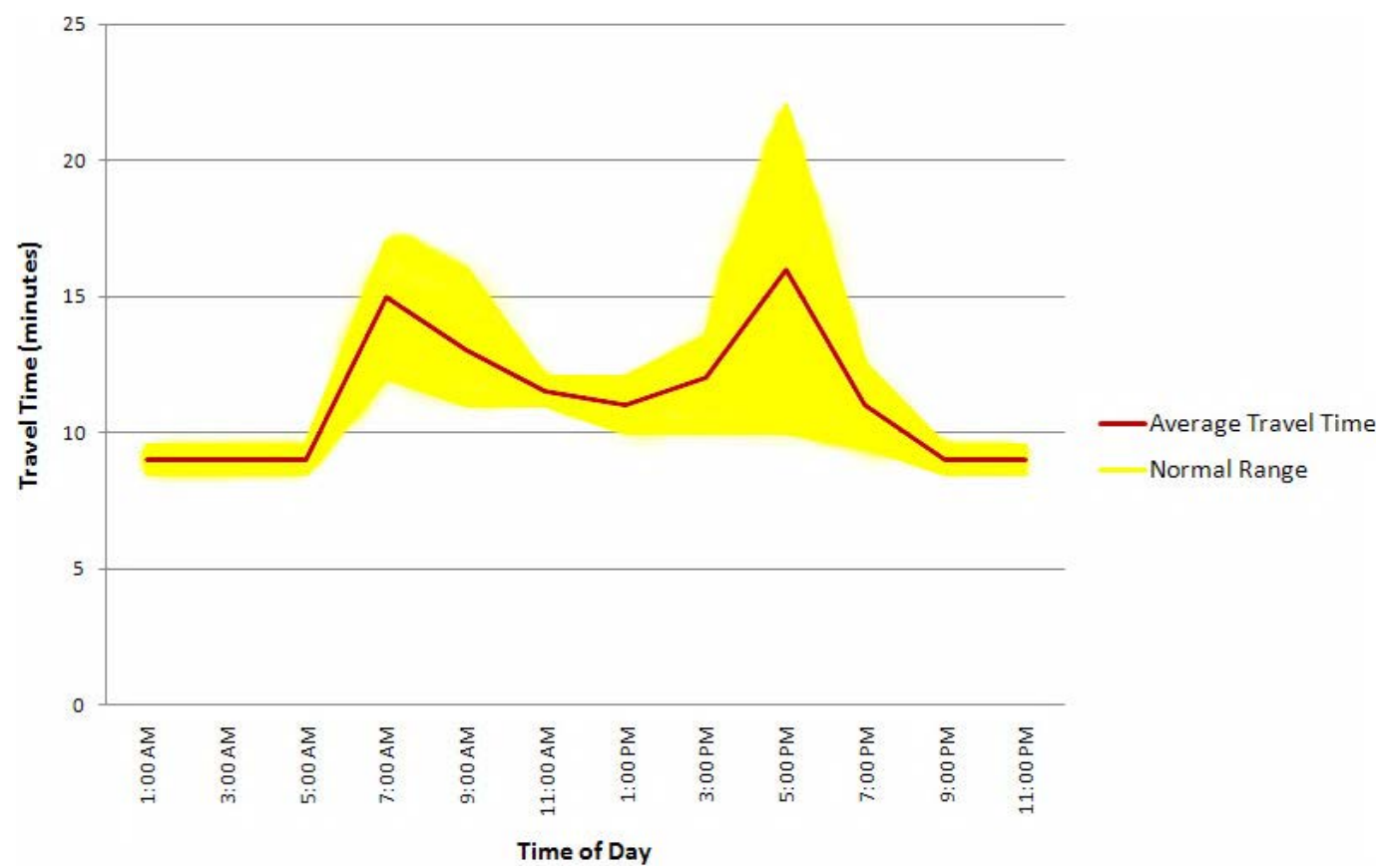

Figure 6.2. Monday travel time example.

Overall, researchers still did not believe that participants were truly separating the concepts of real-time travel data and historical travel data. Furthermore, the travel examples based on everyday scenarios did not effectively impose penalties for being wrong or late. The research team decided to make major revisions to the discussion guide; the revised version presented travel situations that varied according to whether or not they were constrained by either departure or arrival time. The team also decided that the presentation of alternative graphic displays of travel time were not productive in generating lexicon terms, so those images were not included in the final version of the discussion guide.

\section{Discussion Guide Development: Phase 4}

The final discussion guide was divided into several topics, as seen in Table 6.1. It began with separate discussions about (a) nonrecurring conditions and incidents that affect spot travel time and (b) recurring conditions that relate to network reliability and general variability of travel time on segments. Once the difference between the two concepts was established in participants' minds, the facilitators described a potential traveler information system that used historical data to predict trip times. The research team felt that by presenting details of a specific type of travel information system that used system reliability data, the subsequent discussion could be more fruitful. After a brief description of such a system, participants were asked directly whether they saw value in such a system. The researchers then presented trip scenarios to determine when people would use reliability information and what words they would choose to describe situations and fill in sentences. The trip scenarios were established after discussions with L14 partner Noblis and the L02 project team (Establishing Monitoring Programs for Mobility and Travel

Table 6.1. Final Focus Group Discussion Guide Topics

\begin{tabular}{|l|l|l|l|}
\hline & \multicolumn{1}{|c|}{ Constrained Arrival Time } & Constrained Departure Time & \multicolumn{1}{|c|}{ Unconstrained } \\
\hline Unfamiliar destination & Appointment with new doctor & na & $\begin{array}{l}\text { Weekend evening party locally } \\
\text { Weekend getaway 3 hours away }\end{array}$ \\
\hline Familiar destination & Regular commute & $\begin{array}{l}\text { Choosing day of the week for } \\
\text { class after work }\end{array}$ & $\begin{array}{c}\text { Picking up produce from farm } \\
\text { co-op anytime Saturday }\end{array}$ \\
\hline Task insertion & na & na & $\begin{array}{c}\text { Stopping by neighbors' house to } \\
\text { feed cat }\end{array}$ \\
\hline
\end{tabular}

Note: $\mathrm{na}=$ not applicable. 
Table 6.2. Focus Group Participant Demographics

\begin{tabular}{|c|c|c|c|c|c|c|c|c|c|c|c|c|c|}
\hline & 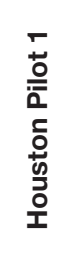 & 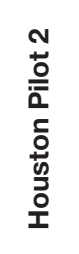 & 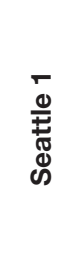 & 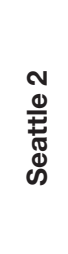 & 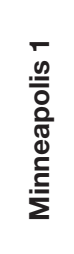 & 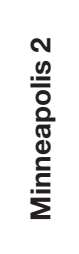 & $\begin{array}{l}\frac{\pi}{\pi} \\
\frac{\pi}{\pi} \\
\frac{\pi}{<}\end{array}$ & 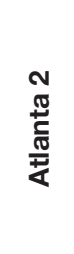 & 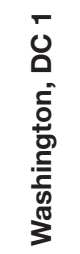 & 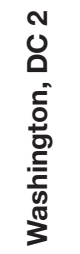 & 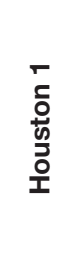 & 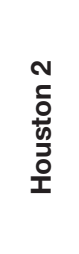 & $\begin{array}{l}\frac{0}{\pi} \\
\stackrel{\pi}{0}\end{array}$ \\
\hline $\begin{array}{l}\text { Total number of } \\
\text { participants }^{a}\end{array}$ & 10 & 10 & 11 & 10 & 10 & 10 & 9 & 10 & 9 & 10 & 9 & 8 & 116 \\
\hline $\begin{array}{l}\text { Number of } \\
\text { males }\end{array}$ & 5 & 6 & 1 & 4 & 3 & 4 & 5 & 5 & 5 & 4 & 3 & 4 & 49 \\
\hline $\begin{array}{c}\text { Number of } \\
\text { females }\end{array}$ & 5 & 4 & 8 & 5 & 7 & 6 & 4 & 5 & 4 & 6 & 6 & 4 & 64 \\
\hline $\begin{array}{c}\text { Average age of } \\
\text { participants }\end{array}$ & 35.9 & 46.3 & 30.4 & 37.0 & 38.4 & 38.6 & 42.9 & 36.7 & 36.1 & 36.3 & 43.8 & 32.4 & 37.9 \\
\hline
\end{tabular}

a Not all participants chose to fill out their information, so the number of males and females may not add up correctly.

${ }^{b}$ Not all participants chose to fill out their information, so the average is calculated from those who did.

Time Reliability). The scenarios varied in their time constraint and in the familiarity of the destination. One scenario involved using the system to decide whether or not the traveler had time to insert a task along the way to work-a use case identified by the L 02 team. More thorough descriptions of these tasks and the discussion questions are presented in Appendix C.

\section{Participants}

Ten focus group sessions were conducted in five cities across the nation: Atlanta, Houston, Minneapolis, Seattle, and Washington, D.C. Table 6.2 shows the demographic breakdown of all the participants. In Houston, focus group sessions were conducted at the TTI office. In all other cities, sessions were held in conference rooms at local hotels.

Recruitment criteria for participation were as follows: must be aged 18 to 80 , possess a valid driver's license, and commute to work regularly. The research team worked to select a representative sample of drivers who either drove to new locations regularly or had time constraints that affected their trips, such as children's schedules.

\section{Results Summary}

According to feedback from the focus groups, the majority of participants appeared to believe the system was a good idea and could be helpful. However, as the scenarios were introduced, the researchers had difficulty determining whether anyone would actually use it. As shown in Table 6.1, participants were presented several scenarios during the session that depicted constrained and unconstrained situations. Participants seemed most likely to use the system for pre-trip planning of constrained, unfamiliar trips within a known community or trips within or to an unfamiliar location. For the unconstrained trip scenarios, overwhelmingly, most participants would not use it; and weekends seemed to be the time it would be used the least.

In general, participants believed the travel time reliability (TTR) system would be valuable in some instances but would be immensely more valuable if it contained real-time data in addition to historical data. They felt that a TTR system would be useful when planning a trip to an unfamiliar location when they had significant time constraints on one or both ends of the trip. However, they were concerned that planning a trip on the basis of historical information would not be accurate if it was not updated with current road and traffic conditions. They also expressed concern that the predictions provided by the reliability system would not be more accurate than existing mapping tools. Many said they make their actual travel decisions on the day of the event and check traffic daily. Hence, they would not need to use the system to predict future trips. Some participants also said that they would prefer a "focus on coordinating getting information together with real-time information than calculating odds." This strong desire, and possible need, for real-time information could indicate distrust in a TTR-type system. Distrust of the system was further assessed by the open-ended surveys when determining how much additional time participants would add to the given travel time and why they added it.

When asked how they currently planned a trip, most participants said they used a mapping tool such as Google Maps, 
MapQuest, their GPS, or another system to give them a starting travel time. However, they all factored additional time onto the mapping tool estimate. They expected to calculate their own time and were not bothered by having to do this. They also indicated they did not know how the mapping tools came up with their time estimates. (At the time the focus groups were conducted, travel times provided by online mapping tools were generally based on the average of the posted speed limits along the mapped route; real-time traffic information was not yet included in calculations.) Drivers relied heavily on personal experience, and many simply assumed an hour to get to a new destination. Overall, the participants had a good understanding that traffic is not the same every time of day or every day of the week; they understood that many variables can affect traffic, and the existing systems are lacking in taking that into effect. Researchers used the computer survey and open-ended survey to expand on how drivers decide how much time to allow for a trip, particularly the additional time they add, and how various travel time terms could influence that decision.

Overall, participants preferred to enter an exact address into a mapping tool rather than simply choosing a starting city or general area. They also wanted the ability to specify a trip calculation based on time of departure or time of arrival. The computer survey continued investigation on this topic by addressing the preferred terminology to be used for the departure and arrival times.

When prompted, participants wanted to see multiple route options in their output. They also wanted to be able to compare choices and make their own decisions on which route to take. Participants seemed interested in having the ability to enter a window of time and rely on the system to tell them what time to leave to spend the shortest amount of time on the road. They had no clear preference on text or graphical output; however, several mentioned that both should be used. One participant thought the output "should say 'there is a $90 \%$ probability you are going to make it here in XX minutes, " showing a good understanding of the intention of the system. Another suggested "the longest this trip has taken historically is XX." Both the computer survey and the open-ended survey covered what output would best instill confidence in the system.

The general public may not use this system on a regular basis. Rather, individuals with consistent reasons to use the system may be more likely to use it regularly given the diversity of their daily trips. For example, salespeople, delivery services drivers, or professionals who frequently travel to new locations would be potential active users.

Many groups expressed concern that users would get the system confused with a real-time system and that people would not understand that travel times would be a calculated number and there could still be variation. Participants felt that real-time information was more important than reliability information and would be the most misunderstood component of the system. To investigate this concept further, questions were incorporated into the computer survey to assess understanding of a real-time versus historical-based system.

When asked by the facilitator, most said they would not pay to use the system in website form. Drivers seemed to realize nothing is free and were open to the idea of paying a nominal fee for a cell phone application or GPS addition. Some of the participants stated that they would actively use the system if their employers paid for it. Others suggested selling it to Google or paying some amount to add it to Google's existing navigation system.

In response to the question of who would provide this system to the public, one participant summarized the groups' responses by saying, "If a private company wants to charge a fee, I have no problem with it; but if the government does, I do." As long as access to the system was free, people did not seem to have a strong preference as to whether it was run by a DOT or private enterprise.

\section{Lexicon Information}

When talking about variability in travel time, participants preferred a wide variety of terms. Participants viewed sentences and had the opportunity to fill in the blanks with the term they thought best explained the scenario. TTI divided the sentences into two major groups: (1) sentences concerned with the system's output describing the time of day a driver takes a trip, and (2) sentences concerned with how the system would tell a driver about alternate modes of transportation.

In the first category, participants most often chose general words such as possibly, probably, chance, or likely to describe variability at a certain time of day. Generally, they preferred that those words have a descriptor in front, such a "X\% chance" or "highly likely" to make the term less general. When talking about traffic patterns at a specific time of day, participants used varies, changes, and increases or decreases most often.

In the second category, when discussing the terminology the system would use to suggest different routes or modes of travel, participants used the words faster, more reliable, easier, and likely. When speaking about time added to a trip to ensure on-time arrival, participants' terminology varied greatly with no real consensus. For example, terms like additional time, traffic time, leeway, driving time, just-in-case time, fluff time, and additional drive time all came up as possible descriptors. Unprompted, participants used the words or phrases cushion, allow an additional $X$ minutes for variables, tack on extra, and extra time during discussion. 
Several additional terms came up unprompted in the discussion sessions; many of them specifically addressed reliability information, including reliability factors, plus or minus 10\%, and 9 out of 10 days. Terms that addressed a range of time were approximately, usually, window of time instead of exact time, give or take, variation, cushion, buffer, time frame that's certain, average, and depends.
The most common terms that came up in the focus groups were included in both the computer survey and the openended survey. The computer survey spent time addressing what to call travel times, departure times, and additional times, while the open-ended survey spent extensive time on how the various terms influenced participants' decision making and trust of the system. 


\section{OHAPTtR 7 \\ Usability Surveys}

The usability portion of this project ultimately consisted of three research activities: a multiple-choice survey conducted on a laptop computer, an open-ended survey, and a computerbased experiment intended to simulate daily trips. Each research activity produced different types of data that were analyzed separately and, where applicable, in comparison with one another. The usability study activities were used to achieve the following primary objectives:

- Determine comprehension of and preference for reliability terms.

- Elicit additional terms for potential inclusion in the lexicon.

- Test user acceptance of and trust in the information described by various reliability terms.

- Determine drivers' use of reliability information, the serenity benefits of having access to reliability information, the drivers' willingness to pay for that information, and whether reliability information helps drivers form their own personal historical framework for expected travel times.

The human factor studies planned for Tasks 6 through 10 were presented and approved by the panel in June 2010. They consisted of focus groups and a usability study. As conceived at that time, the usability study used computer-based multiplechoice testing of terminology and website interface features. This objective testing allowed rapid testing of large numbers of participants because data entry and scoring could be automated. The computer survey was developed and conducted in the fall of 2010.

The computer survey presented test items asking participants to select departure times in response to a sample traveler information website that provided total trip time estimates. After approximately half the participants were tested, researchers reviewed interim results and found that a large portion of people were adding their own buffer time on top of the total trip time recommended by the system. Unfortunately, researchers could not determine whether this was because participants lacked interest in the terms used or lacked understanding of how to apply them conceptually in the survey environment. The research team decided to add an open-ended aspect to the survey to investigate this critical question in more depth.

\section{Study Locations}

The usability study activities were conducted in five cities selected on the basis of their traffic congestion rankings in TTI's Annual Mobility Report (Schrank et al. 2011). The presence and availability of transit service and high-occupancyvehicle or high-occupancy/toll (HOV/HOT) lanes was also a factor in selecting the cities. Dallas, Texas; Miami, Florida; Denver, Colorado; San Jose, California; and Hartford, Connecticut, were chosen.

The intent was to ensure that study participants would likely be familiar with the possibility of altering a travel mode or paying a fee to travel on a less-congested roadway. Dallas data collection was conducted in two waves, with half the participants tested at the start of the data collection period and half at the end. The open-ended survey was conducted only in San Jose and Hartford and during the second wave in Dallas.

\section{Study Participants}

Participants for the usability study were recruited through ads on craigslist.org, by word of mouth, and by distributing flyers containing information about the study within the cities. Because the study's focus was regular commuters, drivers under age 60 were targeted. Older drivers were allowed to participate if they still worked outside the home and commuted regularly. Table 7.1 summarizes the numbers of participants that participated in each of the study activities. 
Table 7.1. Usability Study Participants

\begin{tabular}{|c|c|c|c|c|c|c|}
\hline City & $\begin{array}{l}\text { Computer } \\
\text { Survey and } \\
\text { Open-Ended } \\
\text { Survey }\end{array}$ & $\begin{array}{l}\text { Computer } \\
\text { Survey Only }\end{array}$ & $\begin{array}{l}\text { Travel Behavior } \\
\text { Experiment and } \\
\text { Open-Ended } \\
\text { Survey }\end{array}$ & $\begin{array}{l}\text { Travel Behavior } \\
\text { Experiment Only }\end{array}$ & $\begin{array}{l}\text { Open-Ended } \\
\text { Survey Total }\end{array}$ & $\begin{array}{c}\text { Total } \\
\text { Participants } \\
\text { Per City }\end{array}$ \\
\hline Dallas & 31 & 31 & 14 & 1 & 45 & 77 \\
\hline Denver & 0 & 61 & 0 & 15 & 0 & 76 \\
\hline Miami & 0 & 67 & 0 & 10 & 0 & 77 \\
\hline San Jose & 54 & 0 & 20 & 0 & 74 & 74 \\
\hline Hartford & 53 & 3 & 17 & 4 & 70 & 77 \\
\hline Total & 138 & 162 & 51 & 30 & 189 & 381 \\
\hline
\end{tabular}

\section{Computer Survey}

The objectives of the survey were to test comprehension of and preferences for terms used to present reliability information and to test initial concepts for a website presenting this information (to be developed for the field study). A total of 300 drivers participated in the computer survey.

\section{Influence of Focus Group Results}

The focus groups that were conducted in a previous task of the project provided the research team with some information regarding travelers' uses for reliability information. The focus group results also provided the team with a large set of terms that participants preferred or suggested to describe travel time reliability. Those results indicated that drivers perceived the most value in travel time reliability information in the context of an unfamiliar trip with a constrained departure time, arrival time, or both. Focus group participants tended to view realtime information as more useful in most instances; many felt that historical travel time information for a given route would be more relevant for trip planning with real-time conditions figured in. Most stated an unwillingness to pay for such information on a website but were somewhat amenable to a nominal fee for reliability information provided via a mobile or smartphone application or as an addition to a GPS system. Researchers presented a number of potential lexicon terms for travel time reliability and related concepts to the focus groups; some additional terms and phrases were suggested by focus group participants themselves.

In addition to providing several of the terms that were tested in the usability surveys, the focus group results influenced the next phase of the research in several ways:

- A travel behavior laboratory experiment was added to better gauge the potential value of reliability information to drivers.
- An open-ended survey was added to further clarify comprehension of and preferences for various terms.

- Questions were included in the computer-based survey and in the open-ended survey to elicit more potential lexicon terms.

- The research team concluded that participants' lack of fundamental understanding of the reliability concept would not likely be clarified by graphical representation, and so reduced the emphasis on graphical displays for reliability outputs.

- Strong consideration was given to including real-time information along with historical information on the field operational test website.

\section{Survey Development}

The computer-based survey presented participants with multiple-choice questions on concepts and terms associated with the presentation of reliability information. The objectives of the survey were to test comprehension of, and preferences for, terms used to present reliability information as well as to test initial concepts for a website presenting this information that was planned to be developed for the field operational test. A list of specific research questions based on these objectives guided the development of this portion of the usability study:

- What terms should be avoided?

- What are the best terms for general use?

- What input features are the most desired for websites and similar tools?

- What output features are the most desired?

- What output features are the best understood?

- Will drivers use a travel time reliability information website, and when?

- What information should be included in user instructions and frequently asked questions (FAQs)? 


\section{Travel Information Website Reviews}

The first step of the computer survey development was a review of existing travel information websites, including those that offer some type of information regarding historical travel times. The websites were identified through the literature review, expert surveys, and technology and innovation scan activities in Phase 1 of the project. Researchers reviewed 16 websites operated by state DOTs, metropolitan TMCs, airports or airlines, and private-sector vendors. The reviewed websites included the following:

- Arizona 511;

- Lake Michigan Interstate Gateway Alliance;

- Colorado DOT;

- Florida 511;

- Florida DOT, District Four;

- Georgia DOT;

- 511, Los Angeles;

- NAVTEQ Traffic.com;

- Phoenix Valley Metro, Arizona;
- Texas DOT TransGuide, San Antonio;

- 511 Travel Info, San Francisco;

- San Diego Transportation, California;

- Washington State DOT;

- FlightStats; and

- FlightCaster.

The website reviews examined the formats of the home page or the primary traffic information page, the labeling of any links or buttons and banners for accessing information about historical travel time information, the mechanism and labeling for trip information input by the user, and the format and terminology used for the output information. The research team compiled lists of terms and labels for possible testing, including the websites' terms for trip inputs (origin and destination, departure, and arrival times) and outputs (average historical travel time, 95th percentile trip time, recommended departure time). These were compared with terms and phrases from the focus group results. Table 7.2 provides examples of trip information input and output terms collected from reviewed websites.

Table 7.2. Sample Terminology from Reviewed Websites

\begin{tabular}{|c|c|}
\hline Input/Output Type & Terminology Used \\
\hline Origin, destination, road, or route & $\begin{array}{l}\text { Available locations. } \\
\text { Segment start location. Segment end location. } \\
\text { Type in address, intersection, or landmark. } \\
\text { Where does your trip start? Where does your trip end? } \\
\text { A (origin). B (destination). } \\
\text { Select your origin. Select your destination. } \\
\text { Where are you starting from? Where are you going? }\end{array}$ \\
\hline Departure/Arrival/Time window & $\begin{array}{l}\text { Estimated time of arrival. } \\
\text { Depart on time. } \\
\text { Best time to travel. } \\
\text { Leave my starting point at. Arrive at my destination at. } \\
\text { Select day and time for historical data display. } \\
\text { What time do you need to get there? }\end{array}$ \\
\hline Current trip & $\begin{array}{l}\text { Travel time current. } \\
\text { Current travel time. } \\
\text { Leave at [time]. } \\
\text { Drive time now/delay. } \\
\text { Fastest now. } \\
\text { Direct drive. } \\
\text { Current conditions: avg speed, min speed, max speed. }\end{array}$ \\
\hline Historical trip or reliability & $\begin{array}{l}\text { Travel time average. } \\
\text { Average travel time (minutes). } \\
\text { Current travel time (minutes). } \\
\text { Normal range. } \\
\text { Avg overall travel time. } \\
\text { Normal travel time. } \\
\text { Drive time @ speed limit. } \\
\text { Average speed. } \\
\text { Typical travel time. } \\
\text { Your } 95 \% \text { reliable travel time is } 25 \text { minutes. } 95 \% \text { of the } \\
\text { time you would need to leave at } 7: 35 \text { a.m. to arrive } \\
\text { by } 8: 00 \text { a.m. }\end{array}$ \\
\hline
\end{tabular}




\section{Selection of Test Platform}

Initially, the research team intended to conduct the usability study using an interactive mock-up of a travel information website to test input and output terminology associated with travel time reliability information, as well as related screen titles and labels. Elements of some of the reviewed travel time websites-such as overall screen layout, maps of roadways with available travel information, and dialog boxes for user inputswere adapted to portray a fictional urban area. The study as originally conceived would use keystroke logging, eye-tracking, observation of participant inputs, and verbal feedback from participants to test comprehension of and preference for input and output terms related to travel time reliability.

However, as the researchers further defined the types of questions that would best determine participants' comprehension of reliability terms, they realized that an interactive website, even one with limited functions, would not be the most suitable study platform for those questions. The primary concern was that other website usability issues (graphic design, website hierarchy and organization, etc.) could confound the primary research questions regarding terminology and lexicon for the participants. Second, testing several different terms for each of the several website input and output possibilities would necessitate building multiple versions of the website; and that was impractical under the time budgeted for the task.

Ultimately, the research team decided to use a multiplechoice survey to address comprehension and preference questions regarding reliability terms and associated trip information. The survey was conducted on laptops using SuperLab software to present questions and instructions and to collect participant answers. The designs for the mock-up website pages were presented to participants as static pictures to provide context for some of the survey questions. This study format allowed researchers to test multiple participants at once, allowed them to test multiple terms in the context of a single simulated web page, and minimized the effects of website usability issues and biases that did not pertain to the reliability terminology being investigated.

Some of the survey questions asked participants about specific terms used on a sample web page. Preliminary versions of the SuperLab survey showed a split screen with the sample web page on one portion of the screen and the question on the other. During pilot testing, researchers determined that these images were too small and difficult to read on a computer screen and might pose difficulties for participants with reduced vision, or even bifocals. A three-ring binder was created to hold full $8^{1 / 2} \mathrm{in}$. by $11 \mathrm{in}$. color printouts of each of the web page screen shots. The binder pages served as visual aids for most of the questions in the remainder of the survey. Displaying the simulated web pages in the binder had the advantage of allowing participants unlimited viewing time of each image, including the option to look back and forth between the image and the on-screen question.

\section{Paper Prototyping of Web Page Examples Used in Survey}

A human factor usability research technique called paper prototyping was used to develop the simulated websites displayed during the survey. This method was developed in the computer interface design field and allows rapid early-stage testing of web page input and output screens without requiring computer coding. The research team printed screenshots of some of the reviewed travel information websites and cut out individual text and graphic elements such as user input boxes, roadway maps, text links and buttons, and various output formats for trip travel times. Initially these web page pieces were physically arranged and adhered in place as team members agreed on the content and format of each simulated screen. User input was also obtained from other TTI staff members and student workers to help finalize the look of each screen. The team decided that the simulated website would display a fictional city rather than a real location to avoid the possibly confounding effects of selecting a city that happened to be familiar to some participants and not to others. To that end, several road maps from the real traveler information website were composited to create a network of roadways that bore no immediate resemblance to any one city but had the overall look of a metropolitan area. Once each screen's arrangement was finalized, it was recreated using graphics software.

\section{Selection of Terms and Questions}

In deciding which terms to test with participants in the SuperLab survey, the research team began with the various terms and phrases for trip parameters, travel time, and reliability used by the reviewed websites, as well as from the terms mentioned most frequently by participants during focus groups. The research team organized the survey by first defining categories of trip inputs (origin/destination, arrival/departure times, other route/trip preferences, labels for input buttons, map titles) and outputs (historical and current trip times, average versus 95th percentile trip times) that could reasonably be included in a travel time reliability information website. For each of the input and output categories, the team listed (a) potential terms, and (b) questions that could be used to test user comprehension and preference for each of the terms.

The research team developed the survey questions collaboratively over several sessions. An outline format was developed for research team members to list potential travel time, reliability, and other input and output terms and phrases within topic categories (e.g., Website Input Screen-origin/ destination field). For each set of terms, researchers proposed 
Table 7.3. Outline of Computer Survey Questions

\begin{tabular}{|c|c|}
\hline Questions 1-4 & Preconceived understanding of four terms for reliability: reliable, predictable, consistent, best \\
\hline Question 5 & Importance of reliability \\
\hline Questions 6-8 & Understanding of websites for current and future general purposes \\
\hline \multicolumn{2}{|r|}{ Explanation of website and its purpose } \\
\hline Questions 9-10 & Trip planning terms tested by versions: predict trip, plan trip, create trip, get trip \\
\hline Questions 11-12 & Departure and arrival terms \\
\hline Questions 13-14 & Website map titles \\
\hline Questions 15-17 & $\begin{array}{l}\text { Trip time terms by versions: average, typical, historical, estimated, 95th percentile, worst case, } \\
\text { maximum, most common }\end{array}$ \\
\hline Questions 18-20 & Departure time terms by versions: recommended, estimated, 95th percentile, suggested \\
\hline Questions 21-22 & Desire for possible additional time (over the average) \\
\hline Question 23 & Type of travel time metric (average, most common, worst case, etc.) \\
\hline Questions 24-26 & $\begin{array}{l}\text { Fill-in-the-blank terminology: } \\
\text { - It is } \frac{\text { that your trip will take } 45 \text { minutes. }}{\text { - Your trip time may }} \text { - It will take } \\
\text { - It the average time by } 20 \text { minutes. }\end{array}$ \\
\hline Question 27 & 95th percentile term understanding \\
\hline Question 28 & Trust-instilling phrases \\
\hline Question 29 & Will drivers change mode of travel? \\
\hline
\end{tabular}

question types that could be used to test comprehension of and preference for the terms.

A brief outline of the final survey questions is presented in Table 7.3. Questions 1 through 8 were asked before any explanation of the hypothetical website was provided. Those questions were asked to get naïve participants' reactions to terms and input screens as a new user to the system would encounter on an actual site. A description of the motivation behind each set of questions is provided, along with pictures of the stimulus screens and binder pages (if applicable) and results tabulation. Not all possible terms could be tested with every person because the survey would have been too lengthy. Thus, for a small number of questions, multiple versions of the survey were created, as shown in Table 7.4. The participants were divided across four different versions of the survey, Versions A through D.

\section{Research Method}

\section{Participant Demographics}

Researchers surveyed a total of 300 participants. Each person was asked the following demographic and driving questions:

- Gender;

- Age;

- Education;

- How often participant drove on freeways on weekdays between 6 a.m. and 7 p.m.;

- How often participant traveled to a different city and drove in that city;

- Flexibility of participant's arrival time to work each day;

- How much time it took the participant on average to commute to work each day;

Table 7.4. Experimental Design for Survey Questions that Varied Across Versions

\begin{tabular}{|l|l|l|l|l|}
\hline \multirow{2}{*}{$\begin{array}{l}\text { Question } \\
\text { Number }\end{array}$} & \multicolumn{4}{|c|}{ Version of Survey } \\
\cline { 2 - 5 } & \multicolumn{1}{|c|}{ A } & \multicolumn{1}{|c|}{ B } & \multicolumn{1}{c|}{ C } & \multicolumn{1}{c|}{ D } \\
\hline $9-10$ & Predict trip & Plan trip & Create trip & Get trip \\
\hline 15 & Average & Typical & Historical & Estimated \\
\hline 16 & 95th percentile & Worst case & Maximum & Most common \\
\hline $18-20$ & Recommended & Estimated & 95th percentile & Suggested \\
\hline
\end{tabular}


Table 7.5. Gender and Age of Participants by City

\begin{tabular}{|c|c|c|c|c|c|c|c|c|}
\hline Gender & $\begin{array}{c}\text { Age } \\
\text { Range }\end{array}$ & Dallas & Miami & Denver & San Jose & Hartford & Total & Percentage \\
\hline \multirow[t]{5}{*}{ Male } & $18-29$ & 3 & 9 & 16 & 11 & 6 & 45 & 15.00 \\
\hline & 30-39 & 5 & 8 & 6 & 5 & 8 & 32 & 10.67 \\
\hline & $40-49$ & 8 & 7 & 9 & 2 & 5 & 31 & 10.33 \\
\hline & $50-59$ & 7 & 11 & 3 & 5 & 6 & 32 & 10.67 \\
\hline & $60+$ & 3 & 2 & 2 & 3 & 0 & 10 & 3.33 \\
\hline \multirow[t]{5}{*}{ Female } & $18-29$ & 10 & 10 & 8 & 9 & 14 & 51 & 17.00 \\
\hline & 30-39 & 7 & 5 & 5 & 5 & 2 & 24 & 8.00 \\
\hline & $40-49$ & 9 & 6 & 5 & 6 & 11 & 37 & 12.33 \\
\hline & $50-59$ & 8 & 9 & 7 & 6 & 4 & 34 & 11.33 \\
\hline & $60+$ & 2 & 0 & 0 & 2 & 0 & 4 & 1.00 \\
\hline \multicolumn{2}{|l|}{ Total } & 62 & 67 & 61 & 54 & 56 & 300 & 100.00 \\
\hline
\end{tabular}

- How much time it took the participant on average to commute from work each day;

- How much the participant's travel time to and from work could vary from day to day; and

- Participant's travel requirements during the work day.

The gender and age distribution of the participants is shown in Table 7.5. A total of 150 females and 150 males participated in the surveys. Additional information that was collected about the participants, their driving characteristics, and the flexibility of their work schedules is included in Appendix D.

The sample represented a wide variety of education and employment. The percentage of participants with a bachelor's degree, $23 \%$, was close to the comparable U.S. Census number of $27 \%$. The ages of the participants were well distributed across the age range recruited. Ninety-three percent of participants drove daily or a few times a week. Nearly 50\% of the participants had jobs for which they had to arrive at work at a specific time; the remaining participants had some flexibility in when they arrived. Most of the participants did not drive much during the day for their jobs. Our sample purposely included people who did not work outside the home because this group often takes multiple mid-day trips for household errands and may be more flexible in travel planning.

Average daily commute times ranged from less than $10 \mathrm{~min}$ to more than $50 \mathrm{~min}$. The background questions also asked how much a person's travel time varied from day to day. The values for this question depended on the average trip time but also ranged from $5 \mathrm{~min}$ to greater than $30 \mathrm{~min}$. Interestingly, 15 people reported that their $21 \mathrm{~min}$ to $30 \mathrm{~min}$ trip could vary by more than $30 \mathrm{~min}$. Such comments could be an indication that those people misunderstood that the question was asking about additional travel time above the average and instead answered by choosing the category representing their total trip time.

\section{Experimental Design and Procedure}

The survey was developed using the survey software SuperLab. The software allows measurement of keystroke logging. A binder containing printouts of website screenshots was also used for sections of the survey.

All survey questions were answered on a computer using a seven-key response box. Figure 7.1 shows the response box used for the study. The use of a simple response box reduced

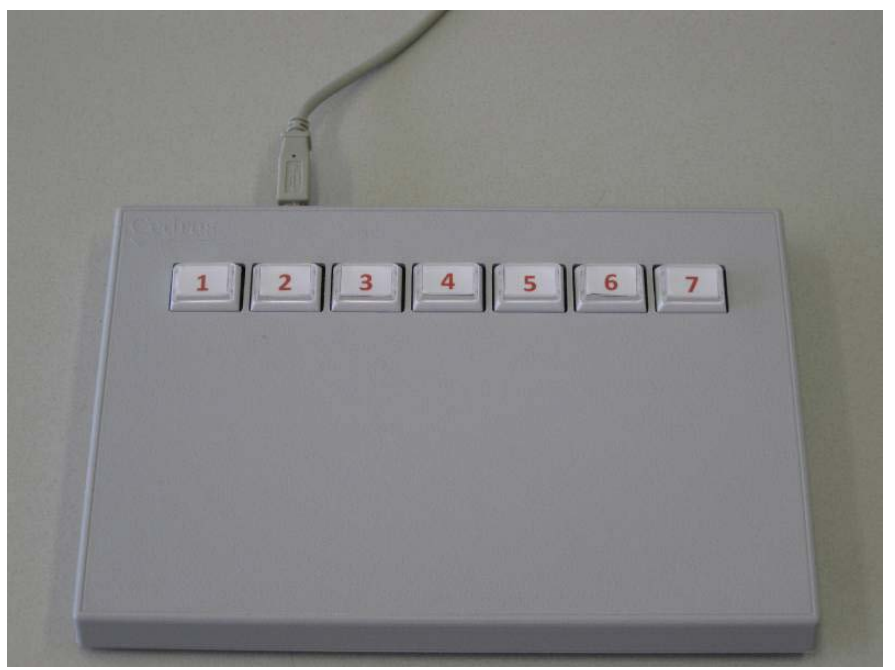

Figure 7.1. Button box used for driver survey response entry. 
entry errors and made completing the survey easier for people who did not routinely use a computer keyboard. The button box limited the survey to multiple-choices questions, with no open-ended answer opportunities.

Before beginning the study, each participant read and signed a consent form explaining the details of the survey and his or her rights as a participant.

At the beginning of the survey, participants were given the following instructions:

- Today you will be viewing website screens in the binder next to you and then will be asked questions about what you see. You will use the button box you see in front of you labeled 1-7 to enter your responses.

- You will only need to flip the binder page when you see the symbol to the right.

- Please raise your hand if you have a question at any time.

The instructions included a picture of an orange book symbol that appeared on the computer screen to alert participants to view a binder slide.

\section{Results}

\section{Questions 1-4: Reliability Concepts}

The first set of survey questions was aimed at uncovering participants' preconceived ideas of four different terms for reliability that were frequently used in the focus groups. Instructions on the computer screen read, "Now we will ask you a set of questions about what words you use to describe the trips you take. (You will not need to use the binder yet)."

Questions 1 through 4 were constructed using short descriptions of four trips, identified with fictional travelers' names (Laura's trip, Bob's trip, Sue's trip, and Tom's trip). The numerical values provided for the described trips all represented an underlying average travel time of $25 \mathrm{~min}$, while representing different travel time reliability concepts. Table 7.6 lists the four trip descriptions and the travel time reliability concept that each description represented.
Table 7.6. Questions 1-4: Trip Descriptions and Corresponding Reliability Concepts

\begin{tabular}{|c|c|}
\hline Trip Description & Reliability Concept \\
\hline $\begin{array}{c}\text { "Laura's trip is } 20 \text { miles and ordinarily } \\
\text { takes } 25 \text { minutes." }\end{array}$ & $\begin{array}{c}\text { Typical travel time (most } \\
\text { frequently occurring) }\end{array}$ \\
\hline $\begin{array}{c}\text { "Bob's trip is } 20 \text { miles and can take } \\
\text { anywhere from } 15 \text { to } 40 \text { minutes." }\end{array}$ & $\begin{array}{c}\text { Low reliability trip with a } \\
\text { large travel time range }\end{array}$ \\
\hline $\begin{array}{c}\text { "Sue's trip is } 20 \text { miles and always takes } \\
\text { less than } 30 \text { minutes." }\end{array}$ & Maximum travel time \\
\hline $\begin{array}{c}\text { "Tom's trip is } 20 \text { miles and can take } \\
\text { anywhere from } 20 \text { to } 30 \text { minutes." }\end{array}$ & $\begin{array}{c}\text { High reliability trip with a } \\
\text { small travel time range }\end{array}$ \\
\hline
\end{tabular}

Each question featured a different underlined term and asked participants to choose which of the four described trips the term best described:

- Question 1: Whose trip is the most reliable?

- Question 2: Whose trip is the most predictable?

- Question 3: Whose trip is the most consistent?

- Question 4: Whose trip is the best trip?

In this way, participants were given a term, such as reliable, and asked which of the described trips best fit that term. The same four trip descriptions were repeated in each of the four questions.

A summary of the responses across this set of questions is shown in Table 7.7. For all four terms seen by participants (reliable, predictable, consistent, and best trip), the trips described as using the typical travel time or the maximum travel time were selected most often. Notably, participants' responses showed little difference in preference for the terms in options 2 and 4 even though the trips described had considerably different travel time ranges: one with a wide range of $25 \mathrm{~min}$ (option 2) and one with a narrow range of $10 \mathrm{~min}$ (option 4). The term consistent was selected equally for trips that varied by a range of $25 \mathrm{~min}$ (option 2) and for a trip with a range of $10 \mathrm{~min}$ (option 4). This result could reflect participants' dislike for examining multiple numbers, expressed as a range.

Table 7.7. Questions 1-4 Response Summary

\begin{tabular}{|l|c|c|c|c|}
\hline Response Choice & $\begin{array}{c}\text { Question 1: } \\
\text { Reliable } \\
\text { (\%) }\end{array}$ & $\begin{array}{c}\text { Question 2: } \\
\text { Predictable } \\
\text { (\%) }\end{array}$ & $\begin{array}{c}\text { Question 3: } \\
\text { Consistent } \\
\text { (\%) }\end{array}$ & $\begin{array}{c}\text { Question 4: } \\
\text { Best Trip } \\
\text { (\%) }\end{array}$ \\
\hline 1. Laura (typical travel time) & 40.00 & 42.33 & 49.33 & 47.67 \\
\hline 2. Bob (large range of travel times) & 8.00 & 10.67 & 5.67 & 8.33 \\
\hline 3. Sue (maximum travel time) & 39.00 & 37.00 & 39.33 & 26.67 \\
\hline 4. Tom (small range of travel times) & 13.00 & 10.00 & 5.67 & 17.33 \\
\hline
\end{tabular}




\section{Question 5: Importance of Trip Planning Factors}

The aim of Question 5 was to understand what factors are important to people when planning a trip, or more specifically, whether or not people even consider reliability when making trip planning decisions. The word predictable was used in the question option to represent reliability and was selected because it was often used in the focus groups. Question 5 asked participants "When taking a trip over 20 miles, which is the most important to you?" The provided response options for this question were as follows:

- Choosing a route that takes the least amount of time on the road;

- Choosing a route that takes the fewest number of miles to get to my destination; and

- Choosing a route that has the most predictable travel time, even though it is more miles.

The majority of the participants, $68.67 \%$, said they would choose a route that takes the least amount of time. Fewer than $20 \%$ selected "most predictable travel time." In retrospect, the wording of this particular first answer option may have led people to discount the importance of predictability when the first option was worded with such certainty. An improved version of this question may have added the phrase "or time" to the last option. These data are presented in further detail in Figure 7.2, which shows the pattern of responses as a function of the respondent's work arrival flexibility as indicated in the preliminary demographics questions. This figure shows predictable travel times are more important for those with inflexible work start times than they are for those with more flexible work schedules. This result is reasonable given that workers with inflexible work start times need to know they will arrive at work on time, because they cannot afford to be late. Results according to average commute time to work are shown in Table 7.8.

\section{Questions 6-8: Terms for Website Trip Planning Feature Links}

In preparation for Question 6, participants received instructions on the computer screen to open the binder to page 1. Page 1 of the binder was a picture representing the home page of the simulated travel information website. This screen was designed to resemble a page that travelers would likely see first when accessing the website of a transportation management center or 511 center, including links for general news, advertisements, social networking options, and assorted menus. The only indication that the simulated website had trip planning information available was a features box with a group of links to "current trip," "future trip," and "traffic alerts." (When reviewing the handful of websites that offered travel time reliability information, the research team had noticed that it was not always obvious where to find that information on the website.) The website images were created by the research team to be generic and did not include real road numbers or destination names.

"When taking a trip over 20 miles, which is the most important to you?"

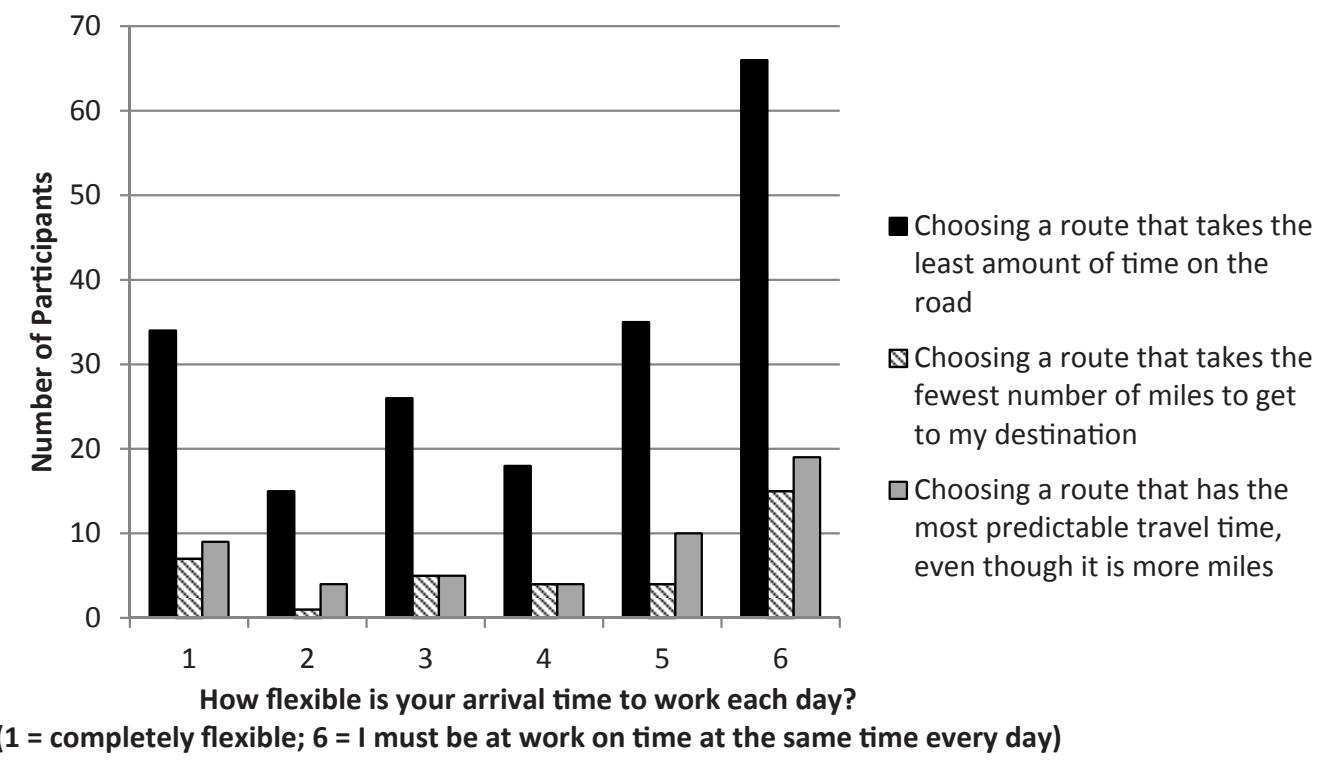

Figure 7.2. Question 5 responses by arrival time flexibility. 
Table 7.8. Question 5 Responses by Average Commute Time to Work

\begin{tabular}{|c|c|c|c|c|c|c|c|}
\hline \multirow[b]{2}{*}{$\begin{array}{l}\text { When taking a trip over } 20 \text { miles, which is the } \\
\text { most important to you? }\end{array}$} & \multicolumn{7}{|c|}{ How much time does it take you on average to commute to work each day? } \\
\hline & $<10 \min$ & $11-20 \mathrm{~min}$ & 21-30 min & $31-40 \mathrm{~min}$ & $41-50 \mathrm{~min}$ & $>50 \mathrm{~min}$ & $\begin{array}{c}\text { I don't work } \\
\text { outside the } \\
\text { home. }\end{array}$ \\
\hline $\begin{array}{l}\text { Choosing a route that takes the least amount of } \\
\text { time on the road. }\end{array}$ & $68 \%$ & $66 \%$ & $70 \%$ & $78 \%$ & $53 \%$ & $69 \%$ & $74 \%$ \\
\hline $\begin{array}{l}\text { Choosing a route that takes the fewest number of } \\
\text { miles to get to my destination. }\end{array}$ & $18 \%$ & $14 \%$ & $13 \%$ & $3 \%$ & $18 \%$ & $23 \%$ & $9 \%$ \\
\hline $\begin{array}{l}\text { Choosing a route that has the most predictable } \\
\text { travel time, even though it is more miles. }\end{array}$ & $15 \%$ & $20 \%$ & $17 \%$ & $19 \%$ & $29 \%$ & $8 \%$ & $17 \%$ \\
\hline$n$ & 40 & 99 & 71 & 37 & 17 & 13 & 23 \\
\hline
\end{tabular}

Websites that offer reliability-based planning tools will need to be careful to distinguish these planning tools from real-time information. Usability benefits might well accrue from designing input and output screens of reliabilitybased planning tools with a look and feel similar to realtime information sites maintained by the same agency. This similarity, however, could result in confusion between what is real time and what are predictions based on historical data. Focus group participants indicated that they would like a system that blended real-time and historical data to make better predictions. They also indicated that they didn't entirely trust solely historical data for predictions and would double-check current conditions on a real-time system before departing. The systems presented in this survey assumed that these two functions would be separate. The researchers felt that it was important to isolate the reliability-based planning tools in the study given the lack of research and knowledge in this area. Without this isolation, the results would be confounded by the influence of real-time information. Future research could investigate the blending of these two information sources-the expressed preference among focus group participants.

For Questions 6 through 8, researchers wanted to briefly assess participants' understanding that the developed website focused on historical trip planning as opposed to real-time information. Questions 6 and 7 asked about the terms current and future to assess participants' ideas of the time frame for planning (i.e., future means later in the day or a future day, not just a few minutes from now). Question 8 approached the future trip concept in a slightly different way by specifically asking about the purpose of entering day and time in the input box. All three questions included the response option "I don't know" because, at this point in the survey, a thorough explanation of reliability had not been provided to the participants. These early questions were intended to gauge participants' naïve understanding of the terms.
Question 6 asked "If you click on the Current Trip button, what do you think you'll get?" Response options for this question were as follows:

- It will take me to a webpage that will allow me to enter information about a trip I want to take right now.

- It will take me to a webpage that will tell me what all the roadways in the area are like right now.

- It will take me to a webpage that will allow me to enter information about a trip I want to take later in the day or tomorrow.

- I don't know.

The responses to Question 6 indicated that about half the participants believed that when they selected the Current Trip link on the website, they would be able to enter and obtain information about their specific trip at that point in time, and approximately $30 \%$ believed the website would tell them about general roadway conditions at that point in time. The response rates indicated some confusion over whether the site would be providing personalized or general information. Given that this was the first question using the binder slides, this result carried less importance, especially considering the results from Question 7.

Question 7 asked about the Future Trip link shown on the simulated webpage: "If you click on the Future Trip button, what do you think you'll get?" A new answer option was added to this question to allow participants to express skepticism at a system's ability to predict future travel times; the response options for this question were therefore as follows:

- It will take me to a webpage that will allow me to enter information about a trip I want to take right now.

- It will take me to webpage that will tell me what all the roadways in the area are like right now. 
- It will take me to a webpage that will allow me to enter information about a trip I want to take later in the day or tomorrow.

- Nothing, because there is no way to predict what a future trip would be like.

- I don't know.

The research team believed that some portion of respondents would likely not be able to conceive of a system that would make predictions beyond current conditions. As expected, $11.15 \%$ of the participants said there was no way to predict what a trip in the future would be like. Encouragingly, approximately $80 \%$ of the participants said the website would allow them to enter information about a trip they wanted to take later in the day or tomorrow. That result indicated a high comprehension of the term future to mean beyond the immediate time frame. Notably, however, participants had just answered the previous question, which contrasted future with current and may have prompted the comparison of the two terms.

Question 8 took a different approach to assessing participants' time frame comprehension. Before they looked at Question 8, participants were told to flip to a binder slide showing a detailed view of the Trip Planner website's input box; that instruction was followed by the statement "This is taken from a website you can use to find out information about a trip you want to take." The input box included input blanks for trip date and time, similar to the one used to enter parameters about a future trip on San Francisco's 511.org Predict-a-Trip web page. Question 8 asked, "Why do you think you enter a time and date when using this website?" Response options were as follows:

- Because the website does not know what day or time it is.

- Because I am wanting information about a trip I am about to take right now.

- Because I am wanting information about a trip I may take in the future.

- I don't know.

More than half the participants said they would be getting information about a trip in the future, but 35\% said they would be getting information about a trip they were about to take at that moment. And there were $9 \%$ who responded that "the website does not know what day or time it is."

These three questions taken together offer different ways to assess drivers' understanding of the real-time nature of a website such as this. Question 7 showed that $81 \%$ of participants understood the term future trip to mean a trip later in the day or tomorrow rather than one with a more immediate departure. The results of Question 8 then were somewhat surprising, as $35 \%$ of participants responded that they thought the purpose of the time input box was to indicate the trip was imminent. Further analysis on the relationship and pattern of responses across the two questions is shown in Table 7.9. Among those who indicated that future trip meant a nonimminent departure, only $52 \%$ thought that the purpose of entering a time and date would be for planning a future trip. This result indicates that the labeling of the input box needs to be consistent and clear. The results from Question 7 indicate that Future Trip may be a better label to confirm that the date and time being entered are for a future trip.

\section{Survey Section Providing Explanation of Sample Travel Time System}

Before continuing with the survey, researchers wanted to make sure that participants had a clear understanding of the website system and that it was using historical data to help plan a trip rather than providing real-time information. What makes reliability information different from real-time information is that it can only be calculated from historical data; historical data provide the travel time frequency distributions from which reliability performance measures are derived. Thus, researchers needed to spell out this distinction for the average driver participating in the surveys. Because so few traveler information websites identified in the literature review currently use historical data to make travel time predictions, researchers were relatively certain that few or none of the participants would have been exposed to them. A lengthy explanation, equating the information and experience of all participants, seemed warranted. The research team developed

Table 7.9. Question 8 Response Choices for One Answer to Question 7

\begin{tabular}{|l|c|c|c|c|}
\hline \multirow{2}{*}{$\begin{array}{l}\text { Participants Who Answered } \\
\begin{array}{l}\text { Question 7, Future Trip } \\
\text { Means ... }\end{array}\end{array}$} & $\begin{array}{c}\text { Why do you think you enter a time and date when using this website? } \\
\text { Boes not know what } \\
\text { day or time it is. }\end{array}$ & $\begin{array}{c}\text { Because I am wanting } \\
\text { information about a trip I am } \\
\text { about to take right now. }\end{array}$ & $\begin{array}{c}\text { Because I am wanting } \\
\text { information about a trip } \\
\text { I may take in the future. }\end{array}$ & $\begin{array}{c}\text { I don't } \\
\text { know. }\end{array}$ \\
\hline $\begin{array}{l}\text { It will take me to a webpage that will } \\
\text { allow me to enter information } \\
\text { about a trip I want to take later } \\
\text { in the day or tomorrow. }\end{array}$ & $9 \%$ & $35 \%$ & $52 \%$ & $4 \%$ \\
\hline
\end{tabular}


the following explanation using examples that performed well in the focus groups:

\section{The remaining survey questions will ask you about the setup and words used in a travel time website.}

This travel time system will make predictions about travel time based on detailed records of freeway conditions that the department of transportation keeps. The system uses data about all trips taken on freeways in the past 5 years and factors in expected delays to predict a very accurate trip time estimate for your specific trip.

It is different from existing mapping tools such as MapQuest because the system knows that travel time varies depending on factors such as traffic, special events, weather, and holidays.

As a driver, this tool would be very helpful in planning a trip you will take in the future. Users have the ability to enter a starting location and destination and the date, day of the week, and time you want to take your trip.

For example, if you had a doctor's appointment and wanted to know how long it would take to get there from your office, the system will give you a different time estimate on Monday morning at 7:30 a.m. than it will if you were to take your trip Wednesday afternoon at 3 p.m.

The important thing to remember is that this is not telling you about traffic now, it is telling you about a specific trip you choose for the future.

\section{Questions 9-10: Terms to Convey Immediacy of Departure}

Questions 9 and 10 continued to test potential terms to be used on a trip planning website but also tested participants' comprehension of the future trip concept as explained above. The binder slide accompanying Question 9 displayed the same trip planning web page with trip inputs for origin, destination, day, and time of a trip, as well as a circled website button. Participants were asked, "What do you think will happen when you fill in the fields in the box and then select the button circled?"

Question 9 was designed to test whether participants could distinguish between the type of information provided by a travel time reliability website and the types of information available through other types of mapping or travel information websites. The answer options provided for Question 9 were as follows:

- The map will display the route with the shortest distance.

- The website will give me step-by-step driving directions for my trip.

- The website will tell me how long my trip will take right now.
- The website will tell me how long my trip will take for the date/time I enter.

- The website will show me if there are any accidents or construction on my trip right now.

- It will do nothing.

- I don't know.

The wording of some of these answers came from comments heard in the focus groups. "The map will display the route with the shortest distance" and "The website will give me step-by-step driving directions for my trip" are answers that would describe a mapping website such as MapQuest or Google Maps; but those websites do not provide historical travel time information. "The website will tell me how long my trip will take right now" and "The website will show me if there are any accidents or construction on my trip right now" could both be descriptors for websites offering real-time traffic information. "It will do nothing" was included as an indicator that a participant might not understand the format or function of the input box.

Question 9 was the first question of the survey to vary by survey version, to present the same question using four different terms. This experimental design offered the opportunity to test multiple options without overwhelming each participant with a lengthy and repetitive survey. The term on the circled button shown in the web page picture in the binder varied among the versions: predict trip, plan trip, create trip, or get trip. Table 7.10 shows a comparison of the overall results for the four versions. For all four versions, the most common response was the correct one- "The website will tell me how long my trip will take for the date/time I enter"-with the term create trip receiving the most correct responses. The second most common response for all versions was "The website will give me step-by-step driving directions for my trip." A few participants who were deciding between those two responses commented to researchers that the response "The website will give me step-by-step driving directions for my trip" could also be correct, given that the website would likely provide both types of information (step-by-step route directions AND the expected travel time for the route). This was to be expected, as most Internet mapping websites do provide both route directions and an estimated travel time. Our survey, however, forced participants to choose a single response.

Because Question 9 had four different versions, Question 10 was added to the survey to give everyone a chance to see all four terms from all of the versions. Thus Question 10 simply asked for participants' preferred term for the button on a website to initiate the trip planning function. Question 10 response options were as follows:

- Predict trip;

- Plan trip;

- Create trip; 
Table 7.10. Question 9 Responses by Version

\begin{tabular}{|c|c|c|c|c|}
\hline Response Choice & $\begin{array}{l}\text { Version A: } \\
\text { Predict Trip (\%) }\end{array}$ & $\begin{array}{l}\text { Version B: } \\
\text { Plan Trip (\%) }\end{array}$ & $\begin{array}{l}\text { Version C: } \\
\text { Create Trip (\%) }\end{array}$ & $\begin{array}{l}\text { Version D: } \\
\text { Get Trip (\%) }\end{array}$ \\
\hline 1. The map will display the route with the shortest distance. & 7.89 & 4.05 & 6.67 & 14.86 \\
\hline 2. The website will give me step-by-step driving directions for my trip. & 17.11 & 27.03 & 16.00 & 20.27 \\
\hline 3. The website will tell me how long my trip will take right now. & 6.58 & 8.11 & 1.33 & 5.41 \\
\hline $\begin{array}{l}\text { 5. The website will show me if there are any accidents or construction on } \\
\text { my trip right now. }\end{array}$ & 1.32 & 1.35 & 0.00 & 0.00 \\
\hline 6. It will do nothing. & 2.63 & 1.35 & 2.67 & 0.00 \\
\hline 7. I don't know. & 1.32 & 2.70 & 1.33 & 1.35 \\
\hline
\end{tabular}

- Get trip;

- Submit;

- Go; and

- $O K$.

The first terms were the items used in the four versions of Question 9. The other three were taken from websites reviewed and were terms typically used on any type of website to initiate action after inputting options.

Before being presented with Question 10, the participants were asked to flip to page 6 in the binder and were told "This page in the binder shows the 'Trip Planner' box. When you fill in the information asked for in the box and hit the circled button with the question marks, the website will show you a route and travel time for the trip you entered." Question 10 asked participants to choose the term that best represented the purpose of the button:

- Predict trip;

- Plan trip;

- Create trip;
- Get trip;

- Submit;

- GO; and

- $\mathrm{OK}$.

As seen in Table 7.11, the most frequently selected terms were predict trip (28\%) and plan trip (25\%). Interestingly, the people who saw those terms in Question 9 (Versions A and B) were no more likely to answer that question correctly than people who saw the other terms, but Question 10 shows an overall preference for them. Normal computer action initiation terms such as $G O$ and $O K$ were not preferred.

The repeating of all possible terms in Question 10 provided an opportunity to assess whether respondents were consistent in their choices or whether they preferred a term in Question 10 over the single term tested in Question 9. Table 7.11 shows preferences expressed in Question 10when shown all the terms - broken out by responses in Question 9-when shown a single term. The table shows that most people preferred a different term than the one they saw in Question 9.

Table 7.11. Question 10 Responses by Term Viewed in Question 9

\begin{tabular}{|l|c|c|c|c|}
\hline \multirow{2}{*}{$\begin{array}{l}\text { Response to } \\
\text { Question 10 } \\
\text { when Selecting } \\
\text { Preferred Term }\end{array}$} & $\begin{array}{c}\text { Version A: } \\
\text { Predict Trip (\%) }\end{array}$ & $\begin{array}{c}\text { Version B: } \\
\text { Plan Trip (\%) }\end{array}$ & $\begin{array}{c}\text { Version C: } \\
\text { Create Trip (\%) }\end{array}$ & $\begin{array}{c}\text { Version D: } \\
\text { Get Trip (\%) }\end{array}$ \\
\cline { 2 - 5 } Predict trip & 36 & 25 & 25 & 27 \\
\hline Plan trip & 25 & 27 & 28 & 19 \\
\hline Create trip & 12 & 13 & 19 & 14 \\
\hline Get trip & 4 & 4 & 7 & 14 \\
\hline Submit & 17 & 15 & 17 & 22 \\
\hline GO & 3 & 8 & 3 & 4 \\
\hline OK & 4 & 8 & 1 & 1 \\
\hline
\end{tabular}




\section{Questions 11-12: Terms to Describe Departure and Arrival Time Constraints}

The background website screenshot for Questions 9 and 10 included a box labeled "Time," which was not specifically part of Questions 9 and 10 and was only briefly mentioned in the explanation. The focus groups had indicated that people need to some plan trips around departure time constraints and other trips around arrival time constraints. Some websites reviewed provided the option to indicate either a departure or arrival time. The survey did not specifically ask for a preference for entering arrival or departure time because the constraints are so trip specific. As a best practice, the researchers recommend that travel information website developers should consider offering departure and arrival constraints as an input option.

Questions 11 and 12 addressed preferences for the term or phrase that best described the time participants would want to start their trip or the time they would want to reach their destination. Terms and phrases that were presented as answer choices in these questions came from the reviewed travel time websites.

For a trip's start time (Question 11), the largest percentage of participants (35\%) preferred the phrase Departing at. Leave at (23\%) and What time will you start your trip? (21\%) ranked second and third in popularity. None of those terms was a clear winner, but the top three preferences all shared the feature of being a specific time rather than an upper limit for a time range for departure time.

With regard to a trip's arrival time, Question 12 asked, "What phrase do you prefer to describe what time to you want to reach your destination?" Response options were as follows:

- Arrive at.

- Arrive by.

- What time do you want to get there?

- What's the earliest you can arrive?

- What's the latest you can arrive?

For the trip arrival time input, $47 \%$ of participants preferred arrive by, and 33\% preferred arrive at. These responses showed a willingness to accept uncertainty in arrival time by choosing $b y$, which can mean "no later than." The two results combined showed a preference for specific departure times but a tolerance for early arrival. An $X^{2}$ test (goodness-of-fit test) showed significant differences between the five responses overall and also between arrive at and arrive by. This means that arrive by is the best term to use to ask for desired arrival time input.

\section{Questions 13-14: Map and Travel Time Output Terminology}

Before moving on to Questions 13 and 14, the participants were asked to flip to page 8 in the binder showing the Trip
Planner site. They were then presented with the following information:

This page in the binder shows the map of the Forest City area. It also shows an "Advanced" link at the bottom of the Trip Planner box. When you select the circled link, you will be taken to a webpage that allows you to enter additional information about the trip you'd like to take. Now flip to page 9. The orange segments on this map indicate where data has been collected on the roadways in the past to predict future travel times. Only the orange segments have this data and are able to be selected for your route. This map does not tell you anything about the current conditions of the roadways.

The website screenshots shown on pages 8 through 10 of the binder displayed a map of roadways. Selected roadways, meant to represent the fictional city's network of freeways and other major roadways, appeared as heavy orange lines; many smaller roads were shown as thin gray lines. In the actual cities and regions that currently collect travel time data, the data are most often collected only on freeways and occasionally on some managed lanes and facilities; they are generally not available for arterial and local streets. Data collection practices may change in the future, but for now, websites need to convey to drivers that a travel time prediction (whether real time or based on historical data) is only valid for roadways on which data are being collected.

The screenshot on page 11 of the binder displayed the same underlying map, but with a superimposed line marking the route of a particular trip. That screenshot accompanied Question 14, which was the first question to address outputs from a travel time reliability website.

The questions that referred to the binder maps attempted to address participants' understanding of the roadway segments in the database. One of the research team's observations during the website review was that labeling output can affect understanding. Thus, Questions 13 and 14 addressed titles for these system coverage maps. Some of the terms and phrases offered as potential map titles were taken from existing travel time websites; additional options were developed by the research team.

Question 13 asked participants to choose a map title, or phrase, to describe the website map before a route had been selected. Available responses for this question were as follows:

- Map of Forest City;

- Road segments with available travel information;

- Road segments with historical data;

- Forest City's available travel information; and

- Forest City's historical data. 
For the map showing all available routes, participants most frequently preferred the two phrases that included available travel information in the title. Forest City's available travel information was preferred by $34 \%$ of participants, and road segments with available travel information was preferred by $32 \%$. Map of Forest City was preferred by $18 \%$ of participants. The phrases containing historical data performed relatively poorly, with $11 \%$ of participants preferring road segments with historical data and 6\% preferring Forest City's historical data.

Beginning with Question 14, the survey began to change focus. Questions 6 through 13 addressed terminology that could potentially become part of a lexicon for travel time reliability information, but those terms pertain to user inputs about potential trips. The next questions focused less on terminology pertaining to website usability and more on terms for conveying travel time reliability. These questions addressed the types of terms that could be used for the outputs of a travel time reliability website or other information delivery system; and these are the terms that would likely form the core of a travel time reliability lexicon.

For Question 14 participants were provided a trip scenario: "This map displays a route based on the start and end locations and the trip times you specified to arrive at your destination at 8:15 a.m." The question then asked participants' their preference for a title term for the output map that displayed a calculated trip route. The options provided for Question 14 were selected from the reliability literature review and the terms used in the focus groups:

- Best route;

- Least variable time;

- Most consistent trip;

- Most reliable trip;

- Most predicable trip;

- Forecasted trip; and

- Historical trip conditions.

The most frequent response was best route, selected by $49 \%$ of participants. This concurred with the focus group feedback that indicated a preference for simple terms.

\section{Questions 15-17: Trip Time Output Terminology}

Existing websites and focus group results provided a large number of terms that could potentially be used to describe projected trip times and the variability of those times. The research team used many of those terms as answer options in Questions 15 through 28.

Questions 15 through 17 tested terms that could be used to describe a projected trip time based on historical information. Questions 15 and 16 were included to test how participants would view a historically based trip time provided by a website (or other information source). Would they assume, on the basis of the terms used, that the given trip time was an average or midpoint of a range, that it was a near-guarantee, or conversely that it would be unreliable?

Because of the number of terms that the research team wished to test, the same question was asked as Question 15 and Question 16; the trip time term tested in each of the questions varied among four survey versions, as shown in Table 7.12.

Question 15 tested the first four terms describing a trip time, varying by survey version: average, typical, historical, or estimated trip time. The question asked participants how often they would expect the trip to take the indicated time of $25 \mathrm{~min}$ or less based on the term used. Five answer choices were shown:

- Almost never (1 day out of 20);

- Less than half the time (5 days out of 20);

- About half the time (10 days out of 20);

- More than half the time (15 days out of 20); and

- Nearly all the time (19 days out of 20).

The answer choice "nearly all the time (19 days out of 20)" was based on the Washington State DOT's travel time reliability website, which provides a 95th percentile trip time based on historical data and in the past expressed 95th percentile as " 19 out of 20 ." The answer choice "about half the time (10 days out of 20)" would be an appropriate certainty level for a website providing a historically based average trip time. "More than half the time (15 days out of 20)" was based on a focus group response. The other two options were added by the research team to allow a full range of confidence levels.

Of the terms tested in Question 15's four versions, version B (typical trip time) scored the highest: $58 \%$ of participants seeing this version indicated they would expect the trip to take 25 minutes or less "most of the time." Average and historical trip time terms scored the highest for participants choosing "nearly all of the time." Notably, although participants preferred the term average, the results from Question 15, Version A, indicated that they misunderstood the mathematical definition of

Table 7.12. Experimental Design for Questions 15 and 16

\begin{tabular}{|l|l|l|l|l|}
\hline \multirow{2}{*}{ Question } & \multicolumn{4}{|c|}{ Version of Survey } \\
\cline { 2 - 5 } & \multicolumn{1}{|c|}{ A } & B & C & D \\
\hline 15 & Average & Typical & Historical & Estimated \\
\hline 16 & 95 th percentile & Worst case & Maximum & Most common \\
\hline
\end{tabular}


How often would you expect this trip to take $\mathbf{2 5}$ minutes or less?

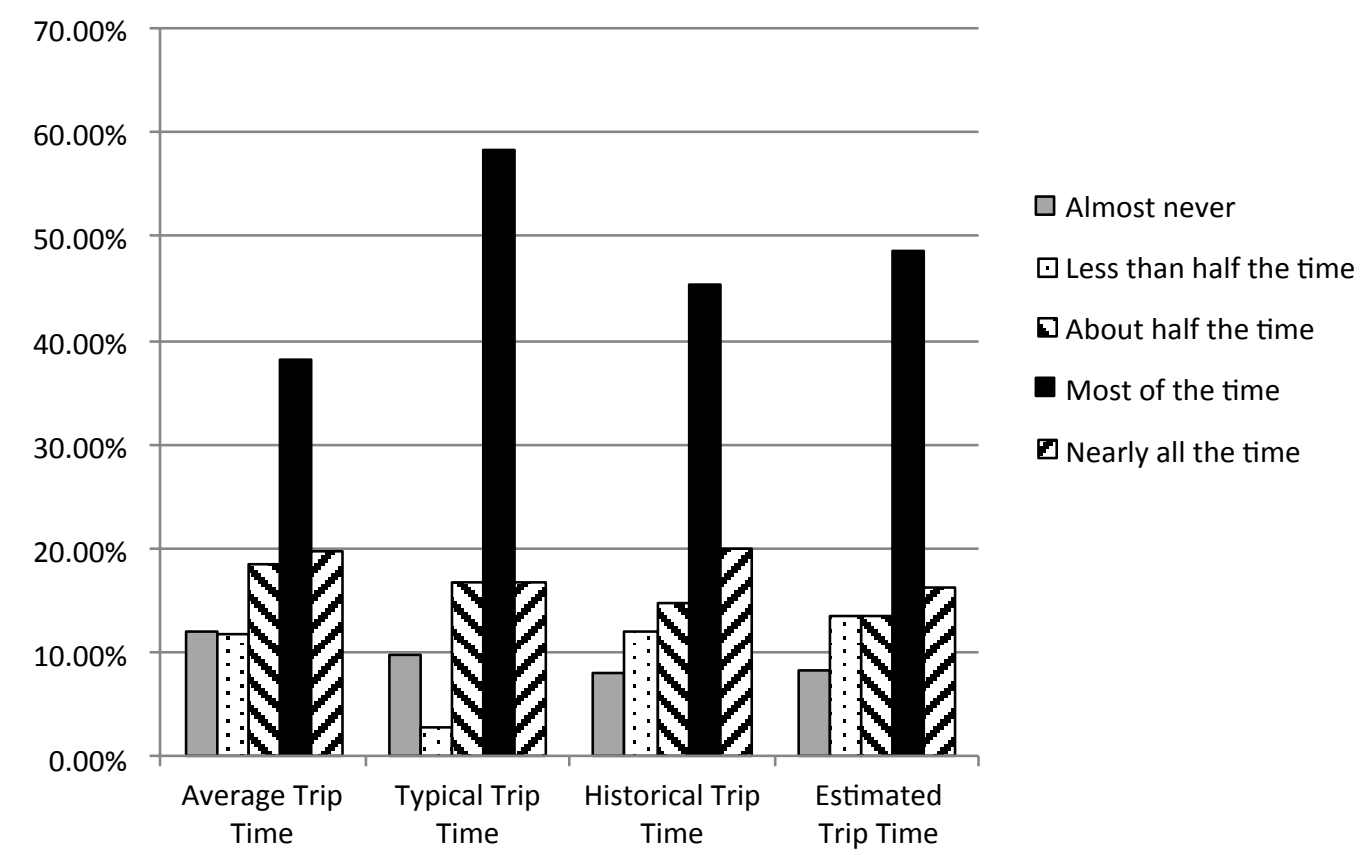

Figure 7.3. Combined responses to Questions 15 and 16.

average. More than half of the participants for this version indicated that average meant that the trip time would be in the expected range most or nearly all of the time; mathematically the correct answer would have been "about half the time," but only $18 \%$ of participants selected that answer.

Question 16 asked the same question providing a different term in the circled region, again varying by version: 95 th percentile, worst case, maximum, or most common trip time.

Of the terms tested in Question 16, the term most common trip time scored the highest percentage for the answer choice "most of the time," with $43 \%$ of participants selecting this response; most common scored the lowest (16\%) for the answer choice "nearly all of the time." The term 95th percentile trip time scored the highest for "nearly all of the time." But even for that term, only $37 \%$ of participants correctly chose the mathematical meaning of the term.

Because the eight reliability terms were spread across two questions, Figure 7.3 is helpful for showing how responses to Questions 15 and 16 compared across the terms tested. If a traveler information service provider wishes to label a trip time prediction that is derived from the 95 th percentile times, these data suggest that the terms maximum trip time and 95th percentile trip time would be the best. However, note that focus group results showed a strong dislike of the statistical-sounding term 95th percentile. Also note how poorly the term worst case did in this question. The 95th percentile could indeed be thought of as a near-worst-case travel time prediction, but when the worst case term was used on the output box, many people thought that it would take $25 \mathrm{~min}$ or less rarely, when it was intended to convey the opposite. If the worst-case travel time is reported as $25 \mathrm{~min}$, then the trip will nearly always take less than $25 \mathrm{~min}$.

Another way to examine these results is to look at the number of responses to the highest two categories: "most" and "nearly all." If the purpose of the term is to convey that this is the trip time estimate that the system has the most confidence in, then these two highest categories could be considered a success. Using this metric, the term typical does the best job of conveying that the provided trip time will encompass the actual trip time most or nearly all of the time, as shown in Table 7.13.

The experimental design purposely allowed each participant to see only two of the eight terms being evaluated to avoid bias and reduce survey length. Question 17 allowed

Table 7.13. Responses for "Most" or "Nearly All" for Questions 15 and 16

\begin{tabular}{|l|c|}
\hline $\begin{array}{l}\text { Term from } \\
\text { Questions 15 and 16 }\end{array}$ & $\begin{array}{c}\text { Total Responses for "Most" } \\
\text { or “Nearly All” of the Time (\%) }\end{array}$ \\
\hline Average & 57.90 \\
\hline Typical & 75.00 \\
\hline Historical & 65.33 \\
\hline Estimated & 64.87 \\
\hline 95th percentile & 64.47 \\
\hline Worst case & 38.66 \\
\hline Maximum & 53.33 \\
\hline Most common & 59.46 \\
\hline
\end{tabular}


participants to indicate their preference among terms from the larger set. Seven of the eight travel time terms from Questions 15 and 16 were presented to participants in Question 17 (a maximum of seven terms could be tested in any given question because of the seven answer choices available with the button pad). Given this limitation, the research team members decided to drop most common trip time from the preference rating question because they believed that an agency would not likely want to use trip time statistical mode in its traveler information. When asked to choose a preferred term to grammatically modify trip time, $43 \%$ of participants chose estimated, and $33 \%$ chose average.

\section{Questions 18-20: Planning and Buffer Time Terminology}

Questions 18 through 20 presented travel time reliability information as a recommended departure time rather than as an estimated trip time. Many current map and navigation systems simply provide an estimated trip time, and users must subtract that time from the desired arrival time to calculate when to leave (or add it to the desired departure time to calculate when they will arrive). The questions in this section began by providing participants with the scenario that the website was "telling you the time to leave, and the map displays a route based on the start and end locations and the time of day you specified." In this way, Questions 18 through 20 explored whether participants understood that the website had already included a buffer time in its total trip time estimate. From the focus groups, researchers knew that the majority of people would not trust a system that provided an overall trip time and would add their own buffer on top of it. This inclination could pose a real problem for systems that use reliability data to calculate a predicted trip time which accounts for the variability and cushion time needed. If users add their own buffer on top of those estimates, they would most likely arrive far too early.

Question 18 presented alternative ways of expressing the technical concept of planning time, which represents the total travel time that should be allowed. Planning time is calculated by some researchers as an index and by others as an actual time representing the sum of some central tendency or freeflow condition plus some buffer time. Question 19 asked about terms that could be used for buffer time, which FHWA defines as the extra time, or time cushion, that travelers add to their average travel time.

The Washington State DOT's travel time website for the Seattle area provides a recommended departure time based on the origin, destination, day of travel, and time of arrival specified by the traveler. Similar to Questions 15 and 16, Question 18 tested different terms describing a provided departure time to see what the effect of each of those terms was on the credibility of the departure time provided to participants. For this question, the research team assumed the provided departure time to be based on a 95th percentile trip time.

The binder slide for Question 18 showed a circled departure time term that varied across the four survey versions: recommended, estimated, 95th percentile, or suggested departure time. All those terms appear on travel time websites and/or were mentioned in the focus groups. The question asked participants how often they would expect to arrive at their destination on time if they left at the departure time provided by the fictional website. Table 7.14 includes the overall results for the four versions; the same results are illustrated in Figure 7.4.

Comparing the four versions with the four different departure time terms, researchers found that only about 39\% of participants said they would expect to arrive on time "nearly all of the time" when the term 95th percentile was used, demonstrating a lack of understanding of the mathematical meaning of the term. The small number of people who selected "nearly all of the time" as a response is troubling and indicates some lack of trust in the accuracy of such predications. However, across the four different departure time terms, a majority_-though not overwhelming —of respondents realized that they would arrive on time "most of the time" given the information provided. Although no term stood out clearly, they could all be used on a website, albeit with a descriptor. Furthermore, given that only a fraction of respondents understood the term 95th percentile, researchers believe that most

\section{Table 7.14. Responses to Question 18}

\begin{tabular}{|c|c|c|c|c|}
\hline Response Choice & $\begin{array}{c}\text { Version A: } \\
\text { Recommended } \\
\text { Departure Time (\%) }\end{array}$ & $\begin{array}{c}\text { Version B: } \\
\text { Estimated } \\
\text { Departure Time (\%) }\end{array}$ & $\begin{array}{c}\text { Version C: } \\
\text { 95th Percentile } \\
\text { Departure Time (\%) }\end{array}$ & $\begin{array}{c}\text { Version D: } \\
\text { Suggested } \\
\text { Departure Time (\%) }\end{array}$ \\
\hline 1. Almost never ( 1 day out of 20 ) & 2.67 & 5.33 & 4.00 & 1.37 \\
\hline 2. Less than half the time (5 days out of 20 ) & 4.00 & 4.00 & 2.67 & 5.48 \\
\hline 3. About half the time ( 10 days out of 20 ) & 18.67 & 10.67 & 13.33 & 19.18 \\
\hline 4. Most of the time ( 15 days out of 20 ) & 58.67 & 65.33 & 41.33 & 52.05 \\
\hline 5. Nearly all the time (19 days out of 20 ) & 17.33 & 14.67 & 38.67 & 23.29 \\
\hline
\end{tabular}


If you departed from your starting point at the time shown, how often would you expect to arrive at your destination on time?

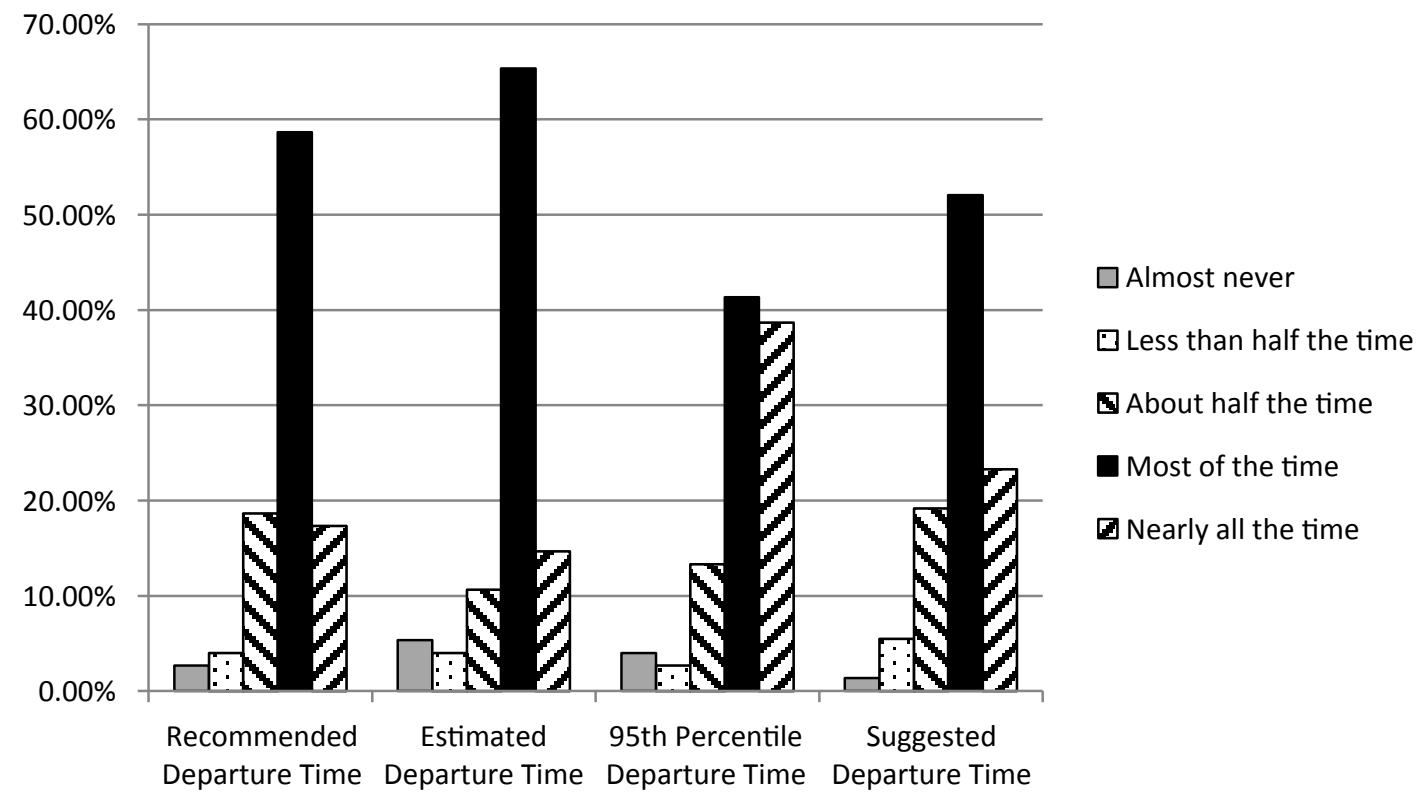

Figure 7.4. Illustration of responses to Question 18: planning time terms.

participants would build in their own buffer time, even though it is already included in the time provided.

As a follow up, Question 19 tried to determine what additional travel time, if any, participants would add depending on the term used to describe the provided departure time. Participants were asked, "If it is important that you arrive at your destination at 8:15, what time would you leave for this trip?" Possible responses were in 5-min increments from 7:30 (20 min before the departure time provided by the website) to 8:05 (15 min after the departure time provided by the website). This question was included as an additional indicator of how participants viewed the credibility of the provided departure time. Would people add additional buffer time to the provided total trip time?
Comparison of the results for all versions of Question 19 are shown in Table 7.15 and in Figure 7.5. Regardless of the term used, a majority of participants chose to leave earlier than the departure time provided by the fictional website. That result is important to note because the terms tested were proposed as surrogates for planning time, which presumably already includes a buffer time. The results indicate either a lack of understanding or a lack of trust in the departure times provided by such a system and are consistent with many of the comments made by the focus groups as well.

Because each person only saw one potential term in the previous two questions, depending on his or her survey version, Question 20 presented a list of departure time terms (recommended departure time, estimated departure time, 95th percentile

Table 7.15. Question 19 Visuals and Responses by Version

\begin{tabular}{|l|c|c|c|c|}
\hline Response Choice & $\begin{array}{c}\text { Version A: } \\
\text { Recommended } \\
\text { Departure Time (\%) }\end{array}$ & $\begin{array}{c}\text { Version B: } \\
\text { Estimated } \\
\text { Departure Time (\%) }\end{array}$ & $\begin{array}{c}\text { Version C: } \\
\text { 95th Percentile } \\
\text { Departure Time (\%) }\end{array}$ & $\begin{array}{c}\text { Version D: } \\
\text { Suggested } \\
\text { Departure Time (\%) }\end{array}$ \\
\hline 1. 20 minutes before departure time shown & 35.53 & 36.00 & 29.33 & 35.14 \\
\hline 2. 10 minutes before departure time shown & 27.63 & 34.67 & 34.67 & 35.14 \\
\hline 3. 5 minutes before departure time shown & 14.47 & 20.00 & 16.00 & 18.92 \\
\hline 4. At departure time shown & 18.42 & 4.00 & 1.33 & 6.76 \\
\hline 5. 5 minutes after departure time shown & 2.63 & 1.33 & 0.00 & 2.70 \\
\hline 6. 10 minutes after departure time shown & 0.00 & 4.00 & 1.30 & 1.35 \\
\hline 7. 15 minutes after departure time shown & 1.32 & 0.00 & & 2.00 \\
\hline
\end{tabular}


If it is important that you arrive at your destination at 8:15, what time would you leave for this trip?

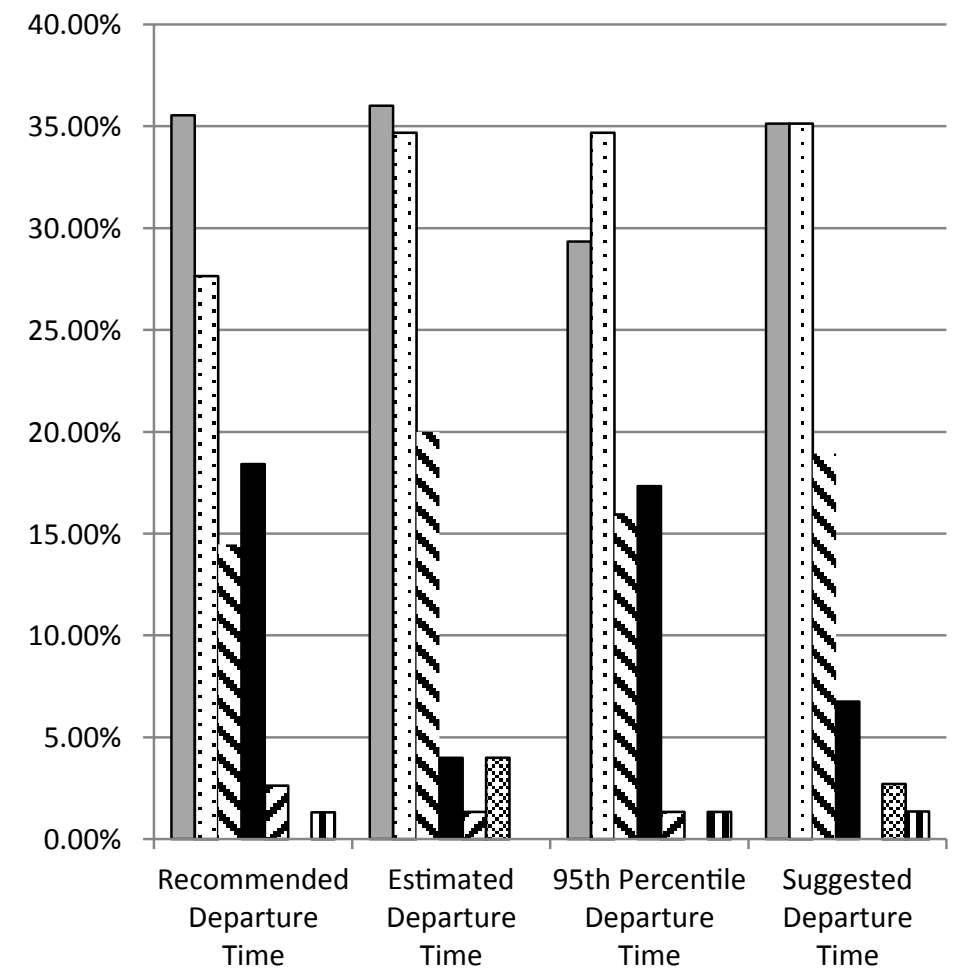

$\square 20$ minutes before
departure time
shown
$\square 10$ minutes before
departure time
shown
$\mathbf{\nabla}$ minutes before
departure time
shown
at the departure
time shown
$\square 5$ minutes after
departure time
shown
10 minutes after
departure time
shown
$\square 15$ minutes after
departure time
shown

Figure 7.5. Illustration of Question 19 responses: Buffer time terms.

departure time, and suggested departure time) and asked participants to choose the term they most preferred. Almost half of the participants preferred recommended departure time. The term 95 th percentile departure time was favored by less than $5 \%$ of participants. The research team noted that these preferences were markedly different from the terms that showed the best comprehension in Question 18. The results of further analysis can be seen in Table 7.16, which shows a cross-tabulation of preferences expressed in Question 20 as a function of response to Question 18 (Question 18 asked more directly about comprehension of the term). Among those who saw recommended departure time in their comprehension question, $57 \%$ still preferred that term over the alternatives. Across all versions, recommended departure time received most of the shifts in preference: $40 \%$ to $45 \%$ of people preferred the term to the one they saw in their own version of the survey.

\section{Questions 21-22: Buffer Time Terminology}

These questions were included to explore participants' understanding of buffer time and whether they wanted an information system to provide a buffer time value. Questions 21 and 22 assessed participants' desire to be told the amount of potential variability associated with a predicted trip time.

Table 7.16. Responses to Question 20 as a Function of Response to Questions 18 and 19

\begin{tabular}{|l|c|c|c|c|}
\hline \multirow{2}{*}{ Preferred Term in Question 20 } & \multicolumn{4}{|c|}{ Term Viewed in Questions 18 and 19 } \\
\cline { 2 - 5 } & $\begin{array}{c}\text { Version A: } \\
\text { Recommended } \\
\text { Departure Time (\%) }\end{array}$ & $\begin{array}{c}\text { Version B: } \\
\text { Estimated } \\
\text { Departure Time (\%) }\end{array}$ & $\begin{array}{c}\text { Version C: } \\
\text { 95th Percentile } \\
\text { Departure Time (\%) }\end{array}$ & $\begin{array}{c}\text { Version D: } \\
\text { Suggested } \\
\text { Departure Time (\%) }\end{array}$ \\
\hline Recommended departure time & 57 & 45 & 43 & 43 \\
\hline Estimated departure time & 14 & 28 & 23 & 19 \\
\hline 95th percentile departure time & 4 & 3 & 9 & 1 \\
\hline Suggested departure time & 25 & 24 & 25 & 36 \\
\hline
\end{tabular}


Question 21 presented participants with an assumption: the trip time provided by the fictional website was an average time for the specified trip, based on past travel time information, and the actual trip time had some chance of being longer than average, depending on roadway conditions and nonrecurring congestion factors. Participants were asked if they would want to know how much additional time their trip could take, beyond the average time provided. Almost 9 out of 10 participants wanted to know how much additional time a trip could take beyond the average time provided.

Question 22 asked participants which term they preferred to describe the additional time. From the options providedbuffer, departure window, leeway, cushion, and extraapproximately $33 \%$ of the participants chose the term extra, while another $28 \%$ favored departure window.

Because the concepts of average and buffer time are so critical to reliability information, the research team created an openended survey to examine this issue further. That study is presented in Chapter 8.

\section{Question 23: Preference for Travel Time Distribution Output}

Question 23 examined what type of travel time metric drivers would prefer to receive from a system using historical trip time data as its resource. This question reminded participants that the fictional website's system for predicting trip times was based on historical data and, with this in mind, asked what type of trip time calculation they would want the system to provide. The response options provided were designed to represent different travel time metrics that could be calculated from historical travel time data:

- An average time (statistical mean);

- A worst-case time (95th percentile);

- A range of times it could take (5th percentile time to 95th percentile time);

- The best-case time it could take (5th percentile time);

- The most likely time it would take (statistical mode, most frequently occurring value); and

- The time where half the trips would take longer and half would take shorter (statistical median).

The most frequently expressed preferences were for an average time (31\% of participants), a range of times the trip could take (29\%), or the most likely time the trip would take (24\%). Although average was the most frequent response, that must be interpreted in light of the results of Question 15, which showed many participants interpreted the mathematical meaning of average incorrectly. The low popularity of worst case and best case contrasted somewhat with many of the comments heard during the focus groups. There, participants indicated they would like the worst case to be presented, and they would make adjustments on the basis of their individual risk tolerance.

\section{Questions 24-26: Travel Time Variability Terminology}

Questions 24 through 26 explored additional terms and phrases that could be used to express travel time variability. These questions arose from similar concepts discussed in the focus groups, and the terms presented as options in these questions also came primarily from focus group responses. Each question provided participants with a fill-in-the-blank sentence and a list of reliability terms from which to select to complete the sentence. In each case, participants were advised to select the term that would give them the most confidence about the trip time information provided.

Question 24 presented a trip time derived from historical data using the sentence "It is ___ that your trip will take 45 minutes." Response options for this question were forecasted, anticipated, estimated, probable, likely, reliable, and predicted. More than half of the participants (56\%) preferred estimated to complete the sentence. Question 25 presented a sentence describing the potential variability of a trip time: "Your trip time may ___ from the average time by 20 minutes." Of the options provided-differ, vary, fluctuate, change, go up or down, increase or decrease, and deviate - 59\% of participants selected vary to complete the sentence. Question 26 began with the sentence "It will take 20 minutes to make your trip." Of the terms presented as options to complete this sentence-about, approximately, give or take, an estimate of, around, roughly, and an average of $-69 \%$ of participants preferred approximately.

\section{Question 27: 95th Percentile Trip Time Terminology}

Researchers wanted to further explore drivers' understanding of the term 95th percentile trip time because it is commonly used by traffic engineers but may be misunderstood by the general public. Question 27 asked, "What does 95\% time mean to you?" The response options for this question were as follows:

- It will take $95 \%$ of the provided trip time to arrive.

- 95 times out of 100 it will take the provided time to arrive.

- The system is $95 \%$ confident that its provided time is correct to arrive.

- 95 times out of 100 it will take at or less than the provided time to arrive.

- The time it takes you to complete $95 \%$ of the distance of your trip.

- I don't know.

Four of the six response options provided were developed by the research team to represent what the team members felt 
could be likely or possible misconceptions about the term. The correct answer is "95 times out of 100 it will take at or less than the provided time to arrive." Only $14 \%$ of participants chose this answer. The most commonly selected answer (44\% of participants) was "95 times out of 100 it will take the provided time to arrive." That result may indicate that those participants misunderstood the meaning; the answer implies that the provided time is the same for every trip, rather than being the upper end of a time range. The second most frequently selected answer (32\% of participants) was "The system is $95 \%$ confident that its provided time is correct to arrive." While the second group chose a description that is not technically correct, they still had a general understanding that the chances of arriving within the given time are high. In terms of travel behavior decisions, that level of understanding may be acceptable.

\section{Question 28: System Trust Terminology}

Researchers wanted to determine the best wording to explain that the website system's travel time estimation is trustworthy and additional time does not need to be added to the provided trip time. Question 28 asked what statement best communicated this concept. The response options were all based on a 95th percentile trip time (for simplicity, the usual modifier specifying that the trip time could be less than the 95th percentile time was not mentioned):

- It is $95 \%$ reliable that your travel time will be 45 minutes.

- 19 out of 20 times your travel time will be 45 minutes.

- Your maximum trip time is 45 minutes.

- There is low variability to your trip time of 45 minutes.

- The worst-case travel time is 45 minutes.

- The system is very certain that your trip will take 45 minutes.

The wording of one of the options ("19 out of 20 times. ...") was taken from a travel website; the wording of the other options was developed by the research team.

Most participants chose a statement using the phrase "Your maximum trip time is..." (selected by $38 \%$ ) or "It is $95 \%$ reliable that your travel time will be...." (selected by $34 \%$ ). The remaining phrases were rarely selected.

Researchers compared individuals' responses for Questions 27 and 28. Table 7.17 shows this relationship and presents a contingency table for the two items. Among the people who answered Question 27 correctly (Q: What does $95 \%$ time mean to you? A: 95 times out of 100 it will take at or less than the provided time to arrive.), only $12 \%$ thought the phrase " 19 out of 20 " was the best wording to convey that users need

Table 7.17. Responses to Question 28 as a Function of Response to Question 27

\begin{tabular}{|c|c|c|c|c|c|c|}
\hline \multirow[b]{2}{*}{$\begin{array}{l}\text { Answered } \\
\text { Question } 27-\text { What } \\
\text { does } 95 \% \text { time } \\
\text { mean to you? }\end{array}$} & \multicolumn{6}{|c|}{$\begin{array}{c}\text { Responses to Question } 28-\text { Which of these statements uses wording that best explains that the provided travel } \\
\text { time given to you is trustworthy and you don't need to add additional time? }\end{array}$} \\
\hline & $\begin{array}{c}\text { It is } 95 \% \text { reliable } \\
\text { that your travel } \\
\text { time will be } \\
45 \text { minutes. (\%) }\end{array}$ & $\begin{array}{c}19 \text { out of } 20 \\
\text { times your travel } \\
\text { time will be } \\
45 \text { minutes. }(\%)\end{array}$ & $\begin{array}{l}\text { Your maximum } \\
\text { trip time is } \\
45 \text { minutes. (\%) }\end{array}$ & $\begin{array}{l}\text { There is low } \\
\text { variability to } \\
\text { your trip time of } \\
45 \text { minutes. (\%) }\end{array}$ & $\begin{array}{l}\text { The worst-case } \\
\text { travel time is } \\
45 \text { minutes. (\%) }\end{array}$ & $\begin{array}{l}\text { The system is } \\
\text { very certain that } \\
\text { your trip will take } \\
45 \text { minutes. (\%) }\end{array}$ \\
\hline $\begin{array}{l}\text { It will take } 95 \% \text { of } \\
\text { the provided trip } \\
\text { time to arrive. }\end{array}$ & 42.11 & 15.79 & 31.58 & 0.00 & 0.00 & 10.53 \\
\hline $\begin{array}{l}95 \text { times out of } 100 \\
\text { it will take the } \\
\text { provided time to } \\
\text { arrive. }\end{array}$ & 31.30 & 12.21 & 41.98 & 1.53 & 7.63 & 5.34 \\
\hline $\begin{array}{l}\text { The system is } 95 \% \\
\text { confident that its } \\
\text { provided time is } \\
\text { correct to arrive. }\end{array}$ & 38.14 & 4.12 & 36.08 & 5.15 & 4.12 & 12.37 \\
\hline $\begin{array}{l}95 \text { times out of } 100 \\
\text { it will take at or } \\
\text { less than the pro- } \\
\text { vided time to } \\
\text { arrive. }\end{array}$ & 34.15 & 4.88 & 36.59 & 4.88 & 9.76 & 9.76 \\
\hline $\begin{array}{l}\text { The time it takes you } \\
\text { to complete } 95 \% \\
\text { of the distance of } \\
\text { your trip. }\end{array}$ & 0.00 & 25.00 & 25.00 & 0.00 & 25.00 & 25.00 \\
\hline I don't know. & 0.33 & 0.33 & 1.00 & 0.00 & 0.33 & 0.67 \\
\hline
\end{tabular}


not add their own buffer time- though the two are mathematically equivalent. The people who understood $95 \%$ mathematically, as evidenced by their responses to Question 27, preferred the phrases $95 \%$ reliable or maximum trip time to convey trustworthiness.

\section{Question 29: Travel Mode Shift Information}

Currently, most travel time websites provide travel time (and sometimes reliability information) for roadway driving only. If a website does provide estimated times for a transit or managed-lane trip, those times are located on a different page of the website, and travel time and/or reliability comparisons are not automatic. The data underlying route planners and realtime information for different modes are most often provided by different agencies. If reliability information will indeed have any effect on mode choice as envisioned by the profession, then a comparison of modes' reliability may need to be presented to users. Before such cross-agency communication and interoperability is developed, the survey sought to assess users' likelihood of changing modes at all.

As a final question then, the survey asked participants if they would change their transportation mode if the system showed a different mode would be quicker for that day. Encouragingly, three out of four participants (76\%) said that they would change their mode if they could.

\section{Important Findings}

\section{Understanding of Reliability and Travel Time Distribution Concepts}

- The large majority (90\%) of participants had a desire for information that a planned trip had a chance of taking longer than average (Question 21). When asked to select a term to describe that additional time, only $10 \%$ selected buffer time, which is the term used by professionals in the travel time reliability field.

- Only $18 \%$ of participants understood average to mean "about half the time (10 days out of 20)"; others interpreted it to mean "most of the time (15 days out of 20)" (Question 15). Although that is not necessarily an incorrect interpretation of the term, it could be incorrect if the underlying distribution of travel times is skewed, as is typical. Designers of traveler information systems and performance measures need to be sensitive to the layperson's understanding of such terms as average and distinguish the technical use from the everyday use.

- Likewise, only $37 \%$ of participants understood 95 th percentile to mean "nearly all the time, 19 days out of 20" (Question 15). Although this term was the most successful of the eight tested in conveying " 19 days out of 20 ," less than $40 \%$ of participants selected it-which is troubling. Because 95th percentile is such a critical and common performance metric for reliability calculations, travel information designers and providers need to be sensitive to this finding. As indicated by the focus groups as well, most people do not like statistical-sounding terms such as this. The term typical did the best job of conveying that the system-provided trip time would encompass the actual trip time most or nearly all of the time.

- When asked directly what $95 \%$ time meant, $44 \%$ of participants correctly answered that it means that 95 times out of 100 , it would take the provided amount of time to make a trip. Another 32\% thought that the 95\% term meant the system was $95 \%$ confident in its prediction. Those findings again speak to the need to use a more everyday term, even though it may not be technically correct. A glossary or frequently asked questions (FAQ) section of a traveler information system could provide the technical definition.

- The response pattern of most participants indicated that, when given a total trip time estimate by the system (which was based on typical time plus some buffer time), they would still add their own buffer time onto the system estimate (Questions 18 and 19). Regardless of the term used, a majority of participants chose to leave earlier than the departure time provided by the fictional website. This is important to note because the terms tested were proposed as surrogates for planning time, which presumably already includes a buffer time. These results indicate either a lack of understanding or a lack of trust in the departure times provided by such a system. This agrees with many of the comments made during the focus groups as well.

- When asked directly about system trust and the need to add one's own buffer time "just in case," participants were split between preferring maximum trip time and 95\% reliable that your travel time will be $x x$ minutes. Again, this indicates that some portion of the population is interpreting the $95 \%$ phrase to refer to system confidence and accuracy, not a point on a travel time distribution (Question 28).

- If reliability information will have any effect on mode choice as envisioned by the profession, then a comparison of modes' reliability may need to be presented to users. Before such cross-agency communication and interoperability is developed, the survey sought to assess users' likelihood of changing modes at all. Encouragingly, three out of four participants (76\%) said that they would change their mode if they could.

\section{Website Interface Design}

- The use of future trip as a button label, section header, or navigation button conveys that the trip being planned is not imminent and will be based on historical, rather than 
real-time, travel time data. The term is particularly appropriate for home page locations to invite users to enter the trip planning section of the website. Question 7 showed that $81 \%$ of participants understood the term future trip to mean a trip later in the day or tomorrow rather than one with a more immediate departure. The results from Question 7 also indicate that future trip may be the best label to use on input screens for date and time to reinforce that the trip being planned is a future trip.

- To initiate action on trip planning systems, terms like predict trip and plan trip are preferred over standard action labels such as $O K$ or Go (see results for Questions 9 and 10). Terms like predict trip and plan trip could be used at the bottom of trip detail input screens.

- For trip detail input screens, users preferred the term arrive by as a prompt for entering their desired arrival time. This term could be used as the stem for a drop-down menu from which a user would select a time from a list (Question 12). The term is short enough to be useful for full websites, mobile website front-ends, and smartphone applications.

- For the map showing all available routes, participants most frequently preferred the two phrases that included available travel information in the title. Notably, phrases that contained historical data performed relatively poorly.
- For output screens, the term best route was preferred as a map title or output section header (Question 14). This concurred with the focus group feedback that indicated a preference for simple terms.

- Across all versions, recommended departure time received most of the shifts in preference, meaning that $40 \%$ to $45 \%$ of people preferred this term to the one they saw in their own version of the survey (Question 20).

- In reporting the output of travel time calculations, reliability information could be conveyed through the following phrases (the word in italics was the most popular term of those tested in the survey):

○ It is estimated that your trip will take 45 minutes.

O Your trip time may vary from the average time by 20 minutes.

○ It will take approximately 20 minutes to make your trip.

Throughout the survey, researchers repeatedly found a disconnect between the technical terms used by professionals and the layperson's understanding of and preference for those terms. This finding was evident in the focus group comments as well. The lexicon and guidebook that are part of the L14 project hope to bridge this gap so that professionals can more easily communicate these concepts to transportation system users. 


\section{онартеп 。 \\ Open-Ended Survey}

The objective of the open-ended survey was to determine whether drivers could correctly interpret both an indicator of normal trip time and an indicator of reliability (buffer time or total trip time estimate). Conceptually, a driver's average or normal travel (trip) time plus the buffer time the driver adds for contingencies (uncertainty of conditions) equals the driver's estimated total trip time for planning purposes:

\section{normal trip time + buffer time $=$ total trip time estimate}

TTI researchers developed the open-ended, paper-based survey to determine which combinations of terms representing a normal trip time, a buffer time, and the ultimate trip time to plan for would be best for conveying trip time reliability information.

\section{Research Method}

\section{Survey Design}

The following potential terms were tested for comprehension and preference in conveying the normal travel time at a particular time of day:

- Average travel time;

- Estimated travel time;

- Expected travel time; and

- Typical travel time.

Terms tested to represent the buffer time for a given trip (as in the preceding equation) included the following:

- Added time;

- Cushion time;

- Extra time; and

- Recommended cushion time.

Finally, the terms tested to represent the total trip time that drivers should plan for to protect themselves against being late (i.e., "total trip time estimate" in the preceding equation) were the following:

- 95th percentile travel time;

- The majority of the time the travel time is $\mathrm{xx}$ minutes or less;

- Most of the time the travel time is xx minutes or less; and

- Travel time for planning.

These terms were based on focus group results and the expertise and experience of the TTI research team, or they were being evaluated in the usability studies. Some terms initially considered in the focus groups and usability surveys were not evaluated here to keep this part of the study short enough to mesh with the current surveys and experiments already in progress at the time. The terms worst-case and maximum were not tested here, as agency concerns regarding liability and credibility would likely preclude them from using such absolute terms. Similarly, the term most common could be perceived by motorists in a way that would reduce agency credibility and so was not evaluated in this portion of the surveys. Negative feedback from focus groups led researchers to remove the terms buffer and leeway from analysis. The term departure window was considered to be adequately examined in the existing usability study. The term added travel time was not included in the initial survey and was not offered by focus group participants; however, researchers believed that participants might not have suggested it because a version of the term was used by facilitators to explain the concept. Thus, added travel time was evaluated here.

Researchers presented terms to participants in the context of two scenario-based, multipart questions. Scenario 1 presented participants with a term for the normal trip time and a term for the buffer time (as defined in the above equation). Scenario 2 presented participants with a term for the normal trip time and a term for the total trip time estimate. Because of the large number of terms to be tested in these scenarios, 
Table 8.1. Experimental Design for Open-Ended Survey

\begin{tabular}{|c|c|c|c|c|c|}
\hline \multirow{2}{*}{$\begin{array}{l}\text { Survey } \\
\text { Version }\end{array}$} & \multicolumn{2}{|c|}{ Scenario 1 Terms } & \multicolumn{2}{|c|}{ Scenario 2 Terms } & \multirow{2}{*}{$\begin{array}{l}\text { Number of } \\
\text { Participants }\end{array}$} \\
\hline & Normal Trip Time & Buffer Time & Normal Trip Time & Total Trip Time Estimate & \\
\hline A & Average & Cushion & Expected & 95th percentile & 12 \\
\hline B & Average & Extra & Typical & Trip time for planning & 11 \\
\hline C & Average & Added & Estimated & Most of the time & 12 \\
\hline D & Expected & Recommended cushion & Average & Majority of the time & 12 \\
\hline E & Expected & Cushion & Average & 95th percentile & 12 \\
\hline $\mathbf{F}$ & Expected & Extra & Typical & Most of the time & 12 \\
\hline G & Expected & Added & Estimated & Trip time for planning & 12 \\
\hline $\mathbf{H}$ & Typical & Recommended cushion & Expected & Majority of the time & 12 \\
\hline I & Typical & Cushion & Average & Trip time for planning & 12 \\
\hline $\mathbf{J}$ & Typical & Extra & Expected & 95th percentile & 12 \\
\hline K & Typical & Added & Estimated & Most of the time & 12 \\
\hline $\mathbf{L}$ & Estimated & Recommended cushion & Typical & Majority of the time & 12 \\
\hline M & Estimated & Cushion & Average & Most of the time & 12 \\
\hline $\mathbf{N}$ & Estimated & Extra & Expected & Trip time for planning & 12 \\
\hline 0 & Estimated & Added & Typical & 95th percentile & 11 \\
\hline $\mathbf{P}$ & Average & Recommended cushion & Estimated & Majority of the time & 11 \\
\hline
\end{tabular}

not every term could be tested in every combination with every participant. A total of 16 versions of the survey were created, as shown in Table 8.1. Participants were divided across the 16 survey versions, with 11 or 12 participants taking each version.

\section{Participants}

The open-ended survey was administered to participants who had completed either the computer survey or travel behavior laboratory experiment. Thus, a total of 189 participants completed open-ended surveys in five cities. Table 8.2 lists the number of open-ended survey participants in each city. Detailed demographic information on the participants was not recorded for this survey but was believed to be representative of the driving populations in each location.

\section{Procedure}

A researcher administered the open-ended survey to participants who had completed either the computer survey or the travel behavior experiment. The researcher read the questions to the participant and recorded the participant's answers. The individual interviews took place in a separate room, away from the computer testing, and took $15 \mathrm{~min}$ to 20 min to complete.
For some of the questions, cards describing the travel scenario were placed in front of the participants to lessen their working memory load. The interviewer read aloud the text on the card, and the participant was allowed to re-read the text if desired and examine the questions and answer alternatives while discussing the item. The survey materials from Version A are presented in Appendix E.

Survey participants were presented with two hypothetical scenarios. Each scenario placed participants in a hotel in the morning, and their destination was a meeting in a downtown office building. They were able to get travel time

Table 8.2. Number of Open-Ended Survey Participants per City

\begin{tabular}{|l|c|c|c|}
\hline City & $\begin{array}{c}\text { Participated in } \\
\text { Computer-Based } \\
\text { Survey }\end{array}$ & $\begin{array}{c}\text { Participated in } \\
\text { Travel Behavior } \\
\text { Experiment }\end{array}$ & $\begin{array}{c}\text { Total } \\
\text { Participants } \\
\text { per City }\end{array}$ \\
\hline Dallas & 31 & 14 & 45 \\
\hline Denver & 0 & 0 & 0 \\
\hline Miami & 0 & 0 & 0 \\
\hline San Jose & 54 & 20 & 74 \\
\hline Hartford & 53 & 17 & 70 \\
\hline Total & 138 & 51 & 189 \\
\hline
\end{tabular}


information from a hotel website before they left the hotel for the meeting.

Scenario 1 read as follows. Blank spaces were replaced with one of the four terms being evaluated to convey the normal trip time and with one of the four terms representing the buffer time.

Imagine that you are in a hotel in an unfamiliar city and have an important business meeting first thing this morning at a downtown office building. You will need to leave the hotel during the peak period in order to arrive at the business meeting. You are trying to decide how much travel time to allow for your drive to downtown. You cannot be late to the meeting, but you do not want to arrive too early. You know that you can park right next to the building and do not need to allow additional time to search for parking. You also know that you can get current travel time information from the hotel website. Before you drive to your downtown meeting, you check the website and obtain the following information...

The_travel time to downtown is 40 minutes
and time to downtown is 30 minutes.
the t__ to the

As shown in the following text, scenario 2 was presented in an identical format but with the normal travel time to downtown set at $30 \mathrm{~min}$ instead of $40 \mathrm{~min}$ and a total trip time estimate (representing the sum of the normal time and a buffer time) set at $55 \mathrm{~min}$. Again, the blanks were replaced with one of the four terms being evaluated to convey the normal trip time and one of the four terms representing the total trip time estimate. As noted in Table 8.1, for each survey version, different terms were used to represent the normal trip time in scenario 1 and scenario 2.

Imagine that you are again in a hotel in another unfamiliar city and have an important business meeting this morning at a downtown office building. As before, you will need to leave the hotel during the peak period in order to arrive at the business meeting. You are trying to decide how much travel time to allow for your drive to downtown. You cannot be late to the meeting, but you do not want to arrive too early. You know that you can park right next to the building and do not need to allow additional time to search for parking. You also know that you can get current travel time information from the hotel website. Before you drive to your downtown meeting, you check the website and obtain the following information...

\section{The travel time to downtown is $\mathbf{3 0}$ minutes and}

[or less].
After each scenario was provided to a participant (with the blanks replaced with the appropriate terms), the participant was asked the following questions:

- How much time would you allow for your drive to downtown?

- How did you decide on that time?

- What does the (average, estimated, expected, or typical) travel time mean to you about how long your drive will take?

- For scenario 1: What does the (added, cushion, extra, or recommended cushion) time mean to you about how long your drive will take?

- For scenario 2: What does the (95th percentile time, majority of the time, most of the time, or travel time for planning time) mean to you about how long your drive will take?

- How likely are you to reach downtown in (70 for scenario 1, 55 for scenario 2) minutes or less?

- For scenario 1: If the (added, cushion, extra, or recommended cushion) time had been 10 minutes, how much time would you have allowed for your drive? Why? (Note: The response implying the desired use of that term would be $50 \mathrm{~min}$.)

- For scenario 2: If the (95th percentile time, majority of the time, most of the time, or travel time for planning time) had been 45 minutes, how much time would you have allowed for your drive? Why? (Note: The response implying the desired use of that term would be $45 \mathrm{~min}$.)

At the end of each scenario, the participants were shown the remaining possible terms being tested to convey uncertainty and asked to indicate whether each one meant the same thing as the term they had originally seen in the website message. If not, participants were asked to explain why the term was different. Finally, participants were asked which term they preferred to convey travel time uncertainty information.

After participants answered questions concerning both scenarios, they were asked whether they preferred the travel time uncertainty information in the first scenario (buffer time) or the second scenario (total trip time estimate).

\section{Results}

The following sections discuss the use of the buffer time terms and total trip time estimate terms by participants when determining the total trip time they would allow for each scenario provided. To evaluate effectiveness, researchers examined the distribution of participant-selected trip times relative to the total trip time intended by the message. For this evaluation, the greater the percentage of participants selecting travel times within the 10-min to 15-min range around that total trip time value, the more effective the message was deemed. Trip times much below the intended total trip time would result in motorists being late more frequently than 
desired; and trip times much higher would result in excessively early arrival times for the majority of trips made.

\section{Use of Buffer Time in Total Trip Time Estimation}

Researchers analyzed the responses to the questions regarding how much time the participants would allow for their trip. They initially looked at each pair of normal trip time and buffer terms to determine whether the normal trip time term affected the use of the buffer term. Researchers did not identify any clear trends that indicated the normal trip time term affected the use of the buffer term, so the data for each buffer term were aggregated across the normal trip time terms for further analysis.

Researchers explored how the type of buffer term used may influence the extent to which motorists do or do not incorporate the amount of buffer time presented in a message into their travel plans. At the simplest level of analysis, researchers envisioned four possible ways in which motorists would use the times presented in scenario 1 (the normal trip time and the buffer value) to estimate the time they would allow for their trip:

- Participants could ignore the buffer value and base their trip time strictly on the normal trip time value provided.

- Participants could ignore the normal trip time value and base their trip time strictly on the buffer time value provided (implying confusion as to the intent of the buffer term).

- Participants could combine the normal trip time and the buffer time values to come up with the trip time they would allow for (the intended use of the buffer time value).
- Participants could add some time other than the buffer time value to the normal trip time to come up with the trip time they would allow for (the amount of time added may or may not be influenced by the buffer term).

The percentage of participants who selected various trip times in each of those categories is presented in Table 8.3. Overall, few participants indicated they would allow for only the normal trip time. Researchers could not determine whether that resulted from being provided a buffer time or that was simply the participants' normal trip planning behavior. In addition, very few participants stated they would only allow for the buffer time provided in the website message, indicating that only a small percentage of participants were confused by the intent of the buffer term. Among the four buffer terms, recommended cushion time resulted in the greatest percentage of participants adding the buffer time to the normal trip time (28\%), followed closely by added time (23\%). When the term cushion time was used, only $8 \%$ of participants added the normal trip time and buffer time to determine the amount of time they should allow for the trip. Still, the majority of participants added some other value to the normal trip time when choosing the trip time they would allow for in scenario 1.

Additional insights into the trip times that participants indicated they would allow for (as a function of the trip time uncertainty term) are presented in Table 8.4. As expected, the percentages for the "35-44 min" and "25-34 min" groups are similar to those of the "used normal trip time only" and "used buffer term only" rows in Table 8.3, respectively. In addition, those in the "65-74 min" column correlate with the "added normal trip time and buffer time values together" column in Table 8.3. The distribution of trip times that would be allowed

Table 8.3. Participant Use of Buffer Term When Planning a Trip

\begin{tabular}{|c|c|c|c|c|c|}
\hline \multirow[b]{3}{*}{ Participant Use of Buffer Term } & \multicolumn{5}{|c|}{ Percentage of Participants } \\
\hline & \multicolumn{4}{|c|}{ Buffer Term Viewed } & \multirow[b]{2}{*}{ Average } \\
\hline & $\begin{array}{c}\text { Added } \\
(n=47)\end{array}$ & $\begin{array}{l}\text { Cushion } \\
(n=48)\end{array}$ & $\begin{array}{c}\text { Extra } \\
(n=47)\end{array}$ & $\begin{array}{l}\text { Recommended } \\
\text { Cushion }(n=47)\end{array}$ & \\
\hline Used normal trip time only & 4 & 17 & 4 & 6 & 8 \\
\hline Used buffer term only & 2 & 4 & 2 & 6 & 4 \\
\hline $\begin{array}{l}\text { Added normal trip time and } \\
\text { buffer time values together }\end{array}$ & 23 & 8 & 15 & 28 & 19 \\
\hline $\begin{array}{l}\text { Added normal trip time and } \\
\text { some other value together }\end{array}$ & 66 & 67 & 66 & 55 & 63 \\
\hline Total & $95^{a}$ & $96^{b}$ & $87^{c}$ & $95^{d}$ & $94^{e}$ \\
\hline
\end{tabular}

a Does not add up to $100 \%$ because researchers categorized $5 \%$ of responses as "other."

${ }^{b}$ Does not add up to $100 \%$ because researchers categorized $4 \%$ of responses as "other."

cDoes not add up to $100 \%$ because researchers categorized $13 \%$ of responses as "other."

dDoes not add up to $100 \%$ because researchers categorized $5 \%$ of responses as "other."

e Does not add up to $100 \%$ because researchers categorized $6 \%$ of responses as "other." 
Table 8.4. Distribution of Trip Times Allowed for 70-Minute Trip

\begin{tabular}{|c|c|c|c|c|c|}
\hline \multirow[b]{3}{*}{$\begin{array}{l}\text { Trip Times Allowed } \\
\text { for } 70 \text {-min Trip }\end{array}$} & \multicolumn{5}{|c|}{ Percentage of Participants } \\
\hline & \multicolumn{4}{|c|}{ Buffer Term Viewed } & \multirow[b]{2}{*}{ Average } \\
\hline & $\begin{array}{l}\text { Added } \\
(n=47)\end{array}$ & $\begin{array}{l}\text { Cushion } \\
(n=48)\end{array}$ & $\begin{array}{c}\text { Extra } \\
(n=47)\end{array}$ & $\begin{array}{c}\text { Recommended } \\
\text { Cushion }(n=47)\end{array}$ & \\
\hline$<25 \min$ & & 2 & 4 & & 2 \\
\hline $25-34 \mathrm{~min}$ & 4 & 6 & 6 & 8 & 6 \\
\hline 35-44 min & 6 & 17 & 6 & 8 & 9 \\
\hline $45-54 \min$ & 6 & 32 & 11 & 11 & 15 \\
\hline $55-64 \min$ & 28 & 27 & 24 & 30 & 27 \\
\hline $65-74 \min$ & 24 & 8 & 19 & 28 & 20 \\
\hline $75-84 \min$ & 11 & 8 & 15 & 13 & 12 \\
\hline$\geq 85 \min$ & 21 & & 15 & 2 & 9 \\
\hline Total & 100 & 100 & 100 & 100 & 100 \\
\hline
\end{tabular}

Note: Normal trip time $=40 \mathrm{~min}$; buffer time provided $=30 \mathrm{~min}$.

shows some small differences by type of trip time uncertainty term used.

Stated another way, Figure 8.1 shows the cumulative distribution of trip times that participants would allow-as a function of the buffer time used. Among the participants who saw the term recommended cushion, $70 \%$ chose a trip time that was within 15 min of the total trip time provided in the website message. Thus, recommended cushion time is apparently the term best able to encourage selection of the trip time which most closely aligns with the amount of trip time uncertainty that the operating agency would be attempting to convey. When the terms added time and extra time were shown, a greater percentage of participants chose travel times that exceeded the 70-min intended value. That result suggests a potentially lower level of confidence implied by those two terms and led participant to allow more buffer time than was

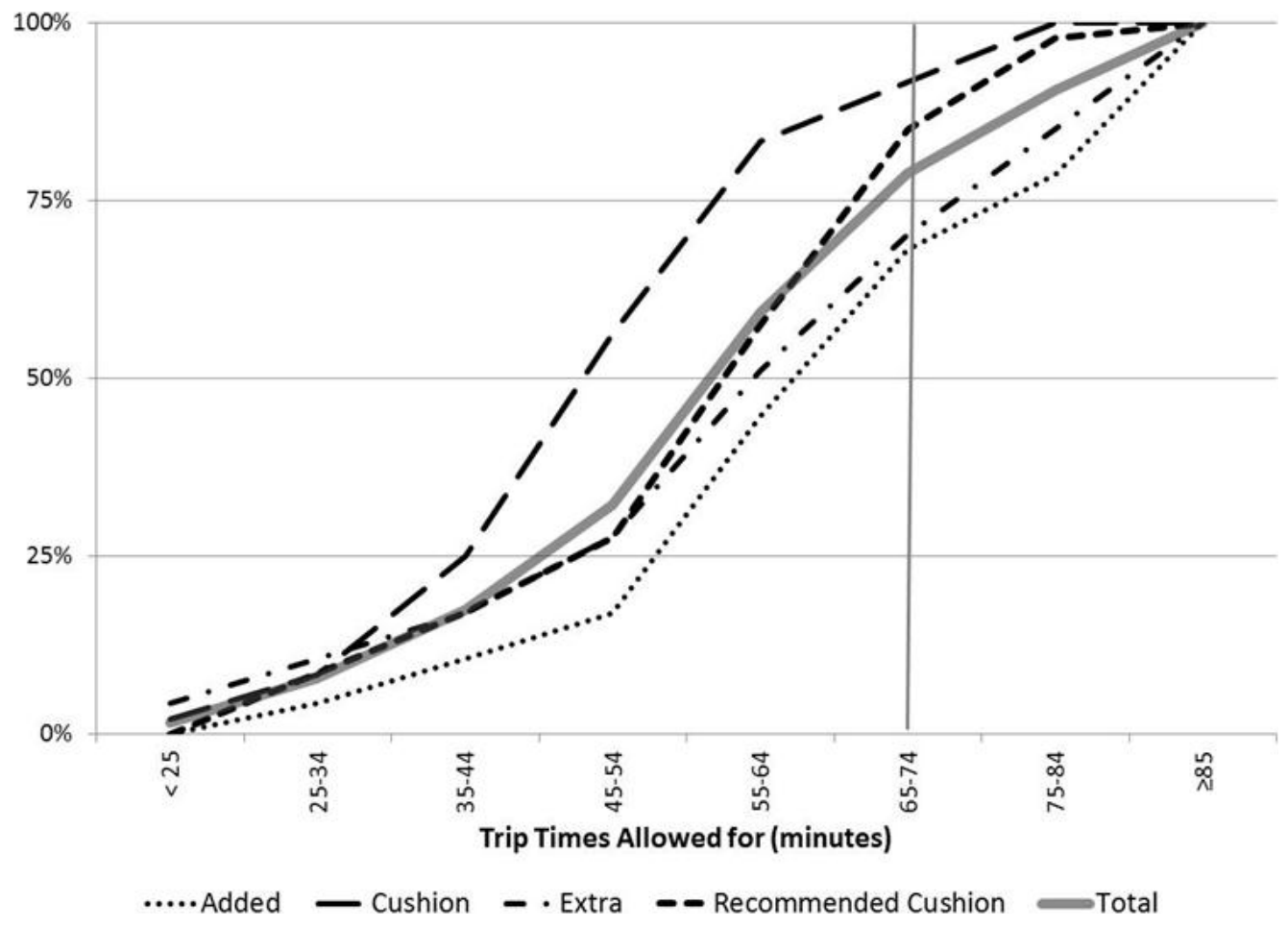

Figure 8.1. Cumulative distribution of trip times allowed for 70-minute trip. 
Table 8.5. Participant Confidence in Arriving at Destination in 70 Minutes or Less

\begin{tabular}{|l|c|c|c|c|c|}
\hline \multirow{2}{*}{} & \multicolumn{5}{|c|}{ Percentage of Participants } \\
\cline { 2 - 6 } Participant Confidence & \multicolumn{5}{|c|}{ Buffer Term Viewed } \\
\cline { 2 - 6 } & $\begin{array}{c}\text { Added } \\
(\boldsymbol{n}=\mathbf{4 7})\end{array}$ & $\begin{array}{c}\text { Cushion } \\
(\boldsymbol{n}=\mathbf{4 8})\end{array}$ & $\begin{array}{c}\text { Extra } \\
(\boldsymbol{n}=\mathbf{4 7})\end{array}$ & $\begin{array}{c}\text { Recommended } \\
\text { Cushion }(\boldsymbol{n}=\mathbf{4 7})\end{array}$ & Average \\
\hline Absolutely certain (100\% chance) & 21 & 17 & 15 & 23 & 19 \\
\hline Very likely (95\% chance) & 53 & 52 & 55 & 62 & $\mathbf{5 6}$ \\
\hline Probably (75\% chance) & 19 & 21 & 26 & 11 & $\mathbf{1 9}$ \\
\hline Unsure (50/50 chance) & 7 & 8 & 4 & 2 & $\mathbf{5}$ \\
\hline Doubtful (less than 50/50 chance) & 0 & 2 & 0 & 2 & $\mathbf{1 0 0}$ \\
\hline Total & $\mathbf{1 0 0}$ & $\mathbf{1 0 0}$ & $\mathbf{1 0 0}$ & $\mathbf{1 0 0}$ & $\mathbf{1 0}$ \\
\hline
\end{tabular}

Note: Normal trip time $=40 \mathrm{~min}$; buffer time provided $=30 \mathrm{~min}$.

presented in the message. Participants also had less confidence in the term cushion time, but instead of allowing more time than presented, they chose a value less than the sum of the normal trip time and buffer time. That result suggests the term cushion time may convey a sense of excessive uncertainty in the trip time, greater than desired.

The same trends regarding the confidence participants expressed in use of the trip time uncertainty terms are evident in Table 8.5. It shows participant responses to the question "How likely are you to arrive at your destination in 70 minutes or less?" Overall, $85 \%$ of participants viewing recommended cushion time responded "absolutely certain (100\% chance)" or "very likely (95\% chance)." The percentage of participants selecting those two categories for the other buffer terms were added time (74\%), extra time (70\%), and cushion time (69\%).

Next, participants were asked how much time they would have allowed if the normal trip time remained at $40 \mathrm{~min}$, but the trip time uncertainty value was only $10 \mathrm{~min}$. The responses to that question are summarized in Table 8.6 and shown in Figure 8.2. The responses made clear that drivers are not likely to have a high degree of confidence in small buffer values, regardless of the term used. Although recommended cushion time again led the greatest percentage of participants to select the sum of the normal and uncertainty trip time values (i.e., 50 minutes), a large portion of participants chose trip times longer than that amount. Furthermore, responses did not vary significantly as a function of trip time uncertainty term used.

Table 8.6. Distribution of Trip Times Allowed for 50-Minute Trip

\begin{tabular}{|c|c|c|c|c|c|}
\hline \multirow[b]{3}{*}{$\begin{array}{l}\text { Trip Times Allowed } \\
\text { for } 50 \text {-Min Trip }\end{array}$} & \multicolumn{5}{|c|}{ Percentage of Participants } \\
\hline & \multicolumn{4}{|c|}{ Buffer Term Viewed } & \multirow[b]{2}{*}{ Average } \\
\hline & $\begin{array}{c}\text { Added } \\
(n=47)\end{array}$ & $\begin{array}{l}\text { Cushion } \\
(n=48)\end{array}$ & $\begin{array}{c}\text { Extra } \\
(n=47)\end{array}$ & $\begin{array}{l}\text { Recommended } \\
\text { Cushion }(n=47)\end{array}$ & \\
\hline$<25 \min$ & 2 & 6 & 9 & 9 & 6 \\
\hline 25-34 min & 2 & 4 & 0 & 2 & 2 \\
\hline $35-44 \min$ & 0 & 10 & 2 & 6 & 5 \\
\hline $45-54 \min$ & 38 & 35 & 38 & 45 & 39 \\
\hline $55-64 \min$ & 45 & 33 & 43 & 30 & 38 \\
\hline $65-74 \min$ & 9 & 6 & 4 & 4 & 6 \\
\hline $75-84 \min$ & 2 & 2 & 2 & 4 & 3 \\
\hline Total & $98^{a}$ & $96^{b}$ & $98^{c}$ & 100 & $99^{d}$ \\
\hline
\end{tabular}

Note: Normal trip time $=40 \mathrm{~min}$; buffer time provided $=10 \mathrm{~min}$

a Does not add up to $100 \%$ because $2 \%$ of participants did not provide an answer.

${ }^{b}$ Does not add up to $100 \%$ because $4 \%$ of participants did not provide an answer.

${ }^{c}$ Does not add up to $100 \%$ because $2 \%$ of participants did not provide an answer.

${ }^{a}$ Does not add up to $100 \%$ because $1 \%$ of participants did not provide an answer. 


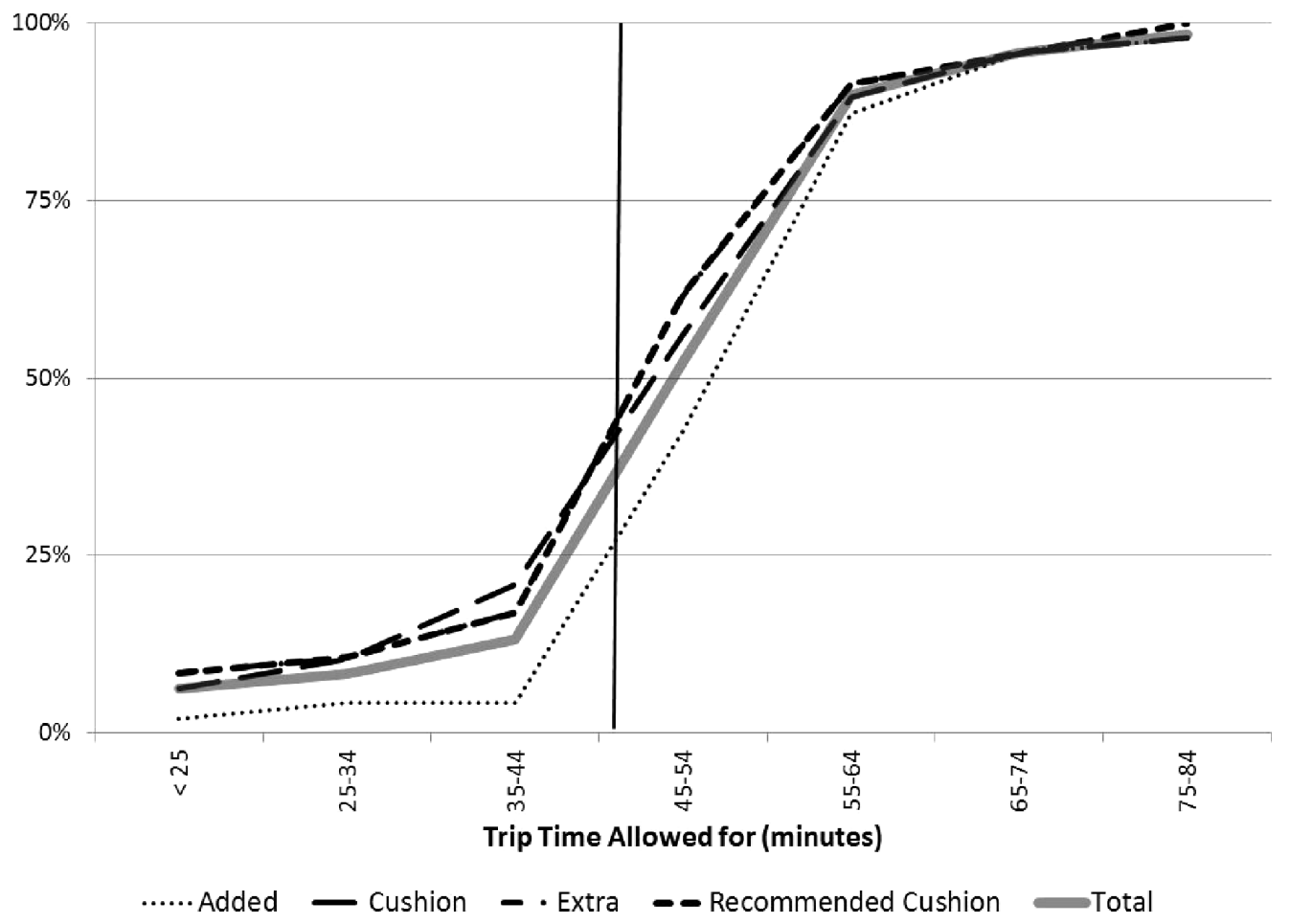

Figure 8.2. Cumulative distribution of trip times allowed for 50-minute trip (normal trip time $=40 \mathrm{~min}$, buffer $=10 \mathrm{~min}$ ).

These results imply that drivers may have difficulty accepting and trusting a route-specific buffer value that is smaller than the generic buffer they have developed through years of driving experiences and other influences.

\section{Similar Meanings and Preference of Buffer Terms}

Another portion of the survey addressed whether participants thought the buffer term provided in the scenario 1 website message meant the same thing as the remaining buffer terms (those not originally viewed by the participant). The percentage of participants who indicated that various terms implied the same meaning is provided in Table 8.7. Overall, two clear trends emerged. Participants who saw either added time or extra time rated the other term as having a similar meaning. Likewise, those who saw either cushion time or recommended cushion time viewed the other term as having the same meaning. As shown in Figure 8.2, added time and extra time resulted in similar trends, so it is not surprising that these two terms

Table 8.7. Assessment of Similar Meanings of Buffer Terms

\begin{tabular}{|c|c|c|c|c|c|}
\hline \multirow[b]{3}{*}{ Terms Compared } & \multicolumn{5}{|c|}{ Percentage of Participants } \\
\hline & \multicolumn{4}{|c|}{ Buffer Term Viewed } & \multirow[b]{2}{*}{ Average } \\
\hline & $\begin{array}{l}\text { Added } \\
(n=47)\end{array}$ & $\begin{array}{l}\text { Cushion } \\
(n=48)\end{array}$ & $\begin{array}{c}\text { Extra } \\
(n=47)\end{array}$ & $\begin{array}{l}\text { Recommended } \\
\text { Cushion }(n=47)\end{array}$ & \\
\hline Added means the same as Cushion & 43 & 50 & & & 46 \\
\hline Added means the same as Extra & 81 & & 66 & & 73 \\
\hline $\begin{array}{l}\text { Added means the same as } \\
\text { Recommended Cushion }\end{array}$ & 43 & & & 43 & 43 \\
\hline Cushion means the same as Extra & & 50 & 40 & & 45 \\
\hline $\begin{array}{l}\text { Cushion means the same as } \\
\text { Recommended Cushion }\end{array}$ & & 73 & & 72 & 73 \\
\hline $\begin{array}{l}\text { Extra means the same as } \\
\text { Recommended Cushion }\end{array}$ & & & 36 & 49 & 43 \\
\hline
\end{tabular}

Note: Shaded cells indicate combinations that were not evaluated. 
Table 8.8. Preferences Among Buffer Terms

\begin{tabular}{|l|c|c|c|c|c|}
\hline \multirow{2}{*}{ Preferred Term } & \multicolumn{5}{|c|}{ Percentage of Participants } \\
\cline { 2 - 6 } & \multicolumn{5}{|c|}{ Buffer Term Viewed } \\
\cline { 2 - 6 } & $\begin{array}{c}\text { Added } \\
(n=47)\end{array}$ & $\begin{array}{c}\text { Cushion } \\
(\boldsymbol{n}=\mathbf{4 8})\end{array}$ & $\begin{array}{c}\text { Extra } \\
(\boldsymbol{n}=\mathbf{4 7})\end{array}$ & $\begin{array}{c}\text { Recommended } \\
\text { Cushion }(\boldsymbol{n}=\mathbf{4 7})\end{array}$ & \multirow{2}{*}{ Average } \\
\hline Added & 17 & 17 & 15 & 15 & 16 \\
\hline Extra & 15 & 10 & 13 & 32 & 17 \\
\hline Cushion & 34 & 25 & 45 & 30 & 33 \\
\hline Recommended cushion & 32 & 48 & 26 & 23 & 32 \\
\hline Added or extra & 2 & 0 & 0 & 0 & 1 \\
\hline None & 0 & 0 & 3 & 0 & 100 \\
\hline Total & 100 & 100 & 100 & 100 & 1 \\
\hline
\end{tabular}

were considered to have similar meanings. However, the trip times allowed by participants for cushion time and recommended cushion time (shown in Table 8.7) were not similar.

The percentage of participants who preferred each of the terms tested is shown in Table 8.8. Overall, the results did not indicate a clear preference for any of the terms: no single term was preferred by more than one-third of the participants, on average. However, the preference data did reflect that pairs of terms were viewed as having similar meanings: cushion time and recommended cushion time were preferred over added time or extra time.

\section{Use of Total Trip Time Estimate}

Again, researchers analyzed the responses to the questions regarding how much time participants would allow for their trip. They initially looked at each normal trip time or total trip time estimate pair to determine whether the normal trip time terms affected the use of the total trip time estimate term. Researchers did not identify any clear trends that indicated the normal trip time term affected the use of the total trip time estimate term, so the data for each total trip time estimate term were aggregated across the normal trip time terms for further analysis.

The next analysis explored how the total trip time estimate terms influence the extent to which motorists may or may not use the total trip time provided to plan their travel. At the simplest level of analysis, researchers envisioned four possible ways in which drivers would use the times presented in scenario 2 (the normal trip time and the total trip time values) to estimate how much time they would allow for their trip:

- Participants could ignore the total trip time estimate and base their trip time strictly on the normal trip time value provided.
- Participants could ignore the normal trip time value provided and base their trip time strictly on the total trip time estimate provided (the intended use).

- Participants could combine the normal trip time and the total trip time estimate to come up with the trip time they would allow for (the intended use of the buffer time term).

- Participants could add some other time (their own buffer) to the normal trip time or the total trip time estimate to come up with the trip time they would allow for (the amount of other time added may or may not be influenced by the total trip time estimate).

The percentage of participants who selected trip times in each of those categories is presented in Table 8.9. Few participants indicated they would allow for only the normal trip time provided in the website message. In addition, few participants combined the values for the two terms provided. Among the four total trip time estimate terms, majority of the time resulted in the greatest percentage of participants basing their trip time strictly on the total trip time estimate provided. Still, on average, $37 \%$ of participants added some other value to the total trip time estimate to come up with the trip time they would allow for in scenario 2 .

Additional insights into the trip times that participants indicated they would allow for (as a function of the trip time uncertainty term) are presented in Table 8.10. The majority of the trip time values were in the " $45-54$ min" and "55-64 min" groups, which were within 10 min of the total trip time estimate value ( $55 \mathrm{~min}$ ). Those times reflect the responses of participants who used the total trip time estimate, the total trip time estimate plus some other small time, or the normal trip time plus some other larger time.

The distribution of trip times that participants would allow for shows some small differences by type of total trip time estimate term used. The distribution is shown in Figure 8.3. 
Table 8.9. Use of Total Trip Time Estimate for Trip Planning

\begin{tabular}{|c|c|c|c|c|c|}
\hline \multirow[b]{3}{*}{$\begin{array}{l}\text { Participant Use of Total Trip } \\
\text { Time Estimate }\end{array}$} & \multicolumn{5}{|c|}{ Percentage of Participants } \\
\hline & \multicolumn{4}{|c|}{ Total Trip Time Estimate Term Viewed } & \multirow[b]{2}{*}{ Average } \\
\hline & $\begin{array}{l}\text { 95th Percentile } \\
\quad(n=46)\end{array}$ & $\begin{array}{c}\text { Majority of the } \\
\text { Time }(n=48)\end{array}$ & $\begin{array}{l}\text { Most of the } \\
\text { Time }(n=48)\end{array}$ & $\begin{array}{l}\text { Travel Time for } \\
\text { Planning }(n=47)\end{array}$ & \\
\hline Used normal trip time only & 4 & 4 & 0 & 4 & 3 \\
\hline Used total trip time estimate only & 24 & 37 & 25 & 31 & 29 \\
\hline $\begin{array}{l}\text { Added normal trip time and total } \\
\text { trip time estimate values together }\end{array}$ & 7 & 0 & 0 & 2 & 2 \\
\hline $\begin{array}{l}\text { Added normal trip time and some } \\
\text { other value together }\end{array}$ & 13 & 15 & 29 & 40 & 24 \\
\hline $\begin{array}{l}\text { Added total trip time estimate and } \\
\text { some other value together }\end{array}$ & 50 & 40 & 38 & 19 & 37 \\
\hline Total & $98^{a}$ & $96^{b}$ & $92^{c}$ & $96^{d}$ & $95^{e}$ \\
\hline
\end{tabular}

a Does not add up to $100 \%$ because researchers categorized $2 \%$ of the responses as "other."

b Does not add up to $100 \%$ because researchers categorized $4 \%$ of the responses as "other."

"Does not add up to $100 \%$ because researchers categorized $8 \%$ of the responses as "other."

"Does not add up to $100 \%$ because researchers categorized $4 \%$ of the responses as "other."

e Does not add up to $100 \%$ because researchers categorized $5 \%$ of the responses as "other."

According to these data, the term majority of the time resulted in the largest percentage of participants (79\%) choosing times within $10 \mathrm{~min}$ of the total trip time estimate ( $55 \mathrm{~min}$ ) conveyed in the website message for scenario 2. When the terms most of the time and 95th percentile were shown, a greater percentage of participants chose travel times that exceeded the total trip time estimate presented in the message $(23 \%$ and $35 \%$, respectively). This results suggests participants may have less confidence in these two terms and that led to decisions to allow more time than was presented in the message. Conversely, many participants who viewed the term travel time for planning chose to use a value less than the total trip time estimate in deciding how much time to allow. This result suggests that the term travel time for planning may convey more of a sense of a maximum trip time.

Table 8.11 shows participants' confidence in how likely they would be to arrive at the destination in $55 \mathrm{~min}$ or less for the trip time uncertainty terms used in scenario 2 . Strangely, the term most of the time resulted in the largest percentage of participants (75\%) selecting "absolutely certain (100\% chance)" or "very

Table 8.10. Distribution of Trip Times Allowed for 55-Minute Trip

\begin{tabular}{|c|c|c|c|c|c|}
\hline \multirow[b]{3}{*}{$\begin{array}{l}\text { Trip Times } \\
\text { Allowed For }\end{array}$} & \multicolumn{5}{|c|}{ Percentage of Participants } \\
\hline & \multicolumn{4}{|c|}{ Total Trip Time Estimate Term Viewed } & \multirow[b]{2}{*}{ Average } \\
\hline & $\begin{array}{l}\text { 95th Percentile } \\
\quad(n=46)\end{array}$ & $\begin{array}{c}\text { Majority of the } \\
\text { Time }(n=48)\end{array}$ & $\begin{array}{l}\text { Most of the } \\
\text { Time }(n=48)\end{array}$ & $\begin{array}{c}\text { Travel Time for } \\
\text { Planning }(n=47)\end{array}$ & \\
\hline$<25 \min$ & 2 & 0 & 0 & 0 & 1 \\
\hline $25-34 \min$ & 4 & 4 & 4 & 8 & 5 \\
\hline $35-44 \min$ & 7 & 2 & 4 & 11 & 6 \\
\hline $45-54 \min$ & 7 & 11 & 25 & 23 & 16 \\
\hline $55-64 \min$ & 45 & 68 & 44 & 50 & 52 \\
\hline $65-74 \min$ & 11 & 2 & 7 & 4 & 6 \\
\hline $75-84 \min$ & 11 & 9 & 8 & 2 & 7 \\
\hline$\geq 85 \min$ & 13 & 4 & 8 & 2 & 7 \\
\hline Total & 100 & 100 & 100 & 100 & 100 \\
\hline
\end{tabular}

Note: Normal trip time $=30 \mathrm{~min}$; total trip time estimate provided $=55 \mathrm{~min}$. 


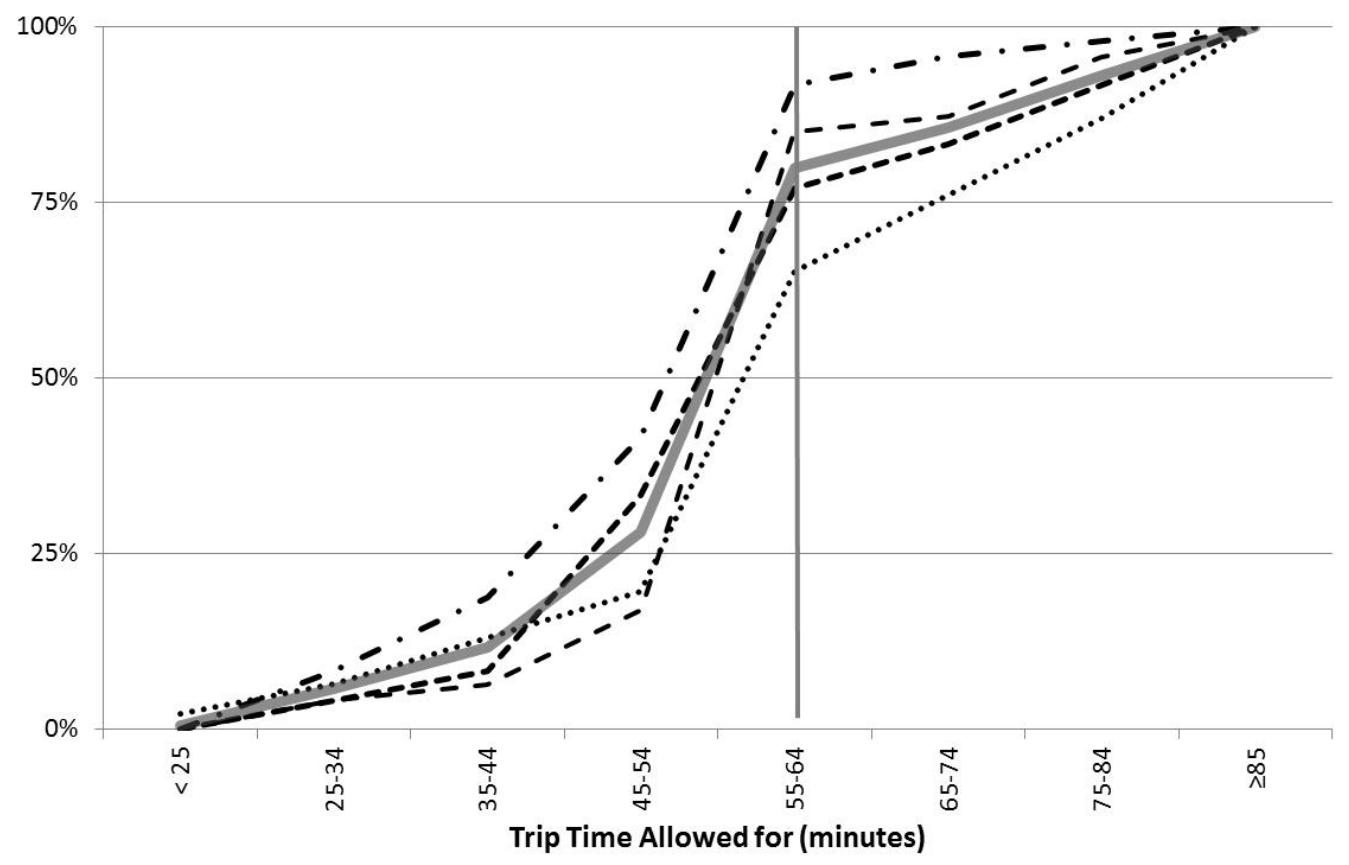

-..995th Percentile - - Majority of the Time - - Most of the Time - Travel Time for Planning

Figure 8.3. Cumulative distribution of trip times allowed for 55-minute trip (normal trip time $=30 \mathrm{~min}$; total trip time estimate provided $=55 \mathrm{~min}$ ).

likely (95\% chance)." This result was unexpected because $23 \%$ of participants chose a travel time that exceeded the total trip time. For travel time for planning, the percentage of participants selecting those two categories was $73 \%$. That result was expected because $92 \%$ of participants chose a travel time that was less than the total trip time estimate. For both majority of the time and 95th percentile, the percentage of participants selecting the same two categories corresponded to only $62 \%$ and $54 \%$, respectively.

The final question for this scenario asked participants how much trip time they would allow if the normal trip time remained at $30 \mathrm{~min}$ but the total trip time estimate was $45 \mathrm{~min}$ (10 min less than in the original scenario 2). The responses to that question are summarized in Table 8.12 and shown in Figure 8.4. The majority of the trip time values were in the "45-54 min" and "35-44 min" groups, which was again within $10 \mathrm{~min}$ of the total trip time estimate value ( $45 \mathrm{~min}$ ). This result shows that most of the participants did shift the trip time they would allow for to match the total trip time estimate presented. However, for all of the trip time uncertainty terms used, a larger percentage of participants chose a trip time longer than the total trip time estimate, showing less confidence in the smaller total

Table 8.11. Participant Confidence in Arriving at Destination in 55 Minutes or Less

\begin{tabular}{|c|c|c|c|c|c|}
\hline \multirow[b]{3}{*}{ Participant Confidence } & \multicolumn{5}{|c|}{ Percentage of Participants } \\
\hline & \multicolumn{4}{|c|}{ Total Trip Time Estimate Term Viewed } & \multirow[b]{2}{*}{ Average } \\
\hline & $\begin{array}{l}\text { 95th Percentile } \\
\quad(n=46)\end{array}$ & $\begin{array}{c}\text { Majority of the } \\
\text { Time }(n=48)\end{array}$ & $\begin{array}{l}\text { Most of the } \\
\text { Time }(n=48)\end{array}$ & $\begin{array}{l}\text { Travel Time for } \\
\text { Planning }(n=47)\end{array}$ & \\
\hline Absolutely certain ( $100 \%$ chance) & 2 & 9 & 21 & 13 & 11 \\
\hline Very likely (95\% chance) & 54 & 53 & 54 & 60 & 56 \\
\hline Probably (75\% chance) & 20 & 27 & 17 & 15 & 20 \\
\hline Unsure (50/50 chance) & 17 & 9 & 8 & 10 & 11 \\
\hline Doubtful (less than $50 / 50$ chance) & 7 & 2 & 0 & 2 & 2 \\
\hline Total & 100 & 100 & 100 & 100 & 100 \\
\hline
\end{tabular}

Note: Normal trip time $=30 \mathrm{~min}$; total trip time estimate provided $=55 \mathrm{~min}$. 
Table 8.12. Distribution of Trip Times Allowed for 45-Minute Trip

\begin{tabular}{|c|c|c|c|c|c|}
\hline \multirow[b]{3}{*}{$\begin{array}{l}\text { Trip Times } \\
\text { Allowed For }\end{array}$} & \multicolumn{5}{|c|}{ Percentage of Participants } \\
\hline & \multicolumn{4}{|c|}{ Total Trip Time Estimate Term Viewed } & \multirow[b]{2}{*}{ Average } \\
\hline & $\begin{array}{l}\text { 95th Percentile } \\
\quad(n=46)\end{array}$ & $\begin{array}{c}\text { Majority of the } \\
\text { Time }(n=48)\end{array}$ & $\begin{array}{c}\text { Most of the } \\
\text { Time }(n=48)\end{array}$ & $\begin{array}{c}\text { Travel Time for } \\
\text { Planning }(n=47)\end{array}$ & \\
\hline$<25 \min$ & 4 & 0 & 2 & 2 & 2 \\
\hline 25-34 min & 4 & 4 & 2 & 4 & 4 \\
\hline $35-44 \min$ & 0 & 11 & 15 & 17 & 11 \\
\hline $45-54 \min$ & 48 & 49 & 44 & 50 & 48 \\
\hline $55-64 \min$ & 26 & 28 & 27 & 25 & 26 \\
\hline $65-74 \min$ & 3 & 2 & 2 & 0 & 1 \\
\hline $75-84 \min$ & 13 & 4 & 4 & 2 & 6 \\
\hline$\geq 85 \min$ & 2 & 2 & 4 & 0 & 2 \\
\hline Total & 100 & 100 & 100 & 100 & 100 \\
\hline
\end{tabular}

Note: Normal trip time $=30 \mathrm{~min}$; total trip time estimate provided $=45 \mathrm{~min}$.

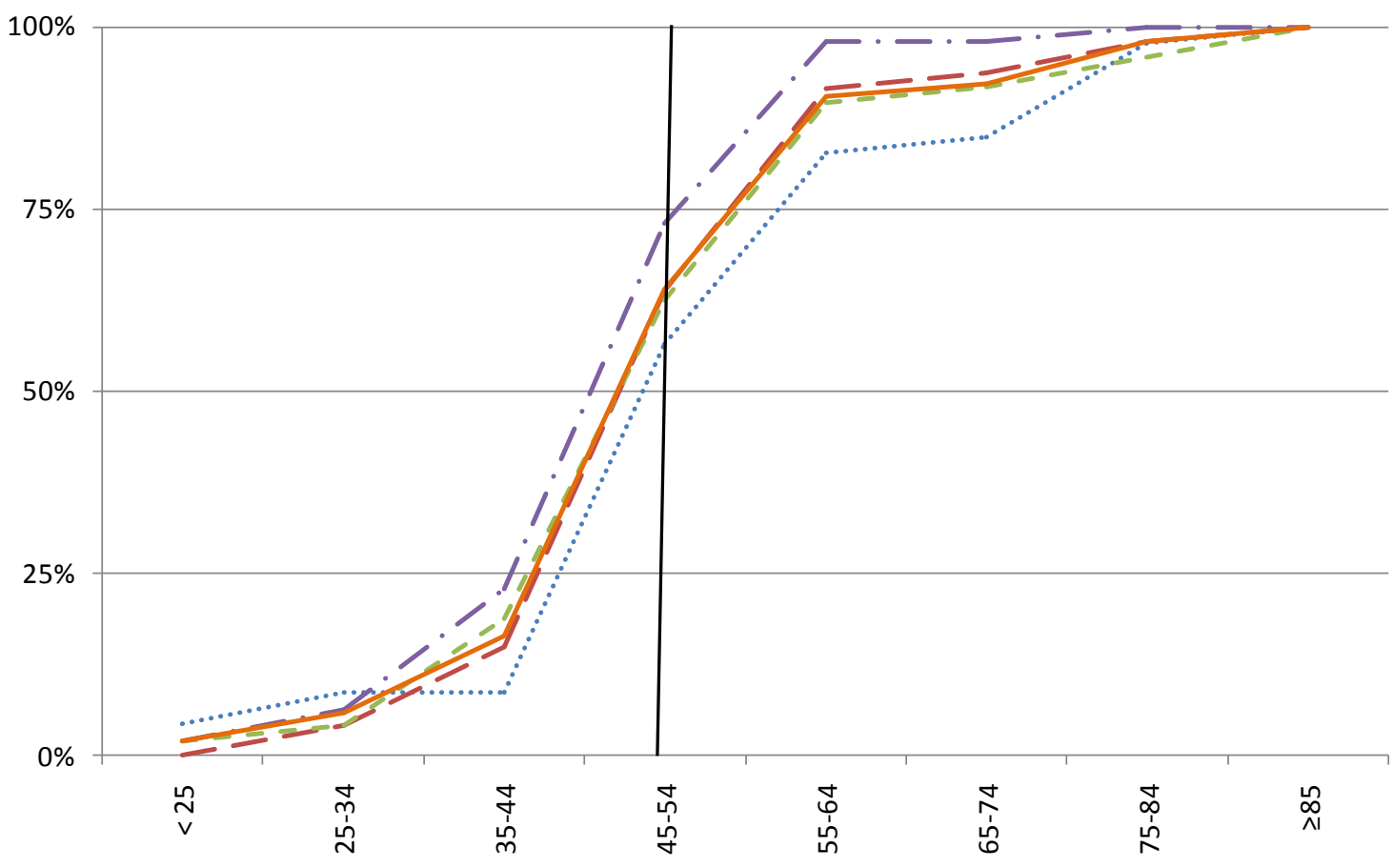

Trip Time Allowed for (minutes)

.... 95th Percentile - Majority of the Time -- Most of the Time $-\cdot$ Travel Time for Planning - Total

Figure 8.4. Cumulative distribution of trip times allowed for 45-minute trip (normal trip time = 30 min; total trip time estimate provided $=\mathbf{4 5} \mathrm{min}$ ). 
Table 8.13. Assessment of Similar Meanings of Total Trip Time Estimate Terms

\begin{tabular}{|c|c|c|c|c|c|}
\hline \multirow[b]{3}{*}{ Terms Compared } & \multicolumn{5}{|c|}{ Percentage of Participants } \\
\hline & \multicolumn{4}{|c|}{ Total Trip Time Estimate Term Viewed } & \multirow[b]{2}{*}{ Average } \\
\hline & $\begin{array}{l}\text { 95th Percentile } \\
\qquad(n=46)\end{array}$ & $\begin{array}{l}\text { Majority of the } \\
\text { Time }(n=48)\end{array}$ & $\begin{array}{l}\text { Most of the } \\
\text { Time }(n=48)\end{array}$ & $\begin{array}{l}\text { Travel Time for } \\
\text { Planning }(n=47)\end{array}$ & \\
\hline 95th percentile means the same as majority of the time & 68 & 45 & & & 56 \\
\hline 95th percentile means the same as most of the time & 49 & & 54 & & 52 \\
\hline $\begin{array}{l}\text { 95th percentile means the same as travel time for } \\
\text { planning }\end{array}$ & 13 & & & 32 & 22 \\
\hline $\begin{array}{l}\text { Majority of the time means the same as most of } \\
\text { the time }\end{array}$ & & 91 & 79 & & 85 \\
\hline $\begin{array}{l}\text { Majority of the time means the same as travel time } \\
\text { for planning }\end{array}$ & & 26 & & 47 & 36 \\
\hline $\begin{array}{l}\text { Most of the time means the same as travel time for } \\
\text { planning }\end{array}$ & & & 29 & 64 & 46 \\
\hline
\end{tabular}

Note: Shaded cells indicate combinations that were not evaluated.

trip time estimate value. Here again, researchers inferred that drivers may have difficulty accepting and trusting a total trip time estimate that is closer to the normal trip time.

\section{Meanings of and Preference for Total Trip Time Estimate Terms}

Next, researchers asked whether participants thought the total trip time estimate term provided in the scenario 2 website message meant the same thing as the remaining total trip time estimate terms. The percentage of participants who indicated that various terms implied the same meaning is provided in Table 8.13. Overall, one clear trend emerged: participants who saw either majority of the time or most of the time rated the other term as having a similar meaning. This result was not surprising considering the similar trends for these two terms shown in Figure 8.1. No other term combinations were viewed as being similar by most participants.
The percentage of participants who preferred each of the terms tested is shown in Table 8.14. These findings revealed no clear consensus as to the most preferred total trip time estimate term. However, the preference data did reflect that majority of the time and most of the time were similarly preferred.

\section{Preference for Travel Time Uncertainty Terms}

Participants were also asked whether they preferred the travel time uncertainty information in the first scenario (buffer time) or the second scenario (total trip time estimate). Overall, $60 \%$ of participants preferred to be provided the normal travel time and the total trip time estimate (scenario 2). Of these participants, about half preferred the total trip estimate term because the total number was provided (i.e., they did not have to add multiple values). Another 20\% thought the total value would be more accurate or exact than the two pieces of information that had to be added together.

Table 8.14. Preference for Total Trip Time Estimate Terms

\begin{tabular}{|c|c|c|c|c|c|}
\hline \multirow[b]{3}{*}{ Preferred Term } & \multicolumn{5}{|c|}{ Percentage of Participants } \\
\hline & \multicolumn{4}{|c|}{ Total Trip Time Estimate Term Viewed } & \multirow[b]{2}{*}{ Average } \\
\hline & $\begin{array}{l}\text { 95th Percentile } \\
\qquad(n=46)\end{array}$ & $\begin{array}{c}\text { Majority of the } \\
\text { Time }(n=48)\end{array}$ & $\begin{array}{l}\text { Most of the } \\
\text { Time }(n=48)\end{array}$ & $\begin{array}{l}\text { Travel Time for } \\
\text { Planning }(n=47)\end{array}$ & \\
\hline 95th percentile & 21 & 15 & 10 & 13 & 15 \\
\hline Majority of the time & 34 & 30 & 63 & 30 & 39 \\
\hline Most of the time & 26 & 38 & 17 & 47 & 32 \\
\hline Travel time for planning & 15 & 17 & 10 & 8 & 12 \\
\hline 95th percentile or majority of the time & 2 & 0 & 0 & 0 & 1 \\
\hline None & 2 & 0 & 0 & 2 & 1 \\
\hline Total & 100 & 100 & 100 & 100 & 100 \\
\hline
\end{tabular}


Table 8.15. Assessment of Similar Meanings of Normal Travel Time Terms

\begin{tabular}{|l|c|c|c|c|c|}
\hline \multirow{2}{*}{ Terms Compared } & \multicolumn{4}{|c|}{ Percentage of Participants } \\
\cline { 2 - 5 } & \multicolumn{3}{|c|}{ Normal Travel Time Term Viewed } \\
\cline { 2 - 5 } & $\begin{array}{c}\text { Average } \\
(\boldsymbol{n}=\mathbf{4 8})\end{array}$ & $\begin{array}{c}\text { Estimated } \\
(\boldsymbol{n}=\mathbf{4 7})\end{array}$ & $\begin{array}{c}\text { Expected } \\
(\boldsymbol{n}=\mathbf{4 8})\end{array}$ & $\begin{array}{c}\text { Typical } \\
(\boldsymbol{n}=\mathbf{4 6})\end{array}$ & \multirow{2}{*}{ Average } \\
\hline Average means the same as estimated & 63 & 66 & & & 64 \\
\hline Average means the same as expected & 42 & & 48 & & 45 \\
\hline Average means the same as typical & 71 & & & 70 & 70 \\
\hline Estimated means the same as expected & & 68 & 83 & & 76 \\
\hline Estimated means the same as typical & & 40 & & 37 & 39 \\
\hline Expected means the same as typical & & & 52 & 43 & 49 \\
\hline
\end{tabular}

Note: Shaded cells indicate combinations that were not evaluated.

\section{Similar Meanings of and Preference for Normal Trip Time Terms}

Researchers also asked whether participants thought the normal trip time term provided in the scenario 2 website message meant the same thing as the remaining normal trip time terms. As shown in Table 8.15, on average, $76 \%$ of participants viewed the terms estimated and expected as having a similar meaning. Likewise, $70 \%$ of participants viewed average as similar to typical. No other clear trends emerged.

The results of the participants' preferences for the normal travel time terms are summarized in Table 8.16. These findings revealed no clear consensus as to the most preferred normal travel time term.

\section{Conclusions}

\section{Buffer Time Terms}

- Approximately $20 \%$ of participants added the normal travel time and buffer time to determine the amount of time they should allow for the trip. The particular buffer time term used may have had a small influence on this value. The term recommended cushion time resulted in the greatest percentage of participants adding the buffer time to the normal trip time, while the term cushion time resulted in the least.

- Participants who saw recommended cushion time were more likely to select a total trip time that was clustered closely around the 70 -min total trip time implied by the website message. This term also produced the highest confidence among participants that they would arrive at their destination in 70 min or less. Even though recommended cushion time and cushion time were viewed as having a similar meaning, participants who saw cushion time were more likely to choose a trip time less than $70 \mathrm{~min}$, implying that participants had less trust in this term.

- Participants viewed the terms added time and extra time as having a similar meaning. In addition, those who saw added time or extra time were more likely to choose a trip time that exceeded $70 \mathrm{~min}$. This result suggests that they had somewhat less confidence in these terms and so added even more time to ensure on-time arrival; or it may suggest that they did not fully understand the intent of these time values.

- Results did not indicate a clear preference for any of the buffer terms. The terms recommended cushion time and cushion time were selected most frequently, but only by about one-third of the participants for each.

Table 8.16. Preference for Normal Travel Time Terms

\begin{tabular}{|c|c|c|c|c|c|}
\hline \multirow[b]{3}{*}{ Preferred Term } & \multicolumn{5}{|c|}{ Percentage of Participants } \\
\hline & \multicolumn{4}{|c|}{ Normal Travel Time Term Viewed } & \multirow[b]{2}{*}{ Average } \\
\hline & $\begin{array}{r}\text { Average } \\
(n=48)\end{array}$ & $\begin{array}{c}\text { Estimated } \\
(n=47)\end{array}$ & $\begin{array}{c}\text { Expected } \\
(n=48)\end{array}$ & $\begin{array}{c}\text { Typical } \\
(n=46)\end{array}$ & \\
\hline Average & 13 & 30 & 13 & 24 & 20 \\
\hline Estimated & 58 & 21 & 52 & 33 & 41 \\
\hline Expected & 16 & 40 & 25 & 39 & 30 \\
\hline Typical & 13 & 9 & 10 & 4 & 9 \\
\hline Total & 100 & 100 & 100 & 100 & 100 \\
\hline
\end{tabular}


- When the buffer time was reduced from $30 \mathrm{~min}$ to $10 \mathrm{~min}$, participants did not reduce their total trip time estimate by that same amount. This result suggests that participants did not totally trust the buffer time values, regardless of the term used. Trip time providers may have difficulty getting drivers to accept a trip uncertainty value that is less than the general uncertainty values they have learned through their own experiences when making trip time decisions.

\section{Total Trip Time Estimate Terms}

- Results of the survey indicated that approximately $30 \%$ of participants used a total trip time measure for determining the amount of time they should allow for a particular trip. The particular total trip time term used may have had a small influence on this value. The term majority of the time resulted in the greatest percentage of participants who strictly used the total trip time measure, while the term 95th percentile resulted in the least.

- The term majority of the time resulted in a greater percentage of participants who would select the time provided, or a value near it, when deciding how much time to allow for a particular trip. Even though participants judged the terms majority of the time and most of the time to have similar meanings, participants who saw most of the time were more likely to choose a trip time that exceeded $55 \mathrm{~min}$, implying a lower level of confidence for this term. Participants who saw the term 95th percentile were also more likely to choose a travel time that exceeded $55 \mathrm{~min}$. Conversely, participants who viewed the term travel time for planning were more likely to choose a value less than $55 \mathrm{~min}$, suggesting that this term may convey more of a sense of a maximum trip time; if drivers relied on this term, they might be late more often than they had hoped.

- Participants showed no clear preference among the total trip time terms. The terms majority of the time and most of the time were selected most frequently but only by about one-third of the participants for each.

- Changing the value of the total trip time estimate (relative to the normal trip time estimate) did affect the time participants chose to allow for a trip. However, as with the buffer time terms, participants' selection of a time to allow for a trip appeared to depend on how the value of the total trip time estimate compared with their own personal buffer time that had developed over time on the basis of their own experiences. Terms that implied a buffer of less than 20 min appeared to be less trusted by drivers.

\section{Comparison of Travel Time Uncertainty Information}

- Use of a buffer time or a total trip time estimate resulted in similar levels of confidence in arriving on time at a destination. Overall, $75 \%$ and $67 \%$ of participants, respectively, indicated that their trip would certainly or very likely take less than the value shown, further indicating that they recognized that value as an upper limit to how long a trip might take.

- A slight preference was detected among participants for the use of total trip time terms over the use of buffer terms. This result most likely occurred because many drivers would prefer not to have to do the math (adding a normal trip time and a buffer time) to estimate the time they should allow for a trip.

\section{Comprehension of Terms for Normal Travel Time}

- None of the four terms evaluated to convey normal travel time appeared to affect participants' selection and use of the buffer time or total trip time estimates.

- Participants viewed the terms estimated and expected as having similar meanings. In addition, participants judged the terms average and typical to have similar meanings. No other clear trends emerged as to whether participants believed that the various normal travel time terms had similar meanings.

- No clear trends emerged with regard to participant preference for the normal travel time terms. The terms estimated and expected garnered the most support but only by about one-third or so of the participants for each.

\section{Recommendations}

- Because the four normal travel time terms evaluated performed similarly and no clear preference was identified, researchers believe that any of the four terms may be used to convey the normal travel time.

- Both types of travel time uncertainty terms (buffer time and total trip time estimate) similarly influenced the extent to which motorists might or might not use the travel time uncertainty in their travel time decisions. However, participants did prefer to be provided with the total trip time estimate. Researchers think that providing the total trip time estimate decreases driver workload and the potential for math errors, which could otherwise lead users to distrust the information provided by an agency. Thus, a total trip time estimate term appears to be preferable to a buffer term when trying to convey trip time uncertainty information. Furthermore, researchers recommend the use of the term majority of the time to describe the total trip time estimate.

- The above notwithstanding, if an agency still desires to use a buffer time to convey travel time uncertainty, researchers recommend the use of the term recommended cushion time. 


\section{онАВтеп \\ Travel Behavior Laboratory Experiment}

Utility is a measure of absolute or relative satisfaction. Conversely, disutility is a measure of dissatisfaction. In the case of travel, disutility assessments translate the dissatisfaction associated with travel into quantified and monetized measures. Trip disutility is the complex and collective bottom-line outcome that takes into account interactions among traveler needs and preferences, trip purpose, travel conditions, perceived and objective trip time, and travel time uncertaintyin addition to the direct costs of travel, such as fuel or fares. Trip disutility functions assign value, or cost per unit, for specific components associated with trips, such as travel time and schedule delay. Valuations based on reductions in travel disutility are central to cost-benefit analyses for transportation investments and, more recently, for traffic operations decisions such as value pricing of high-occupancy toll lanes.

Many studies have assessed the value of travel time, schedule delay, and reliability associated with travel; and a number have further refined the valuation to reflect specific traveler and modal characteristics. Fewer studies further assess reductions in trip disutility resulting from the provision of traveler information. What is still relatively unexplored is the monetized reduction in trip disutility associated with the provision of travel time reliability information. Travel time reliability information, as defined in the SHRP 2 L14 effort, encompasses a broad range of information that describes underlying trip time variability and other contextual data travelers use to manage delay and on-time performance. The information encompasses three general categories: (a) descriptions of the statistical variation in travel time dependent on departure time choice, (b) data regarding on-time performance and lateness risk by route and destination, and (c) contextual information to interpret cueing throughout the travel experience to allow travelers to better assess travel time and lateness risk both pre-trip and en route. Travelers who make repeated similar trips at the same time of day accumulate an internalized assessment of trip travel time variability with which to gauge lateness risk. Relative to those familiar trips, researchers expect that-for unfamiliar trips- travelers will perceive a larger benefit from travel time reliability information.

The travel behavior laboratory experiment described in this chapter aimed to measure the perceived value of trip reliability information and how that valuation changes with increasing trip familiarity. This assessment of how reliability information can reduce travel disutility included the consideration of a particular aspect, trip serenity, or the reduction of stress en route associated with potential late arrival. The experiment captured variations in reported stress associated with on-time performance at trip start, while en route, and at trip end among scenarios with varying levels of traveler information and trip outcomes.

The objectives of the experiment were twofold: (a) to assess the value participants placed on traveler information, specifically reliability information, within the context of a simulated time-constrained trip; and (b) to determine whether having reliability information helped speed the transition from an unfamiliar commuter to an experienced commuter. The underlying conjecture in the experiment was that providing reliability information would allow an unfamiliar traveler to manage the trade-off between on-time performance and travel budget (time allocated to travel) more effectively and with less stress. Put another way, the provision of reliability information to travelers unfamiliar with underlying travel time variability patterns was expected to both improve overall trip outcomes (reduction of early and late schedule delay, better on-time performance, and reduced delay) and reduce perceptional disutility associated with those improved outcomes. Three experimental hypotheses were posed:

1. Provision of accurate reliability information will result in improved on-time performance and lower generalized travel disutility compared with a control group receiving no reliability information.

2. While travel outcomes improve with the provision of reliability information, the perceived value of the reliability 
information will underestimate the realized benefit in terms of reduced delay, improved on-time reliability, and reduced stress.

3. The benefits of reliability information will decline over time as both experimental and control subjects learn and understand the underlying travel time variability. That is, the benefit from reliability information during the first weeks will be larger than during the last weeks.

To meet experimental objectives and to best fit within the overall SHRP 2 L14 project, Noblis selected a trip simulation approach to the experiment. The experimental strategy and corresponding evaluation tools were prepared by Noblis, while the experiment was administered by the TTI staff. Data generated by the experiment were analyzed by Noblis in support of the SHRP 2 L14 Task 12, Development of Utility Functions.

The experiment presented 80 participants with a scenario in which they were commuting in an unfamiliar city with a constrained arrival time (with penalties for both late and early arrival) for two work weeks (10 commutes). Participants had access to one of three additive levels of travel time information for their planned route and optional route deviations: real-time advisory messages only, real-time advisories plus real-time travel time information, or real-time advisories plus both real-time and reliability (historical travel time range) information. Participants selected from among three departure time options and two potential alternate route options to arrive at their destination on time. All participants received the real-time advisory messages (from simulated DMSs deployed along the simulated route); a subset of participants had to actively acquire the higher levels of real-time and reliability information by pressing an interface button. The experiment was conducted in the same five cities (Dallas, Denver, Hartford, Miami, and San Jose) as the SHRP 2 L14 computer survey.

\section{Background}

Within the SHRP 2 L14 project, two activities - a driver simulation study and a field test-were expected to generate data that would be leveraged to measure the perceived value of trip reliability information - the goal of Task 12, Development of Utility Functions. However, the elimination of the simulator testing along with a recasting of planned field testing precluded the acquisition of data critical to the completion of Task 12. At the same time, findings from Phase 1 focused attention on quantifying the relative value of reliability information. Because of these changes in project direction, Noblis and TTI proposed an experiment using subjects traversing a simulated trip to meet the goals of Task 12. The experiment was designed to assess the serenity benefits from trip reliability information and to translate the value of trip reliability information through assessment of trip disutility levels. The experiment was also designed to work within the implementation framework of the existing SHRP 2 L14 computer survey task. The experiment was developed by Noblis and deployed by TTI in concert with the planned computer surveys.

\section{Literature Review}

The valuation of individual's travel has been of interest in transportation research for decades, with earliest valuations focusing on the travel time component of travel. Valuation of travel time for work trips expanded to valuation by travel mode and to nonwork trips. Since the 1960s, studies through econometric, stated preference, and revealed preference models have assessed valuation of travel. Over time, researchers have developed more complex frameworks for this valuation to more truly reflect actual valuation by travelers by considering factors such as travel conditions (e.g., congestion, reliability) and traveler characteristics (e.g., trip purpose, scheduling flexibility).

Vickery (1963) suggested expanding travel valuation beyond travel time to consider the concept that, for the work commute, the penalty for being early would be less than for being late. The concept of time constraints on a trip and travelers' preference for a specific arrival time was furthered by Gaver (1968), who hypothesized that trip disutility is a linear function of travel time and early and late schedule delays. Small (1982) built on earlier work using a scheduling delay framework to explicitly tie departure time choice to the utility function of a traveler. Using data from 527 San Francisco Bay Area auto commuters, Small formulated a model with specific components for travel time, early arrival, late arrival, and a fixed late-arrival penalty. Hendrickson and Plank (1984), using journey-to-work data from Pittsburgh, deconstructed the valuation to differentiate free-flow travel time, congested travel time, modal costs, linear early and late arrival, and quadratic terms for early and late arrival. Their work confirmed that, for the work trip, late arrival has far greater disutility compared with early arrival and that, once an individual is quite late for work, additional late time has relatively little additional disutility.

A parallel theoretical framework was proposed by Jackson and Jucker (1982), in which trip disutility is a function of the expected travel time and travel time variance; the latter reflects the valuation of reliability but without differentiation for trip arrival outcome. Polak (1987) proposed alternatives in which the variability term can be quadratic or exponential (for travelers with absolute risk aversion). Black and Towriss (1993) then extended this work to develop a reliability ratio as the ratio of the value of reliability to the value of time.

Noland and Small (1995) combined those two approaches by explicitly including the uncertainty of travel time in the trip scheduling approach through a random variable that reflects the travel time variability. Small et al. (1999) conducted surveys 
of thousands of households in southern California and found that the value individuals place on travel time and trip reliability varies depending on the level of congestion. Furthermore, on the basis of this large stated preference survey, researchers found that a quadratic term for early schedule delay best reflects observations. Below are the form and estimated parameters of the 1999 research by Small et al. Both were applied to the current experiment to compare perceived benefit measures with utility-based outcomes.

$$
c=\alpha T+\beta_{S D E}(S D E)+2 \beta_{S D E 2}(S D E)^{2}+\gamma(S D L)+\theta D_{L}
$$

where

$$
\begin{aligned}
c & =\text { cost of travel (disutility) } \\
T & =\text { travel time } \\
S D E & =\text { schedule delay early } \\
S D L & =\text { schedule delay late } \\
D_{L} & =\text { late-arrival index }= \begin{cases}1 & \text { if } S D L>0 \\
0 & \text { otherwise }\end{cases}
\end{aligned}
$$

The estimates of the parameters are

$$
\begin{aligned}
\alpha= & \$ 0.0564 / \mathrm{min} \text { (linear cost of in-vehicle travel time) } \\
\beta_{S D E}= & -\$ 0.023 / \mathrm{min} \text { (linear component of quadratic early } \\
& \text { cost) } \\
\beta_{S D E 2}= & \$ 0.005 / \mathrm{min} \text { (quadratic component of quadratic early } \\
& \text { cost) } \\
\gamma= & \$ 0.310 / \mathrm{min} \text { (linear cost of late arrival) } \\
\theta= & \$ 2.87 \text { (one-step penalty for arriving late) }
\end{aligned}
$$

Small et al. (2005) found that commuters differ substantially in how they value travel time and reliability, and that the average valuation of both is quite high and is considerably higher when measured in real as opposed to hypothetical scenarios. Recent work has explored variants in the form of the early- and late-arrival components as well as the form by which to represent travel time variance (e.g., Bates et al. 2001; Tilahun and Levinson 2010). Other research addressed the inclusion of risk attitudes in trip scheduling models (Senbil and Kitamura 2004; Li 2010) and extended the formulation to consider trip chains (Jenelius et al. 2011).

With the growth in public real-time traveler information in the 1990s, travel valuation research expanded to valuation of traveler information, as it not only provides trip makers the ability to save travel time but also provides serenity benefits and the ability to shift activities to accommodate trip uncertainty. Many studies have estimated the system-level travel time savings from traveler information through network simulation models (e.g., Wunderlich et al. 1999), while others have focused on willingness to pay through stated preference surveys (e.g., Khattak et al. 2003).

Zhang and Levinson (2006) focused on the benefits of reductions in driver uncertainty when traveler information is provided at the beginning of a trip. In that study, travelers were given information (static value of travel time) for multiple routes. Travelers were asked to make a trip using multiple route options. Participant travel was recorded through GPS units, and participants were then asked to rank order travel among the multiple routes as well as provide ratings on the quality of the trip. Using regression, multinomial, and rank-ordered models, the authors founds that travelers had perception biases and systematically misperceived certain aspects of their trip. Furthermore, accurate information was valued only for commute and event trips, and not for other trip purposes, such as recreation.

Basu and Maitra (2010) applied a stated preference approach to compare traveler (both taxi and private vehicle) valuation of traffic information on a variable message sign. Information was presented in two forms: instantaneous travel time and an additive level of information, which was a categorical variable for variation in instantaneous travel time from the previous estimate. The application of two different types of logit models resulted in valuation of travel time variation at par or greater than the value of travel time for private-car trip makers. For taxi trip makers, both models indicated that valuation of travel time variation was greater than travel time.

\section{Experiment Plan}

Several iterations of an initial experimental design were considered by the Noblis team. Constraints of overall testing session length and resources required for participant sample size restricted the number of factorial combinations that could be tested. The final chosen form focused specifically on making an initially unfamiliar, routine, time-constrained trip. The travel behavior experiment was programmed using Visual Basic for Applications (VBA) within the Microsoft Excel environment. Several iterations of quality assurance and testing were completed on the multiple laptop computers used in the field to create accurate visual appearance and macro execution given the varying performance levels and settings among field laptops.

The experiment was conducted in five cities-Dallas, Denver, Hartford, Miami, and San Jose-over a period of 4 months and acquired information from 80 participants. Experiment participants were recruited through advertisements on craigslist.org. Table 9.1 presents the number of participants by city. The cities were selected to include a diverse survey population that had experience with varying levels of traffic congestion, commute lengths, and highway network connectivity.

Figure 9.1 presents the screenshots of the Excel-based interface that served as the starting point for experiment participants, as well as a sample experiment screenshot. Each participant was seated in front of a dedicated laptop with a mouse and was required to maneuver the mouse to click 
Table 9.1. Responses in Commute Experiment, by City and Type of Information Received

\begin{tabular}{|l|c|c|c|c|c|}
\hline City & Experiment Dates & $\begin{array}{c}\text { Participant } \\
\text { Count }\end{array}$ & VMS & VMS + RT & $\begin{array}{c}\text { VMS + RT + } \\
\text { HISTORIC }\end{array}$ \\
\hline Dallas & $2 / 14 / 2011,2 / 15 / 2011$ & 15 & 6 & 5 & 4 \\
\hline Denver & $12 / 8 / 2010,12 / 9 / 2010$ & 15 & 3 & 4 & 8 \\
\hline Hartford & $1 / 18 / 2011-1 / 20 / 2011$ & 21 & 9 & 7 & 5 \\
\hline Miami & $11 / 30 / 2010-12 / 2 / 2010$ & 10 & 1 & 3 & 6 \\
\hline San Jose & $1 / 11 / 2011-1 / 13 / 2011$ & 19 & 7 & 5 & 7 \\
\hline & Total & $\mathbf{8 0}$ & $\mathbf{2 6}$ & $\mathbf{2 4}$ & $\mathbf{3 0}$ \\
\hline
\end{tabular}

Note: VMS = variable message sign (also known as changeable or dynamic message sign, CMS or DMS), $\mathrm{RT}=$ real-time travel time, HISTORIC = reliability or historical travel time range information .

on interface buttons, radio buttons, or drop-down menus. Participants did not use the keyboard during the experiment. On arrival at the survey room, participants were seated and provided a brief explanation about the intent of the experiment. Participants completed a pre-experiment survey of 10 complex questions, followed by a group-led experiment and a post-experiment four-question survey. Table 9.2 summarizes the information requested in the three sections of the experiment.

Participants independently completed the pre-experiment survey ("Begin Survey" button in Figure 9.1) and waited for a moderator to indicate the start of the commute experiment ("Begin Experiment" button in Figure 9.1). The pre-experiment survey requested socioeconomic, travel purpose, roadway usage, and schedule integrity questions. The socioeconomic

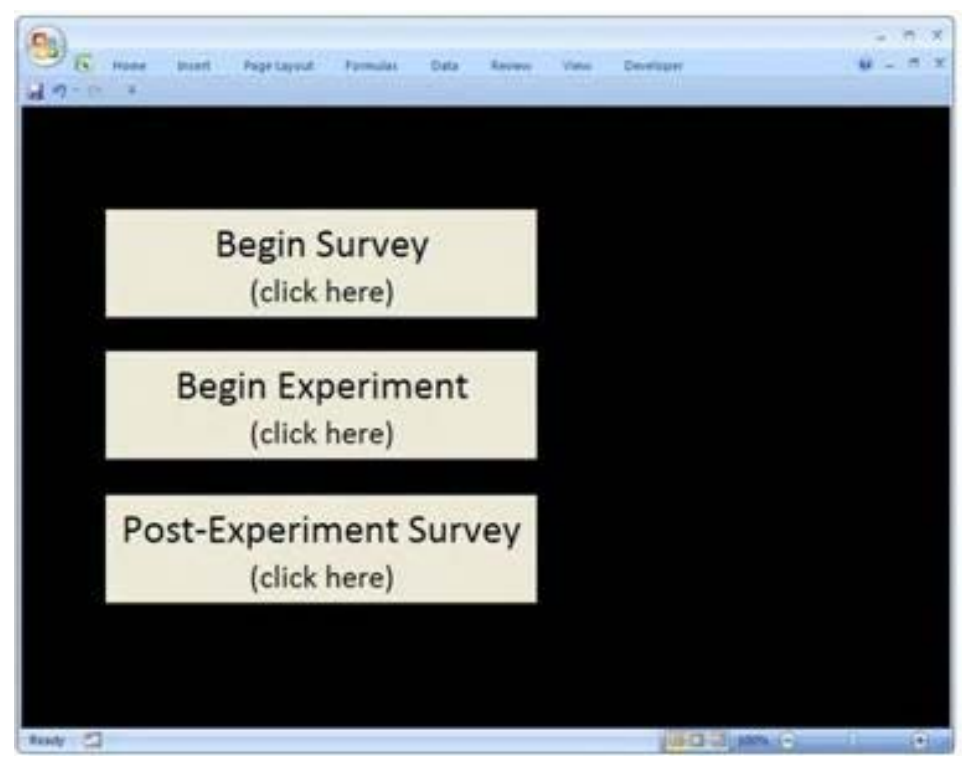

Figure 9.1. Interface view when participants were seated for the experiment.

information framed the characteristics of participants and can be compared against the population at large or with other future experimental groups. The travel purpose, schedule integrity, and roadway usage questions together identified the types of trips for which on-time arrival was most important to participants, the frequency with which those trips were made, and whether the trips for which on-time arrival was most critical were made on roadways where traveler information is usually available. The detailed descriptions and outcomes of the pre-experiment survey are presented later in this chapter.

The moderator began the commute experiment with instructions to the participant group. The moderator read aloud the contextual setup text presented in the experiment and informed participants that different individuals would have

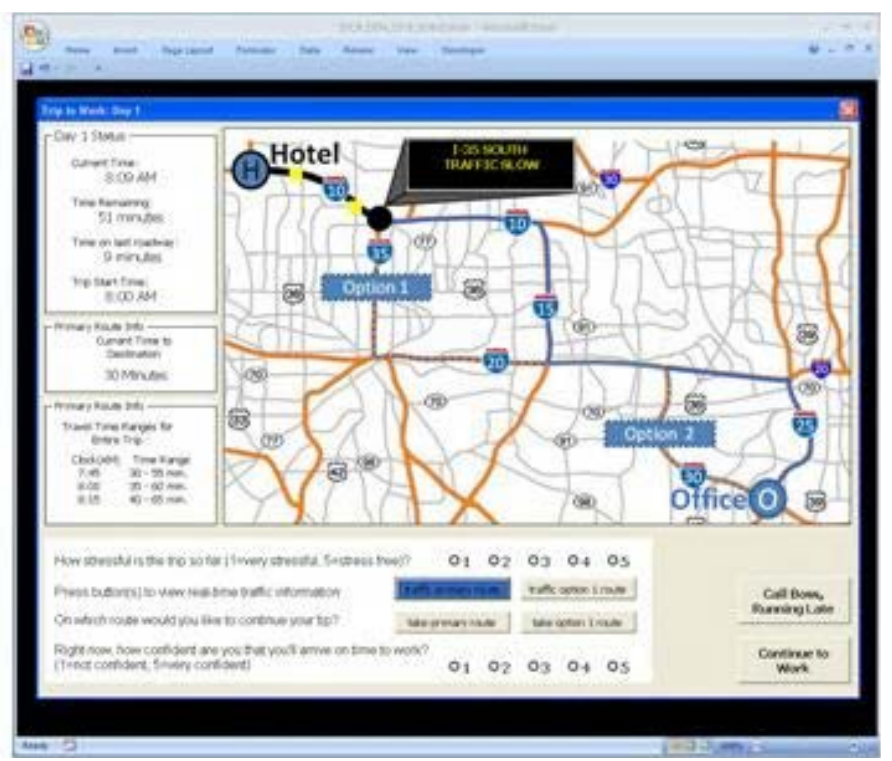


Table 9.2. Information Acquired from Experiment

\begin{tabular}{|c|c|c|}
\hline Pre-Survey Information Acquired & Experiment Information Acquired & Post-Survey Information Acquired \\
\hline $\begin{array}{l}\text { - Gender, age, education } \\
\text { - Travel frequency by trip purpose (work, educa- } \\
\text { tion, childcare, medical, recurring social) } \\
\text { By Trip Purpose for trips with frequency selection } \\
\text { other than "none": } \\
\text { - On-time arrival importance } \\
\text { - Average travel time } \\
\text { - "Bad day" additional time Quantification of "late" } \\
\text { - Willingness to pay travel time guarantee } \\
\text { - Willingness to pay knowledge of late impending } \\
\text { arrival en-route time guarantee }\end{array}$ & $\begin{array}{l}\text { Participants selected the following for the begin- } \\
\text { ning and subsequent segments of each trip } \\
\text { (as applicable): } \\
\text { - whether to view traffic information } \\
\text { - willingness to pay amount for information viewed } \\
\text { - start time for trip } \\
\text { - route selection } \\
\text { - level of confidence for an on-time arrival } \\
\text { - level of stress for the trip thus far } \\
\text { At the end of each trip, participants also selected } \\
\text { the following: } \\
\text { - overall level of stress for the entire trip } \\
\text { - level of happiness with trip outcome } \\
\text { - overall level of usefulness of traffic information } \\
\text { - willingness to pay amount for all the information } \\
\text { viewed during this trip. }\end{array}$ & $\begin{array}{l}\text { - Usefulness of traffic info at } \\
\circ \text { beginning of experiment } \\
\circ \text { end of experiment } \\
\text { - Sources of traffic information } \\
\text { participant uses for their travel } \\
\text { (radio, television, computer, } \\
\text { handheld or dashboard devices) }\end{array}$ \\
\hline
\end{tabular}

access to differing levels of traveler information. Participants were instructed not to share their successful or failed commute strategies with each other to prevent participants with lower levels of traveler information from acquiring insight afforded to participants with more precise traveler information. The moderator supported participants for the first day of travel by presenting on large visual images the location of specific types of information and decision sets. Thereafter, participants used experiment information and made commute decisions at their own pace to complete commutes to work. Although the intent of the experiment was to have participants complete 20 commute days ( 1 commute month), time and resource constraints required curtailing the experiment to 10 commute days.

Participants indicated willingness to pay for traveler information, on-time arrival confidence level, trip stress level, satisfaction level with trip outcome, and traveler information usefulness for each of the 10 completed trips. Data supporting the experiment were designed to represent a moderate level of trip variability as well as temporal consistency among departure time, traveler information, and trip experiences. The details related to the simulation data set along with the outcomes from the commute experiment are presented later in this chapter.

As with the pre-experiment survey, the moderator instructed participants to complete the post-experiment survey independently. The post-experiment survey was brief, consisting of four questions. This survey asked participants to rate the usefulness of traveler information at the beginning and toward the end of the experiment. That information was used to corroborate participants' responses within the commute experiment. The format and findings of the post-experiment survey are presented later in this chapter.

\section{Pre- and Post-Experiment Surveys}

The pre-experiment and post-experiment surveys developed a baseline of participant characteristics that were useful in comparing participant characteristics with the population at large. Key data acquired through the surveys included gender, age, educational level, and traveler information usage. Additionally, the survey questions aimed to explore whether the participant population made trips that would benefit most from traveler information - that is, trips that were sufficiently long, required stringent on-time arrivals, and used facilities for which such information would be available (i.e., highways). Participants with these routine trip-making characteristics were likely to better internalize trade-offs requested in the simulation experiment, and their responses were more likely to reflect true trip decision making. The specific text of pre- and post-experiment questions and the response options for questions are presented in Appendix F.

The survey questions also enabled researchers to develop lateness thresholds, that is, to explore what participants perceived as arriving late according to their specification for on-time arrival importance and trip purpose. The thresholds can be applied in future work to refine the form of utility functions.

Willingness to pay for guaranteed travel time (zero trip variability or $100 \%$ trip reliability) and willingness to pay for knowledge of late arrival were both assessed to compare with sensitivities observed through the experiment. A priori, participants with high on-time arrival importance were expected to be willing to pay more for this guarantee or arrival outcome information.

At the end of the simulation experiment, the survey asked participants what their most important objective was when making departure time decisions (calling family, reducing 
parking fee, avoiding late arrival, arriving exactly on time). The survey also asked how useful they found the traveler information at the beginning and end of the trip. The data provided a clearer holistic understanding of what drove participants' departure choices and whether a value loss occurred in their rating of traveler information usefulness.

\section{Experiment Description}

The key goals of the experimental design were (a) to assess the value participants placed on traveler information-and the reliability information component-within the context of a simulated time-constrained trip; and (b) to determine whether having reliability information helped speed the transition from an unfamiliar commuter to an experienced commuter. The expectation was that participants with higher levels of traveler information would assign more positive valuation to questions regarding usefulness of traffic information, trip stress, and trip outcome satisfaction during the first week while they were unfamiliar with the trip variability. Furthermore, the expectation was that those with higher levels of information were likely to perform better on event and non-event days because higher levels of information would speed the transition from unfamiliar to experienced commuter. In making these determinations, researchers conducted comparisons of mean valuation among participants with different levels of information and computation of utility values based on trip outcomes.

Appendix F presents the narrative given on screen and communicated verbally to prepare participants for the simulation experiment. In essence, participants were in a new town for work and had to arrive on time to work or otherwise incur a \$25 late-arrival fee. Once during the simulation experiment, participants could inform their employer of a late arrival and avoid the late-arrival fee. Early arrival cost (parking cost) was introduced at $\$ 4.00$ per hour, and an incentive for later departure was introduced in the form of time spent video-chatting with family back home. Participants were asked to select among three departure times (7:45 a.m., 8:00 a.m., and 8:15 a.m.) and multiple routes for a simulated trip to work over a 2-week period, totaling 10 commutes.

Participants received one of three levels of information: L1 participants received only qualitative information pre-trip and en route. L2 participants received the same qualitative information as L2 and also could view a real-time estimate of route-specific travel time to their destination. Real-time information was presented in the simulation at 5-min increments. L3 participants had access to the same information as L2 and also could view static travel time reliability information.

Figure 9.2 presents the trip network and identifies the waypoints along the trip where qualitative DMS messages were presented. The image in Figure 9.2 is from the simulation tool for a participant with L3 traveler information, after the participant had clicked on the "traffic primary route" button.

A qualitative DMS message was visible at the start of the trip ( $\mathrm{H}$ in Figure 9.2) and at two waypoints along the trip. The DMS message sets are presented in Appendix F along with their frequency of use. Participants chose route options and whether to view additional travel information, if available to them, at Waypoints 1 and 2. During the commute, participants had information (left side of Figure 9.2) regarding the current simulation time, time remaining for them to complete their trip on time, time to traverse the previous segment of roadway, and when they had started their trip.

Specific questions regarding levels of trip stress and on-time arrival confidence had to be completed at each waypoint and at the beginning and end of each trip, as relevant. The specific types of data collected through the simulation experiment are presented in Appendix F.

Among the 10 commute days, half were non-event days and half were event days, as listed in Table 9.3. The order of days remained consistent among all participants. Commute times were designed for temporal and route consistency and were reflective of event and non-event days. Table 9.4 and Table 9.5 present average travel times and range of travel time by time of departure and route, for non-event and event days, respectively. The specific travel times for each day are presented in Appendix F. Given the simulation travel times, the optimal departure time to meet the schedule-constrained arrival of 9:00 a.m. was 8:15 a.m. for non-event days and 8:00 a.m. on all event days except for day 8 , the heavy rain day. On that day, a 7:45 a.m. departure time was required for an on-time arrival.

The DMS, real-time, and reliability data that were presented to participants aligned with the commute times they experienced. That is, the quality of the traveler information was good. Only on days 5 and 10 did the real-time travel time estimate at the beginning of the trip differ significantly from experience, and that was because accidents were simulated to occur after trip departure.

\section{Pre- and Post-Experiment Survey Findings}

The pre- and post-experiment surveys provided insight on the characteristics and preferences of participants. All 80 participants completed the surveys. A greater subset of participants made work and medical trips with some frequency compared with school, child-care, or other routine trips. Work and medical trips more often use highways, are longer than school or child-care trips, and have higher requirements for on-time arrival than child-care or other routine trips. Consequently, trips made to work and medical appointments are ideal for using traveler information. However, familiarity with traffic for routine work trips will likely make reliability information 


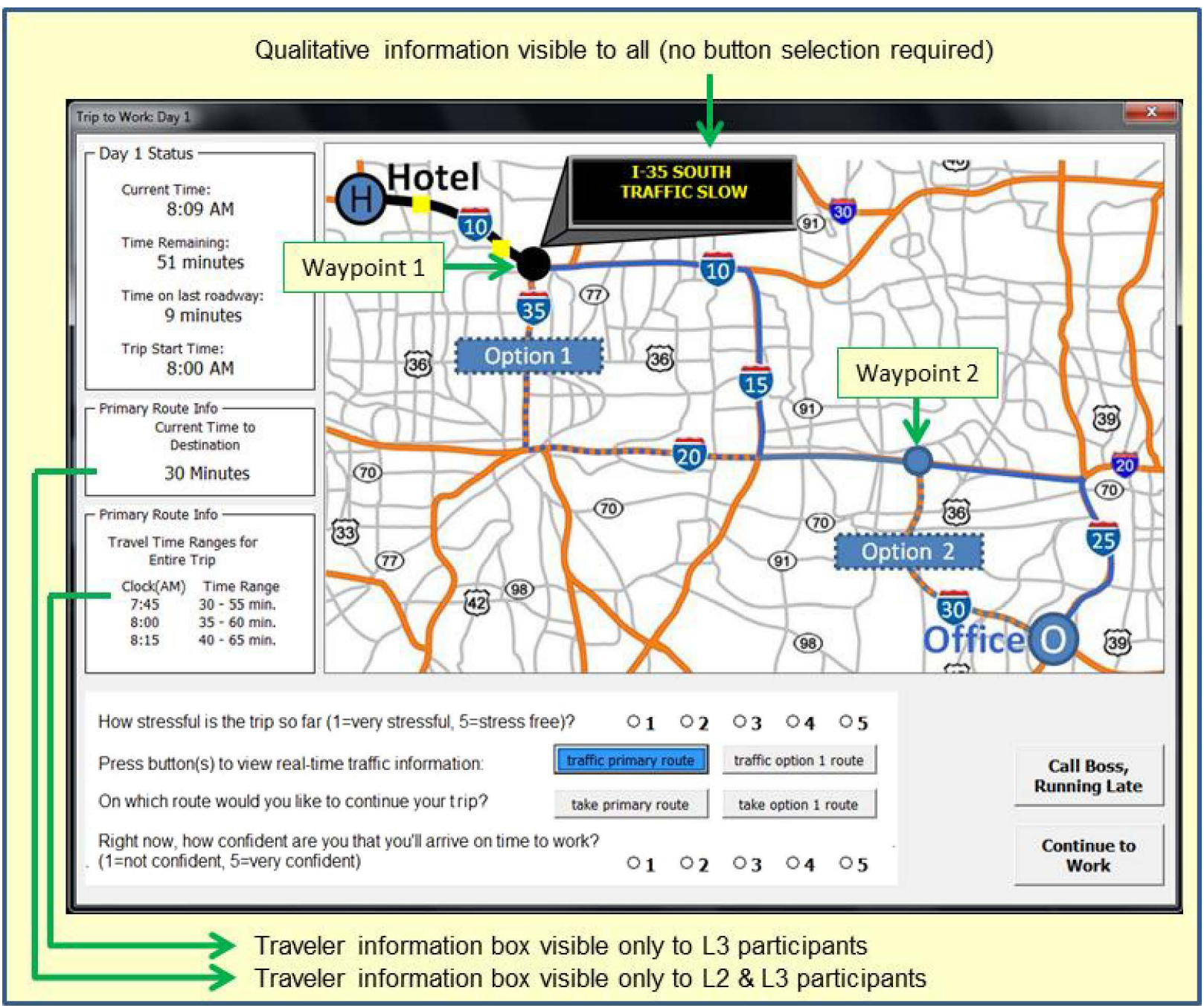

Figure 9.2. Road network for trip simulation.

\section{Table 9.3. Description of Simulated Commutes}

\begin{tabular}{|l|l|c|c|}
\hline Simulation Day & \multicolumn{1}{|c|}{ Description of Day } & Ideal Departure Time $^{a}$ & Alternate Route Far Better $^{b}$ \\
\hline Week 1, Day 1 & Non-event traffic day & $8: 15$ a.m. & No \\
\hline Week 1, Day 2 & Higher than usual traffic, moderate delays all routes & $8: 00$ a.m. & No \\
\hline Week 1, Day 3 & Non-event traffic day & $8: 15$ a.m. & No \\
\hline Week 1, Day 4 & Non-event traffic day & $8: 15$ a.m. & No \\
\hline Week 1, Day 5 & Minor accident at 7:50 a.m. affecting all routes, minor delays & $8: 00$ a.m. & Yes \\
\hline Week 2, Day 6 & $\begin{array}{c}\text { Blocked lane on primary route from 7:15 a.m. to 8:00 a.m., } \\
\text { moderate delays }\end{array}$ & $8: 00$ a.m. & No \\
\hline Week 2, Day 7 & Non-event traffic day & $8: 15$ a.m. & No \\
\hline Week 2, Day 8 & Heavy rain day, significant delays on all routes & $8: 45$ a.m. & No \\
\hline Week 2, Day 9 & Non-event traffic day & $8: 15$ a.m. & No a.m. \\
\hline Week 2, Day 10 & Minor accident at 8:00 a.m. affecting primary route & & \\
\hline
\end{tabular}

${ }^{a}$ Ideal departure time avoids late arrival and minimizes excessive early arrival.

${ }^{b}$ Alternate route is equivalent for most days, superior for one simulated day. 
Table 9.4. Non-Event Day Travel Time Profiles

\begin{tabular}{|l|c|c|c|c|}
\hline \multirow{2}{*}{$\begin{array}{l}\text { Departure } \\
\text { Time }\end{array}$} & \multicolumn{4}{|c|}{$\begin{array}{c}\text { Average and Range of Travel Time } \\
\text { by Route for Non-Event Days }\end{array}$} \\
\cline { 2 - 5 } & Primary & Option 1 & Option 2 & Option 1 \& 2 \\
\hline 7:15 AM & $32.8(31-35)$ & $36.4(34-39)$ & $34.2(33-36)$ & $37.6(35-41)$ \\
\hline 8:00 AM & $37.2(34-41)$ & $40.4(38-43)$ & $39.4(36-43)$ & $42.0(38-43)$ \\
\hline 8:15 AM & $41.2(38-45)$ & $44.6(40-47)$ & $43.6(41-47)$ & $45.6(44-49)$ \\
\hline
\end{tabular}

a lower value add-on compared with real-time information. Conversely, when planning nonroutine work or medical appointment trips, reliability information will provide a higher add-on value compared with only real-time information.

What participants meant by late was far more precise (within 2 min of scheduled arrival time) for those making work ( $41 \%$ of participants) and child-care trips (53\% of participants); all participants making school trips selected a lateness threshold of $10 \mathrm{~min}$ or less. For these participants with a narrow lateness threshold, trip reliability played a significant role in budgeting travel time for their trip. These participants were also willing to pay more for a guarantee of travel time or to know early in a trip that they would arrive late.

Table 9.6 lists the average minutes late participants characterized as arriving late. Across all participants and trip purposes, the average threshold for characterizing a trip as late was $6.7 \mathrm{~min}$ beyond the desired arrival time. As expected, individuals who indicated arriving on time was very important had a far smaller lateness threshold. For example, participants who indicated that an on-time arrival at work was very important (category 5) believed that arriving 4.9 min past their scheduled arrival time was considered late, whereas this value was $17.0 \mathrm{~min}$ for participants who indicated that on-time arrival at work was somewhat important.

Given that the experiment simulated a work trip, participants were asked to identify how frequently they sought out traffic information for this type of trip. More than $90 \%$ of participants who made work trips also sought out traffic information once or more per week. Among the participants that made daily work trips, $40 \%$ also sought out traffic information daily.
Participants often used multiple media for acquiring traffic information. Among the 80 participants, nearly $60 \%$ used radio, while television, computer, and handheld or dashboard devices were used by $43 \%, 28 \%$, and $23 \%$, respectively.

The expectation was that eliminating trip variability would be valued far more than learning en route that arrival would be delayed. According to survey responses, however, participants were willing to pay only slightly more to eliminate travel time variability than to be forewarned of a late arrival. As expected, more important on-time arrivals translated to a greater willingness to pay. Furthermore, among the various trip purposes, participants were willing to pay most to eliminate trip variability for work trips and to know of a late work arrival. On average, across all trips, participants indicated that they would be willing to pay $\$ 0.85$ per trip to eliminate travel time variability and $\$ 0.72$ to be informed of a late arrival. Table 9.7 lists participants' average willingness to pay by trip purpose and by importance of on-time arrival.

Participants' valuation (perceived usefulness) of the traffic information solicited through the post-experiment survey was neutral to somewhat useful at the start of the experiment. The neutrality diminished with transference more to the "somewhat useful" category and the "rarely/not useful" categories. The relative valuation between the three levels of information received was only moderately different. Approximately $20 \%$ of participants who received DMS-only information (L1) negatively rated its usefulness. Surprisingly, only $8 \%$ of L2 participants negatively rated its usefulness, while $13 \%$ of L3 participants negatively rated its usefulness. The differences in outcomes, however, were not statistically significant.

Table 9.5. Event Day Travel Time Profiles

\begin{tabular}{|l|c|c|c|c|}
\hline \multirow{2}{*}{$\begin{array}{l}\text { Departure } \\
\text { Time }\end{array}$} & \multicolumn{4}{|c|}{$\begin{array}{c}\text { Average and Range of Travel Time } \\
\text { by Route for Event Days }\end{array}$} \\
\cline { 2 - 5 } & Primary & Option 1 & Option 2 & Option 1 \& 2 \\
\hline 7:15 AM & $47.6(37-64)$ & $45.8(37-61)$ & $48(37-63)$ & $46.6(38-62)$ \\
\hline 8:00 AM & $56.4(47-66)$ & $53.6(45-66)$ & $56(42-68)$ & $52.4(38-65)$ \\
\hline 8:15 AM & $57.8(47-71)$ & $56.4(39-71)$ & $57.8(47-73)$ & $55.6(42-74)$ \\
\hline
\end{tabular}


Table 9.6. Average Threshold for Late Arrival, by Importance of On-Time Arrival and Trip Purpose (min)

\begin{tabular}{|l|c|c|c|c|c|c|}
\hline \multirow{2}{*}{ Arrival Importance } & \multicolumn{5}{|c|}{ Trip Destination by Purpose } & \multirow{2}{*}{ All Trips } \\
\cline { 2 - 7 } & Work & School & Child Care & Medical & Other & A. \\
\hline 1 & na & 4.8 & 20.0 & na & 15.0 & 9.0 \\
\hline 2 & na & 10.0 & 25.0 & 5.0 & 22.5 & 17.0 \\
\hline 3 & 17.0 & 6.0 & 7.0 & 7.1 & 9.0 & 9.2 \\
\hline 4 & 5.1 & 5.6 & 11.8 & 7.3 & 10.7 & 8.3 \\
\hline 5 & 4.9 & 6.0 & 3.3 & 5.7 & 5.5 & 5.1 \\
\hline Average lateness threshold & 5.8 & 5.9 & 6.0 & 6.2 & 9.3 & 6.7 \\
\hline
\end{tabular}

Note: na $=$ not applicable. In the first column, $1=$ unimportant and $5=$ very important.

\section{Pre-Experiment Survey, Sample Statistics}

The participants' median age range was 40 to 49 years. Nearly half had a college degree or higher, whereas only $25 \%$ of the U.S. population (age 25 and older) has completed a college or postgraduate degree. While most participants made work trips (94\%) or trips for a medical appointment (94\%), a few participants did indicate that they never made these types of trips (6\%). Conversely, $55 \%$ and $60 \%$ of participants never made school-related or child-care trips, respectively. In addition, although nearly all participants made work-related trips, only $60 \%$ commuted daily to work. Furthermore, only $13 \%$ commuted daily to either school or child care. As expected, no participant made daily medical-related trips. Figures summarizing basic statistics related to participants' gender, age, education, and types of trips made are included in Appendix G.

Approximately $25 \%$ of participants that made trips to work, school, and medical appointments traveled using mostly highways. Only 9\% and 17\% of participants who made childcare and other routine trips, respectively, did so primarily through highway travel. Most frequently, trips were made through a combination of travel on highways and signalized roadways. Given the road-type use trends among participants, and because traveler information is available mostly on highways, approximately a third of participants (those using primarily signalized and neighborhood roads) who made a specific type of trip would likely not have traveler information relevant to their trip. Figure 9.3 displays these results.

Participants unanimously indicated that for every type of trip they made, arriving on time was between "somewhat important" and "very important," based on a 5-point scale, with 3 equaling "somewhat important" and 5 equaling "very important." All 36 experiment participants (100\%) who indicated they made school trips identified arriving on time as very important. Nearly all ( $86 \%$ and $88 \%$, respectively) experiment participants who indicated they made trips to

Table 9.7. Willingness to Pay for Trip Reliability and Information on Late Arrival, by Importance of On-Time Arrival and Trip Purpose

\begin{tabular}{|c|c|c|c|c|c|c|c|c|c|c|}
\hline \multirow{2}{*}{$\begin{array}{l}\text { Arrival } \\
\text { Importance } \\
\text { (1 = unimportant, } \\
5=\text { very } \\
\text { important) }\end{array}$} & \multicolumn{5}{|c|}{$\begin{array}{l}\text { Eliminate Trip Variability } \\
\text { (Guaranteed Travel Time) }\end{array}$} & \multicolumn{5}{|c|}{$\begin{array}{l}\text { Know You're Going to Be Late } \\
\text { (Late Arrival Traveler Information) }\end{array}$} \\
\hline & $\begin{array}{l}\text { Work } \\
\text { Trips }\end{array}$ & $\begin{array}{l}\text { School } \\
\text { Trips }\end{array}$ & $\begin{array}{l}\text { Child-Care } \\
\text { Trips }\end{array}$ & $\begin{array}{l}\text { Medical } \\
\text { Trips }\end{array}$ & $\begin{array}{l}\text { Other } \\
\text { Trips }\end{array}$ & $\begin{array}{l}\text { Work } \\
\text { Trips }\end{array}$ & $\begin{array}{l}\text { School } \\
\text { Trips }\end{array}$ & $\begin{array}{l}\text { Child-Care } \\
\text { Trips }\end{array}$ & $\begin{array}{l}\text { Medical } \\
\text { Trips }\end{array}$ & $\begin{array}{l}\text { Other } \\
\text { Trips }\end{array}$ \\
\hline 1 & na & na & na & na & na & na & na & na & na & na \\
\hline 2 & na & na & na & na & na & na & na & na & na & na \\
\hline 3 & $\$ 0.55$ & na & na & $\$ 0.78$ & $\$ 0.30$ & $\$ 0.30$ & na & na & $\$ 0.58$ & $\$ 0.25$ \\
\hline 4 & $\$ 0.52$ & $\$ 0.75$ & na & $\$ 0.23$ & $\$ 0.34$ & $\$ 0.59$ & $\$ 0.54$ & na & $\$ 0.45$ & $\$ 0.45$ \\
\hline 5 & $\$ 1.38$ & $\$ 1.18$ & $\$ 1.17$ & $\$ 0.98$ & $\$ 0.73$ & $\$ 1.23$ & $\$ 0.91$ & $\$ 0.86$ & $\$ 0.90$ & $\$ 0.14$ \\
\hline Average WTP & $\$ 1.20$ & $\$ 0.93$ & $\$ 1.05$ & $\$ 0.77$ & $\$ 0.37$ & $\$ 1.08$ & $\$ 0.73$ & $\$ 0.73$ & $\$ 0.74$ & $\$ 0.28$ \\
\hline
\end{tabular}

Note: $\mathrm{WTP}=$ willingness to pay na $=$ not applicable, because there were fewer than five observations (fewer than five people were willing to pay for the information). 


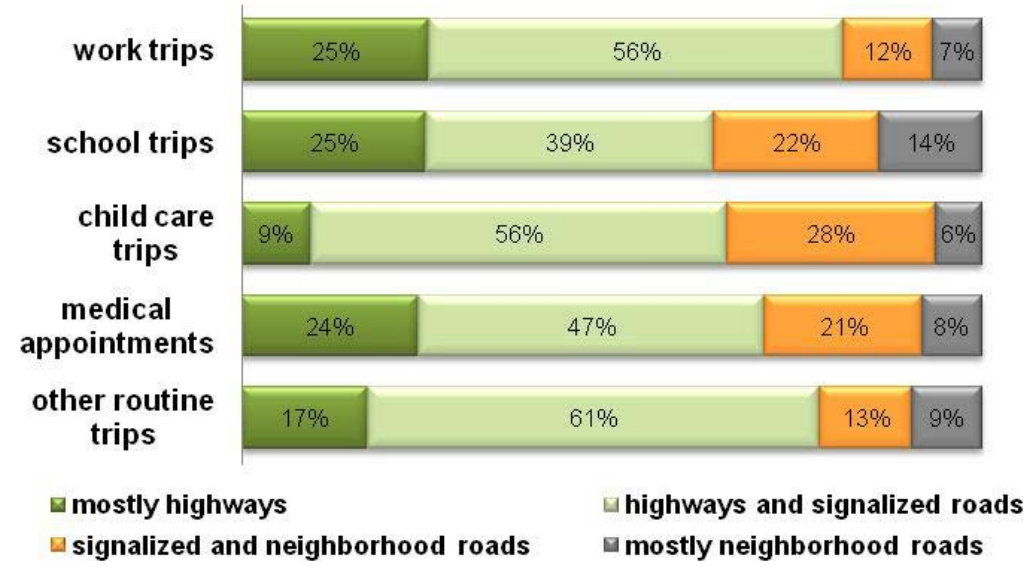

Figure 9.3. Types of roads participants use for specific trip types.

work or medical appointments also identified arriving on time as very important. Figure 9.4 presents a chart summarizing participants' ratings for on-time arrival importance by trip type.

Figure 9.5 presents a chart summarizing participants' normal trip time for the subset of participants that made a specific trip type. Most participants' (58\%) average worktrip time ranged between $10 \mathrm{~min}$ and $30 \mathrm{~min}$, while $30 \%$ of participants who traveled to work experienced an average travel time greater than $30 \mathrm{~min}$. Participants' medical appointments appeared to have a similar average trip time distribution to participants' work trips. Average travel times for work and medical-related trips were generally greater than average travel times for school and child-care trips.

Figure 9.6 presents a chart summarizing participants' additional travel time on a bad day. More than $50 \%$ of participants who made child-care trips experienced very low levels of trip variability ( $0 \mathrm{~min}$ to $10 \mathrm{~min}$ of extra travel time), while only $41 \%$ making work trips had the same low level of trip variability. One in four participants who made work trips indicated that on a bad day, their trip can take 30 or more additional minutes beyond their average trip time; only one participant had that level of variability for his or her child-care trip.

Yet, participants defined late far more precisely for work and child-care trips compared with other trip types. One in five participants who made work trips categorized arriving late as arriving even $1 \mathrm{~min}$ beyond his or her scheduled arrival time, and another one in five set the late-arrival bar at $2 \mathrm{~min}$ within the scheduled arrival time. Late arrivals for child-care trips were perceived by participants as even more time sensitive. These outcomes are presented in Figure 9.7.

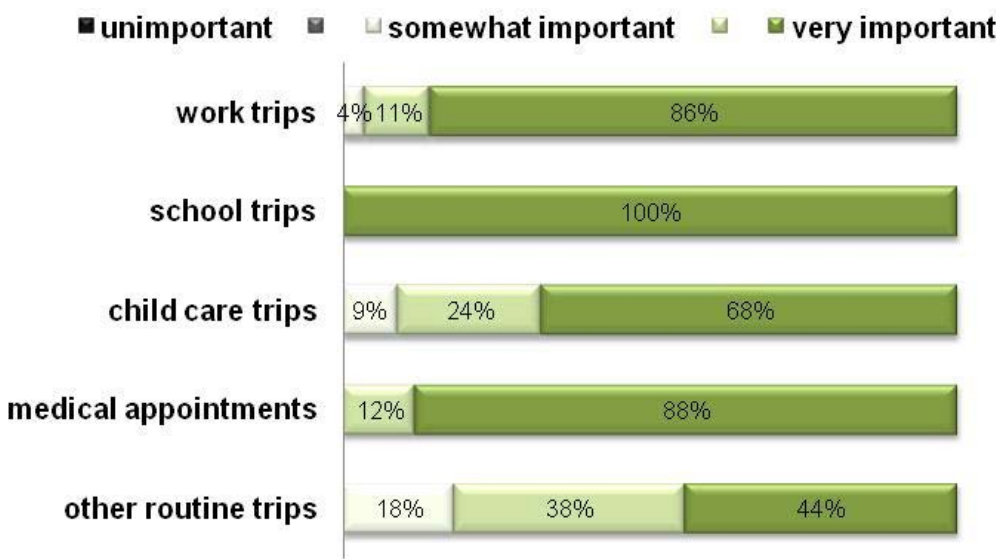

Figure 9.4. Participants' rating of importance of on-time arrival, by trip purpose. 


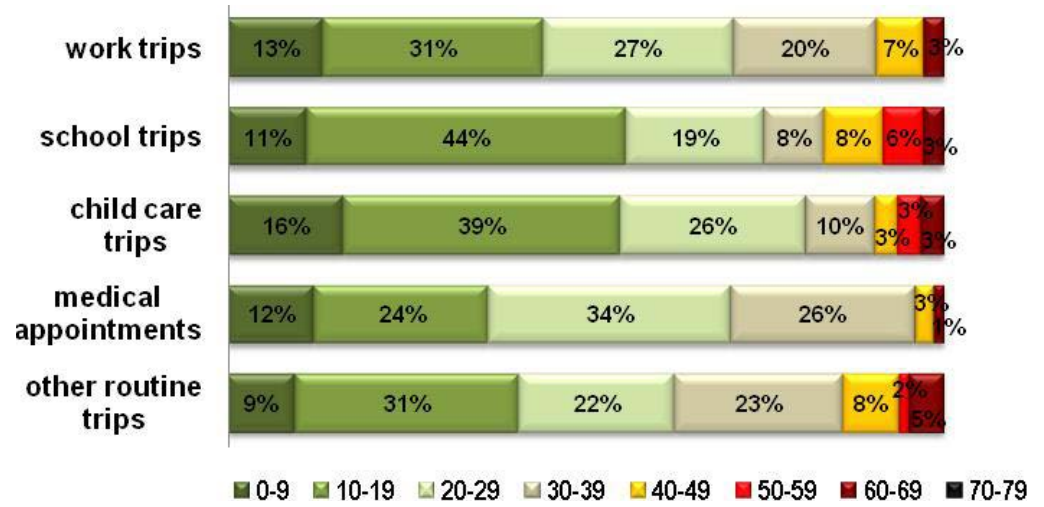

Figure 9.5. Average trip duration, by trip type.

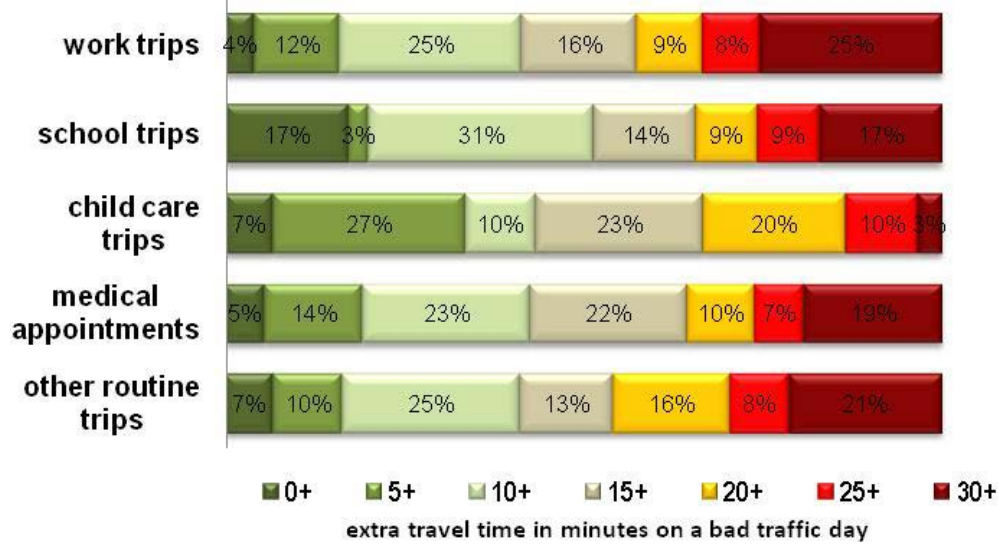

Figure 9.6. Trip time variability, by trip type.

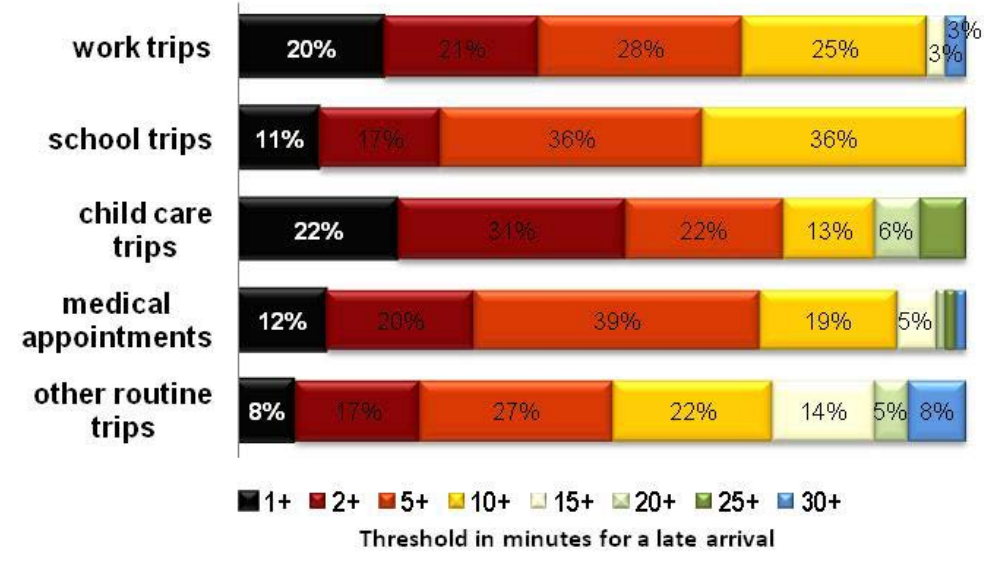

Figure 9.7. Various definitions of late arrival, by trip type. 


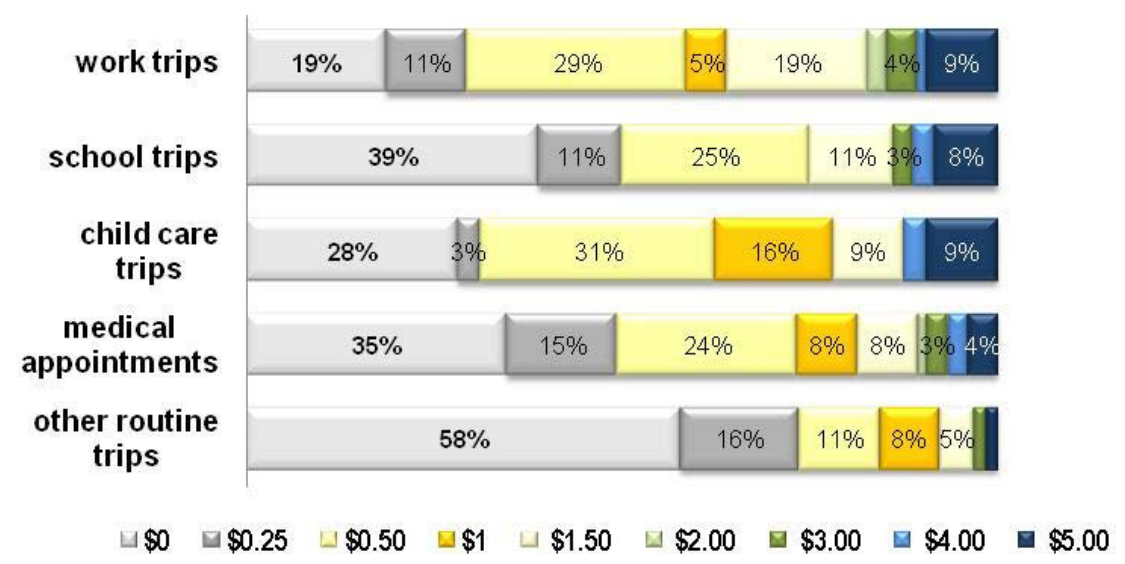

Figure 9.8. Willingness to pay for guaranteed travel time, by trip type.

Figure 9.8 presents a chart summarizing participants' willingness to pay for a guaranteed travel time to their destination. More than $80 \%$ of participants were willing to pay something to eliminate the variability in travel to work, but only $42 \%$ of participants who made other routine trips were willing to pay money to reduce variability on those trips. Such trips have a far lower on-time arrival importance. On average, participants who made work trips were willing to pay $\$ 1.20$ per trip to eliminate trip variability. For child-care trips, the value was $\$ 1.05$; and for school-related trips, the value was $\$ 0.93$.

Surprisingly, nearly as many people (76\%) were willing to pay for information showing that they would arrive late for a work trip as were willing to pay to reduce their work-trip time variability $(81 \%)$. Furthermore, they were willing to pay nearly as much for the information ( $\$ 1.08$ versus $\$ 1.20$ ), even though the information would not improve their travel time or trip outcome. The average willingness-to-pay amount for school, child-care, and medical trips was the same, at $\$ 0.73$ per trip. Figure 9.9 presents a chart summarizing participants' willingness to pay for late-arrival knowledge.

\section{Post-Experiment Survey}

Participants were asked four brief questions after completing the simulation experiment. The second question asked how they perceived the usefulness of traveler information at the start of the simulation and after completing the experiment. Figure 9.10 and Figure 9.11 present participants' responses.

At the beginning of the experiment, about half of the participants in the L1 and L2 groups were neutral (48\% and $52 \%$, respectively) about the usefulness of the traveler information; fewer believed it was somewhat useful (44\%

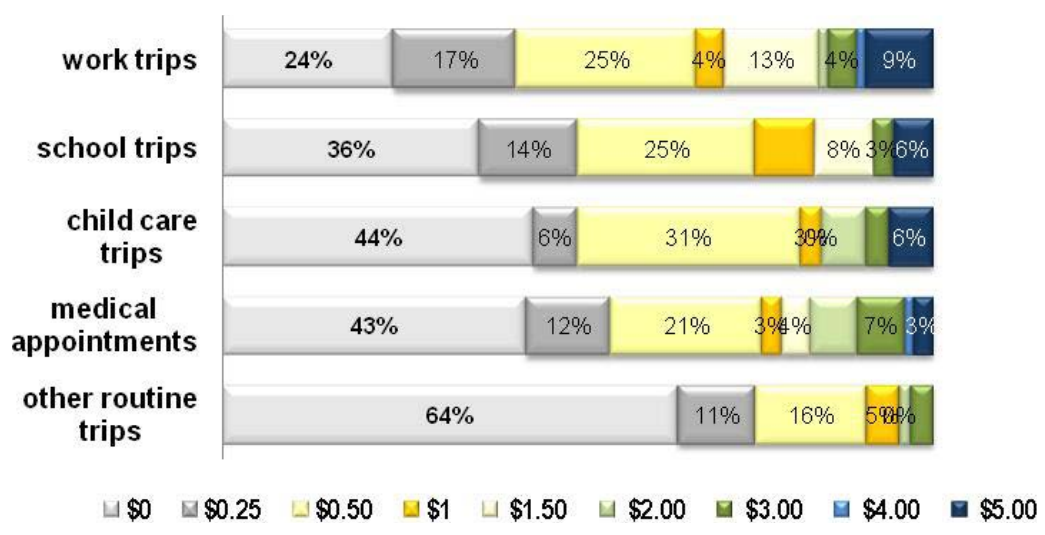

Figure 9.9. Willingness to pay for late-arrival knowledge, by trip type. 


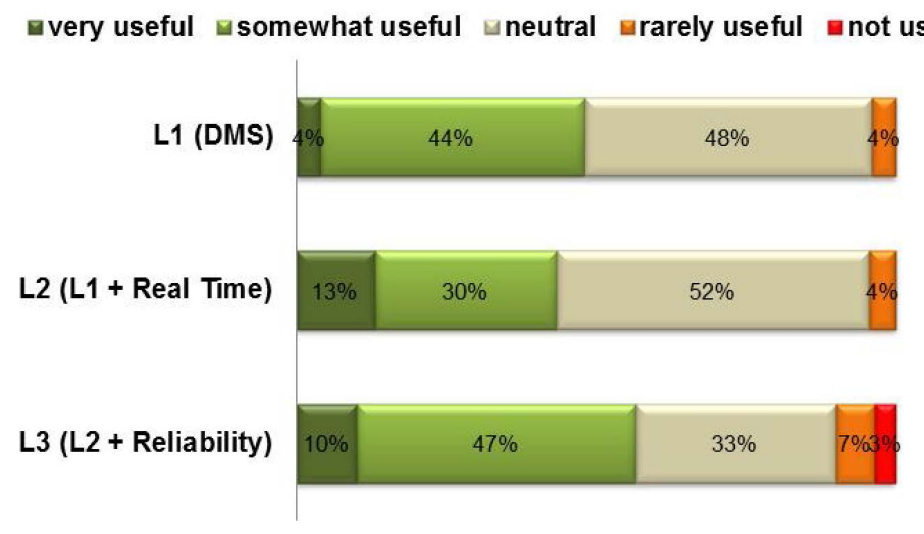

Figure 9.10. Ratings of traveler information usefulness at beginning of experiment.

and 30\%, respectively). Many more L3 participants rated the information as somewhat to very useful (57\%) than rated it neutrally $(33 \%)$. At the end of the experiment, the L1 and L2 groups who were neutral shifted significantly more toward the positive and slightly toward the negative. The L3 group had a far less pronounced shift to the positive.

During the post-experiment survey, participants also revealed how frequently they sought out traffic information for the work trip and the types of information they used to acquire this information. Participants' responses were aggregated and are presented in Figure 9.12 and Figure 9.13. Most participants frequently sought out traffic information, and $30 \%$ did so every day. Most used multiple media to acquire the information. More than half of the participants who sought out traffic information for the work commute used the radio.

\section{Experimental Results}

Participants who received all three information sets (qualitative messages on roadside message signs along route, realtime travel time at 5-min granularity, and static travel time ranges based on time of departure) were referred to as receiving L3 information. Participants who received the qualitative messages and real-time travel time information were referred to as receiving $\mathrm{L} 2$ information. And participants who received only the qualitative messages were referred to as receiving L1 information.

Participants with L3 information were more conservative in their departure time decisions than the L1 and L2 groups on non-event days. This result likely occurred because the reliability information that supplemented L3 participants' real-time information increased their awareness of trip variability and led them to budget more travel time. Only $23 \%$ of

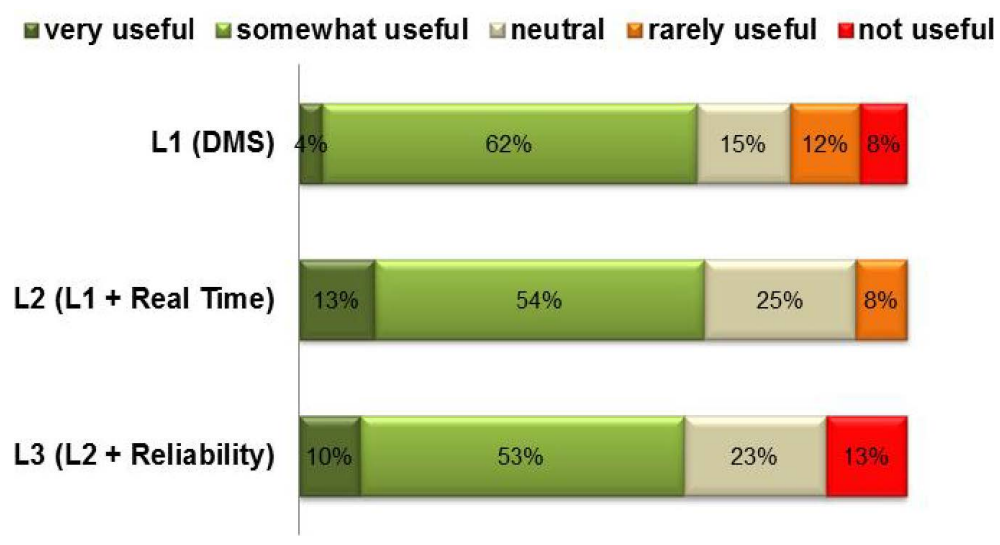

Figure 9.11. Ratings of traveler information usefulness at end of experiment. 


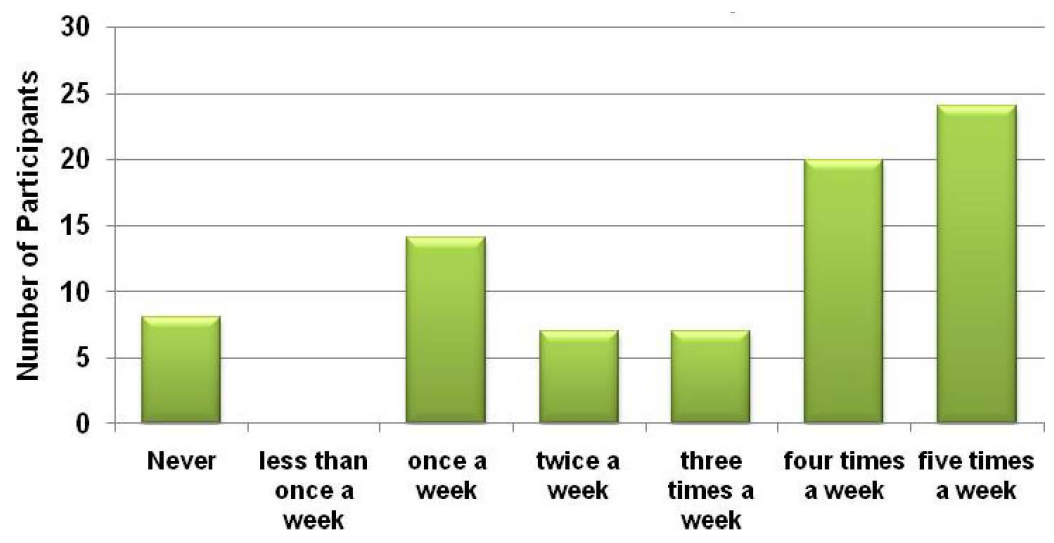

Figure 9.12. Frequency of seeking traffic information for work trips.

participants with reliability information departed at 8:15 a.m. on non-event days, while $30 \%$ and $45 \%$ of participants receiving L1 and L2 information, respectively, departed at 8:15 a.m. Conversely, L2 participants budgeted the least travel time for their trips. Table 9.8. summarizes participants' departure time decisions by information level for event and non-event days.

The trip objective for participants was to depart no earlier than 8:00 a.m. so they could spend that time checking in on family. At the same time, participants were asked to arrive by 9:00 a.m. to work to avoid a $\$ 25$ late-arrival work penalty but not much earlier to avoid parking fees of $\$ 4.00$ per hour. Note that all of the motivations constructed for these simulated trips were hypothetical, and no variations in participant compensation were tied to performance within the experiment. Given that L3 participants chose to depart earlier than L1 and L2 participants, they more often missed time with their family. L3 participants missed morning family time on average 2.6 out of 10 days, while L2 participants missed on average 2.0 out of 10 days.

Participants with reliability information (L3) arrived late 1.5 days out of 10 on average, while their counterparts with L2 and L1 information arrived late on average 2.1 days out of 10 . The average total late-arrival cost for participants—based on the experimental framework and 10 commute days-was $\$ 45$, $\$ 39$, and $\$ 28$ for L1, L2, and L3 participants, respectively. The average early arrival costs were $\$ 11, \$ 9$, and $\$ 11$ for L1, L2, and L3 participants, respectively. Table 9.9 breaks down late and early costs by week and by event and non-event days. Based on the cost layouts presented to experiment participants, those receiving reliability information in addition to the other sets of information (L3 participants) had the overall lowest schedule offset cost of $\$ 38.61$. In-vehicle travel time costs are not a component of the calculations presented in Table 9.9.

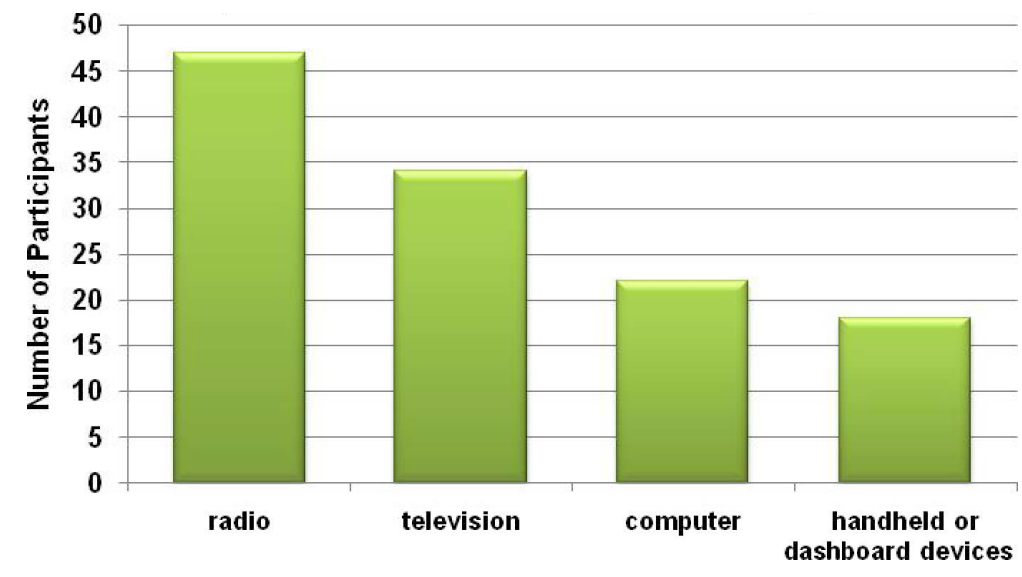

Figure 9.13. Media used in acquiring traffic information for work trips. 
Table 9.8. Aggregate Departure Decisions for Groups with Different Traveler Information

\begin{tabular}{|l|c|c|c|c|c|c|}
\hline \multirow{2}{*}{$\begin{array}{l}\text { Departure } \\
\text { Time }\end{array}$} & \multicolumn{3}{|c|}{ Non-Event Days } & \multicolumn{3}{c|}{ Event Days } \\
\cline { 2 - 6 } & $\begin{array}{c}\text { L1 } \\
\text { (DMS) }\end{array}$ & $\begin{array}{c}\text { L2 } \\
\text { (DMS + RT) }\end{array}$ & $\begin{array}{c}\text { L3 } \\
\text { (DMS + RT + REL) }\end{array}$ & $\begin{array}{c}\text { L1 } \\
\text { (DMS) }\end{array}$ & $\begin{array}{c}\text { L2 } \\
\text { (DMS + RT) }\end{array}$ & $\begin{array}{c}\text { L3 } \\
\text { (DMS + RT + REL) }\end{array}$ \\
\hline $7: 45$ & $16 \%$ & $7 \%$ & $17 \%$ & $30 \%$ & $34 \%$ & $34 \%$ \\
\hline $8: 00$ & $54 \%$ & $48 \%$ & $60 \%$ & $51 \%$ & $40 \%$ & $48 \%$ \\
\hline $8: 15$ & $30 \%$ & $45 \%$ & $23 \%$ & $19 \%$ & $26 \%$ & $18 \%$ \\
\hline
\end{tabular}

Note: DMS = dynamic message sign; RT = real-time travel time; and REL = reliability information.

\section{Confidence in On-Time Arrival Was Tempered with Reliability Information}

On average, participants' level of confidence in an on-time arrival at the beginning of each trip was equivalent among the three traveler information groups and did not vary significantly from week 1 to week 2. At Waypoint 1 (first decision point), for non-event days, arrival confidence on average was nearly the same as at the beginning of the trip. However, on event days, participants' levels of confidence in on-time arrival decreased by $8 \%, 21 \%$, and $13 \%$ for L1, L2, and L3 participants, respectively. Having quantitative information (L2) on delay caused a significant decrease (4.2 to 3.3, average from trip start to Waypoint 1) in level of confidence. Knowing overall variability of the trip in addition to having quantitative information (L3) mitigated this degradation in confidence (4.1 to 3.5, average trip start to Waypoint 1 ).

At Waypoint 2 (second decision point), the trend was similar to Waypoint 1 in that arrival confidence was lower for event days compared with non-event days. L1 participants continued to have less confidence in an on-time arrival from Waypoint 1 to Waypoint 2. L2 participants' average level of confidence increased from Waypoint 1 to Waypoint 2; at
Waypoint 2, they often passed the event causing delay and had additional information increasing their confidence in an on-time arrival. The average level of confidence for L3 participants was equivalent from Waypoint 1 to Waypoint 2. Figure 9.14 presents graphically the average change in level of confidence in an on-time arrival from the beginning of the trip to Waypoints 1 and 2.

\section{Reliability Information Reduced Pre-Trip and En Route Stress for Unfamiliar Trips}

The level of serenity along trips was solicited through the questions asking about levels of stress at specific points along the trip. At the first waypoint, participants generally selected values representing neutral (value of 3 ) to stress-free travel (value of 5). Higher levels of traveler information generally did not equate to less stress at the first trip waypoint. Interestingly, for participants receiving L1 information, the first commute day was the day with the highest average level of stress at the first waypoint, followed by the eighth commute day (bad weather and highest travel times). For L2 participants, the average stress level at Waypoint 1 was nearly equivalent

Table 9.9. Schedule Offset Costs Based on Experiment Framework

\begin{tabular}{|l|c|c|c|c|c|c|c|c|c|}
\hline \multicolumn{7}{|c|}{ Costs for Schedule Offset Based on Experimental Framework } \\
\hline & \multicolumn{2}{|c|}{ Late Arrival Cost } & \multicolumn{2}{c|}{ Early Arrival Cost } & \multicolumn{3}{c|}{ Total Cost $^{\star}$} \\
\cline { 2 - 11 } & L1 & L2 & L3 & L1 & L2 & L3 & L1 & L2 & L3 \\
\hline All 10 Simulation Days & $\$ 45.19$ & $\$ 38.54$ & $\$ 27.50$ & $\$ 10.36$ & $\$ 8.88$ & $\$ 11.11$ & $\$ 55.55$ & $\$ 47.42$ & $\$ 38.61$ \\
\hline Week One & $\$ 18.27$ & $\$ 20.83$ & $\$ 15.83$ & $\$ 5.52$ & $\$ 4.05$ & $\$ 5.46$ & $\$ 23.79$ & $\$ 24.89$ & $\$ 21.29$ \\
\hline Week Two & $\$ 26.92$ & $\$ 17.71$ & $\$ 11.67$ & $\$ 4.84$ & $\$ 4.82$ & $\$ 5.65$ & $\$ 31.76$ & $\$ 22.53$ & $\$ 17.32$ \\
\hline Non-Event Days & $\$ 10.58$ & $\$ 9.38$ & $\$ 5.00$ & $\$ 6.40$ & $\$ 4.86$ & $\$ 6.77$ & $\$ 16.98$ & $\$ 14.24$ & $\$ 11.77$ \\
\hline Event Days & $\$ 34.62$ & $\$ 29.17$ & $\$ 22.50$ & $\$ 3.95$ & $\$ 4.01$ & $\$ 4.34$ & $\$ 38.57$ & $\$ 33.18$ & $\$ 26.84$ \\
\hline
\end{tabular}

* Total cost includes only early and late schedule offset and not in-vehicle travel time. 


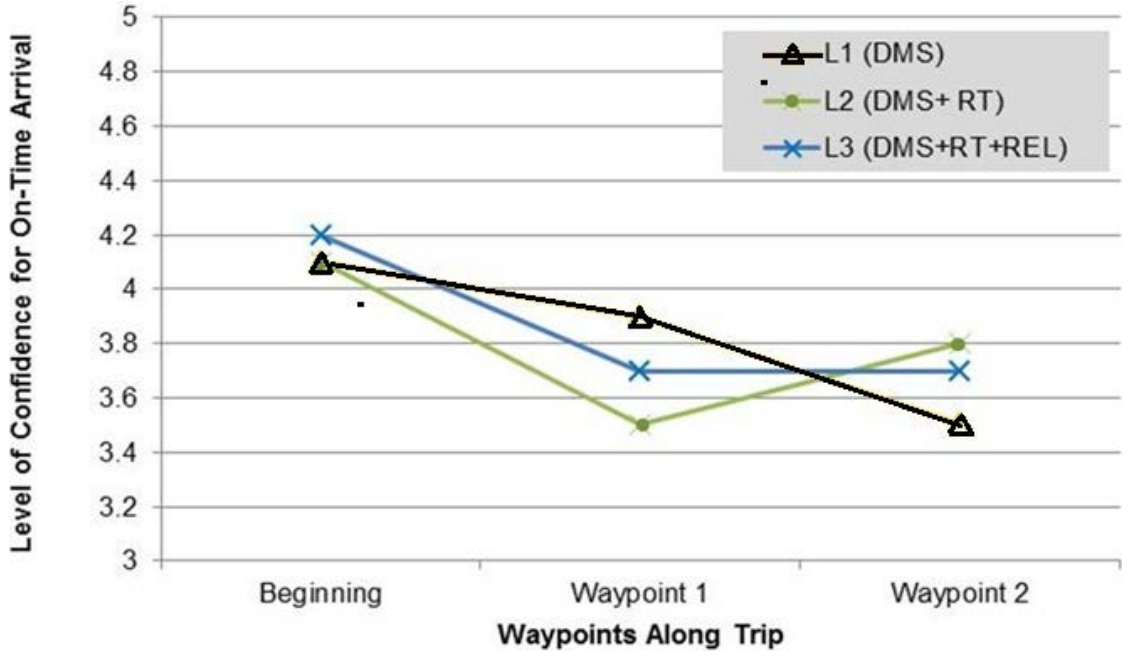

Figure 9.14. Average on-time arrival confidence during trips on event days.

for the first commute day and event day 6. On most event days, L3 participants found travel more stressful at Waypoint 1 compared with the first commute day. The higher serenity levels for the first commute day (at the beginning of the trip) for L3 participants coupled with their higher willingness to pay for traveler information at this waypoint suggested that reliability information for unfamiliar trips has value.

The level of serenity ( $1=$ very stressful, $5=$ stress free $)$ selected by participants at the end of the trip was nearly equivalent to that at the first waypoint across all non-event days (average rating of 4.1). Further inspections revealed that the level of serenity (stress reduction) was lower (by $0.4-0.5$ points) for non-event days in the first week compared with non-event days in the second week. This finding again illustrates the learning curve with unfamiliar trips. Furthermore, as expected, participants' level of serenity for event days was on average $20 \%$ lower than on non-event days (4.1 versus 3.2). Average differences among groups with different information levels did not prove significant for end-of-trip serenity levels. The question about happiness associated with trip outcome yielded findings that were nearly identical to the question about level of stress information at trip end.

\section{Willingness to Pay for Traveler Information at Beginning of Trip Reflected Value of Reliability Information}

The possible range of willingness to pay that participants could select at the beginning of the trip was $\$ 0.00$ to $\$ 1.50$. At the beginning of each trip day, participants' willingness to pay for traveler information was slightly higher on average for L3 information (\$0.61) compared with the other two groups
(\$0.54 for L1, \$0.55 for L2). Furthermore, the willingness to pay trend line had a negative slope for L3 participants. Providing reliability bands, in addition to real-time travel times, helped participants become familiar with the work trip more quickly; consequently, their willing to pay for information at the beginning of the trip diminished further into the experiment. Conversely, the willingness to pay trend line had a positive slope for L2 participants, suggesting that the realtime information was continuing to help them gain an inherent understanding of the trip's variability. Figure 9.15 presents these trends; however, these trends likely would have changed had participants completed additional simulation days.

The possible range of willingness to pay from which participants could select at the end of the trip was $\$ 0.00$ to $\$ 5.00$. The average among the three groups was $\$ 2.30$. Surprisingly, this measure did not show significant sensitivity in aggregate from week 1 to week 2 or for event and non-event days. Participants were, on average, willing to pay only slightly more ( $\$ 0.02$ to $\$ 0.07$ ) for traveler information on event days compared with non-event days; however, these differences were not statistically significant. Further, participants receiving L1 information were, on average, willing to pay $\$ 0.15$ to $\$ 0.17$ more per trip compared with participants receiving L2 and L3 information.

\section{Traveler Information Provided Trip Serenity Even When Trip Outcome Could Not Be Improved}

Traveler information can benefit travelers even when the information itself does not reduce travel time by allowing 


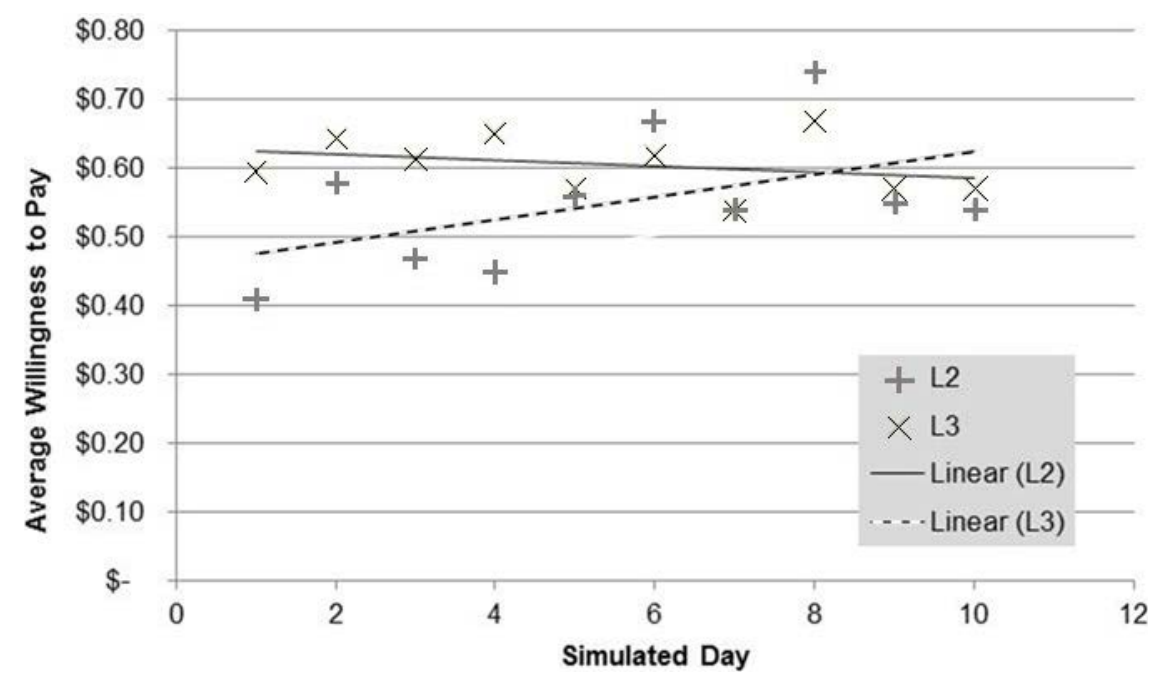

Figure 9.15. Trend in willingness to pay (at beginning of trip) across simulation days.

travelers to adjust their schedule to accommodate a late arrival. In the simulation experiment, participants were allowed an opportunity to simulate a call to work to inform their employer that they would be arriving late (and avoid the \$25 late-arrival penalty). This penalty avoidance could be used only once during the simulated 2 -week period. Researchers compared the trip characteristics and trip experience for each participant who used this opportunity against another trip made by the same individual in which the outcome was also a late arrival but the call option was not exercised. The expectation was that having the opportunity to call the employer would provide a level of serenity that would translate into lower trip stress, greater happiness with trip outcome, and higher perceived usefulness of traveler information compared to a comparable trip without the call option.

Among the 80 participants, 33 arrived late and chose to inform their employer of a late arrival. Of those 33, 11 called their employer at the first waypoint, and 22 did so at the second waypoint. Of the 33 participants, 7 did not arrive late any other day. Consequently, the sample set became 26 because a point for comparison was not available for those 7 participants. A few of the 26 participants arrived late on multiple days. For this circumstance, the day with an arrival delay similar to the day they chose to call their employer was selected for a paired comparison.

Of the 26 paired trips analyzed, 6 pairs were made by participants who received L1 traveler information, 11 pairs were made by participants who received $\mathrm{L} 2$ traveler information, and the remaining 8 were made by participants who received L3 traveler information. Twenty of the 26 paired trips had the same departure time. In three pairs, the trip during which the call to employer was made began at 8:00 a.m., while its counterpart began at 8:15 a.m. The reverse occurred for the remaining three pairs.

On average, the 26 trips for which a call for late arrival was made required $5 \mathrm{~min}$ of additional in-vehicle time and ended on arrival 5 min earlier than their paired counterparts. When able to call their employer regarding a late arrival, participants found the trip less stressful, were happier with their trip outcome, and found traveler information more useful. The difference with regard to happiness with trip outcome was statistically significant at the $99 \%$ level, and the difference in the usefulness of traveler information was statistically significant at the $95 \%$ level. All other differences were statistically significant at the $90 \%$ level with the exception of willingness to pay, where the difference was significant at the $80 \%$ level. Outcomes of the paired analyses are presented in Table 9.10.

The difference in travel times and arrival delay were statistically significant between the paired sets, which suggested that the trips may not actually have been equivalent with regard to travel times and arrival outcome. On further investigation, researchers observed that, for 14 of the 26 paired trips, the difference in arrival delay was greater than $10 \mathrm{~min}$ and that those differences may have clouded the assessment of serenity benefits. Therefore, a second analysis with only 12 paired trips was conducted.

The more focused analysis confirmed that knowledge of a late arrival en route provided a serenity benefit as captured by lower trip stress, greater happiness with trip outcome, and higher rating for traveler information usefulness. In this analysis, the difference among pairs was not statistically significant for in-vehicle travel time and average minutes late. Furthermore, for this sample, the average difference in willingness to 
Table 9.10. Value of Late-Arrival Information, Expanded Sample Size

\begin{tabular}{|c|c|c|c|}
\hline \multirow[b]{2}{*}{$\begin{array}{l}\text { Trip Metrics } \\
\text { Sample Size }=26 \text { Paired Trips }\end{array}$} & \multicolumn{2}{|c|}{ Average Trip Value } & \multirow{2}{*}{$\begin{array}{l}\text { Statistical Sig. } \\
\text { of Differences } \\
\text { in Paired Data }\end{array}$} \\
\hline & $\begin{array}{l}\text { Participants } \\
\text { Phoned Ahead }\end{array}$ & $\begin{array}{c}\text { Paired } \\
\text { Counterpart }\end{array}$ & \\
\hline Average in-vehicle travel time (minutes) & 61.2 & 56.3 & $95 \%$ \\
\hline Average minutes late & 13.9 & 18.8 & $95 \%$ \\
\hline Trip Stressfulness ( 1 = very stressful, $5=$ stress free) & 1.92 & 1.81 & $75 \%$ \\
\hline Happiness with trip outcome ( $1=$ unhappy, $5=$ very happy $)$ & 2.12 & 1.31 & $99 \%$ \\
\hline Usefulness of traveler information ( $1=$ not useful, $5=$ very useful) & 3.88 & 3.19 & $99 \%$ \\
\hline Willingness to pay for traveler information $(\$ 0-\$ 5)$ & $\$ 2.73$ & $\$ 2.50$ & $85 \%$ \\
\hline
\end{tabular}

pay for traveler information was $\$ 0.25$ and was statistically significant. These findings are presented in Table 9.11.

\section{Application of Results in Travel Utility Functions}

A typical method for valuation of trips to work involves the application of the cost function (utility model) proposed by Small et al. (1999). This model, based on a population of commuters along SR-91 in California, estimates that invehicle travel time costs $\$ 3.38$ per hour, which the authors professed is low. They attributed the low value to participants placing greater interest on trip variability trade-offs. The cost per hour for late arrival is $\$ 18.60$, with a $\$ 2.87$ onestep penalty for arriving late. Furthermore, the model's cost for early arrivals provides a quadratic penalty for very early arrivals.

Application of this utility model, and model parameters, to all trips simulated in this experiment yielded an average cost of $\$ 5.25$ per trip, or $\$ 52.55$ for the set of 10 trips. This cost includes early- and late-arrival costs as well as in-vehicle travel time. Trip costs were slightly lower for L3 participants compared with L1 and L2, but the differences were not statistically significant. Table 9.12 breaks down late and early costs by week and by event and non-event days. As expected, total per-participant trip costs on event days were far greater than costs on non-event days, and the differences were statistically significant. Difference in cost among different traveler information groups was not statistically significant for event days.

The schedule offset costs as outlined in the experiment and the trip costs based on Small et al.'s model admittedly are inherently different in form; consequently, they cannot be directly compared. Small et al.'s model has a greater penalty for very early arrivals and lesser penalty for late arrivals compared with the simplified framework of this experiment. Furthermore, the total costs based on the experimental framework do not include in-vehicle travel time.

What does prove useful from this experiment is participants' valuation of trip serenity. Given participants' stated willingness to pay for traveler information - and differences in this value when participants could simulate a call to their employer while having the same trip outcome- the research team suggests adding a term to the Small et al. model to include a cost discount for the serenity benefit of traveler information. The proposed model is this:

$$
c=\alpha T+\beta_{S D E}(S D E)+2 \beta_{S D E 2}(S D E)^{2}+\gamma(S D L)+\theta D_{L}+\lambda D_{I}
$$

Table 9.11. Value of Late-Arrival Information, Specific Paired Comparison

\begin{tabular}{|c|c|c|c|}
\hline \multirow[b]{2}{*}{$\begin{array}{l}\text { Trip Metrics } \\
\text { Sample Size = } 12 \text { Paired Trips }\end{array}$} & \multicolumn{2}{|c|}{ Average Trip Value } & \multirow{2}{*}{$\begin{array}{l}\text { Statistical Sig. } \\
\text { of Differences } \\
\text { in Paired Data }\end{array}$} \\
\hline & $\begin{array}{l}\text { Participants } \\
\text { Phoned Ahead }\end{array}$ & $\begin{array}{c}\text { Paired } \\
\text { Counterpart }\end{array}$ & \\
\hline Average in-vehicle travel time (minutes) & 58.2 & 56.4 & $75 \%$ \\
\hline Average minutes late & 8.2 & 7.7 & $75 \%$ \\
\hline Trip Stressfulness ( $1=$ very stressful, $5=$ stress free) & 2.33 & 1.75 & $93 \%$ \\
\hline Happiness with trip outcome ( $1=$ unhappy, $5=$ very happy) & 2.50 & 1.50 & $99 \%$ \\
\hline Usefulness of traveler information ( $1=$ not useful, $5=$ very useful) & 4.25 & 3.25 & $99 \%$ \\
\hline Willingness to pay for traveler information $(\$ 0-\$ 5)$ & $\$ 2.75$ & $\$ 2.50$ & $96 \%$ \\
\hline
\end{tabular}


Table 9.12. Application of Small et al. Utility Framework

\begin{tabular}{|c|c|c|c|c|c|c|c|c|c|}
\hline \multicolumn{10}{|c|}{ Costs for Schedule Offset Based on Small et al. Framework } \\
\hline & \multicolumn{3}{|c|}{ Late Arrival Cost } & \multicolumn{3}{|c|}{ Early Arrival Cost } & \multicolumn{3}{|c|}{ Total Cost ${ }^{\star}$} \\
\hline & L1 & L2 & L3 & L1 & L2 & L3 & L1 & L2 & L3 \\
\hline All 10 Simulation Days & $\$ 10.84$ & $\$ 11.87$ & $\$ 9.69$ & $\$ 24.08$ & $\$ 18.52$ & $\$ 25.02$ & $\$ 53.76$ & $\$ 50.62$ & $\$ 53.05$ \\
\hline Week One & $\$ 5.69$ & $\$ 7.91$ & $\$ 6.37$ & $\$ 14.05$ & $\$ 9.10$ & $\$ 13.06$ & $\$ 28.62$ & $\$ 27.19$ & $\$ 28.40$ \\
\hline Week Two & $\$ 5.14$ & $\$ 3.96$ & $\$ 3.32$ & $\$ 10.03$ & $\$ 9.42$ & $\$ 11.97$ & $\$ 25.14$ & $\$ 23.43$ & $\$ 24.65$ \\
\hline Non-Event Days & $\$ 1.89$ & $\$ 1.48$ & $\$ 0.82$ & $\$ 15.64$ & $\$ 10.35$ & $\$ 16.31$ & $\$ 24.11$ & $\$ 19.80$ & $\$ 23.43$ \\
\hline Event Days & $\$ 8.95$ & $\$ 10.39$ & $\$ 8.86$ & $\$ 8.44$ & $\$ 8.16$ & $\$ 8.71$ & $\$ 29.65$ & $\$ 30.83$ & $\$ 29.61$ \\
\hline
\end{tabular}

* Total cost includes late and early schedule offset plus in-vehicle travel time.

where

$\begin{aligned} c & =\text { cost of travel (disutility) } \\ T & =\text { travel time } \\ S D E & =\text { schedule delay early } \\ S D L & =\text { schedule delay late } \\ D_{L} & =\text { late-arrival index }= \begin{cases}1 & \text { if } S D L>0 \\ 0 & \text { otherwise }\end{cases} \\ D_{I} & =\text { late-arrival information index }= \begin{cases}1 & \text { if late-arrival } \\ \text { information } & \text { is acquired } \\ 0 & \text { otherwise }\end{cases} \end{aligned}$

Based on this model form, the $\lambda$ reflects the reduction in trip cost from having information en route that the outcome of the trip will be a late arrival. The proposed value $\lambda$ according to the findings of this study, would be $-\$ 0.25$. This value is based on an average trip length of $55 \mathrm{~min}$, with an approximate travel time range of $45 \mathrm{~min}$ to $70 \mathrm{~min}$. On average, participants received traveler information of late arrival $35 \mathrm{~min}$ into the trip, which was $20 \mathrm{~min}$ before their trip end and $15 \mathrm{~min}$ before their planned arrival.

Note that, in the pre-experiment survey, participants' average willingness to pay for knowledge of a late arrival for the trip to work ranged from $\$ 0.30$ to $\$ 1.23$, depending on how important on-time arrival was for the group. Overall, average willingness to pay was $\$ 1.08$ based on a 10 -point scale from $\$ 0.00$ to $\$ 5.00$. Compared with the findings from the experiment, these findings illustrate that stated willingness to pay was somewhat higher than what was measured in the simulation; but both the stated and the observed significance of information on late arrivals was valuable.

\section{Conclusions and Next Steps}

This section summarizes experimental findings with respect to the three key experimental hypotheses. In addition, a number of next steps are identified for possible inclusion in possible follow-up experimentation.
The first hypothesis stated that provision of accurate reliability information would result in improved on-time performance and lower generalized travel disutility compared with a control group receiving no reliability information. Findings from the study supported this hypothesis. Participants who received reliability information were on time more frequently ( $85 \%$ versus $79 \%$ ) and had lower total late- and early-arrival penalties associated with the simulated trip ( $\$ 38.61$ versus $\$ 47.42$ and $\$ 55.55$ in the two control groups). Both findings were statistically significant with $95 \%$ confidence despite the relatively small sample size of the experiment.

The second hypothesis proposed that, while travel outcomes would improve with the provision of reliability information, the perceived value of the reliability information would underestimate the realized benefit in terms of reduced delay, improved on-time reliability, and reduced stress. Findings from the study supported this hypothesis as well. Measured perceptions of benefit associated with reliability information were not commensurate with the observed improvements in trip outcomes. Changes in the value of information and stress reduction, although generally favorable to reliability information, were not statistically significant. The implication is that although reliability information can be useful in managing trip time variability, the apparent value of the information is lost in the context of learning over time. The experiment's subjects likely internalized the reliability information as one element in the learning process, rather than considering its value outside of the learning process. Providers of reliability information may face an uphill battle in measuring perceptions of the effects of reliability information even when such information is useful in improving trip outcomes.

The third hypothesis was that the benefits of reliability information would decline over time as both experimental and control subjects learned and internalized an understanding of underlying travel time variability. That is, the benefit from reliability information in the first few weeks would be larger than in the last few weeks. Findings from the study were 
inconclusive on this point. Participants with access to reliability information did see total early- and late-arrival penalties decline from week 1 to week 2 of the simulated trip-making. Control groups who did not receive this information were mixed, with one group realizing a reduction in total costs and another seeing a rise in those costs. Interestingly, participants' willingness to pay for reliability information declined over time in the simulation experiment, indicating that experience within the simulated trip began to offset the reliance on provided reliability information.

According to the post-experiment survey, for groups without reliability information, many more individuals perceived a positive overall usefulness of traveler information at the end of the experiment than at the beginning. In contrast, in the group with reliability information, the swing toward the positive proved more muted. This finding suggests that as the groups without reliability information used the real-time information to learn about trip variability, more individuals found value in the information. For those with reliability information, trip variability knowledge was already present; consequently, its usefulness remained consistent from the beginning to the end of the experiment. Overall, however, a 2-week period was observed to be too short to make any definitive statements regarding this hypothesis. 


\section{сонатте 10 \\ Enhanced Laboratory Experiment}

Reliability information encompasses a broad range of information that describes underlying trip variability and other contextual data travelers use to manage delay and on-time performance. Included in this category are (a) information describing the statistical variation in travel time dependent on a departure time choice, (b) data describing on-time performance and lateness risk by route and destination, and (c) contextual information to interpret cueing throughout the travel experience. All of these types of information allow travelers to better assess travel time and lateness risk both pre-trip and en route.

The SHRP 2 L14 project prepared a lexicon for transportation operations professionals, agency public information officers, and the research community to provide guidance on the provision of reliability information. The lexicon is intended both to orient the reader regarding the concept and potential value of reliability information and to provide guidance on the terms, graphics, and delivery media used to provide reliability information.

To aid in the development of the lexicon, researchers completed two parallel activities, each addressing a specific aspect of reliability information. First, coordinated focus groups and survey instruments were used to develop a draft lexicon of phrases that could be used to convey reliability information. Second, a protocol and experimental materials were developed to quantitatively assess the value of reliability information in a travel behavior simulation. The benefits from reliability information were measured in terms of improved traveler on-time performance, reduced total time allocated to travel, and reduced stress related to uncertainty about on-time arrival.

Although the lexicon development effort identified terms and phrases preferred by travelers in describing travel time reliability, it did not quantitatively evaluate the effectiveness of reliability-related terms and phrases in improving on-time reliability. The initial travel behavior laboratory experiment showed that providing reliability information does improve ontime performance and reduce delays (even in the presence of real-time condition information). However, that initial experiment did not include a systematic assessment of alternative terms, phrases, or delivery media for reliability information.

The goal of this enhanced laboratory experiment was to quantitatively evaluate - through a travel simulation - the effectiveness of reliability-related terms, phrases, and delivery media in reducing traveler stress and improving on-time arrival reliability. Additionally, the experiment solicited participants' perceptions of information value and understandability. Further, the experiment tested the hypothesis that reliability information will expedite the transition from unfamiliar traveler to routine commuter.

This chapter defines the experimental plan and outlines the specific experiments and surveys that were implemented to meet the scope and hypotheses described in the following section. It also summarizes the findings from the pre-experiment survey, focusing on the demographic and trip-making characteristics of study participants. Additional chapter content includes detailed findings from the first simulation experiment (experiment 1), highlighting the valuation and quantitative benefits from different forms of pre-trip reliability information; a summary of the findings from the second experiment (experiment 2), quantifying the change in reliability information valuation and benefits over a 1-month simulation period; and an exploration of the ratings and rankings on the usefulness and complexity of the reliability forms specifically for use with experiment 1 and for use in general trip planning associated with unfamiliar trips. The final section presents the findings of the experiment with regard to the defined hypotheses and suggests future research options.

\section{Experiment Scope and Hypotheses}

Reliability information can be useful to any traveler. However, one finding from earlier research using focus groups indicated that reliability information can be most usefully targeted at 
unfamiliar travelers who are planning or executing a trip which has a high level of uncertainty regarding travel time and on-time performance. Unfamiliar travelers are most likely to underestimate or overestimate travel times and have the highest stress associated with on-time reliability-precisely because they have not made these trips many times and accumulated experience related to travel time, delays, and the underlying pattern of variation on potential travel routes. Furthermore, reliability information is expected to prove effective at expediting the transition from unfamiliar traveler to routine commuter. Consequently, this set of experiments focused on the unfamiliar traveler.

Researchers in many disciplines, including traffic operations, have observed that direct assessments of comprehension are often worse than indirect assessments (e.g., freeway lane choice in a driving simulator in response to a particular guide sign design). The travel behavior simulation approach used in this experiment provided a way to indirectly assess comprehension and can be thought of as a type of revealed preference survey as well. The study examined three key hypotheses:

1. Provision of accurate reliability information (in an easy-tounderstand format) will result in improved on-time performance and lower generalized travel disutility compared with a control group receiving no reliability information.

a. While travel outcomes improve with the provision of reliability information, the perceived value of the reliability information will underestimate the realized benefit in terms of reduced delay, improved on-time reliability, and reduced stress.

$b$. Provision of reliability information using different textual, graphical, and auditory forms will result in differences in both accrued on-time reliability benefits and perceived benefit. The differences among experimental groups are expected to be smaller than between any group and the control (no reliability information) group.

2. Experimental subjects receiving contextual information on underlying variation with numeric indicators reinforced with en route information (reliability signposting) will have improved on-time performance compared with both an experimental group that receives reliability information but no contextual information as well as a control group that receives no reliability information.

3. The benefits of reliability information will decline over time as both experimental and control subjects learn and understand the underlying travel time variability. That is, the benefit from reliability information during the first weeks will be larger than during the last weeks.

Phrases describing reliability that were identified as preferred by travelers in the lexicon were quantitatively evaluated in a controlled experiment to answer the hypotheses proposed above. The specific phrases, graphics, and delivery media evaluated in the experiment were selected on the basis of the lexicon findings and accepted by the SHRP 2 L14 technical expert task group. The specific reliability-related terms, phrases, and delivery media assessed in this experiment are listed as follows:

1. Text-based 95th percentile travel time referred to as "the majority of the time";

2. Text-based average and 95th percentile travel time referred to as "estimated travel time and extra time for unexpected delays";

3. Text-based 20th, average, and 95th percentile travel times referred to as "good day," "typical day," and "bad day";

4. Text and visual/colored signposting with travel time ranges associated with color;

5. Graphical average and 95th percentile arrival times with a legend identifying data as "estimated and extra time";

6. Graphical 20th, average, and 95th percentile arrival times with a legend identifying data as "good day," "typical day," and "bad day"; and

7. Voice-based 95th percentile travel time referred to as "the majority of the time."

The seven presentations of reliability data were introduced to subsets among 240 participants from three metropolitan regions: Chicago, Illinois; Houston, Texas; and Washington, D.C. The reliability information presented to experiment participants did not vary from day to day but did reflect the historical reliability of travel along a route by time of day. In addition to the reliability information, all participants received pre-trip and en route advisory message sets that reflected radio and qualitative dynamic message sets.

\section{Experimental Plan}

Two experiments were developed and implemented to address the study hypotheses. Both experiments used the framework of the travel behavior simulation approach developed in previous efforts; however, the implementation of this framework differed by the type of travel and information provided. The content of each experiment was developed to work within the constraints of overall testing session length (90 min). Within the framework of the experiments, participants selected a departure time based on pre-trip information, experienced travel time as a function of trip duration, and rated the value of the information simulated from day to day.

In both experiments, the reliability information presented to participants did not vary from day to day but instead reflected the historical reliability of travel along a route. In addition to differing levels of reliability information, all participants received pre-trip and en route advisory messages that reflected qualitative dynamic message sets and radio messages, albeit in text format. In both experiments, participants 
selected a departure time within a 30-min window to minimize schedule offset for a morning commute along a single route on freeway facilities.

The experiments were conducted in three cities-Chicago, Houston, and Washington, D.C.- - over a period of 2 months and acquired information from approximately 240 participants. Experiment participants were recruited through advertisements on craigslist.org. Requirements for participation included a valid U.S. driver's license; fluency in reading, writing, and speaking English; a commute to work that included $20+$ minutes of freeway travel; and familiarity with online mapping tools such as Google Maps or MapQuest. The last of these requirements was included to ensure that participants would have proficiency in the use of computers and familiarity with viewing maps, which were the basis for visually presenting the travel simulation.

Table 10.1 presents the number of participants by city. The cities were selected to ensure a diverse survey population that had experiences with high levels of traffic congestion, commute lengths, and highway network connectivity.

Each participant was provided and used a dedicated laptop with a mouse and headphones. Participants were required to use the mouse to click on interface buttons, radio buttons, or drop-down menus. Participants did not use the keyboard for any part of the experiment. Upon arrival in the survey room, participants were seated and given a brief explanation about the intent of the experiment. Participants in both experiments completed the same pre-experiment survey of 12 multipart questions, followed by the group-led experiment and varying post-experiment surveys. Appendix G lists the specific data types requested and generated from the pre-experiment survey questions.

The travel behavior simulation was programmed using VBA within the Microsoft Excel environment. Several iterations of quality assurance and testing were completed on the multiple laptop computers used in the field to create and support consistent visual appearance and accurate macro execution based on varying performance levels and settings among field laptops. Figure 10.1 presents the screenshots of the Excel-based interface, which served as the starting point for experiment participants for experiments 1 and 2. The screenshots are identical with the exception of the post-game survey, which was implemented within experiment 1 but was not relevant to experiment 2 .

Participants independently completed the pre-experiment survey ("Begin Survey" button in Figure 10.1) and waited for a moderator to jointly commence the commute experiment ("Begin Experiment" button in Figure 9.1). The pre-experiment survey requested socioeconomic, travel purpose, roadway usage, and schedule integrity information. This socioeconomic information framed the characteristics of participants and can be compared against the population at large or with other future experimental groups. The travel purpose, schedule integrity, and roadway usage questions together identified the types of trips for which on-time arrival was most important to participants, the frequency with which these trips were made, and whether the trips for which on-time arrival was most critical

Table 10.1. Experiment Layout and Sample Size for Two Distinct Experiments

\begin{tabular}{|l|c|c|c|c|c|c|}
\hline & \multicolumn{3}{|c|}{$\begin{array}{c}\text { Experiment 1: } \\
\text { 5 Cities, } \\
\text { 1 Week/City" } \\
\text { Implementation } \\
\text { Version \# }\end{array}$} & $\begin{array}{c}\text { Experiment 2: } \\
\text { 1 City, 4 Weeks } \\
\text { Implementation } \\
\text { Version \# }\end{array}$ \\
\cline { 2 - 8 } & $\mathbf{1}$ & $\mathbf{2}$ & $\mathbf{3}$ & $\mathbf{4}$ & $\mathbf{5}$ & $\mathbf{6}$ \\
\hline Planned pontent (A-H) & 90 & 30 & 30 & 30 & 30 & 30 \\
\hline Valid "good" participant count & 98 & 29 & 25 & 33 & 30 & 30 \\
\hline A. Control (no reliability info) & $\checkmark$ & $\checkmark$ & $\checkmark$ & $\checkmark$ & $\checkmark$ & na \\
\hline B. Textual 95th & $\checkmark$ & na & $\checkmark$ & na & na & na \\
\hline C. Textual Avg + 95th & $\checkmark$ & $\checkmark$ & $\checkmark$ & $\checkmark$ & na & na \\
\hline D. Textual 20th + Avg + 95th & $\checkmark$ & $\checkmark$ & na & $\checkmark$ & na & na \\
\hline E. Visual and textual signposting & $\checkmark$ & na & na & na & na & na \\
\hline F. Graphical presentation of "C" & na & $\checkmark$ & $\checkmark$ & na & na & na \\
\hline G. Graphical presentation of " $D$ " & na & $\checkmark$ & na & $\checkmark$ & na & $\checkmark$ \\
\hline H. Auditory presentation of "B" & na & na & $\checkmark$ & $\checkmark$ & na & na \\
\hline
\end{tabular}

Note: $\boldsymbol{\checkmark}=$ included; na $=$ not applicable.

${ }^{a}$ City refers to travel in a simulated city for participants and not to city of experiment implementation. 

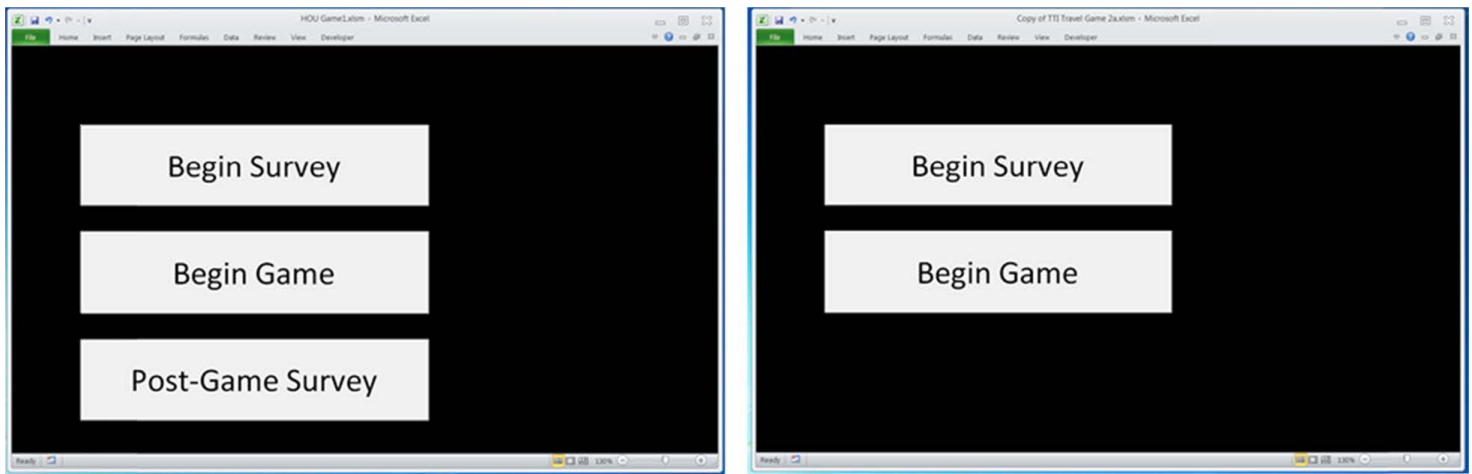

Figure 10.1. Beginning screenshot for experiments 1 (left) and 2 (right).

were made on roadways where traveler information was usually available. The detailed descriptions and outcomes of the preexperiment survey are presented later in this chapter.

The commute experiment began with the moderator giving instructions to the participant group. The moderator read aloud the contextual setup text presented in the experiment and informed participants that different individuals would have access to differing levels of traveler information. Participants selected departure time, their confidence in arriving on time, and usefulness of trip information before beginning each trip. The interface presented the movement of a vehicle along segments of the defined route. The duration of the vehicle travel in the experiment paralleled (by a factor) the simulated trip duration based on the selected departure. For example, a 30-min trip required the participant to view progress over $6 \mathrm{~s}$, while a 60 -min trip required the participant to view progress over $12 \mathrm{~s}$.

At the end of each simulated trip, participants were provided trip outcome statistics in the form of simulated arrival time, trip duration, schedule offset, and cost of travel. At the end of each trip, participants were also asked to indicate willingness to pay for traveler information, trip stress level, and traveler information usefulness. Figure 10.2 presents two screenshots of the Excel-based interface for making the initial trip departure decisions and for the trip experience for a specific day of commute. Figure 10.2 also illustrates the implementation of the highway message board.

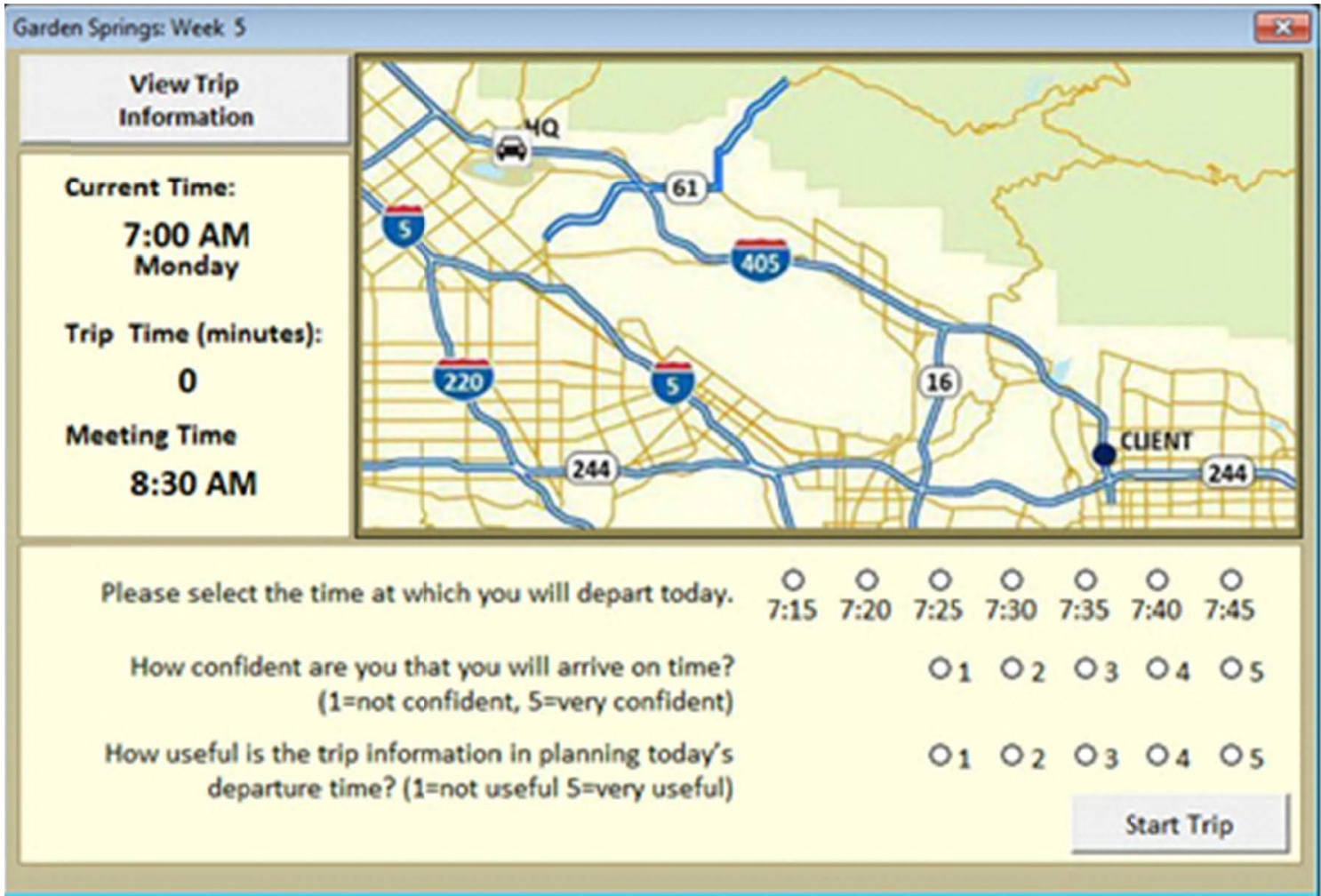

Figure 10.2. Screenshots of trip departure decisions and travel along route. 
The moderator supported participants for the first day of travel by presenting on large visual images the location of specific types of information and decision sets. Thereafter, participants independently navigated the information and made commute decisions at their own pace to complete commutes to work over a 1-week period. Participants were still given the option of moderator support for navigating the experiment but not for interpreting reliability information.

Travel time and radio message data supporting the experiment were designed to represent a moderate level of trip variability as well as temporal consistency among departure time, traveler information, and trip experiences. The outcomes from the commute experiment are presented later in this chapter.

\section{Pre-Experiment Survey}

The pre-experiment survey was delivered to all participants and developed a baseline of participant characteristics that were useful for comparing participant characteristics with the population at large. Key data acquired through the surveys for this comparison included gender, age, educational level, and traveler information usage. Additionally, the survey questions aimed to explore whether the participant population made trips that would benefit most from traveler information - that is trips that were sufficiently long, required stringent on-time arrivals, and used facilities where such information would be available (i.e., highways). Participants with these routine tripmaking characteristics were likely to better internalize tradeoff requested in the simulation experiment, and their responses were more likely to reflect true trip decision making. The specific text of pre-experiment questions and the response options for questions are presented in Appendix $\mathrm{H}$.

The survey questions also enabled the development of lateness thresholds - that is, they allowed researchers to explore what participants perceived as arriving late based on participants' specification for on-time arrival importance and trip purpose. These thresholds can be applied in future work to refine the form of utility functions.

Willingness to pay for guaranteed travel time (zero trip variability or $100 \%$ trip reliability) and willingness to pay for knowledge of late arrival were both assessed to compare with sensitivities observed through the experiment. A priori, that participants with high on-time arrival importance were expected to be willing to pay more for this guarantee or arrival outcome information.

\section{Experiment 1 Description}

The first experiment framed the simulation as that of traveling for work to five different cities and commuting for 5 weekdays from each city's regional headquarters to a morning client meeting that was $40 \mathrm{~min}$ to $60 \mathrm{~min}$ from headquarters. Each participant experienced four of the seven reliability-related data, a specific term each for a week. During one week, participants received no reliability-related data. The order of the type of data received was randomly defined. The visual make-up of highway and arterials for each of the simulated cities was fictional and did not parallel any actual city. This was done to remove the potential for participants' affiliation and familiarity with traffic in actual cities.

The goal of the first experiment was to quantitatively evaluate the effectiveness of reliability-related terms, phrases, and delivery media in reducing traveler stress and improving ontime arrival reliability. Additionally, the first experiment solicited perceptions of information value and understandability. The specific reliability-related terms, phrases, and delivery media assessed were compared with a baseline without reliability information. The baseline and reliability terms tested in this experiment are listed below. The visual delivery of these message sets through the simulated commute is presented in Appendix H.

- No reliability information (baseline);

- Text-based 95th percentile travel time referred to as "the majority of the time";

- Text-based (textual) average and 95th percentile travel time referred to as "estimated travel time and extra time for unexpected delays";

- Text-based 20th, average, and 95th percentile travel time referred to as "good day," "typical day," and "bad day";

- Text and visual/colored signposting with travel time ranges associated with color;

- Graphical average and 95th percentile arrival time with a legend identifying data as "estimated and extra time";

- Graphical 20th, average, and 95th percentile arrival time with a legend identifying data as "good day," "typical day," and "bad day"; and

- Voice-based 95th percentile travel time referred to as "the majority of the time."

On the basis of the experimental design outlined in Table 10.1, six unique city networks were developed such that a participant experienced a different visual network/city in each of the 5 weeks of commutes. Table 10.2 identifies for each information type the name of the associated simulated city and the specific off-peak travel times, meeting time, and departure options available.

A narrative was presented on screen and communicated verbally to prepare participants for the simulation experiment. In essence, participants were in a new town for work and had to arrive on time to work or otherwise incur a \$25 late-arrival fee. Early arrival cost (parking cost) was introduced at $\$ 12.00$ per hour. Participants were asked to select among seven departure times and multiple routes for a simulated travel to work over a 
Table 10.2. Reliability Information Types, Simulated Cities, and Departure Options

\begin{tabular}{|c|c|c|c|c|c|c|c|}
\hline $\begin{array}{l}\text { Info } \\
\text { Code }\end{array}$ & Reliability Information Type & City & City Name & $\begin{array}{l}\text { Off-Peak } \\
\text { Travel Time }\end{array}$ & $\begin{array}{l}\text { Meeting } \\
\text { Time }\end{array}$ & \multicolumn{2}{|c|}{$\begin{array}{c}\text { Departure Time } \\
\text { Range } \\
\text { (Earliest-to-Latest) }\end{array}$} \\
\hline A & Baseline & 1 & Prairie Cliffs & 30 & 8:30 a.m. & 7:25 a.m. & 7:55 a.m. \\
\hline B & Text-based 95th percentile travel time & 2 & Garden Springs & 30 & 8:00 a.m. & 6:55 a.m. & 7:25 a.m. \\
\hline C & Text-based average +95 th percentile travel time & 3 & Port Frederick & 40 & 8:30 a.m. & 7:15 a.m. & 7:45 a.m. \\
\hline D & Text: good, typical, bad day & 4 & Sioux Rapids & 50 & 9:00 a.m. & 7:35 a.m. & 8:05 a.m. \\
\hline E & Reliability signpost & 5 & Harrisonville & 30 & 9:00 a.m. & 7:55 a.m. & 8:25 a.m. \\
\hline $\mathrm{F}$ & Graphical average +95 th percentile travel time & 5 & Harrisonville & 40 & 8:00 a.m. & 6:45 a.m. & 7:15 a.m. \\
\hline G & Graphical: good, typical, bad day & 2 & Garden Springs & 50 & 8:30 a.m. & 7:05 a.m. & 7:35 a.m. \\
\hline $\mathrm{H}$ & Audio: 95th percentile travel time & 6 & New Glaxtonberg & 40 & 9:00 a.m. & $7: 45$ a.m. & 8:15 a.m. \\
\hline
\end{tabular}

5-week period, totaling 25 commutes. This narrative and the visual of each simulated city are included in Appendix $\mathrm{H}$.

For each week, or conversely, for each reliability information type, participants experienced one good traffic day, two typical traffic days, one day somewhat worse than typical, and one bad day. The order of the 5 days varied from city to city, while the order of the cities varied from participant to participant. Travel times generally increased with later departure options. Appendix $\mathrm{H}$ presents the data that fueled participant trip experiences by city, day of the week, and time of day.

Participants in this experiment made two valuations and a departure time decision pre-trip, as well as three valuations at the end of each simulated commute. Additionally, at the end of each week, participants provided five additional valuations, both of which repeated inquiries pre- and post-trip. When making end-of-week valuations, participants were provided summary statistics for the week's travel and a visual of the reliability information made available to them that week. Participants were also instructed to share their achievement expressed through cost of travel at the end of each week to encourage investment in engaging in the simulated decision making.

The pre-trip, post-trip, and end-of-week valuations were based on a 5-point scale with 1 representing the least value and 5 representing the greatest value. The valuations were as follows:

- Pre-trip usefulness of traveler information ( $1=$ not useful, $5=$ very useful);

- Pre-trip on-time arrival confidence $(1=$ not confident, $5=$ very confident);

- Post-trip usefulness of traveler information ( $1=$ not useful, 5 = very useful);

- Post-trip level of stress in completing the trip $(1=$ not stressful, 5 = very stressful);

- Post-trip willingness to pay for information per trip ( $\$ 0$, $\$ 1, \$ 2, \$ 3, \$ 4)$;
- End-of-week helpfulness of information in reducing commute stress ( $1=$ not at all, $5=$ absolutely);

- End-of-week usefulness of information overall $(1=$ not useful, $5=$ very useful);

- End-of-week helpfulness of information in better managing departure decisions ( $1=$ not at all, $5=$ absolutely);

- End-of-week level of difficulty in understanding information $(1=$ very easy, $5=$ very difficult $)$; and

- End-of-week willingness to pay per trip for information viewed $(\$ 0, \$ 1, \$ 2, \$ 3, \$ 4)$.

At the end of this experiment, participants were instructed to complete the Excel-based post-trip survey. The survey presented the set of four reliability terms used during the experiment and asked participants to rate the relative value of each form of reliability information with regard to usefulness and complexity. Findings from the post-experiment survey as well as other postexperiment metrics are presented later in this chapter.

\section{Experiment 2 Description}

The second experiment framed the simulation as that of traveling to a new city for work and making daily departure time decisions for a month (4 weeks, 5 days a week). This second experiment tested the hypothesis that reliability information will expedite the transition from unfamiliar traveler to routine commuter. Here, half of the participants received graphical 20th, average, and 95th percentile arrival time reliability information with a legend identifying data as "good day," "typical day," and "bad day." This reliability information was labeled type $G$ and is presented in Table 10.2. The other half of the participants did not receive reliability information, which is presented in Table 10.2 by information code A. Both groups of participants received pre-trip and en route advisory messages that reflected qualitative dynamic message sets and radio messages, albeit in text format. 
The process by which participants received information was similar to that described in the opening narrative included in Appendix H. Differences included the fact that reliability information type A used the same data and simulated city layout as information type G, New Glaxtonberg. The travel time and radio message data viewed in experiment 2 were the same as those in experiment 1 . The transition screen of simulated commutes from week to week was that of a weekly planner rather than an aircraft as used in experiment 1. Finally, slight differences were made with regard to weekly summaries.

End-of-week valuations and post-experiment valuations were not collected for this experiment given that the objective was to evaluate trip decisions and outcomes. In lieu of valuations, and based on post-hoc analysis of experiment times for completion of experiment 2 at the first city execution of the experiment (Washington, D.C.), the team chose to implement a second, paper survey instrument for experiment 2 participants as well as for experiment 1 participants who finished earlier than others. That survey is also included in Appendix H.

\section{Pre-Experiment Survey Findings}

The pre-experiment surveys completed through the Excelbased interface provided insight on the characteristics and travel sensitivities of participants. The intent of the preexperiment survey was also to determine how well suited the general travel characteristics of participants were to the simulation scenarios they experienced in the experiment. All participants completed the pre-experiment surveys $(n=251)$. The findings from the survey are summarized in the following subsections and highlighted here.

Overall, many participants did make long trips for work and other trip purposes. They often indicated having high ontime arrival importance as well as low thresholds for late arrivals. More individuals valued reliability (guaranteed travel time) and were willing to pay more for a reliable travel time compared with their valuation of late-arrival knowledge. Participants frequently used traveler information for their work commutes. Participant characteristics exhibited in the preexperiment survey paralleled the simulation scenarios that were presented to participants through the experiment. Consequently, participants were well suited for and were likely to respond effectively to the simulation experiment.

\section{Demographic and Trip-Making Characteristics}

Experiment participants leaned toward the male gender and were generally older and more highly educated compared with national averages. Nearly $60 \%$ of participants were male, and the median age range among all participants was 40 years to 49 years. Close to half of all participants had a college degree or higher, whereas only $25 \%$ of the U.S. population (age 25 and older) has completed a college or postgraduate degree. The implications of the demographic differences between the study participants and the population at large are unclear. A more highly educated sample may be better able to interpret traveler information; however, an older population may be less familiar with technology applications.

Participants were asked to record the frequency with which they made work, child-care, school, medical, and other routine trips. Trip-making frequency choices presented to participants included never, less than once a month, 1 to 3 times a month, once a week, 2 to 4 times a week, and daily. All participants made trips for child care $(100 \%)$, and nearly all made trips for work (98\%), medical appointments $(90 \%)$, or other trips (92\%) with some frequency. Conversely, $69 \%$ of participants never made school-related trips. Additionally, although nearly all participants made work-related trips, only $71 \%$ commuted daily to work, while $57 \%$ made daily trips for child care. As expected, only $2 \%$ of participants made daily medicalrelated trips, and $8 \%$ made daily school-related trips. The goal was to solicit individuals for participation who routinely commuted to work; and according to the aforementioned statistics, this goal was achieved.

Figures summarizing the specific distributions for gender, age, and education level, and summarizing participant trip types as discussed above, are included in Appendix I. Supplemental figures for the following section are also contained in Appendix I.

\section{Traveler Information and Road Use Characteristics}

Participants also revealed how frequently they sought out traffic information for their work trip and the media they used to acquire the information. Participants' responses were aggregated and are presented in Figure 10.3. When making work trips, most $(81 \%)$ sought out traffic information with some frequency, and more than $30 \%$ did so five or more times a week. Most used multiple forms of media to acquire this information. More than $60 \%$ of participants who sought out traffic information for the work commute consulted the radio. Two out of five participants used their desktop or laptop computer or mobile device, such as a smartphone or tablet. This usage supersedes vehicle dashboard systems for acquisition of traveler information. Given these high rates of information usage, the expectation was that participants would relate well to the experimental scenarios which presented traveler information to use in planning a work trip.

Given that the experiment simulated travel along highways, the pre-experiment survey requested information on the types of facilities participants used in making routine trips for 

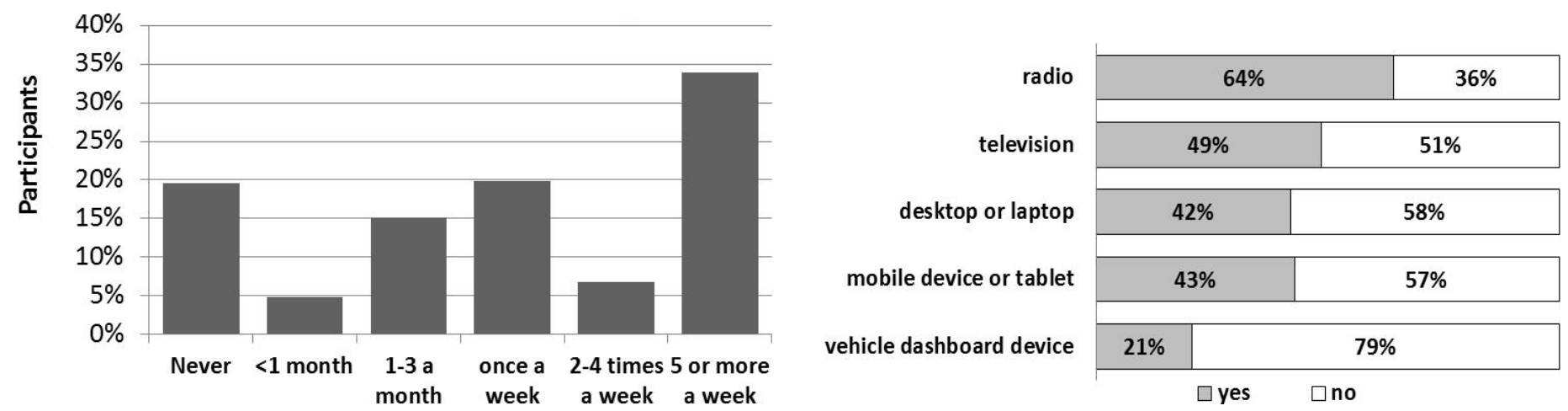

Figure 10.3. Frequency (left) and types of traffic information (right) used for work trips (percent of participants).

work, child care, school, and medical and other needs. Among individuals who made work trips, $88 \%$ used highways or a combination of highways and arterials on their work commute. Highways were used less often for other types of trips compared with work trips. For child-care, school, and medical trip makers, $45 \%$ to $51 \%$ of participants also used highways or a combination of highways and arterials. Transit was used by a handful of participants for all trip purposes except child care (for which transit was not used by any participants).

\section{Importance and Definition of Arriving on Time}

Participants were asked to rate the importance of an on-time arrival and were also asked about their sensitivity to arriving late. Participants overwhelmingly indicated that for work, school, child care, and medical appointments, arriving on time was between "somewhat important" and "very important," based on a 5 -point scale ( $1=$ not important, $5=$ very important). Among participants who indicated that they made work trips, $84 \%$ suggested that arriving on time was very important. Arriving on time to school, child-care, or medical destinations was less frequently cited as very important. Between $64 \%$ and $68 \%$ of individuals making school, child-care, and medical trips indicated that arriving on time was very important for their trips.

Participants' ratings for importance of arriving on time did not parallel their definition for late arrival. While $84 \%$ indicated on-time arrival was very important for work trips, only $23 \%$ defined late as arriving beyond the planned arrival time ( 1 or more minutes late). The meaning of late was more precise for work and child-care trips compared with the other trip purposes. Within the simulation experiment, the exact meaning for late arrival was modeled as 1 or more minutes lateparallel to $23 \%$ of participants for work trips. Almost the same proportion of child-care trip makers (24\%) also categorized arriving late as arriving a minute beyond their scheduled arrival time. More than $80 \%$ of participants believed that arriving 10 or more minutes beyond a scheduled arrival was considered late. The most frequent threshold for arriving late was cited as $5 \mathrm{~min}$ beyond the scheduled arrival time.

Table 10.3 lists what participants, on average, defined as arriving late by the importance they placed on arriving on

Table 10.3. Average Threshold for Late Arrival, by On-Time Arrival Importance and Trip Purpose (min)

\begin{tabular}{|l|r|c|c|c|c|c|}
\hline \multirow{2}{*}{$\begin{array}{l}\text { Arrival Importance } \\
\text { (sample size) }\end{array}$} & \multicolumn{5}{|c|}{ Trip Destination by Purpose } & \\
\cline { 2 - 7 } & Work & School & Child Care & Medical & Other & \multirow{2}{*}{ All Trips } \\
\hline $1(3)$ & 5.0 & na & na & 6.0 & 15.6 & 11.5 \\
\hline $2(7)$ & 25.1 & 19.2 & 22.0 & 11.3 & 15.4 & 18.5 \\
\hline $3(9)$ & 20.4 & 12.6 & 17.9 & 19.5 & 17.5 & 17.9 \\
\hline $4(21)$ & 15.8 & 14.6 & 16.2 & 14.1 & 15.5 & 15.1 \\
\hline $5(206)$ & 12.2 & 12.6 & 11.1 & 11.9 & 12.6 & 12.1 \\
\hline Average lateness threshold & 13.1 & 13.4 & 12.5 & 13.1 & 15.2 & 13.6 \\
\hline
\end{tabular}

Note: na $=$ not applicable. In the first column, $1=$ unimportant and $5=$ very important. 
time. Across all participants and trip purposes, the average threshold for characterizing a trip as late was $13.6 \mathrm{~min}$ beyond the desired arrival time. As expected, individuals who indicated arriving on time was very important had a far smaller lateness threshold. For example, participants who indicated that an on-time work arrival was very important (category 5) believed that arriving on average $12.1 \mathrm{~min}$ past their scheduled arrival time was considered late, whereas this value was 15.8 minutes for participants who indicated that on-time arrival at work was somewhat important (category 4).

\section{Travel Times and Trip Variability}

Two pre-survey questions addressed participants' normal trip time by trip purpose and the additional time that participants' trips might take on a bad day of traffic. According to participants' responses, on average, trips made to work required the greatest travel time, while trips made for child care required the least travel time. The largest share of participants required on average $20 \mathrm{~min}$ to $40 \mathrm{~min}$ to complete a trip. At least a few participants indicated that travel took $60 \mathrm{~min}$ or more on average to their work, school, child-care, medical, or other destination. Among participants who made work trips by car, $42 \%$ had an average work-trip time that was similar to the times presented in the experiment $(40 \mathrm{~min}$ or greater), while another $45 \%$ traveled slightly lower work-trip times, ranging from $20 \mathrm{~min}$ to $40 \mathrm{~min}$.

Work trips had by far the greatest level of variability for participants, as is evidenced by the $42 \%$ who indicated their work-trip time could increase by as much as $30 \mathrm{~min}$ on a bad traffic day. Child-care trips had the least magnitudes of variability, with $52 \%$ of participants indicating an additional time under $15 \mathrm{~min}$.

As a trend, participants noting longer average trip times also noted having greater magnitudes in travel time increases for bad traffic days. Figure 10.4 presents a three-dimensional visual of participants' additional travel time on a bad day compared with their average travel time specifically for work trips. The highest peaks indicate that for work trips, the greatest frequency of response was for trips that took $30 \mathrm{~min}$ to 39 min on average, with an extra time of 30 min or more on bad traffic days. The next two most frequent replies were for work trips that were $40 \mathrm{~min}$ to $49 \mathrm{~min}$ or even $60+\min$ on average, with trip variability of $30+\min$ on a bad day. These three most frequent response categories are highlighted in black in Figure 10.4 and represent $31 \%$ of all participant responses for work trips.

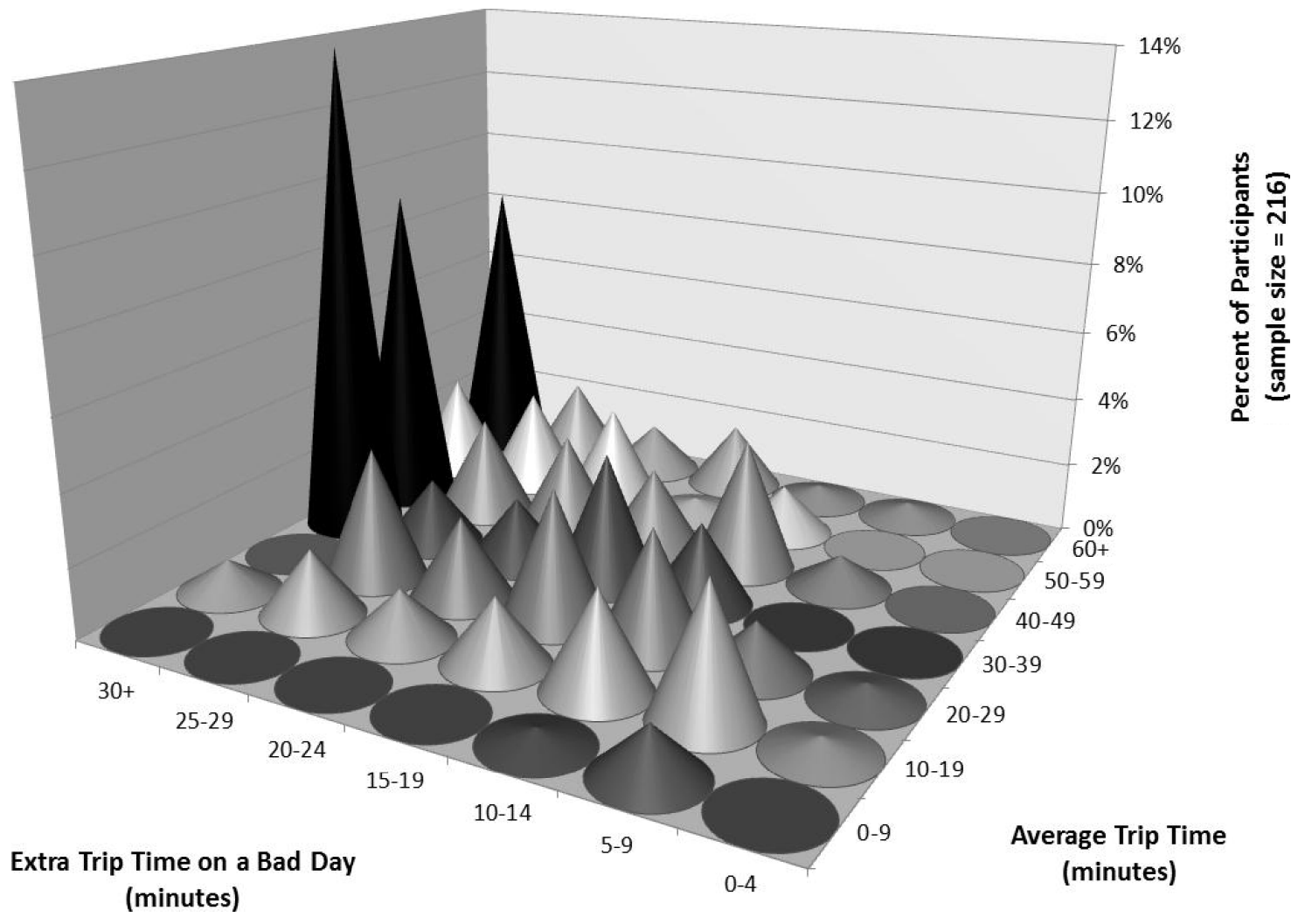

Figure 10.4. Average travel time and extra time entries for work trips. 


\section{Valuation of Reliability and Serenity}

Two more survey questions gauged participants' willingness to pay for a guaranteed travel time to their destination and their willingness to pay for knowledge of a late-arrival trip outcome. The first question placed a value on a guaranteed arrival time (i.e., a reliable travel time) and measured individuals' willingness to pay to eliminate uncertainty in travel times. The second question placed a value on the knowledge of an adverse trip arrival outcome (i.e., a late arrival) en route. Although an individual cannot change their trip outcome, they can use this information to adjust their subsequent activities and inform individuals of schedule offsets. Further, sometimes information, even if not acted upon, provides travelers with a greater level of serenity simply by knowing what is to come. The knowledge question, consequently, placed a value on this serenity effect.

Approximately $68 \%$ of individuals indicated they were willing to pay to ensure a reliable travel time. Participants more often were willing to pay for this guaranteed time for work trips than for other trip purposes. For example, $20 \%$ of individuals who made work trips indicated that they would be willing to pay $\$ 4.00$ to $\$ 5.00$ per trip for a guaranteed travel time. This value was only $10 \%$ for school trips. Of course, work trips had a far higher on-time arrival importance. On average, participants who made work trips were willing to pay $\$ 1.69$ per trip to eliminate trip variability. Table 10.4 summarizes the average valuation for guaranteed travel time and knowledge of late arrivals among participants for specific trip types.

The majority of individuals found value in knowing they would be late for work, school, child-care, and medical trips. Fewer individuals were willing to pay for knowledge of a late arrival $(60 \%)$ compared with reducing trip time variability $(68 \%)$. Further, the amounts individuals were willing to pay for knowledge of a late arrival were somewhat lower than for a guaranteed travel time. On average, participants were willing to pay $\$ 0.87$ for late-arrival information en route versus $\$ 1.20$ for a guaranteed travel time. This makes sense given that a

Table 10.4. Average Willingness to Pay for Reliable Travel and Late-Arrival Knowledge, by Trip Purpose Based on Replies to Pre-Experiment Survey

\begin{tabular}{|l|c|c|}
\hline Trip Purpose & $\begin{array}{c}\text { Value of } \\
\text { Reliable Travel }\end{array}$ & $\begin{array}{c}\text { Value of Late-Arrival } \\
\text { Knowledge }\end{array}$ \\
\hline Work trips & $\$ 1.69$ & $\$ 1.27$ \\
\hline School trips & $\$ 1.05$ & $\$ 0.89$ \\
\hline Child-care trips & $\$ 1.28$ & $\$ 1.23$ \\
\hline Medical appointments & $\$ 1.10$ & $\$ 0.82$ \\
\hline Other routine trips & $\$ 0.81$ & $\$ 0.27$ \\
\hline Average & $\$ 1.20$ & $\$ 0.87$ \\
\hline
\end{tabular}

guaranteed travel time mitigates adverse trip arrival outcomes, while the knowledge of a late arrival does not change an adverse trip outcome.

\section{Experiment 1 Findings: Effectiveness and Valuation of Reliability Terms for Unfamiliar Trips}

Participants in this experiment made two valuations and a departure time decision pre-trip, as well as three valuations at the end of each simulated commute. Additionally, at the end of each week, participants provided five additional valuations, both of which repeated inquiries pre- and post-trip. These valuations were based on a 5-point scale, with 1 representing the least value and 5 representing the greatest value. The valuations are as follows:

- Pre-trip usefulness of traveler information ( $1=$ not useful, 5 = very useful);

- Pre-trip on-time arrival confidence ( $1=$ not confident, $5=$ very confident);

- Post-trip usefulness of traveler information ( $1=$ not useful, 5 = very useful);

- Post-trip level of stress in completing the trip $(1=$ not stressful, $5=$ very stressful);

- Post-trip willingness to pay for information per trip ( $\$ 0$, $\$ 1, \$ 2, \$ 3, \$ 4)$;

- End-of-week helpfulness of information in reducing commute stress ( $1=$ not at all, $5=$ absolutely);

- End-of-week usefulness of information overall $(1=$ not useful, 5 = very useful);

- End-of-week helpfulness of information in better managing departure decisions ( $1=$ not at all, $5=$ absolutely);

- End-of-week level of difficulty in understanding information ( 1 = very easy, $5=$ very difficult); and

- End-of-week willingness to pay per trip for information viewed $(\$ 0, \$ 1, \$ 2, \$ 3, \$ 4)$.

In addition to the user-provided valuation, the experiment yielded trip outcome metrics that included average minutes of early schedule delay if early, average minutes of late schedule delay if late, and frequency of late arrivals per week. The week's schedule offset costs were also computed on the basis of the predefined costs for early and late arrivals.

These metrics were compared pairwise between the specific reliability information (types $\mathrm{B}$ through $\mathrm{H}$, as defined in Table 10.2) and the baseline traveler information (type A), which did not provide reliability information. The sample size for comparisons varied for the specific pairs; specific findings are presented in the next subsection and summarized in tabular formats. In the second subsection, the three 
reliability constructs (types B through D) are compared with their counterparts, delivered using either auditory or graphical methods.

\section{Pairwise Comparisons for Reliability Terms Against the Baseline}

This section presents results from pairwise comparisons for participants who completed simulated commutes for both the reliability term being tested and the baseline information, which did not contain reliability data. A summary of trends are first discussed. Each of the seven following subsections presents the detailed pairwise comparisons in the order below:

1. Text-based 95th percentile travel time information compared with no reliability information;

2. Text-based average and 95th percentile travel time information compared with no reliability information;

3. Text-based 20th percentile, average, and 95th percentile travel time information compared with no reliability information;

4. Signposting reliability information;

5. Graphical average and 95th percentile travel time information compared with no reliability information;

6. Graphical 20th percentile, average, and 95th percentile travel time information compared with no reliability information; and

7. Auditory 95th percentile information compared with no reliability information.

Comparisons with the baseline pre-trip information revealed that trip outcomes were more positive for participants using the text-based and auditory reliability information and somewhat better for those using the graphical average and 95th percentile data. Trip outcomes when using the graphical good, typical, and bad information as well as the reliability signposting were not significantly better than the baseline.

Trip outcomes did not correlate with willingness to pay for information. Furthermore, participants' responses regarding willingness to pay for information viewed varied significantly when asked post-trip compared with when asked at the end of the week. For example, although participants performed better when using text-based 95th percentile reliability information than when using the baseline, participants' willingness to pay for the information was not significantly higher than baseline when asked at the end of the week. When asked post-trip, participants were willing to pay approximately $\$ 0.16$ per trip more for the reliability information compared with the baseline. Alternately, participants did not have better trip outcomes when using the graphical good, typical, and bad information, but they were willing to pay $\$ 0.42$ per trip more compared with the baseline information when asked post-trip.
Ratings for information usefulness when asked post-trip were either lower than the baseline or otherwise not significantly different, even when trip outcomes were better with the reliability information. One reason for this phenomenon may be that when participants were late, they faulted more strongly the reliability information if available; however, in the absence of reliability information, they may have faulted inherent traffic rather than the baseline radio information. Consequently on late-arrival days, usefulness ratings were far lower in the presence of reliability information. Once participants sufficiently disassociated with the immediate trip outcome, they tended to rate the reliability information usefulness higher compared with the baseline information. A similar outcome was observed in ratings for post-trip stress reduction compared with the end-of-week rating for the reliability information's effectiveness at reducing stress.

With regard to ratings on the level of difficulty in understanding the information presented, all but two forms of information did not yield statistically significant differences between the baseline and reliability information. The signposting and the graphical good, typical, and bad information had ratings differentials when compared against the baseline, which suggests that those forms of information are more difficult to understand.

\section{Text-Based 95th Percentile Reliability Information Versus Baseline (Type B Versus Type A)}

Among all experiment participants, 123 individuals made a week ( 5 days) of simulated commutes to work using the textbased 95th percentile reliability information (type B) and also made a week (5 days) of simulated commutes to work using no reliability information (type A). The specific text used to deliver reliability information was "the majority of the time." The simulated commute decisions were pairwise analyzed for trip outcome and valuations of information. For both weeks, participants received radio messages pre-trip and viewed dynamic message signs along the trip route. Analysis outcomes are presented in Table 10.5.

The trip outcomes for participants using type B information included average lower frequency of late arrival (40\% fewer late arrivals) and lower magnitudes of late delay ( $0.6 \mathrm{~min}$ less) at the expense of higher magnitudes of early arrivals (3.1 min earlier) when compared with the use of type A information. For trip outcome with regard to the schedule offset costs, as defined for the experiment, participants using the type B information had a total week's cost that was $21 \%$ lower (\$10.02) compared with the week they did not receive reliability information.

Participants having access to type B information generally departed a few minutes earlier (3.9 min), had a higher pretrip on-time arrival confidence rating (3.6 versus 3.4 ), and 
Table 10.5. Trip Decisions, Outcomes, and Valuations of Text-Based 95th Percentile Reliability Data Compared with Baseline

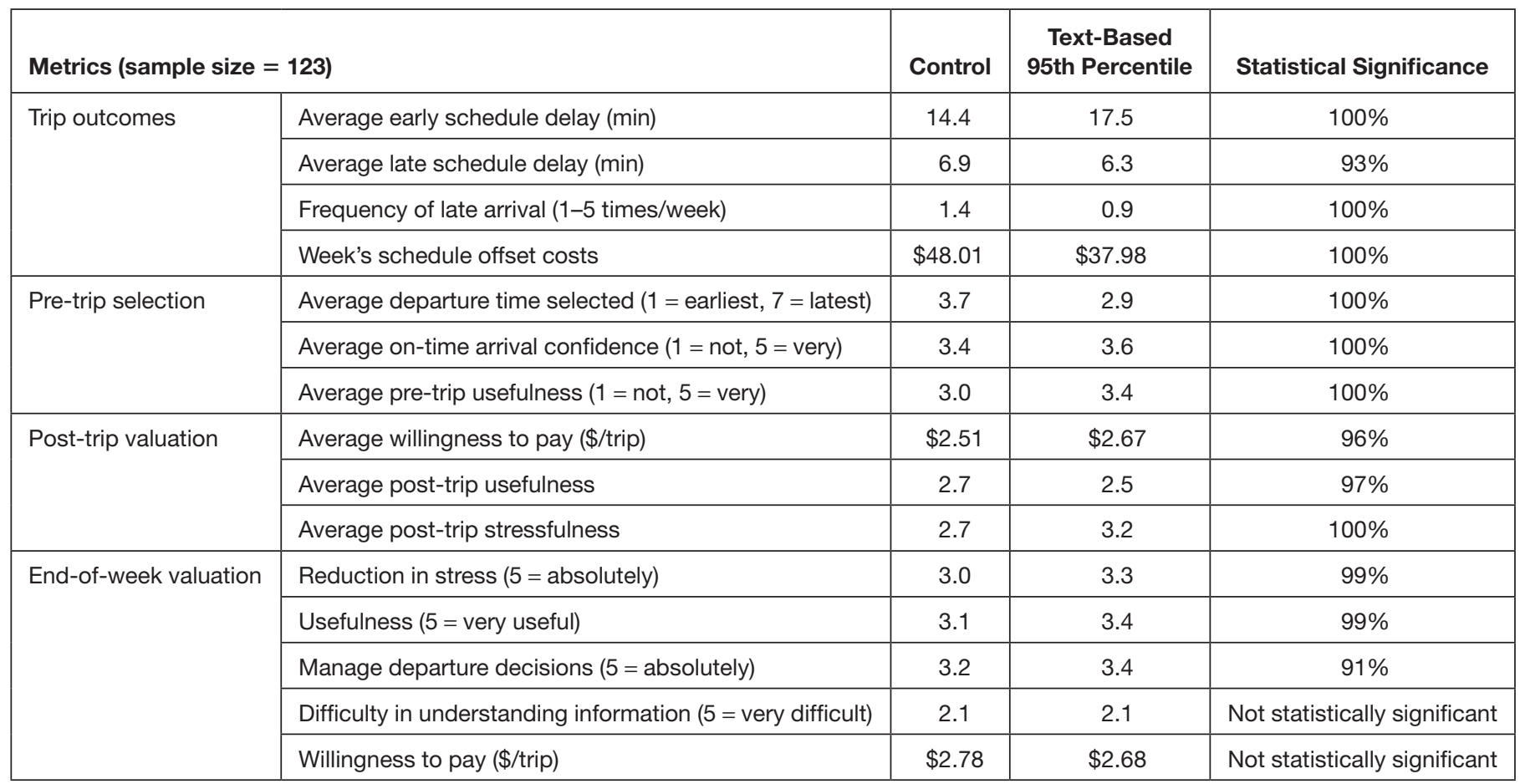

had a higher information usefulness rating (3.4 versus 3.0). As expected, participants' end-of-week ratings regarding pretrip information reducing stress, proving useful, and helping manage departure decisions were higher for type B (95th percentile reliability information) compared with the baseline.

An unexpected and conflicting finding was that participants gave the reliability information a higher usefulness rating pre-trip but a lower rating post-trip compared with the no reliability information scenario. Yet, at the end of the week, they again rated reliability information as more useful. Participants' may have penalized the reliability information for poor trip outcomes while attributing poor trip outcomes to normal congestion when they did not have reliability data. At the end of the week, participants came to recognize through valuation a higher usefulness for reliability information. Similarly, when asked at the end of the week, individuals were willing to pay on average $\$ 0.10$ more for type B information; however, this differential did not prove statistically significant. Yet, for the same question at the end of each trip, participants were willing to pay about $\$ 0.15$ more per trip for type B information, and this differential was statistically significant.

Finally, when asked how difficult it was to understand the traveler information, participants rated both type $\mathrm{A}$ and type B nearly identically. That is, participants did not find understanding the "majority of the time" reliability information any more difficult than understanding the radio message without reliability information.

\section{Text-Based Average and 95th Percentile Reliability Information Versus Baseline (Type C Versus Type A)}

Among all experiment participants, 185 individuals made a week (5 days) of simulated commutes to work using the textbased average and 95th percentile reliability information (type C) and also made a week (5 days) of simulated commutes to work using no reliability information (type A). The specific text used to deliver reliability information was "estimated travel time, and extra time for unexpected delays." The simulated commute decisions were pairwise analyzed to evaluate trip outcome and valuations of information. For both weeks, participants also received radio messages pre-trip and viewed dynamic message signs along the trip route. Analysis outcomes are presented in Table 10.6.

The trip outcomes for participants using type $\mathrm{C}$ information included average lower frequency of late arrival (28\% fewer late arrivals) and lower magnitudes of late delay ( $2.7 \mathrm{~min}$ less on average) at the expense of slightly higher magnitudes of early arrivals ( 1.7 min earlier) when compared with the use of type A information. For trip outcome with regard to the schedule offset costs, as defined for the experiment, participants using the type C information had a total week's cost that was $17 \%$ lower (\$8.20) compared with the week they did not receive reliability information.

Participants having access to type $\mathrm{C}$ information generally departed a few minutes earlier $(2.5 \mathrm{~min})$, had a higher 
Table 10.6. Trip Decisions, Outcomes, and Valuations of Text-Based Average and 95th Percentile Reliability Data Compared with Baseline

\begin{tabular}{|c|c|c|c|c|}
\hline \multicolumn{2}{|c|}{ Metrics (sample size = 185) } & \multirow{2}{*}{$\begin{array}{c}\text { Control } \\
14.4\end{array}$} & \multirow{2}{*}{$\begin{array}{c}\begin{array}{c}\text { Text-Based } \\
\text { Average }+ \\
\text { 95th Percentile }\end{array} \\
16.1\end{array}$} & \multirow{2}{*}{$\begin{array}{c}\begin{array}{c}\text { Statistical } \\
\text { Significance }\end{array} \\
99 \%\end{array}$} \\
\hline Trip outcomes & Average early schedule delay (min) & & & \\
\hline & Average late schedule delay (min) & 7.4 & 5.0 & $100 \%$ \\
\hline & Frequency of late arrivals (1-5 times/week) & 1.5 & 1.1 & $100 \%$ \\
\hline & Week's schedule offset costs & $\$ 49.29$ & $\$ 41.07$ & $100 \%$ \\
\hline \multirow[t]{3}{*}{ Pre-trip selection } & Average departure time selected $(1=$ earliest, $7=$ latest $)$ & 3.7 & 3.1 & $100 \%$ \\
\hline & Average on-time arrival confidence ( $5=$ very confident) & 3.4 & 3.6 & $100 \%$ \\
\hline & Average pre-trip usefulness ( 5 = very useful) & 3.0 & 3.5 & $100 \%$ \\
\hline \multirow[t]{3}{*}{ Post-trip valuation } & Average willingness to pay (\$/trip) & $\$ 2.34$ & $\$ 2.65$ & $100 \%$ \\
\hline & Average post-trip usefulness ( 5 = very useful) & 2.7 & 2.5 & $99 \%$ \\
\hline & Average post-trip stressfulness ( $5=$ very stressful) & 2.7 & 3.5 & $100 \%$ \\
\hline \multirow[t]{5}{*}{ End-of-week valuation } & Reduction in Stress (5 = absolutely) & 3.0 & 3.5 & $100 \%$ \\
\hline & Usefulness (5 = very useful) & 3.1 & 3.6 & $100 \%$ \\
\hline & Manage departure decisions ( 5 = absolutely) & 3.2 & 3.7 & $100 \%$ \\
\hline & Difficulty in understanding information ( $5=$ very difficult) & 2.0 & 2.2 & $\begin{array}{l}\text { Not statistically } \\
\text { significant }\end{array}$ \\
\hline & Willingness to pay (\$/trip) & $\$ 2.61$ & $\$ 2.83$ & $99 \%$ \\
\hline
\end{tabular}

pre-trip on-time arrival confidence rating (3.6 versus 3.4), and had a higher information usefulness rating ( 3.5 versus 3.0). As expected, participants' end-of-week ratings regarding pre-trip information reducing stress, proving useful, and helping manage departure decisions were higher for type $\mathrm{C}$ compared with the baseline.

Conflicting outcomes were observed between post-trip and end-of-week valuations for information usefulness and effectiveness in reducing stress. Participants gave the reliability information type $\mathrm{C}$ a higher usefulness rating pre-trip but a lower rating post-trip ( 3.5 versus 2.5 ) compared with the no reliability information week ratings of (3.0 versus 2.7). Yet, at the end of the week, they again rated the type $\mathrm{C}$ reliability information as more useful (3.6 versus 3.1). Participants' may have penalized the reliability information for poor trip outcomes while attributing poor trip outcomes to normal congestion when they did not have reliability data. Once away from the immediate disappointment of a poor trip outcome, participants came to recognize the usefulness of the information and provided a valuation for reliability information. Similarly, at the end of each trip with type $\mathrm{C}$ information, participants indicated on average higher levels of stress compared with the end of each trip with no reliability information (3.5 versus 2.7 ); yet at the end of the week, they generally assigned higher ratings to the value of type $\mathrm{C}$ information in reducing stress (3.5 versus 3.0 ).
When asked at the end of the week, individuals were willing to pay on average $\$ 0.22$ more for type $\mathrm{C}$ information compared with the baseline; however, for the same question at the end of each trip, participants were willing to pay about $\$ 0.31$ more per trip. Finally, when asked how difficult it was to understand the traveler information, participants rated type $\mathrm{C}$ slightly more challenging to understand ( 2.2 versus 2.0) compared with the baseline information; however, this difference was not statistically significant. Thus, participants did not find understanding the "estimated travel time, and extra time for unexpected delays" reliability information any more difficult than understanding the radio message without reliability information.

\section{Text-Based 20th Percentile, Average, and 95th Percentile Reliability Information Versus Baseline (Type D Versus Type A)}

Among all experiment participants, 160 individuals made a week ( 5 days) of simulated commutes to work using the textbased 20th percentile, average, and 95th percentile reliability information (type D) and also made a week (5 days) of simulated commutes to work using no reliability information (type A). The specific text used to deliver reliability information was "good day, typical day, and bad day." The simulated 
commute decisions were pairwise analyzed to evaluate trip outcome and valuations of information. For both weeks, participants also received radio messages pre-trip and viewed dynamic message signs along the trip route. Analysis outcomes are presented in Table 10.7.

The trip outcomes for participants using type D information included average lower frequency of late arrival $(26 \%$ fewer late arrivals) and lower magnitudes of late delay ( $3.4 \mathrm{~min}$ less on average) when compared with the use of type A information. For the week using type D information, participants' early schedule delay was 0.8 min less than for the week using type A information; however, this outcome was not statistically significant. These trip outcomes resulted in a 13\% lower $(\$ 6.60)$ total week's cost compared with the week participants did not receive reliability information.

Participants when having access to type D information did not depart significantly earlier than when having type A information. They did have a higher on-time arrival confidence and rated the information they viewed as more useful (3.5 versus 3.0) pre-trip compared with the week when they made pre-trip decisions without reliability information. At the end of each trip made using type D information, participants were willing to pay on average $\$ 0.25$ more per trip for the information. This differential in willingness to pay, however, was not observed when asked at the end of the week.
Conflicting outcomes were observed between post-trip and end-of-week valuations for information usefulness and effectiveness in reducing stress. Participants gave the reliability information type $\mathrm{D}$ a higher usefulness rating pre-trip and at the end of the week, but a lower rating post-trip compared with the no reliability information week ratings. Participants' may have penalized the reliability information for poor trip outcomes while attributing poor trip outcomes to normal congestion when they did not have reliability data. Once away from the immediate disappointment of a poor trip outcome, participants came to recognize the usefulness of the information and provided a valuation for reliability information.

Similarly, at the end of each trip, participants indicated, on average, higher levels of stress when using type D information compared with the end of each trip with no reliability information (3.5 versus 2.7); yet at the end of the week, they generally provided higher ratings to the value of type $\mathrm{D}$ information in reducing stress (3.6 versus 3.0).

Finally, when asked how difficult it was to understand the traveler information, participants rated type D slightly more challenging to understand (2.1 versus 2.0 ) compared with the baseline information; however, this difference was not statistically significant. That is, participants did not find understanding the "good day, typical day, and bad day" reliability information any more difficult than understanding the radio message without reliability information.

Table 10.7. Trip Decisions, Outcomes, and Valuations of Text-Based 20th Percentile, Average, and 95th Percentile Reliability Data Compared with Baseline

\begin{tabular}{|c|c|c|c|c|}
\hline \multicolumn{2}{|c|}{ Metrics (sample size $=160$ ) } & \multirow{2}{*}{$\begin{array}{c}\text { Control } \\
14.5\end{array}$} & \multirow{2}{*}{$\begin{array}{c}\text { Text-Based } \\
\text { 20th Percentile }+ \\
\text { Average }+ \\
\text { 95th Percentile } \\
13.7\end{array}$} & \multirow{2}{*}{$\begin{array}{l}\text { Statistical Significance } \\
\text { Not statistically significant }\end{array}$} \\
\hline Trip outcomes & Average early schedule delay (min) & & & \\
\hline & Average late schedule delay (min) & 7.4 & 6.0 & $100 \%$ \\
\hline \multirow[t]{3}{*}{ Pre-trip selection } & Average departure time selected $(1=$ earliest, $7=$ latest $)$ & 3.7 & 3.6 & Not statistically significant \\
\hline & Average on-time arrival confidence $(5=$ very confident $)$ & 3.4 & 3.6 & $100 \%$ \\
\hline & Average pre-trip usefulness ( $5=$ very useful) & 3.0 & 3.5 & $100 \%$ \\
\hline \multirow[t]{5}{*}{ End-of-week valuation } & Reduction in stress (5 = absolutely) & 3.0 & 3.6 & $100 \%$ \\
\hline & Usefulness ( 5 = very useful) & 3.1 & 3.6 & $100 \%$ \\
\hline & Manage departure decisions ( $5=$ absolutely) & 3.3 & 3.7 & $100 \%$ \\
\hline & Difficulty in understanding information ( $5=$ very difficult) & 2.0 & 2.1 & Not statistically significant \\
\hline & Willingness to pay (\$/trip) & $\$ 2.59$ & $\$ 2.69$ & Not statistically significant \\
\hline
\end{tabular}




\section{Reliability Signposting Versus Baseline (Type E Versus Type A)}

The concept of signposting is one in which individuals are provided a color-coded trip path that reflects historical levels of congestion along key road segments of the trip route. Along with the color-coded historical congestion, a travel time range is provided. A total of 98 participants completed both a week of simulated commutes using the reliability signposting data (type E) and a week having the control information (type A).

No statistically significant difference emerged in trip outcomes with regard to late arrivals or magnitude of late schedule delay. Further, this was the one form of reliability information that exhibited a statistically significant higher rating for difficulty in understanding the information presented. In addition, although reliability signposting ratings for end-of-week usefulness and ability to reduce stress were higher than the control, the differences did not prove statistically significant. Table 10.8 summarizes the key metrics for reliability signposting.

\section{Graphical Average and 95th Percentile Reliability Information Versus Baseline (Type F Versus Type A)}

Among all experiment participants, 54 individuals made a week ( 5 days) of simulated commutes to work using the graphical average and 95th percentile reliability information (type F) and also made a week (5 days) of simulated commutes to work using no reliability information (type A). The specific graphic used to deliver reliability information was a bar chart showing estimated travel time and extra time for unexpected delays, using departure time as the $\mathrm{x}$-axis and arrival time as the y-axis. The graphic is presented in Appendix H, Figure H.6. The simulated commute decisions were pairwise analyzed to evaluate trip outcome and valuations of information. For both weeks, participants also received radio messages pre-trip and viewed dynamic message signs along the trip route. Analysis outcomes are presented in Table 10.9.

The trip outcomes for participants using type $\mathrm{F}$ information proved statistically significant above the 80th percentile but not at the 95th percentile. This might be explained by the smaller sample size involved in the evaluation. Nonetheless, for the week participants used type F information, they did on average have a lower frequency of late arrival (16\% fewer late arrivals) and lower magnitudes of late delay (1.0 min less on average) without greater magnitudes of early arrivals when compared with the week with type A information. For trip outcome with regard to the schedule offset costs, as defined for the experiment, participants using the type $\mathrm{F}$ information had a total week's cost that was 9\% lower (\$7.70) compared with the week they did not receive reliability information.

Participants having access to type $\mathrm{F}$ information had a higher pre-trip on-time arrival confidence rating (3.6 versus 3.3) and a higher pre-trip information usefulness rating (3.6 versus 3.1). As expected, participants' end-of-week ratings regarding pre-trip information reducing stress, proving useful, and helping manage departure decisions were significantly higher for type F compared with the baseline.

Table 10.8. Trip Decisions, Outcomes, and Valuations of Reliability Signposting Compared with Baseline

\begin{tabular}{|c|c|c|c|c|}
\hline \multicolumn{2}{|c|}{ Metrics (sample size $=98$ ) } & \multirow{2}{*}{$\begin{array}{c}\text { Control } \\
14.6\end{array}$} & \multirow{2}{*}{$\begin{array}{c}\begin{array}{c}\text { Reliability } \\
\text { Signposting }\end{array} \\
17.1\end{array}$} & \multirow{2}{*}{$\begin{array}{c}\text { Statistical Significance } \\
100 \%\end{array}$} \\
\hline Trip outcomes & Average early schedule delay (min) & & & \\
\hline & Average late schedule delay (min) & 7.0 & 6.6 & Not statistically significant \\
\hline & Frequency of late arrival (1-5 times/week) & 1.8 & 1.8 & Not statistically significant \\
\hline & Week's schedule offset costs & $\$ 48.57$ & $\$ 46.95$ & Not statistically significant \\
\hline \multirow[t]{3}{*}{ Pre-trip selection } & Average departure time selected $(1=$ earliest, $7=$ latest $)$ & 3.7 & 3.4 & $100 \%$ \\
\hline & Average on-time arrival confidence ( $5=$ very confident) & 3.5 & 3.4 & Not statistically significant \\
\hline & Average pre-trip usefulness ( $5=$ very useful) & 3.0 & 3.3 & $100 \%$ \\
\hline \multirow[t]{3}{*}{ Post-trip valuation } & Average willingness to pay (\$/trip) & $\$ 2.57$ & $\$ 2.69$ & Not statistically significant \\
\hline & Average post-trip usefulness ( $5=$ very useful) & 2.6 & 2.6 & Not statistically significant \\
\hline & Average post-trip stressfulness ( $5=$ very stressful) & 2.9 & 3.2 & $100 \%$ \\
\hline \multirow[t]{5}{*}{ End-of-week valuation } & Reduction in stress (5 = absolutely) & 3.0 & 3.1 & Not statistically significant \\
\hline & Usefulness (5 = very useful) & 3.1 & 3.3 & Not statistically significant \\
\hline & Manage departure decisions ( 5 = absolutely) & 3.3 & 3.3 & Not statistically significant \\
\hline & Difficulty in understanding information ( $5=$ very difficult) & 2.1 & 2.5 & $99 \%$ \\
\hline & Willingness to pay (\$/trip) & $\$ 2.77$ & $\$ 2.76$ & Not statistically significant \\
\hline
\end{tabular}


Table 10.9. Trip Decisions, Outcomes, and Valuations of Graphical Average and 95th Percentile Reliability Information Compared with Baseline

\begin{tabular}{|c|c|c|c|c|}
\hline \multicolumn{2}{|c|}{ Metrics (sample size $=54$ ) } & \multirow{2}{*}{$\begin{array}{c}\text { Control } \\
14.7\end{array}$} & \multirow{2}{*}{$\begin{array}{c}\text { Graphical Average } \\
+ \text { 95th Percentile }\end{array}$} & \multirow{2}{*}{$\begin{array}{l}\text { Statistical Significance } \\
\text { Not statistically significant }\end{array}$} \\
\hline Trip outcomes & Average early schedule delay (min) & & & \\
\hline & Frequency of late arrival (1-5 times/week) & 1.5 & 1.2 & $84 \%$ \\
\hline & Week's schedule offset costs & $\$ 49.35$ & $\$ 41.63$ & $91 \%$ \\
\hline \multirow{2}{*}{ Pre-trip selection } & Average on-time arrival confidence ( 5 = very confident $)$ & 3.3 & 3.6 & $100 \%$ \\
\hline & Average pre-trip usefulness ( 5 = very useful) & 3.1 & 3.6 & $100 \%$ \\
\hline \multirow[t]{2}{*}{ Post-trip valuation } & Average willingness to pay (\$/trip) & $\$ 2.39$ & $\$ 2.73$ & $97 \%$ \\
\hline & Average post-trip usefulness ( $5=$ very useful) & 2.7 & 2.6 & Not statistically significant \\
\hline \multirow{4}{*}{ End-of-week valuation } & Usefulness (5 = very useful) & 3.2 & 4.0 & $100 \%$ \\
\hline & Manage departure decisions ( 5 = absolutely) & 3.0 & 4.0 & $100 \%$ \\
\hline & Difficulty in understanding information ( $5=$ very difficult) & 1.9 & 2.0 & Not statistically significant \\
\hline & Willingness to pay (\$/trip) & $\$ 2.48$ & $\$ 2.70$ & Not statistically significant \\
\hline
\end{tabular}

Conflicting outcomes were observed between post-trip and end-of-week valuations for information usefulness. Participants rated graphical reliability information type $\mathrm{F}$ higher with regard to usefulness pre-trip and end of week, but posttrip rating differences between type $\mathrm{F}$ and type $\mathrm{A}$ information were not statistically significant. Participants' may have penalized the reliability information for poor trip outcomes while attributing poor trip outcomes to normal congestion when they did not have reliability data. Once away from the immediate disappointment of a poor trip outcome, participants came to recognize the usefulness of the information and provided a more positive valuation for reliability information. Similarly, at the end of each trip with type F information, participants indicated on average higher levels of stress compared with the end of each trip with no reliability information (3.7 versus 2.6); yet at the end of the week, they generally provided higher ratings to the value of type $\mathrm{F}$ information in reducing stress (3.8 versus 2.9 ).

When asked at the end of the week, participants rated willingness to pay for information higher for type $\mathrm{F}$ compared with type A; however, this difference was not statistically significant. For the same question at the end of each trip, participants were willing to pay about $\$ 0.34$ more per trip. Finally, when asked how difficult it was to understand the traveler information, participants rated type F slightly more challenging to understand (2.0 versus 1.9) compared with the baseline information; however, this difference was not statistically significant. That is, participants did not find the graphical reliability information any more difficult to understand than the radio message without reliability information.

\section{Graphical 20th Percentile, Average, and 95th Percentile Reliability Information Versus Baseline (Type G Versus Type A)}

Among all experiment participants, 62 individuals made a week (5 days) of simulated commutes to work using the graphical 20th percentile, average, and 95th percentile reliability information (type G). The same 62 individuals also made a week (5 days) of simulated commutes to work using no reliability information (type A). The specific graphic used to deliver reliability information was a bar chart with "good day," "typical day," and "bad day," using departure time as the x-axis and arrival time as they-axis. The graphic is presented in Appendix $\mathrm{H}$, Figure H.7. For both weeks, participants also received radio messages pre-trip and viewed dynamic message signs along the trip route. Analysis outcomes are presented in Table 10.10.

Participants rated graphical information as more useful than the control pre-trip; however, differences in trip outcomes for participants that used type $\mathrm{G}$ and type A information were not statistically significant at the $95 \%$ level. Neither differences in selected departure times nor on-time arrival 
Table 10.10. Trip Decisions, Outcomes, and Valuations of Graphical 20th Percentile, Average, and 95th Percentile Reliability Information Compared with Baseline

\begin{tabular}{|c|c|c|c|c|}
\hline \multicolumn{2}{|c|}{ Metrics (sample size = 62) } & \multirow{2}{*}{ Control } & \multirow{2}{*}{$\begin{array}{c}\text { Graphical } \\
\text { 20th Percentile + } \\
\begin{array}{c}\text { Average + } \\
\text { 95th Percentile }\end{array} \\
14.4\end{array}$} & \multirow{2}{*}{$\begin{array}{l}\text { Statistical Significance } \\
\text { Not statistically significan }\end{array}$} \\
\hline Trip outcomes & Average early schedule delay (min) & & & \\
\hline & Average late schedule delay (min) & 8.2 & 9.4 & $91 \%$ \\
\hline & Frequency of late arrivals ( $1-5$ times/week) & 1.6 & 1.6 & Not statistically significant \\
\hline & Week's schedule offset costs & $\$ 51.82$ & $\$ 50.85$ & Not statistically significant \\
\hline \multirow[t]{3}{*}{ Pre-trip selection } & Average departure time selected $(1=$ earliest, $7=$ latest $)$ & 3.7 & 3.8 & Not statistically significant \\
\hline & Average on-time arrival confidence ( $5=$ very confident) & 3.3 & 3.4 & Not statistically significant \\
\hline & Average pre-trip usefulness ( 5 = very useful) & 3.1 & 3.4 & $98 \%$ \\
\hline \multirow[t]{3}{*}{ Post-trip valuation } & Average willingness to pay ( $\$ /$ trip) & $\$ 2.02$ & $\$ 2.44$ & $99 \%$ \\
\hline & Average post-trip usefulness ( $5=$ very useful) & 2.7 & 2.8 & Not statistically significant \\
\hline & Average post-trip stressfulness $(5=$ very stressful) & 2.7 & 3.2 & $99 \%$ \\
\hline \multirow[t]{5}{*}{ End-of-week valuation } & Reduction in stress (5 = absolutely) & 3.0 & 3.2 & Not statistically significant \\
\hline & Usefulness (5 = very useful) & 3.2 & 3.4 & Not statistically significant \\
\hline & Manage departure decisions ( 5 = absolutely) & 3.2 & 3.3 & Not statistically significant \\
\hline & Difficulty in understanding information ( $5=$ very difficult) & 1.9 & 2.3 & $99 \%$ \\
\hline & Willingness to pay (\$/trip) & $\$ 2.26$ & $\$ 2.35$ & Not statistically significant \\
\hline
\end{tabular}

confidence ratings were statistically significant. Surprisingly, when asked post-trip, participants were willing to pay $\$ 0.42$ more per trip for type $\mathrm{G}$ information. This willingness to pay more for type $\mathrm{G}$ information did not materialize when asked at the end of the week. End-of-week valuations related to stress, usefulness, or managing departure decisions was slightly higher for type $\mathrm{G}$ information; however, differences were not statistically significant.

Most noticeable, when asked how difficult it was to understand the traveler information, participants rated type $\mathrm{G}$ more challenging to understand (2.3 versus 1.9) compared with the baseline information, and this difference was statistically significant. That is, participants found understanding this three-tiered graphical reliability information more difficult than understanding the radio message without reliability information.

\section{Auditory 95th Percentile Reliability Information Versus Baseline (Type $H$ Versus Type A)}

Among all experiment participants, 25 individuals made a week (5 days) of simulated commutes to work using the auditory 95th percentile reliability information (type $\mathrm{H}$ ) and also made a week (5 days) of simulated commutes to work using no reliability information (type A). The delivery of auditory reliability information was achieved through waveform audio file format (WAV) files played while participants wore headphones. The visual of the interface is shown in Appendix H, Figure H.8. As participants shifted the departure time bar, they heard the reliability messages. The text they heard was the same as the text written in Appendix H, Figure H.2, but it was delivered through audio instead. The simulated commute decisions were pairwise analyzed for trip outcome and valuations of information. For both weeks, participants also received radio messages pre-trip (delivered through text as in the other comparisons) and viewed dynamic message signs along the trip route. Analysis outcomes are presented in Table 10.11.

Participants having access to type $\mathrm{H}$ information departed on average a few minutes earlier ( $3.5 \mathrm{~min})$. Consequently, when early, they arrived on average $2.6 \mathrm{~min}$ earlier; and when late, they were on average 4 min less late than their baseline counterparts. The auditory information enabled participants to reduce the frequency of late arrivals by $37 \%$ and reduce the overall schedule offset costs by $\$ 7.19$, or $17 \%$.

Participants rated type $\mathrm{H}$ information higher than baseline for on-time arrival confidence and trip usefulness pre-trip. Further, participants were on average willing to pay $\$ 0.64$ per trip more for the type $\mathrm{H}$ information compared with the baseline when asked at the end of each trip. When asked again at the end of the week, the differential in willingness to pay was only $\$ 0.12$ per trip, and this differential did not prove 
Table 10.11. Trip Decisions, Outcomes, and Valuations of Auditory 95th Percentile Reliability Information Compared with Baseline

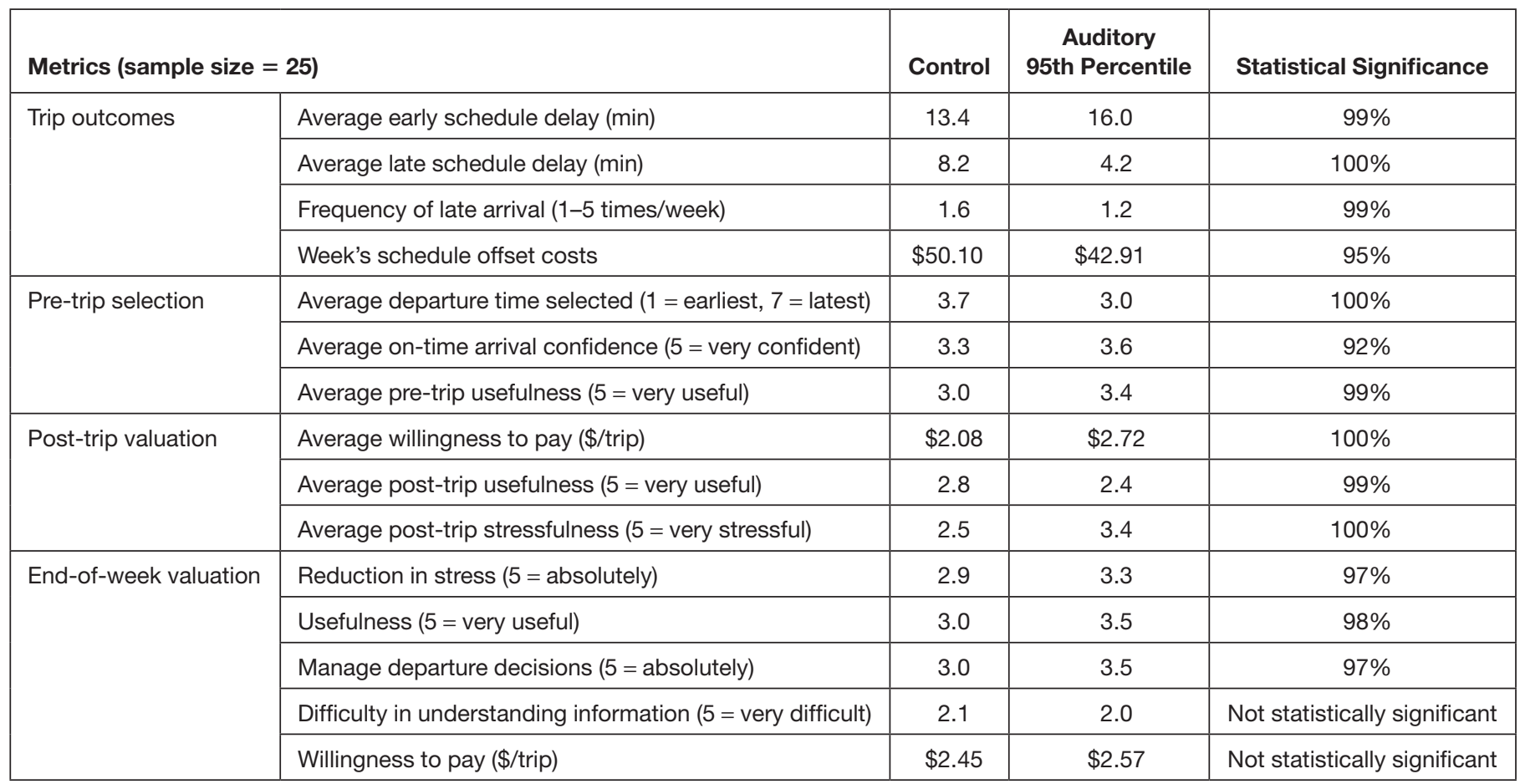

statistically significant. Still, participants' end-of-week ratings regarding pre-trip information reducing stress, being useful, and helping manage departure decisions were higher for type $\mathrm{H}$ compared with the baseline.

An unexpected and conflicting outcome was that participants gave the reliability information a higher usefulness rating pre-trip but a lower rating post-trip compared with the no reliability information scenario. Yet, at the end of the week, they again rated reliability information as more useful. Participants' may have penalized the reliability information for poor trip outcomes while attributing poor outcomes to normal congestion when they did not have reliability data. At the end of the week, they came to recognize through valuation a higher usefulness for reliability information.

When asked how difficult it was to understand the traveler information, participants rated both type $\mathrm{H}$ and type A nearly identically. That is, participants did not find understanding the auditory "majority of the time" reliability information any more difficult than understanding the radio message without reliability information.

\section{Comparison of Equivalent Reliability Information Pairs}

The reliability content presented to participants textually in types B, C, and D was also presented in a second format. Type $\mathrm{B}$ reliability information was also presented in audio format (type $\mathrm{H}$ ), while the information in types $\mathrm{C}$ and $\mathrm{D}$ was presented in graphical formats (type $\mathrm{F}$ and type $\mathrm{G}$, respectively). These three pairs were compared to examine whether a specific format of delivery resulted in different trip outcomes and information valuations.

The only difference that was both statistically and practically significant when comparing the text-based versus auditory 95th percentile reliability information (types $\mathrm{B}$ and $\mathrm{H}$ ) was that individuals were willing to pay about $\$ 0.21$ more post-trip for the text-based information than the auditory information. This outcome might be attributable to participants who-hoping to complete the experiment faster- preferred the quick read compared with the longer auditory delivery of information. In a real-world environment, individuals would probably be multitasking and might prefer auditory traveler information.

Comparing the graphic and text-based versions of average and 95th percentile reliability data also failed to yield statistically significant valuations and outcomes, with two exceptions. The graphical data correlated with a slightly later departure time and consequently lower magnitudes of early schedule delay compared with the text-based version; yet higher magnitude of late schedule delay for the graphical data did not prove statistically significant. The graphical data were also rated slightly higher for reducing trip stress at the end of the week.

Comparing the graphical and text-based versions of 20th percentile, average, and 95th percentile reliability data did yield statistically significant differences in trip departure 
decisions and outcomes. When using graphical information, participants were late more often and had higher magnitudes of late schedule offsets but not higher magnitudes of early schedule offsets. Thus, the week's trip cost was $\$ 7.33$ higher compared to when participants used text-based information. Although the text-based presentation of reliability information (type D) yielded far better trip outcomes compared with the graphical presentation (type $G$ ), differences in willingness to pay for the information were not statistically significant.

The following subsections provide pairwise analyses in the following order:

1. Text-based 95th percentile versus auditory 95th percentile reliability information (type $\mathrm{B}$ versus type $\mathrm{H}$ );

2. Text-based versus graphical average and 95th percentile reliability information (type $\mathrm{C}$ versus type $\mathrm{F}$ ); and

3. Text-based versus graphical 20th percentile, average, and 95th percentile reliability information (type $\mathrm{D}$ versus type $\mathrm{G})$.

\section{Text-Based 95th Versus Auditory 95th Percentile Reliability Information (Type $B$ Versus Type $H$ )}

Among all experiment participants, 25 individuals completed a week (5 days) of simulated commutes to work using the text-based 95th percentile reliability information (type B) and another week (5 days) of simulated commutes using auditory 95th percentile reliability information (type $\mathrm{H}$ ). These simulated commute decisions were pairwise analyzed to evaluate trip outcome and valuations of information. For both weeks, participants also received radio messages pre-trip and viewed dynamic message signs along the trip route. Analysis outcomes are presented in Table 10.12.

The expectation was that reading text-based 95th percentile traveler information and listening to the same information would enable participants to have equivalent trip outcomes. This proved to be true (shown in Table 10.12), as differences in pre-trip selection, trip outcomes, post-trip valuation, and end-of-week valuation were either by and large not statistically significant or significant in magnitude. The magnitudes of early arrival when participants were early did result in a statistically significant difference; however, the difference was less than $2 \%$ and consequently not practically significant.

The only difference that was both statistically and practically significant was that individuals were willing to pay about $\$ 0.21$ more post-trip for the text-based information compared with the auditory information. Although not statistically significant, participants were also willing to pay $\$ 0.27$ more at the end of the week for the text-based information. This was likely because the text could be read far more quickly compared with having

\section{Table 10.12. Trip Decisions, Outcomes, and Valuations of Text-Based Versus Auditory 95th Percentile Reliability Information}

\begin{tabular}{|c|c|c|c|c|}
\hline \multicolumn{2}{|c|}{ Metrics (sample size $=25$ ) } & $\begin{array}{l}\text { Text-Based } \\
\text { 95th Percentile }\end{array}$ & $\begin{array}{c}\text { Auditory } \\
\text { 95th Percentile }\end{array}$ & Statistical Significance \\
\hline \multirow[t]{4}{*}{ Trip outcomes } & Average early schedule delay (min) & 16.6 & 16.9 & $95 \%$ \\
\hline & Average late schedule delay (min) & 5.8 & 4.3 & Not statistically significant \\
\hline & Frequency of late arrival (1-5 times/week) & 1.2 & 1.1 & Not statistically significant \\
\hline & Week's schedule offset costs & $\$ 43.72$ & $\$ 43.26$ & Not statistically significant \\
\hline \multirow[t]{3}{*}{ Pre-trip selection } & $\begin{array}{l}\text { Average departure time selected }(1=\text { earliest, } \\
7=\text { latest })\end{array}$ & 2.9 & 2.9 & Not statistically significant \\
\hline & $\begin{array}{l}\text { Average on-time arrival confidence }(1=\text { not, } \\
5=\text { very) }\end{array}$ & 3.6 & 3.6 & Not statistically significant \\
\hline & Average pre-trip usefulness ( $1=$ not, $5=$ very) & 3.3 & 3.5 & Not statistically significant \\
\hline \multirow[t]{3}{*}{ Post-trip valuation } & Average willingness to pay (\$/trip) & $\$ 2.76$ & $\$ 2.55$ & $95 \%$ \\
\hline & Average post-trip usefulness & 2.5 & 2.5 & Not statistically significant \\
\hline & Average post-trip stressfulness & 3.0 & 3.4 & Not statistically significant \\
\hline \multirow[t]{5}{*}{ End-of-week valuation } & Reduction in stress ( $5=$ absolutely) & 3.1 & 3.2 & Not statistically significant \\
\hline & Usefulness (5 = very useful) & 3.2 & 3.5 & Not statistically significant \\
\hline & Manage departure decisions ( $5=$ absolutely) & 3.4 & 3.5 & Not statistically significant \\
\hline & $\begin{array}{l}\text { Difficulty in understanding information } \\
\text { ( } 5=\text { very difficult })\end{array}$ & 2.4 & 2.0 & $92 \%$ \\
\hline & Willingness to pay (\$/trip) & $\$ 2.68$ & $\$ 2.41$ & Not statistically significant \\
\hline
\end{tabular}


to listen to the information in the experiment. Participants wanted to finish the experiment as quickly as possible and wanted to minimize the time required to acquire the information provided. In real-world situations, the auditory delivery could prove more valuable, as measured by willingness to pay, given that individuals are likely to be multitasking when listening to traveler information.

\section{Text-Based Versus Graphical Average and 95th Percentile Reliability Information (Type C Versus Type F)}

Among all experiment participants, 54 individuals completed a week (5 days) of simulated commutes to work using the text-based average and 95th percentile reliability information (type C) and another week (5 days) of simulated commutes using the graphical display of this reliability information (type F). The simulated commute decisions were pairwise analyzed to evaluate trip outcome and valuations of information. For both weeks, participants also received radio messages pre-trip and viewed dynamic message signs along the trip route. Analysis outcomes are presented in Table 10.13.

Reading text-based reliability information or interpreting this information through a graphical presentation should have enabled participants to have equivalent trip outcomes. However, this was predicated on the expectation that individuals would be able to correctly interpret information independent of delivery media, which often was not the case.
Participants, when using the text-based information, chose to depart on average slightly earlier (on average 2 min earlier), and consequently arrived on average $3.1 \mathrm{~min}$ earlier compared with when they had access to the graphical form of the same information. Further, when using the graphical format, participants on average indicated a higher stress reduction compared with the text-based information (3.8 versus 3.4) when asked at the end of the week. These differences were statistically significant.

Differences in trip outcomes as well as pre- and post-trip valuations for all other metrics were not statistically significant. Although differences did not prove statistically significant, average participant ratings for post-trip and end-of-week information usefulness were slightly higher for the graphical presentation of information.

\section{Text-Based Versus Graphical 20th Percentile, Average, and 95th Percentile Reliability Information (Type D Versus Type G)}

Among all experiment participants, 62 individuals completed a week (5 days) of simulated commutes to work using the textbased 20th percentile, average, and 95th percentile reliability information (type D) and another week (5 days) of simulated commutes using the graphical display of the same reliability information (type G). These simulated commute decisions were pairwise analyzed to evaluate trip outcome and valuations of information. For both weeks, participants also received radio

Table 10.13. Trip Decisions, Outcomes, and Valuations of Text-Based Versus Graphical Average and 95th Percentile Reliability Information

\begin{tabular}{|c|c|c|c|c|}
\hline \multicolumn{2}{|c|}{ Metrics (sample size $=54$ ) } & \multirow{2}{*}{$\begin{array}{c}\text { Text-Based } \\
16.8\end{array}$} & \multirow{2}{*}{$\begin{array}{c}\text { Graphical } \\
13.7 \\
\end{array}$} & \multirow{2}{*}{$\begin{array}{c}\text { Statistical Significance } \\
100 \%\end{array}$} \\
\hline Trip outcomes & Average early schedule delay (min) & & & \\
\hline & Average late schedule delay (min) & 5.3 & 5.9 & Not statistically significant \\
\hline & Frequency of late arrival (1-5 times/week) & 1.2 & 1.2 & Not statistically significant \\
\hline & Week's schedule offset costs & $\$ 44.43$ & $\$ 41.63$ & Not statistically significant \\
\hline \multirow[t]{3}{*}{ Pre-trip selection } & Average departure time selected ( $1=$ earliest, $7=$ latest) & 3.1 & 3.5 & $97 \%$ \\
\hline & Average on-time arrival confidence ( $1=$ not, $5=$ very $)$ & 3.6 & 3.6 & Not statistically significant \\
\hline & Average pre-trip usefulness ( $1=$ not, $5=$ very) & 3.6 & 3.6 & Not statistically significant \\
\hline \multirow[t]{3}{*}{ Post-trip valuation } & Average willingness to pay (\$/trip) & $\$ 2.57$ & $\$ 2.73$ & Not statistically significant \\
\hline & Average post-trip usefulness & 2.5 & 2.6 & Not statistically significant \\
\hline & Average post-trip stressfulness & 3.5 & 3.7 & Not statistically significant \\
\hline \multirow[t]{5}{*}{ End-of-week valuation } & Reduction in stress ( 5 = absolutely) & 3.4 & 3.8 & $99 \%$ \\
\hline & Usefulness ( 5 = very useful) & 3.8 & 4.0 & Not statistically significant \\
\hline & Manage departure decisions ( $5=$ absolutely $)$ & 3.7 & 4.0 & Not statistically significant \\
\hline & Difficulty in understanding information ( $5=$ very difficult) & 2.2 & 2.0 & Not statistically significant \\
\hline & Willingness to pay (\$/trip) & $\$ 2.78$ & $\$ 2.70$ & Not statistically significant \\
\hline
\end{tabular}


messages pre-trip and viewed dynamic message signs along the trip route. Analysis outcomes are presented in Table 10.14.

The hypothesis was that reading text-based reliability information or interpreting this information through a graphical presentation would enable participants to have equivalent trip outcomes. This was predicated on the expectation that individuals would be able to correctly interpret information independent of delivery media, which proved not to be the case with reliability data type $G$ presented graphically.

Individuals rated the text-based information as more effective in managing departure decisions compared with the same information presented graphically (3.8 versus 3.3 ). Further, individuals, when given the text-based information, had less frequent late arrivals and lower magnitudes of late schedule delay compared with when they were given the information graphically. The overall week's schedule offset cost for graphical information was $\$ 7.30$ (17\%) higher than the same information presented textually.

Pre-trip on-time arrival confidence and information usefulness ratings did not exhibit any statistically significant difference. Post-trip, individuals rated the usefulness of the graphical reliability information higher (2.8 versus 2.6$)$ than the text-based reliability information. At the end of each week, the only statistically significant difference in valuation was that the text-based information was rated as more effective in reducing stress and managing departure decisions; no statistically significant difference appeared in participants' willingness to pay for either form of reliability information.

\section{Experiment 2 Findings: Reliability Data Expedites Learning Curve for Trip Familiarity}

The objective of experiment 2 was to determine whether having reliability information helped speed the transition from an unfamiliar traveler to an experienced commuter. The underlying conjecture was that providing reliability information allows an unfamiliar traveler to manage the trade-off between on-time performance and travel budget (time allocated to travel) more effectively and potentially with less stress. Put another way, a priori, the provision of reliability information to travelers unfamiliar with underlying travel time variability patterns was expected to improve overall trip outcomes (reduction of early and late schedule delay, and better on-time performance) and reduce perceptional disutility associated with those outcomes. Additionally, the benefits of reliability information was expected to decline over time as both experimental and control subjects learned and internalized an understanding of underlying travel time variability. Thus, the benefit from reliability information in the first weeks would be larger than in the last weeks.

A total of 32 individuals completed experiment 2 without reliability information (type A), and another 30 individuals completed the experiment with graphical reliability information that provided 20th percentile, average, and 95th percentile

Table 10.14. Trip Decisions, Outcomes, and Valuations of Text-Based Versus Graphical 20th Percentile, Average, and 95th Percentile Reliability Information

\begin{tabular}{|c|c|c|c|c|}
\hline \multicolumn{2}{|c|}{ Metrics (sample size $=62$ ) } & \multirow{2}{*}{$\begin{array}{c}\text { Text-Based } \\
14.0\end{array}$} & \multirow{2}{*}{$\begin{array}{c}\text { Graphical } \\
14.4\end{array}$} & \multirow{2}{*}{$\begin{array}{l}\text { Statistical Significance } \\
\text { Not statistically significant }\end{array}$} \\
\hline Trip outcomes & Average early schedule delay (min) & & & \\
\hline & Average late schedule delay (min) & 6.1 & 9.4 & $100 \%$ \\
\hline & Frequency of late arrival (1-5 times/week) & 1.3 & 1.6 & $99 \%$ \\
\hline & Week's schedule offset costs & $\$ 43.52$ & $\$ 50.85$ & $99 \%$ \\
\hline \multirow[t]{3}{*}{ Pre-trip selection } & Average departure time selected ( $1=$ earliest, $7=$ latest) & 3.5 & 3.8 & $98 \%$ \\
\hline & Average on-time arrival confidence ( $5=$ very confident) & 3.5 & 3.4 & Not statistically significant \\
\hline & Average pre-trip usefulness ( $5=$ very useful) & 3.4 & 3.4 & Not statistically significant \\
\hline \multirow[t]{3}{*}{ Post-trip valuation } & Average willingness to pay (\$/trip) & $\$ 2.37$ & $\$ 2.44$ & Not statistically significant \\
\hline & Average post-trip usefulness ( $5=$ very useful) & 2.6 & 2.8 & $97 \%$ \\
\hline & Average post-trip stressfulness ( $5=$ very stressful) & 3.4 & 3.2 & Not statistically significant \\
\hline \multirow[t]{5}{*}{ End-of-week valuation } & Reduction in stress ( $5=$ absolutely) & 3.6 & 3.2 & $99 \%$ \\
\hline & Usefulness ( 5 = very useful) & 3.6 & 3.4 & Not statistically significant \\
\hline & Manage departure decisions ( $5=$ absolutely) & 3.8 & 3.3 & $100 \%$ \\
\hline & Difficulty in understanding information ( $5=$ very difficult) & 2.1 & 2.3 & Not statistically significant \\
\hline & Willingness to pay (\$/trip) & $\$ 2.40$ & $\$ 2.35$ & Not statistically significant \\
\hline
\end{tabular}


travel times (type G) categorized as "good, typical, and bad." Both types $A$ and $G$ received the same radio message as text and had the same departure time options with the same potential travel time experiences. The same set of data was presented from week to week with one good traffic day, two typical traffic days, and two bad traffic days. The order of these days was different from week 1 through week 4 but was consistent from participant to participant. In effect, the same level of trip variability occurred from week to week.

The four trip outcome metrics are summarized in the grouped chart presented in Figure 10.5. Outcomes included weekly averages for magnitude of early schedule delay when early, magnitude of late schedule delay when late, frequency of late arrival, and total schedule offset cost for the week's commute. Given the smaller and nonpairwise sample size, the differences from week to week were statistically significant between week 1 and week 2 and to a lesser extent from week 2 to week 3 with regard to schedule offset costs and frequency of late arrival. Likewise, differences from week to week were statistically significant from week 1 to week 2 for pre-trip usefulness. Other differences from week to week were not statistically significant but were practically significant.

Providing reliability information allows unfamiliar travelers to manage the trade-off between on-time performance and travel budget (time allocated to travel) more effectively. Overall outcomes in terms of reduction of early and late schedule delay and lower frequencies of late arrival were observed week after week for participants using type $G$ information compared with participants who were not provided reliability information. Furthermore, trip outcome benefits from reliability information in the first weeks were larger than in the last weeks. The benefits of reliability information did decline from the 1st week to the $3 \mathrm{rd}$ week, while benefits from the 3 rd week to the 4 th week were relatively stable.

Whereas trip outcome benefits from provision of reliability information to unfamiliar travelers are generally clear, this information does not always translate to reduced perceptional disutility associated with those outcomes. Figure 10.6 summarizes the pre-trip on-time arrival confidence, posttrip valuation of stress and information usefulness, and average willingness to pay for reliability information. Pre-trip, participants with reliability information expressed higher on-time arrival confidence from week to week. Surprisingly, post-trip, participants with reliability information rated trip usefulness nearly equivalently to participants without reliability information. Even more, participants assigned higher trip stress values and lower willingness to pay for trips made with reliability information.
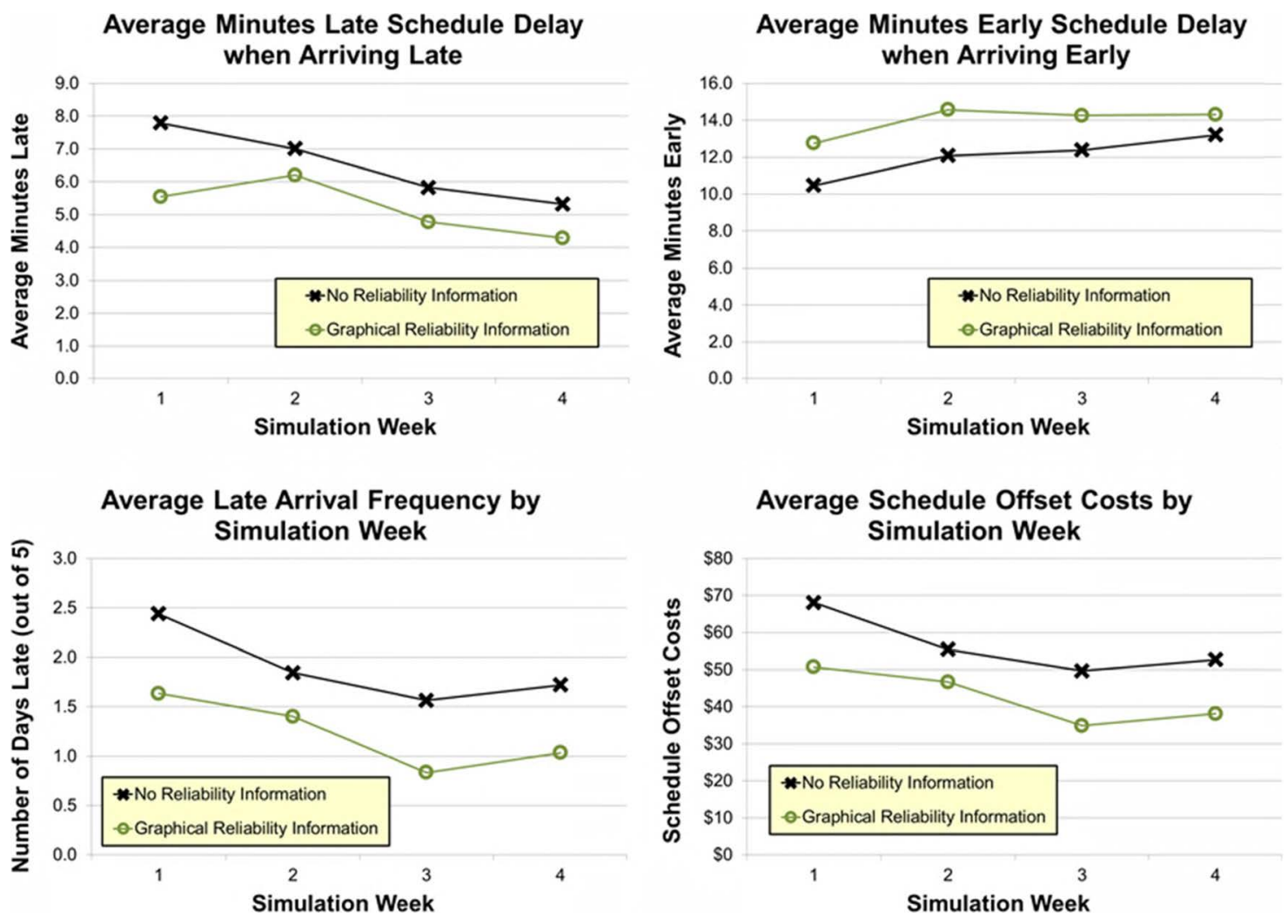

Figure 10.5. Changes in schedule offset from week 1 through week 4. 


\section{Post-Experiment Survey Findings}

Participants who completed experiment 1 involving multiple forms of reliability information completed a post-experiment survey within the Excel-based environment. The survey asked participants to use a seven-point scale to rate the complexity and usefulness of the four types of reliability information in the simulation, paying attention to the relative ratings among the information types. This was the first opportunity for participants in experiment 1 to view the four sets of reliability information together on one screen. Findings from this postexperiment survey are presented in the first subsection below.

Additionally, individuals recruited in Chicago and Houston were asked to complete a paper-based survey requesting that they rank order the six visual forms of information (audio excluded). They were also asked to rate three additional factors:

- The complexity of the information presented (same as experiment 1 post-survey);

- The individual's ease of understanding the information presented; and

- The usefulness of reliability information specific to unfamiliar trips.
The second factor focused on the individual, while the first factor focused on the general. Individuals were expected to rate their ease of understanding information more positively, even when rating the complexity of the information as high. The third factor focused on the usefulness beyond the work trips in experiment 1 to all trips for which individuals were unfamiliar with the underlying trip variability. Findings from this Chicago-Houston post-experiment survey are presented in the second subsection below.

\section{Findings from Experiment 1 Post-Survey: Rating of Reliability Form Complexity and Usefulness}

Figure 10.7 summarizes the outcome of the post-experiment survey. The figure shows the average ratings given for usefulness (left axis, outer bars) and information complexity (right axis, inner bars). Note that, for all types of information, the usefulness rating is greater than the complexity rating. The greater the difference between the two bars, the more effective the information. According to the post-experiment survey findings, the 95th and average graphical information was most effective (2.2 rating difference between usefulness and complexity), followed by audio 95th percentile reliability information (2.1 rating difference) and text-based 95th percentile

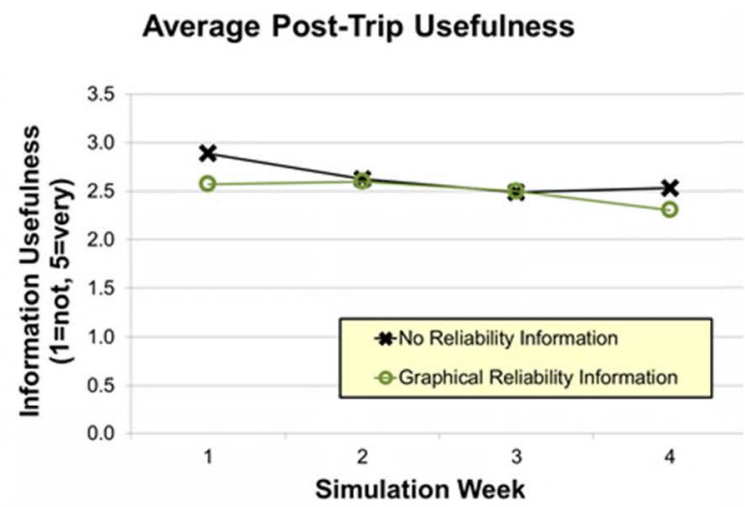

Average Willingness to Pay Per Trip

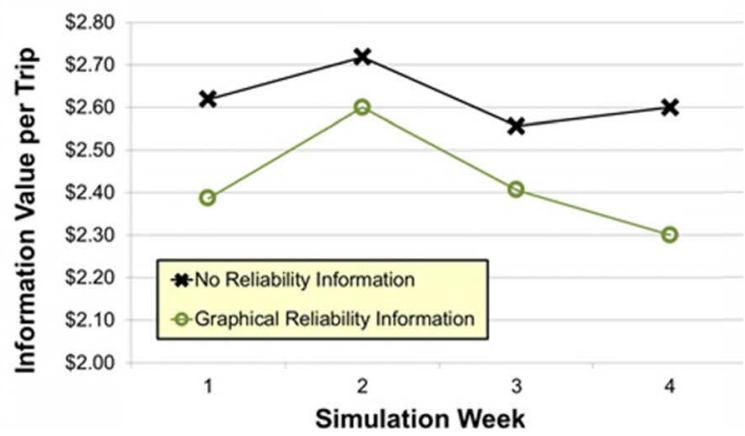

Figure 10.6. Changes in trip valuations from week 1 through week 4. 


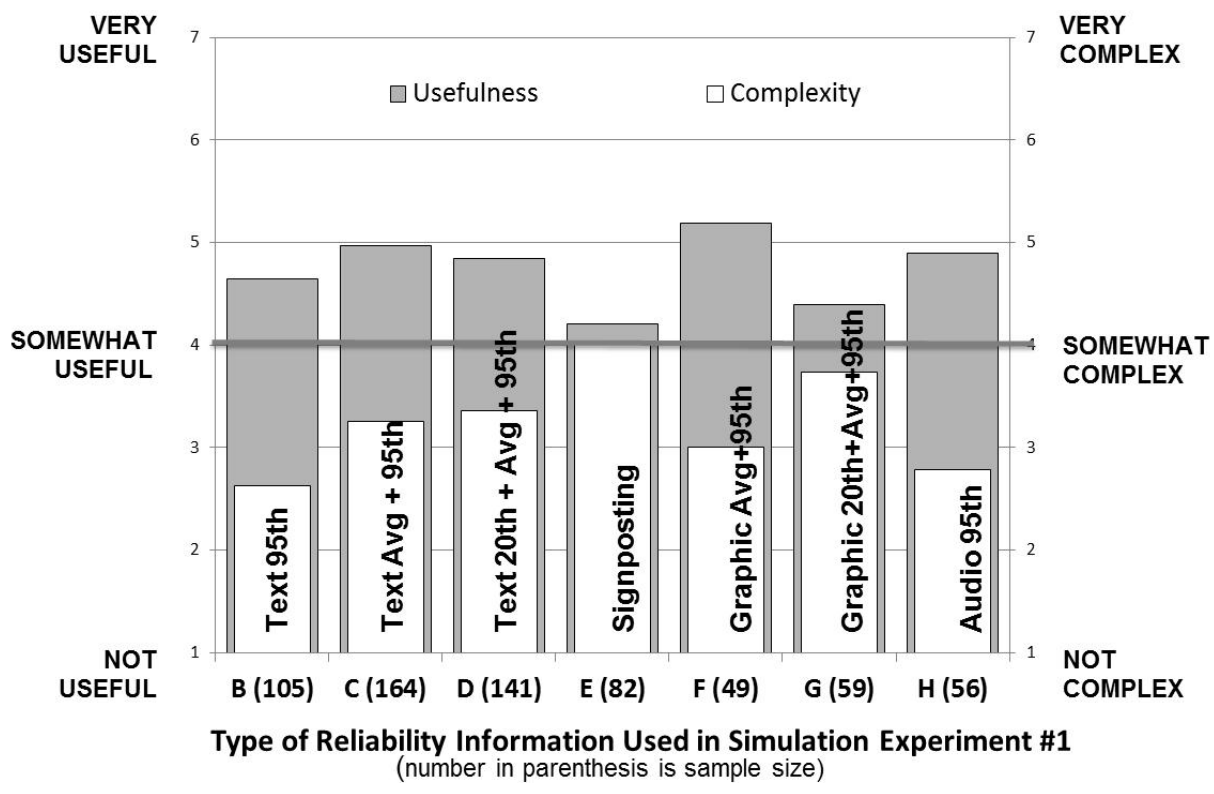

Figure 10.7. Average complexity and usefulness ratings of reliability terms in experiment 1.

reliability information (2.0 rating difference). These findings paralleled observations from experiment 1 individual ratings.

The reliability signposting and the graphical presentation of good-, typical-, and bad-day reliability information were the two forms of information with the highest complexity ratings and lowest usefulness ratings. This paralleled observations from the experiment 1 individual ratings. Consequently, although the ratings were based on only limited exposure (five trips) to each information form, these two delivery forms for reliability information were least recommended.

\section{Findings from Chicago-Houston Post-Experiment Survey}

Of the 183 participants in the Chicago and Houston experiments, seven did not complete the paper survey. Of the 176 remaining individuals who did complete the paper survey, 122 participants completed experiment 1,42 participants completed experiment 2 , and 12 participants were excluded from experiment analyses because they did not provide complete or realistic responses within experiment 1 or 2 .

The overall ranking of the six types of reliability information with regard to information usefulness and complexity is presented in Figure 10.8 and Figure 10.9, respectively. The relative ranking for information types clearly indicates that the text-based average and 95th percentile reliability information ranked highest for usefulness among terms, followed by the text-based 20th percentile, average, and 95th percentile reliability terms. Third was the graphical average and 95th percentile reliability terms. This ranking of usefulness differed from the ratings summarized earlier: many individuals did not in fact use the reliability terms but did rank them, which accounts for the difference. Further, this ranking was for all unfamiliar trips compared with specifically work-trip ratings in Table 10.10. Nonetheless, the top three reliability terms were the same in both surveys (excluding audio).

What was surprising was the bipolar response to the more complex good, typical, and bad graphical term. Nearly $25 \%$ of participants ranked this reliability term first (most useful), while $40 \%$ ranked this term sixth (least useful). Response rates were the same for experiment 1 users and experiment 2 users for this reliability term. Clearly, people either strongly preferred or strongly disliked the three-level graphical form.

Rankings of reliability terms based on information complexity yielded similar results to rankings based on usefulness, as shown in Figure 10.10. The least complex forms of information were the text-based average and 95th percentile information along with the text-based 20th percentile, average, and 95th percentile information. The most complex forms of information included the graphic with three levels of travel time and the signposting concept.

Overall ratings for reliability term complexity confirmed that the graphical three-tier form of reliability information and reliability signposting were selected on average as most complex, lowest ease in understanding, and lowest usefulness. Conversely, the graphical and text-based average and 95th percentile 


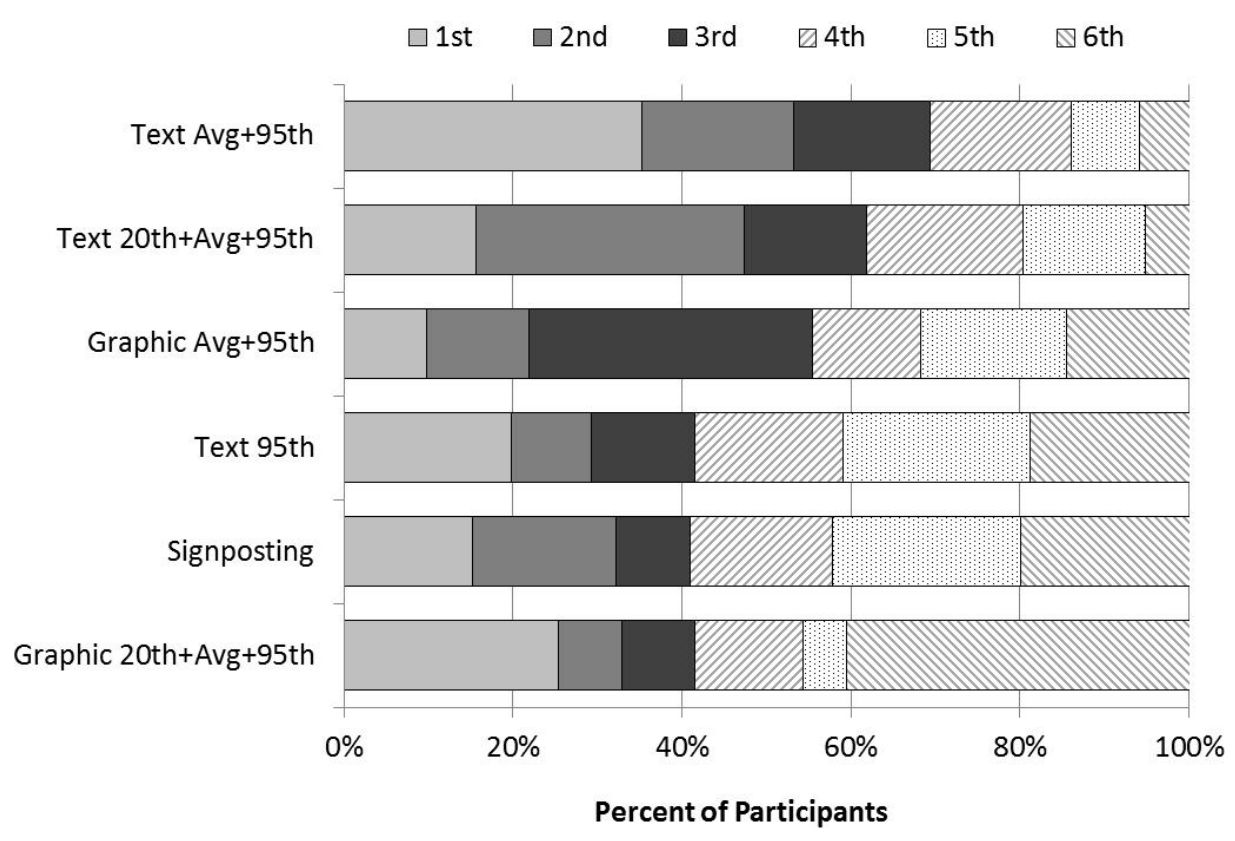

Figure 10.8. Rank ordering of reliability terms based on information usefulness. (1st $=$ most useful)

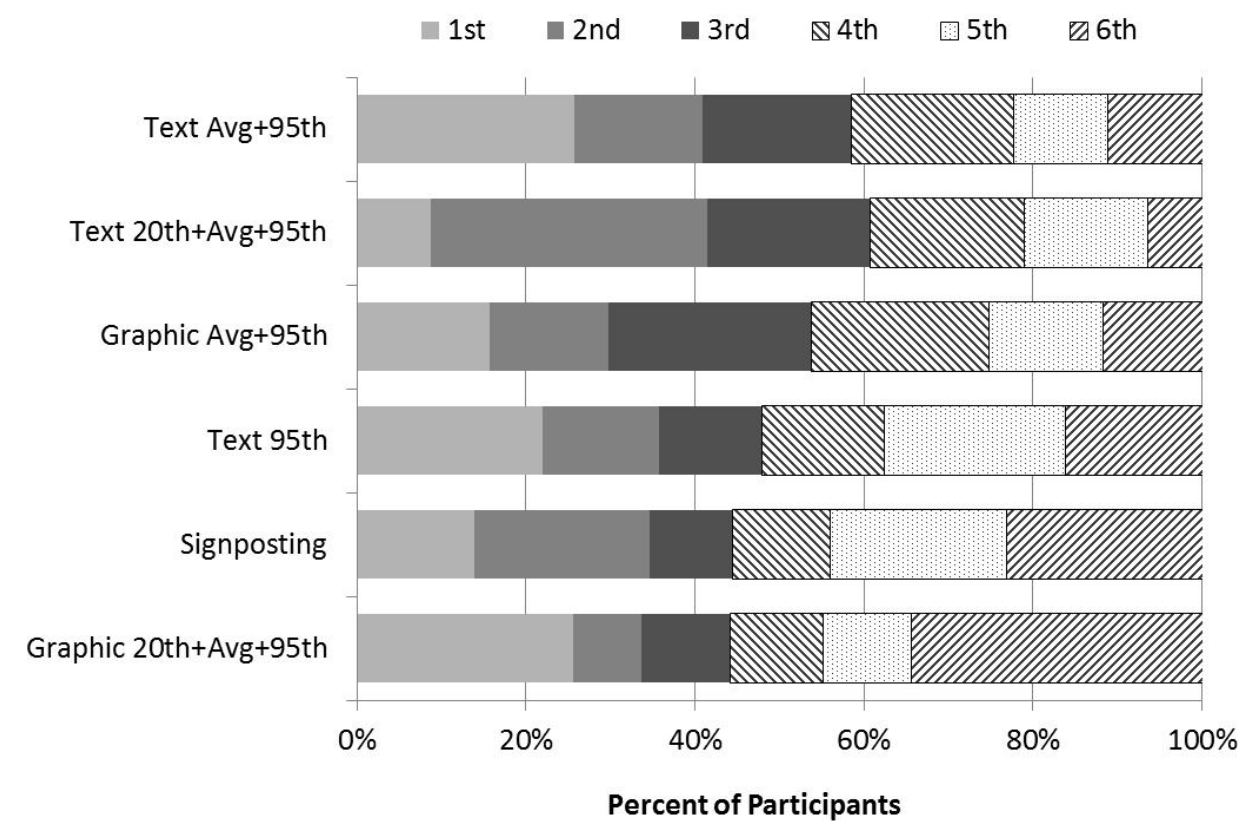

Figure 10.9. Rank ordering of reliability terms based on information complexity. (1st = least complex) 


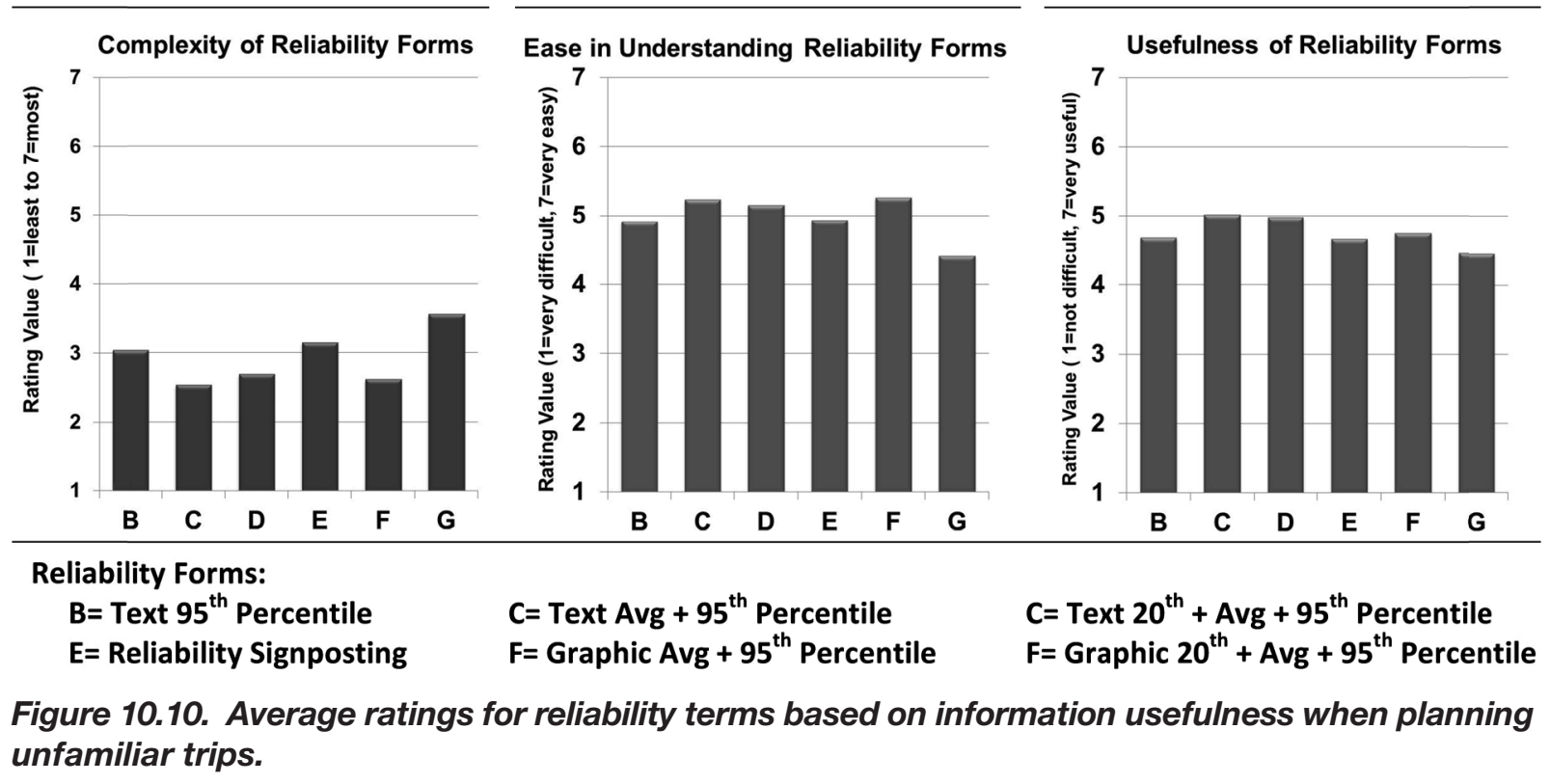

reliability forms were least complex and easiest to understand. Figure 10.10 summarizes the average ratings for perceived complexity, understandability, and usefulness of reliability terms.

\section{Conclusions and Looking Forward}

In this enhanced laboratory experiment, researchers conducted two sets of experiments across three cities involving approximately 240 individuals. The plan was to quantitatively assess the effectiveness of specific reliability terms in improving trip outcomes and to identify whether the valuation and perceived usefulness suggested by participants correlated with their quantitative trip outcomes. Travel time valuations traditionally are based on experiments in which participants make trade-offs between money, time, and other factors. The goal of these two experiments was to determine the relative revealed performance and stated preferences for different types of reliability information. In addition, the findings provide quantitative estimates of the value of reliability information in reducing the frequency of late arrivals and identify a greater willingness to pay for information of some types.

In the experiments, the value of reliability information to drivers was based on trip cost as predefined in each experiment, including costs of early and late arrival. The overall trip cost was noted as significantly higher for those without reliability information. The value of knowing one will be late for a trip (serenity benefit) was determined on the basis of participants' willingness to pay for information at the end of a trip.
Researchers developed three hypotheses, and findings related to each of these hypotheses are described below.

Hypothesis 1 stated that provision of accurate reliability information (in an easy-to-understand format) would result in improved on-time performance and lower generalized travel disutility compared with a control group receiving no reliability information. Hypothesis 1 was strongly supported. Of the seven different forms of delivery of reliability information tested, users presented with five of the options demonstrated statistically significant reductions in weekly schedule offset costs compared with the control group receiving no reliability information. These five were also the simplest of the forms of reliability information, focusing on average and 95th percentile travel time values, delivered in various forms. Participants receiving these simple forms of reliability information reduced schedule offset costs by $9 \%$ to $21 \%$ compared with the control group.

Hypothesis 1a posited that while travel outcomes improve with the provision of reliability information, participants' perceived value of the reliability information would underestimate the realized benefit in terms of reduced delay, improved on-time reliability, and reduced stress. Hypothesis la was strongly supported. For each of the simple forms of reliability information tested, improvements in trip outcomes were clear and statistically significant. For example, frequency of late arrivals declined $16 \%$ to $40 \%$ when participants received reliability information in these forms compared with when they did not receive reliability information. Reduction in stress reported at the end of each week was also statistically significant, in a similar range from $10 \%$ to $31 \%$. However, participant willingness to pay for reliability information compared 
with willingness to pay for baseline (real-time) information was often not statistically significant. For example, participants receiving the simple text +95 th percentile reliability information reduced late arrivals by $40 \%$ and reported a $10 \%$ reduction in stress. However, the same participants were willing to pay on average only $\$ 0.10$ more for reliability information ( $\$ 2.78$ versus $\$ 2.68$ per trip), a difference too small to be statistically significant.

Hypothesis $1 \mathrm{~b}$ stated that provision of reliability information using different text-based, graphical, and auditory forms would result in differences in both accrued on-time reliability benefits as well as perceived benefits. These differences among experimental groups were expected to be smaller than between any group and the control (no reliability information) group. Hypothesis $1 \mathrm{~b}$ was supported. Provision of simple forms of reliability information had similar results whether provided in text-based, graphical, or auditory form. The more complex graphical and signposting concepts were not effective.

Hypothesis 2 proposed that experimental subjects receiving contextual information on underlying variation with numeric indicators reinforced with en route information (reliability signposting) would have improved on-time performance compared with both an experimental group that received reliability information but no contextual information as well as a control group that received no reliability information. Hypothesis 2 was not supported. The signposting concept was not successful for participants in the management of trip outcomes and stress reduction. To some degree, this was because of the complexity of the presentation and the brevity with which participants were required to learn and interpret information content. Signposting may still be a valuable concept to pursue in reliability information provision, but work remains on how to make this delivery method more accessible.

Hypothesis 3 posited that the benefits of reliability information would decline over time as both experimental and control subjects learned and understood the underlying travel time variability. That is, the benefit from reliability information during the first weeks would be larger than during the last weeks. Hypothesis 3 was supported. Participants using reliability information were equally effective in managing trip outcomes (late arrivals, schedule delays, and offset costs) in the first week of exposure to unfamiliar travel time variability patterns as their counterparts without reliability information were after 4 weeks. Within the 4 -week constraints of the experiment, both reliability information users and control group counterparts reduced offset costs through week three, at which time costs leveled off. That said, the difference in realized offset costs between the two groups was still significant even in week three and week four, roughly $25 \%$ ( $\$ 40$ versus $\$ 50$ ). This finding implies that reliability information still has value at 4 weeks of experience, and presumably may still have value longer than 4 weeks since the gap in performance between week one and week four between the two groups narrowed only from $40 \%$ to $25 \%$. 


\section{Lexicon Development}

The measurement of travel time reliability is an emerging practice. However, a few measures appear to have technical merit and are easily understood by nontechnical audiences. Most of these measures compare days with high travel times with days with average travel times. Four recommended measures are as follows:

- 90th or 95th percentile travel time;

- Buffer index;

- Planning time index; and

- Frequency with which congestion exceeds some expected threshold (Texas A\&M Transportation Institute with Cambridge Systematics, Inc. 2006).

\section{Frequently Used Terms}

The 90th or 95th percentile travel time is a time identified for a specific travel route that indicates how bad delay will be on the heaviest travel days (Texas A\&M Transportation Institute with Cambridge Systematics, Inc. 2006). These travel times are reported in minutes and seconds and are thought to be easily understood by commuters familiar with their trips. For this reason, this measure is ideally suited for traveler information. This measure has the disadvantage of not being easily compared across trips, as most trips will have different lengths. Nor can it be easily used to combine route or trip travel times into a subarea or citywide average. Several reliability indices are presented below that enable comparisons or combinations of routes or trips with different lengths.

The buffer index represents the extra time cushion (or buffer) that most travelers add to their average travel time when planning trips to account for unforeseen delays and to ensure on-time arrival (Texas A\&M Transportation Institute with Cambridge Systematics, Inc. 2006). The buffer index is expressed as a percentage, and its value increases as reliability gets worse. For example, a buffer index of $40 \%$ means that for a 20-min average travel time, a traveler should budget an additional $8 \mathrm{~min}(20 \mathrm{~min} \times 40 \%=8 \mathrm{~min})$ to ensure on-time arrival most of the time. In this example, the eight extra minutes is called the buffer time. The buffer index is computed as the difference between the 95th percentile travel time and average travel time, divided by the average travel time.

The planning time index represents the total travel time that a traveler should expect or plan on when an adequate buffer time is included (Texas A\&M Transportation Institute with Cambridge Systematics, Inc. 2006). The planning time index differs from the buffer index in that it includes typical delay as well as unexpected delay. Thus, the planning time index compares near-worst-case travel time to a travel time in light or freeflow traffic. For example, a planning time index of 1.60 means that for a 15-min trip in light traffic, the total time that should be planned for the trip is $24 \mathrm{~min}(15 \mathrm{~min} \times 1.60=24 \mathrm{~min})$. The planning time index is useful because it can be directly compared with the travel time index (a measure of average congestion) on similar numeric scales. The planning time index is computed as the 95 th percentile travel time divided by the free-flow travel time.

From a data perspective, using continuous travel time data is the only way to establish reliability patterns empirically. Although predictive methods-such as the ones being developed by the project team for the SHRP 2 L03 project titled Analytic Procedures for Determining the Impacts of Reliability Mitigation Strategies-may be used in a reliability monitoring system when these data are unavailable, only continuously collected travel time data can produce the actual travel time distribution from which all reliability metrics are derived. For example, the reliability metrics being used in the SHRP 2 L03 project are all derivatives of the travel time distribution.

What is clear is the lack of agreement within the professional field on the terms to be used or what the mathematical calculations for each of the terms should be. If the professionals cannot reach consensus on the technical terms, then the general public certainly will not do so. The purpose of the L14 project was to discover what terms the layperson would use to refer to travel 
time reliability concepts and to encourage the use of those terms in communications with transportation system users.

\section{Terminology Assessment}

The most basic considerations for trip reliability information relate to the points during a trip when reliability information should be provided, the content of the reliability information to be provided, and how content differs as a trip is made from planning and origin to ultimate destination. Another consideration is how reliability information needs differ for travelers with familiarity and experience making a recurrent trip compared with travelers making a trip without the benefit of dayto-day experience of the trip's reliability. Likewise, how might transmission media and message content differ according to the needs of different driver types and trip purposes (e.g., older drivers or newer drivers, commercial vehicle operators or carpool organizers)? Furthermore, what innovations can assist in efficiently meeting these varying needs?

The literature review, expert interviews, and technology scan completed in Phase 1 of the project identified the reliability terms that the transportation profession uses to describe the travel time reliability of a transportation system. The initial list, shown in Table 11.1, was drawn primarily from the FHWA Travel Time Reliability information brochure (Texas A\&M Transportation Institute with Cambridge Systematics, Inc. 2006) and the TTI Urban Mobility Report (Schrank et al. 2011). The list also includes user interface terms identified through the review of traveler information websites conducted in preparation for the surveys. The human factor studies (focus group discussions, a computer-based multiple-choice survey, an openended survey, the initial travel behavior laboratory study, and the enhanced laboratory study) were intended to discover what terms the layperson would use and understand to refer to travel time reliability concepts, to determine to what extent travel time reliability information would inform travel decisions, and to ascertain the value of this information to system users.

Terms for some of the listed parameters were not tested in the human factor studies: (a) terms that have few or no logical alternatives and are considered by the research team to be words or phrases that laypeople would readily recognize, (b) terms pertaining to reliability measures that would be unlikely to be used

\section{Table 11.1. Proposed Travel Time Terms and Concepts to Be Included in Lexicon}

\begin{tabular}{|c|c|}
\hline Technical Term & Technical Definition \\
\hline 95th percentile & $\begin{array}{l}\text { The point on a travel time frequency distribution at which } 95 \% \text { of the trips made would arrive at or before the } \\
\text { identified time }\end{array}$ \\
\hline Arrival time & The time at which a traveler would arrive after a trip \\
\hline Average travel time & $\begin{array}{l}\text { An average of all travel times calculated over a specified time interval for a specified trip or roadway segment } \\
\text { (The period of time over which the average is calculated is not consistent within the profession.) }\end{array}$ \\
\hline Buffer index & $\begin{array}{l}\text { A multiplier that represents the extra time or time cushion a traveler must add to his or her average travel } \\
\text { time when planning trips to ensure on-time arrival }\end{array}$ \\
\hline Buffer time & The average travel time multiplied by the buffer index \\
\hline Delay time & The amount of extra time spent traveling due to congestion \\
\hline Departure time & The time at which a traveler would depart for a trip \\
\hline Free-flow travel time & Travel time for a trip under free-flow conditions (level of service A) \\
\hline Peak travel time & The free-flow travel time added to the delay time \\
\hline Planning time & The free-flow travel time multiplied by the planning time index \\
\hline Planning time index & A multiplier that represents how much total time a traveler should allow to ensure on-time arrival \\
\hline Recommended departure time & $\begin{array}{l}\text { A time of departure calculated by a traveler information system that would ensure an on-time arrival for a } \\
\text { given level of risk tolerance }\end{array}$ \\
\hline Recommended route & $\begin{array}{l}\text { A route between two points calculated by a traveler information system that would ensure an on-time arrival } \\
\text { for a given level of risk tolerance }\end{array}$ \\
\hline Reliability & Consistency or dependability in travel times, as measured from day to day or across different times of day \\
\hline Total trip time & The total time a trip would take, door to door \\
\hline Travel time index & Peak travel time/free-flow travel time \\
\hline Travel time range & $\begin{array}{l}\text { The range of travel times that can be expected and could be anchored by any two points on the travel time } \\
\text { frequency distribution }\end{array}$ \\
\hline Trend information & An indication that congestion is changing \\
\hline
\end{tabular}


by laypeople (e.g., buffer and travel time indices), or (c) terms that were close parallels to other tested parameters (e.g., planning time, which is similar in output to 95th percentile trip time). The following sections describe the terminology tested in the various human factor studies and results that influenced the development of the lexicon.

\section{5th Percentile Travel Time}

The 95th percentile travel time is a time identified for a specific travel route that indicates how long a given trip could take on the heaviest travel days (Texas A\&M Transportation Institute with Cambridge Systematics, Inc. 2006). The following terms for communicating 95th percentile travel times were discussed in the focus groups and/or tested in one or both of the surveys:

- 95th percentile trip time;

- Majority of the time;

- Most of the time;

- Travel time for planning;

- Maximum trip time;

- Worst-case trip time; and

- $X$ out of $Y$ days (e.g., 19 out of 20 days).

Of these terms, maximum trip time and worst-case trip time were not tested because of potential credibility concerns on the part of a public agency. The phrase 19 out of 20 days was not tested in surveys but is a probability expression that has been shown in the literature to be more readily understood by the general population than percentages or percentiles.

Majority of the time, used as part of the sentence "The majority of the time your trip will take $X X$ minutes or less," was most likely to be interpreted correctly by participants as representing a trip time that would apply to unusually heavy traffic and unusual delays. This phrase was used to describe 95th percentile travel times in the enhanced laboratory study.

The term 95th percentile was not well understood by survey participants, and participants who were presented with a 95th percentile trip time were less confident about arriving on time compared with participants who viewed the same trip time described with other tested terms (e.g., majority of the time, most of the time). Participants viewing 95th percentile trip time were likely to add their own buffer time on top of the total trip time provided.

Most of the time, used as part of the sentence "Most of the time your trip will take _ minutes or less," produced the greatest (expressed) confidence in arriving by the time shown; but participants still tended to add their own buffer time to the time provided.

Participants given a trip time described as travel time for planning were more likely to view that time as a maximum trip time or worst-case scenario rather than the 95th percentile time that was intended.

\section{Arrival Time}

Alternate terms for arrival time-the time that a traveler arrives at his or her destination after a trip-were not tested because the phrase is commonly used. However, phrases that a traveler might use to describe a desired arrival time were presented in the computer survey.

For a scenario in which a traveler would enter a preferred arrival time into a travel time calculator (to receive a recommended departure time), the survey offered the following phrases:

- Arrive by;

- Arrive at;

- What time do you want to get there?

- What's the earliest you can arrive? and

- What's the latest you can arrive?

By a statistically significant margin, the largest percentage of participants preferred arrive by, with arrive at the second most frequently selected option. These responses showed a willingness to accept either an on-time or an early arrival, since by can mean "no later than." The other three phrases were selected much less frequently by participants. The research team concluded from the survey results that arrive by is the best of the tested terms to use to ask for desired arrival time input.

\section{Average Travel Time}

The technical definition of average travel time is an average of all travel times calculated over a specified time interval for a specified trip or roadway segment. (The period of time over which the average is calculated is not consistent within the profession.) Terms to communicate average travel time were discussed in focus groups and tested in both surveys:

- Average travel time;

- Estimated travel time;

- Expected travel time;

- Typical travel time; and

- Historical travel time.

Average, estimated, expected, and typical travel time were all terms that were mentioned by focus group participants. Historical travel time is used by some travel time websites to distinguish an average trip time based on past travel time data. In the open-ended survey, researchers found no clear preference for or effect on comprehension among the terms average, estimated, typical, and expected travel times. However, in 
the computer-based survey, estimated travel time was preferred by the largest number of participants, followed by average travel time. Typical travel time and historical travel time were selected least frequently by participants in the computer-based survey.

Estimated travel time was selected to describe a calculated average travel time in the enhanced laboratory study.

Average travel time was addressed in two additional ways in the focus groups and in the computer survey. The sentence "It will take__ 20 minutes to make your trip" was presented to focus groups to elicit potential terms for describing average trip time. Responses included about, an estimate of, approximately, around, an average of, roughly, give or take, and at least. When tested in the computer-based survey, approximately was preferred by a majority of participants, followed by about, an estimate of, and an average of.

When the sentence "It is ___ that your trip will take $45 \mathrm{~min}$ utes" was completed by focus group participants and in the computer survey, estimated was preferred the highest number of participants, followed by likely and predicted.

\section{Buffer Index}

The buffer index represents the extra time cushion (or buffer) that most travelers add to their average travel time when planning trips to account for unforeseen delays and to ensure on-time arrival (Texas A\&M Transportation Institute with Cambridge Systematics, Inc. 2006). The buffer index is expressed as a percentage, and its value increases as reliability gets worse. For example, a buffer index of $40 \%$ means that for a 20 -min average travel time, a traveler should budget an additional $8 \mathrm{~min}$ ( $20 \mathrm{~min} \times 40 \%=8 \mathrm{~min}$ ) to ensure on-time arrival most of the time. In this example, the eight extra minutes is called the buffer time. The buffer index is computed as the difference between the 95th percentile travel time and average travel time, divided by the average travel time.

Terminology for the buffer index was not tested in the human factor studies, as this is a metric that is unlikely to be used by roadway users.

\section{Buffer Time}

Buffer time is defined as the average travel time multiplied by the buffer index. When speaking about the additional time added to a trip to ensure on-time arrival, focus group participants suggested terms and phrases including additional time, traffic time, leeway, driving time, just-in-case time, fluff time, additional drive time, cushion, allow an additional $X$ minutes for variables, tack on extra, and extra time. Terms that were tested in one or both surveys included the following:

- Added time;

- Buffer time;
- Cushion;

- Departure window;

- Extra time;

- Leeway; and

- Recommended cushion.

Of the tested terms, extra time was preferred by the most participants in the computer survey, followed by departure window; in the open-ended survey, recommended cushion, added time, and extra time all performed well. Buffer time was preferred by the fewest number of participants in the computer survey and so was not tested in the open-ended survey. Despite the popularity of departure window in the computer survey, the research team does not recommend its use as a synonym for buffer time.

Extra time was used to describe buffer time in the travel time information provided to participants in the enhanced laboratory study.

\section{Delay Time}

Terminology for delay time was not tested in the human factor studies; instead, terms were tested for the related concept of buffer time.

\section{Departure Time}

Focus group participants wanted the ability to specify a trip calculation based on time of departure or time of arrival. The computer survey continued investigation on this topic by addressing the preferred terminology to be used for the departure and arrival times. Terms and phrases tested in the survey included the following:

- Departing at.

- Leave at.

- What time will you start your trip?

- Leave by.

- Departing by.

- What's the earliest you can start your trip?

- What's the latest you can start your trip?

Departing at, leave at, and what time will you start your trip? were the top three terms selected by participants, showing a preference for specific departure times versus a range of potential departure times (as could be implied by the other four tested phrases).

\section{Free-Flow Travel Time}

Terminology for free-flow travel time (i.e., travel time for a trip under free-flow conditions) was not tested in the focus 
groups or surveys. In the enhanced laboratory study, one of the graphical travel time information formats included projected trip times on a great day for travel speeds, along with corresponding times for average/typical and bad days. The "great day" trip time was intended to represent free-flow travel time.

\section{Peak Travel Time}

Terminology for peak travel time (free-flow travel time added to delay time) was not tested in the human factor studies. Terms for the similar concept of 95th percentile travel time were tested instead.

\section{Planning Time}

Terminology for planning time (free-flow travel time multiplied by the planning time index) was not tested in the human factor studies. Terms for the similar concept of 95th percentile travel time were tested instead; travel time for planning was one of the alternatives tested to represent 95th percentile travel time.

\section{Planning Time Index}

The planning time index is used to calculate the total travel time that a traveler should expect or plan on when an adequate buffer time is included (Texas A\&M Transportation Institute with Cambridge Systematics, Inc. 2006). The planning time index differs from the buffer index in that it includes typical delay as well as unexpected delay. Thus, the planning time index compares near-worst-case travel time with a travel time in light or free-flow traffic. For example, a planning time index of 1.60 means that for a 15 -min trip in light traffic, the total time that should be planned for the trip is $24 \mathrm{~min}(15 \mathrm{~min} \times 1.60=24 \mathrm{~min})$. The planning time index is computed as the 95th percentile travel time divided by the free-flow travel time.

Terminology for planning time index was not tested in the human factor studies, as this is a metric that is unlikely to be used by roadway users.

\section{Recommended Departure Time}

Recommended departure time is defined as the time of departure calculated by a traveler information system that would ensure an on-time arrival for a given level of risk tolerance. The following terms were tested in the computer-based survey to describe this calculated time of departure:

- Recommended departure time;

- Estimated departure time;

- 95th percentile departure time; and

- Suggested departure time.
Of the tested terms, recommended departure time was preferred most frequently by survey participants, followed by suggested departure time and estimated departure time; 95th percentile departure time was the least preferred.

\section{Recommended Route}

A recommended route in the context of travel time reliability is defined as the route between two points calculated by a traveler information system that would ensure an on-time arrival for a given level of risk tolerance. Terms tested in the computer survey to describe a route provided to a traveler by a traveler information system included the following:

- Best route;

- Forecasted trip;

- Most reliable trip;

- Most predictable trip;

- Most consistent trip;

- Historical trip conditions; and

- Least variable time.

Of the tested terms, the most frequently preferred was best route, followed by forecasted trip and most reliable trip. While the term recommended route was not tested in the surveys, its similarity to participant-preferred terms like recommended departure time and recommended cushion likely indicates that recommended route would also be a strong candidate.

\section{Reliability}

Terms for both reliability and variability were discussed in focus groups and tested in the computer survey. Most often, focus group participants chose general words such as possibly, probably, chance, or likely to describe variability at a certain time of day. Generally, they preferred that those words have a descriptor in front, such as "X\% chance" or "highly likely," to make the term less general. When talking about traffic patterns at a specific time of day, participants used varies, changes, and increases/decreases most often. Focus group participants preferred the terms reliable and consistent when describing the reliability of a roadway or mode.

The computer survey described four different fictional trips that were actually trip times presented in different ways: a typical/average trip time, a maximum trip time, a small trip time range, and a large trip time range. Participants were then asked to select a term that they felt described each of those trip times:

- Predictable;

- Reliable;

- Consistent; and

- Best. 
All four terms were treated similarly by participants: they were selected to describe the typical and maximum trip times much more frequently than to describe either of the trip time ranges.

Terms for trip time variability were also tested in the computer survey, using the sentence "Your trip time may __ from the average trip time by 15 minutes." Response options included the following:

- Vary;

- Differ;

- Fluctuate;

- Change;

- Go up or down;

- Increase or decrease;

- Deviate; and

- Be longer or shorter.

Of these options, survey participants preferred vary most frequently, by far.

\section{Total Trip Time}

Terminology for total trip time was not tested in the human factor studies because the phrase is commonly used and few synonyms exist.

\section{Travel Time Savings}

Terminology for travel time savings was not tested in the human factor studies because the phrase is commonly used and few synonyms exist.

\section{Travel Time Range}

In focus groups, terms used to compete the sentence "It will take __ 10 to 30 minutes to make your trip" were about, approximately, between, around, on average, likely, anywhere from, somewhere between, usually, and ideally.

In the computer survey, two hypothetical trips for which travel time ranges were provided were not as frequently described by participants as reliable, predictable, or consistent compared with trips for which a single (typical/average or 95th percentile) trip time was provided.

Historical travel time information in the first travel behavior laboratory study was presented in the form of trip time ranges.

\section{Trend Information}

Terms for trend information (an indication that congestion is changing) were not tested in the human factor studies. Travel planning websites that were reviewed during focus group preparation and survey development often indicated trend information graphically (if they indicated it at all).

\section{Lexicon Format}

The research team identified several key elements of a lexicon entry that were deemed necessary to completely present each term. The elements are as follows:

- Technical Term - the formal travel time reliability term to be defined;

- Definition-a definition of the term within the reliability framework;

- Usage - a general description of when an agency might use the reliability term or for what purpose it would use the term in the traveler information system; and

- Recommendation - the ranking of the messages and/or terms to be used in order of preference:

○ Best-represents the term(s), phrase(s), and/or format(s) that performed the best in the human factor studies and will most likely yield the desired behavioral results when conveyed to system users;

O Adequate - represents term(s), phrase(s), and/or format(s) that performed reasonably well in the human factor studies and will not likely present significant comprehension problems for system users; and

$\circ$ Avoid_-represents terms(s), phrase(s), and/or format(s) that did not perform well in the human factor studies or are recommended to avoid for noted reasons.

- Alternate Phrase - an alternative term or phrases of different lengths that would work on some technology platforms but not on others; and

- Information Technology Platforms - identification of appropriate media and technology interfaces for each alternative. The list of technology platforms could continue to evolve as new media are introduced. These might include portable navigation devices, Connected Vehicle (formerly IntelliDrive) on-board equipment, and advanced car stereo or satellite radio systems. An initial list is included in Table 11.2 and includes

$\circ$ Web - intended to mean full website format viewed from a full-sized personal computer screen in a full-featured Internet browser.

- Mobile Web —intended to mean a website format viewed from mobile devices such as smartphones and tablet computers.

- Text-including short message service (SMS) text messages and social network text messages, such as Twitter, viewed on a mobile device.

- Mobile Application — specially designed user interfaces optimized to work on a specific smartphone operating 
Table 11.2. Lexicon Format

\begin{tabular}{|c|c|c|c|c|c|c|c|}
\hline Technical Term & \multicolumn{7}{|c|}{ 95th Percentile } \\
\hline Definition & \multicolumn{7}{|c|}{$\begin{array}{l}\text { The point on a travel time frequency distribution at which } 95 \% \text { of the trips made would arrive at or } \\
\text { before the identified time }\end{array}$} \\
\hline Usage & \multicolumn{7}{|c|}{ To describe the longest time a driver can expect a trip to take } \\
\hline & \multirow[b]{2}{*}{$\begin{array}{l}\text { Alternate } \\
\text { Phrase }\end{array}$} & \multirow[b]{2}{*}{$\begin{array}{l}\text { Wording Context/ } \\
\text { Additional Information }\end{array}$} & \multicolumn{5}{|c|}{ Information Technology Platforms } \\
\hline Recommendation & & & Web & $\begin{array}{l}\text { Mobile } \\
\text { Web }\end{array}$ & Text & $\begin{array}{c}\text { Mobile } \\
\text { Application }^{a}\end{array}$ & $\begin{array}{l}\text { Dynamic } \\
\text { Message Sign }\end{array}$ \\
\hline Best & & & $\checkmark$ & $\checkmark$ & $J^{b}$ & $\mathfrak{J}^{b}$ & $\mathbf{x}$ \\
\hline Adequate & & & $\checkmark$ & $\checkmark$ & $J^{b}$ & $J^{b}$ & $\mathbf{x}$ \\
\hline Avoid & & & na & na & na & na & na \\
\hline
\end{tabular}

Note: $\checkmark=$ suitable for this message; $X=$ not recommended for this message; na $=$ not applicable.

a Mobile Web and Mobile Application did not include auditory messages.

${ }^{b}$ Underlined terms to be removed from this platform (in the Wording Context/Additional Information column); other phrase shortening may be possible depending on user preference.

system. These "apps" include user input and output screens and data entry mechanisms, such as drop-down text boxes and scrolling menus, specifically designed for the touchscreen or keyboard supported by that operating system.

- Dynamic Message Sign—roadside dynamic message sign.

An example format for the data elements the research team identified for travel time reliability is illustrated in Table 11.2. This structure organizes the data elements in a way that can be applied to both reliability terms at a concept level and user interface phrases and terms. This structure also provides a convenient check box matrix indicating the platforms for which each variant of the term is recommended.

\section{Limitations of Lexicon Information}

Note that the studies conducted in this project were laboratory studies, and none of these terms was tested in a field environment. Only in a field test with specific detailed travel behavior data can researchers determine the true impacts and benefits of the use of travel time reliability information on behavior and resulting trip performance. Note especially that nowhere in the various human factor studies were the phrases suggested for display on DMSs tested specifically as being displayed in that format or as en route information. The phrases suggested for display on DMSs were developed by the research team on the basis of the results discussed for the related terminologies. The team developed these phrases using the general guidance for DMS message development provided in the Manual on Uniform Traffic Control Devices (MUTCD). Also note that the formatting of these travel time messages is very different from the standard messages used by state transportation agencies on DMSs. For many of the reliability terms, their use on a DMS would present various challenges to the traveler including the following:

- Drivers are conditioned to see real-time travel information displayed on DMSs on freeway corridors, and reliability information may confuse them when placed on DMSs.

- Any reliability information displayed on a DMS would need to be relative to the specific location of the sign on the freeway facility, as drivers would have begun their trips from various locations in the region's transportation network.

- Messages providing departure time or buffer time information are not appropriate for DMSs because travelers would need to see the messages before starting their trip, not en route.

\section{Travel Time Reliability Lexicon}

The following tables (Table 11.3 through Table 11.10) present the specific lexicon of phrases for each travel time reliability term tested in the various human factors studies. 
Table 11.3. Travel Time Reliability Lexicon for 95th Percentile

\begin{tabular}{|c|c|c|c|c|c|c|c|}
\hline Technical Term & \multicolumn{7}{|l|}{ 95th Percentile } \\
\hline Definition & \multicolumn{7}{|c|}{$\begin{array}{l}\text { The point on a travel time frequency distribution at which } 95 \% \text { of the trips made would be arrive at or less than the iden- } \\
\text { tified time }\end{array}$} \\
\hline Usage & \multicolumn{7}{|c|}{ To describe the longest time a driver can expect a trip to take } \\
\hline \multirow[b]{2}{*}{ Recommendation } & \multirow[b]{2}{*}{ Alternate Phrase } & \multirow[b]{2}{*}{$\begin{array}{l}\text { Wording Context/Additional } \\
\text { Information }\end{array}$} & \multicolumn{5}{|c|}{ Information Technology Platforms } \\
\hline & & & Web & $\begin{array}{l}\text { Mobile } \\
\text { Web }\end{array}$ & Text & $\begin{array}{l}\text { Mobile } \\
\text { Application }\end{array}$ & $\begin{array}{l}\text { Dynamic } \\
\text { Message } \\
\text { Sign }\end{array}$ \\
\hline \multirow[t]{3}{*}{ Best } & \multirow[t]{2}{*}{ Majority of the time } & $\begin{array}{l}\text { "The majority of the time, your trip will } \\
\text { take } X \text { minutes or less." }\end{array}$ & $\checkmark$ & $\checkmark$ & $\sqrt{a}$ & $\boldsymbol{J}^{a}$ & $\mathbf{X}$ \\
\hline & & $\begin{array}{l}\text { MAJORITY OF TIME TRIP TO } \\
\text { [DESTINATION] X MIN OR LESS }\end{array}$ & $\checkmark$ & $\checkmark$ & $\checkmark$ & $\checkmark$ & $J^{b}$ \\
\hline & 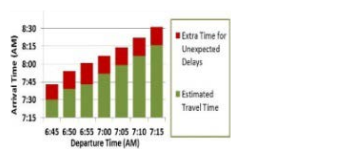 & $\begin{array}{l}\text { Graphical representation of the aver- } \\
\text { age }+95 \text { th percentile }\end{array}$ & $\checkmark$ & $\checkmark$ & $\mathbf{X}$ & $\checkmark$ & $\mathbf{X}$ \\
\hline \multirow[t]{4}{*}{ Adequate } & \multirow[t]{2}{*}{ Most of the time } & $\begin{array}{l}\text { "Most of the time, your trip will take X } \\
\text { minutes or less." }\end{array}$ & $\checkmark$ & $\checkmark$ & $\mathfrak{J}^{a}$ & $\mathfrak{J}^{a}$ & $\mathbf{X}$ \\
\hline & & $\begin{array}{l}\text { MOST OF THE TIME TRIP TO } \\
\text { [DESTINATION] X MIN OR LESS }\end{array}$ & $\checkmark$ & $\checkmark$ & $\checkmark$ & $\checkmark$ & $J^{b}$ \\
\hline & Travel time for planning & $\begin{array}{l}\text { "Travel time for planning is } X \text { minutes } \\
\text { or less." }\end{array}$ & $\checkmark$ & $\checkmark$ & $\sqrt{ }^{a}$ & $\mathfrak{J}^{a}$ & $\mathbf{x}$ \\
\hline & 95th percentile trip time & $\begin{array}{l}\text { "The } 95 \text { th percentile trip time is } X \\
\text { minutes or less." } \\
\text { Provide description such as "19 out of } \\
20 \text { days." }\end{array}$ & $\checkmark$ & $\checkmark$ & $J^{a}$ & $\sqrt{ }^{a}$ & $\mathbf{x}$ \\
\hline \multirow[t]{3}{*}{ Avoid } & Maximum trip time & \multirow{3}{*}{$\begin{array}{l}\text { Agency concerns regarding liability } \\
\text { and credibility }\end{array}$} & \multirow[t]{3}{*}{ na } & \multirow[t]{3}{*}{ na } & \multirow[t]{3}{*}{ na } & \multirow[t]{3}{*}{ na } & \multirow[t]{3}{*}{ na } \\
\hline & Most common trip time & & & & & & \\
\hline & Worst-case trip time & & & & & & \\
\hline
\end{tabular}

Note: $\mathfrak{V}=$ suitable for this message; $X=$ not recommended for this message; na = not applicable.

a Underlined terms to be removed for this platform; other phrase shortening may be possible depending on user preference.

${ }^{b}$ The formatting of this travel time message is very different from the standard messages used by state transportation agencies on DMSs. 
Table 11.4. Travel Time Reliability Lexicon for Arrival Time

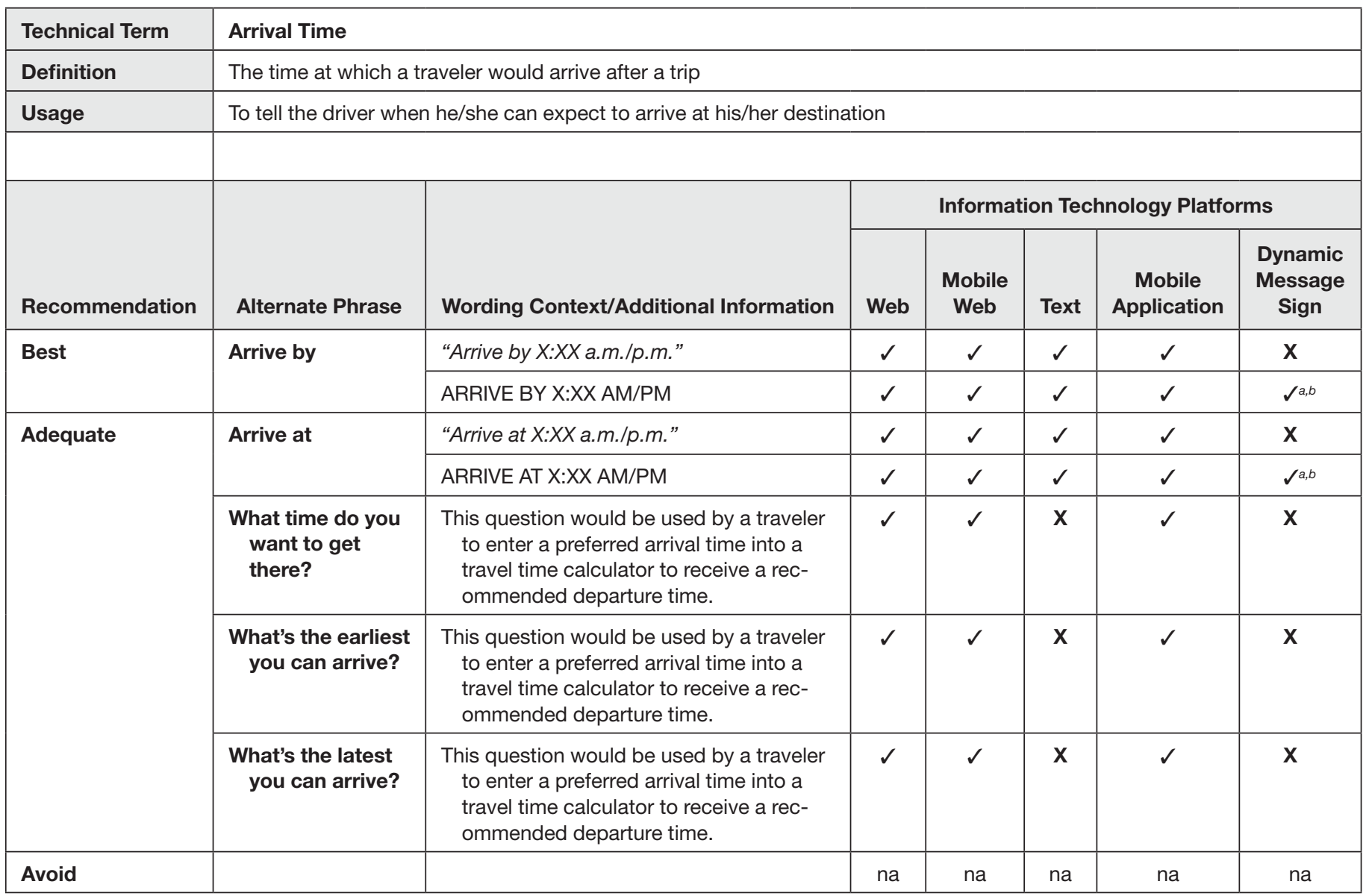

Note: $\checkmark$ = suitable for this message; $X=$ not recommended for this message; na = not applicable.

a The formatting of this travel time message is very different from the standard messages used by state transportation agencies on DMSs.

${ }^{b}$ Term may present ambiguity to the viewers, as they would not see a specific destination. 
Table 11.5. Travel Time Reliability Lexicon for Average Travel Time

\begin{tabular}{|c|c|c|c|c|c|c|c|}
\hline Technical Term & \multicolumn{7}{|l|}{ Average Travel Time } \\
\hline Definition & \multicolumn{7}{|c|}{ An average of historical travel times calculated over a specified time interval for a specified trip or roadway segment } \\
\hline Usage & \multicolumn{7}{|c|}{ To describe the typical travel time a driver can expect a trip will take } \\
\hline & \multirow[b]{2}{*}{ Alternate Phrase } & & \multicolumn{5}{|c|}{ Information Technology Platforms } \\
\hline Recommendation & & $\begin{array}{l}\text { Wording Context/Additional } \\
\text { Information }\end{array}$ & Web & $\begin{array}{c}\text { Mobile } \\
\text { Web }\end{array}$ & Text & $\begin{array}{c}\text { Mobile } \\
\text { Application }\end{array}$ & $\begin{array}{l}\text { Dynamic } \\
\text { Message } \\
\text { Sign }\end{array}$ \\
\hline \multirow[t]{5}{*}{ Best } & \multirow[t]{3}{*}{ Estimated travel time } & "Estimated travel time is $X$ minutes." & $\checkmark$ & $\checkmark$ & $\boldsymbol{J}^{a}$ & $J^{a}$ & $\mathbf{x}$ \\
\hline & & $\begin{array}{l}\text { "It is estimated that your trip will take } X \\
\text { minutes." }\end{array}$ & $\checkmark$ & $\checkmark$ & $\boldsymbol{J}^{a}$ & $\mathfrak{J}^{a}$ & $\mathbf{x}$ \\
\hline & & $\begin{array}{l}\text { EST THAT TRIP TO [DESTINATION] } \\
\text { WILL TAKE X MIN }\end{array}$ & $\checkmark$ & $\checkmark$ & $\checkmark$ & $\checkmark$ & $\mathfrak{J}^{b}$ \\
\hline & \multirow[t]{2}{*}{$\begin{array}{l}\text { Approximate travel } \\
\text { time }\end{array}$} & $\begin{array}{l}\text { "It will take approximately } X \text { minutes to } \\
\text { make your trip." }\end{array}$ & $\checkmark$ & $\checkmark$ & $\sqrt{ }^{a}$ & $\mathfrak{J}^{a}$ & $\mathbf{x}$ \\
\hline & & APPROX X MIN TO [DESTINATION] & $\checkmark$ & $\checkmark$ & $\checkmark$ & $\checkmark$ & $J^{b}$ \\
\hline \multirow[t]{5}{*}{ Adequate } & Typical travel time & "Typical travel time is $\underline{X}$ minutes." & $\checkmark$ & $\checkmark$ & $\boldsymbol{J}^{a}$ & $\boldsymbol{J}^{a}$ & $\mathbf{x}$ \\
\hline & 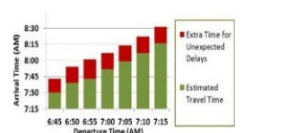 & $\begin{array}{l}\text { Graphical representation of the average } \\
+95 \text { th percentile (typical day and } \\
\text { bad day) }\end{array}$ & $\checkmark$ & $\checkmark$ & $\mathbf{x}$ & $\checkmark$ & $x$ \\
\hline & 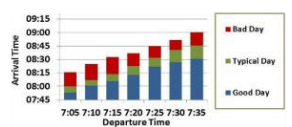 & $\begin{array}{l}\text { Graphical representation of the 20th } \\
\text { percentile + average }+95 \text { th percentile } \\
\text { (good, typical, and bad day) }\end{array}$ & $\checkmark$ & $\checkmark$ & $\mathbf{x}$ & $\checkmark$ & $\mathbf{x}$ \\
\hline & Average travel time & "Average travel time is $X$ minutes." & $\checkmark$ & $\checkmark$ & $\boldsymbol{J}^{a}$ & $\mathfrak{J}^{a}$ & $x$ \\
\hline & Expected travel time & "Expected travel time is $X$ minutes." & $\checkmark$ & $\checkmark$ & $\sqrt{ }^{a}$ & $\sqrt{ }^{a}$ & $\mathbf{x}$ \\
\hline Avoid & Historical travel time & $\begin{array}{l}\text { Difficult to determine relevance with no } \\
\text { comparison to real-time information }\end{array}$ & na & na & na & na & na \\
\hline
\end{tabular}

Note: $\checkmark$ = suitable for this message; $\mathrm{X}=$ not recommended for this message; na = not applicable.

a Underlined terms to be removed for this platform; other phrase shortening may be possible depending on user preference.

${ }^{b}$ The formatting of this travel time message is very different from the standard messages used by state transportation agencies on DMSs. 
Table 11.6. Travel Time Reliability Lexicon for Buffer Time

\begin{tabular}{|c|c|c|c|c|c|c|c|}
\hline Technical Term & \multicolumn{7}{|l|}{ Buffer Time } \\
\hline Usage & \multicolumn{7}{|c|}{ To describe how much extra time a driver should plan for a trip he/she wishes to take } \\
\hline Recommendation & Alternate Phrase & Wording Context/Additional Information & \multicolumn{5}{|c|}{ Information Technology Platforms } \\
\hline \multirow[t]{2}{*}{ Best } & \multirow[t]{2}{*}{ Extra time } & "Extra time for trip is $X$ minutes." & $\checkmark$ & $\checkmark$ & $\mathfrak{S}^{a}$ & $\mathfrak{J}^{a}$ & $\mathbf{X}$ \\
\hline & & EXTRA TIME TO [DESTINATION] IS X MIN & $\checkmark$ & $\checkmark$ & $\checkmark$ & $\checkmark$ & $\mathbf{x}$ \\
\hline Adequate & Added time & "Added time for trip is $X$ minutes." & $\checkmark$ & $\checkmark$ & $\mathfrak{J}^{a}$ & $\boldsymbol{J}^{a}$ & $\mathbf{x}$ \\
\hline \multirow[t]{4}{*}{ Avoid } & Cushion & \multirow[t]{4}{*}{ Preference shown for other terms } & \multirow[t]{4}{*}{ na } & \multirow[t]{4}{*}{ na } & \multirow[t]{4}{*}{ na } & \multirow[t]{4}{*}{ na } & \multirow[t]{4}{*}{ na } \\
\hline & Buffer time & & & & & & \\
\hline & $\begin{array}{r}\text { Departure } \\
\text { window }\end{array}$ & & & & & & \\
\hline & Leeway & & & & & & \\
\hline
\end{tabular}

Note: $\boldsymbol{V}=$ suitable for this message; $X=$ not recommended for this message; na $=$ not applicable.

a Underlined terms to be removed for this platform; other phrase shortening may be possible depending on user preference.

Table 11.7. Travel Time Reliability Lexicon for Departure Time

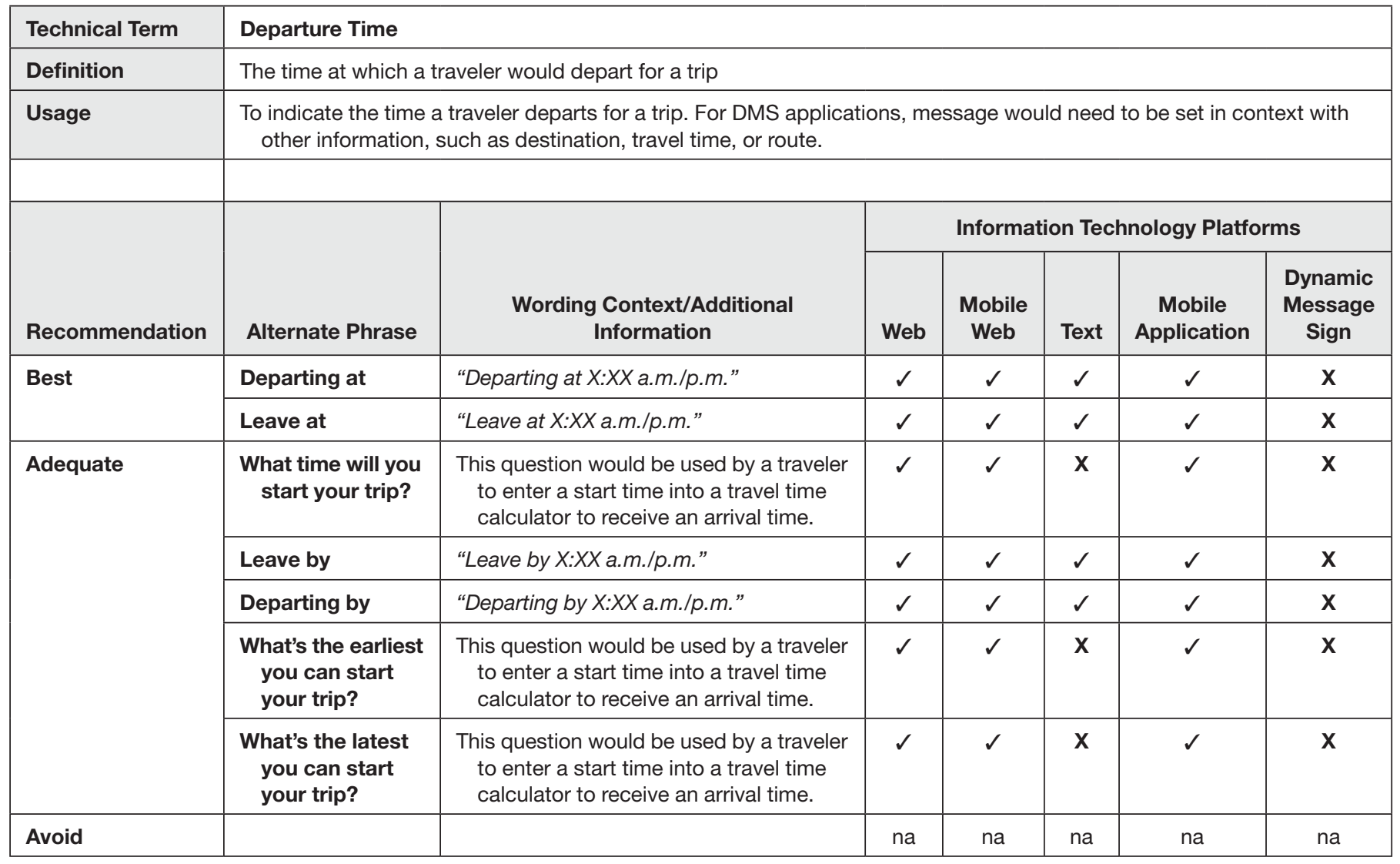

Note: $\checkmark=$ suitable for this message; $\mathrm{X}=$ not recommended for this message; na $=$ not applicable. 


\section{Table 11.8. Travel Time Reliability Lexicon for Recommended Departure Time}

\begin{tabular}{|c|c|c|c|c|c|c|c|}
\hline Technical Term & \multicolumn{7}{|c|}{ Recommended Departure Time } \\
\hline Definition & \multicolumn{7}{|c|}{$\begin{array}{l}\text { A time of departure calculated by a traveler information system that would ensure an on-time arrival for a given level of risk } \\
\text { tolerance. }\end{array}$} \\
\hline Usage & \multicolumn{7}{|c|}{$\begin{array}{l}\text { To indicate the time a driver should depart for a trip to ensure he/she arrives at his/her destination on time. For DMS } \\
\text { applications, message would need to be set in context with other information, such as destination, travel time, or } \\
\text { route. }\end{array}$} \\
\hline \multirow[b]{2}{*}{ Recommendation } & \multirow[b]{2}{*}{ Alternate Phrase } & \multirow[b]{2}{*}{ Wording Context/Additional Information } & \multicolumn{5}{|c|}{ Information Technology Platforms } \\
\hline & & & Web & $\begin{array}{c}\text { Mobile } \\
\text { Web }\end{array}$ & Text & $\begin{array}{c}\text { Mobile } \\
\text { Application }\end{array}$ & $\begin{array}{l}\text { Dynamic } \\
\text { Message } \\
\text { Sign }\end{array}$ \\
\hline Best & $\begin{array}{l}\text { Recommended } \\
\text { departure time }\end{array}$ & $\begin{array}{l}\text { "Recommended departure time is } X: X X \\
\text { a.m./p.m." }\end{array}$ & $\checkmark$ & $\checkmark$ & $\sqrt{ }^{a}$ & $\sqrt{ }^{a}$ & $\mathbf{x}$ \\
\hline \multirow[t]{3}{*}{ Adequate } & $\begin{array}{l}\text { Suggested } \\
\text { departure time }\end{array}$ & "Suggested departure time is $X: X X$ a.m./p.m." & $\checkmark$ & $\checkmark$ & $\boldsymbol{J}^{a}$ & $\boldsymbol{J}^{a}$ & $\mathbf{x}$ \\
\hline & $\begin{array}{l}\text { Estimated } \\
\text { departure time }\end{array}$ & "Estimated departure time is $X: X X$ a.m./p.m." & $\checkmark$ & $\checkmark$ & $\sqrt{ }^{a}$ & $\sqrt{ }^{a}$ & $\mathbf{x}$ \\
\hline & $\begin{array}{l}\text { 95th percentile } \\
\text { departure time }\end{array}$ & $\begin{array}{l}\text { "The } 95 \text { th percentile departure time is } X: X X \\
\text { a.m./p.m." } \\
\text { Provide description such as " } 19 \text { out of } 20 \text { days." }\end{array}$ & $\checkmark$ & $\checkmark$ & $\sqrt{ }^{a}$ & $\boldsymbol{J}^{a}$ & $\mathbf{x}$ \\
\hline Avoid & & & na & na & na & na & na \\
\hline
\end{tabular}

Note: $\checkmark$ = suitable for this message; $X=$ not recommended for this message; na $=$ not applicable.

a Underlined terms to be removed for this platform; other phrase shortening may be possible depending on user preference.

${ }^{b}$ Can be used if term is NOT being used to mean average trip time. 
Table 11.9. Travel Time Reliability Lexicon for Recommended Route

\begin{tabular}{|c|c|c|c|c|c|c|c|}
\hline Technical Term & \multicolumn{7}{|l|}{ Recommended Route } \\
\hline Definition & \multicolumn{7}{|c|}{$\begin{array}{l}\text { A route between two points calculated by a traveler information system that would ensure an on-time arrival for a given } \\
\text { level of risk tolerance }\end{array}$} \\
\hline Usage & \multicolumn{7}{|c|}{ To describe the route a driver should take for a planned trip to ensure he/she arrives on time to his/her destination } \\
\hline & \multirow[b]{2}{*}{ Alternate Phrase } & \multirow[b]{2}{*}{$\begin{array}{l}\text { Wording Context/Additional } \\
\text { Information }\end{array}$} & \multicolumn{5}{|c|}{ Information Technology Platforms } \\
\hline Recommendation & & & Web & $\begin{array}{l}\text { Mobile } \\
\text { Web }\end{array}$ & Text & $\begin{array}{l}\text { Mobile } \\
\text { Application }\end{array}$ & $\begin{array}{l}\text { Dynamic } \\
\text { Message } \\
\text { Sign }\end{array}$ \\
\hline \multirow[t]{2}{*}{ Best } & \multirow[t]{2}{*}{ Best route } & "Best route is via [facility]." & $\checkmark$ & $\checkmark$ & $\boldsymbol{J}^{a}$ & $J^{a}$ & $\mathbf{x}$ \\
\hline & & $\begin{array}{l}\text { BEST ROUTE TO [DESTINATION] TAKE } \\
\text { [FACILITY] }\end{array}$ & $\checkmark$ & $\checkmark$ & $\checkmark$ & $\checkmark$ & $\mathfrak{S}^{b}$ \\
\hline \multirow[t]{8}{*}{ Adequate } & \multirow[t]{2}{*}{ Forecasted trip } & "Forecasted trip is via [facility]." & $\checkmark$ & $\checkmark$ & $\mathfrak{J}^{a}$ & $\mathfrak{J}^{a}$ & $x$ \\
\hline & & $\begin{array}{l}\text { FORECASTED TRIP TIME VIA [FACILITY] } \\
\quad \text { X MIN }\end{array}$ & $\checkmark$ & $\checkmark$ & $\checkmark$ & $\checkmark$ & $\mathfrak{J}^{b}$ \\
\hline & \multirow[t]{2}{*}{ Most reliable trip } & "Most reliable trip is via [facility]." & $\checkmark$ & $\checkmark$ & $\boldsymbol{J}^{a}$ & $\sqrt{a}$ & $x$ \\
\hline & & $\begin{array}{l}\text { MOST RELIABLE TRAVEL TIME TO } \\
\text { [DESTINATION] TAKE [FACILITY] }\end{array}$ & $\checkmark$ & $\checkmark$ & $\checkmark$ & $\checkmark$ & $J^{b}$ \\
\hline & \multirow[t]{2}{*}{ Most predictable trip } & "Most predictable trip is via [facility]." & $\checkmark$ & $\checkmark$ & $\sqrt{ }^{a}$ & $\sqrt{a}$ & $x$ \\
\hline & & $\begin{array}{l}\text { MOST PREDICTABLE TRAVEL TIME TO } \\
\text { [DESTINATION] TAKE [FACILITY] }\end{array}$ & $\checkmark$ & $\checkmark$ & $\checkmark$ & $\checkmark$ & $J^{b}$ \\
\hline & \multirow[t]{2}{*}{ Most consistent trip } & "Most consistent trip is via [facility]." & $\checkmark$ & $\checkmark$ & $\mathfrak{J}^{a}$ & $\boldsymbol{J}^{a}$ & $\mathbf{x}$ \\
\hline & & $\begin{array}{l}\text { MOST CONSISTENT TRAVEL TIME TO } \\
\text { [DESTINATION] TAKE [FACILITY] }\end{array}$ & $\checkmark$ & $\checkmark$ & $\checkmark$ & $\checkmark$ & $\mathfrak{J}^{b}$ \\
\hline \multirow[t]{2}{*}{ Avoid } & $\begin{array}{c}\text { Historical trip } \\
\text { conditions }\end{array}$ & \multirow{2}{*}{$\begin{array}{l}\text { Difficult to determine relevance with no } \\
\text { comparison to real-time information; } \\
\text { preference shown for other terms }\end{array}$} & \multirow[t]{2}{*}{ na } & \multirow[t]{2}{*}{ na } & \multirow[t]{2}{*}{ na } & \multirow[t]{2}{*}{ na } & \multirow[t]{2}{*}{ na } \\
\hline & Least variable time & & & & & & \\
\hline
\end{tabular}

Note: $\checkmark$ = suitable for this message; $X=$ not recommended for this message; na $=$ not applicable.

${ }^{a}$ Underlined terms to be removed for this platform; other phrase shortening may be possible depending on user preference.

${ }^{b}$ The formatting of this travel time message is very different from the standard messages used by state transportation agencies on DMSs. 
Table 11.10. Travel Time Reliability Lexicon for Reliability

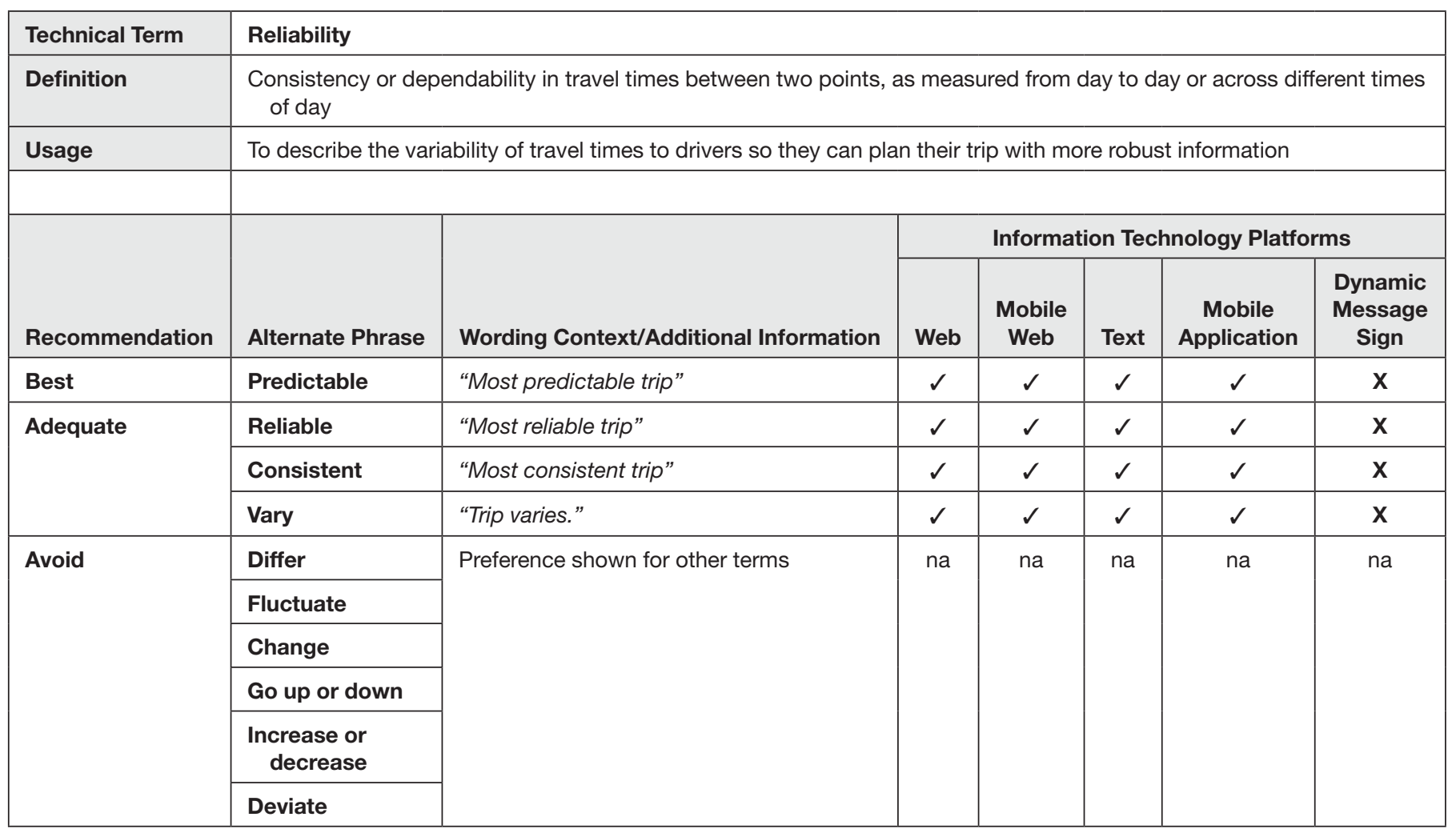

Note: $\checkmark=$ suitable for this message; $\mathrm{X}=$ not recommended for this message; na = not applicable. 


\section{CHAPTER 12}

\section{Final Remarks}

The SHRP 2 Reliability program aims to improve trip time reliability by reducing the frequency and effects of events that cause travel times to fluctuate in an unpredictable manner. As the program planning document points out, congestion caused by unreliable, or nonrecurring, events is roughly as extensive as congestion caused by routine bottlenecks (Cambridge Systematics, Inc. 2003). Nonrecurring events such as crashes, work zones, special events, and weather disrupt normal traffic flow by causing reduced speeds, lane closures, and erratic driving maneuvers.

Travel time reliability information includes static data about traffic speeds or trip times that capture historical variations from day to day and enable individuals to understand the level of variation in traffic. Unlike real-time travel time information, which provides a current snapshot of trip conditions and travel time, reliability information can be used to plan and budget in advance for a trip. A key component to addressing the reliability issue related to urban mobility is conveying this reliability-related information to system users so that they can make informed decisions about their travel. The challenge for transportation professionals lies in selecting the best means of conveying that information so that it is usable and effective. The goal of this research project was to examine what combination of words, numbers, and other features of user information messages, along with communications methods and technology platforms, best communicates information about travel time and reliability to travelers so that they can make optimal travel choices from their own point of view. Such choices include whether to take a trip or not, departure time, mode choice, and route choice.

This report provides a comprehensive description of an increasingly detailed series of human factor experiments and the development of a utility function. With input from a literature review, expert interviews, and a scan of technology and innovation, the report provides key information and insight into (a) how individuals comprehend and interpret travel time reliability information, (b) how they use that information to make trip decisions, and (c) how reliability terms can be phrased to reach the highest percentage of travelers so that their travel decisions yield some benefit to them.

The most important product of this study is the lexicon of travel time reliability terms. The research team identified several key elements of a lexicon entry that were deemed necessary to completely present each term and how it might be used within the transportation community.

To that end the research team developed a structure for the lexicon which organizes the data elements in a way that can be applied to both reliability terms at a concept level and user interface phrases and terms. This structure also provides a convenient check box matrix indicating the platforms for which each variant of the term is recommended.

\section{Study Limitations}

Note that the studies conducted in this project were laboratory studies, and none of the terms was tested in a field environment. Only in a field test with specific detailed travel behavior data can researchers determine the true impacts and benefits of the use of travel time reliability information on behavior and resulting trip performance. Note specially that nowhere in the various human factor studies were the phrases suggested for display on DMSs tested specifically as being displayed in that format or as en route information. The phrases suggested for display on DMSs were developed by the research team on the basis of the results discussed for the related terminologies. The team developed these phrases using the general guidance for DMS message development provided in the MUTCD. Also note that the formatting of these travel time messages is very different from the standard messages used by state transportation agencies on DMSs. 


\section{Key Findings on Travel Time Reliability Terminology}

The human factors studies found that several of the reliability terms commonly used and understood by transportation professionals-such as 95th percentile, buffer time, buffer index, and even average trip time - are not necessarily preferred or consistently understood by travelers. The results of the focus groups, the computer survey, and the open-ended survey indicated that participants did not like statisticalsounding terms and were likely to interpret those terms inaccurately. For instance, significant numbers of participants interpreted 95th percentile trip time as being a time about which a trip prediction system was $95 \%$ confident, or estimated that an average trip time would apply to approximately $75 \%$ of trips rather than approximately $50 \%$ of trips. In most cases, no single term or phrase for a given reliability concept was a "slam dunk," that is, understood and preferred by a large majority of participants. However, generally one to three terms in each category tested reasonably well with study participants, and the research team feels those terms are good candidates for pilot testing or further testing in field studies. The lexicon lists the tested terms for eight categories of travel time reliability information (as well as sample graphics for some information categories) along with the research team's recommendations regarding their use.

\section{Key Study Observations on User Behavior}

After careful assessment of the aforementioned travel time reliability terms and the results obtained in the various human factor studies and experiments conducted throughout the course of the L14 project, the research team established three key hypotheses related to the use and value of travel time reliability information from the user's perspective. These hypotheses were tested in the enhanced laboratory study. The following sections highlight the three hypotheses and the results from the study - all of which were combined with the results from the other human factor experiments to develop the lexicon.

\section{Hypothesis 1}

The first hypothesis stated that the provision of accurate reliability information (in an easy-to-understand format) would result in improved on-time performance and lower generalized travel disutility compared with a control group receiving no reliability information.

Findings from the first laboratory study supported this hypothesis. Experimental participants who received reliability information were on time more frequently ( $85 \%$ versus
$79 \%)$ and had lower total late and early arrival penalties associated with the simulated trip ( $\$ 38.61$ versus $\$ 47.42$ and $\$ 55.55$ in the two control groups). Both findings were statistically significant with $95 \%$ confidence despite the relatively small sample size of the experiment.

The results of the enhanced laboratory study strongly supported this hypothesis as well. Of the seven different forms of delivery of reliability information tested in this experiment, users presented with five of the options demonstrated statistically significant reductions in weekly schedule offset costs (i.e., costs established in the experiment for early and late arrivals) compared with the control group receiving no reliability information. These five were also the simplest of the forms of reliability information, focusing on average and 95th percentile travel time values, delivered in various forms. Participants receiving these simple forms of reliability information reduced schedule offset costs by $9 \%$ to $21 \%$ compared with the control group.

\section{Hypothesis 1a}

Hypothesis la stated that while travel outcomes improve with the provision of reliability information, participants' perceived value of the reliability information would underestimate the realized benefit in terms of reduced delay, improved on-time reliability, and reduced stress.

The enhanced laboratory study results strongly supported this hypothesis. For each of the simple forms of reliability information tested, improvements in trip outcomes were clear and statistically significant. For example, frequency of late arrivals declined $16 \%$ to $40 \%$ when participants received reliability information in these forms compared with when they did not receive reliability information. Reduction in stress reported at the end of each week was also statistically significant, in a similar range from $10 \%$ to $31 \%$. However, participant willingness to pay for reliability information compared with willingness to pay for baseline (real-time) information was often not statistically significant. For example, participants receiving the simple text +95 th percentile reliability information reduced late arrivals by $40 \%$ and reported a $10 \%$ reduction in stress. However, these same participants were willing to pay on average only $\$ 0.10$ more for reliability information ( $\$ 2.78$ versus $\$ 2.68$ per trip), a difference too small to be statistically significant.

\section{Hypothesis $1 b$}

Hypothesis $1 \mathrm{~b}$ stated that the provision of reliability information using different text-based, graphical, and auditory forms would result in differences in both accrued on-time reliability benefits and perceived benefits. The study team expected these 
differences among experimental groups would be smaller than between any group and the control (no reliability information) group.

The enhanced laboratory study supported this hypothesis. Provision of simple forms of reliability information had similar results whether provided in text-based, graphical, or auditory form. The more complex graphical and signposting concepts were not effective.

\section{Hypothesis 2a}

Hypothesis 2a (tested in the first laboratory study) proposed that while travel outcomes improved with the provision of reliability information, the perceived value of the reliability information would underestimate the realized benefit in terms of reduced delay, improved on-time reliability, and reduced stress.

Findings from the first laboratory study supported this hypothesis. Measured perceptions of benefit associated with reliability information were not commensurate with the observed improvements in trip outcomes. Changes in the value of information and stress reduction, although generally favorable to reliability information, were not statistically significant. The implication is that although reliability information can be useful in managing trip time variability, the apparent value of this information is lost in the context of learning over time. The experimental subjects likely internalized the reliability information as one element in the learning process, rather than considering its value outside of their learning process. Providers of reliability information may face an uphill battle in measuring participants' perceptions of reliability information effects even when such information is useful in improving trip outcomes.

\section{Hypothesis 2b}

Hypothesis $2 \mathrm{~b}$ (tested in the second laboratory study) stated that experimental subjects receiving contextual information on underlying variation with numeric indicators reinforced with en route information (reliability signposting) would have improved on-time performance compared with both an experimental group that received reliability information but no contextual information as well as a control group that received no reliability information.

Hypothesis 2 was not supported by the enhanced laboratory study. The signposting concept was not successful for participants in the management of trip outcomes and stress reduction. To some degree, this was because of the complexity of the presentation. Signposting may still be a valuable concept to pursue for providing reliability information, but work remains to make this delivery method more accessible.

\section{Hypothesis 3}

Hypothesis 3 stated that the benefits of reliability information would decline over time as both experimental and control subjects learned and understood the underlying travel time variability. That is, the benefit from reliability information during the first weeks would be larger than during the last weeks.

Findings from the first laboratory study were inconclusive. Participants with access to reliability information did see total early- and late-arrival penalties decline from week 1 to week 2 of the simulated trip-making. Control groups who did not receive this information were mixed, with one group realizing a reduction in total costs and another seeing a rise in costs. Interestingly, participants' willingness to pay for reliability information declined over time in the simulation experiment, indicating that experience within the simulated trip began to offset the reliance on provided reliability information.

The enhanced laboratory study supported hypothesis 3 . Participants using reliability information were equally effective in managing trip outcomes (late arrivals, schedule delays, and offset costs) in the first week of exposure to unfamiliar travel time variability patterns as their counterparts without reliability information were after 4 weeks. Within the 4 -week constraints of the experiment, both reliability information users and control group counterparts reduced offset costs through week three, at which time costs leveled off. That said, the difference in realized offset costs between the two groups was still significant even in week three and week four, roughly $25 \%$ ( $\$ 40$ versus $\$ 50$ ). This finding implies that reliability information still has value at 4 weeks of experience and presumably may still have value longer than 4 weeks since the gap in performance between week one and week four between the two groups narrowed only from $40 \%$ to $25 \%$.

\section{Potential Next Steps}

Given the complexity of the travel time reliability concept and the myriad ways the information may affect system users, system operators, and service providers, the project team identified several potential issues that can be addressed in further detail and refined through additional investigation. These issues are discussed in the following sections.

\section{Graphical Formats for Reliability Information}

Two graphical formats were tested in this study's second laboratory experiment as alternatives for presenting reliability information to drivers. These two formats were rated by participants as being "more complex" and therefore less easy to use than the same information presented in a text format. However, other graphical formats may prove useful as alternative 
or supplemental methods for communicating reliability information to drivers. Further research should be conducted to assess the potential usefulness and usability of "star" ratings, Harvey Balls, and other graphical formats for conveying reliability information.

\section{Reliability Information in the Context of More Complex Trip Planning}

The research team looked only at single-occupancy highway trips with time of departure choice. The more complex the range of travel choices available to the user (with low overall travel time variance correlation), the more valuable reliability information will be in reducing late trip arrivals and schedule offset costs.

\section{Mechanisms of Reliability Information Under-Valuation by Users}

Although this work makes clear that travelers do not attribute improved trip outcomes to access to reliability information, the reason why is not clear. Participants may have seen the experiment as a game in which they were actively learning and discounted inputs to their learning process compared with an assessment of their own innate powers of deduction. A set of structured experiments to uncover the mechanisms of the perception of travel time reliability information can be constructed to investigate this interesting result.

\section{Predictive Reliability Information and the Experienced Traveler}

One tantalizing morsel from the second (enhanced) experiment calls into question the assumption from the focus group activity that reliability information will have value primarily for unfamiliar travelers. In the second experiment, the difference in schedule offset costs between users of reliability information and the control group declined from $40 \%$ in the first week to $25 \%$ in the fourth week. The experiment begs the question of how many weeks would be required until the performance of the two groups was the same, or if indeed such a convergence would actually occur. This may imply that there is some inherent value in providing accurate data to the users even if they have acclimated themselves to the information in a nonquantitative way. Another key observation is that the underlying patterns of travel time variation did not change in the experiment; therefore, there may be a value in predicting trends in travel time variability and tailoring reliability information even for the most experienced traveler.

\section{Impact of Reliability Information on Broader Range of Travel-Related Choices}

The provision of reliability information may have benefits in other choices not studied in this experiment. These choices might include decisions on telework - both the practical value of telework on a regular basis and dynamic telework decisions to remain at home rather than risk being en route at the time of a critical meeting (whether in-person or virtual). Other decisions potentially informed by reliability information include a home purchase or new job with travel-related impacts, as well as facility location decisions for businesses and supply chain managers.

\section{Monetization of Reliability Information Impacts}

The first traveler behavior laboratory experiment looked specifically at the monetization of reliability information impacts and derived a parameter for serenity benefits associated with knowing as early as possible about possible trip outcomes (late or otherwise). Additional work in this area suggested by the experience with the enhanced laboratory experiment includes the potential development of utility functions that cover a broader range of serenity impacts, as well as a new class of multimodal functions addressing more complex trip chains and tours. Further, the development and documentation of practical methods of data collection for the local calibration of reliability information-sensitive utility functions is another valuable extension to this research. Additional exploration of serenity impacts under constrained and unconstrained rescheduling options would also be of value using an experimental structure similar to the one designed for this study.

\section{Traveler Responses to Increased Travel Time Reliability}

A before-and-after study of traveler responses to changes in travel time reliability along particular corridors could provide a real-world assessment of the value travelers place on reliability. Such a study would involve selecting corridor segments that have recently or will soon experience a significant change in travel time reliability as a result of an operational change, such as initiation of congestion pricing or the opening of a parallel facility. Surveys of travelers along the corridor before (ideally) and after the change would examine travel patterns and preferences and the value respondents place on travel time reliability; in the case of a newly established priced facility, this value could be indexed to the amount that travelers would be willing to pay to travel on the more reliable facility. Simulation-based tests similar to those conducted for this study (described in Chapter 9 and Chapter 10) would be 
another approach to further assessing the relative value of and responses to travel time reliability information within different trip situations. Either simulation-based or field-test studies would examine drivers' trade-off decisions for travel routes, times, and modes, through stated and/or revealed preferences.

\section{Use of Reliability Information by the Freight Industry}

Commercial drivers plan routes primarily on the basis of cost-effectiveness and tend to select the most direct route (based on distance) or a route that allows them to avoid traffic congestion or other obstructions. Drivers and dispatchers consider time of day, traffic patterns in major metropolitan areas, and construction when planning routes and when considering route diversions during a trip. A driver who delivers to regular repeat customers will often develop "usual" routes and will stick to them unless conditions dictate otherwise. If a driver has a time-sensitive delivery, the travel time along a given route becomes more important, and the driver and the company will be more likely to opt for a toll facility or other route option that provides a more reliable trip time (Higgins et al. 2013). The research team hypothesizes, therefore, that commercial drivers would not only value TTR information, but would also be better able (compared with commuters) to express that value monetarily. Research should be conducted to examine the potential valuation and use of travel time reliability information by the freight industry.

\section{Reliability Information in Public Transit}

The human factors studies and utility function development conducted in this study focused on drivers; however, the literature indicates that reliability information is also valuable to transit riders. Similar research should be developed to further examine the effects of information about transit travel time and arrival reliability on riders' mode decisions, departure time decisions, stress levels, and satisfaction with the transit service.

\section{Combining Real-time and Reliability Information}

Feedback from the focus groups and computer survey indicated that travelers consider real-time travel time information to be a valuable and even necessary addition to historical data when planning trips. Research is needed to determine how best to combine real-time and historical travel time information to provide the most useful and accurate information to travelers. SHRP 2 Project L15A, Forecasting and Delivery of Highway Travel Time Reliability Information, developed a prototype of a forecasting website (http://MyRoadTripAdvisor .com) that predicts travel time for a given route on the basis of both historical patterns and current conditions, including incidents, weather, and work zones. The website offers registered users the options to save frequent trips by name and to have travel time forecasts for scheduled trips "pushed" to them by e-mail, text message, or telephone. This demonstration project provided real-time and travel time reliability information for portions of I-66 in Northern Virginia. Because the two projects (L14 and L15A) were conducted during the same time period, different sets of terminology were developed for communicating reliability concepts; future research might involve testing MyRoadTripAdvisor with terminology from L14's lexicon, as well as testing additional auditory or graphical options for communicating real-time and reliability information to travelers.

\section{Field Tests of Reliability Terminology}

A field test of the lexicon terminology is one way to implement and validate the results of this project's human factors studies and utility function development, by collecting data about travelers' use of pre-trip and en route reliability information in a real-world environment. A field test would use recommended reliability terms and formats from the lexicon as part of the provided information on a localized travel website, on DMS, and via other media and messaging techniques in a selected city or cities. As mentioned above, the prototype website developed by Project L15A would be a potential starting point for such a field test. 


\section{References}

ABI: Win-Win Partnerships Key to Traffic Data. 2008. TrafficTechnology Today.com. February 29. http://www.traffictechnologytoday.com/ news.php?NewsID=3904. Accessed Oct. 23, 2012.

AirSage. 2012. Using Wireless Signals to Define Locations. http://www .airsage.com/Technology/How-it-works/. Accessed Oct. 23, 2012.

Alkim, T. P., P. H. van der Mede, and W. H. Janssen. 2000. Graphical Route Information on Variable Message Signs. Proc., 10th International Conference on Road Transport Information and Control. Commonwealth Institute, London, pp. 32-36.

Android Applications. 2012. http://androidforums.com/androidapplications/22768-accuracy-google-navigator.html. Accessed Oct. 23, 2012.

Arbaiza, A., and A. Lucas. 2010. ESG4-Mare Nostrum: 3 Main Gears. Presented at Easy Way Forum, Lisbon, Portugal.

Arrington, M. 2009. Google Redefines GPS Navigation Landscape: Google Maps Navigation for Android 2.0. http://www.techcrunch.com/ 2009/10/28/google-redefines-car-gps-navigation-google-mapsnavigation-android/. Accessed Oct. 23, 2012.

Associated Press. 2010. Ford Adding Tweets to Its Sync In-Car Technology. Jan. 8. http://www.thehindu.com/sci-tech/technology/fordadding-tweets-to-its-sync-incar-technology/article77295.ece. Accessed Oct. 23, 2012.

Ban, X., Y. Li, and J. Margulici. 2009. Optimal Use of Changeable Message Signs for Displaying Travel Times. University of California, Berkeley, Calif.

Bar-Gera, H. 2007. Evaluation of a Cellular Phone-Based System for Measurements of Traffic Speeds and Travel Times: A Case Study from Israel. Transportation Research Part C: Emerging Technologies, Vol. 16, No. 6, pp. 380-391.

Basu, D., and B. Maitra. 2010. Stated Preference Approach for Valuation of Travel Time Displayed as Traffic Information on a VMS Board. Journal of Urban Planning and Development, Vol. 136, No. 3, pp. 214-224.

Bates, J., J. Polak, P. Jones, and A. Cook. 2001. The Valuation of Reliability for Personal Travel. Transportation Research Part E, Vol. 37, pp. 191-229.

BBC News. 2007. Predictable Forecast ... Probably? BBC News. Oct. 29. http://news.bbc.co.uk/2/hi/programmes/more_or_less/7067003 .stm. Accessed Oct. 11, 2012.

Berg InSight. 2009. Personal Navigation Devices. Berg InSight, Gothenburg, Sweden.

Bertini, R. L., and S. Tantiyanagulchai. 2004. Transit Buses as Traffic Probes: Use of Geolocation Data for Empirical Evaluation. In
Transportation Research Record: Journal of the Transportation Research Board, No. 1870, Transportation Research Board of the National Academies, Washington, D.C., pp. 35-45.

Biehl, M., and B. Halpern-Felsher. 2001. Adolescents' and Adults' Understanding of Probability Expressions. Journal of Adolescent Health, Vol. 28, pp. 30-35.

Black, I., and J. Towriss. 1993. Demand Effects of Travel Time Reliability. Technical Report, Center for Logistics and Transportation, Craneld Institute of Technology, London.

Blumenthal, M. 2009. Google Replaces Tele Atlas Data in US with Google StreetView Data. October 12. http://blumenthals.com/blog/2009/ 10/12/google-replaces-tele-atlas-data-in-us-with-google-data/. Accessed Oct. 23, 2012.

Cambridge Systematics, Inc. 2007. SHRP 2 Project L03: Analytic Procedures for Determining the Impacts of Reliability Mitigation Strategies. Transportation Research Board of the National Academies, Washington, D.C.

Cambridge Systematics, Inc. 2006. AASHTO Freight Transportation Bottom Line Report: Freight Demand and Logistics. AASHTO, Washington, D.C.

Cambridge Systematics, Inc. 2003. Providing a Highway System with Reliable Travel Times: Study 3-Reliability. Transportation Research Board, Washington, D.C.

Cambridge Systematics, Inc. with Texas Transportation Institute. 2005. Traffic Congestion and Reliability: Trends and Advanced Strategies for Congestion Mitigation. FHWA, U.S. Department of Transportation, Washington, D.C.

Center for Advanced Transportation Technology. 2008. Bluetooth Traffic Monitoring Technology: Concept of Operation \& Deployment Guidelines. Center for Advanced Transportation Technology, University of Maryland, Blacksburg, Va.

ClearChannel. 2009. Clear Channel Radio's Total Traffic Network Now Reaches More than 125 Million Users. Nov. 3. http://www.clearchannel .com/MediaAndEntertainment/PressRelease.aspx?PressReleaseID=25 $28 \&$ KeyWord=Clear+Channel+Radio $\%$ E2\%80\%99s+Total+Traffic + Network+Now+Reaches+More+Than+125+Million+Users. Accessed Oct. 23, 2012.

Concas, S., and A. Kolpakov. 2009. Synthesis of Research on Value of Time and Value of Reliability. Center for Urban Transportation Research, University of South Florida, Tampa, Fla.

Cooney, M. 2009. Ballmer Gets His New Car Delivered by Ford CEO. May 27. http://www.networkworld.com/community/node/42189. Accessed Oct. 23, 2012. 
Cunningham, W. 2009. Inrix Offers Free Traffic App for iPhone. Aug. 7. http://reviews.cnet.com/8301-13746_7-10305540-48.html. Accessed Oct. 23, 2012.

Dudek, C. L. 2008. Changeable Message Sign Displays During Non-Incident, Non-Roadwork Periods. NCHRP, Transportation Research Board, National Research Council, Washington, D.C.

Dudek, C. L. 2006. Dynamic Message Sign Message Design and Display Manual. Texas Department of Transportation, Austin, Tex.

Dudek, C. L. 2004. Changeable Message Sign Operation and Messaging Handbook. FHWA, U.S. Department of Transportation, Washington, D.C.

Dudek, C. L. 2001. Variable Message Sign Operations Manual. New Jersey Department of Transportation, Trenton, N.J.

Dudek, C. L., and R. D. Huchingson. 1991. Use of Changeable Message Signs for Displaying Travel Time and Other Time-Related Messages. Dudek \& Associates, Bryan, Tex.

Eby, D. 2009. Technology and Distracted Driving Panel. Distracted Driving Summit. U.S. Department of Transportation, Washington, D.C.

Farivar, C. 2006. Vodafone, TomTom Partner to Create Real-Time Traffic Data Network. Oct. 27. http://mobile.engadget.com/2006/10/27/ vodafone-tomtom-partner-to-create-real-time-traffic-data-network/. Accessed Oct. 23, 2012.

FHWA. 2004. Request for Interpretation: 2-55(I)—CMS Display of Travel Time. Manual on Uniform Traffic Control Devices (MUTCD), U.S. Department of Transportation, Washington, D.C. May 17. http://mutcd.fhwa.dot.gov/resources/interpretations/pdf/2_551 .pdf. Accessed Oct. 22, 2012.

FHWA. 2003. Status of the Nation's Highways, Bridges, and Transit: 2002 Conditions and Performance Report. U.S. Department of Transportation, Washington, D.C.

FHWA. 2002. Federal Highway Administration Fiscal Year 2003 Performance Plan. http://www.fhwa.dot.gov/reports/2003plan/fy03fhwa plan.pdf. Accessed Oct. 22, 2012.

Fontaine, M. D., and B. L. Smith. 2007. Investigation of the Performance of Wireless Location Technology-Based Traffic Monitoring Systems. Journal of Transportation Engineering, March, pp. 157-165.

Gal, I. 2002. Adults' Statistical Literacy: Meanings, Components, Responsibilities. International Statistical Review, Vol. 70, No. 1.

Gaver, D. 1968. Headstart Strategies for Combating Congestion. Transportation Science, Vol. 2, No. 2, pp. 172-181.

Governors Highway Safety Association. 2012. Cell Phone and Texting Laws. http://www.ghsa.org/html/stateinfo/laws/cellphone_laws .html. Accessed Oct. 12, 2012.

Hendrickson, C., and E. Plank. 1984. The Flexibility of Departure Times for Work Trips. Transportation Research 18A, pp. 25-36.

Hicks, B., and M. Carter. 2000. What Have We Learned About Intelligent Transportation Systems? Chapter 3: What Have We Learned About ITS? Arterial Management. FHWA, U.S. Department of Transportation, Washington, D.C.

Higgins, L., A. Nelson, T. Geiselbrecht, and B. Ullman. 2013. Understanding the Decision-Making Process for Drivers Faced with Lane Restrictions or Closures on Wisconsin Highways. WisDOT ID No. 0092-11-15. Wisconsin Department of Transportation, Sheboygan, Wis.

Houston TranStar. 2012. Houston TranStar Traffic Map. http://traffic .houstontranstar.org/layers. Accessed Oct. 12, 2012.

Houston TranStar. 2010. Houston TranStar 2010 Annual Report. The Houston TranStar Consortium, Houston, Tex.

INRIX. 2009. INRIX Delivers Real-Time Access to 10,000 Traffic Cameras. http://www.inrix.com/pressrelease.asp?ID=66. Accessed Oct. 23, 2012.

INRIX. 2012a. INRIX Triples Real-Time Traffic Flow Coverage in North America to Over 160,000 Miles. http://www.inrix.com/pressrelease .asp?ID=68. Accessed Oct. 23, 2012.
INRIX. 2012b. Public Sector Solutions. http://www.inrix.com/solutions public.asp. Accessed Oct. 23, 2012.

INRIX Traffic Pro. 2010. Jan. 7. http://www.navigadget.com/index .php/2010/01/07/inrix-traffic-pro. Accessed Oct. 23, 2012.

Jackson, W., and J. Jucker. 1982. An Empirical Study of Travel Time Variability and Travel Choice Behavior. Transportation Science, Vol. 16, pp. 460-475.

Jenelius, E., L.-G. Mattsson, and D. Levinson. 2011. Traveler Delay Costs and Value of Time with Trip Chains, Flexible Activity Scheduling and Information. Transportation Research Part B, Vol. 45, pp. 789-807. http://rational.ce.umn.edu/Papers/Traveller_disruptions_costs.pdf. Accessed June 17, 2014.

Khattak, A., Y. Yim, and L. Stalker. 2003. Willingness to Pay for Travel Information. Transportation Research 11C, pp. 137-159.

Knoblauch, R., M. Nitzburg, R. Seifert, H. McGee, and K. Daily. 1995. Uniform Traffic Control and Warning Messages for Portable Changeable Message Signs. FHWA, U.S. Department of Transportation, Washington, D.C.

Kothuri, S. M., K. Tufte, H. Hagedorn, R. L. Bertini, and D. Deeter. 2007. Survey of Best Practices in Real Time Travel Time Estimation and Prediction. Compendium of Technical Papers, Institute of Transportation Engineers, District 6 Annual Meeting. Institute of Transportation Engineers, Portland, Ore.

Lam, T. C., and K. A. Small. 2001. The Value of Time and Reliability: Measurement from a Value Pricing Experiment. Transportation Research Part E (Pergamon Press, Elsevier, Inc.), Vol. 37, pp. 231-251.

Lerner, N., J. Singer, and R. Huey. 2004. Animation and Color in Changeable Message Signs Use for Traffic Control Device Applications. FHWA, U.S. Department of Transportation, Washington, D.C.

Lerner, N., J. Singer, E. Robinson, R. Huey, and J. Jennes. 2009. Driver Use of En Route Real-Time Information. FHWA, U.S. Department of Transportation, Washington, D.C.

Li, Z. 2010. Embedding Risk Attitudes in a Scheduling Model: Application to the Study of Commuting Departure Time. Transportation Science, Vol. 46, No. 2, pp. 170-188.

Liu, H. X., A. Danczyk, R. Brewer, and R. Starr. 2008. Evaluation of Cell Phone Traffic Data in Minnesota. In Transportation Research Record: Journal of the Transportation Research Board, No. 2086, Transportation Research Board of the National Academies, Washington, D.C., pp. 1-7.

Luoma, J., and P. Rama. 2001. Comprehension of Pictographs for Variable Message Signs. Traffic Engineering and Control, Feb.

Mannering, F., and M. Hamed. 1990. Occurrence, Frequency, and Duration of Commuters' Work-to-Home Departure Delay. Transportation Research 24B, pp. 99-109.

Martin, S. 2009a. Waze-Driver Generated Mapping Program. May 19. http://www.gpslodge.com/archives/026315.php. Accessed Oct. 23, 2012.

Martin, S. 2009b. INRIX: I-95 Corridor Coalition Confirms Accuracy. Feb. 17. http://www.gpslodge.com/archives/024605.php. Accessed Oct. 23, 2012.

Martin, S. 2007. JVC Exits GPS/PND Market. May 18. http://www .gpslodge.com/archives/011290.php. Accessed Oct. 23, 2012.

Montgomery, D. 2009. Will GPS-enabled Smartphones Avoid Handset Slowdown? Jan.21.http://smartphone.biz-news.com/news/2009/01/ 21/0009. Accessed Oct. 23, 2012.

NAVTEQ. 2012. Navteq. http://www.navteq.com/. Accessed Oct. 23, 2012.

Ngo, D. 2009. Navigon GPS iPhone App to Get Live Traffic. Sept. 16. http://reviews.cnet.com/8301-19512_7-10354874-233.html. Accessed Oct. 23, 2012.

NHTSA. 2012. Distracted Driving. http://www.distraction.gov/. Accessed Oct. 23, 2012. 
Niccolai, J. 2007. Nokia Buys Navtec Mapping Service for \$8.1 Billion. Oct. 1. http://www.pcworld.com/article/137887/nokia_buys_navtec_ mapping_service_for_81_billion.html. Accessed Oct. 23, 2012.

Noland, R., and K. Small. 1995. Travel Time Uncertainty, Departure Time Choice, and the Cost of the Morning Commutes. Transportation Research Record, Vol. 1493, pp. 150-158.

Paling, J. 2003. Strategies to Help Patients Understand Risks. British Medical Journal, Vol. 327, pp. 745-748.

Peirce, S., and J. Lappin. 2004. Why Don't More People Use Advanced Traveler Information? Evidence from the Seattle Area. Research and Innovative Technology Administration, Bureau of Transportation Statistics. Presented at 83rd Annual Meeting of the Transportation Research Board, Washington, D.C. http://ntl.bts.gov/lib/jpodocs/ repts_te/14004_files/14004.pdf. Accessed Oct. 11, 2012.

Peng, Z. R., D. Yu, and E. Beimborn. 2002. Transit User Perceptions of the Benefits of Automatic Vehicle Location. In Transportation Research Record: Journal of the Transportation Research Board, No. 1791, Transportation Research Board of the National Academies, Washington, D.C., pp. 127-133.

Perk, V., J. Flynn, and J. Volinski. 2008. Transit Ridership, Reliability, and Retention. National Center for Transit Research, University of South Florida, Tampa, Fla.

Phoenix Tightens Travel Time Estimates. 2008. TrafficTechnology Today.com. July 25. http://www.traffictechnologytoday.com/news .php?NewsID=7061. Accessed Oct. 22, 2012.

Polak, J. 1987. A More General Model of Individual Departure Time Choice. PTRC Summer Annual Meeting, Proceedings of Seminar C.

Price, M., R. Cameron, and P. Butow. 2007. Communicating Risk Information: The Influence of Graphical Display Format on Quantitative Information Perception-Accuracy, Comprehension and Preference. Patient Education and Counseling, Vol. 69, pp. 121-128.

Privat, L. 2009. Traffic Cast to Power Tom Tom Connected PND in the U.S. April 1. http://www.gpsbusinessnews.com/TrafficCast-to-powerTomTom-connected-PND-in-the-U-S_a1434.html. Accessed Oct. 23, 2012.

Privat, L. 2008. Magellan Consumer Sold to MiTAC. Dec. 15. http://www .gpsbusinessnews.com/Magellan-consumer-sold-to-MiTAC_a1232 .html. Accessed Oct. 23, 2012.

PRR, Inc. 2005. WSDOT 511 IVR Survey and Usability Testing Results. http://www.wsdot.wa.gov/NR/rdonlyres/596DD86C-340E-4B108660-22805F9AE10E/0/511Survey.pdf.

Ramsey, D. 2009. New iPhone Traffic App Delivers Personalized Traffic Reports to California Commuters. Feb. 17. http://www.physorg.com/ news154117861.html. Accessed Oct. 23, 2012.

Rao, L. 2009. Aha Mobile Launches New Version of Traffic and Road Entertainment iPhone App. Sept. 30. http://www.techcrunch.com/2009/ 09/30/aha-mobile-launches-new-version-of-traffic-and-roadentertainment-iphone-app/. Accessed Oct. 23, 2012.

Real-Time System Management Information Program. 2010. http://www .gpo.gov/fdsys/pkg/FR-2011-07-19/html/2011-17986.htm. Accessed Oct. 23, 2012.

RITA. 2012a. Deployment Statistics: 2004-2007 Deployment Tracking Survey Results. http://www.itsdeployment.its.dot.gov/itsprevious years/. Accessed Oct. 23, 2012.

RITA. 2012b. ITS Standards Program. http://www.standards.its.dot .gov/. Accessed Oct. 23, 2012.

RITA. 2012c. Knowledge Resources-Lessons Learned. http://www.its lessons.its.dot.gov/its/benecost.nsf/Lesson?OpenForm\&61C6488E EAB07920852576320051D9F0^Home. Accessed Oct. 23, 2012.

Roizen, B. 2009. How Will Aha Mobile Make Money? Dec. 3. http://vator .tv/news/show/2009-12-03-how-will-aha-mobile-make-money. Accessed Oct. 23, 2012.
Rutgers Department of Transportation. 2012. On-Time Performance Stats. Rutgers, The State University of New Jersey, New Brunswick, N.J. http://parktran.rutgers.edu/ontimeschedule.shtml. Accessed Oct. 12, 2012.

San Antonio TransGuide. 2012. http://www.transguide.dot.state.tx.us/ITS_ $\mathrm{WEB} /$ Frontend/default.html? $\mathrm{r}=\mathrm{SAT} \& \mathrm{p}=\mathrm{San} \% 20$ Antonio $\& \mathrm{t}=\mathrm{dms}$. Accessed Oct. 22, 2012.

Schafer, R.-P., K.-U. Thiessenhusen, E. Brockfeld, and P. Wagner. 2002. Analysis of Travel Times and Routes on Urban Roads by Means of Floating Car Data. Presented at European Transport Conference 2002, Homerton College, Cambridge, England.

Schouten, W., M. van Lieshout, and W. Spit. 1998. VMS in the Polder: The Emergence of a Standardized Modular Information System to Control, Support and Promote Initiatives for VMS in Holland in a Flexible Way. Proc., Ninth International Conference on Road Transport Information and Control, IEE, London, pp. 222-226.

Schrank, D., T. Lomax, and B. Eisele. 2011.2011 Annual Urban Mobility Report. Texas A\&M Transportation Institute, College Station, Tex.

Schwartz, J. 2009. People's Misperceptions Cloud Their Understanding of Rainy Weather Forecasts. EurekAlert. April 14. http://www.eurekalert .org/pub_releases/2009-04/uow-pmc041409.php. Accessed Oct. 11, 2012.

Seattle Area Travel Times. 2012. Washington State Department of Transportation. http://www.wsdot.com/traffic/Seattle/traveltimes/ 95reliable.aspx. Accessed Oct. 12, 2012.

Senbil, M., and P. Kitamura. 2004. Reference Points in Departure Time Choice: A Prospect Theoretic Test of Alternative Decision Frames. Journal of Intelligent Transportation Systems, Vol. 8, No. 1, pp. 19-31.

Small, K. 1982. The Scheduling of Consumer Activities: Work Trips. American Economic Review, Vol. 72, No. 3, pp. 467-479.

Small, K., R. Noland, X. Chu, and D. Lewis. 1999. Valuation of Travel Time Savings and Predictability in Congested Conditions for Highway User-Cost Estimation. NCHRP Report 431.

Small, K., C. Winston, and J. Yan. 2005. Uncovering the Distribution of Motorists' Preferences for Travel Time and Reliability. Econometrica, Vol. 73, pp. 1367-1382.

Task Group 09. 2009. VMS Harmonisation in Europe. Conference of European Directors of Roads, Paris.

Techie-Menson, J. P. 2001. (GRIPs), Will They Work in the United Kingdom? Traffic Engineering and Control, September.

Teigen, K. H. 1988. The Language of Uncertainty. Acta Psychologica, Vol. 68, pp. 27-28.

Texas A\&M Transportation Institute. 2012. Anonymous Wireless Address Matching for Traffic Information. http://ttihouston.tamu.edu/blue tooth/projects.aspx. Accessed Oct. 23, 2012.

Texas A\&M Transportation Institute with Cambridge Systematics, Inc. 2006. Travel Time Reliability: Making It There On Time, All the Time. FHWA, U.S. Department of Transportation, Washington, D.C. http://ops.fhwa.dot.gov/publications/tt_reliability/brochure/index .htm. Accessed Oct. 11, 2012.

Tilahun, N., and D. Levinson. 2010. Contacts and Meetings: Location, Duration and Distance Traveled. TRB 89th Annual Meeting Compendium of Papers, Paper No. 10-3912, Washington, D.C.

Traffax Inc. 2012. The BluFax Concept. http://www.traffaxinc.com/ content/blufax-concept. Accessed Oct. 23, 2012.

Traffic Vizzion. 2012. http://www.vizzion.com/index.htm. Accessed Oct. 23, 2012.

TrafficCast. 2012. BlueTOAD. http://www.trafficcast.com/products/ view/blue-toad/. Accessed Oct. 23, 2012.

TrafficGauge, Inc. 2012. http://www.trafficgauge.com/cell_blackberry .html. Accessed Oct. 23, 2012. 
Transit App Gallery. 2012. http://www.citygoround.org/apps/. Accessed Oct. 23, 2012.

Travel Midwest Stats. 2012. http://www.travelmidweststats.com/. Accessed Oct. 22, 2012.

Tryhorn, C. 2009. Nice Talking to You ... Mobile Phone Use Passes Milestone. The Guardian, March 2. http://www.guardian.co.uk/ technology/2009/mar/03/mobile-phones1. Accessed Oct. 23, 2012.

TSC Staff. 2006. Navteq Buys Traffic.com. Nov. 6. http://www.thestreet .com/story/10320080/navteq-buys-trafficcom.html. Accessed Oct. 23, 2012.

University of California, Berkeley. 2012. Mobile Millennium. http:// www.traffic.berkeley.edu/. Accessed Oct. 23, 2012.

Vickery, W. 1963. Pricing in Urban and Suburban Transport. American Economic Review, Vol. 53, No. 2, pp. 452-465.

Vochin, A. 2009. Navigon Exits the North American PND Market. May 5. http://gadgets.softpedia.com/news/Navigon-Exits-the-NorthAmerican-PND-Market-2687-01.html. Accessed Oct. 23, 2012.

Volvo. 2006. Volvo 'IDIS' Helps the Driver Avoid Distractions in Busy Situations. Nov. 1. Volvo IDIS Helps the Driver Avoid Distraction in Busy Situations. Accessed Oct. 23, 2012.

Wallsten, T. S., D. V. Budescu, R. Zwick, and S. M. Kemp. 1993. Preferences and Reasons for Communicating Probabilistic Information in Verbal or Numerical Terms. Bulletin of the Psychonomic Society, Vol. 31, pp. 135-138.
Washington Times. 2009. States Send Traffic Tweets to Motorists. Sept. 22. http://www.washingtontimes.com/news/2009/sep/22/states-sendtraffic-tweets-to-motorists/. Accessed Oct. 23, 2012.

Wasson, J. S., J. R. Sturdevant, and D. M. Bullock. 2008. Real-Time Travel Time Estimates Using Media Access Control Address Matching. ITE Journal, June, pp. 20-23.

Westwood One. 2009. Westwood One's Metro Traffic Goes Live with the Most Comprehensive Video Traffic Information Network in the Country. Feb. 25. http://corporate.trafficland.com/library/ww1_vds.pdf. Accessed Oct. 23, 2012.

Wunderlich, K. 1996. An Assessment of Pre-trip and En Route ATIS Benefits in a Simulated Regional Urban Network, 3rd World Congress on Intelligent Transportation Systems.

Wunderlich, K. 2011. Integrated Corridor Management (ICM) Program: Calculating Multi-Modal Corridor Performance Measures from Simulation Outputs. 18th ITS World Congress, Orlando, Florida.

Wunderlich, K., J. A. Bunch, and J. J. Larkin. 1999. ITS Impacts Assessment for Seattle MMDI Evaluation: Modeling Methodology and Results. Final Report for FHWA. Mitretek Systems, McLean, Virginia. http:// ntl.bts.gov/lib/jpodocs/repts_te/11323.pdf. Accessed June 17, 2014.

Zeis, A. 2009. Traffic.com Releases Free App for Blackberry. Oct. 22. http:// crackberry.com/free-traffic-com-app. Accessed Oct. 23, 2012.

Zhang, L., and D. Levinson. 2006. Determinants of Route Choice and Value of Traveler Information: Field Experiment. TRB 85th Annual Meeting Compendium of Papers, Paper No. 06-1714, Washington, D.C. 


\section{Glossary}

95th percentile trip time: Time identified for a specific travel route that indicates how bad delay will be on the heaviest travel days; $95 \%$ of trips along this route will take no more than this time to complete.

average trip time: Time identified for a specific travel route that is the average or statistical mean of all recorded trip times along the route over a specified time period (e.g., over 1 month or 1 year).

buffer index (BI), mean-based: The difference between the 95th percentile travel time and the average travel time, normalized by the average travel time.

buffer index, median-based: The difference between the 95th percentile travel time and the median travel time, normalized by the median travel time.

buffer time: The time that is calculated by multiplying the average trip time for a specific travel route by the buffer index. failure/on-time measures, median-based: Percentage of trips with travel times less than $1.1 \times$ median travel time and/or $1.25 \times$ median travel time.

failure/on-time measures, speed-based: Percentage of trips with travel times less than $50 \mathrm{mph}, 45 \mathrm{mph}$, and/or $30 \mathrm{mph}$. Speed is the space-mean speed over the study section.

misery index (modified): The average of the top 5\% worst travel times divided by the free-flow travel time.

planning time indices: 95 th, 90 th, and 80 th percentile travel times divided by the free-flow travel time.

skew statistic: The ratio of (90th percentile travel time minus the median) to (the median minus the 10th percentile).

utility function: A measure of absolute or relative satisfaction or dissatisfaction, expressed as the value or cost per defined unit. 


\section{APPENDIX A}

\section{General Literature Review}

\section{Overview}

In cities where congestion in the transportation system is commonplace, drivers are accustomed to the congestion; they expect and plan for some increase in travel time, particularly during peak driving times. Many system users either adjust their schedules to avoid peak hours or budget extra time to allow for unexpected traffic congestion or incidents. However, problems arise when travel times are much higher than anticipated. Most travelers are less tolerant of unexpected travel time increases because those longer travel times cause travelers to be late for work or important meetings, to miss appointments, or to incur extra child-care fees. Moreover, unexpected delays in the transportation of goods by a freight carrier or shipper can result in disruption in just-in-time delivery and manufacturing processes and can cause the carrier or shipper to lose money and a competitive edge (Texas A\&M Transportation Institute with Cambridge Systematics, Inc. 2006).

Transportation professionals most commonly discuss travel time reliability in terms of historical average travel times calculated over periods of a year or longer, as illustrated in Figure A.1. A typical definition for travel time reliability is the following:

The consistency or dependability in travel times, as measured from day to day or across different times of the day.

Most travelers do not experience the same average travel time each day, however. As shown in Figure A.2, travelers experience and remember something much different than the average throughout a year of commutes. Their travel times may vary greatly from day to day, and they remember the few bad days they suffered through unexpectedly longer travel times. Research has shown that travel time reliability information can provide transportation system users with a more complete picture of the expected travel time along a particular route. The challenge is how to communicate that reliability information effectively to road and transit system users so that they understand it clearly.

Another example illustrating travel time reliability is shown in Figure A.3, which shows travel time data from a major commuter route in Seattle, Washington. Without congestion along the route, travel times are about $12 \mathrm{~min}$ (e.g., see President's Day in Figure A.3). On all other weekdays, the average travel time is $18 \mathrm{~min}$. But when traffic incidents and weather combine to cause unexpected congestion, travel times may be 25 min or more, or $39 \%$, longer than usual. Commuters who travel this route must plan for this variability if they want to arrive on time. If they plan their commute on the basis of the average travel time, they will be late half the time and early the other half of the time. In other words, commuters have to build in a time cushion or buffer to their trip planning to account for the variability. If they build in a buffer, they will arrive early on some days. That is not necessarily a bad thing, but the extra time is still carved out of their day-time they could be using on pursuits other than commuting.

\section{Travel Time Reliability Metrics}

The measurement of travel time reliability is an emerging practice. However, a few measures appear to have technical merit and are thought to be easily understood by nontechnical audiences. Most of these measures compare high travel time days with average travel time days. Four recommended measures are 90th or 95th percentile travel time, buffer index, planning time index, and frequency with which congestion exceeds an expected threshold (Texas A\&M Transportation Institute with Cambridge Systematics, Inc. 2006).

The 90th or 95th percentile travel time is a time identified for a specific travel route and indicates how bad the delay will be on the heaviest travel days (Texas A\&M Transportation Institute with Cambridge Systematics, Inc. 2006). These travel times are reported in minutes and are thought to be easily understood by commuters familiar with their trips. Therefore, 


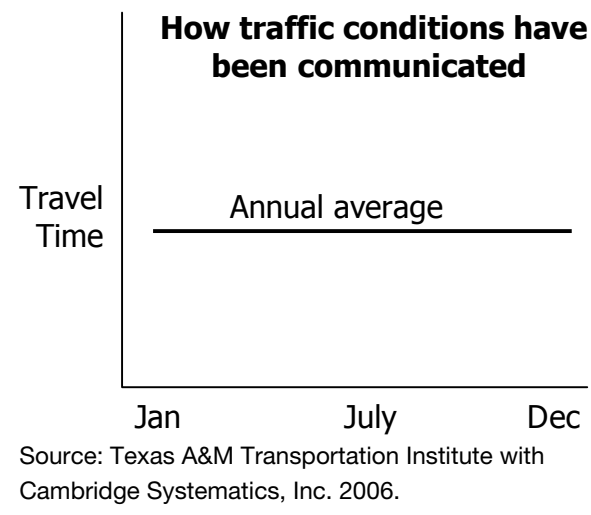

Figure A.1. Average travel time used by professionals.

this measure is ideally suited for traveler information. It has the disadvantage of not being easily compared across trips, because most trips will have different lengths. Nor can this measure be used to easily combine route or trip travel times into a subarea or citywide average.

Two indices that enable comparisons or combinations of routes or trips with different lengths are the buffer index and the planning time index. The buffer index represents the extra time cushion (or buffer) that most travelers add to their average travel time when planning trips to account for unforeseen

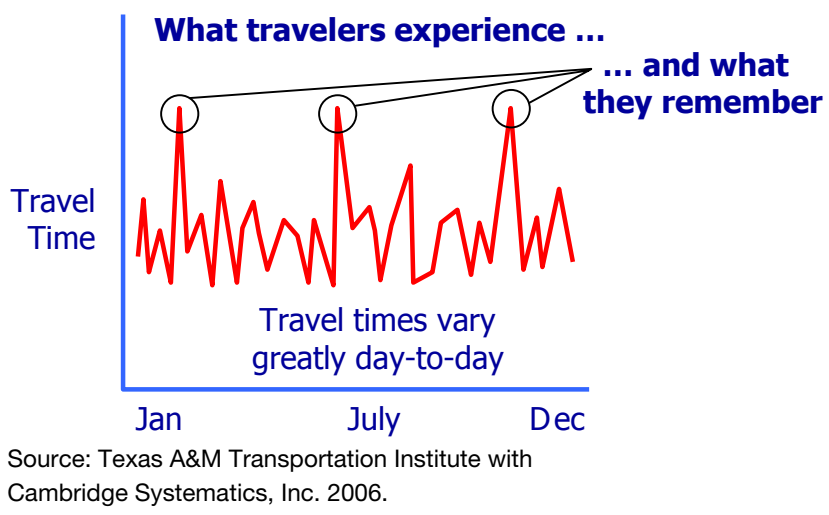

Figure A.2. Traveler travel time experiences.

delays and to ensure on-time arrival (Texas A\&M Transportation Institute with Cambridge Systematics, Inc. 2006). The buffer index is expressed as a percentage and its value increases as reliability gets worse. For example, a buffer index of $40 \%$ means that, for a 20-min average travel time, a traveler should budget an additional $8 \mathrm{~min}(20 \mathrm{~min} \times 40 \%=8 \mathrm{~min})$ to ensure on-time arrival most of the time. In this example, the eight extra minutes is called the buffer time. The buffer index is computed as the difference between the 95th percentile travel time and average travel time, divided by the average travel time.

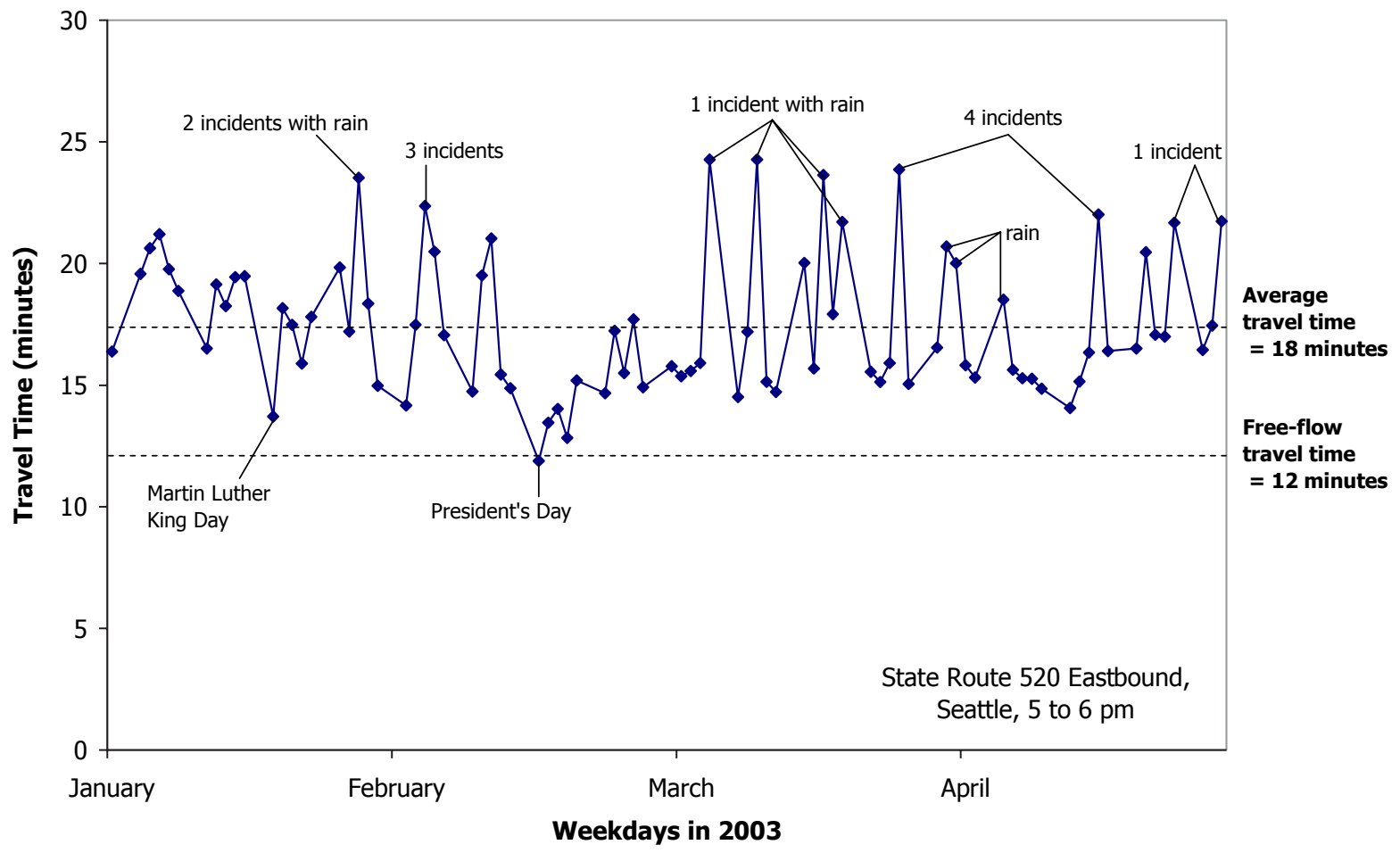

Source: Cambridge Systematics, Inc. with Texas Transportation Institute 2005.

Figure A.3. Example of commuters planned trips based on the worst days, not the average day. 
Table A.1. Recommended Reliability Performance Metrics from SHRP 2 Project LO3

\begin{tabular}{|c|c|c|}
\hline Reliability Performance Metric & Definition & Unit \\
\hline Buffer index (BI), mean-based & $\begin{array}{l}\text { The difference between the 95th percentile travel time and the average travel time, } \\
\text { normalized by the average travel time }\end{array}$ & Percent \\
\hline Buffer index, median-based & $\begin{array}{l}\text { The difference between the 95th percentile travel time and the median travel time, } \\
\text { normalized by the median travel time }\end{array}$ & Percent \\
\hline Failure or on-time measures, median-based & $\begin{array}{l}\text { Percentage of trips with travel times less than } 1.10 \times \text { median travel time and/or } 1.25 \times \\
\text { median travel time }\end{array}$ & Percent \\
\hline Failure or on-time measures, speed-based ${ }^{a}$ & Percentage of trips with travel times less than $50 \mathrm{mph}, 45 \mathrm{mph}$, and/or $30 \mathrm{mph}$ & Percent \\
\hline Misery index (modified) & The average of the top $5 \%$ worst travel times divided by the free-flow travel time & None \\
\hline Planning time indices & 95th, 90th, and 80th percentile travel times divided by the free-flow travel time & None \\
\hline Skew statistic & $\begin{array}{l}\text { The ratio of (90th percentile travel time minus the median) to (the median minus the } \\
\text { 10th percentile) }\end{array}$ & None \\
\hline
\end{tabular}

a Speed is the space-mean speed over the study section.

Source: Cambridge Systematics, Inc. (2007a).

The planning time index represents the total travel time that a traveler should expect or plan on when an adequate buffer time is included (Texas A\&M Transportation Institute with Cambridge Systematics, Inc. 2006). The planning time index differs from the buffer index in that it includes typical delay as well as unexpected delay. Thus, the planning time index compares near-worst-case travel time to a travel time in light or free-flow traffic. For example, a planning time index of 1.60 means that, for a 15-min trip in light traffic, the total time that should be planned for the trip is $24 \min (15 \mathrm{~min} \times$ $1.60=24 \mathrm{~min}$ ). The planning time index is useful because it can be directly compared with the travel time index (a measure of average congestion) on similar numeric scales. The planning time index is computed as the 95th percentile travel time divided by the free-flow travel time.

From a data perspective, continuous travel time data is the only way to establish reliability patterns empirically. Although predictive methods (e.g., the SHRP 2 L03 project Analytic Procedures for Determining the Impacts of Reliability Mitigation Strategies) may be used in a reliability monitoring system when the data are unavailable, only continuously collected travel time data can produce the actual travel time distribution from which all reliability metrics are derived. For example, the reliability metrics being used in the SHRP 2 L03 project, as shown in Table A.1, are all derivatives of the statistical distribution of travel times.

At present, transportation experts do not agree on the terms to be used or what the mathematical calculations of each term should be. If transportation professionals can't come to consensus on the technical terms, then the general public certainly will not do so. The purpose of the L14 project is to determine what terms the layperson uses to refer to travel time reliability concepts and to encourage the use of those terms in communications with transportation system users.

\section{Importance of Travel Time Reliability}

Travel time reliability is significant to many transportation system users, whether they are vehicle drivers, transit riders, freight shippers, or even air travelers. Good and consistent system reliability is a valuable service that can be provided on privately operated and publicly operated highways alike. Because reliability is so important to transportation system users, transportation planners, operators, and decision makers should consider travel time reliability a key performance measure.

\section{Travel Time Reliability and Highway Travel}

Travel time reliability is valuable to traffic professionals because it better quantifies the benefits of traffic management and operation activities than simple averages. For example, consider a typical before-and-after study that attempts to quantify the benefits of an incident management or ramp metering program. The improvement in average travel time may appear modest, as shown on the left side of Figure A.4. However, reliability measures will show a much greater improvementas illustrated on the right side of Figure A.4-because they show the effect of improving the worst few days of unexpected delay and will be much more meaningful to the transportation system users.

For drivers, travel time reliability information can be valuable when they are selecting a route. For example, the value of travel time reliability was assessed through a mail survey, trip diaries, and loop-detector data by Lam and Small (2001) soon after the first high-occupancy/toll (HOT) lane opened on State Route 91 in Riverside, California. The researchers found that, for women in this study, the value of travel time reliability was actually higher than simple travel time information. 

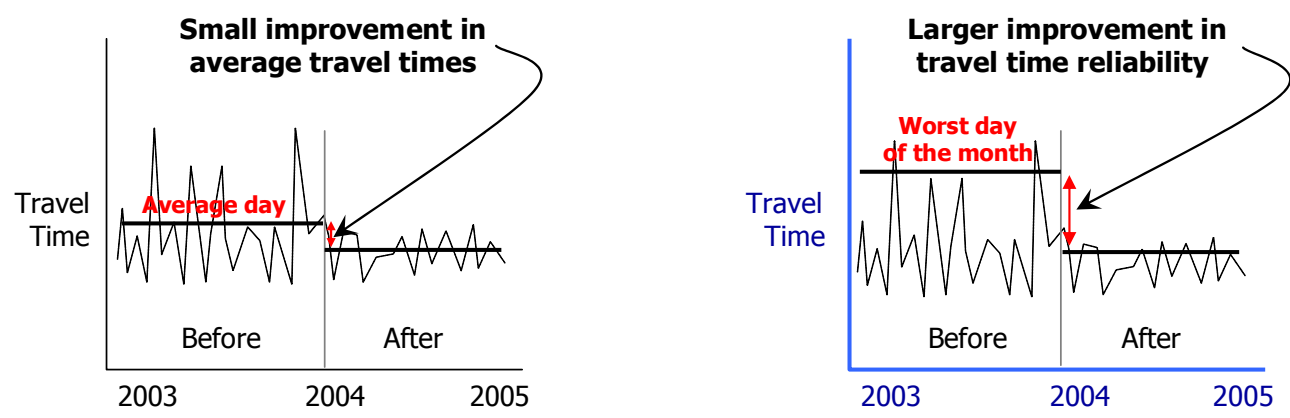

Source: Texas A\&M Transportation Institute with Cambridge Systematics, Inc. 2006.

Figure A.4. Reliability measures capture the benefits of traffic management.

For men, the value of time was roughly $50 \%$ higher than the value of reliability information. The reasons for this difference were not clear from the data collected, though some have interpreted the data to indicate that women have more time critical commitments related to child-care trips. For this study, the researchers defined travel time as the 90th percentile travel time minus the median travel time. The authors discuss further how the transponder usage records of participants show that few drivers habitually used the HOT lane. Rather, people made the decision whether to pay for the HOT lane on a daily basis depending on trip purpose and traffic conditions. In applications such as HOT lanes, travel time reliability information may be useful en route to help drivers make the purchase decision to use the HOT lanes. The influence of pre-trip and en route travel information on route decisions has been demonstrated in other studies: An evaluation of the Washington State DOT's 511 travel information system in 2005 found that $21 \%$ of respondents changed their original travel plans on the basis of information they got from the 511 system (PRR, Inc. 2005). Drivers on an Orlando, Florida, toll road who stated that they used information from the state's 511 service or from dynamic message signs (DMSs) that displayed estimated delay times for the road were more likely to change their route in response to unexpected congestion.

A review of research on travel time and travel time reliability conducted by the Center of Urban Transportation Research (University of South Florida) includes the finding that most travelers value trip time reliability at least as much as actual trip time. In fact, when travelers' arrival and departure times were inflexible because of the nature of the trip, the value of reliability was as much as three times that of trip time (Concas and Kolpakov 2009).

\section{Travel Time Reliability and Transit}

Studies of transit ridership have shown that trip time reliability (including the reliability of a rider's wait time at transit stops) is more important to retaining riders than the trip and waiting times themselves. Wait-time reliability is particularly important, as transit riders tend to perceive time spent waiting for a transit vehicle as being longer than an equivalent amount of time spent riding in the vehicle. Real-time information that allows transit riders to schedule their own arrival at a transit stop and/or to monitor the wait time remaining until the vehicle's arrival increases rider confidence in the service (Perk et al. 2008). Transit passengers surveyed in two cities ranked knowledge of when their bus would arrive and knowledge that it would arrive on time as the two most important factors affecting their decision to ride transit (Peng et al. 2002).

\section{Travel Time Reliability and Freight}

In terms of economic value, reliability is probably more important to freight carriers and shippers than to personal travelers. With the rise in just-in-time deliveries (largely as a replacement for extensive warehousing), providing dependable (reliable) service has become extremely valuable while failure to provide dependable service can increase costs considerably (Cambridge Systematics, Inc. 2007a). For example, improvements in transportation reliability play an important role in reducing inventory in the chemical supply chain for freight shippers. Because of the many nodes in the supply chain, upwards of one-third of all chemical inventory is in transit at any point in time. Inventory managers keep safety or buffer supplies to cushion against variability of inbound arrivals, and the amount of safety supplies increases with the degree of unreliability and the number of stocking locations (Cambridge Systematics, Inc. 2007a). However, the capacity to receive chemical supplies is limited by the size of the liquid storage silos. Balancing capacity with demand is a challenge. As transportation reliability decreases, wait time, dead freight, and cost increases (Cambridge Systematics, Inc. 2006).

\section{Real-Time Travel Information: State of the Practice}

Real-time travel time messages have been in use in the United States for well over a decade, ever since traffic monitoring and integration systems became available and reliable. The most commonly used media for these messages are dynamic message 
signs (DMSs) and transportation agency websites; but the widespread use of cell phones and other mobile devices is prompting a growing number of transportation agencies and providers to offer real-time updates on transportation conditions and options via e-mails, text messages, and Twitter feeds.

Real-time travel time estimates are most often provided for a particular roadway segment or a particular transit route on the basis of recent travel speeds or conditions. Some agencies also provide travel time comparisons among two or more routes/roadways to help travelers make decisions about the route or transportation mode to take. Most recent and most rare are the information sources that advise travelers about travel time reliability - that is, the likelihood that the estimated travel time for a particular trip or trip segment will be dependable. The following subsections describe some of the real-time travel information messages that are being provided to travelers on DMSs, on websites, and via mobile devices, as well as some of the lessons learned about providing travel information.

\section{Dynamic Message Signs}

Two Department of Transportation surveys of state and local agencies in 2007 found that incident reports were the most common form of real-time traffic information provided to travelers in large metropolitan areas in the United States, followed by travel times and then travel speeds (U.S. Government Accountability Office 2009). Dudek reported in 2008 that travel time information was displayed on DMSs by 18 departments of transportation (DOTs) in the United States. He cited two primary reasons why travel time information was not displayed on CMSs by some state DOTs: (1) infrastructure or software was not available and (2) congestion was not a problem (Dudek 2008).

The Georgia DOT began posting travel time messages on DMSs in 1998, using the qualitative descriptors "moving very well," "moving well," "moving slowly," and "moving very slowly." Responding to requests from Georgia drivers for more precise terminology, the Georgia DOT used its travel management software (NaviGAtor) to generate approximate travel times along roadway segments. Drivers now see a threeline message: the name of a destination (such as a highway exit), the distance to that destination in miles, and a travel time range based on the average speed along the roadway segment. The Georgia DOT does not post travel times for distances greater than 15 miles, because the accuracy of the time range decreases at greater distances (Dudek 2008). Other signs in the Atlanta region alert drivers that travel time information can be obtained by dialing 511 anywhere in the state (NaviGAtor 511 Real-Time Traffic Map 2012).

DMSs of the type developed for the Georgia DOT are also being used by the Oregon and Tennessee DOTs. The Oregon DOT provides travel time estimates on highways in the
Portland area using travel time ranges such as "12-15 MIN" (FHWA 2005a). Ranges of travel times are also used by Tennessee's SmartWay intelligent transportation system in the Nashville and Knoxville areas (FHWA 2005b), by the Illinois State Toll Highway Authority on Chicago-area highways (FHWA 2005c), and by the Texas DOT in the San Antonio area (FHWA 2005d).

Travel time ranges are just one way to express the travel time reliability of a highway segment. A California PATH program study in 2009 found that most commuters surveyed (71\%) preferred that travel time estimates be displayed as an exact number of minutes rather than as a range of minutes (29\%) (Ban et al. 2009). A similar preference was found among Arizona commuters. On the basis of commuter feedback, the Arizona DOT changed the format of its travel time estimation signs to provide "to the minute" precision rather than 5-min estimation windows (Phoenix Tightens Travel Time Estimates 2008).

A study of DMS messaging performed by Battelle for the FHWA in 2004 recommended including the distance in miles along with travel time. The study found that distance information is particularly useful to travelers who are unfamiliar with the area and enables them to mentally estimate the amount of delay from the distance coupled with the estimated travel time (PBS\&J 2004).

Real-time travel time messaging tends to be most effective on a road on which travel times are likely to change with reasonable frequency. If travel times are too static, drivers tend to view the messages as static rather than dynamic and therefore less credible (Meehan 2005). This "freshness factor" may hold true for travel time reliability information as well. Some agencies such as Houston TranStar provide a time stamp (e.g., "Travel time - to US 59-6 min at 10:10") to their travel time signs and web-based information to assure users that the information is current (Houston TranStar 2012a).

Messages on DMSs on highways in the United Kingdom (UK) change from travel time estimates (number of miles and minutes to given destinations under normal traffic flow conditions) to travel delay descriptions and estimates (e.g., "Segment name - Accident - 15 minutes delay") in response to roadway incidents. Delay time estimates are based on "typical traffic profiles" of individual road segments according to time of day and known traffic generators (Traffic England Traffic Map 2012).

Some agencies have started to show comparative travel times to certain destinations via different routes. The Washington State DOT recently installed new travel time signs in the Seattle area showing side-by-side travel time estimates for two different routes to a common destination (Washington State DOT 2012a). Signs such as this would be natural places to add information about travel time reliability along the two routes. Signs showing comparative travel times in generalpurpose and HOT lanes are another location where travel 
time reliability could be added and could prove useful to motorists making route decisions during a trip. Consideration must be given, however, to the amount of information that drivers can read while passing a sign. The 2009 DMS study recommended that travel times for HOV lanes and general-purpose lanes not be provided together on one sign, as this could result in too much information for drivers to process during the time they have to look at the sign (Ban et al. 2009). This recommendation may be mitigated by other conclusions of the study, including the finding that travelers who see travel time messages on DMSs on their regular route can begin to anticipate elements of the messages and therefore read and understand them in less time than they would otherwise need (Ban et al. 2009).

The first travel time signs in use for a managed lane facility were on a HOT lane along I-15 in San Diego. Research has shown that users consistently overestimate their travel time savings (Brownstone and Small 2009). For this reason, agencies have been reluctant to post travel times in managed lanes for fear that, when actual comparative travel times are shown, drivers might not choose to use the managed lane. Other research has shown, however, that drivers value the trip time reliability offered by managed lanes (Lam and Small 2001).

The Long Island Expressway uses the words Average Travel Time on their signs showing travel times to multiple destinations along a single route (see Figure A.5). However, the original request to FHWA to deviate from the Manual on Uniform Traffic Control Devices showed signs with the term Estimated Travel Time (FHWA 2004).

The presentation of travel time is not limited to highways and highway travel. The Wisconsin DOT provides highway travel times to specified destinations via the freeway on selected

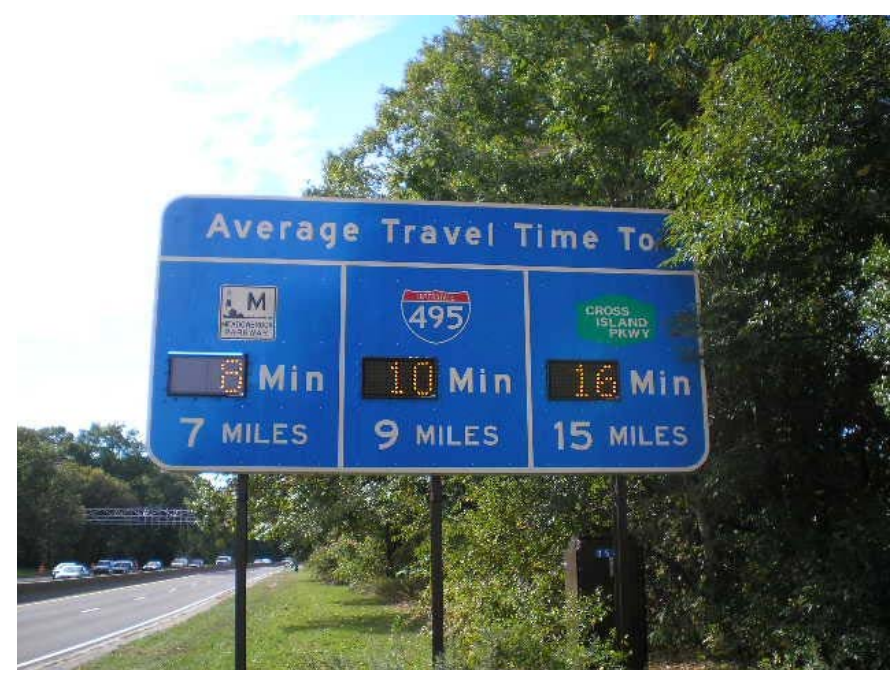

Source: FHWA 2004.

\section{Figure A.5. Northern state parkway signs on Long Island.}

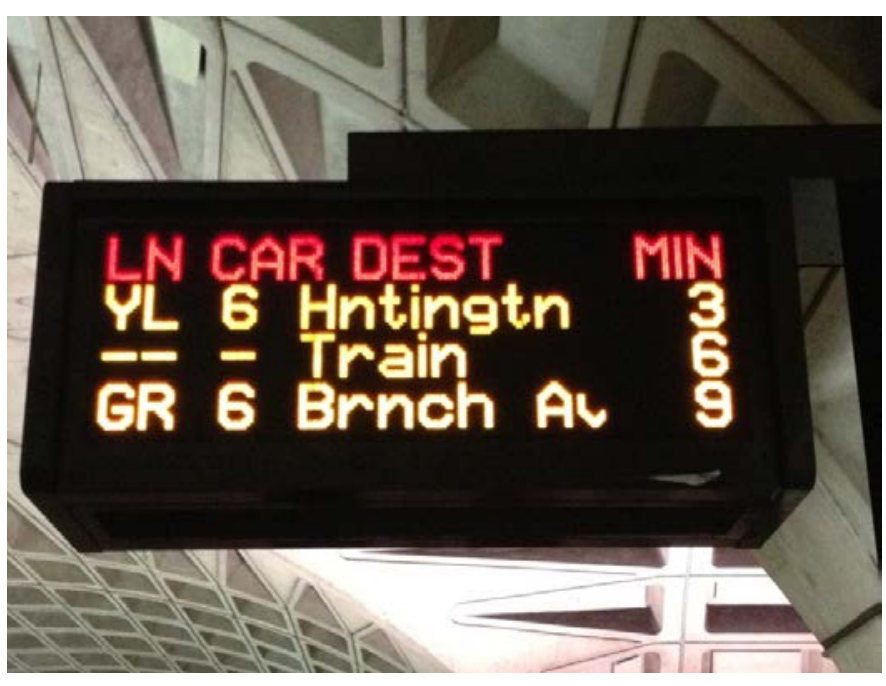

Source: TTI.

\section{Figure A.6. Next-train arrival sign at a Washington, D.C., Metro station.}

arterials before freeway entrance ramps to provide drivers with information to make route choices (Peng et al. 2004). A DMS pilot program in the San Francisco Bay Area provides travelers with both highway and Caltrain (transit) travel times to selected destinations, along with the arrival time of the next train (Mortazivi 2009).

Real-time bus and train arrival information is available in increasing numbers of U.S. cities, posted on DMS at transit centers and on transit websites. Some transit providers also provide real-time notifications about route delays and diversions. A real-time train arrival sign at one of Washington, D.C.'s, Metro stations is shown in Figure A.6.

Real-time arrival signs tend to be viewed positively by transit customers. Customer surveys conducted by transit agencies in the United States and abroad found that real-time arrival information at transit stops made riders feel more confident, particularly at night, and even improved riders' overall perception of the quality of transit service provided (Schweiger 2003).

\section{Travel Websites}

Many state DOT transportation management centers (TMCs) and partner transportation agencies provide users with realtime travel information via websites. The format and features of these websites vary considerably. For example, the California Department of Transportation (Caltrans) reproduces the travel time information displayed on DMS on Los Angeles area freeways via its TMC website (California Department of Transportation District 7 2012).

The Tennessee SmartWay website (Tennessee Department of Transportation 2012) and the Utah DOT CommuterLink 
website (Utah Commuterlink 2012) both provide real-time travel information to online users by posting real-time photos of the travel time DMS signs, as well as color-coded highway maps showing road conditions (hazardous, patches of ice/snow, flooded), traffic flow, incident and construction locations and descriptions, and real-time camera views of highway locations. The UK highways website features the same types of information and also provides advance notification of future construction sites and expected future events (such as holiday travel) that are likely to affect roadway conditions and traffic speeds (Traffic England Traffic Map 2012).

The roadway information website provided by the Ontario, Canada, Ministry of Transportation displays a similar traffic map, with green, yellow, and red traffic flow categories labeled "moving well (75 km/h and above)," "moving slowly (40 to $75 \mathrm{~km} / \mathrm{h}$ )," and "very slow (less than $40 \mathrm{~km} / \mathrm{h}$ )" (Ontario Ministry of Transportation 2012).

Travel time reliability information is starting to make appearances on transportation websites. The Wisconsin DOT website provides a table of current and "normal" travel times for Milwaukee-area highways. Travel times that are $20 \%$ or more above normal are shown in bold print (Kothuri et al. 2007). The Washington State DOT provides a similar trip time table, also including times for the same roadway segments if the HOV lane is used (Washington State DOT 2012b).

The travel information website for the Gary-ChicagoMilwaukee corridor also displays a table of current and average travel times and traffic speeds for highways along the corridor (RoadStats, LLC 2012). The user can click on the average travel time number for each segment to view a graph detailing the most recently collected travel time, the average travel time for all historical data samples, and the normal range of travel time values by time period over a 24 -hour period each day. The graph also includes three speed thresholds, indicating what the travel time would be for the segment with no traffic congestion (traffic moving at $55 \mathrm{mph}$ or higher), with moderate traffic congestion ( $54 \mathrm{mph}$ to $35 \mathrm{mph}$ ), and with heavy traffic congestion (34 mph to $15 \mathrm{mph}$ ) (RoadStats, LLC 2012).

The Washington State DOT has recently added a feature to its travel time website that displays 95th percentile travel times (Washington State DOT 2012a). A user enters an original-destination pair from a drop-down menu containing names of suburbs, and the system displays a text message as shown in Figure A.7. The Driving Times feature on the San Francisco Bay Area's 511 website also allows users to enter the origin and destination of their driving trip; the website then generates multiple potential routes for the trip, displaying the current and typical/historical trip times for each route, along with a table of minimum, maximum, and average current traffic speeds (and typical historical speed) on each of the route's roadway segments (see Figure A.8). The site's Predict-a-Trip feature allows users to view the typical traffic speeds and travel times of the same route options for some future trip by entering the day and time period (511 SF Bay 2012).

Many airlines now provide on-time performance histories for particular flight numbers and times that can be viewed by customers making online reservations. In addition, thirdparty websites compile information from multiple airlines and airports to provide estimates, or forecasts, about a flight's on-time performance. The FlightCaster website tracks both current delays and historical on-time performance for U.S. domestic flights to estimate a specific flight's departure time; six delay factors are also shown on the forecast, with colorcoded icons to signal potential problems (FlightCaster 2012). Similarly, the FlightStats website shows historical on-time performance information for airline routes using the named categories on-time, late, very late, excessive, cancelled, and diverted along with the percentage of flights in each category. The percentages are also shown on a bar graph (FlightStats 2012).

\section{WSDOT}

\section{Best Time To Leave}

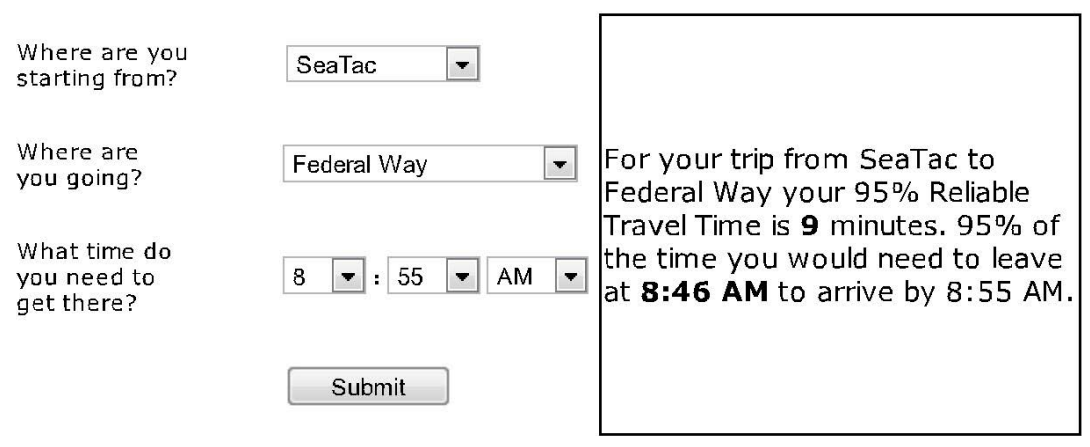

Figure A.7. Travel time reliability display from Washington State DOT website. 


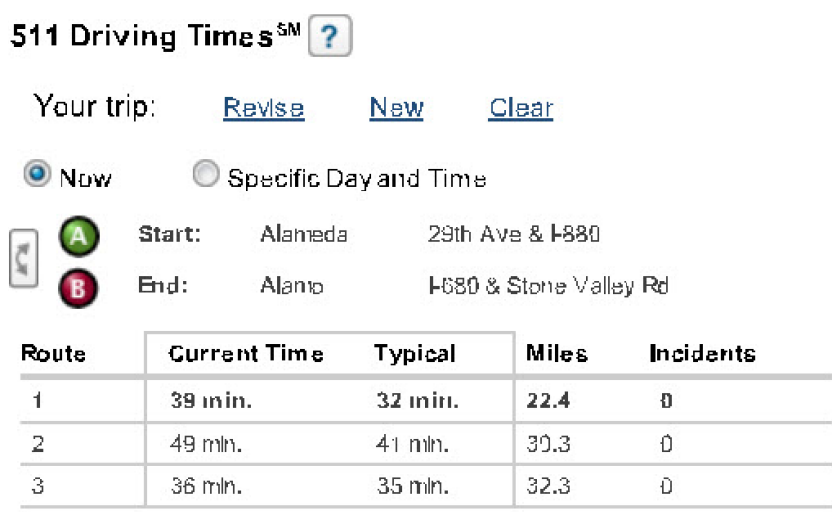

\begin{tabular}{|c|c|c|c|}
\hline Route 1 & Eurrent Speed & Typical & lhaidehts \\
\hline $18 B 0 \mathrm{~N}$ & $42 \mathrm{mph}+$ & 43 mpht & $\sqrt{3}$ \\
\hline 1-980 E & 58 mph+ & 56 moh+ & ] \\
\hline $\mathrm{C} 4-24 \mathrm{E}$ & $57 \mathrm{miph+}$ & 55 riplit & $\eta$ \\
\hline GA-24 E-t580 S Ranp & 50 niplit & 55 molit & $\eta$ \\
\hline $1-6805$ & 27 mpht & 38 mplit & $\sqrt{2}$ \\
\hline
\end{tabular}

Source: 511 SF Bay 2012

Figure A.8. Driving times and traffic speeds for input origin-destination pair in San Francisco Bay Area.

Route-by-route reliability information is generated by many transit systems for planning purposes, but is only rarely provided as part of transit customer information. Rutgers University in New Jersey has posted on-time performance history information for its bus routes, including percentages for on-time, early, and late arrivals (Rutgers Department of Transportation 2012). More transit systems may follow, especially if traveler demand for this information grows. Evidence of increasing demand for transit reliability information includes the provision of a performance dashboard for TriMet transit routes in the Portland region (TriMet 2012).

\section{E-mails, Texts, Tweets: Mobile Device Messaging}

In addition to accessing the Caltrans website for travel times in the Los Angeles area, motorists may also subscribe to a free service that provides the same information on their mobile device. Similarly, Houston TranStar offers free, personalized e-mail alerts to its system users regarding incidents and travel times on Houston area freeways. The alerts can be sent to any device capable of receiving e-mail or text messages, including personal computers, mobile phones, personal digital assistants, and text pagers (Houston TranStar 2012b). A similar messaging service is provided by the Regional Transportation Commission of Southern Nevada's Freeway and Arterial System of Transportation (FAST) program (Regional Transportation
Commission of Southern Nevada 2012). The Arkansas State Highway Department has begun using Twitter to notify motorists about highway conditions (Arkansas State Highway and Transportation Department 2012).

The Washington Metropolitan Area Transit Authority's (WMATA) "e-alerts" provide information about service delays or disruptions on Washington, D.C.'s, Metrorail, MetroAccess paratransit services, and elevators at Metro's transit centers (Washington Metropolitan Area Transit Authority 2012). WMATA has also begun to broadcast these alerts via Twitter, though soon after the new medium was adopted, subscribers realized they were receiving only partial messages. The partial messages were caused by limitations on Twitter message length; the longer messages that had been developed for an e-mail format were being truncated. WMATA is looking for ways to provide the same information to its Twitter subscribers using shorter messages (Hohmann 2009).

The Bay Area Rapid Transit system in San Francisco provides real-time service information to its passengers via its mobile website (for those with access to an Internet connection), emailed and text-messaged service advisories, and most recently via Twitter updates (Rhodes 2009). Boston's “T Alerts" provide the same service for passengers on Massachusetts Bay Transportation Authority buses and trains (Massachusetts Bay Transportation Authority 2012).

\section{Communicating Reliability Information: Other Research}

The challenge with conveying travel time reliability information to the user is ensuring that they understand the message. Cognitive science has shown that most people are not good at understanding statistical concepts and applying them to everyday situations such as medical diagnoses, gambling odds, and variability in stochastic processes such as traffic (Gal 2002). Statistical literacy is related to overall aptitude with numbers, literacy, and cultural components. Research has shown significant cultural differences in understanding statistical concepts, and those related to risk in particular (Wright et al. 1978).

Communicating probabilities or risks using only qualitative language can lead to misunderstandings, simply because the listener (or reader) may ascribe a different meaning to a descriptive word than was intended. The English language has a multitude of terms for concepts of uncertainty and risk, but attempts to systematically map them to numerical probabilities have failed (Teigen 1988). Research has shown that people switch between numerical (e.g., 50-50 chance) and verbal (e.g., probably) in unpredictable ways controlled more by grammar than by probability values (Wallsten et al. 1993). In one study, tests of various probability terms (e.g., certainly, 
definitely, possibly, probably, rarely) with adolescents and young adults indicated that individual definitions of the terms were not consistent enough to convey information effectively to the general public. Absolute numbers, such as percentages or percentage ranges, were recommended instead of qualitative language (Biehl and Halpern-Felsher 2001).

Some suggestions and recommendations for communicating risk and probability to the public come from two nontransportation fields: weather forecasting and medicine. Although most people are familiar with weather forecasts on television and in other media, the probabilities used in those forecasts (e.g., 20\% chance of rain) are not widely understood. In a study comparing several weather report formats, $43 \%$ of participants correctly interpreted a weather forecast that included symbolic icons depicting a weather condition (such as rain) and graphs showing the percent likelihood of that condition. When forecast information included graphs that showed the chance of rain AND the chance of "no rain," the number of participants correctly understanding the forecast rose to 52\% (Schwartz 2009). An experiment conducted with university students in the United Kingdom found that participants who were given a graph of forecast temperatures that included information about the probability, or uncertainty, of those temperatures answered questions about the forecast more accurately than the participants who were given the temperature graph by itself (BBC News 2007).

A medical diagnosis or a decision about possible courses of treatment usually involves probabilistic data (the probability that a test result is accurate, the likelihood of various outcomes of a treatment). In a 2003 article for the British Medical Journal, several techniques were recommended for helping patients understand the risks and benefits associated with medical treatments:

- Avoid the use of purely descriptive terms; supplement qualitative language with numbers.

- Use a consistent denominator/numerical scale.

- Provide both positive and negative outcomes (e.g., $3 \%$ chance of negative outcome AND 97\% chance of positive outcome).

- Express probabilities as absolute numbers (75\% of cases have Outcome A, 25\% have Outcome B) rather than in relative terms (three times as many cases have Outcome A as have Outcome B ...).

- Use visual aids such as pie charts and graphs to illustrate probabilities (Paling 2003).

Studies examining both doctors' and patients' comprehension of probability-based information have found that many people understand frequencies (e.g., 19 out of 20) better than percentages or proportions ( $95 \%$ or 0.95 ). Presenting probabilities related to cancer screenings as a set of frequencies rather than as a set of percentages resulted in quicker and more accurate comprehension of those probabilities by study participants, particularly if several probabilities had to be considered in tandem (Hanoch 2004).

People presented with quantitative health risk information in pictograph formats perceived the information more accurately when it was presented in one compound graph (in which the proportions and percentages of the potential outcomes add up to $100 \%$ ) than when the same information was presented as two side-by-side graphs (Price et al. 2007).

The goal for establishing a lexicon to convey travel time reliability information is quite similar to that of establishing a lexicon to convey downstream traffic state information (i.e., what are traffic conditions downstream?) on a DMS, which TTI researchers addressed in the late 1970s. Through a series of standard and innovative laboratory experiments, researchers developed and tested numerous text and graphical representations of downstream traffic conditions (Dudek et al. 1978). Research results showed that traffic descriptors should be displayed only for unusual traffic conditions (e.g., due to an accident). Displaying traffic descriptors during normal, recurrent peak-period traffic conditions was discouraged. In addition, the acceptable ways of conveying traffic state information were different in small cities than in large cities (Dudek and Huchingson 1986).

As seen in the state-of-the-practice examples, a variety of terms are currently being used to describe travel times and the likelihood or reliability of those times. Average, historical, 95\% reliable, and typical are just some of the terms used, and they may have different meanings to drivers depending on the context in which they are used.

A variety of formats is also seen for the estimated travel times, as previously discussed. Early studies warned practitioners about the presentation of travel time information (whether in terms of actual times, delays, time saved, etc.) because of the potential for the information to be refuted by travelers and thus reduce credibility of the system with drivers. More recent research suggests that drivers recognize (to some degree) the inherent variability and potential for change in travel time information (Dudek et al. 2000). Furthermore, such variance does not reduce the information's credibility among drivers, nor does it reduce the desire for such information. As an example, both of the formats of travel time displays shown in Figure A.9 were equally understood to imply an approximate travel time that may not be exactly what is experienced by the driver reading that information at a particular point along the route. As mentioned previously, some drivers prefer the single time value format (e.g., $20 \mathrm{~min}$ ) over a range of times, even though they know that time may vary somewhat. 
TRAVEL TIME TO DOWNTOWN

AT 7:20 AM

20 MINUTES

TRAVEL TIME TO DOWNTOWN:

20-25 MINUTES

Source: Dudek and Huchingson 1991.

Figure A.9. Comprehended travel time displays.

\section{Bibliography}

511 SF Bay. 2012. http://traffic.511.org/. Accessed Oct. 12, 2012.

Arkansas State Highway and Transportation Department. 2012. Road Conditions. http://www.arkansashighways.com/roads/roads.aspx. Accessed Oct. 12, 2012.

Ban, X., Y. Li, and J. Margulici. 2009. Optimal Use of Changeable Message Signs for Displaying Travel Times. University of California, Berkeley, Calif.

BBC News. 2007. Predictable Forecast ... Probably? BBC News. Oct. 29. http://news.bbc.co.uk/2/hi/programmes/more_or_less/7067003 .stm. Accessed Oct. 11, 2012.

Biehl, M., and B. Halpern-Felsher. 2001. Adolescents' and Adults' Understanding of Probability Expressions. Journal of Adolescent Health, Vol. 28, pp. 30-35.

Brownstone, D., and K. Small. 2009. Valuing Time and Reliability: Assessing the Evidence from Road Pricing Demonstrations. Transportation Research Part A: Policy and Practice, Vol. 39, No. 4, pp. 279-293.

California Department of Transportation District 7. 2012. Travel Times Information. http://www.dot.ca.gov/dist07/travel/travel_times/index .php. Accessed Oct. 29, 2012.

Cambridge Systematics, Inc. 2007a. SHRP 2 Project L03: Analytic Procedures for Determining the Impacts of Reliability Mitigation Strategies. Transportation Research Board of the National Academies, Washington, D.C.

Cambridge Systematics, Inc. 2007b. Identification of the Reliability Performance Metrics to Be Used. Transportation Research Board of National Academies, Washington, D.C.

Cambridge Systematics, Inc. 2006. AASHTO Freight Transportation Bottom Line Report: Freight Demand and Logistics. AASHTO, Washington, D.C.

Cambridge Systematics, Inc., with Texas Transportation Institute. 2005. Traffic Congestion and Reliability: Trends and Advanced Strategies for Congestion Mitigation. FHWA, U.S. Department of Transportation, Washington, D.C. http://ops.fhwa.dot.gov/congestion_report/ congestion_report_05.pdf. Accessed Oct. 22, 2012.

Concas, S., and A. Kolpakov. 2009. Synthesis of Research on Value of Time and Value of Reliability. Center for Urban Transportation Research, University of South Florida, Tampa, Fla.

Dudek, C. L. 2008. Changeable Message Sign Displays During Non-Incident, Non-Roadwork Periods. NCHRP, Transportation Research Board, National Research Council, Washington, D.C.
Dudek, C. L., and R. D. Huchingson. 1991. Use of Changeable Message Signs for Displaying Travel Time and Other Time-Related Messages. Dudek \& Associates, Bryan, Tex.

Dudek, C. L., and R. D. Huchingson. 1986. Manual on Real-Time Motorist Information Displays. FHWA, U.S. Department of Transportation, Washington, D.C.

Dudek, C. L., R. D. Huchingson, R. J. Koppa, and M. L. Edwards. 1978. Human Factors Requirements for Real-Time Motorist Information Displays. FHWA, U.S. Department of Transportation, Washington, D.C.

Dudek, C., N. Trout, S. Booth, and G. L. Ullman. 2000. Improved Dynamic Message Sign Messages and Operations. Texas A\&M Transportation Institute, College Station, Tex.

FHWA. 2005a. Travel Time Messaging on Dynamic Message SignsPortland, OR. U.S. Department of Transportation, Washington, D.C. http://ops.fhwa.dot.gov/publications/travel_time_study/portland/ portland_ttm.htm. Accessed Oct. 22, 2012.

FHWA. 2005b. Travel Time Messaging on Dynamic Message SignsNashville, TN. U.S. Department of Transportation, Washington, D.C. http://ops.fhwa.dot.gov/publications/travel_time_study/nashville/ nashville_ttm.htm. Accessed Oct. 22, 2012

FHWA. 2005c. Travel Time Messaging on Dynamic Message SignsChicago, IL. U.S. Department of Transportation, Washington, D.C. http://ops.fhwa.dot.gov/publications/travel_time_study/chicago/ chicago_ttm.htm. Accessed Oct. 22, 2012.

FHWA. 2005d. Travel Time Messaging on Dynamic Message SignsHouston, TX. U.S. Department of Transportation, Washington, D.C. http://ops.fhwa.dot.gov/publications/travel_time_study/houston/ houston_ttm.htm. Accessed Oct. 22, 2012.

FHWA. 2004. Request for Interpretation: 2-55(I)-CMS Display of Travel Time. Manual on Uniform Traffic Control Devices (MUTCD). U.S. Department of Transportation, Washington, D.C. May 17. http://mutcd.fhwa.dot.gov/resources/interpretations/pdf/2_551 .pdf. Accessed Oct. 22, 2012.

FlightCaster. 2012. FlightCaster Inc. http://www.flightcaster.com/. Accessed Oct. 12, 2012.

FlightStats. 2012. Historical On-time Performance Rating. FlightStats, Inc. http://www.flightstats.com/go/FlightRating/flightRatingBy Route.do. Accessed Oct. 29, 2012.

Gal, I. 2002. Adults' Statistical Literacy: Meanings, Components, Responsibilities. International Statistical Review, Vol. 70, No. 1.

Hanoch, Y. 2004. Improving Doctor-Patient Understanding of Probability in Communicating Cancer-Screening Test Findings. Journal of Health Communication, Vol. 9, pp. 327-335.

Hohmann, J. 2009. Metro Tweets Far from Short and Sweet (or Decipherable). Washington Post, Aug. 21. http://www.washingtonpost .com/wp-dyn/content/article/2009/08/20/AR2009082004250_pf .html. Accessed Oct. 29, 2012.

Houston TranStar. 2012a. Houston TranStar Dynamic Message Signs. http://traffic.houstontranstar.org/dms/dms_by_roadway.html. Accessed Oct. 12, 2012.

Houston TranStar. 2012b. Houston TranStar Traffic Alerts. http://traffic .houstontranstar.org/trafficalert/. Accessed Oct. 12, 2012.

Kothuri, S., K. Tufte, H. Hagedorn, R. L. Bertini, and D. Deeter. 2007. Survey of Best Practices in Real Time Travel Time Estimation and Prediction. Compendium of Technical Papers, Institute of Transportation Engineers, District 6 Annual Meeting. Institute of Transportation Engineers, Portland, Ore.

Lam, T., and K. A. Small. 2001. The Value of Time and Reliability: Measurement from a Value Pricing Experiment. Transportation Research Part E, Vol. 37, pp. 231-251.

Massachusetts Bay Transportation Authority. 2012. Welcome to T-Alerts. http://www.talerts.com/. Accessed Oct. 12, 2012. 
Meehan, B. 2005. Travel Times on Dynamic Message Signs. ITE Journal, Vol. 75, No. 9, pp. 23-27.

Mortazivi, A. E. 2009. Commuter Travel Time Information System: Displaying Transit Messages on Changeable Message Signs. Presented at 88th Annual Meeting of the Transportation Research Board (CD-ROM). Washington, D.C.

NaviGAtor 511 Real-Time Traffic Map. 2012. Georgia Department of Transportation. http://www.georgia-navigator.com/realtimetraffic .html. Accessed Oct. 22, 2012.

Ontario Ministry of Transportation. 2012. Interactive Map. http://whttp:// www.mto.gov.on.ca/english/traveller/trip/map.shtml?ll=43 $.696424,-79.459648 \& z=10$. Accessed Oct. 29, 2012.

Paling, J. 2003. Strategies to Help Patients Understand Risks. British Medical Journal, Vol. 327, pp. 745-748.

PBS\&J. 2004. Amber, Emergency, and Travel Time Messaging Guidance for Transportation Agencies. FHWA, U.S. Department of Transportation, Washington, D.C. http://www.ops.fhwa.dot.gov/TravelInfo/ resources/cms_rept/cmspractices.pdf.

Peng, Z., N. Guequierre, and J. C. Blakeman. 2004. Motorist Response to Arterial Variable Message Signs. In Transportation Research Record: Journal of the Transportation Research Board, No. 1899, Transportation Research Board of the National Academies, Washington, D.C., pp. 55-63.

Peng, Z., D. Yu, and E. Beimborn. 2002. Transit User Perceptions of the Benefits of Automatic Vehicle Location. In Transportation Research Record: Journal of the Transportation Research Board, No. 1791, Transportation Research Board of the National Academies, Washington, D.C., pp. 127-133.

Perk, V., J. Flynn, and J. Volinski. 2008. Transit Ridership, Reliability, and Retention. National Center for Transit Research, University of South Florida, Tampa, Fla.

Phoenix Tightens Travel Time Estimates. 2008. TrafficTechnologyToday .com. July 25. http://www.traffictechnologytoday.com/news.php? NewsID=7061. Accessed Oct. 22, 2012.

Price, M., R. Cameron, and P. Butow. 2007. Communicating Risk Information: The Influence of Graphical Display Format on Quantitative Information Perception-Accuracy, Comprehension and Preference. Patient Education and Counseling, Vol. 69, pp. 121-128.

PRR, Inc. 2005. WSDOT 511 IVR Survey and Usability Testing Results. http://www.wsdot.wa.gov/NR/rdonlyres/596DD86C-340E-4B108660-22805F9AE10E/0/511Survey.pdf. Accessed Oct. 11, 2012.

Regional Transportation Commission of Southern Nevada. 2012. Freeway Traffic Alerts. http://www.rtcsnv.com/planning-engineering/ freeway-arterial-system-of-transportation-fast/freeway-traffic-alerts/. Accessed Oct. 12, 2012.

Rhodes, S. 2009. Survey Finds Strong Demand for BART Mobile Applications. Bay Area Rapid Transit. Feb. 20. http://www.bart.gov/news/ articles/2009/news20090126.aspx. Accessed Oct. 29, 2012.

RoadStats, LLC. 2012. Travel Midwest Stats. GCM Travel Website. http://www.travelmidweststats.com/. Accessed Oct. 22, 2012.
Rutgers Department of Transportation. 2012. On-Time Performance Stats. Rutgers, The State University of New Jersey, New Brunswick, N.J. http://parktran.rutgers.edu/ontimeschedule.shtml. Accessed Oct. 12, 2012.

Schwartz, J. 2009. People's Misperceptions Cloud Their Understanding of Rainy Weather Forecasts. EurekAlert. April 14. http://www .eurekalert.org/pub_releases/2009-04/uow-pmc041409.php. Accessed Oct. 11, 2012.

Schweiger, C. 2003. Real-Time Bus Arrival Information Systems: A Synthesis of Transit Practice. Transportation Research Board, Washington, D.C.

Tailor, P. 2006. Journey Time Reliability and Network Monitoring. Highways Agency, Department for Transport, London.

Teigen, K. 1988. The Language of Uncertainty. Acta Psychologica, Vol. 68, pp. 27-28.

Tennessee Department of Transportation. 2012. TDOT SmartWay. http://www.tdot.state.tn.us/tdotsmartway/. Oct. 12, 2012.

Texas A\&M Transportation Institute with Cambridge Systematics, Inc. 2006. Travel Time Reliability: Making It There On Time, All the Time. FHWA, U.S. Department of Transportation, Washington, D.C. http://ops.fhwa.dot.gov/publications/tt_reliability/brochure/ index.htm. Accessed Oct. 11, 2012.

Traffic England. 2012. UK Highways Agency. http://www.trafficengland .com/index.aspx. Accessed Oct. 12, 2012.

Traffic England Traffic Map. 2012. UK Highways Agency. http://www .trafficengland.co.uk/map.aspx?isTrafficAlert=true\&lat=53.357971 \&lon=-2.333874. Accessed Oct. 22, 2012.

TriMet. 2012. Performance Dashboard. TriMet. http://trimet.org/about/ dashboard.htm. Accessed Oct. 29, 2012.

U.S. Government Accountability Office. 2009. Surface Transportation: Efforts to Address Highway Congestion Through Real-Time Traffic Information Systems Are Expanding but Face Implementation Challenges. U.S. Government Accountability Office, Washington, D.C. http://www.gao.gov/assets/100/96486.pdf. Accessed Oct. 12, 2012.

Utah Commuterlink. 2012. Utah Department of Transportation. http:// www.utahcommuterlink.com. Accessed Oct. 12, 2012.

Wallsten, T., D. V. Budescu, R. Zwick, and S. M. Kemp. 1993. Preferences and Reasons for Communicating Probabilistic Information in Verbal or Numerical Terms. Bulletin of the Psychonomic Society, Vol. 31, No. 2, pp. 135-138.

Washington Metropolitan Area Transit Authority. 2012. Alerts \& Advisories. http://www.wmata.com/rider_tools/metro_service_status/rail_ Bus.cfm?. Accessed Oct. 12, 2012.

Washington State Department of Transportation. 2012a. Seattle Area Travel Times. http://www.wsdot.com/traffic/Seattle/traveltimes/ 95reliable.aspx. Accessed Oct. 12, 2012.

Washington State Department of Transportation. 2012b. Best Time To Leave. http://www.travelmidweststats.com/. Accessed Oct. 29, 2012.

Wright, G., L. D. Phillips, P. C. Whalley, G. T. Choo, K. Ng, I. Tan, et al. 1978. Cultural Differences in Probabilistic Thinking. Journal of Cross-Cultural Psychology, Vol. 9, No. 3, pp. 285-299. 


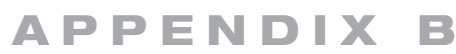 \\ Telephone Interview Guide for Expert Interviews}

\section{Contact Information}

Contact Person:

Company/Agency: Position:

Telephone Number:

Email:

Date \& Time of Survey:

\section{Introduction}

Hello. My name is and I am with the Texas Transportation Institute.

The Texas Transportation Institute is currently working on a SHRP 2 (Strategic Highway Research Program) project for the Transportation Research Board to recommend the best approaches for disseminating travel time reliability information to travelers.

One of the first steps in our study is to gather information about the state-of-practice of disseminating travel time and travel time reliability information. We are gathering the information via telephone interviews with a select group of private companies and public agencies regarding their experiences.

You are being contacted because you have been identified as the person in your company/agency who can help us with the information we are seeking.

If the contact expresses that they are not the correct person to talk to:

Can you please give me a different person in your company/agency that would be the right person to talk with about travel time reliability information?

Contact Person:

Position: Telephone Number:

Email:

Researcher Note: Anticipated Responses are indicated in Blue. Other instructions in Red.

(Researchers are trying to identify how the participating entity views travel time reliability and current state-of-the-practice. Focus should be on format and wording of this information.) 


\section{Practice, Definitions, and Users}

1. In your own words, how do you define travel time reliability (nontechnical definition)?

Let them express their definition before we get into technical perspectives below. Pay close attention to the specific words/phrases used by the participant.

Make sure they give a definition of travel time reliability rather than a definition for travel time.

Researchers may provide FHWA definition once they have responded to this question as a clarification on our use of the phrase of travel time reliability.

FHWA Definition: "The consistency or dependability in travel times as measured from day-to-day or across different times of day."

2. Do you see travel time reliability as a static (or historical) data projection or as near real-time data (changing with conditions at the time)?

"Static" - driven primarily by historical data (e.g., using 6 months of data to estimate the 95th percentile travel time)

"Real-time" - estimates that can change based upon conditions at the time the user requests the travel time information

Another way of stating this: Is the TT reliability information about the trip at this point in time or about how this trip "performs" all the time?

3. In your opinion, does travel time reliability information have value to the public or your customers? Yes/No

a. If "yes" - Explain what you believe the value to be.

b. If "no" - Why not?

Is there another form of information that is more valuable to the public or your customers?

4. In your opinion, does travel time reliability information have value to transportation operations staff and/or to public officials?

5. Do you currently disseminate travel time reliability information? Yes/No

If "no," has this type of information been considered for use by your company/agency? Yes/No

a. If “yes" - Why was this type of information not used?

b. If "no" - Is there a specific reason that your company/agency is not considering the use of travel time reliability information?

If “no" to Question \#5 - skip to Question \#15. If “yes" - continue with \#6.

6. What travel time reliability information do you provide to the public and/or to your customers?

Specific measures provided (95th percentile, "best/worst" estimate, some sort of confidence level, average condition with an additional attribute [increasing, decreasing, remaining the same], etc.) 
Be sure to identify the form and wording they are using for these measures. Ask for specific examples if possible (could be that they send you a document or plan).

7. What travel time reliability information do you provide to agency management or to public officials?

8. How is travel time reliability defined and computed for what is provided (as identified in Question \#5)?

Refer specifically to examples they have given to gain understanding of how they are defining reliability.

For example, is reliability simply a form of the confidence they have on the average conditions estimate or does it truly incorporate information from the last 6 months or year. It could also be a specific calculation method.

9. Who are your current users (or customers) and what are the primary uses of the travel time reliability information you provide?

Traveler information for typical commuters (software tools, text messages, maps), media outlets, etc.

If customer is not end user (or commuter) - Is it possible for me to contact your customer(s) to discuss further with them how they are using the provided travel time reliability information? Get Contact Information

\section{Applications}

10. How are you currently communicating travel time reliability information to your users (e.g., what media, format, phrasing, etc.)?

Software packages and/or trip planners for subscribers, text messages, etc.

Accessible via computer, phone, etc.

Include media methods such as social networking sites.

11. Do you communicate (i.e., phrase or format) travel time reliability information to your users differently based on the method of communication (for example: desktop computers vs. phone application)? Yes/No

If "yes," explain the differences (or changes)?

Specifically address the different communication tools identified in Question 10. Ask for examples to be provided (possibly through e-mail).

Identify if the information dissemination is a "push" (or automatic) system or a "pull" (user has to seek out the information) system.

Identify distinctions in the graphical user interface and the level-of-detail that can be provided for different screen sizes, etc. Also identify differences in wording based on space (character) limitations, etc.

12. If information distributed nationally - Does the form of communication or terminology you use change based on the area (region) it is being distributed to?

Example: the term XXXXX may be understood in New York but not in Houston.

Or Seattle may prefer a desktop application where Miami prefers a phone-in system. 
13. Can we get samples of the travel time reliability information you provide?

Examples: Website, software, subscription service, or e-mail.

14. What information are you providing if there is a catastrophe (e.g., unexpected road closures/failures, airport closure)?

Here we want to know how they handle unexpected system changes and "bad news" on the system. What do they communicate to users when experiencing extremely bad situations (relatively low/unknown reliability)?

15. Are you currently measuring your effectiveness of conveying travel time reliability information to users? Yes/No

16. If “yes" - What measures of effectiveness are used?

Examples: tracking of website hits and/or feedback, commuter or other customer feedback to office, customer satisfaction (possibly through a survey)

Might also ask feedback on the number of users signed up as a surrogate effectiveness measure.

\section{Future Directions and Challenges}

17. Do you think there is a market or use for providing travel time reliability information? Yes/No

18. If "yes" - What do you think this market is?

Business or planning applications, transit, general commuter

19. If " $n o$ " - What do you believe would need to change to create this market?

(i.e., what specific market forces at work?, expressed interest from a specific stakeholder(s)?, policy changes, at national or local level, etc.).

20. What is your agency's/company's short-term (e.g., the next year or next product cycle) vision for reporting travel time reliability to your users?

What would you like to provide? What might change? Recognize that private sector will be reluctant to discuss.

21. What is your agency's/company's long-term vision (beyond 5 years) for reporting travel time reliability to your users?

What would you like to provide? What might change? Recognize that private sector will be reluctant to discuss.

22. What are your agency's/company's challenges that keep you from "rolling out" travel time reliability according to your vision in the near-term (within five years) and beyond five years as identified in Questions \#14 and \#15?

Typical challenges include financial resources, data limitations, data screening/reduction, identifying user needs, satisfying the needs of users of different communication platforms.

\section{Additional Comments}

23. In your opinion, what are the potential impacts of providing effective travel time reliability information in terms of:

24. Customer satisfaction, 
25. Impact on travel choices, and/or

26. Impact on system performance and reliability?

27. Impact on management and public officials?

This will include their opinions and might be supported by feedback from users (commuters, media outlets), transit operators, transportation agency staff, others.

With public sector or transit participants identify good and bad impacts to their organization/agency.

28. Do you have any notable lessons learned that you would like to share related to estimating and/or communicating travel time reliability?

Where are the difficulties/challenges? What advice they might provide to others?

29. Do you have any additional comments or opinions that would help us understand the state-of-the-practice in reporting travel time reliability?

30. Are you aware of other agencies/companies with whom we should speak about travel time reliability information? Yes/No If “yes," do you have contact information for them?

\section{Closing}

Thank you for your time and your support of this research effort. If you have any questions regarding this study, please feel free to contact me at [provide phone \#] or the Principal Investigator for this project, Susan Chrysler, at 979-862-3928. 


\section{APPENOIX O \\ Detailed Focus Group Summary}

\section{Introductory Questions}

This appendix includes the slides and script used to conduct each of the focus groups, along with a summary of comments. The session began with the facilitator welcoming the group (Figure C.1) and reviewing the ground rules for the focus group, which are shown in Figure C.2.

After reviewing the ground rules, the participants introduced themselves using the prompts in Figure C.3. This gave the group a chance to relax, to get to know each other, and to become comfortable with participating in the discussion. They referenced their responses to the questionnaire they filled out when they arrived to give the facilitator an idea of the types of situations in which timeliness was important. The prompts and questions from the group facilitator follow as italicized headings.

\section{What causes travel time variation?}

Discussion began with a brainstorming activity that required participants to list factors and events that made them early or late to their destination. The facilitator created a list on the screen of everything that was said. After the initial list was generated, the facilitator pointed out the "day to day" or single occurrences and asked what things affected the time it took to make a regular or recurring trip. Responses initially focused on events such as accidents, weather, and personal factors. Once the focus shifted to repeating factors, the responses focused on time of day, traffic levels, events, and holidays. Once the focus of the discussion shifted to predictable events, the facilitator moved on to the next question.

\section{And how did you decide what time to leave to get here today?}

This question prompted a discussion about use of online mapping tools and navigation systems to help plan trips. Individuals were familiar with some type of online mapping tool and had used one at least once. A few participants even had the print-out with directions to the focus group location with them. Several participants stated that they used personal experience and familiarity with the area and main roads to determine their leaving time.

Overwhelmingly, participants stated they added additional time to starting travel time given to them by the system. They were not bothered by having to do this. Drivers relied heavily on personal experience; several typically assume an hour to get to a new destination. One participant said, "I always give myself 1 hour no matter where it is. There is nowhere I am going to go that's going to take me anywhere past an hour to get to so if it's a doctor's appointment, if it's a job interview (etc.), I give myself an hour." In Seattle, one participant said that the down side of arriving early is that she "could have done something else" with her time. Most acknowledged that there is a cost associated with arriving early but still prefer to be significantly early as opposed to late. The open-ended survey looked deeper into this additional time that drivers add and studied whether they followed trends. For example, do drivers always add the same amount of time to a trip, or do they add a time proportional to the estimated trip time? This questioning can help assess a driver's confidence in a TTR system.

For the most part, participants had not considered how MapQuest or another mapping tool calculated the estimated trip time. Some answered that it was a factor of distance and speed limit, while others thought someone had driven each route and recorded it.

The topic of accuracy was addressed next and participants were asked:

MapQuest tells you the same travel time all the time. Is travel time always the same at different times of day? Days of week?

At this point, participants began to think about recurring influences in terms of their individual trip. Differences in traffic at certain times of day and certain days of the week were discussed. Most groups said traffic was worse on Mondays and Fridays. Holidays, special events, and construction were all mentioned as well. Overall, the participants had a 


\section{Welcome!}

Thank you for participating in today's focus group conducted by

Texas Transportation Institute

Figure C.1. Welcome (Slide 1).

\section{Ground Rules:}

- Onlyone person should talk at a time

- Refrain from having side conversations

- Speak loudly and clearly

- Share your honest opinions (even negative)

- Turn off your cell phones

Figure C.2. Ground rules (Slide 2).

\section{Introductions:}

- First name

- Type of transportation you use during your commute

- 1-2 items from your questionnaire that you indicated were important to arrive on time to

- How long it took you to get here today

Figure C.3. Introductions (Slide 3). good understanding that traffic is not the same at every time of day or day of the week, and understood that many variables can affect traffic and the existing systems.

One participant spoke about uncertainty in general: "[It's] hard to guesstimate a time because every situation, every day is different. This weekend won't be like next and this Monday won't be like next Monday."

The open-ended survey expanded on this topic by using different terms and phrases to describe travel time and then asked participants how certain they were that they would get to work on time.

\section{Is MapQuest estimate of time accurate?}

When asked, participants said MapQuest times were not accurate; on the basis of their own experience, they added additional time to the estimate. Some even said they used two mapping sources, averaged them, and then added additional time. Drivers relied heavily on personal knowledge; several simply assume an hour to get to a new destination.

They overwhelmingly agreed that MapQuest and other existing mapping tools do not factor any of the variables, predictable or not, into their trip time calculations. In fact, most participants had never considered how MapQuest or another mapping tool calculated the estimated trip time. Some answered that it was a factor of distance and speed limit, while others thought someone had driven each route and recorded it.

\section{Terms to Identify Travel Time Variation}

The facilitator asked what words or phrases participants would use to describe the fluctuation in time it takes to make a trip. Participants brainstormed words and phrases out loud before being shown the slide in Figure C.4. Responses included unknown, approximately, unpredictable, variable, average traffic time, flexible travel time, additional, at least, and delay.

Next, participants were asked to identify terms that best fit in the sentences shown in Figure C.4. This exercise forced participants to use terms that would be tested in future studies and considered for the lexicon.

The first sentence in Slide 4_- "It will take__ 20 minutes to make your trip" - was intended to determine which words best describe trip time estimation. Responses varied greatly, but about, approximately, and at least were all mentioned in at least three of the cities. Other words or phrases used to complete this sentence were minimum, average, more than, over, roughly, usually, and estimated. This question was also included in the computer survey, and these responses from the focus groups helped form the multiple choice responses for the survey.

The second sentence in Slide 4 was written to gain insight into words participants use to describe a range of time. Terms 
1. It will take ______ 20 minutes to make your trip.

2. It will take 10 to 30 minutes to make your trip.

3. of the time it takes 20 minutes to make your trip.

\section{Figure C.4. Sentence completion (Slide 4).}

used to complete the sentence were about, approximately, between, around, on average, likely, anywhere from, somewhere between, usually, and ideally.

The third sentence participants were asked to complete was "___ of the time it takes 20 minutes to make you trip." Responses included the terms most, some, half, seldom, majority, often, a good portion, $75 \%$ of the time, and $1 / 8$ of the time.

\section{Travel Time Reliability System}

A hypothetical travel time reliability (TTR) system was introduced to participants as a "system that could make better predictions about your expected travel time but considering all the factors we listed." The explanation served to transition from factors that affect a trip to the value of travel time reliability information. The system was explained as one that

- Users could enter specific "to" and "from" destinations into.

- Would use more factors than just distance and speed limit.

- Would use sensors in the road to continuously gather data.

- Would have a record of weather conditions, road construction, and accidents.

Given this initial introduction, almost all participants said such a system would be valuable. However, as the discussion continued, participants began to express primary interest in real-time information. They did see a value for the historical reliability information in certain instances, primarily for people who were new in an area or traveled often. However, they didn't see it as a tool for daily use. In fact, one participant said that he would rather "focus on coordinating getting the information together with real time info than calculating the odds." To represent this suggested usage and to appeal to most drivers, researchers used this information to create the scenario in the open-ended survey that involved an unfamiliar, yet constrained trip.

Most participants felt that a travel time reliability system would be most useful before they began their trip. However, some felt it would be valuable during a trip, giving them the ability to change routes. Almost all saw the value of pre-trip planning and the cost involved in wasting time. They felt that online access through a website would be the most effective. However, they also mentioned other methods of accessing the information, such as smartphone applications and global positioning system (GPS) units. The desire to have this information available on mobile devices demonstrated that participants did not appreciate the distinction between historical and real-time data.

Participants seemed to believe that this system would be helpful in several specific situations. Evacuations, moving to new areas, buying a house, or general travel to unfamiliar areas were all mentioned as situations where they would possibly want access to historical information. Overwhelmingly, participants agreed that the system did not have any value when used for daily trips. Weekends seemed to be the time it would be utilized the least.

In Seattle, participants repeatedly discussed the idea that HOV lanes saved time. However, they never touched on the greater reliability of those lanes. When asked directly whether the HOV lane was faster or just less extreme, four people responded that it was simply less extreme. One person said that "information about the extremes of different lanes is useful" information.

Participants were still hesitant to consider that the time estimates produced by a travel time reliability system would be accurate. This reluctance made clear that education would be critical to the understanding and application of reliability information. Concern was expressed about taking valuable time to plan a trip in advance-only to begin the drive and encounter an incident that would have been realized with real-time information.

If the system provides a travel time based on a 95th percentile or worst-case situation, then drivers will need to be taught that they do not need to add the same buffer time as before. Given the widespread lack of trust in any system, such an education effort may be challenging; most participants did believe that a system based on historical data would be more accurate than the systems they currently use. Future tasks in this project assessed participants' understanding of the term 95th percentile as well as similar concepts and terms.

\section{System Input and Output}

The participants were shown an example of an input screen (Figure C.5) and then asked additional questions about the input and output options they would prefer. 
Input Screen Example

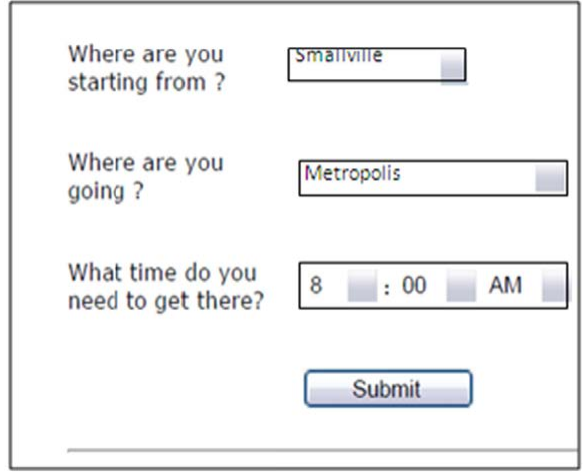

Figure C.5. Input screen (Slide 5).

What would you like to be able to input/personalize? Is "what time do you want to arrive" the right question?

What type of information would you like our new system to provide you with?

Do you want the system to tell you how sure it is of its estimate?

When asked what they would like to be able to input or personalize, participants came up with several additions to make the input screen more specific. All groups mentioned a more specific Origin/Destination entry box. Some wanted to be able to enter the zip codes, and some wanted to enter specific addresses in addition to the city. Although researchers noted that participants want flexibility in their input, they realize that most TTR system Origin/Destinations will be limited by the data available on the roadways. This is the case for the Houston field operational test.

Most drivers wanted the ability to choose between "What time are you leaving?" and "What time do you want to arrive?" Participants mentioned that the time constraint may come on either end of the trip and has the potential to affect their travel time estimate. Other specific entry items included the ability to specify the date or day of the week, the best- and worst-case scenario travel times, and a suggestion of a different and more efficient departure time. The specific phrasing of these questions was covered in the computer survey; however, the preference between a departure or an arrival time option was not discussed because it is dependent on the conditions of the trip.

All participants agreed that the system should provide multiple route options. It should recommend one route but also show alternate routes with the same level of detail. One participant said that the output should give the trip in distance as well as the estimated time so that users can make their own trip time adjustment. Researchers discussed whether to include this functionality in the field operational test, but it may not be feasible to implement with the website system that has already been developed.

When the research team asked participants if they wanted the system to tell how sure it is of its estimate, reaction was mixed. Several participants expressed hesitation in trusting a system that was not certain of its estimate. Others felt that they would like to know how confident they could be that the predicted time would allow them to arrive at their destination on time.

What should the output look like? Just words, a graph, a map?

Groups were split on whether the output information should be shown as a graph, picture, map, text, or a combination of any of those. Most mentioned that color coding is helpful, and travel time information should be provided for the return trip as well. In Houston, both groups suggested including landmarks to aid navigation, and all groups mentioned being given multiple route options so they could choose the best fit for them. One participant said that the ability to get specific driving directions sent to his cell phone would be beneficial.

When prompted, participants in Seattle liked the idea of getting real-time alerts for their current trips. All agreed that they would like travel time information for surface streets so that they had a choice about taking the freeway. They also mentioned the possibility of adding parking information to the system because that is the element that provides the greatest variability in their daily trips.

\section{Use Cases}

To determine under which scenarios travel time reliability information is valuable, examples of four scenarios were introduced. One of them was a constrained unfamiliar trip, one was a constrained familiar trip, another was an unconstrained unfamiliar trip, and the last was an unconstrained familiar trip.

\section{Scenario 1: Constrained Unfamiliar}

The first scenario presented was an appointment with a new doctor.

You have an appointment with a specialist you have never seen before on Thursday and you are not familiar with the area of town the office is located in. You have to tell your boss by Tuesday what time you will need to get off work on Thursday so you need to plan ahead.

This scenario was another instance in which a large number of participants said that they would rely on estimates from mapping tools such as a GPS or MapQuest, and they all would add time to those estimates. Others would rely on their previous knowledge of the area, and several stated they would simply leave an hour in advance. Unprompted, one participant did mention that "the downside of being somewhere 
early is that you could have done something else." However, all agreed that they would rather be early than late to important events such as appointments.

The majority of the participants replied that they would potentially use the system in a situation such as this one. One person said, "It would be good if we were going somewhere we didn't know anything about traffic. [I would] already know the traffic patterns here, so if I was traveling, it would be more useful." However, most said they would still be skeptical of the estimated trip time and would add additional time. Because participants seemed to favor a TTR system for a constrained unfamiliar trip, the open-ended survey's scenario was modeled after this criterion.

\section{Scenario 2: Constrained Familiar}

The second example (constrained familiar) involved making a decision about aerobics class. Depending on the make-up of the group, this example was changed to an activity for a young child that requires taking them and picking them up somewhere.

You are trying to decide whether to sign up for the Monday/ Wednesday or the Tuesday/Thursday aerobics class at your new gym. Class starts at 6 pm and you can't leave work until 5 pm each day.

Generally, groups in all cities mentioned that they could assume traffic is heavier on Mondays and Fridays, so they would avoid those days. This highlighted their awareness of variability.

Overall, the consensus was that people would not use the new system to help make this decision. Although a handful said they might check it to make sure no special events were occurring (e.g., a large church that meets on Wednesday evenings), the majority felt that they would "plan around their life, not traffic." Several also said they did not believe the difference in trip time each day would vary enough to justify the time spent checking the system for a short-term class. One individual suggested he would rather ask someone familiar with the route and base his estimates and decision on that information rather than checking the system.

\section{Scenario 3: Unconstrained Unfamiliar}

One example of an unconstrained unfamiliar local trip is going to a birthday party.

You are going to a friend's birthday party. The party starts at $7 \mathrm{pm}$ and is at a house you have not been to before.

For this example, participants overwhelmingly agreed that they would not use the reliability system. Most said all they would need was basic directions from the GPS unit in-car or from an existing mapping tool, and time was not critical. "If you are a couple of minutes late in this situation, it is not as critical as catching a plane or work or an appointment." One individual mentioned that he might use the system to get a map and check construction status; but overall, this situation did not warrant the use of a pre-planning function.

\section{Scenario 4: Unconstrained Unfamiliar}

The other example of an unconstrained unfamiliar trip is one that requires travel to an area you are not familiar with. Trip destinations were changed for each focus group city to represent a destination that was within several hours of participants' homes.

You are taking a trip to Mt. Bachelor to go skiing and have to drive through Portland to get there. You are not familiar with Portland but have heard that there are times when the traffic is heavy and travel times are slow.

Participants were asked if they would use a TTR system to determine which day of the week or time of day they would want to drive through Portland. The results from this scenario were difficult to gauge because participants did not seem to have strong opinions. Although most saw the value of a TTR system in this situation, they were still not sure they would take the time to consult it. Some said the trip was simply a convenience, so time was not critical; instead of consulting historical information, they would just use a map. Others said that there would need to be a significant time savings before they would change the time they chose to leave. Still another said she might use the system simply to determine what the traffic situation was, although it would have little or no bearing on her departure time decision. Participants also recommended that the system have the potential to include rest stops and trip planning features in addition to peak traffic alerts to increase system usefulness.

In some cities, when time allowed, this example was used to introduce the possibility of the system helping to determine mode change. Participants were asked directly if they would use the system to choose whether to take the train or the bus instead. Most said they would not. However, in cities with established forms of alternative transportation, some participants seemed open to considering the idea of mode change. One individual said, "[I] would probably still take the bus if I had been planning on it, but I might take the bus that leaves half an hour earlier than the one I'd usually take." Overall, participants said that the decision to change mode would be motivated by schedule and convenience more often than strictly by time.

\section{Scenario 5: Unconstrained Familiar}

The unconstrained familiar trip example involved participating in a food co-op. 
You participate in a food co-op and have to go pick your box up every Saturday morning sometime between 8 am and $2 \mathrm{pm}$. The produce is distributed in a church parking lot 20 minutes from your house.

The primary response to this scenario was that traffic is not the same on weekends, and if they were going to use any system, participants would check a source that provided real-time data. Almost everyone said that they would not use this system because Saturday plans vary from weekend to weekend, and they would base the pickup time solely on their personal schedule for the day. They would not necessarily go at the same time each week. Several agreed that the system might be valuable if they could enter a time window and the system had the capability to tell them the most efficient time to make the trip.

\section{Scenario 6: Task Insertion}

This scenario inserted a task: choosing a day of the week to feed the neighbor's cat.

Your neighbor is attending overseas training for their job and will be gone for 3 months. The families on your street are volunteering to take care of their cat one day a week. You are asked to pick a morning you are available to feed the pet every week. You realize the traffic is typically worse on some days than others.

None of the participants said that they would use the system in this situation. All felt that they would choose a day solely on the basis of their personal schedule, not on traffic. Some also felt that the trip from home to work would be very familiar, so they would know from experience what the best day to add a task would be. One participant said, "It would take longer to use the system than to just [feed the cat]."

\section{Scenario 7: Comparing Two Routes with Different Reliability Values}

To get an understanding of the words that participants would use when talking about these types of trips and the kind of information a TTR system would provide, a scenario was presented comparing two routes (Figure C.6). Participants were asked to describe the differences.

Bob and Fred are neighbors who work at the same office building but they take different routes to work. They constantly argue about whose route is better, so they decide to keep track of their arrival times for 2 weeks. They leave their houses at the same time every morning. Here are the results:

These values were chosen because both routes have an identical mean, but the standard deviation of Bob's way was 1.254 and the standard deviation of Fred's way was 4.675.

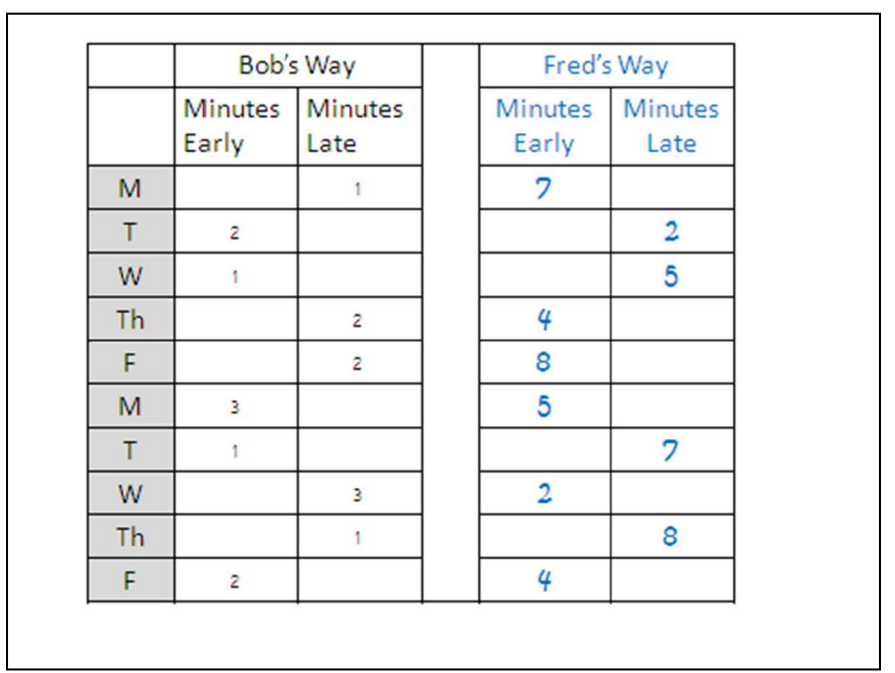

Figure C.6. Bob's and Fred's timecards (Slide 6).

Overall, participants thought that Bob's route was better even though Fred was only late four times compared with Bob's five times. Unprompted, they said Fred had unreliable factors, was more erratic, and had more variance, while Bob's route is more reliable, predictable, and consistent. One participant said the two could not be compared because "late is late and early is early." Another said he would choose Fred's route because he was early by more.

Participants were shown Figure C.7 and asked to fill in the blank to describe the routes Fred and Bob take.

Bob's way is reliable, more efficient, predictable, better, safer, shorter, more direct, better probability, more consistent, faster, quicker, less problematic, less risky than Fred's way.

Bob's way is than Fred's way.

Take Bob's way because it's

Figure C.7. Bob's and Fred's sentence completion (Slide 7). 
Take Bob's way because it [or it's] works, reliable, consistent, a better way, dependable, precise, more thorough, unbelievable, less likely to be late, more efficient, doesn't fluctuate.

These terms provided by the focus group participants were also used in the questions asked in the computer survey.

\section{Fill-in-the-Blank Activity}

The next section of the discussion was intended to gauge the terms used by drivers to describe the uncertainty in traveling. A total of nine fill-in-the-blank sentences were presented. For each one, participants were asked to write down their initial responses for completing the sentence on a blank sheet of paper. The words they wrote down were discussed and then a list of words, previously generated by the research team and shown in red on slides, was shown. After both the participants' initial responses and the list of word choices were shown, each participant indicated their favorite term for that particular sentence or concept. Because of time constraints, not all sentences were discussed in every city. Figures C.8 through C.18 follow.

Chance and possibility were the two terms most preferred by participants in all cities for the sentence shown in Figure C.9. Several participants suggested that chance was broad and should be qualified by saying $X \%$ chance, slight chance, or good chance. Other popular terms included certainty, likelihood, and probability.

Referring to Figure C.10, varies, changes, and increases/ decreases were the preferred terms for completing this sentence describing variability by day. Words such as congested, fluctuates, lightens, and stinks were also suggested.

No specific term received overwhelming support in the sentence shown in Figure C.11. In most cities, no word or

\section{System tells you about the time of day of your trip}

Figure C.8. Topic slide (Slide 8).
1. There is a that travel time will be worse at $4 \overline{\mathrm{PM} \text { than }}$ at $2 \mathrm{PM}$ on Fridays.
Chance
Good Chance
$80 \%$ Chance
Possibility
Likelihood
Certainty
Probability

The list of words below the sentence was shown after participants contributed their initial responses to promote further discussion.

\section{Figure C.9. Sentence 1 (Slide 9).}

phrase was voted on by more than three people. In Seattle, the phrases is most congested, is slow, and gets better/worse were each preferred by two individuals. Other suggestions were is historically bad, peaks, and fluctuates. Three participants in Houston thought that increases was the best word to complete the sentence. Atlanta had the most variability, as nine terms received multiple votes. Is terrible and is worst were the top two choices, while lousy, awful, horrible, is longer/shorter, congested, and chaos rounded out the group. The Washington, D.C., participants preferred congested or is longer/shorter by a narrow margin; and Minneapolis participants felt that

2. Travel time on Main street from Monday to Tuesday.

$$
\begin{aligned}
& \text { Varies } \\
& \text { Is Different } \\
& \text { Changes } \\
& \text { Fluctuates } \\
& \text { Gets Better } \\
& \text { Is Worse } \\
& \text { Is } 20 \% \text { worse }
\end{aligned}
$$

The list of words below the sentence was shown after participants contributed their initial responses to promote further discussion.

Figure C.10. Sentence 2 (Slide 10). 
3. On Mondays, travel time on Main Street between $8 \mathrm{AM}$ and $10 \mathrm{AM}$.

Varies
Fluctuates
Changes
Gets Better
Loosens up
Doesn't change much

The list of words below the sentence was shown after participants contributed their initial responses to promote further discussion.

Figure C.11. Sentence 3 (Slide 11).

increases/decreases was the best term to describe traffic in that time window.

Congested, possible, and likely were the most used terms to fill in the blank shown in Figure C.12. Horrible, grid lock, certain, expected, extremely slow, and unpredictable were also mentioned as words to describe this situation.

Participants thought that the terms faster and more reliable were the best descriptors to fit the scenario in Figure C.14.

Unprompted, the majority of terms provided by participants for the sentence in Figure C.15 dealt with time. For

4. Travel time on that road is on Friday afternoons at 4:30 PM.

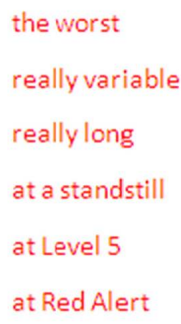

The list of words below the sentence was shown after participants contributed their initial responses to promote further discussion.

Figure C.12. Sentence 4 (Slide 12).

\section{System tells you about other ways to travel}

\section{Figure C.13. Topic slide (Slide 13).}

example, faster, slower, easier, and quicker were mentioned. After participants viewed the prompts, they still believed those terms to be the best though they liked more consistent after it was shown as well.

The majority of participants who viewed the sentence in Figure C.16 chose likely or unlikely as the word that best described the uncertainty. Doubtful, probable, and typical were also mentioned as options.

A variety of responses was given to fill in the blank in the sentence in Figure C.17, and they depended on personal preference. Terms that were mentioned that were not prompted were additional time, traffic time, parking time, wiggle room, leeway, window, driving time, just in case time,

\begin{tabular}{|l|}
\hline 5. Highway A is ____ than Highway B. \\
better \\
more reliable \\
more certain \\
more consistent \\
more predictable \\
less variable \\
less volatile \\
less risky
\end{tabular}

The list of words below the sentence was shown after participants contributed their initial responses to promote further discussion.

Figure C.14. Sentence 5 (Slide 14). 
6. Taking the train is than taking Highway $\mathrm{A}$.

better
more reliable
more certain
more consistent
more predictable
less variable
less volatile
less risky

The list of words below the sentence was shown after participants contributed their initial responses to promote further discussion.

Figure C.15. Sentence 6 (Slide 15).

gap time, fluff time, flex time, human error, allowance, lead time, and additional drive time. Leeway was mentioned in several groups and was well liked in Seattle, Washington, D.C., and Minneapolis. Extra time seemed to be the most popular term among all cities.

The sentence in Figure C.18 also resulted in a variety of answers. Know, expect, and predict appeared to be the three most preferred responses. One participant even recommended using the phrase know from experience. Other well-liked answers were believe and assume.

7. A friend tells you it will take 20-45 minutes to get to the airport. It is that it will take you 25 minutes.

likely
expected
more or less certain
a good guess
typical

The list of words below the sentence was shown after participants contributed their initial responses to promote further discussion.

Figure C.16. Sentence 7 (Slide 16).
8. Your neighbor told you it would take 20 minutes to get there, but you added 10 minutes of and left 30 minutes early.

$$
\begin{aligned}
& \text { buffer time } \\
& \text { padding } \\
& \text { cushion } \\
& \text { safety factor } \\
& \text { extra time } \\
& \text { safety net } \\
& \text { comfortzone }
\end{aligned}
$$

The list of words below the sentence was shown after participants contributed their initial responses to promote further discussion.

Figure C.17. Sentence 8 (Slide 17).

\section{Value and Understanding of the System}

To conclude the session, participants were asked several questions about the overall value of the system and who the users of the system might be. Participants said that primary users of the system would be individuals who drive in new locations on a regular basis. Specific occupations mentioned in the groups were salesmen, delivery drivers, and businessmen who travel to new places regularly. Another target audience

9. I that travel time will be worse Monday than it will Tuesday.
predict
expect
forecast
estimate
anticipate
project
guess

The list of words below the sentence was shown after participants contributed their initial responses to promote further discussion.

Figure C.18. Sentence 9 (Slide 18). 
identified by participants was drivers who are new to a city or area. The system could be used in determining how and when to leave for work or appointments or to help in decisions about where to buy a house or accept a job.

When asked which element of the system would be most understood by users of a TTR system, all of the participants expressed concern that drivers would assume they were being presented with real-time information. They felt that the concept of historical travel time data would confuse drivers, especially once they realized it has no real-time component. Some participants said that without a firm grip on reliability information, drivers would not understand what made this system different from any of the other options currently available. However, this misunderstanding could be rectified with driver education and clear explanations on the website. Other participants were concerned about the system presenting information in terms of percentages because they felt the average driver does not understand what a percentage is telling them or where the data came from.

To help researchers understand how much users value the information provided by a TTR system, participants were asked if they would pay to use the system. Overwhelmingly, drivers said that they would not pay for the use of a website. There are other free options available that are working well for them right now, and they could not justify the expense. However, most said that they might pay a single or annual nominal fee for the convenience of a cell phone application or an upgrade to their GPS package. A few people said that they would pay for separate software. One participant mentioned that companies might be willing to pay for use of the system if they employed a large number of people who drove to new places often. In that case, the time and money savings would justify the expense of the information. When asked whether they would pay for use during a certain situation or circumstance, several said that they would.

\section{Thank you for coming}

More information about the project can be found here:

Transportation Research Board Strategic Highway Research Program Project L14: http://144.171.11.40/cmsfeed/TRBNet ProjectDisplay.asp?ProjectID=2343

Washington DOT currently has a similar system http://www.wsdot.wa.gov/Traffic/Seattle/ TravelTimes/reliability/

Figure C.19. Wrap-up slide (Slide 19).

The final question asked whether the public department of transportation or a private company should provide this travel time reliability information. Initially, participants expressed no strong opinion on this topic, but by the end of the discussion, they were leaning toward private companies. They felt that private companies would have the resources to respond more quickly to demands and more motivation to maintain and improve the system because it was their source of income. When prompted, participants were open to the idea of a private company charging for use of the travel time data because it must make a profit. Although they felt that the government would have more access to information about construction, they were not confident that the system would be as user friendly or well maintained. A few participants still felt that it was the government's responsibility to provide this information.

A final slide closed the session (Figure C.19). 


\section{Computer Survey Questions and Results Tables}

\section{Questions 1-4: Reliability Concepts}

Figure D.1 displays the computer screen participants viewed for Question 1. Questions 2 through 4 varied the underlined term-predictable, consistent, and best trip-as shown in Table D.1.

\section{Question 5: Importance of Trip Planning Factors}

Figure D.2 displays the computer screen participants viewed for Question 5, and Table D.2 summarizes the question responses.

\section{Questions 6-8: Terms for Website Trip Planning Feature Links}

Figure D.3 displays the binder page that participants viewed before answering Question 6. Figures D.4, D.5, and D.6 show the computer screen visuals and results for Questions 6, 7, and 8, respectively. Tables D.3, D.4, and D.5 present the corresponding response summaries.

\section{Questions 9-10: Terms to Convey Immediacy of Departure}

Figure D.7 displays the visuals, and Table D.6 summarizes the results for Question 9, Version A. Other versions of this question substituted other terms for the red circled term Predict Trip shown in this figure, as reflected in the table. Figure D. 8 displays the visuals, and Table D.7 summarizes the results for Question 10.

\section{Questions 11-12: Terms to Describe Departure and Arrival Time Constraints}

Figure D.9 shows the visuals, and Table D.8 summarizes the responses for Question 11. Figure D.10 and Table D.9 show the visuals and responses for Question 12.

\section{Questions 13-14: Map and Travel Time Output Terminology}

Figures D.11 and D.12 display the binder pages presented to participants before Question 13. Figure D.13 displays the visuals and Table D.10 summarizes the results for Question 13. Figure D.14 and Table D.11 display the visuals and results for Question 14.

\section{Questions 15-17: Trip Time Output Terminology}

Figure D.15 displays the visuals for Question 15, Version A. In Versions $\mathrm{B}$ through $\mathrm{D}$ of the survey, the term average trip time was replaced by the terms shown in Table D.12. The table also summarizes the responses.

Figure D.16 displays the visuals for Question 16, Version A. In Versions B through D of the survey, the term average trip time was replaced by the terms shown in Table D.13. The table also summarizes the Question 16 responses.

Figure D.17 displays the visuals, and Table D.14 summarizes the responses for Question 17.

\section{Questions 18-20: Planning and Buffer Time Terminology}

Figure D.18 displays the visuals for Question 18, Version A. In Versions B through D of the survey, the term recommended departure time was replaced by the terms shown in 
The following are examples of 4 different trips that are the same distance:

1: Laura's trip is 20 miles and ordinarily takes 25 minutes

2: Bob's trip is 20 miles and can take anywhere from 15 to 40 minutes

3: Sue's trip is 20 miles and always takes less than 30 minutes

4: Tom's trip is 20 miles and can take anywhere from 20 to 30 minutes

Whose trip is the most reliable?

$$
\begin{array}{|l|l|}
\hline 1 & \text { Laura's } \\
\hline 2 & \text { Bob's } \\
\hline 3 & \text { Sue's } \\
\hline 4 & \text { Tom's } \\
\hline
\end{array}
$$

Figure D.1. Question 1 visual.

Table D.15. The table also summarizes the Question 18 responses.

Figure D.19 displays the visuals for Question 19, Version A. In Versions $\mathrm{B}$ through $\mathrm{D}$ of the survey, the term recommended departure time was replaced by the terms that are shown in Table D.16. The table also summarizes the Question 19 responses.

Figure D.20 displays the visuals, and Table D.17 summarizes the responses for Question 20.

\section{Questions 21-22: Buffer Time Terminology}

Figure D.21 displays the visuals, and Table D.18 summarizes the responses for Question 21.

Figure D.22 displays the visuals, and Table D.19 summarizes the responses for Question 22.

\section{Question 23: Preference for Travel Time Distribution Output}

Figure D.23 displays the visuals, and Table D.20 summarizes the responses for Question 23.

\section{Questions 24-26: Travel Time Variability Terminology}

Figure D.24 displays the visuals, and Table D.21 summarizes the responses for Question 24.

Figure D.25 displays the visuals, and Table D.22 summarizes the responses for Question 25.

Figure D.26 displays the visuals, and Table D.23 summarizes the responses for Question 26.

\section{Question 27: 95th Percentile Trip Time Terminology}

Figure D.27 displays the visuals, and Table D.24 summarizes the responses for Question 27.

\section{Question 28: System Trust Terminology}

Figure D.28 displays the visuals, and Table D.25 summarizes the responses for Question 28.

\section{Question 29: Travel Mode Shift Information}

Figure D.29 displays the visuals, and Table D.26 summarizes the responses for Question 29.

Table D.1. Questions 1-4 Response Summary

\begin{tabular}{|l|c|c|c|c|}
\hline Response Choice & $\begin{array}{c}\text { Question 1: } \\
\text { Reliable (\%) }\end{array}$ & $\begin{array}{c}\text { Question 2: } \\
\text { Predictable (\%) }\end{array}$ & $\begin{array}{c}\text { Question 3: } \\
\text { Consistent (\%) }\end{array}$ & $\begin{array}{c}\text { Question 4: } \\
\text { Best Trip (\%) }\end{array}$ \\
\hline 1. Laura (typical travel time) & 40.00 & 42.33 & 49.33 & 47.67 \\
\hline 2. Bob (large range of travel times) & 8.00 & 10.67 & 5.67 & 8.33 \\
\hline 3. Sue (maximum travel time) & 39.00 & 37.00 & 39.33 & 26.67 \\
\hline 4. Tom (small range of travel times) & 13.00 & 10.00 & 5.67 & 17.33 \\
\hline
\end{tabular}


When taking a trip over 20 miles which is the most important to you?

1 Choosing a route that takes the least amount of time on the road

2 Choosing a route that takes the fewest number of miles to get to my destination

3 Choosing a route that has the most predictable travel time, even though it is more miles
Table D.2. Question 5 Response Summary

\begin{tabular}{|l|c|}
\hline Response Choice & Percentage \\
\hline 1. Least time & 68.67 \\
\hline 2. Fewest miles & 13.00 \\
\hline 3. Most predictable time & 18.33 \\
\hline Total & 100.00 \\
\hline
\end{tabular}

Figure D.2. Question 5 visual.

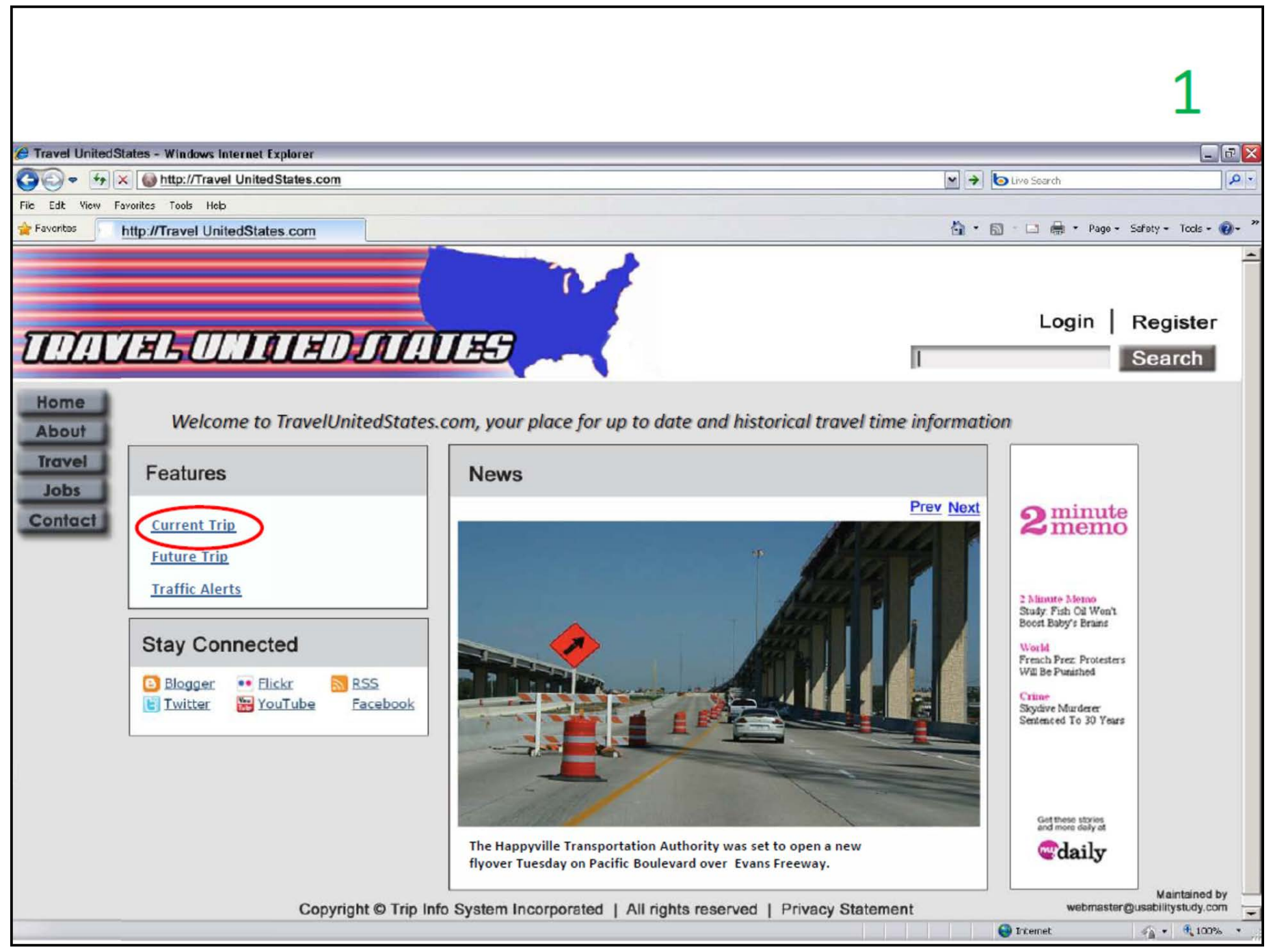

Figure D.3. Visual from binder used to introduce example traveler information website. 


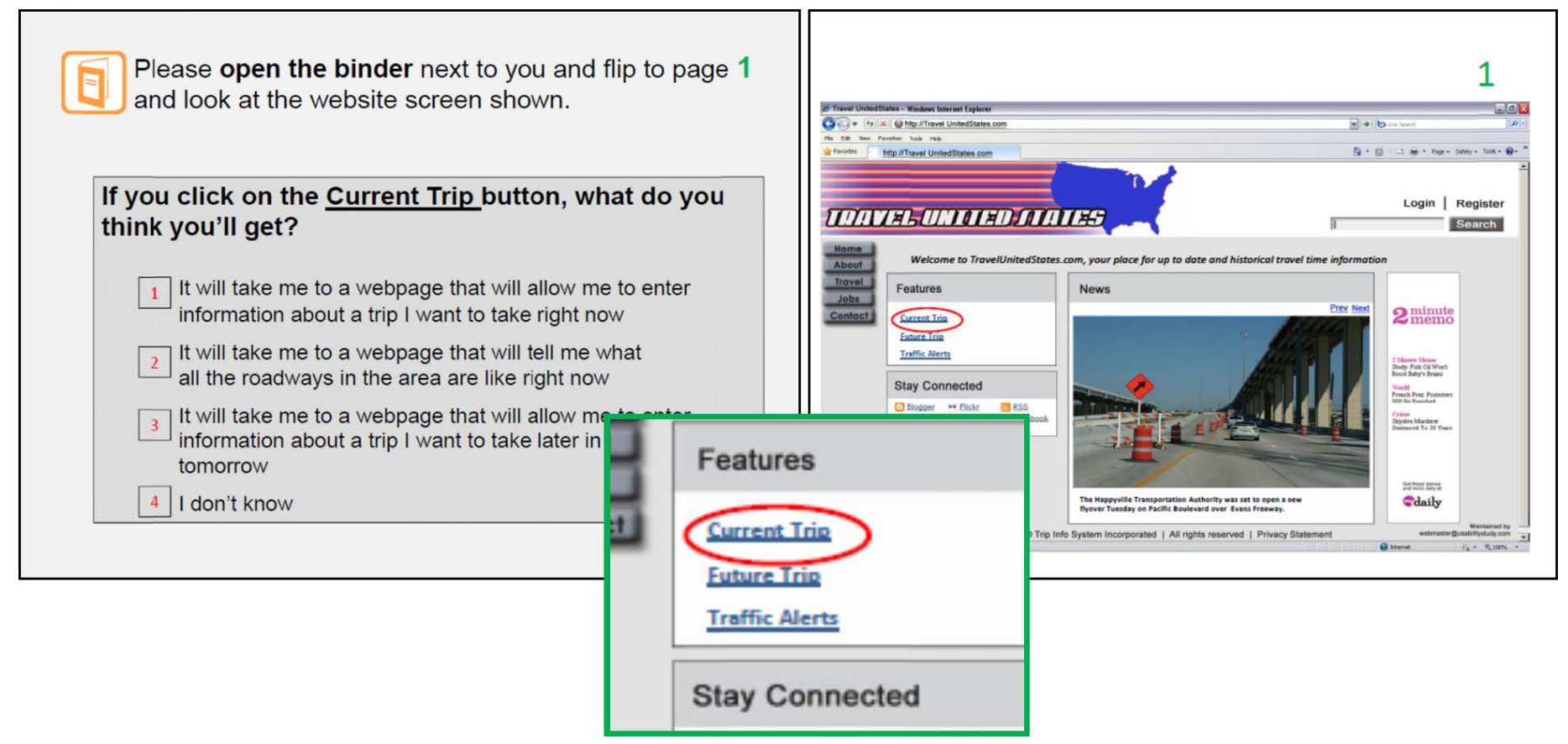

Figure D.4. Question 6 visuals.

Table D.3. Question 6 Response Summary

\begin{tabular}{|l|c|}
\hline Response Choice & Percentage \\
\hline $\begin{array}{l}\text { 1. It will take me to a webpage that will allow me to enter } \\
\text { information about a trip I want to take right now. }\end{array}$ & 53.90 \\
\hline $\begin{array}{l}\text { 2. It will take me to webpage that will tell me what all the } \\
\text { roadways in the area are like right now. }\end{array}$ & 31.97 \\
\hline $\begin{array}{l}\text { 3. It will take me to a webpage that will allow me to enter } \\
\text { information about a trip I want to take later in the day or } \\
\text { tomorrow. }\end{array}$ & 8.18 \\
\hline 4. I don't know. & 100.00 \\
\hline Total & \\
\hline
\end{tabular}


Please flip to page 2 in the binder next to you and look at the website screen shown.

\section{If you click on the Future Trip button, what do you} think you'll get?

1 It will take me to a webpage that will allow me to enter information about a trip I want to take right now

2 It will take me to a webpage that will tell me what all the roadways in the area are like right now

3 It will take me to a webpage that will allow me to enter information about a trip I want to take later in the d tomorrow

4 Nothing, because there is no way to predict what a future trip would be like

5 I don't know
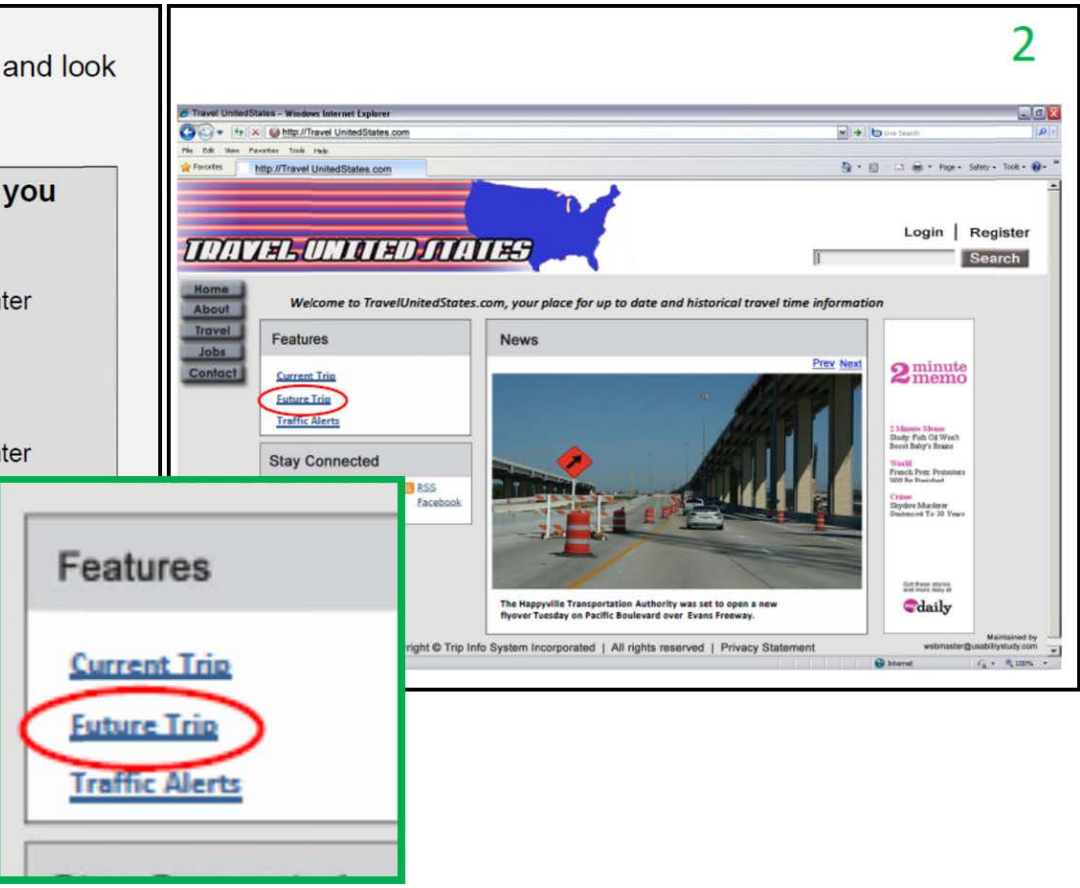

Figure D.5. Question 7 visuals.

Table D.4. Question 7 Response Summary

\begin{tabular}{|l|c|}
\hline Response Choice & Percentage \\
\hline $\begin{array}{l}\text { 1. It will take me to a webpage that will allow me to enter } \\
\text { information about a trip I want to take right now. }\end{array}$ & 1.12 \\
\hline $\begin{array}{l}\text { 2. It will take me to webpage that will tell me what all the } \\
\text { roadways in the area are like right now. }\end{array}$ & 8.83 \\
\hline $\begin{array}{l}\text { 3. It will take me to a webpage that will allow me to enter } \\
\text { information about a trip I want to take later in the day or } \\
\text { tomorrow. }\end{array}$ & 11.15 \\
\hline $\begin{array}{l}\text { 4. Nothing, because there is no way to predict what a } \\
\text { future trip would be like. }\end{array}$ & 1.49 \\
\hline 5. I don't know. & 100.00 \\
\hline Total & \\
\hline
\end{tabular}


Please flip to page 4 in the binder next to you and look at the website screen shown.

Why do you think you enter a time and date when using this website?

1 Because the website does not know what day or time it is

2 Because I am wanting information about a trip I am about to take right now

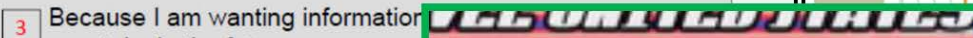
may take in the future

4 I don't know
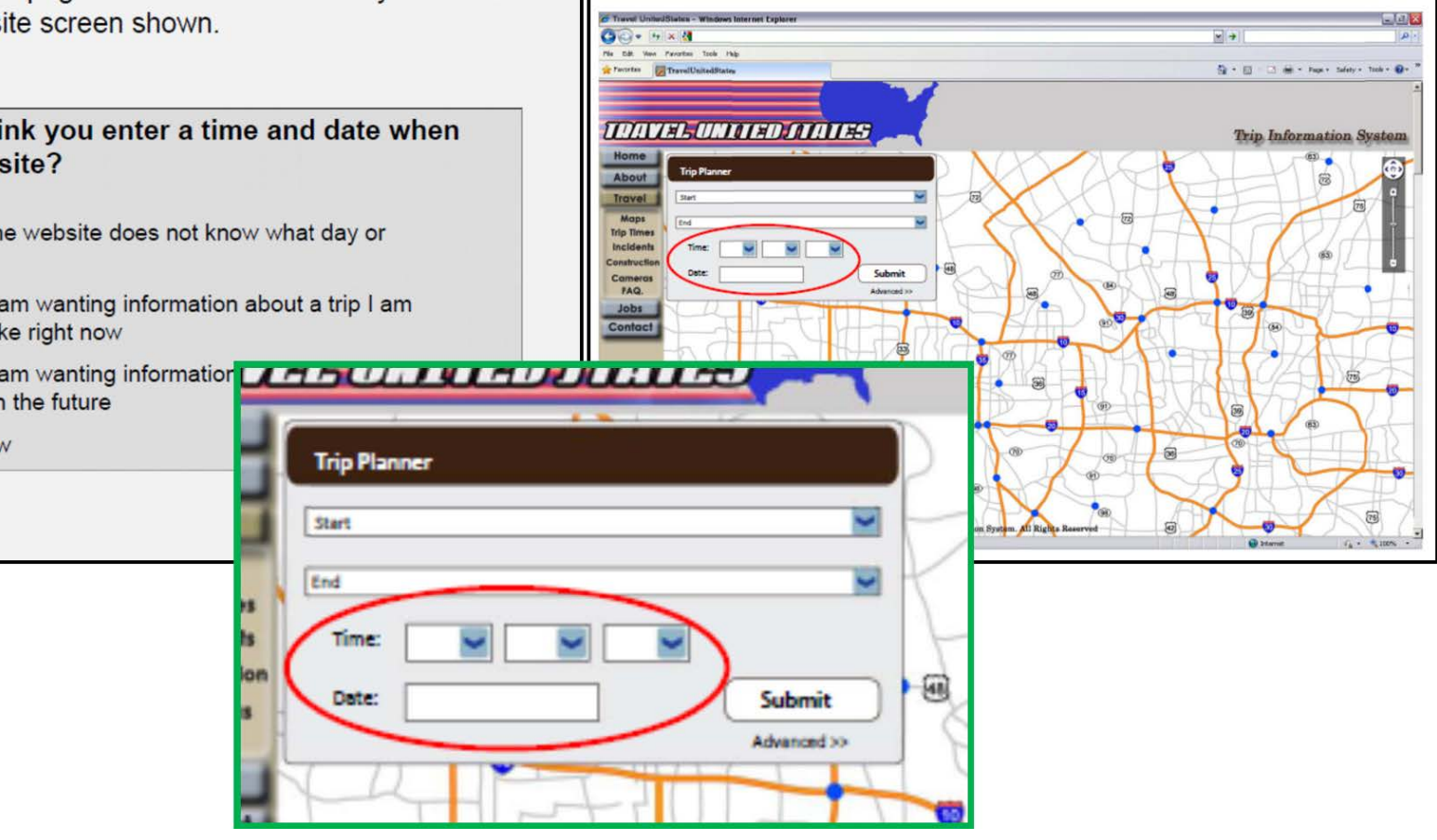

Figure D.6. Question 8 visuals.

Table D.5. Question 8 Response Summary

\begin{tabular}{|l|c|}
\hline Response Choice & Percentage \\
\hline 1. Because the website does not know what day or time it is. & 9.00 \\
\hline $\begin{array}{l}\text { 2. Because I am wanting information about a trip I am about } \\
\text { to take right now. }\end{array}$ & 35.33 \\
\hline $\begin{array}{l}\text { 3. Because I am wanting information about a trip I may take } \\
\text { in the future. }\end{array}$ & 51.67 \\
\hline 4. I don't know. & 4.00 \\
\hline Total & 100.00 \\
\hline
\end{tabular}


Please flip to page 5 in the binder next to you and look at the website screen shown.

What do you think will happen when you fill in the fields in the box and then select the button circled?

1 The map will display the route with the shortest distance

2 The website will give me step by step driving directions for my trip

3 The website will tell me how long my trip will take right now

4 The website will tell me how long my trip will take for the date/time I enter

5 The map will show me if there are any accidents or construction on my trip right now

6 It will do nothing

7 I don't know

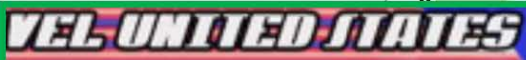

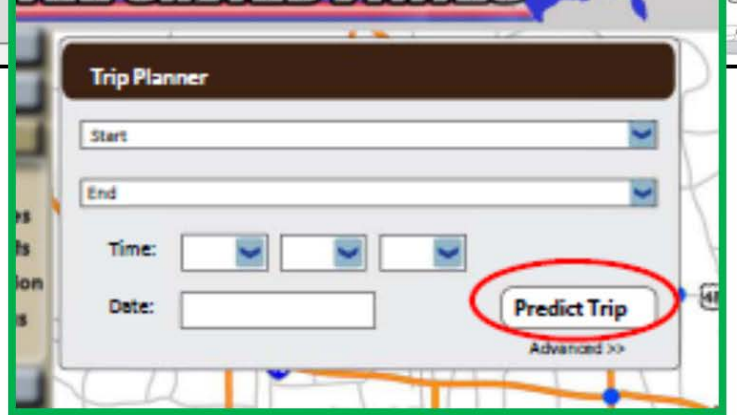

Figure D.7. Question 9 visuals. (Circled term used varied by version.)

Table D.6. Question 9 Response Summary

\begin{tabular}{|c|c|c|c|c|}
\hline Response Choice & $\begin{array}{c}\text { Version A: } \\
\text { Predict Trip (\%) }\end{array}$ & $\begin{array}{l}\text { Version B: } \\
\text { Plan Trip (\%) }\end{array}$ & $\begin{array}{l}\text { Version C: } \\
\text { Create Trip (\%) }\end{array}$ & $\begin{array}{l}\text { Version D: } \\
\text { Get Trip (\%) }\end{array}$ \\
\hline $\begin{array}{l}\text { 1. The map will display the route with the shortest } \\
\text { distance. }\end{array}$ & 7.89 & 4.05 & 6.67 & 14.86 \\
\hline $\begin{array}{l}\text { 2. The website will give me step-by-step driving } \\
\text { directions for my trip. }\end{array}$ & 17.11 & 27.03 & 16.00 & 20.27 \\
\hline $\begin{array}{l}\text { 3. The website will tell me how long my trip will take } \\
\text { right now. }\end{array}$ & 6.58 & 8.11 & 1.33 & 5.41 \\
\hline $\begin{array}{l}\text { 4. The website will tell me how long my trip will take } \\
\text { for the date/time I enter. }\end{array}$ & 63.16 & 56.76 & 72.00 & 58.11 \\
\hline $\begin{array}{l}\text { 5. The website will show me if there are any accidents } \\
\text { or construction on my trip right now. }\end{array}$ & 1.32 & 1.35 & 0.00 & 0.00 \\
\hline 6. It will do nothing. & 2.63 & 1.35 & 2.67 & 0.00 \\
\hline 7. I don't know. & 1.32 & 2.70 & 1.33 & 1.35 \\
\hline
\end{tabular}




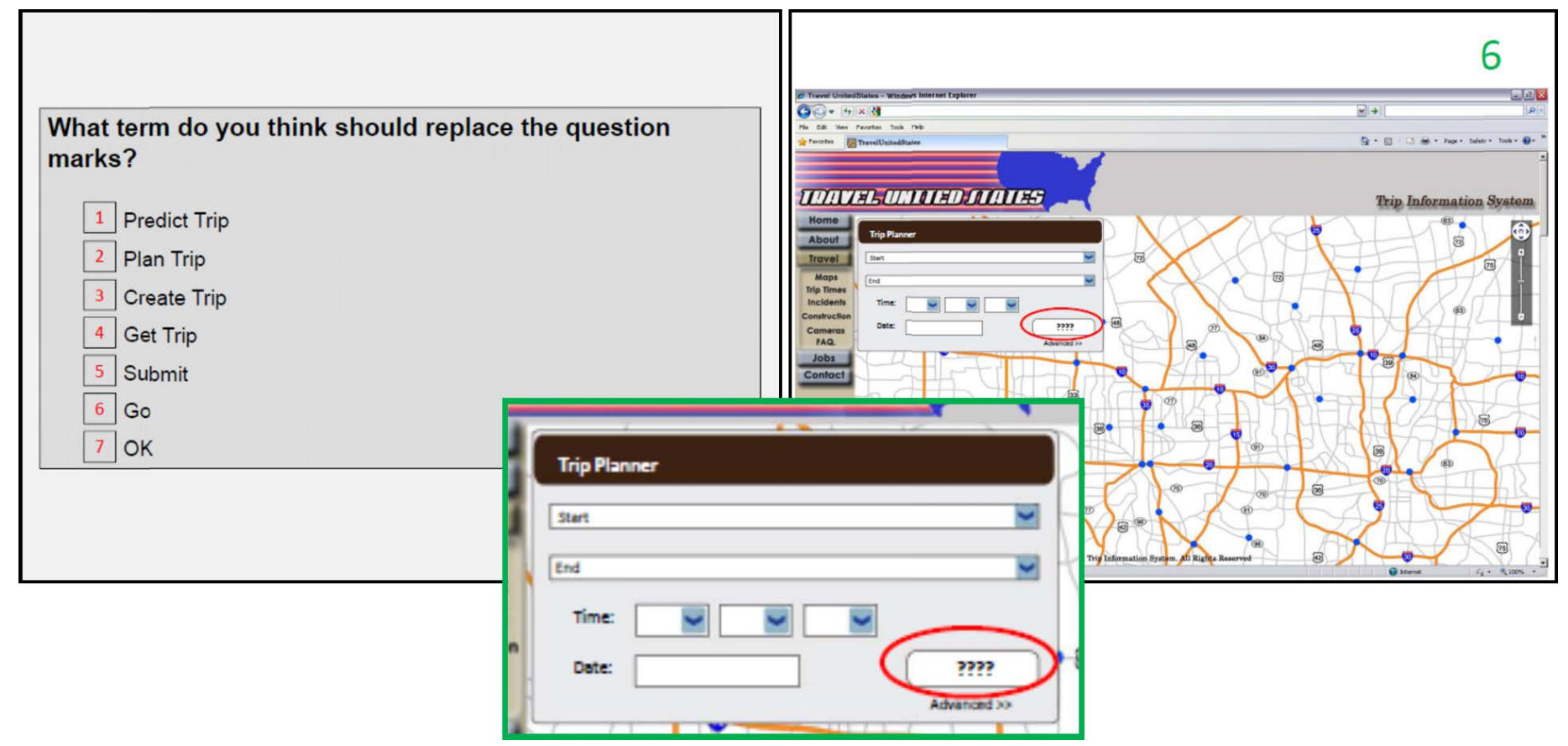

Figure D.8. Question 10 visuals.

Table D.7. Question 10

Response Summary

\begin{tabular}{|l|c|}
\hline Response Choice & Percentage \\
\hline 1. Predict trip & 28.33 \\
\hline 2. Plan trip & 24.67 \\
\hline 3. Create trip & 14.33 \\
\hline 4. Get trip & 7.00 \\
\hline 5. Submit & 17.67 \\
\hline 6. Go & 4.33 \\
\hline 7. OK & 3.67 \\
\hline Total & 100.00 \\
\hline
\end{tabular}


Please flip to page 7 in the binder next to you.

This system knows that travel time varies depending on what time of day it is, so it needs to know what time of day you want to take your trip.

What phrase do you prefer to describe what time you want to start your trip?
1 Leave At
2 Leave By
3 Departing At
4 Departing By
5 What's the earliest you can start your trip?
6 What's the latest you can start your trip?
7 What time will you start your trip?

Figure D.9. Question 11 visual.

*No binder slide viewed*

Table D.8. Question 11 Response Summary

\begin{tabular}{|l|c|}
\hline Response Choice & Percentage \\
\hline 1. Leave at & 23.33 \\
\hline 2. Leave by & 7.00 \\
\hline 3. Departing at & 34.67 \\
\hline 4. Departing by & 8.00 \\
\hline 5. What's the earliest you can start your trip? & 4.33 \\
\hline 6. What's the latest you can start your trip? & 1.33 \\
\hline 7. What time will you start your trip? & 21.33 \\
\hline Total & 100.00 \\
\hline
\end{tabular}


What phrase do you prefer to describe what time you want to reach your destination?
1 Arrve At
2 Arrve By
3 What time do you want to get there?
4 What's the earliest you zan arrive?
5 What's the latest you can arrive?

*No binder slide viewed*

Figure D.10. Question 12 visual.

Table D.9. Question 12 Response Summary

\begin{tabular}{|l|c|}
\hline Response Choice & Percentage \\
\hline 1. Arrive at & 33.33 \\
\hline 2. Arrive by & 47.00 \\
\hline 3. What time do you want to get there? & 14.33 \\
\hline 4. What's the earliest you can arrive? & 1.67 \\
\hline 5. What's the latest you can arrive? & 3.67 \\
\hline Total & 100.00 \\
\hline
\end{tabular}




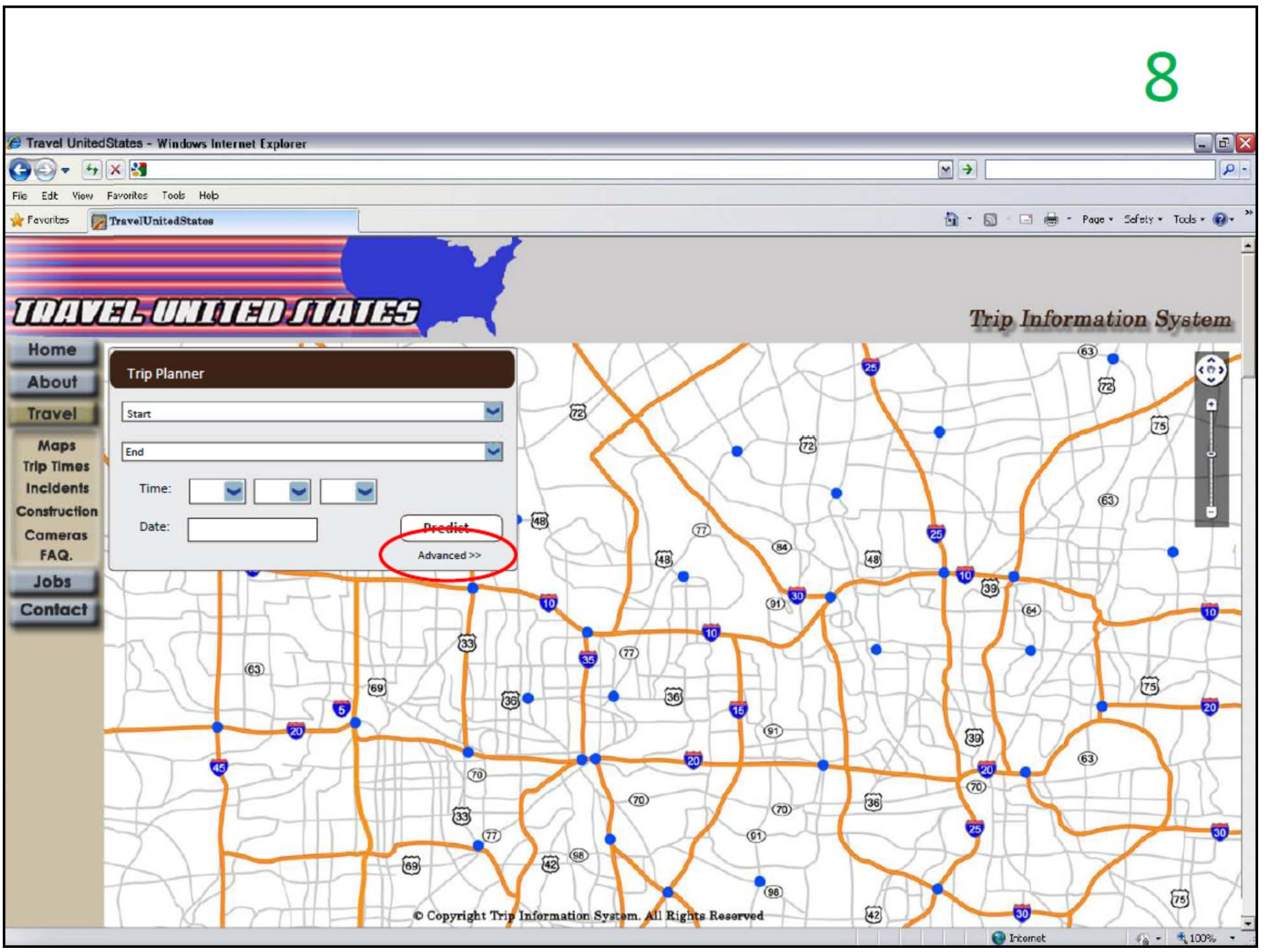

Figure D.11. Binder screenshot introducing map-based output screen. 


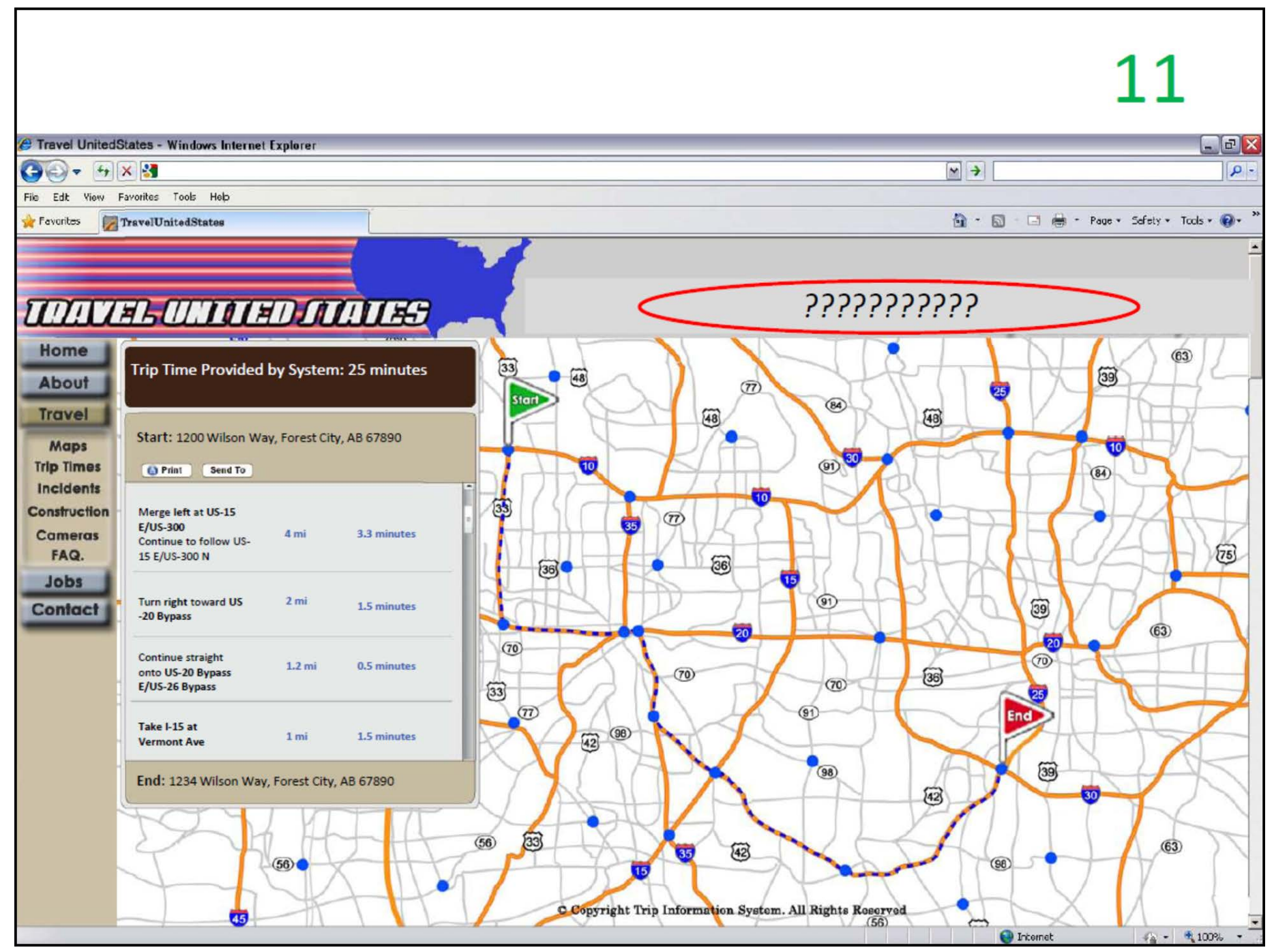

Figure D.12. Binder screenshot of map output showing trip route and travel times. 
Please flip to page 10 in the binder next to you and look at the website screen shown.

What map title do you think should replace the question marks?
1 Map of Forest City
2 Road Segments with Available Travel Information
3 Road Segments with Historical Data
4 Forest City's available travel information
5 Forest City's historical data

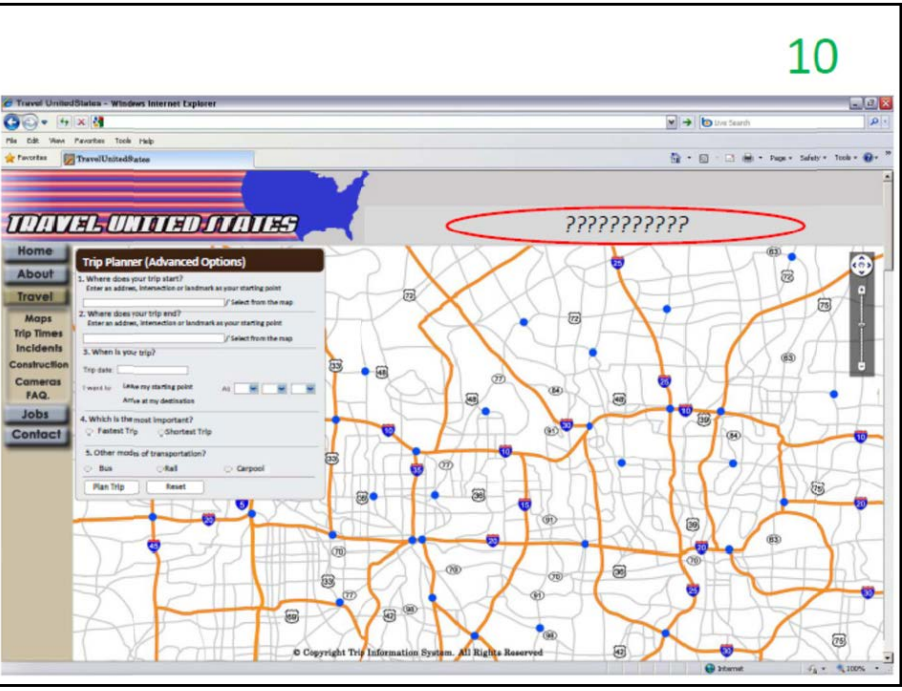

Figure D.13. Question 13 visuals.

Table D.10. Question 13 Response Summary

\begin{tabular}{|l|c|}
\hline Response Choice & Percentage \\
\hline 1. Map of Forest City & 17.67 \\
\hline 2. Road segments with available travel information & 32.00 \\
\hline 3. Road segments with historical data & 10.67 \\
\hline 4. Forest City's available travel information & 33.67 \\
\hline 5. Forest City's historical data & 6.00 \\
\hline Total & 100.00 \\
\hline
\end{tabular}


F Please flip to page 11 in the binder next to you and look at the website screen shown.

This map displays a route based on the start and end locations and the trip times you specified to arrive at your destination at 8:15 a.m.

What map title do you think should replace the question marks?

1 Best Route

2 Least Variable Time

3 Most Consistent Trip

4 Most Reliable Trip

5 Most Predictable Trip

6 Forecasted Trip

7 Historical Trip Conditions

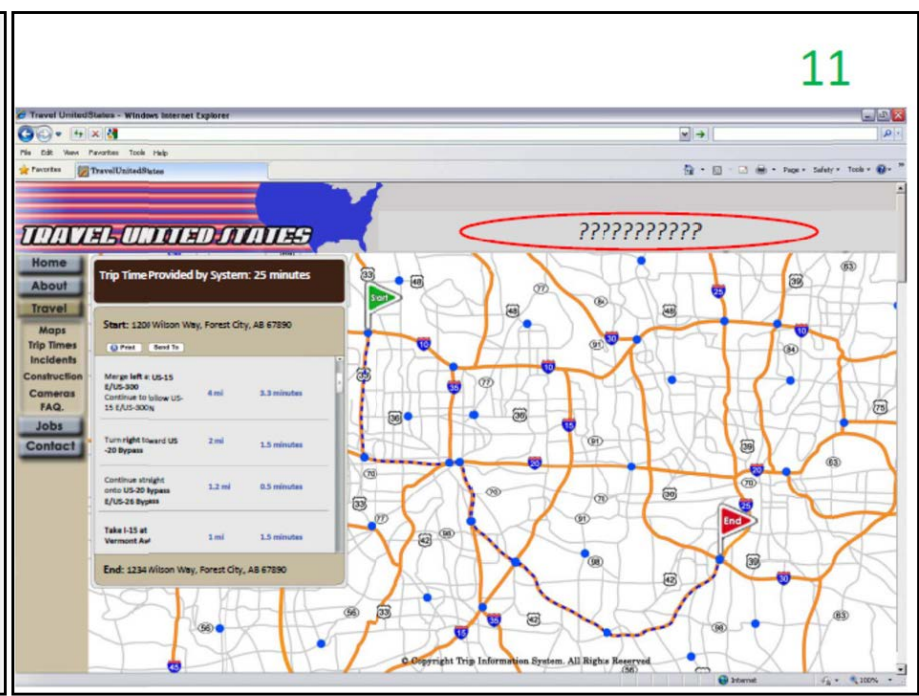

Figure D.14. Question 14 visuals.

Table D.11. Question 14

Response Summary

\begin{tabular}{|l|c|}
\hline Response Choice & Percentage \\
\hline 1. Best route & 49.00 \\
\hline 2. Least variable time & 2.00 \\
\hline 3. Most consistent trip & 6.00 \\
\hline 4. Most reliable trip & 15.00 \\
\hline 5. Most predicable trip & 7.33 \\
\hline 6. Forecasted trip & 18.00 \\
\hline 7. Historical trip conditions & 2.67 \\
\hline Total & 100.00 \\
\hline
\end{tabular}


Please flip to page 12 in the binder next to you and

look at the website screen shown.

Notice the title that is circled.

\section{How often would you expect this trip to take 25 minutes or} less?

1 Almost never (1 day out of 20)

2 Less than half the time ( 5 days out of 20 )

3 About half the time (10 days out of 20 )

4 Most of the time (15 days out of 20 )

Nearly all the time (19 days out of 20 )

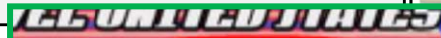

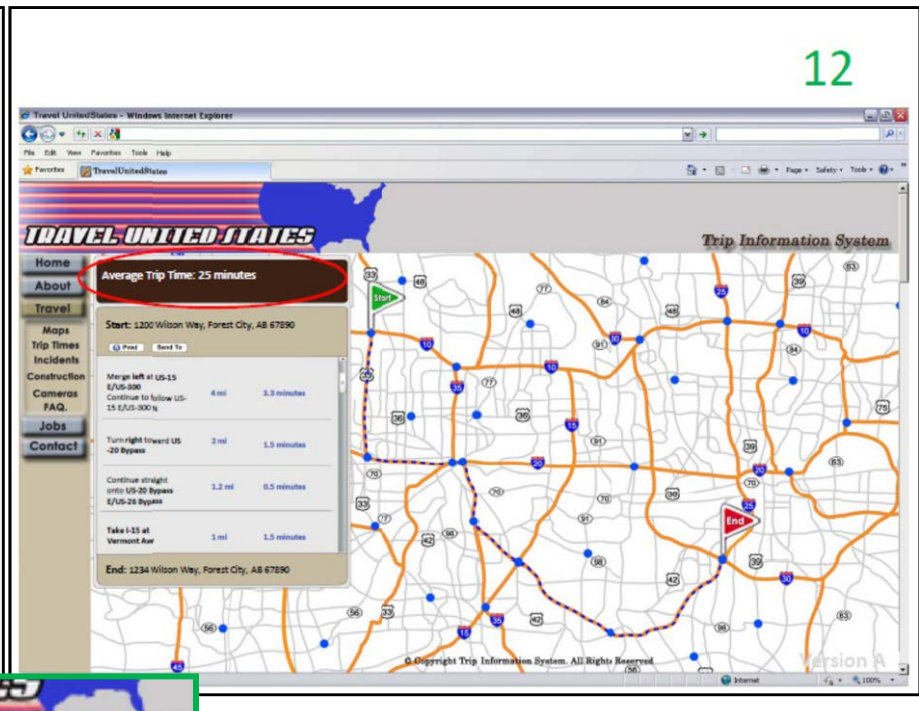

\section{Average Trip Time: 25 minutes}

Start: 1200 Wíbon Way, Forest City, AB 67890

COrmt Sonto

Merge left at Us-15

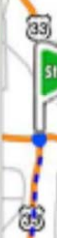

Figure D.15. Question 15 visuals. (Circled term used varied by version.)

Table D.12. Question 15 Response Summary

\begin{tabular}{|c|c|c|c|c|}
\hline Response Choice & $\begin{array}{c}\text { Version A: } \\
\text { Average Trip Time } \\
(\%)\end{array}$ & $\begin{array}{c}\text { Version B: } \\
\text { Typical Trip Time } \\
\text { (\%) }\end{array}$ & $\begin{array}{c}\text { Version C: } \\
\text { Historical Trip Time } \\
\text { (\%) }\end{array}$ & $\begin{array}{c}\text { Version D: } \\
\text { Estimated Trip Time } \\
\text { (\%) }\end{array}$ \\
\hline 1. Almost never (1 day out of 20 ) & 11.84 & 9.72 & 8.00 & 8.11 \\
\hline 2. Less than half the time ( 5 days out of 20 ) & 11.84 & 2.78 & 12.00 & 13.51 \\
\hline 3. About half the time ( 10 days out of 20 ) & 18.42 & 16.67 & 14.67 & 13.51 \\
\hline 4. Most of the time ( 15 days out of 20 ) & 38.16 & 58.33 & 45.33 & 48.65 \\
\hline 5. Nearly all the time ( 19 days out of 20 ) & 19.74 & 16.67 & 20.00 & 16.22 \\
\hline
\end{tabular}


Please flip to page 13 in the binder next to you and look at the website screen shown.

How often would you expect this trip to take 25 minutes or less?

1 Almost never (1 day out of 20 )

2 Less than half the time ( 5 days out of 20 )

3 About half the time ( 10 days out of 20 )

4 Most of the time (15 days out of 20)

5 Nearly all the time (19 days out of 20 )

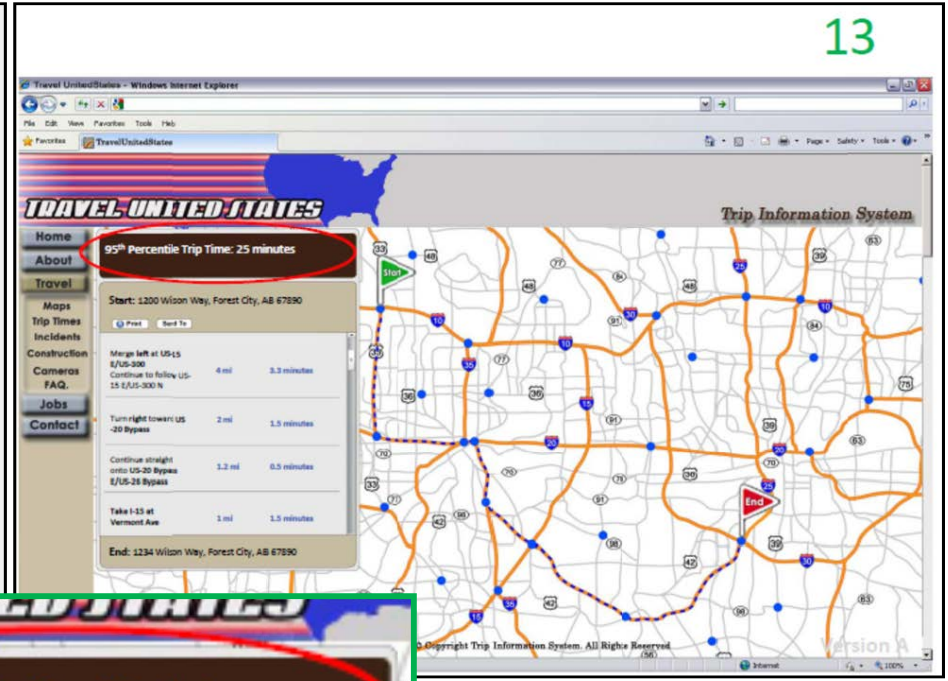

$95^{\text {th }}$ Percentile Trip Time: 25 minutes

Start: 1200 Wileon Way, Forest City, AB 67890

Coprat Bond to

Figure D.16. Question 16 visuals. (Circled term used varied by version.)

Table D.13. Question 16 Response Summary by Version

\begin{tabular}{|l|c|c|c|c|}
\hline Response Choice & $\begin{array}{c}\text { Version A: } \\
\text { 95th Percentile } \\
\text { Trip Time (\%) }\end{array}$ & $\begin{array}{c}\text { Version B: } \\
\text { Worst-Case } \\
\text { Trip Time (\%) }\end{array}$ & $\begin{array}{c}\text { Version C: } \\
\text { Maximum Trip } \\
\text { Time (\%) }\end{array}$ & $\begin{array}{c}\text { Version D: } \\
\text { Most Common } \\
\text { Trip Time (\%) }\end{array}$ \\
\hline 1. Almost never (1 day out of 20) & 9.21 & 16.00 & 4.00 & 9.46 \\
\hline 2. Less than half the time (5 days out of 20) & 10.53 & 32.00 & 21.33 & 13.51 \\
\hline 3. About half the time (10 days out of 20) & 15.79 & 13.33 & 21.33 & 17.57 \\
\hline 4. Most of the time (15 days out of 20) & 27.63 & 17.33 & 24.00 & 43.24 \\
\hline 5. Nearly all the time (19 days out of 20) & 36.84 & 21.33 & 29.33 & 16.22 \\
\hline
\end{tabular}




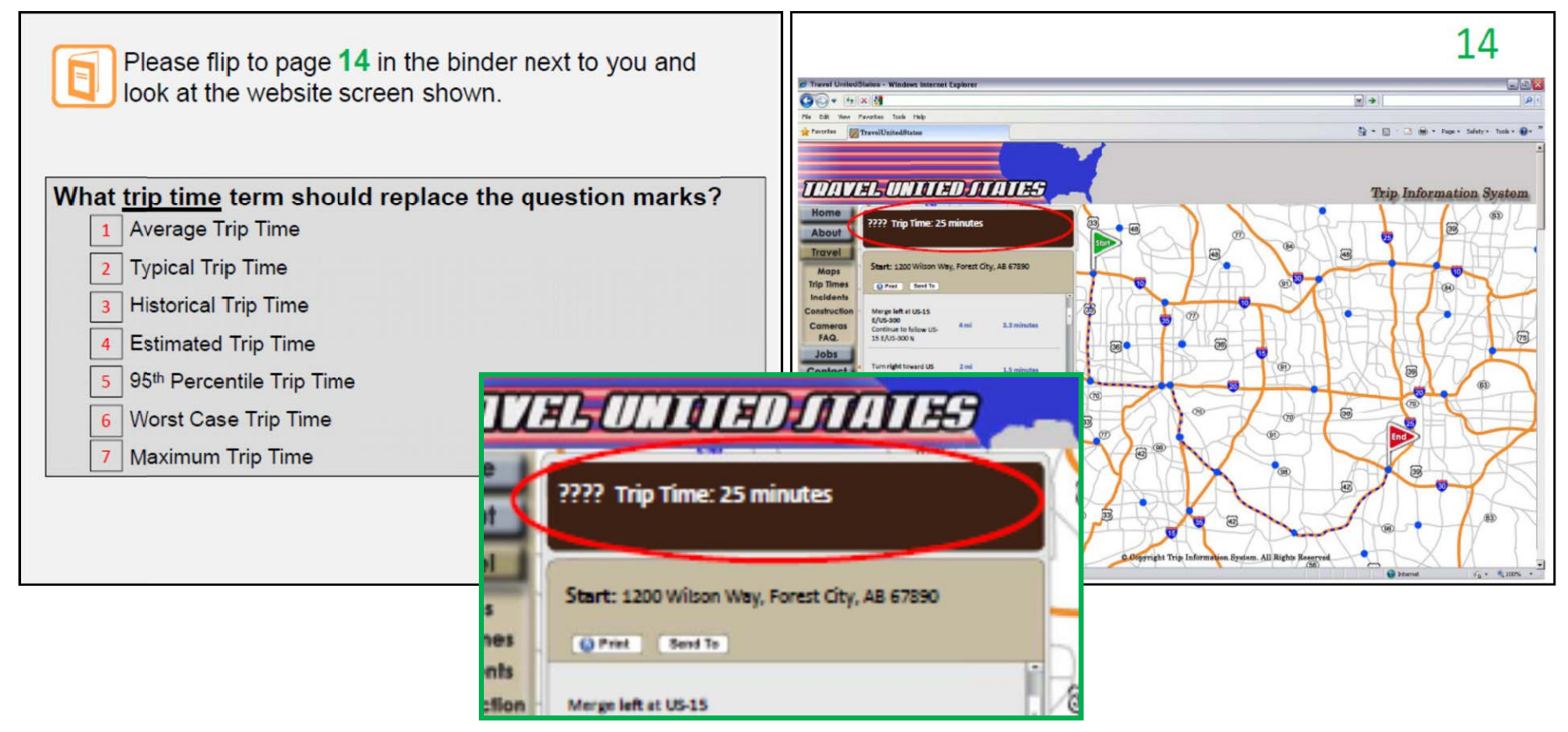

Figure D.17. Question 17 visuals.

Table D.14. Question 17 Response

Summary

\begin{tabular}{|l|c|}
\hline Response Choice & Percentage \\
\hline 1. Average trip time & 33.00 \\
\hline 2. Typical trip time & 9.00 \\
\hline 3. Historical trip time & 4.00 \\
\hline 4. Estimated trip time & 43.33 \\
\hline 5. 95th percentile trip time & 3.00 \\
\hline 6. Worst-case trip time & 1.67 \\
\hline 7. Maximum trip time & 6.00 \\
\hline Total & 100.00 \\
\hline
\end{tabular}


Please flip to page 15 in the binder next to you and lock at the website screen shown.

The circled part is showing you the time to leave, and the map displays a route based on the start and end locations and the time of day you specified.

If you departed from your starting point at the time shown, how often would you expect to arrive at your destination on time?

1 Almost never ( 1 day out of 20 )

2 Less than half the time ( 5 days out of 20 )

3 About half the time ( 10 days out of 20 )

4 Most of the time (15 days out of 20 )

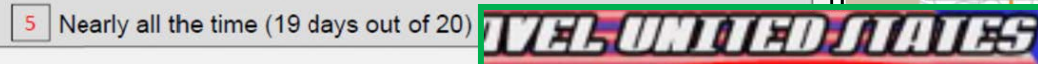

Trip Time Provided by System: 25 minutes

Recommended Departure Time $=7.50$ AM

Start: 1200 Wilbon Way, Forest City, AB 67890

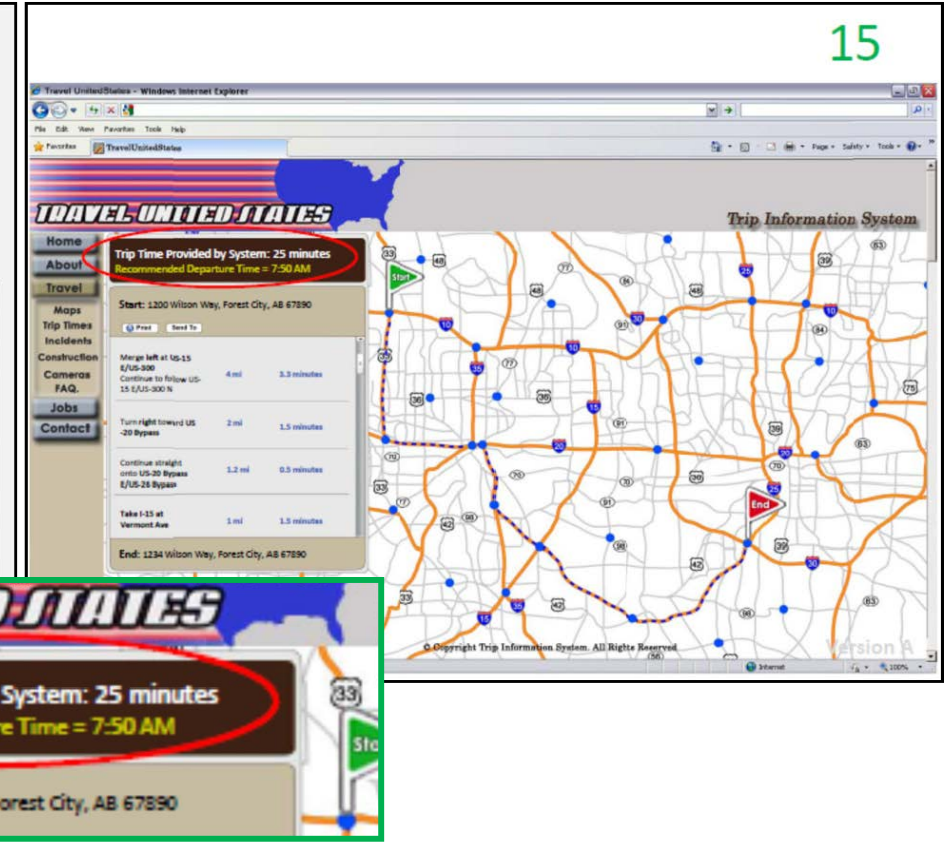

Figure D.18. Question 18 visuals. (Circled term used varied by version.)

Table D.15. Question 18 Response Summary

\begin{tabular}{|c|c|c|c|c|}
\hline Response Choice & $\begin{array}{c}\text { Version A: } \\
\text { Recommended } \\
\text { Departure Time (\%) }\end{array}$ & $\begin{array}{c}\text { Version B: } \\
\text { Estimated } \\
\text { Departure Time (\%) }\end{array}$ & $\begin{array}{c}\text { Version C: } \\
\text { 95th Percentile } \\
\text { Departure Time (\%) }\end{array}$ & $\begin{array}{c}\text { Version D: } \\
\text { Suggested } \\
\text { Departure Time (\%) }\end{array}$ \\
\hline 2. Less than half the time ( 5 days out of 20 ) & 4.00 & 4.00 & 2.67 & 5.48 \\
\hline 3. About half the time ( 10 days out of 20 ) & 18.67 & 10.67 & 13.33 & 19.18 \\
\hline 5. Nearly all the time (19 days out of 20 ) & 17.33 & 14.67 & 38.67 & 23.29 \\
\hline
\end{tabular}


If it is important that you arrive at your destination at 8:15, what time would you leave for this trip?
1 7:30 (20 minutes before departure time shown)
2 7:40 (10 minutes before departure time shown)
3 7:45 (5 minutes before departure time shown)
4 7:50
5 7:55 (5 minutes after departure time shown)
6 8:00 (10 minutes after departure time shown)
7 8:05 (15 minutes after ceparture time shown)

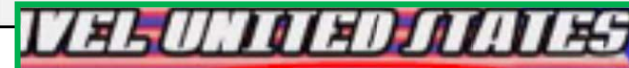

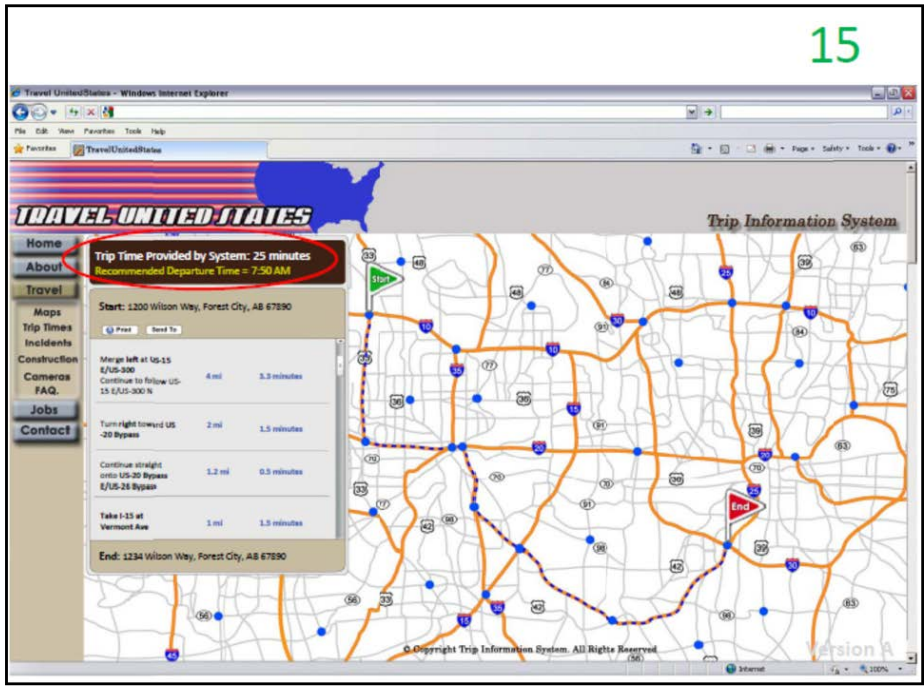

Trip Time Provided by System: 25 minutes

Recommended Departure Time $=750 \mathrm{AM}$

Start: 1200 Wileon Way, Forest City, AB 67890

CPrent Bent to

Figure D.19. Question 19 visuals. (Circled term used varied by version.)

Table D.16. Question 19 Response Summary

\begin{tabular}{|c|c|c|c|c|}
\hline Response Choice & $\begin{array}{c}\text { Version A: } \\
\text { Recommended } \\
\text { Departure Time (\%) }\end{array}$ & $\begin{array}{c}\text { Version B: } \\
\text { Estimated } \\
\text { Departure Time (\%) }\end{array}$ & $\begin{array}{c}\text { Version C: } \\
\text { 95th Percentile } \\
\text { Departure Time (\%) }\end{array}$ & $\begin{array}{c}\text { Version D: } \\
\text { Suggested } \\
\text { Departure Time (\%) }\end{array}$ \\
\hline 2. 10 minutes before departure time shown & 27.63 & 34.67 & 34.67 & 35.14 \\
\hline 3. 5 minutes before departure time shown & 14.47 & 20.00 & 16.00 & 18.92 \\
\hline 5. 5 minutes after departure time shown & 2.63 & 1.33 & 1.33 & 0.00 \\
\hline 6. 10 minutes after departure time shown & 0.00 & 4.00 & 0.00 & 2.70 \\
\hline 7. 15 minutes after departure time shown & 1.32 & 0.00 & 1.33 & 1.35 \\
\hline
\end{tabular}


Please flip to page 16 in the binder next to you and look at the website screen shown.

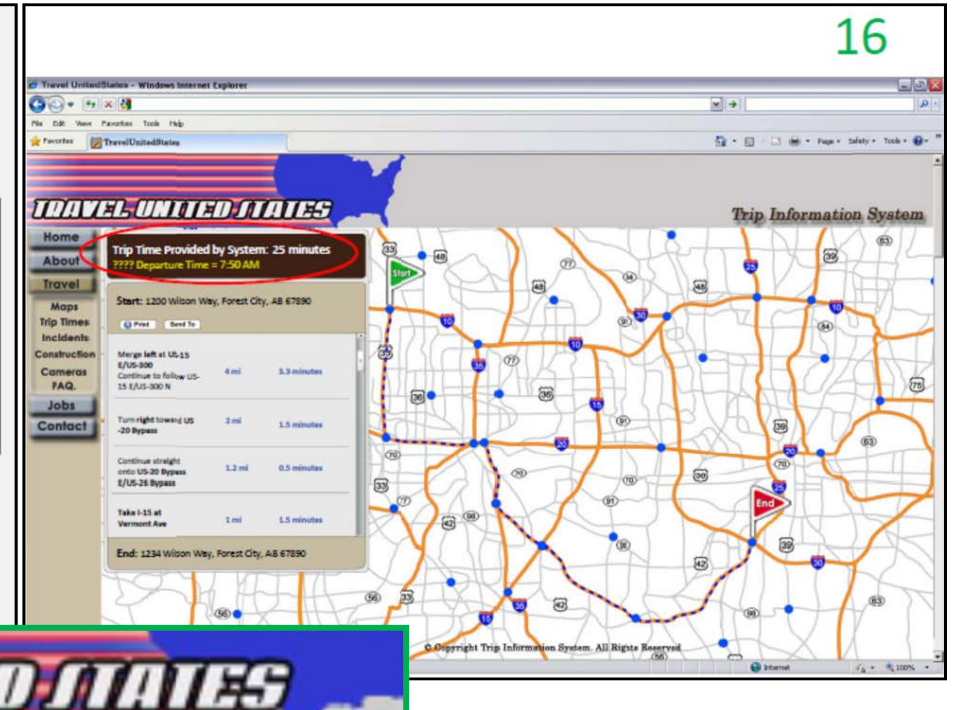

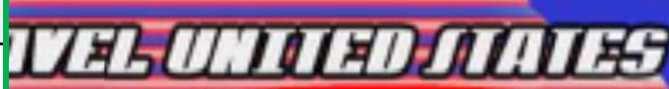

Trip Time Provided by System: 25 minutes

???? Departure Time = 7:50 AM

Start: 1200 Wilson Way, Forest City, AB 67890

Figure D.20. Question 20 visuals.

Table D.17. Question 20

Response Summary

\begin{tabular}{|l|c|}
\hline Response Choice & Percentage \\
\hline 1. Recommended departure time & 47.00 \\
\hline 2. Estimated departure time & 21.00 \\
\hline 3. 95th percentile departure time & 4.33 \\
\hline 4. Suggested departure time & 27.67 \\
\hline Total & 100.00 \\
\hline
\end{tabular}




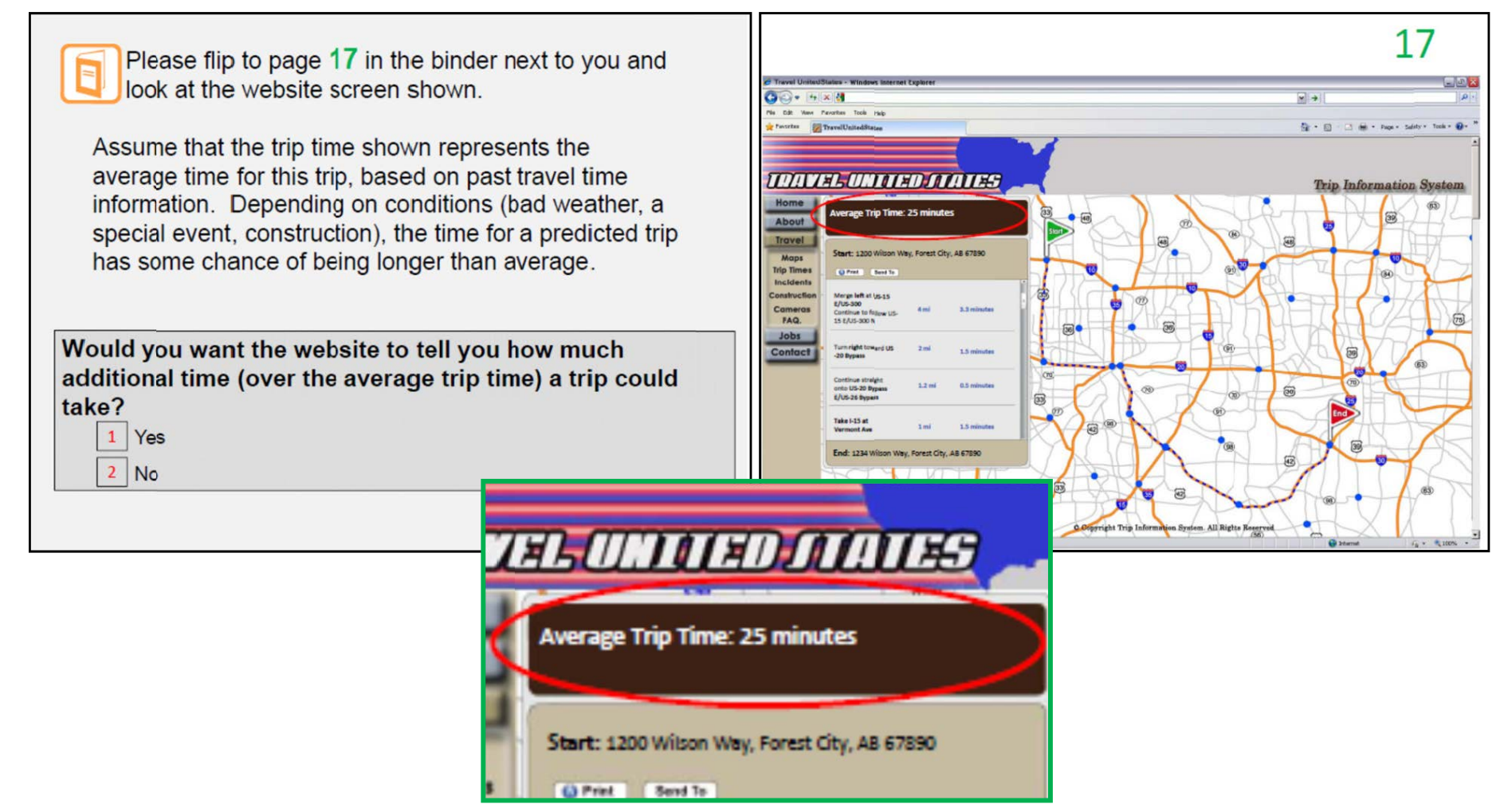

Figure D.21. Question 21 visuals.

Table D.18. Question 21

Response Summary

\begin{tabular}{|l|c|}
\hline Response Choice & Percentage \\
\hline 1. Yes & 88.33 \\
\hline 2. No & 11.67 \\
\hline Total & 100.00 \\
\hline
\end{tabular}




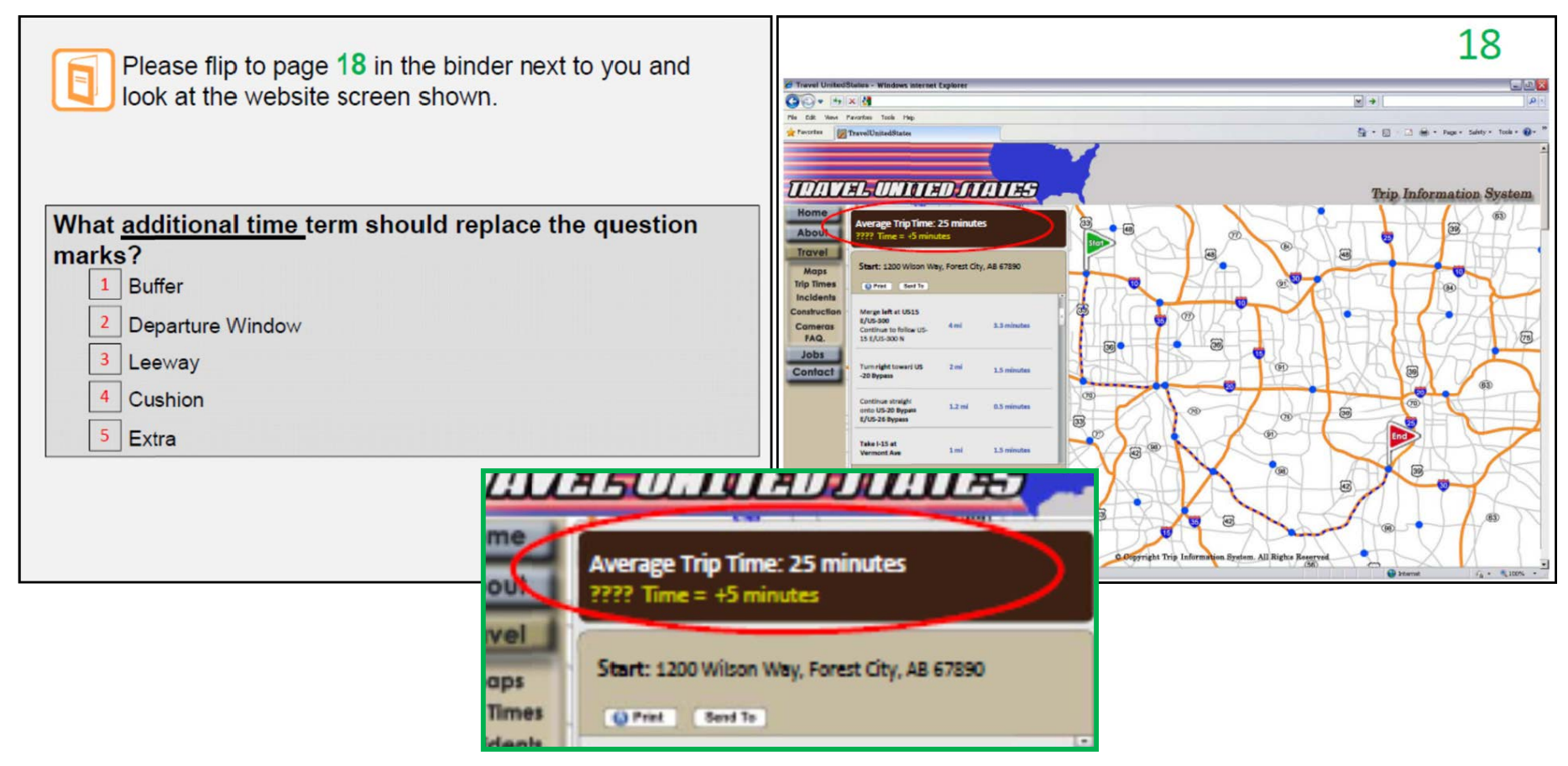

Figure D.22. Question 22 visuals.

Table D.19. Question 22

Response Summary

\begin{tabular}{|l|c|}
\hline Response Choice & Percentage \\
\hline 1. Buffer & 10.33 \\
\hline 2. Departure window & 28.00 \\
\hline 3. Leeway & 15.67 \\
\hline 4. Cushion & 13.33 \\
\hline 5. Extra & 32.67 \\
\hline Total & 100.00 \\
\hline
\end{tabular}


Please flip to page 19 in the binder next to you and look at the website screen shown.

The system is providing you a trip time based on past data collected. With that in mind, what is the most useful information that you would like the system to tell you?
1 An average time
2 A worst case time
3 A range of times it could take (for ex. 20-35 minutes)
4 The best case time it could take
5 The most likely time it will take
6 The time where half the trips would take longer and half would take shorter

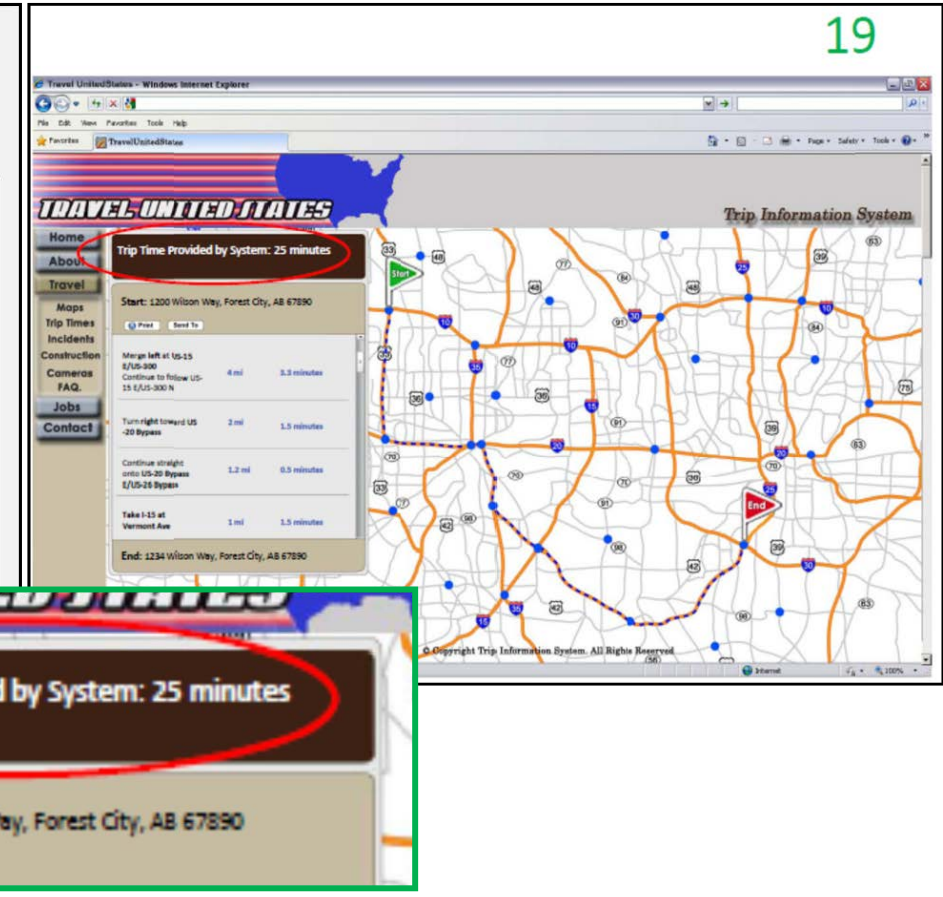

Figure D.23. Question 23 visuals.

Table D.20. Question 23 Response Summary

\begin{tabular}{|l|c|}
\hline Response Choice & Percentage \\
\hline 1. An average time & 30.67 \\
\hline 2. A worst-case time & 8.00 \\
\hline 3. A range of times it could take & 28.67 \\
\hline 4. The best case time it could take & 7.00 \\
\hline 5. The most likely time it will take & 24.33 \\
\hline $\begin{array}{l}\text { 6. The time where half the trips would take longer } \\
\text { and half would take shorter }\end{array}$ & 1.33 \\
\hline Total & 100.00 \\
\hline
\end{tabular}




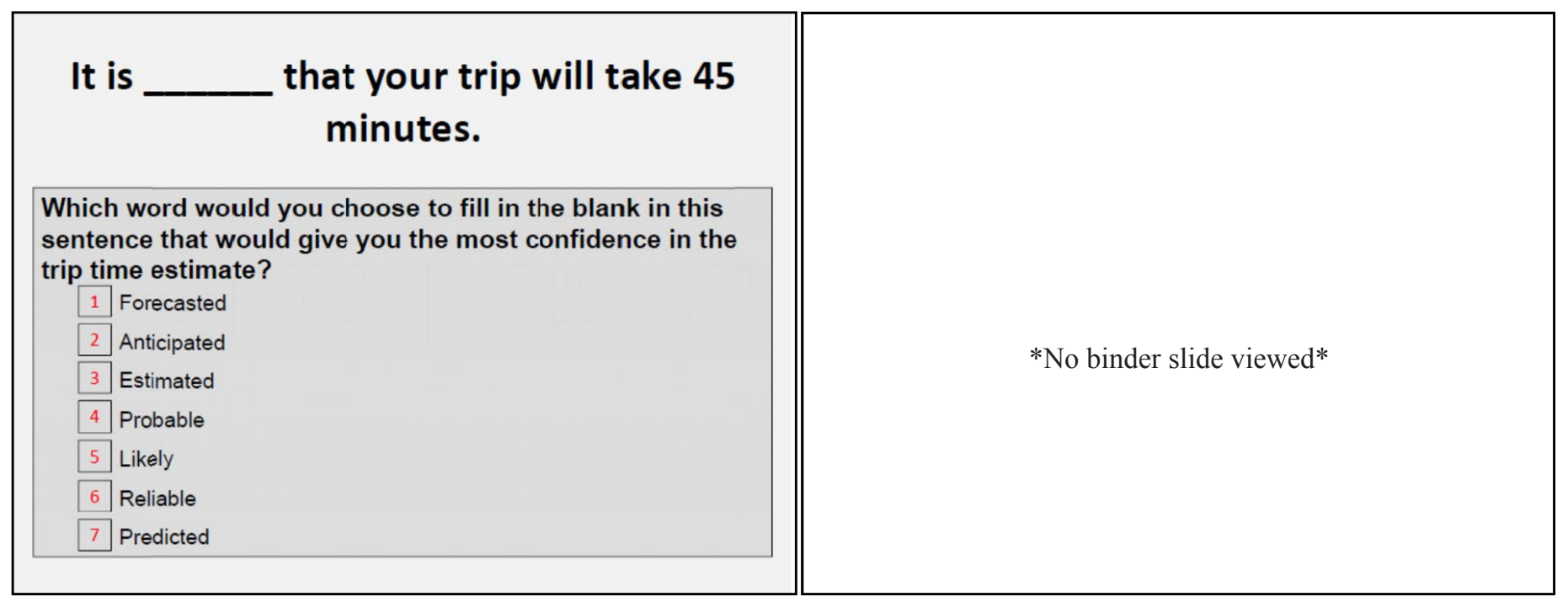

Figure D.24. Question 24 visuals.

Table D.21. Question 24

Response Summary

\begin{tabular}{|l|c|}
\hline Response Choice & Percentage \\
\hline 1. Forecasted & 5.33 \\
\hline 2. Anticipated & 7.33 \\
\hline 3. Estimated & 56.00 \\
\hline 4. Probable & 1.67 \\
\hline 5. Likely & 10.67 \\
\hline 6. Reliable & 6.00 \\
\hline 7. Predicted & 13.00 \\
\hline Total & 100.00 \\
\hline
\end{tabular}




\section{Your trip time may____ from the} average time by 15 minutes.

Which word would you choose to fill in the blank in this sentence that would give you the most confidence in the trip time estimate?
1 Differ
2 Vary
3 Fluctuate
4 Change
5 Go up or down
6 Increase or decrease
7 Deviate

*No binder slide viewed*

Figure D.25. Question 25 visuals.

Table D.22. Question 25

Response Summary

\begin{tabular}{|l|c|}
\hline Response Choice & Percentage \\
\hline 1. Differ & 7.00 \\
\hline 2. Vary & 59.33 \\
\hline 3. Fluctuate & 10.67 \\
\hline 4. Change & 8.33 \\
\hline 5. Go up or down & 0.33 \\
\hline 6. Increase or decrease & 6.00 \\
\hline 7. Deviate & 8.33 \\
\hline Total & 100.00 \\
\hline
\end{tabular}




\section{It will take} 20 minutes to

\section{make your trip.}

Which word would you choose to fill in the blank in this sentence that would give you the most confidence in the trip time estimate?
1 About
2 Approximately
3 Give or Take
4 An estimate of
5 Around
6 Roughly
7 An average of

*No binder slide viewed*

Figure D.26. Question 26 visuals.

Table D.23. Question 26

Response Summary

\begin{tabular}{|l|c|}
\hline Response Choice & Percentage \\
\hline 1. About & 7.33 \\
\hline 2. Approximately & 69.00 \\
\hline 3. Give or take & 1.33 \\
\hline 4. An estimate of & 8.67 \\
\hline 5. Around & 1.33 \\
\hline 6. Roughly & 2.00 \\
\hline 7. An average of & 10.33 \\
\hline Total & 100.00 \\
\hline
\end{tabular}


Please flip to page 21 in the binder next to you and look at the website screen shown.

\section{What does $95 \%$ time mean to you?}

1 It will take $95 \%$ of the provided trip time to arrive

295 times out of 100 it will take the provided time to arrive

3 The system is $95 \%$ confident that its provided time is correct to arrive

4 95 times out of 100 it will take at or less than the provided time to arrive

5 The time it takes you to complete $95 \%$ of the distance of your trip

6 I don't know

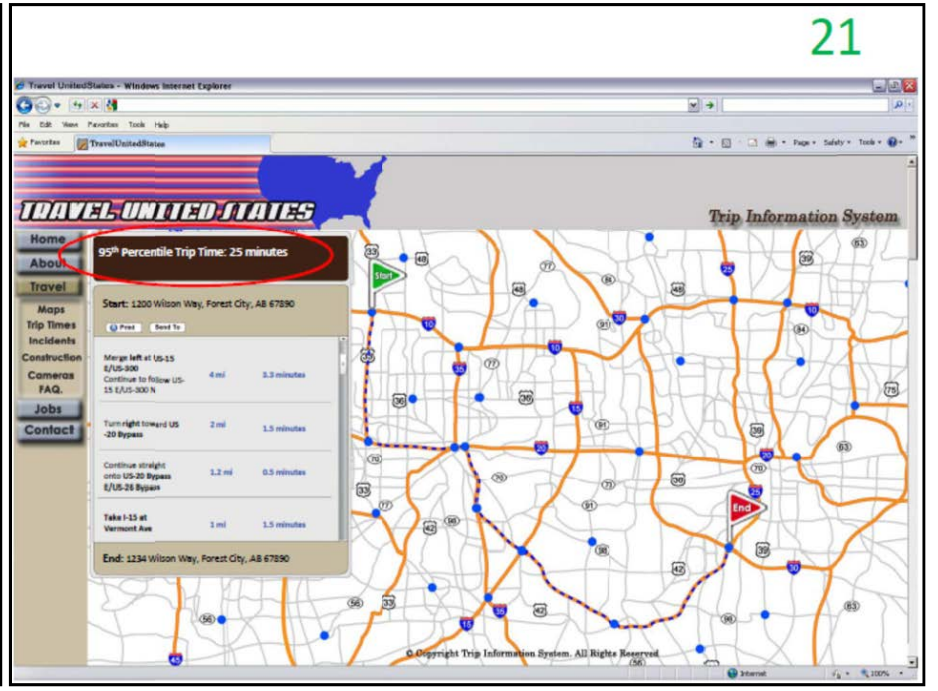

Figure D.27. Question 27 visuals.

Table D.24. Question 27 Response Summary

\begin{tabular}{|l|c|}
\hline Response Choice & Percentage \\
\hline $\begin{array}{l}\text { 1. It will take 95\% of the provided trip time to } \\
\text { arrive. }\end{array}$ & 6.00 \\
\hline $\begin{array}{l}\text { 2. } 95 \text { times out of } 100 \text { it will take the provided time } \\
\text { to arrive. }\end{array}$ & 44.00 \\
\hline $\begin{array}{l}\text { 3. The system is 95\% confident that its provided } \\
\text { time is accurate. }\end{array}$ & 32.33 \\
\hline $\begin{array}{l}\text { 4. } 95 \text { times out of } 100 \text { it will take at or less than the } \\
\text { provided time to arrive. }\end{array}$ & 13.67 \\
\hline $\begin{array}{l}\text { 5. The time it takes you to complete 95\% of the } \\
\text { distance of your trip. }\end{array}$ & 1.33 \\
\hline 6. I don't know. & 100.00 \\
\hline Total & 2.67 \\
\hline
\end{tabular}


Often drivers will add additional time to a travel time they receive from a resource such as Mapquest or their GPS, "just in case".

Which of these statements uses wording that best explains that the provided travel time given to you is trustworthy and you don't need to add any additional time?

1 It is $95 \%$ reliable that your travel time will be 45 minutes.

219 out of 20 times your travel time will be 45 minutes.

3 Your maximum trip time is $\mathbf{4 5}$ minutes.

4 There is low variability to your trip time of 45 minutes.

5 The worst case travel time is 45 minutes.

6 The system is very certain that your trip will take 45 minutes.

*No binder slide viewed*

Figure D.28. Question 28 visuals.

\section{Table D.25. Question 28 Response Summary}

\begin{tabular}{|l|c|}
\hline Response Choice & Percentage \\
\hline $\begin{array}{l}\text { 1. It is } 95 \% \text { reliable that your travel time will be } 45 \\
\text { minutes. }\end{array}$ & 33.67 \\
\hline \begin{tabular}{l} 
2. $\begin{array}{l}19 \text { out of } 20 \text { times your travel time will be } 45 \\
\text { minutes. }\end{array}$ \\
\hline 3. Your maximum trip time is 45 minutes.
\end{tabular} & 3.00 \\
\hline $\begin{array}{l}\text { 4. There is low variability to your trip time of } 45 \\
\text { minutes. }\end{array}$ & 3.00 \\
\hline 5. The worst case travel time is 45 minutes. & 6.67 \\
\hline $\begin{array}{l}\text { 6. The system is very certain that your trip will take } \\
45 \text { minutes. }\end{array}$ & 9.33 \\
\hline Total & 100.00 \\
\hline
\end{tabular}


Please flip to page 23 in the binder next to you and look at the website screen shown.

If you were to select one of the circled options, it would change your mode of travel from your origin and destination

If you have the option to occasionally change your mode, and the system showed that using a different mode would be quicker, would you consider changing your mode for that day?

1 Yes

No
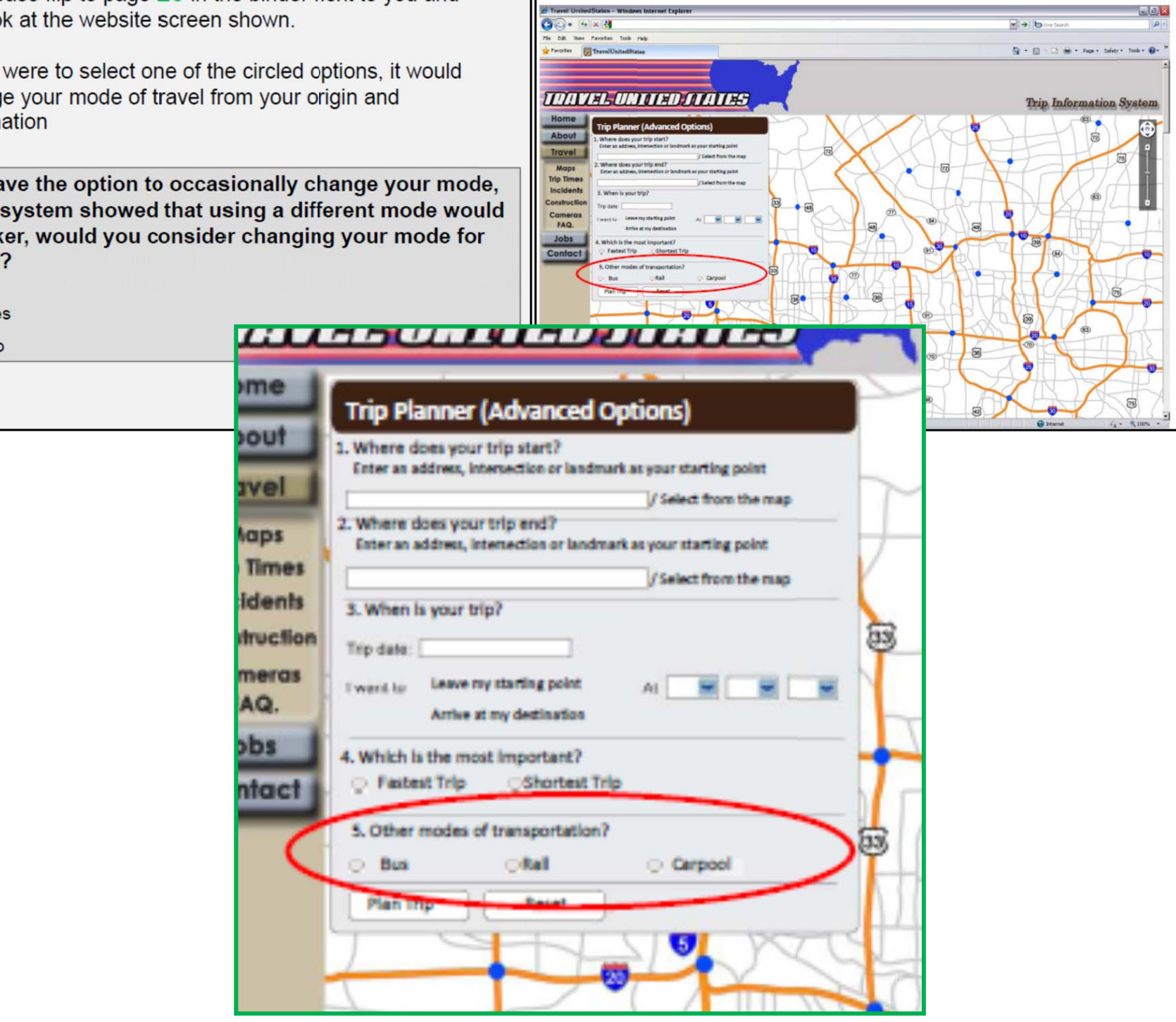

Figure D.29. Question 29 visuals.

Table D.26. Question 29

Response Summary

\begin{tabular}{|l|c|}
\hline Response Choice & Percentage \\
\hline 1. Yes & 76.33 \\
\hline 2. No & 23.67 \\
\hline Total & 100.00 \\
\hline
\end{tabular}




\section{APPENDIX E \\ Open-Ended Survey Materials}

\section{Introduction}

The open-ended survey materials consisted of (1) a survey form that was completed by the researcher and included instructions to the researcher for administering the survey, and (2) a set of cards for each survey question that was presented to the survey participant. The scenario cards for Questions 1 and 2 repeated the travel scenario information that the researcher read aloud to the participant, including two sentences describing travel time parameters. Additional cards for each scenario included a reference card listing five confidence levels, and a total of six cards providing three alternate terms for each of the two travel time parameters in the scenario.

The survey and cards were created in 16 different versions to present different combinations of the travel time reliability terms to the participant pool. Each participant saw only one version of the survey. This appendix contains Version A of the survey, followed by the corresponding cards.

\section{Survey Form and Questions}

Date: City: Subject \#: Survey: A

Study Type: $\square$ Survey $\square$ Experiment Occupation:

Question 1 objective: Determine whether motorists understand an (average, expected, typical, estimated) travel time term and a (cushion, added, extra) time term when presented together, and understand that they can be added together to come up with an arrival-time-constrained travel time.

1. (Show participant Survey A, Question 1 card.) Imagine that you are in a hotel in an unfamiliar city and have an important business meeting first thing this morning at a downtown office building. You will need to leave the hotel during the peak period in order to arrive at the business meeting. You are trying to decide how much travel time to allow for your drive to downtown. You cannot be late to the meeting, but you do not want to arrive too early. You know that you can park right next to the building and do not need to allow additional time to search for parking. You also know that you can get current travel time information from the hotel website. Before you drive to your downtown meeting, you check the website and obtain the following information...

\section{The average travel time to downtown is 40 minutes}

and

the cushion time to downtown is 30 minutes. 
Questions $1 a$ and $1 b$ objective: Determine if motorists will use both the terms above to estimate how much time to allow for a trip. Emphasis should be on determining if they considered the (cushion, added, extra) time at all in their travel time estimate. So, if they use less than 70 minutes or more than 70 minutes, make sure to get them to explain what they added together and why.

a) How much time would you allow for your drive to downtown?

b) How did you decide on that time?

Question 1c objective: Determine if motorists understand that their time may be longer or shorter than what is shown (average, expected, typical, estimated). Facilitator should make sure responses indicate the participants' opinions about whether their particular travel time is likely to be much longer or shorter than this value.

c) What does the average travel time mean to you about how long your drive will take?

Question 1d objective: Determine if motorists understand that the (cushion, added, extra) time represents the additional time it may take if traffic problems or other factors occur that make their trip take longer than normal. Facilitator might need to rephrase the question as "why would an agency try to provide this time to drivers?"

d) What does the cushion time mean to you about how long your drive will take?

Question 1e objective: Determine what probability of a successful arrival (prior to the meeting time) drivers would associate with the two terms.

e) (Show participant the card with the scale below on it.) If you combined the average travel time and cushion time together (70 minutes), how likely are you to reach downtown in 70 minutes or less?

Absolutely certain (100\% chance)

Very likely (95\% chance)

Probably (75\% chance)

Unsure (50/50 chance)

$\square$ Doubtful (less than 50/50 chance)

Why did you select this as your answer?

Question If objective: Determine if small cushions are not likely to be as useful or believed by motorists. Facilitator should make sure the response indicates what, if anything, they do with the 10 minute (cushion, added, extra) time when they make their decision.

f) If the cushion time had been 10 minutes, how much time would you have allowed for your drive? Why? 
Question 1g objectives: Determine whether motorists perceive (cushion, recommended cushion, added, and extra) time to mean the same thing or not, and if different, how are they different. Facilitator should make sure to determine this for all terms, not just compared to term given in scenario above. Facilitator will also obtain the participant's term preference and opinion regarding a better term to use.

g) (Show participant Survey A Card Set\#1.) Which of these terms means the same thing as the cushion time (check all that apply)?
$\square$ Recommended cushion
$\square$ Added
Extra
None of them

For those terms not selected, why are these terms different than the cushion time? If more than one term is not selected, make sure to also determine if those terms have the same or different meaning.

Which term do you like the best and why?

Can you think of a better term to use?

Question 2 objective: Determine whether motorists understand an (average, expected, typical, estimated) travel time term and a (95th percentile, travel time for planning, most of the time less than, majority of the time less than) travel time term when presented together, and understand that the reliability term indicates a recommended travel time to plan for in an arrival-time-constrained situation.

2. (Show participant Survey A, Question 2 card.) Imagine that you are again in a hotel in another unfamiliar city and have an important business meeting this morning at a downtown office building. As before, you will need to leave the hotel during the peak period to arrive at the business meeting. You are trying to decide how much travel time to allow for your drive to downtown. You cannot be late to the meeting, but you do not want to arrive too early. You know that you can park right next to the building and do not need to allow additional time to search for parking. You also know that you can get current travel time information from the hotel website. Before you drive to your downtown meeting, you check the website and obtain the following information...

\section{The expected travel time to downtown is 30 minutes}

\section{and}

\section{the 95th percentile travel time to downtown is 55 minutes.}

Questions $2 a$ and $2 b$ objective: Determine if motorists will use the longer travel time for planning purposes, or if they simply add their own number to the (average, expected, typical, estimated) value.

a) How much time would you allow for your drive to downtown?

b) How did you decide on that time? 
Question 2c objective: Determine if motorists understand that their time may be longer or shorter than what is shown (average, expected, typical, estimated). Facilitator should make sure responses indicate the participants' opinions about whether their particular travel time is likely to be much longer or shorter than this value.

c) What does the expected travel time mean to you about how long your drive will take?

Question 2d objective: Determine if motorists understand that the (95th percentile, travel time for planning, most of the time less than, majority of the time less than) represents the worst-case situation, and that most travel times to downtown do not take this long. Facilitator might need to rephrase the question as "why would an agency try to provide this time to drivers?"

d) What does the statement "the 95th percentile travel time to downtown is 55 minutes" mean to you about how long your drive will take?

Question 2e objective: Determine what probability of a successful arrival (prior to the meeting time) drivers would associate with these terms.

e) (Show participant the card with the scale below on it.) Given the statement "the 95th percentile travel time to downtown is 55 minutes," how likely are you to reach downtown in less than 55 minutes?

Absolutely certain (100\% chance)

Very likely (95\% chance)

Probably (75\% chance)

Unsure (50/50 chance)

$\square$ Doubtful (less than 50/50 chance)

Why did you select this as your answer?

Question $2 f$ objective: Determine if a smaller difference between the (average, expected, typical, estimated) and the (95th percentile travel time, travel time for planning, most of the time less than, majority of the time less than) is less likely to be useful or believed by motorists. Facilitator should make sure the response indicates what, if anything, they do with the smaller time value provided when they make their decision.

f) If the statement had been "the 95th percentile travel time to downtown is 45 minutes," how much time would you have allowed for your drive? Why?

Question $2 g$ objectives: Determine whether motorists perceive (average, expected, typical, estimated) time to mean the same thing or not, and if different, how are they different. Facilitator should make sure to determine this for all terms, not just compared to term given in scenario above. Facilitator will also obtain the participant's term preference and opinion regarding a better term to use.

g) (Show participant Survey A Card Set \#2.) Which of these terms means the same thing as the expected travel time (check all that apply)?

$\square$ Average $\square$ Typical $\square$ Estimated $\square$ None of them 
For those terms not selected, why are these terms different than the expected travel time? If more than one term is not selected, make sure to also determine if those terms have the same or different meaning.

Which term do you like the best and why?

Can you think of a better term to use?

Question $2 h$ objectives: Determine whether motorists perceive the (95th percentile travel time, travel time for planning, most of the time less than, majority of the time less than) to mean the same thing or not, and if different, how are they different. Facilitator should make sure to determine this for all terms, not just compared to term given in scenario above. Facilitator will also obtain the participant's term preference and opinion regarding a better term to use.

h) (Show participant Survey A Card Set \#3.) Which of these terms means the same thing as the 95th percentile travel time is 55 minutes (check all that apply)?

The travel time for planning to downtown is 55 minutes or less.

Most of the time the travel time to downtown is 55 minutes or less.

The majority of the time the travel time to downtown is 55 minutes or less.

$\square$ None of them.

For those terms not selected, why are these statements different than the 95th percentile travel time is 55 minutes? If more than one term is not selected, make sure to also determine if those terms have the same or different meaning.

Which term do you like the best and why?

Can you think of a better term to use?

3. In the first example, you preferred (insert term selected as best in question $1 g$ ), and for the second example, you preferred (insert term selected as best in question $2 h$ ). Which of these two terms do you prefer for indicating the amount of uncertainty in travel time for your trip downtown? Why do you prefer this term?

Do you have any other comments? 


\section{Question 1}

Imagine that you are in a hotel in an unfamiliar city and have an important business meeting first thing this morning at a downtown office building. You will need to leave the hotel during the peak period in order to arrive at the business meeting. You are trying to decide how much travel time to allow for your drive to downtown. You cannot be late to the meeting, but you do not want to arrive too early. You know that you can park right next to the building and do not need to allow additional time to search for parking. You also know that you can get current travel time information from the hotel website. Before you drive to your downtown meeting, you check the website and obtain the following information ...

The average travel time to downtown is 40 minutes

and

the cushion time to downtown is $\mathbf{3 0}$ minutes.

\section{Reference Card Left Visible to Participant as Memory Aid for Confidence Ratings}
Absolutely certain ( $100 \%$ chance)
Very likely (95\% chance)
Probably ( $75 \%$ chance)
Unsure (50/50 chance)
Doubtful (less than $50 / 50$ chance)

\section{Alternate Terms Card Set 1}

The cushion time to downtown is __ minutes.

The added time to downtown is __ minutes.

The extra time to downtown is __ minutes.

The recommended cushion time to downtown is _ minutes.

\section{Alternate Terms Card Set 2}

The average travel time to downtown is __ minutes.

The typical travel time to downtown is _ minutes.

The expected travel time to downtown is _ minutes.

The estimated travel time to downtown is __ minutes. 
Cards for Question 2, Survey Version A

Scenario Card

\section{Question 2}

Imagine that you are again in a hotel in another unfamiliar city and have an important business meeting this morning at a downtown office building. As before, you will need to leave the hotel during the peak period in order to arrive at the business meeting. You are trying to decide how much travel time to allow for your drive to downtown. You cannot be late to the meeting, but you do not want to arrive too early. You know that you can park right next to the building and do not need to allow additional time to search for parking. You also know that you can get current travel time information from the hotel website. Before you drive to your downtown meeting, you check the website and obtain the following information...

The expected travel time to downtown is 30 minutes

and

the 95th percentile travel time to downtown is $\mathbf{5 5}$ minutes.

Reference Card Left Visible to Participant as Memory Aid for Confidence Ratings

\begin{tabular}{|l|}
\hline Absolutely certain $(100 \%$ chance $)$ \\
Very likely (95\% chance) \\
Probably ( $75 \%$ chance) \\
Unsure $(50 / 50$ chance $)$ \\
Doubtful (less than $50 / 50$ chance) \\
\hline
\end{tabular}

\section{Alternate Terms Card Set}

The 95th percentile travel time to downtown is minutes.

The travel time for planning to downtown is minutes or less.

Most of the time the travel time to downtown is minutes or less.

The majority of the time the travel time to downtown is minutes or less. 


\section{Travel Behavior Laboratory Experiment Documentation}

\section{Pre- and Post-Experiment Survey Question and Response Categories}

\begin{tabular}{|c|c|}
\hline & Pre-Experiment Survey Questions \\
\hline & Please select your gender and age range. \\
\hline 2. & Please select your highest level of education. \\
\hline 3. & How frequently do you make the following types of trips? \\
\hline$\overline{4 .}$ & What types of roads do you use when you drive to the following destinations? \\
\hline 5. & For the following types of trips, how \\
\hline 6. & On average, how much time do the following trips take for you to complete by car? \\
\hline 7. & On a bad day, how much more time do the following trips take for you to complete by car? \\
\hline 8. & For the following types of trips, what would you consider as arriving late? \\
\hline & $\begin{array}{l}\text { Say you could be guaranteed a travel time before you make your trip. How much would you } \\
\text { pay for this guarantee when making the following types of trips? }\end{array}$ \\
\hline 10. & $\begin{array}{l}\text { Say you could be informed during the beginning of your trip that you will arrive late. How } \\
\text { much would you pay for this information when making the following types of trips? }\end{array}$ \\
\hline & $\begin{array}{l}\text { Post-Experiment Survey Questions } \\
\end{array}$ \\
\hline & $\begin{array}{l}\text { Which of the following objectives was most important in your departure time decisions at the } \\
\text { beginning and at the end of the experiment? }\end{array}$ \\
\hline 2. & $\begin{array}{l}\text { How useful was traffic information at the beginning of the experiment and at the end of the } \\
\text { experiment? }\end{array}$ \\
\hline 3. & When making work trips in the real world, how often do you seek out traffic information? \\
\hline 4. & $\begin{array}{l}\text { When making work trips in the real world, which of the following sources do you use to get } \\
\text { traffic information? (check all that apply) }\end{array}$ \\
\hline
\end{tabular}




\begin{tabular}{|c|c|}
\hline 1a. Gender & 1b. Age \\
\hline (drop-down menu) & (drop-down menu) \\
\hline female & $18-29$ \\
\hline male & $30-39$ \\
\hline & $40-49$ \\
\hline & $50-59$ \\
\hline & $60-69$ \\
\hline & $70-79$ \\
\hline & $80+$ \\
\hline \multicolumn{2}{|l|}{ 2. Education } \\
\hline \multicolumn{2}{|c|}{ (drop-down menu) } \\
\hline \multicolumn{2}{|c|}{ no high school diploma } \\
\hline \multicolumn{2}{|c|}{ high school diploma } \\
\hline \multicolumn{2}{|c|}{ some college } \\
\hline \multicolumn{2}{|c|}{ Bachelor's degree } \\
\hline \multicolumn{2}{|c|}{ graduate degree } \\
\hline \multicolumn{2}{|c|}{ 3. Frequency of Trips by trip type (matrix) } \\
\hline trip types & frequency options (radio button) \\
\hline to work & never \\
\hline to school/college & less than once a month \\
\hline to childcare pick-up & $1-3$ times a month \\
\hline to medical appointments & once a week \\
\hline \multirow[t]{2}{*}{ to non-work meetings* } & 2-4 times a week \\
\hline & 5 or more times a week \\
\hline \multicolumn{2}{|c|}{${ }^{*}$ meeting for social, hobby, or religious activities } \\
\hline \multicolumn{2}{|c|}{$\begin{array}{l}\text { \#Questions 4-10 exclude trip type categories with frequency response of } \\
\text { "never" in Question } 3 .\end{array}$} \\
\hline \multicolumn{2}{|c|}{ 4. Type of roads used by trip destination (matrix) ${ }^{\#}$} \\
\hline trip types & road options (drop-down menu) \\
\hline to work & mostly highways \\
\hline to school/college & highways and signalized roads \\
\hline to childcare pick-up & signalized and neighborhood roads \\
\hline to medical appointments & mostly neighborhood roads \\
\hline \multirow[t]{2}{*}{ to non-work meetings* } & transit \\
\hline & other means of travel \\
\hline \multicolumn{2}{|c|}{ 5. Importance of arriving at a specific time ${ }^{\#}$} \\
\hline trip types & level of importance (radio buttons) \\
\hline to work & not important \\
\hline to school/college & -intermediate without caption- \\
\hline to childcare pick-up & somewhat important \\
\hline to medical appointments & -intermediate without caption- \\
\hline to non-work meetings* & very important \\
\hline
\end{tabular}




\begin{tabular}{|c|c|}
\hline \multicolumn{2}{|c|}{ 6. Average time to destination when driving in car } \\
\hline Trip Destinations & time range options (drop-down menu) \\
\hline to work & $0-9$ minutes \\
\hline to school/college & $10-19$ minutes \\
\hline to childcare pick-up & $20-29$ minutes \\
\hline to medical appointments & 30 - 39 minutes \\
\hline \multirow[t]{4}{*}{ to non-work meetings* } & $40-49$ minutes \\
\hline & $50-59$ minutes \\
\hline & 1 hour or more \\
\hline & trip not made by car \\
\hline \multicolumn{2}{|c|}{ 7. Additional time to destination on a "bad day" } \\
\hline Trip Destinations & time range options (drop-down menu) \\
\hline to work & 0 - 9 minutes \\
\hline to school/college & $10-19$ minutes \\
\hline to childcare pick-up & 20 - 29 minutes \\
\hline to medical appointments & 30 - 39 minutes \\
\hline \multirow[t]{4}{*}{ to non-work meetings* } & $40-49$ minutes \\
\hline & $50-59$ minutes \\
\hline & 1 hour or more \\
\hline & trip not made by car \\
\hline \multicolumn{2}{|c|}{ 8. How many minutes beyond planned arrival time is considered late } \\
\hline Trip Destinations & time range options (drop-down menu) \\
\hline to work & 1 minute \\
\hline to school/college & 2 - 4 minutes \\
\hline to childcare pick-up & 5 - 9 minutes \\
\hline to medical appointments & $10-14$ minutes \\
\hline \multirow[t]{4}{*}{ to non-work meetings* } & $15-19$ minutes \\
\hline & $20-24$ minutes \\
\hline & $25-29$ minutes \\
\hline & 30 or more minutes \\
\hline \multicolumn{2}{|c|}{ 9. Willingness to pay (WTP) for guaranteed travel time } \\
\hline Trip Destinations & WTP options (drop-down menu) \\
\hline to work & $\$ 0.00$ \\
\hline to school/college & $\$ 0.50$ \\
\hline to childcare pick-up & $\$ 1.00$ \\
\hline to medical appointments & $\$ 1.50$ \\
\hline \multirow[t]{4}{*}{ to non-work meetings* } & $\$ 2.00$ \\
\hline & $\$ 3.00$ \\
\hline & $\$ 4.00$ \\
\hline & $\$ 5.00$ \\
\hline \multicolumn{2}{|c|}{ 10. Willingness to pay (WTP) for late arrival information } \\
\hline Trip Destinations & WTP options (drop-down menu) \\
\hline to work & $\$ 0.00$ \\
\hline to school/college & $\$ 0.50$ \\
\hline to childcare pick-up & $\$ 1.00$ \\
\hline to medical appointments & $\$ 1.50$ \\
\hline \multirow[t]{4}{*}{ to non-work meetings* } & $\$ 2.00$ \\
\hline & $\$ 3.00$ \\
\hline & $\$ 4.00$ \\
\hline & $\$ 5.00$ \\
\hline
\end{tabular}


1a. Most important objective at beginning of trip

code value level of usefulness

$0 \mathrm{n} / \mathrm{a}$

1 calling family

2 reducing parking fee

3 avoiding late arrival

4 arriving exactly on-time

1b. Most important objective at end of trip

code value level of usefulness

0 n/a

1 calling family

2 reducing parking fee

3 avoiding late arrival

4 arriving exactly on-time

2a. Usefulness of traveler information at beginning of experiment

code value level of usefulness

1 not useful

2 -intermediate without caption-

3 somewhat useful

4 -intermediate without caption-

5 very useful

2b. Usefulness of traveler information at end of experiment

code value level of usefulness

1 not useful

2 -intermediate without caption-

3 somewhat useful

4 -intermediate without caption-

5 very useful

3. How often do you seek traveler information

code value level of usefulness

0 Never

1 less than once a week

2 once a week

3 twice a week

4 three times a week

5 four times a week

6 five times a week

4. Types of media used to seek traveler information (all that apply)

code value level of usefulness

$0=-$ no, $1=$ yes $\quad$ radio

$0=-$ no, $1=$ yes $\quad$ television

$0=-$ no, $1=$ yes $\quad$ computer

$0=-$ no, $1=$ yes $\quad$ handheld or dashboard devices 


\section{Simulation Experiment Narrative Screenshots}

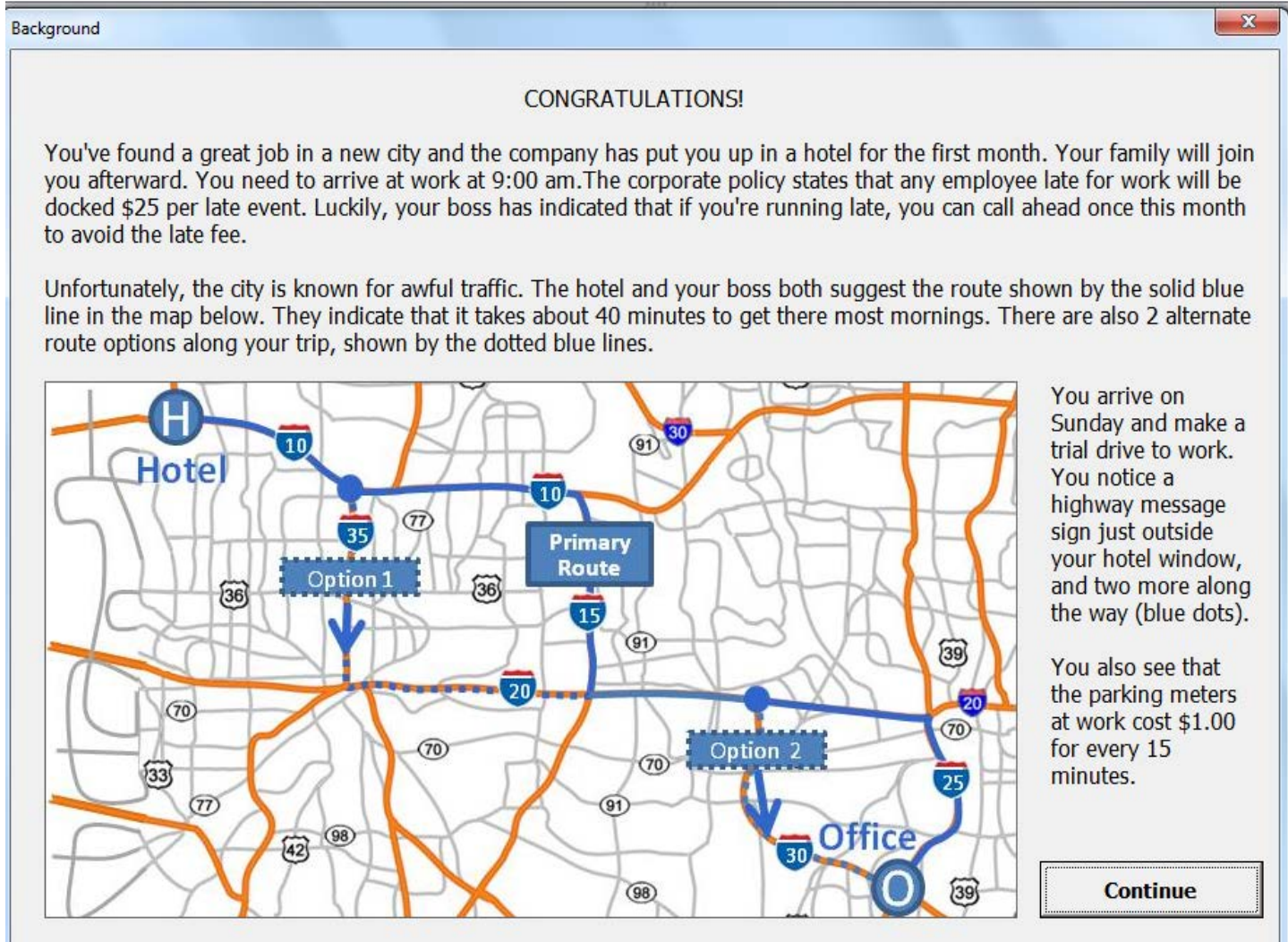

You choose between starting your commute at 7:45, 8:00, or 8:15 in the morning. You want to be on time, but you also don't want to waste money on the meters downtown. Also, your family back home can only call you at 7:45am, so your preference would be to head out at 8:00 am or later.

You subscribe to a traffic information service that you can check every morning at 7:30. The service gives you real-time travel time information. It also gives you travel time ranges based on traffic on your route during the same time of day. For 18 out of 20 trips, the time it will take you to get to work will be within the travel time ranges listed.

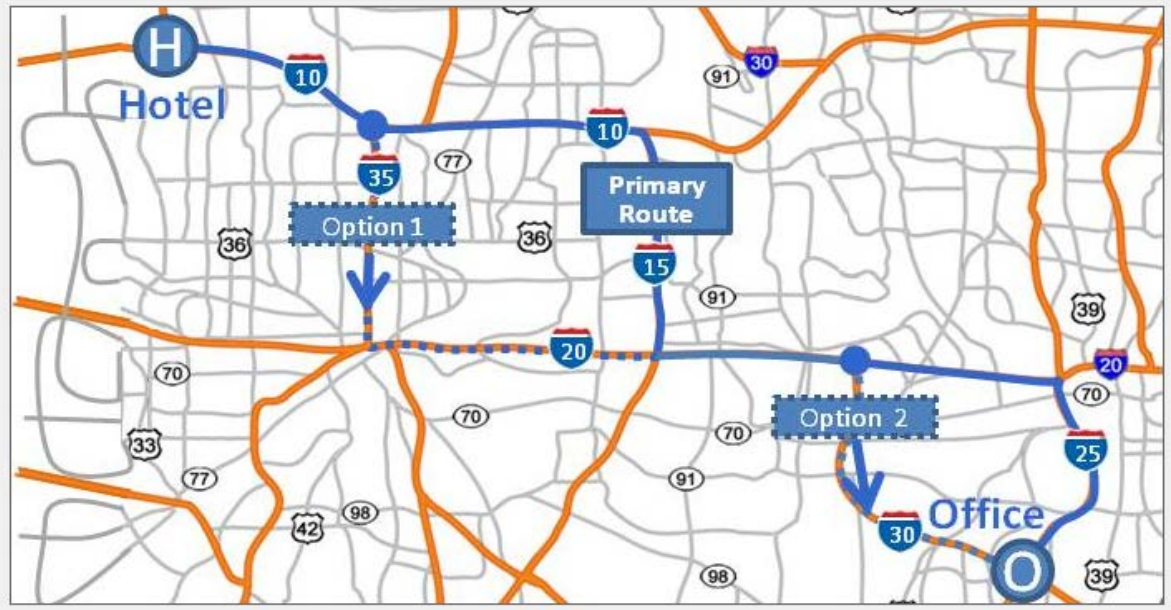

Now, it's 7:30am Monday morning, your first day getting ready for work. You're looking at the message sign on the highway from your hotel window.....let's begin day 1 !

Wait for Moderator

To Begin Day 1 


\begin{tabular}{|c|c|}
\hline \multicolumn{2}{|c|}{ Start Point Qualitative Message Sets } \\
\hline DMS/Radio Messge Sets & Frequency \\
\hline $\begin{array}{c}\text { I-10 NO DELAYS } \\
\text { NEXT 10 MILES }\end{array}$ & 7 \\
\hline $\begin{array}{c}\text { I-10 TRAFFIC SLOWS } \\
\text { NEXT 10 MILES }\end{array}$ & 1 \\
\hline $\begin{array}{c}\text { STALLED VEHICLE } \\
\text { I-10E AT ROUTE 77 }\end{array}$ & 1 \\
\hline $\begin{array}{c}\text { LIMITED VISIBILITY } \\
\text { REDUCE SPEED }\end{array}$ & 1 \\
\hline
\end{tabular}

\begin{tabular}{|c|c|c|c|}
\hline \multicolumn{5}{|c|}{ Waypoint 1 Qualitative Message Sets } \\
\hline \multirow{2}{*}{ DMS/Radio Messge Sets } & \multicolumn{3}{|c|}{ Frequency by Departure Time } \\
\cline { 2 - 4 } & $7: 45$ & $8: 00$ & $8: 15$ \\
\hline $\begin{array}{c}\text { I-10 E NO DELAYS } \\
\text { NEXT 10 MILES }\end{array}$ & 6 & 5 & 4 \\
\hline $\begin{array}{c}\text { I-35 SOUTH } \\
\text { TRAFFIC SLOW }\end{array}$ & 0 & 1 & 1 \\
\hline $\begin{array}{c}\text { I-10 E TRAFFIC SLOWS } \\
\text { NEXT 5 MILES }\end{array}$ & 1 & 1 & 3 \\
\hline $\begin{array}{c}\text { I-20E ACCIDENT } \\
\text { 2 MILES BEFORE I-30 }\end{array}$ & 0 & 1 & 1 \\
\hline $\begin{array}{c}\text { STALLED VEHICLE } \\
\text { I-10E AT ROUTE 77 }\end{array}$ & 1 & 1 & 0 \\
\hline $\begin{array}{c}\text { LIMITED VISIBILITY } \\
\text { REDUCE SPEED }\end{array}$ & 1 & 1 & 1 \\
\hline $\begin{array}{c}\text { I-10 E TRAFFIC SLOWS } \\
\text { NEXT 10 MILES }\end{array}$ & 1 & & \\
\hline
\end{tabular}

\begin{tabular}{|c|c|c|r|}
\hline \multicolumn{3}{|c|}{ Waypoint 2 Qualitative Message Sets From Primary Route } \\
\hline \multirow{2}{*}{ DMS/Radio Messge Sets } & $7: 45$ & $8: 00$ & $8: 15$ \\
\cline { 2 - 4 } & & & 5 \\
\hline $\begin{array}{c}\text { I-20E NO DELAYS } \\
\text { NEXT 5 MILES }\end{array}$ & 9 & 7 & 3 \\
\hline $\begin{array}{c}\text { I-20E TRAFFIC SLOWS } \\
\text { NEXT 5 MILES }\end{array}$ & 0 & 1 & 1 \\
\hline $\begin{array}{c}\text { LIMITED VISIBILITY } \\
\text { REDUCE SPEED }\end{array}$ & 1 & 1 & 1 \\
\hline ACCIDENT AT I-25 S & & & \\
LEFT LANE BLOCKED & 0 & & 1 \\
\hline
\end{tabular}

\begin{tabular}{|c|c|r|r|}
\hline \multicolumn{3}{|c|}{ Waypoint 2 Qualitative Message Sets from Option 1 Route } \\
\hline \multirow{2}{*}{ DMS/Radio Messge Sets } & \multicolumn{3}{|c|}{ Frequency } \\
\cline { 2 - 4 } & $7: 45$ & $8: 00$ & $8: 15$ \\
\hline $\begin{array}{c}\text { I-20E NO DELAYS } \\
\text { NEXT 5 MILES }\end{array}$ & 9 & 7 & 5 \\
\hline $\begin{array}{c}\text { I-20E TRAFFIC SLOWS } \\
\text { NEXT 5 MILES }\end{array}$ & 0 & 1 & 2 \\
\hline $\begin{array}{c}\text { I-30 SOUTH } \\
\text { SLOW TRAFFIC }\end{array}$ & 0 & 0 & 1 \\
\hline $\begin{array}{c}\text { LIMITED VISIBILITY } \\
\text { REDUCE SPEED }\end{array}$ & 1 & 1 & 1 \\
\hline $\begin{array}{c}\text { ACCIDENT AT I-25 } \\
\text { LEFT LANE BLOCKED }\end{array}$ & 0 & 1 & 1 \\
\hline
\end{tabular}


Data Type Acquired in the Simulation Experiment

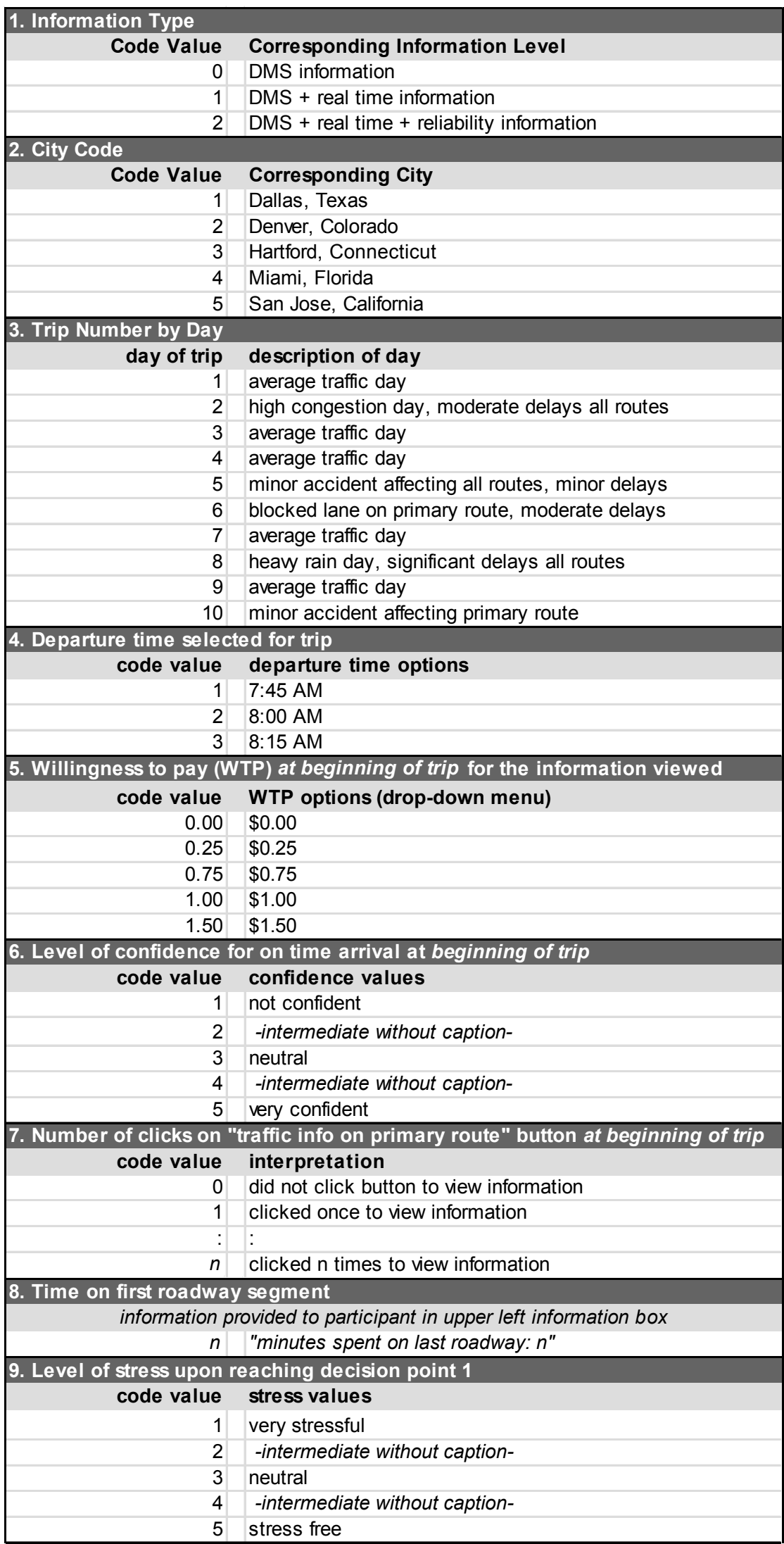




\begin{tabular}{|c|c|}
\hline \multicolumn{2}{|c|}{ 10. Number of clicks on "traffic info on primary route" button at decision point 1} \\
\hline code value & interpretation \\
\hline 0 & did not click button to view information \\
\hline 1 & clicked once to view information \\
\hline : & : \\
\hline$n$ & clicked $\mathrm{n}$ times to view information \\
\hline \multicolumn{2}{|c|}{ 11. Number of clicks on "traffic info on option 1" button at decision point 1} \\
\hline code value & interpretation \\
\hline 0 & did not click button to view information \\
\hline 1 & clicked once to view information \\
\hline & \\
\hline$n$ & clicked $\mathrm{n}$ times to view information \\
\hline \multicolumn{2}{|c|}{ 12. Route selection at decision point 1} \\
\hline code value & route options \\
\hline 1 & primary route \\
\hline 2 & route option 1 \\
\hline \multicolumn{2}{|c|}{ 13. Level of confidence for on time arrival when at decision point 1} \\
\hline code value & confidence values \\
\hline 1 & not confident \\
\hline 2 & -intermediate without caption- \\
\hline 3 & neutral \\
\hline 4 & -intermediate without caption- \\
\hline 5 & very confident \\
\hline \multicolumn{2}{|c|}{ 14. Choice to call work to inform them of a late arrival at decision point 1} \\
\hline code value & route options \\
\hline 0 & did not call to inform of a late arrival \\
\hline 1 & did call to inform of a late arrival \\
\hline \multicolumn{2}{|c|}{ 15. Time on 2 nd roadway segment (primary or option 1) } \\
\hline \multicolumn{2}{|c|}{ information provided to participant in upper left information box } \\
\hline$n$ & "minutes spent on last roadway: $n "$ \\
\hline \multicolumn{2}{|c|}{ 16. Level of stress upon reaching decision point 2} \\
\hline code value & stress values \\
\hline 1 & very stressful \\
\hline 2 & -intermediate without caption- \\
\hline 3 & neutral \\
\hline 4 & -intermediate without caption- \\
\hline 5 & stress free \\
\hline \multicolumn{2}{|c|}{ 17. Number of clicks on "traffic info on primary route" button at decision point 2} \\
\hline code value & interpretation \\
\hline 0 & did not click button to view information \\
\hline 1 & clicked once to view information \\
\hline : & $:$ \\
\hline$n$ & clicked $\mathrm{n}$ times to view information \\
\hline \multicolumn{2}{|c|}{ 18. Number of clicks on "traffic info on option 1" button at decision point 2} \\
\hline code value & interpretation \\
\hline 0 & did not click button to view information \\
\hline 1 & clicked once to view information \\
\hline : & : \\
\hline$n$ & clicked $\mathrm{n}$ times to view information \\
\hline \multicolumn{2}{|c|}{ 19. Route selection at decision point 2} \\
\hline code value & route options \\
\hline 1 & primary route \\
\hline 2 & route option 1 \\
\hline \multicolumn{2}{|c|}{ 20. Level of confidence for on time arrival when at decision point 2} \\
\hline code value & confidence values \\
\hline 1 & not confident \\
\hline 2 & -intermediate without caption- \\
\hline 3 & neutral \\
\hline 4 & -intermediate without caption- \\
\hline 5 & very confident \\
\hline \multicolumn{2}{|c|}{ 21. Choice to call work to inform them of a late arrival at decision point 2} \\
\hline code value & route options \\
\hline 0 & did not call to inform of a late arrival \\
\hline 1 & did call to inform of a late arrival \\
\hline
\end{tabular}




\begin{tabular}{|c|c|}
\hline information & ovided to participant in upper left information box \\
\hline$n$ & "minutes spent on last roadway: $n "$ \\
\hline 23. Overall stressfulnes & of the trip \\
\hline code value & stress values \\
\hline 1 & very stressful \\
\hline 2 & -intermediate without caption- \\
\hline 3 & neutral \\
\hline 4 & -intermediate without caption- \\
\hline 5 & stress free \\
\hline 24. Happiness with trip & utcome \\
\hline code value & happiness value \\
\hline 1 & unhappy \\
\hline 2 & -intermediate without caption- \\
\hline 3 & neutral \\
\hline 4 & -intermediate without caption- \\
\hline 5 & very happy \\
\hline 25. Usefulness of traffic & nformation for this trip \\
\hline code value & usefulness value \\
\hline 1 & not useful \\
\hline 2 & -intermediate without caption- \\
\hline 3 & neutral \\
\hline 4 & -intermediate without caption- \\
\hline 5 & very useful \\
\hline 26. Willingness to pay & traffic information viewed for this trip \\
\hline code value & stress values \\
\hline 0 & $\$ 0.00$ \\
\hline 1 & $\$ 1.00$ \\
\hline 2 & $\$ 2.00$ \\
\hline 3 & $\$ 3.00$ \\
\hline 4 & $\$ 4.00$ \\
\hline 5 & $\$ 5.00$ \\
\hline 27. Total trip time & \\
\hline$n$ & minutes of travel in vehicle \\
\hline 28. Late arrival fee & \\
\hline $\boldsymbol{x}$ & $\$ 25.00$ if late arrival \\
\hline 29. Extra parking cost & \\
\hline$\$ Y$ & $\$ 4.00$ per hour at 15 minute increments \\
\hline
\end{tabular}


Travel Time Profiles by Simulation Day

\begin{tabular}{|c|c|c|c|c|c|}
\hline & \multirow{2}{*}{$\begin{array}{l}\text { Departure } \\
\text { Time }\end{array}$} & \multicolumn{4}{|c|}{ Actual Travel Time on Route Taken } \\
\hline & & primary & Option 1 & Option 2 & Option $1 \& 2$ \\
\hline & $7: 15 \mathrm{AM}$ & 35 & 38 & 34 & 37 \\
\hline & 8:00 AM & 40 & 41 & 40 & 43 \\
\hline ন্ত & $8: 15 \mathrm{AM}$ & 43 & 47 & 43 & 44 \\
\hline \multirow{5}{*}{$\stackrel{\sim}{\stackrel{N}{\widetilde{\pi}}}$} & Departure & \multicolumn{4}{|c|}{ Actual Travel Time on Route Taken } \\
\hline & Time & primary & Option 1 & Option 2 & Option $1 \& 2$ \\
\hline & $7: 15$ AM & 37 & 39 & 39 & 39 \\
\hline & 8:00 AM & 47 & 45 & 48 & 44 \\
\hline & $8: 15$ AM & 47 & 49 & 50 & 49 \\
\hline \multirow{5}{*}{$\stackrel{m}{\underset{\mathbb{\pi}}{\infty}}$} & Departure & \multicolumn{4}{|c|}{ Actual Travel Time on Route Taken } \\
\hline & Time & primary & Option 1 & Option 2 & Option $1 \& 2$ \\
\hline & $7: 15 \mathrm{AM}$ & 34 & 39 & 36 & 41 \\
\hline & 8:00 AM & 41 & 43 & 43 & 43 \\
\hline & $8: 15 \mathrm{AM}$ & 45 & 46 & 47 & 49 \\
\hline \multirow{5}{*}{$\stackrel{+}{\stackrel{\nabla}{\Delta}}$} & Departure & \multicolumn{4}{|c|}{ Actual Travel Time on Route Taken } \\
\hline & Time & primary & Option 1 & Option 2 & Option $1 \& 2$ \\
\hline & $7: 15$ AM & 31 & 36 & 33 & 37 \\
\hline & 8:00 AM & 36 & 41 & 38 & 43 \\
\hline & $8: 15 \mathrm{AM}$ & 41 & 47 & 44 & 46 \\
\hline \multirow{5}{*}{ 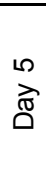 } & Departure & \multicolumn{4}{|c|}{ Actual Travel Time on Route Taken } \\
\hline & Time & primary & Option 1 & Option 2 & Option $1 \& 2$ \\
\hline & $7: 15$ AM & 40 & 43 & 42 & 45 \\
\hline & 8:00 AM & 57 & 59 & 59 & 62 \\
\hline & $8: 15$ AM & 68 & 71 & 72 & 74 \\
\hline \multirow{5}{*}{$\stackrel{0}{\stackrel{0}{\triangle}}$} & Departure & \multicolumn{4}{|c|}{ Actual Travel Time on Route Taken } \\
\hline & Time & primary & Option 1 & Option 2 & Option 1 \& 2 \\
\hline & $7: 15$ AM & 64 & 49 & 63 & 49 \\
\hline & 8:00 AM & 63 & 53 & 63 & 53 \\
\hline & 8:15 AM & 47 & 39 & 47 & 43 \\
\hline \multirow{5}{*}{$\stackrel{\wedge}{\stackrel{\pi}{\Delta}}$} & Departure & \multicolumn{4}{|c|}{ Actual Travel Time on Route Taken } \\
\hline & Time & primary & Option 1 & Option 2 & Option $1 \& 2$ \\
\hline & $7: 15$ AM & 32 & 35 & 35 & 38 \\
\hline & $8: 00 \mathrm{AM}$ & 35 & 39 & 40 & 43 \\
\hline & $8: 15 \mathrm{AM}$ & 38 & 40 & 43 & 45 \\
\hline \multirow{5}{*}{$\stackrel{\infty}{\underset{\mathbb{\pi}}{\Delta}}$} & Departure & \multicolumn{4}{|c|}{ Actual Travel Time on Route Taken } \\
\hline & Time & primary & Option 1 & Option 2 & Option 1 \& 2 \\
\hline & $7: 15$ AM & 58 & 61 & 59 & 62 \\
\hline & 8:00 AM & 66 & 66 & 68 & 65 \\
\hline & $8: 15 \mathrm{AM}$ & 71 & 70 & 73 & 70 \\
\hline \multirow{5}{*}{ 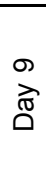 } & Departure & \multicolumn{4}{|c|}{ Actual Travel Time on Route Taken } \\
\hline & Time & primary & Option 1 & Option 2 & Option $1 \& 2$ \\
\hline & $7: 15$ AM & 32 & 34 & 33 & 35 \\
\hline & 8:00 AM & 34 & 38 & 36 & 38 \\
\hline & $8: 15$ AM & 39 & 43 & 41 & 44 \\
\hline \multirow{5}{*}{$\begin{array}{l}\stackrel{0}{a} \\
\frac{\pi}{0}\end{array}$} & Departure & & ctual Trav & Route Ta & \\
\hline & Time & primary & Option 1 & Option 2 & Option 1 \& 2 \\
\hline & $7: 15$ AM & 39 & 37 & 37 & 38 \\
\hline & $8: 00 \mathrm{AM}$ & 49 & 45 & 42 & 38 \\
\hline & $8: 15 \mathrm{AM}$ & 56 & 53 & 47 & 42 \\
\hline
\end{tabular}




\section{Supplemental Figures from Travel Behavior Laboratory Experiment Pre-Survey}

The following figures-Figures G.1 through G.8—summarize statistics related to participants' gender, age, education, and types of trips made. The questions were included in the pre-experiment survey that was part of the first travel behavior laboratory experiment described in Chapter 9 in the report.

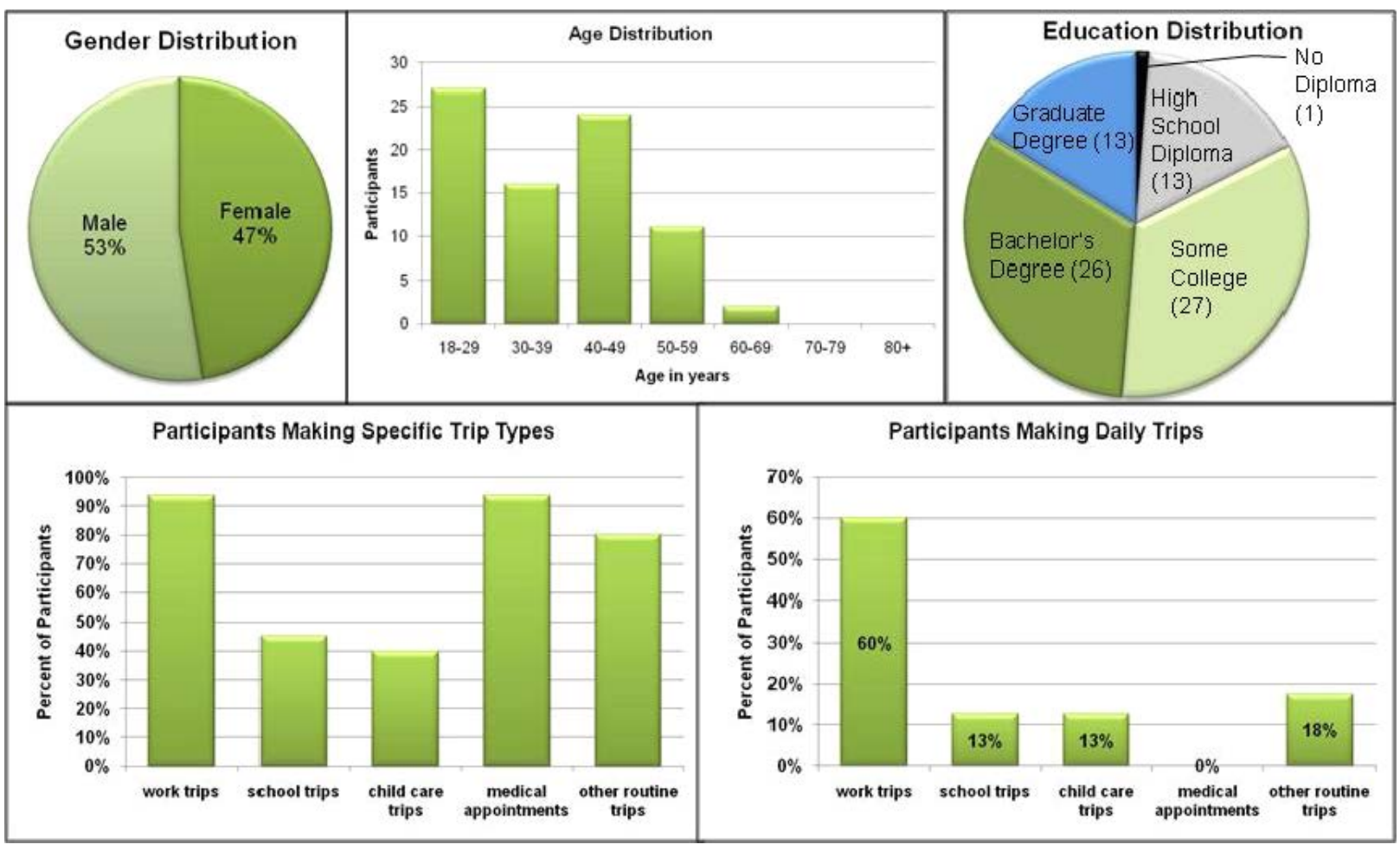

Figure G.1. Demographic and trip-making characteristics of experiment participants. 


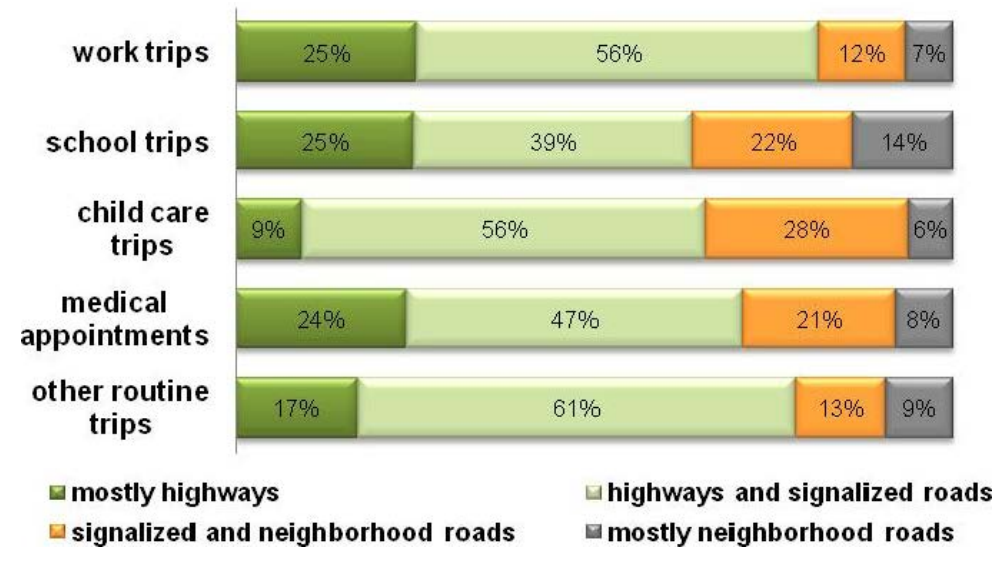

Figure G.2. Types of roads participants use for specific trip types.

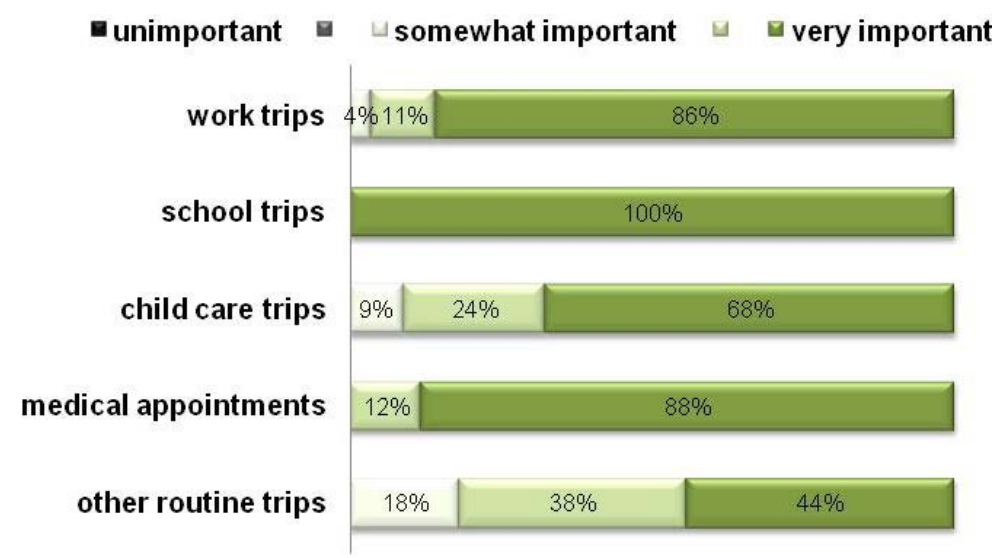

Figure G.3. Participants' rating of importance of on-time arrival, by trip type.

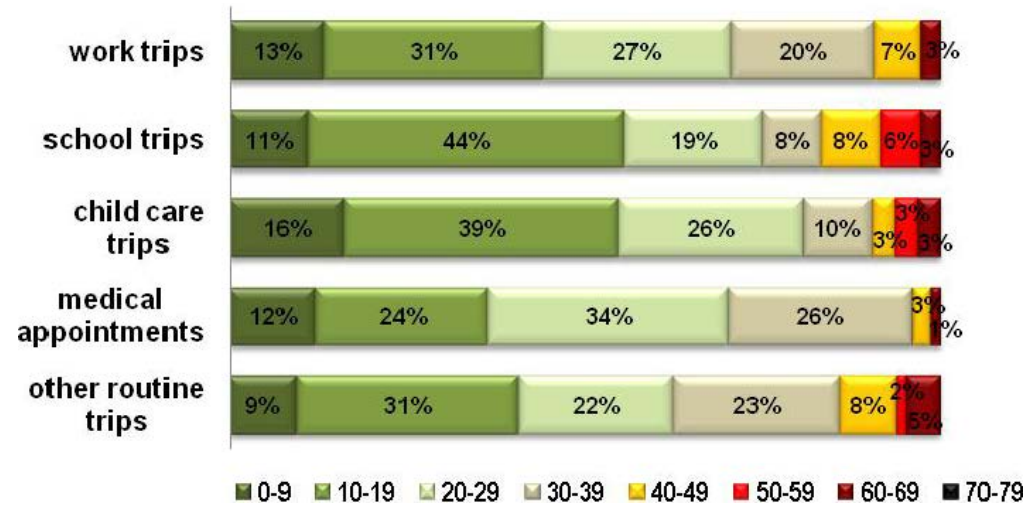

Figure G.4. Average trip duration among participants, by trip type. 


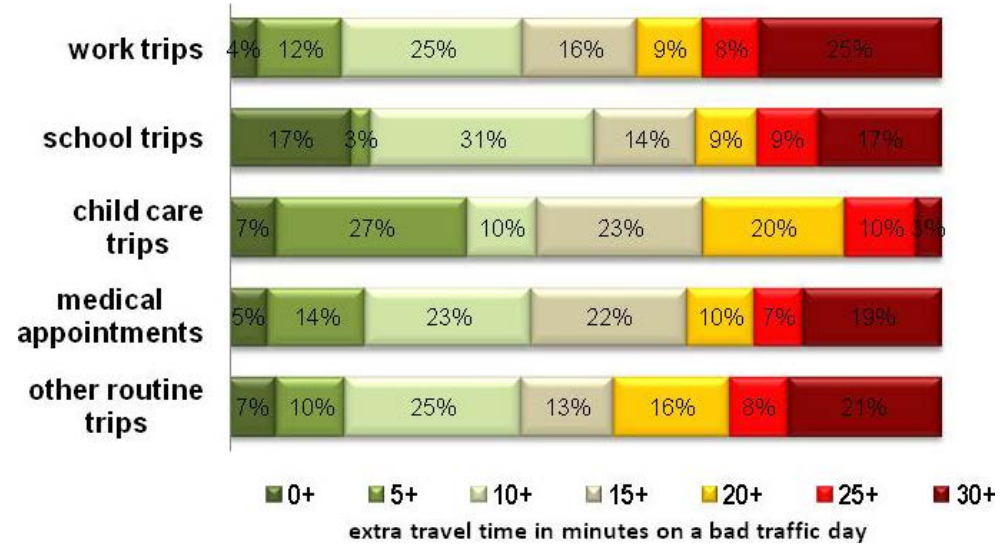

Figure G.5. Trip time variability, by trip type.

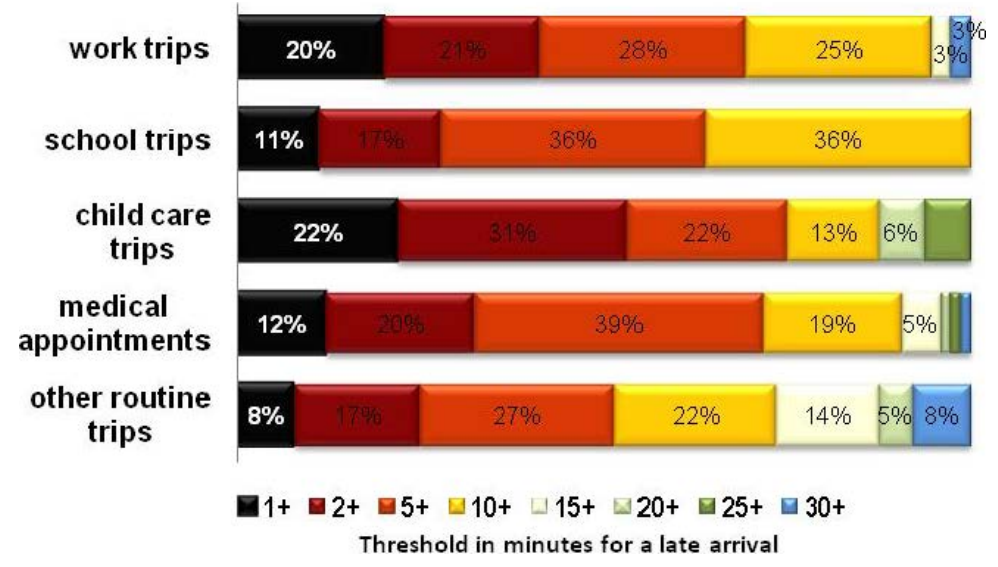

Figure G.6. Various definitions of arriving late, by trip type.

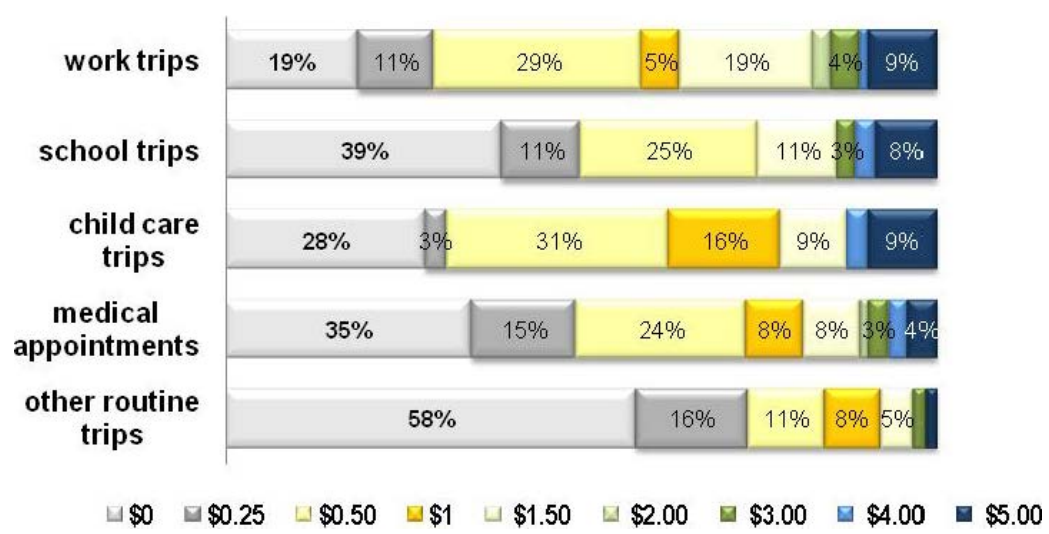

Figure G.7. Willingness to pay for guaranteed travel time, by trip type. 


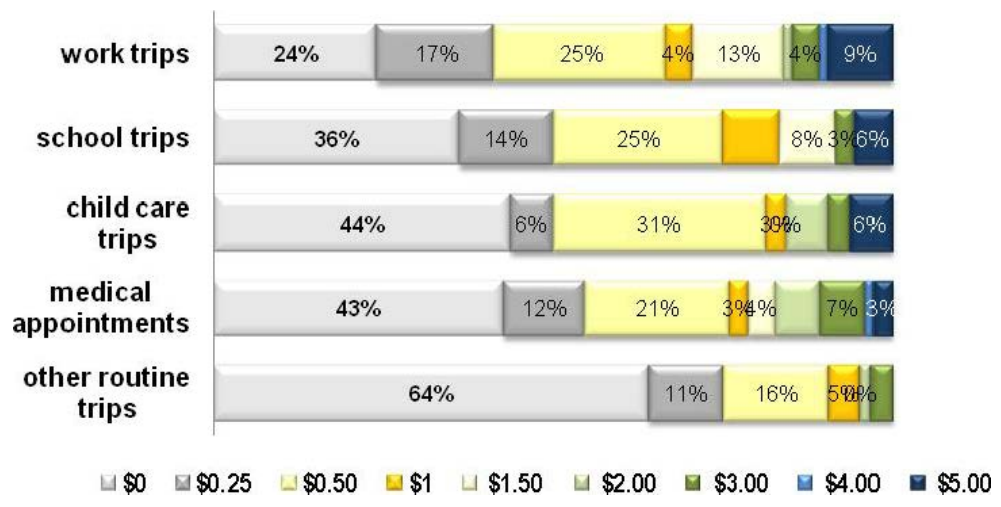

Figure G.8. Willingness to pay for late arrival knowledge, by trip type. 


\section{Enhanced Laboratory Experiment Documentation}

\section{Pre-Experiment Survey Questions 1-12}

The 12 pre-experiment questions presented to participants are listed in Table H.1. These were completed within an Excelbased application.

The potential response levels for each question are outlined in Table H.2.

\section{Visuals of Reliability Forms Presented to Experiment Participants}

Experiment participants had the option of viewing pre-trip information. Figure H.1 through Figure H.8 illustrate the forms of specific reliability data available pre-trip to participants.

\section{Experiment 1 Storyline and Screenshots}

The simulated commute storyline is presented here through screenshots. Figure H.9 presents the first visual once a participant begins the experiment by selecting the "start game" button. The experiment moderators review the introduction and read aloud the content. Once participants click the "begin experiment" button shown in Figure H.9, the first randomly selected city and information type replaces the introduction. That screen, shown in Figure H.10, provides a visual of the fictitious regional traffic network and the locations of the simulated starting point (headquarters [HQ]) and ending point (client) of the daily commute. Also presented are the trip distance, free-flow travel time, desired arrival time, and potential departure time options. When they have reviewed this information, participants click "start week" and are presented the screenshot shown in Figure H.11. From that point, participants select the "view trip information" button. This step is demonstrated by the experiment moderators.

The reliability information is presented next to the map, as shown in Figure H.12. The reliability information with the Harrisonville fictional city illustrates the signposting reliability information. Depending on the type of reliability information presented, the city name and trip information content varies. After closing the information content, individuals choose a departure time, arrival confidence, and information usefulness. Then participants click on "start trip." If any information is incomplete, they are asked to complete it before they can progress.

The participant then views the trip completion information as the vehicle icon progresses through each main roadway illustrated through dashed lines. This is illustrated in Figure H.13. The duration that individuals wait to complete the trip is a function of a baseline number of seconds and an additional time corresponding to the extra time; the extra time is based on that day's predetermined departure-timebased travel time. At intermediary points, DMS messages display information that corresponds with the travel time. Once the simulated trip is completed, summary statistics are emphasized at the bottom of the screen (see Figure H.14); participants indicate the information's usefulness, the level of stress experienced while waiting for the simulated trip to end, and their willingness to pay for the reliability information viewed. Then they click on "next trip" and complete four additional trips, repeating the screenshots displayed in Figures H.11 through H.14.

At the end of five trips, a summary statistic highlights participants' performance over the week, as illustrated through Figure H.15. Participants again answer five questions on reliability information value, usefulness, and ease of use. They then click on "next city" and await further instructions from the 
moderator. During this wait time, they see Figure H.16. At this juncture, the moderator asks participants in the session to share their overall week's cost and experiences to foster a competitive and collaborative environment and to maintain the group's engagement in the experiment. As a group, participants then begin the next city, viewing a different city name and information, similar to the illustration in Figure H.10.

\section{Screenshots of the Six Fictional Cities Developed for Simulated Commutes}

Figure H.17 through Figure H.22 display the fictional cities developed by the researchers for the simulated commutes in the experiment.

Table H.1. Pre-Experiment Survey Questions

\begin{tabular}{|l|}
\hline \multicolumn{1}{|c|}{ Pre-Experiment Survey Questions } \\
\hline 1. Please select your gender and age range. \\
\hline 2. Please select your highest level of education. \\
\hline 3. How frequently do you make the following types of trips? \\
\hline 4. When making work trips, how often do you seek out traffic information? \\
\hline 5. If you seek out traffic information for work trips, what sources do you use? (check all that apply) \\
\hline 6. What types of roads do you use when you drive to the following destinations? \\
\hline 7. For the following types of trips, how important is it for you to arrive at a specific time? \\
\hline 8. On a typical day, how much time do the following trips take you to complete by car? \\
\hline 9. On a bad day, how much more time do the following trips take for you to complete by car? \\
\hline 10. For the following types of trips, what would you consider as arriving late? \\
\hline 11. Say you could be guaranteed a travel time before you make your trip. How much would you pay for \\
this guarantee when making the following types of trips? \\
\hline 12. Say you could be informed during the beginning of your trip that you will arrive late. How much would \\
you pay for this information when making the following types of trips? \\
\hline
\end{tabular}

Table H.2. Potential Pre-Experiment Survey Responses

\begin{tabular}{|c|c|c|}
\hline 1a. Gender & & 1b. Age \\
\hline (drop-down menu) & & (drop-down menu) \\
\hline female & & $18-29$ \\
\hline male & & 30-39 \\
\hline & & $40-49$ \\
\hline & & $50-59$ \\
\hline & & $60-69$ \\
\hline & & $70-79$ \\
\hline & & $80+$ \\
\hline 2. Education & & \\
\hline \multicolumn{3}{|c|}{ (drop-down menu) } \\
\hline \multicolumn{3}{|c|}{ no high school diploma } \\
\hline \multicolumn{3}{|c|}{ high school diploma } \\
\hline \multicolumn{3}{|c|}{ some college } \\
\hline \multicolumn{3}{|c|}{ bachelor's degree } \\
\hline & graduate degree & \\
\hline
\end{tabular}

*Meeting for social, hobby, or religious activities. 
Table H.2. Potential Pre-Experiment Survey Responses (continued)

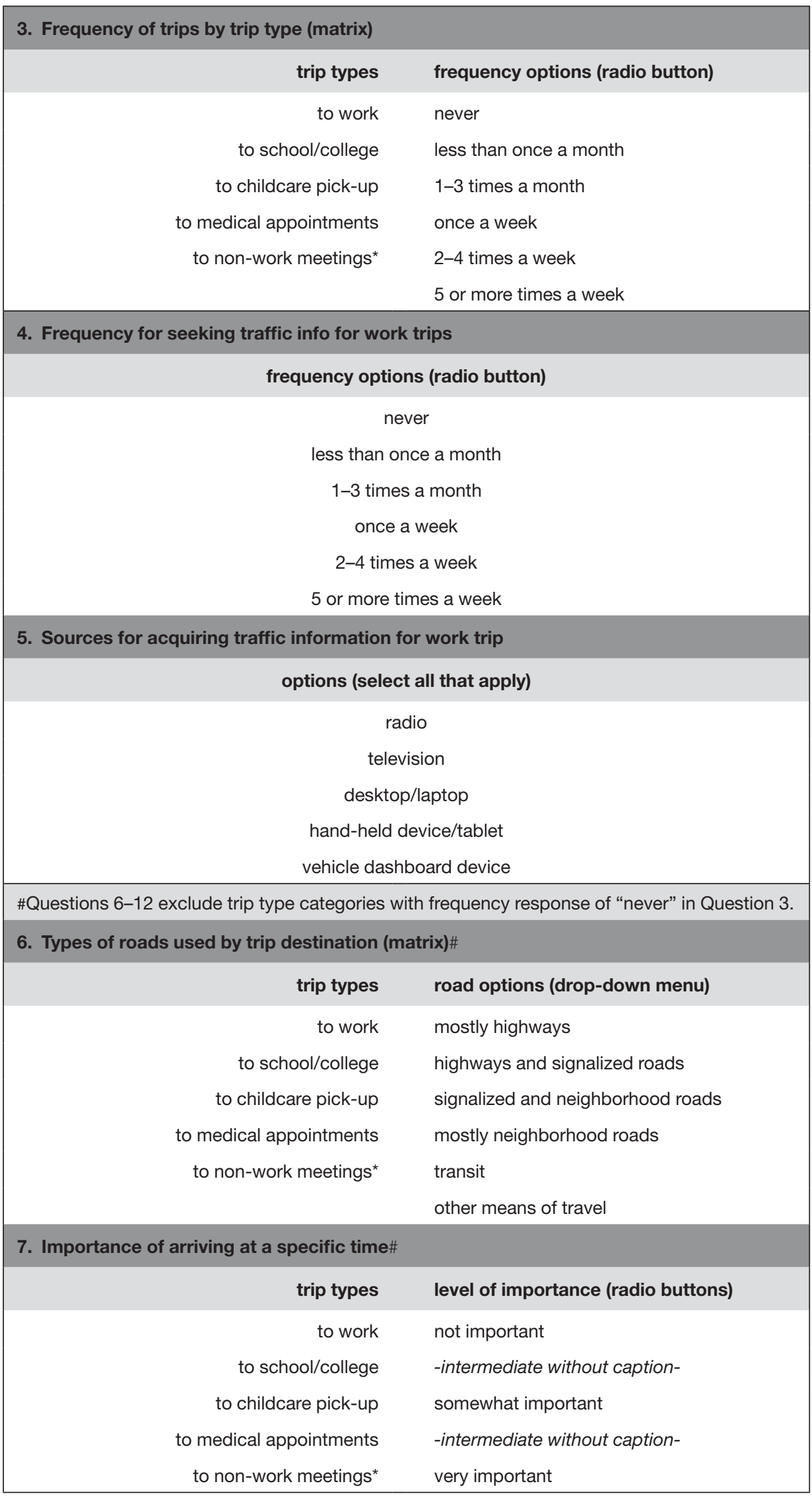


Table H.2. Potential Pre-Experiment Survey Responses (continued)

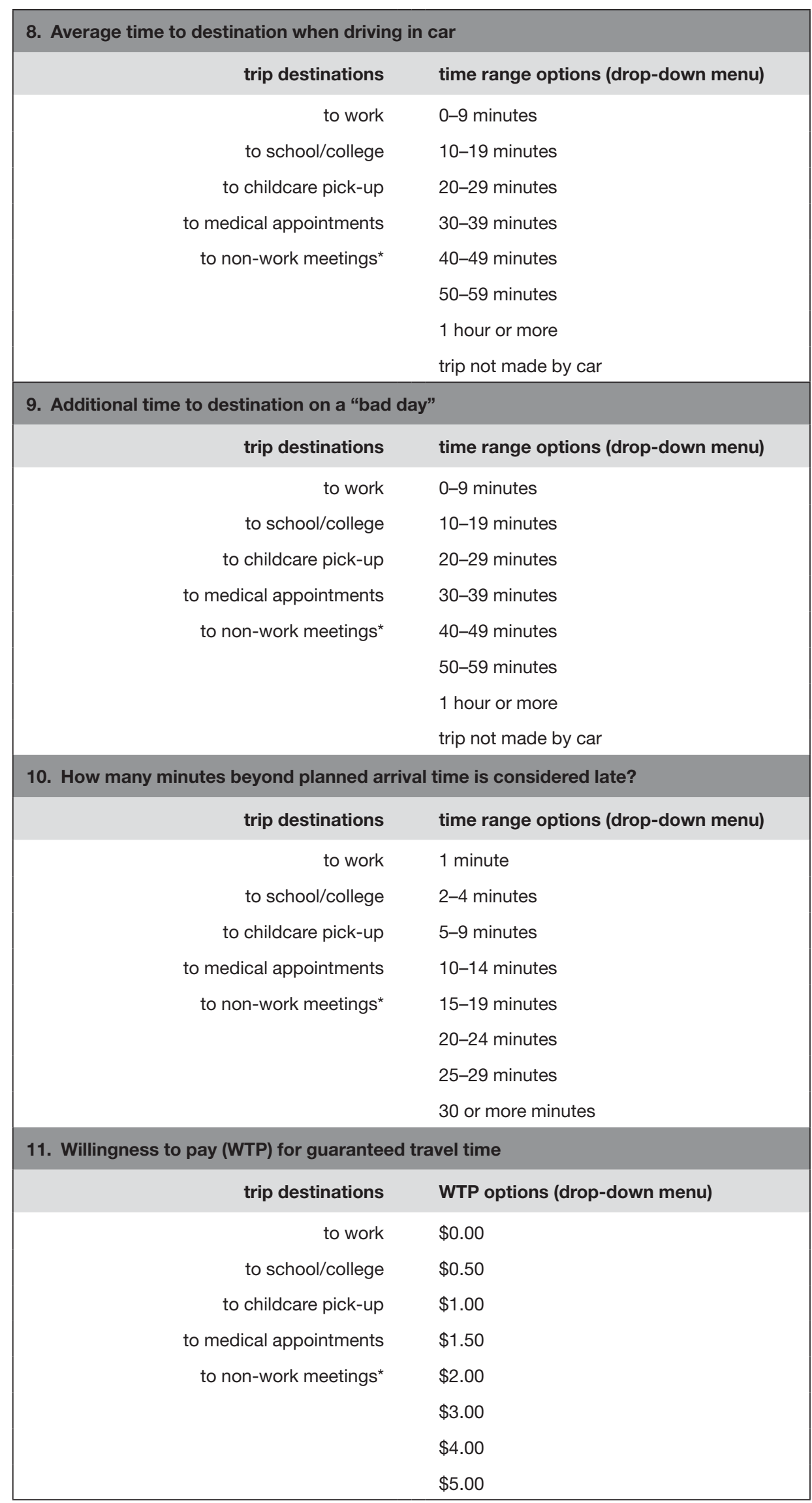

(continued on next page) 
Table H.2. Potential Pre-Experiment Survey Responses (continued)

\begin{tabular}{|rl|}
\hline 12. Willingness to pay (WTP) for late arrival information \\
trip destinations & WTP options (drop-down menu) \\
to work & $\$ 0.00$ \\
to school/college & $\$ 0.50$ \\
to childcare pick-up & $\$ 1.00$ \\
to medical appointments & $\$ 1.50$ \\
to non-work meetings* & $\$ 2.00$ \\
& $\$ 3.00$ \\
& $\$ 4.00$ \\
\hline
\end{tabular}

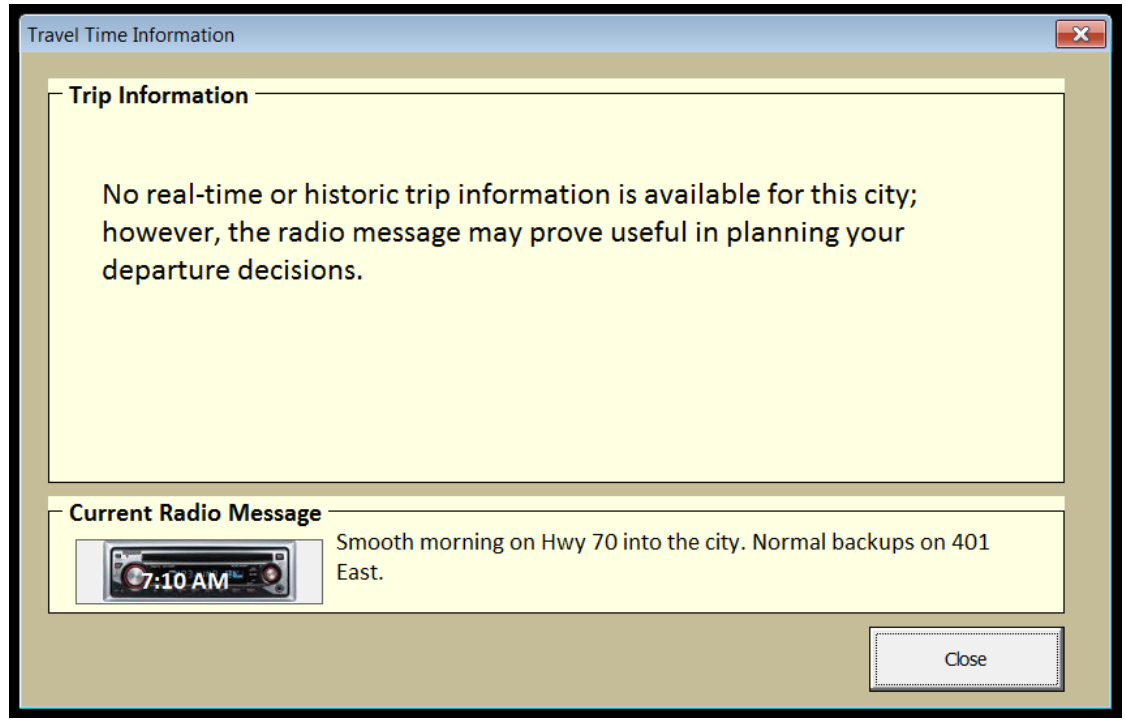

Figure H.1. Information Type A: Only radio message, no reliability data.

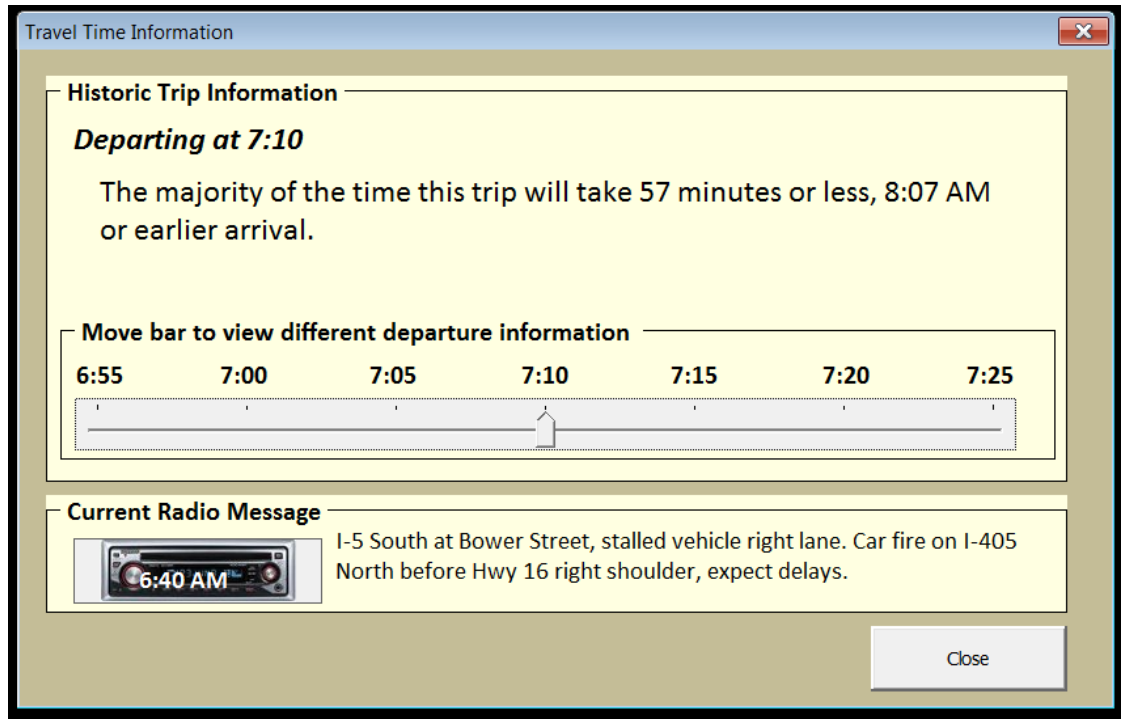

Figure H.2. Information Type B: Textual 95th percentile travel time and arrival times, by time of departure. 


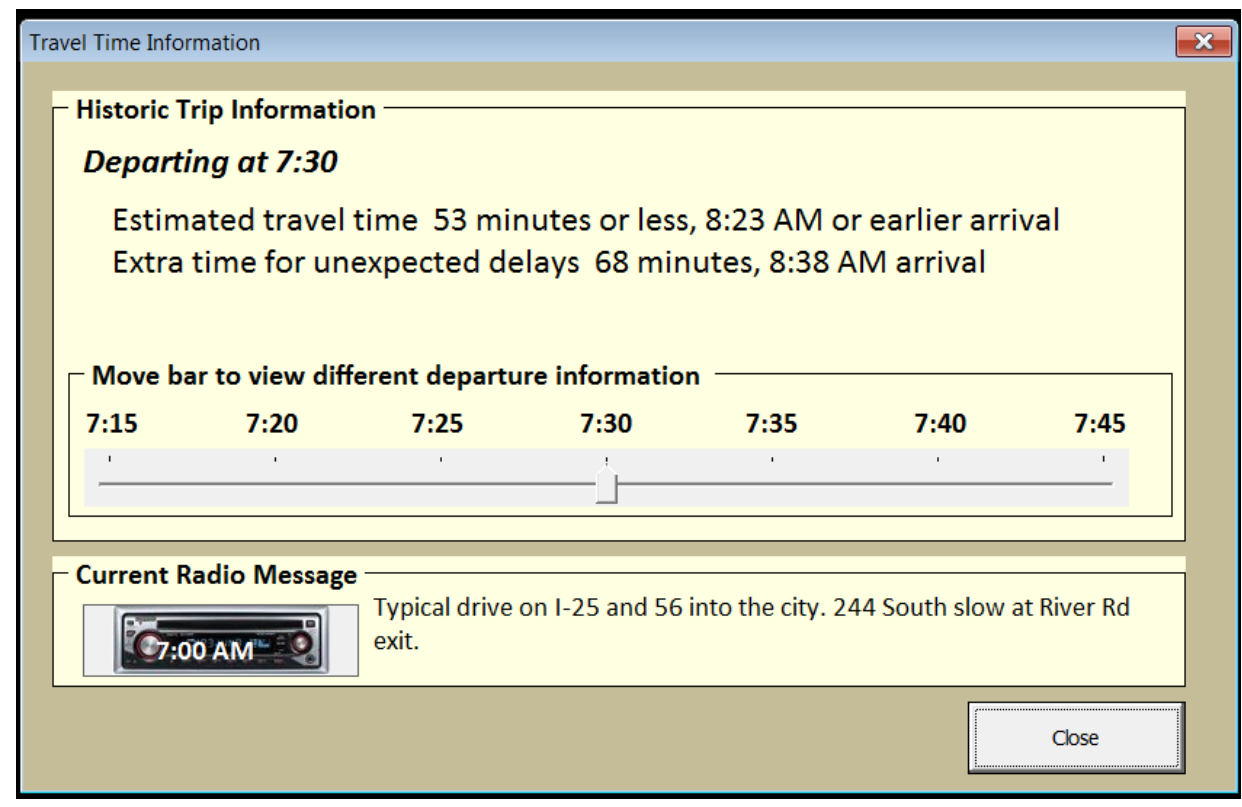

Figure H.3. Information Type C: Textual average and 95th percentile travel and arrival times, by time of departure.

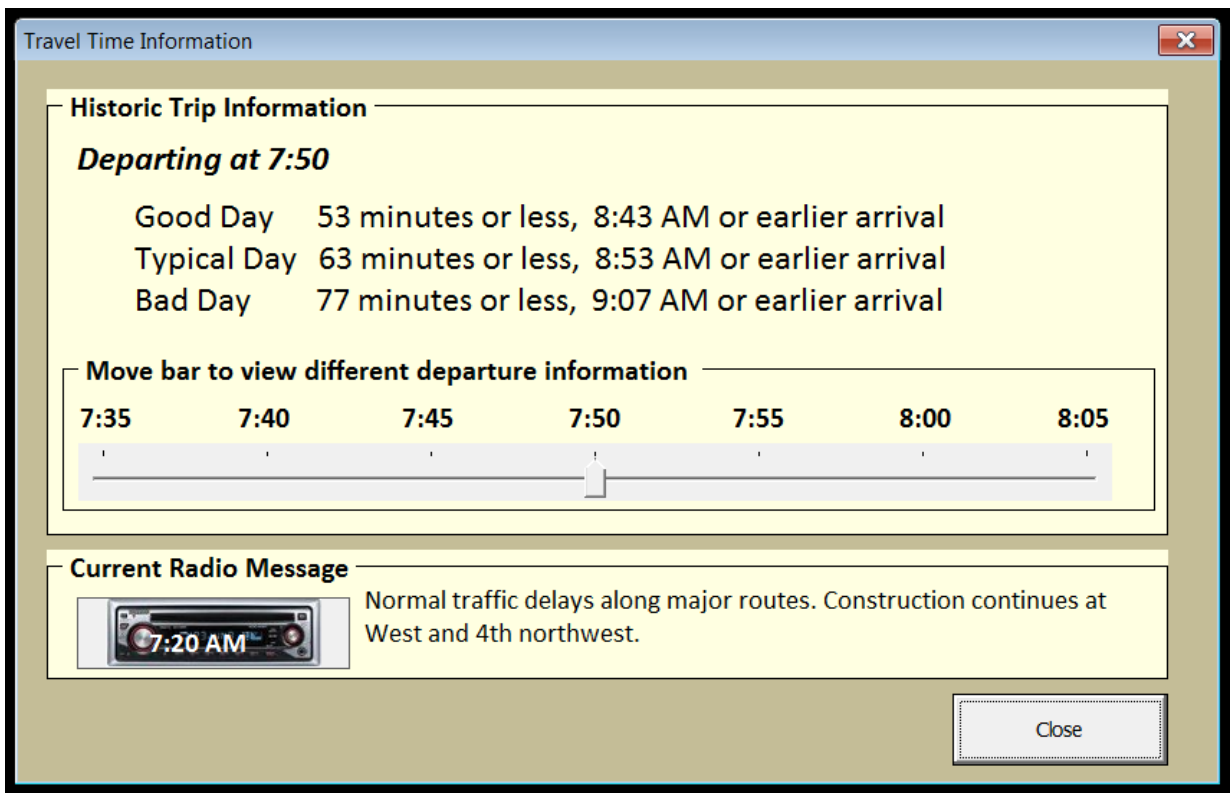

Figure H.4. Information Type D: Textual 20th percentile, average, and 95th percentile travel and arrival times, by time of departure. 


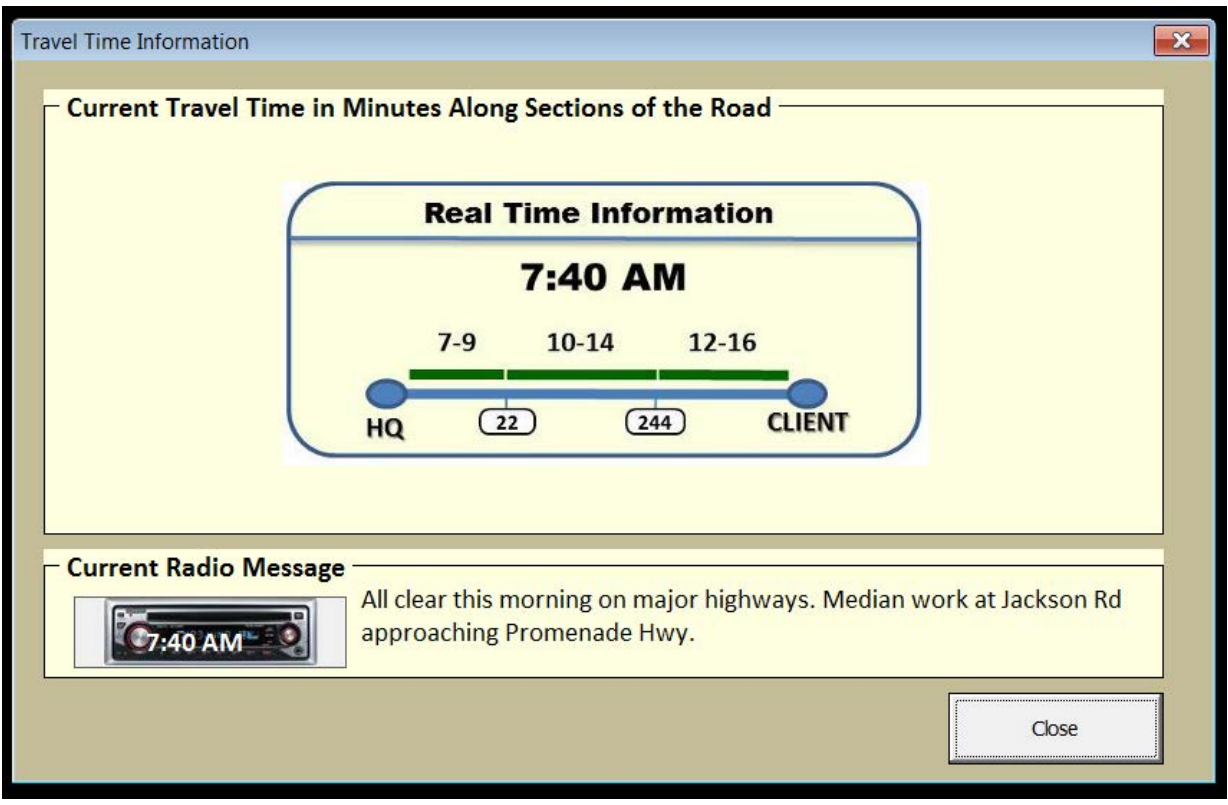

Figure H.5. Information Type E: Visual and textual signposting.

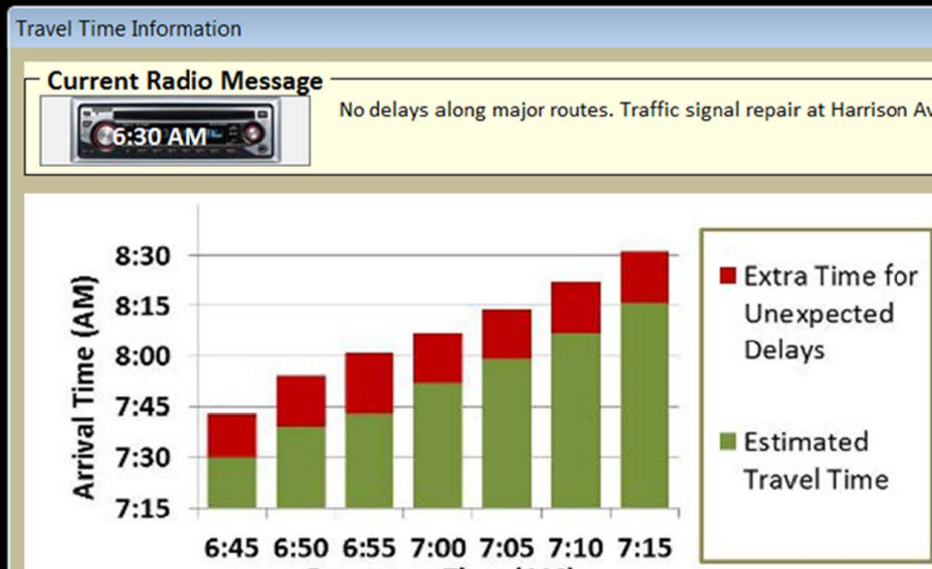
Departure Time (AM)

Figure H.6. Information Type F: Graphical presentation of average and 95th percentile travel and arrival times, by time of departure. 


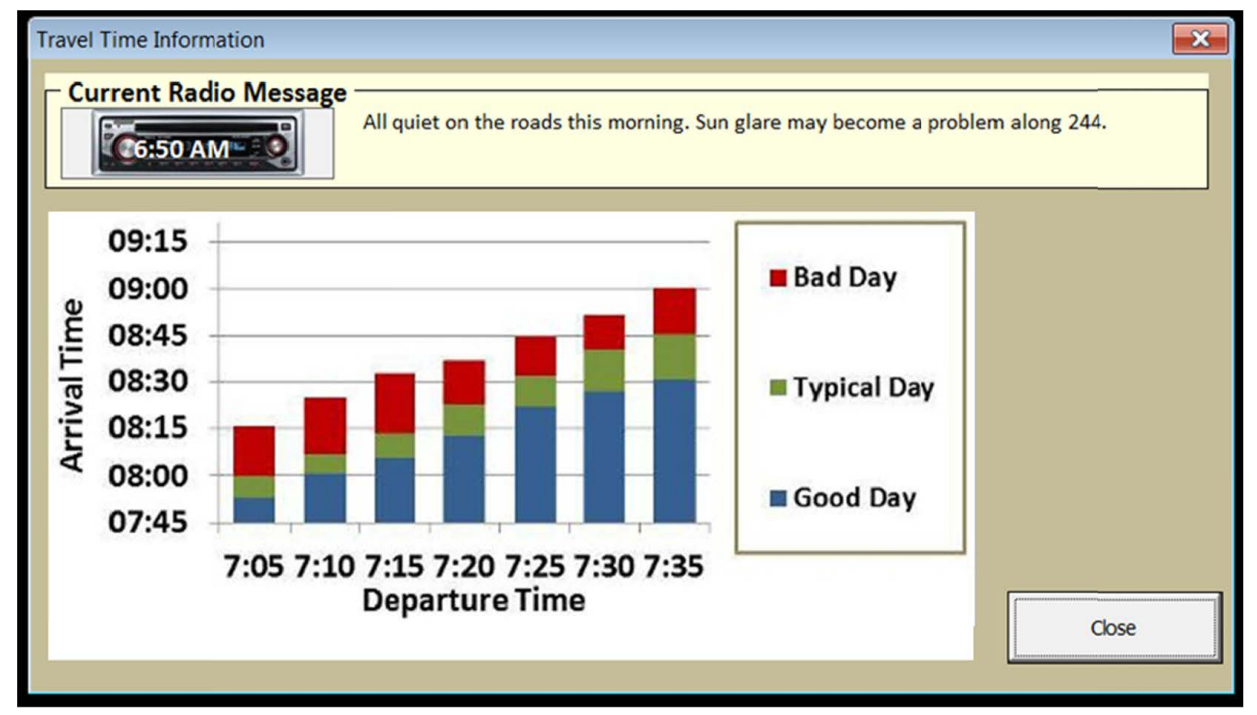

Figure H.7. Information Type G: Graphical presentation of 20th percentile, average, and 95th percentile travel and arrival times, by time of departure.

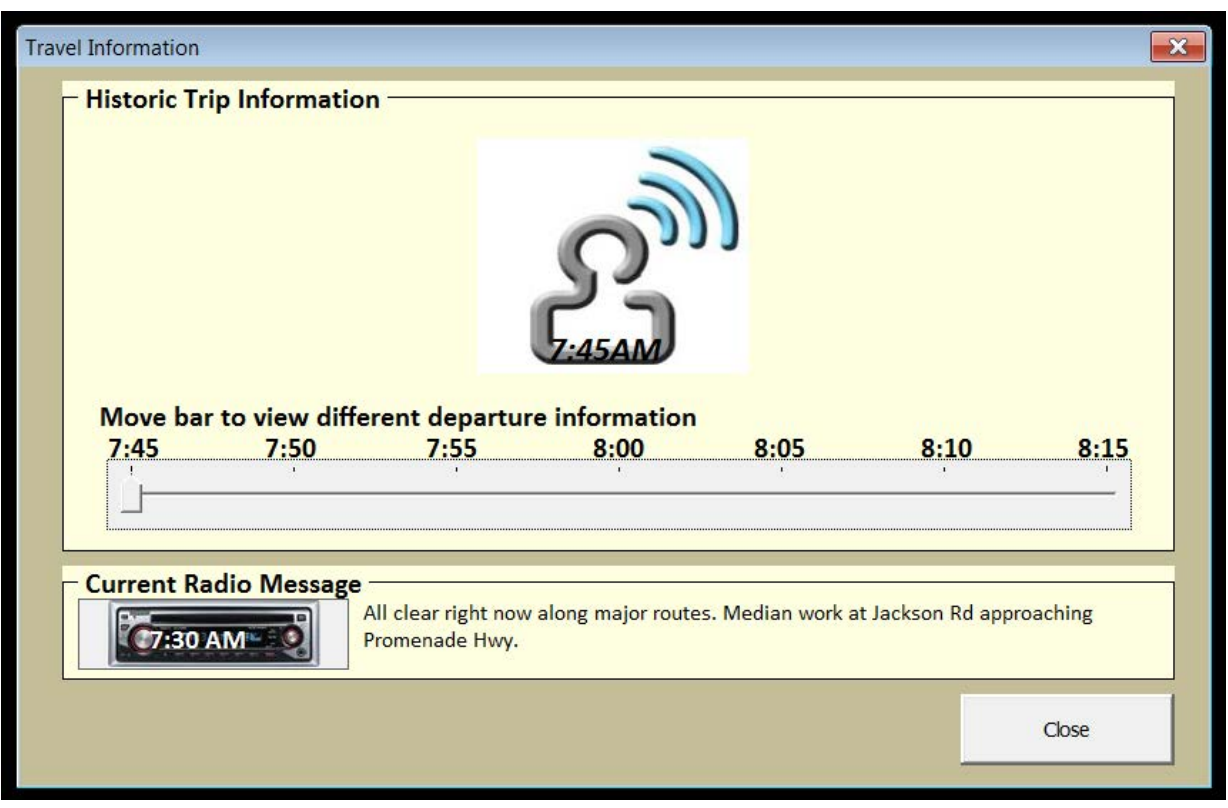

Figure H.8. Auditory 95th percentile travel time and arrival times, by time of departure. 


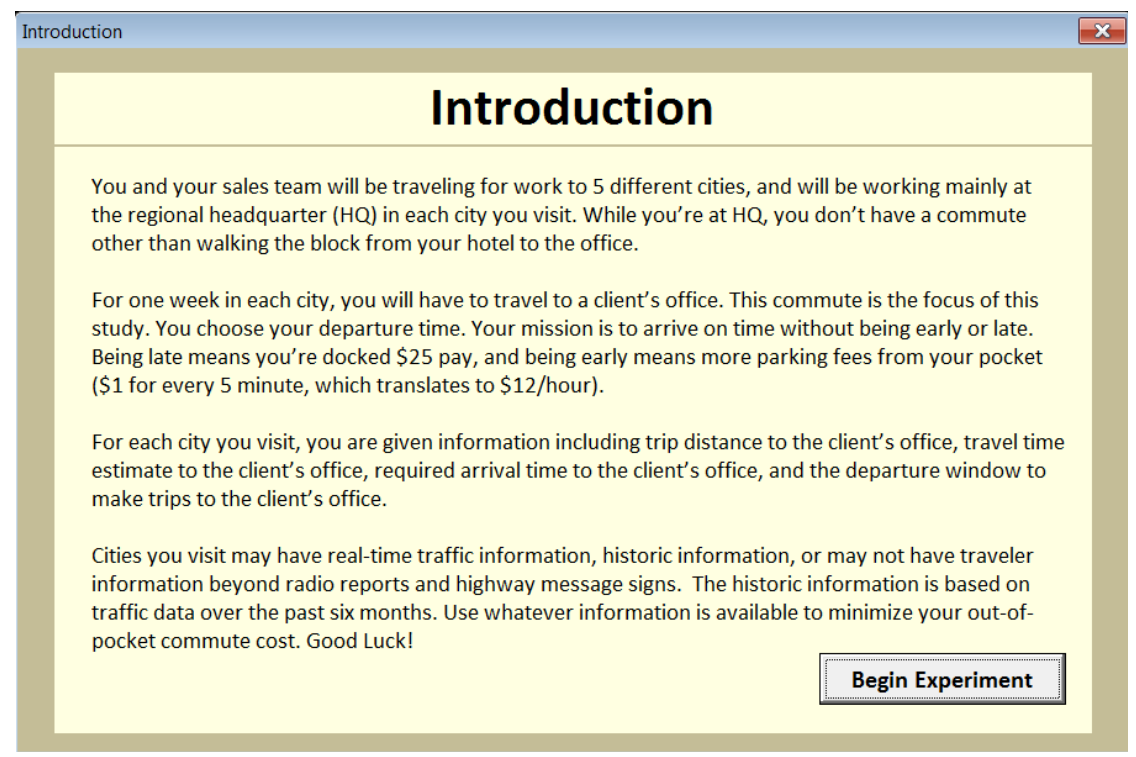

Figure H.9. Introduction screen at start of simulation game.

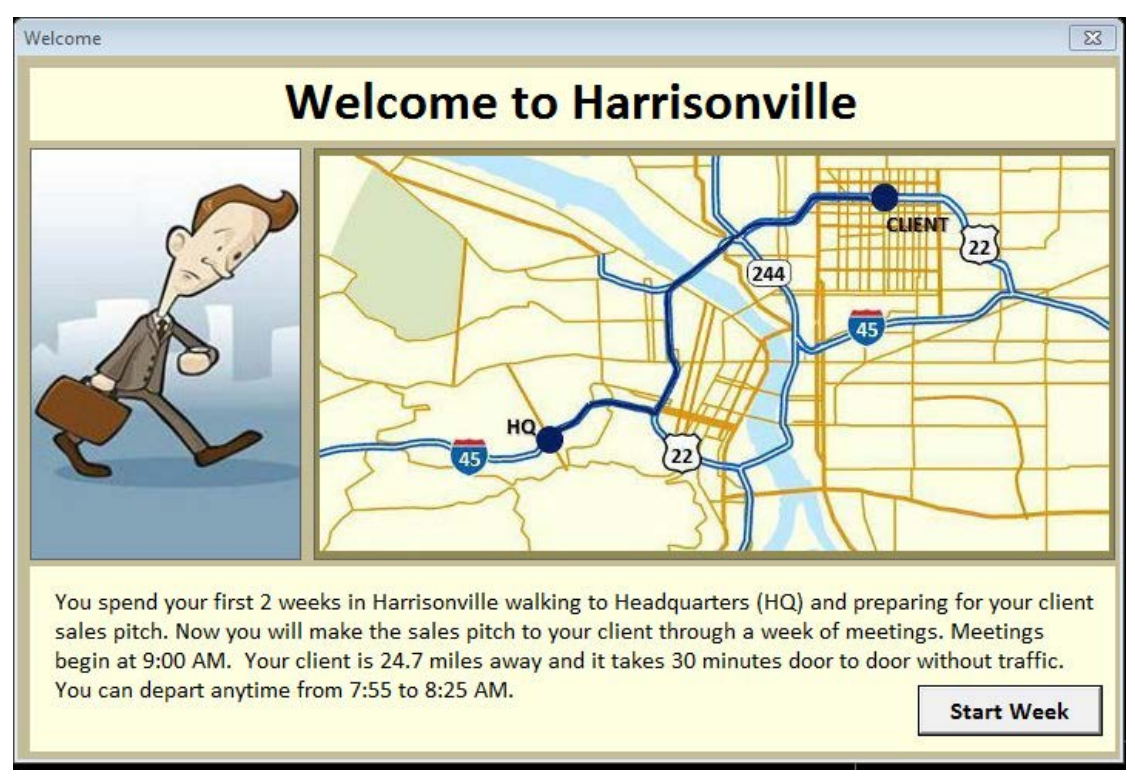

Figure H.10. City introduction screenshot and trip-making details. 


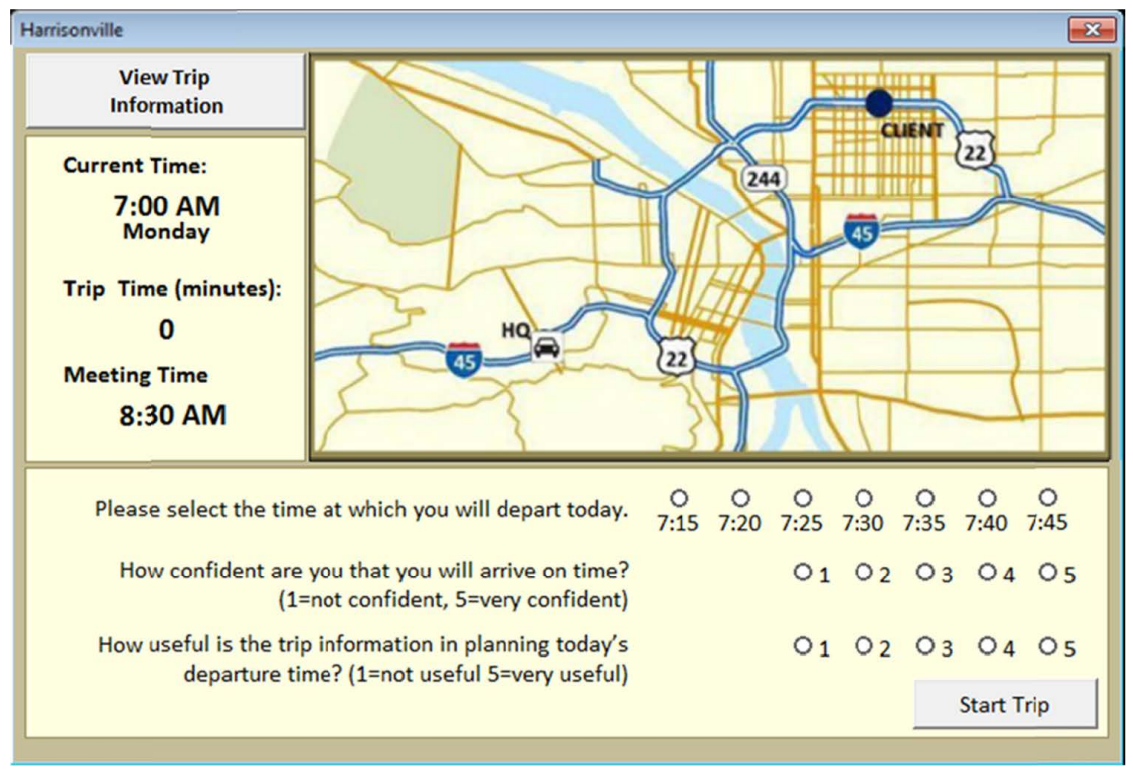

Figure H.11. Trip decision form screenshot.

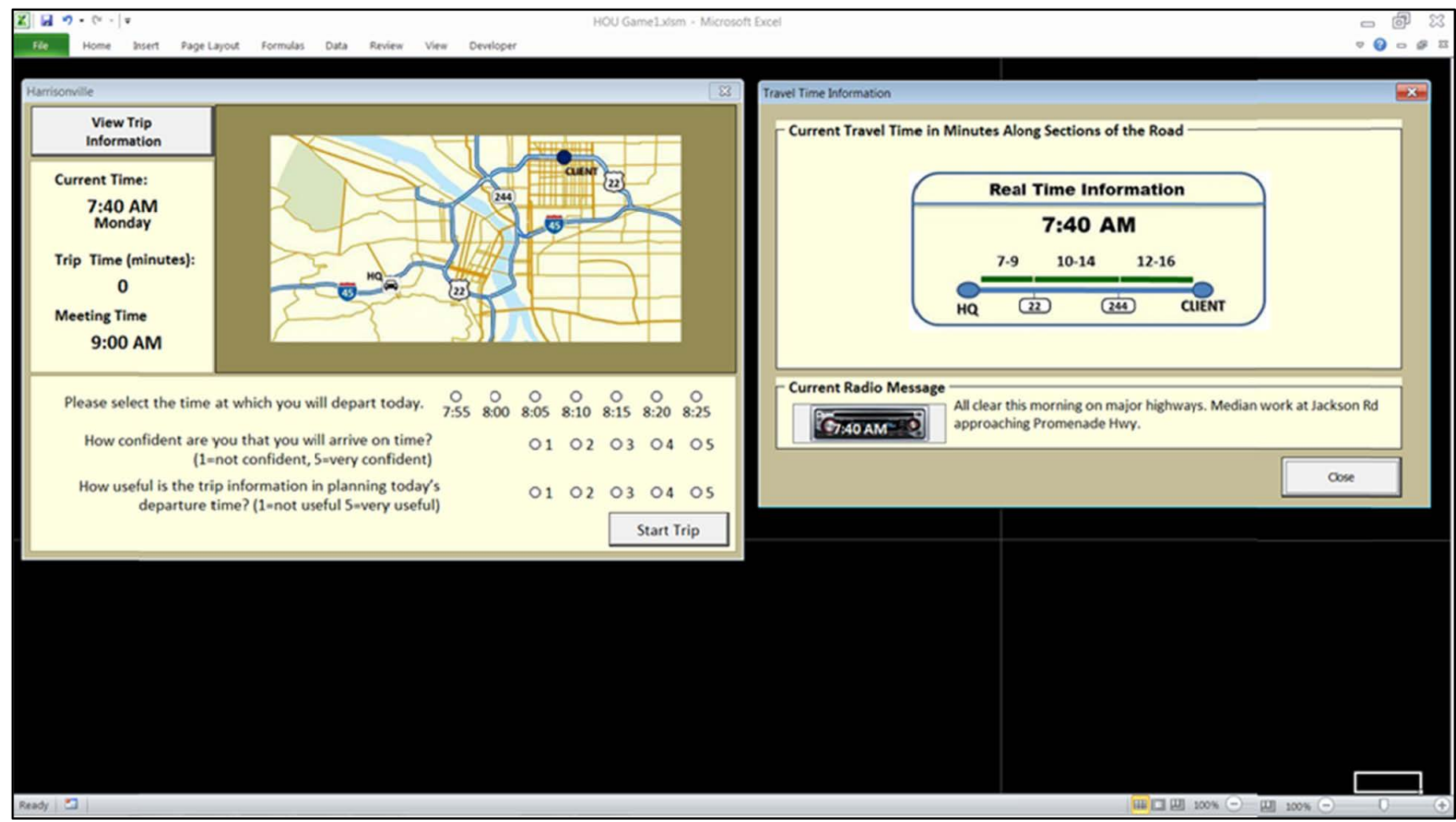

Figure H.12. Screenshot of trip decision form with reliability information being viewed. 


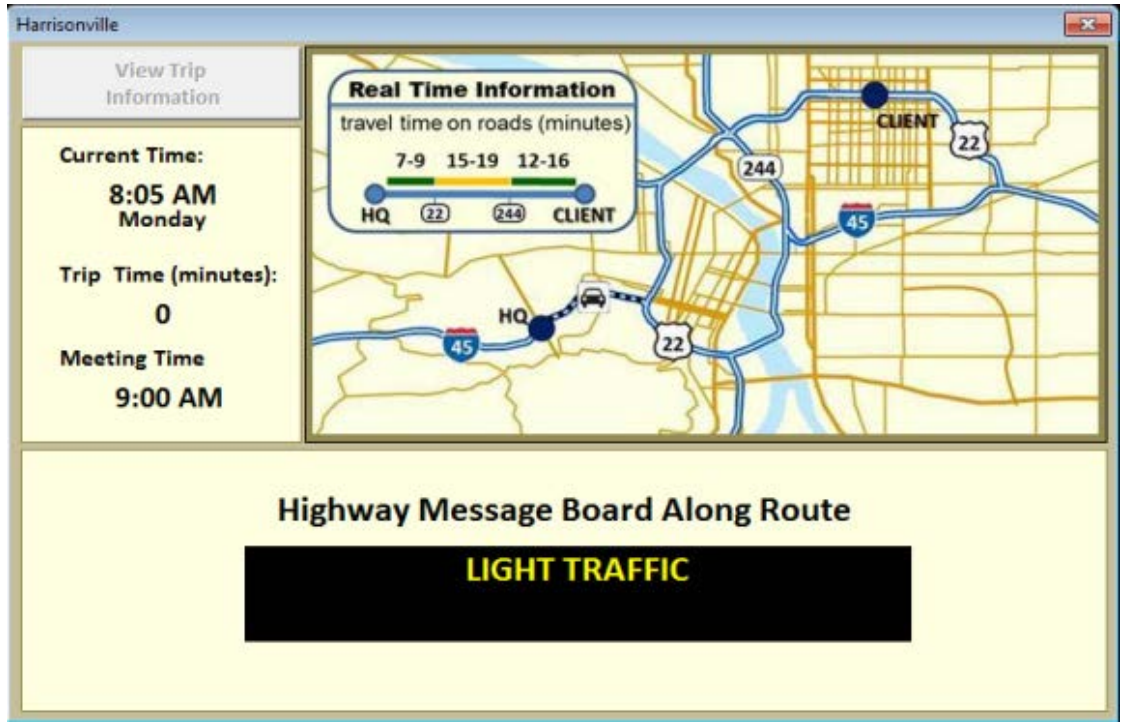

Figure H.13. Screenshot of simulated travel with highway message board.

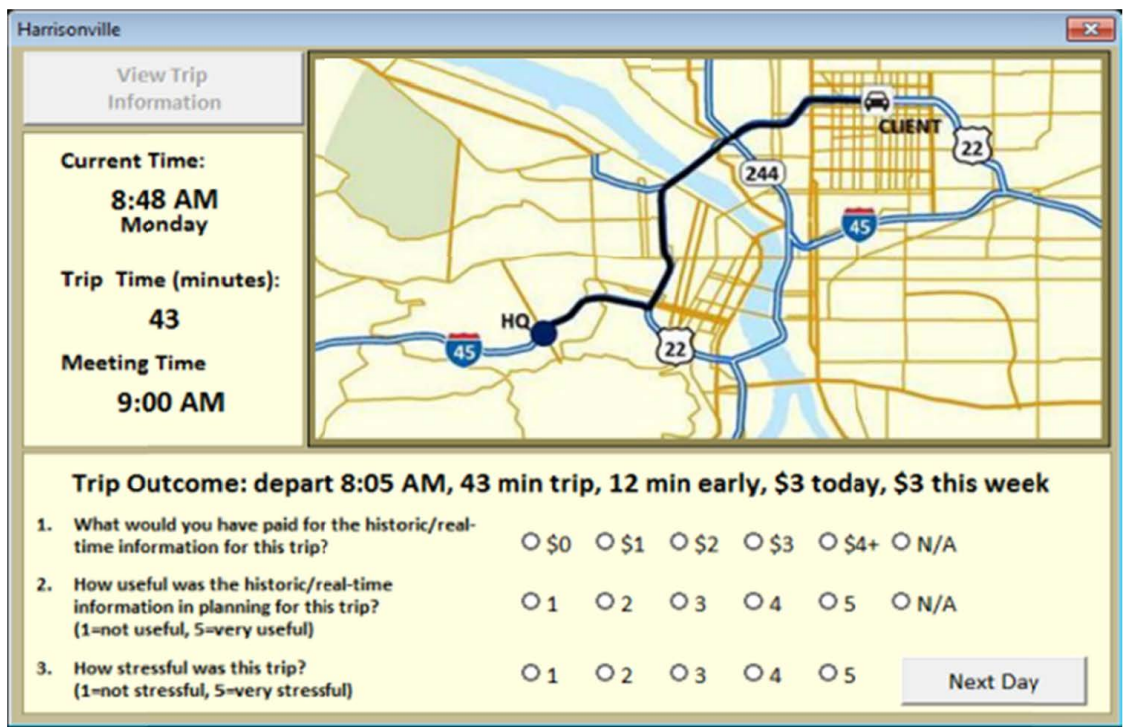

Figure H.14. End-of-trip form proving trip metrics and requesting end-of-trip information. 


\begin{tabular}{|c|c|c|c|c|c|}
\hline \multirow{2}{*}{$\begin{array}{l}\text { Harrisonville Summary } \\
\Gamma \text { Sample of Data Available for this City }\end{array}$} & \multicolumn{5}{|r|}{$-x$} \\
\hline & \multicolumn{5}{|c|}{ WEEK'S SUMMARY } \\
\hline Real Time Information & \multirow{4}{*}{\multicolumn{4}{|c|}{$\begin{array}{l}\text { Days Late: } \\
\text { Total Minutes Late: } \\
\text { Late Arrival Fees: } \\
\text { Total Minutes Early: } \\
\text { Extra Parking Cost: } \\
\text { Total Cost: }\end{array}$}} & \multirow{4}{*}{$\begin{array}{r}2 \\
28 \\
\$ 50 \\
19 \\
\$ 5 \\
\$ 55\end{array}$} \\
\hline 7:40 AM & & & & & \\
\hline $15-19$ & & & & & \\
\hline 244 & & & & & \\
\hline $\begin{array}{l}\text { 1. Did the pre-trip traveler information help reduce commute stress? } \\
\text { (1=not at all, } 5=\text { absolutely) }\end{array}$ & $O_{1}$ & $\mathrm{O}_{2}$ & $\mathrm{O}_{3}$ & $0_{4}$ & 05 \\
\hline $\begin{array}{l}\text { 2. Overall, how useful was the traveler information? } \\
\text { ( } 1=\text { not useful, } 5=\text { very useful) }\end{array}$ & $\mathrm{O}_{1}$ & $\mathrm{O}_{2}$ & $\mathrm{O}_{3}$ & $\mathrm{O}_{4}$ & 05 \\
\hline $\begin{array}{l}\text { 3. Do you feel this information helped you better manage your departure } \\
\text { decisions? ( } 1=\text { not at all, } 5=\text { absolutely })\end{array}$ & $O_{1}$ & $\mathrm{O}_{2}$ & $\mathrm{O}_{3}$ & $\mathrm{O}_{4}$ & 05 \\
\hline $\begin{array}{l}\text { 4. How difficult is it to understand the traveler information? } \\
\text { (1=very easy, } 5=\text { very difficult) }\end{array}$ & $O_{1}$ & $\mathrm{O}_{2}$ & $\mathrm{O}_{3}$ & $0_{4}$ & 05 \\
\hline $\begin{array}{l}\text { 5. How much would you be willing to pay per trip for the information you } \\
\text { viewed this week? }\end{array}$ & $0 \$ 0$ & $O \$ 1$ & $0 \$ 2$ & $0 \$ 3$ & $0 \$ 4$ \\
\hline & & & & \multicolumn{2}{|c|}{ Next City } \\
\hline
\end{tabular}

Figure H.15. End-of-week form requesting information on reliability term.

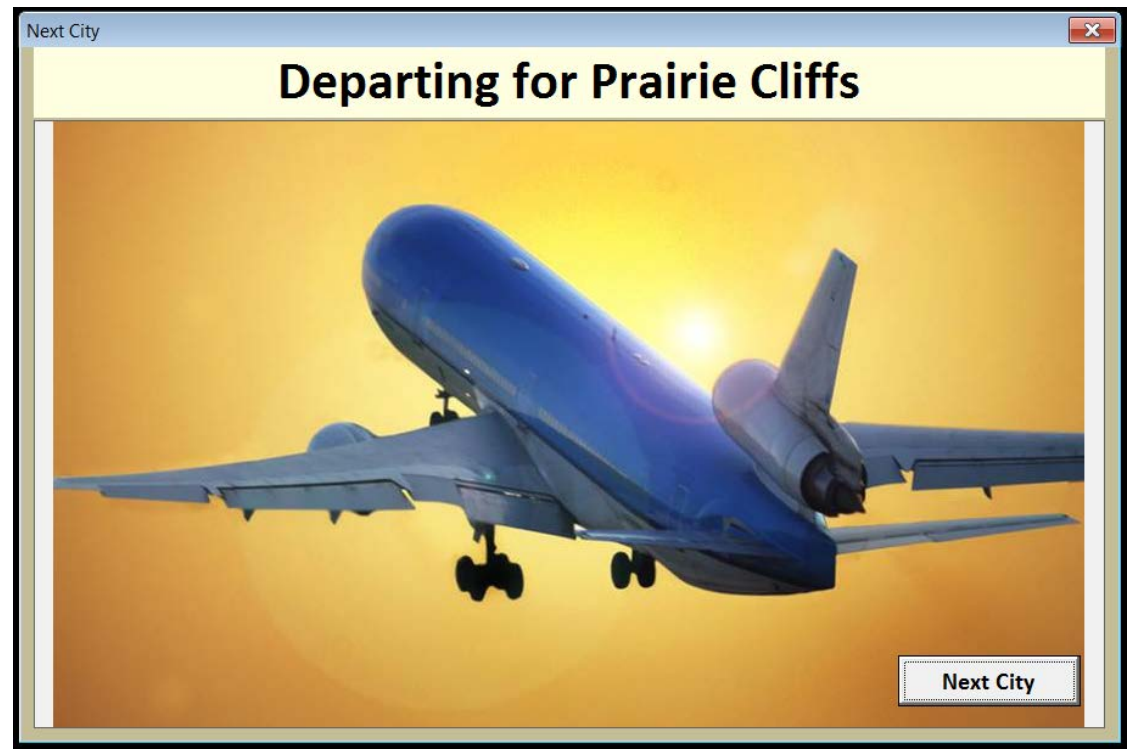

Figure H.16. Transition slide presented between weeks of simulated commute. 


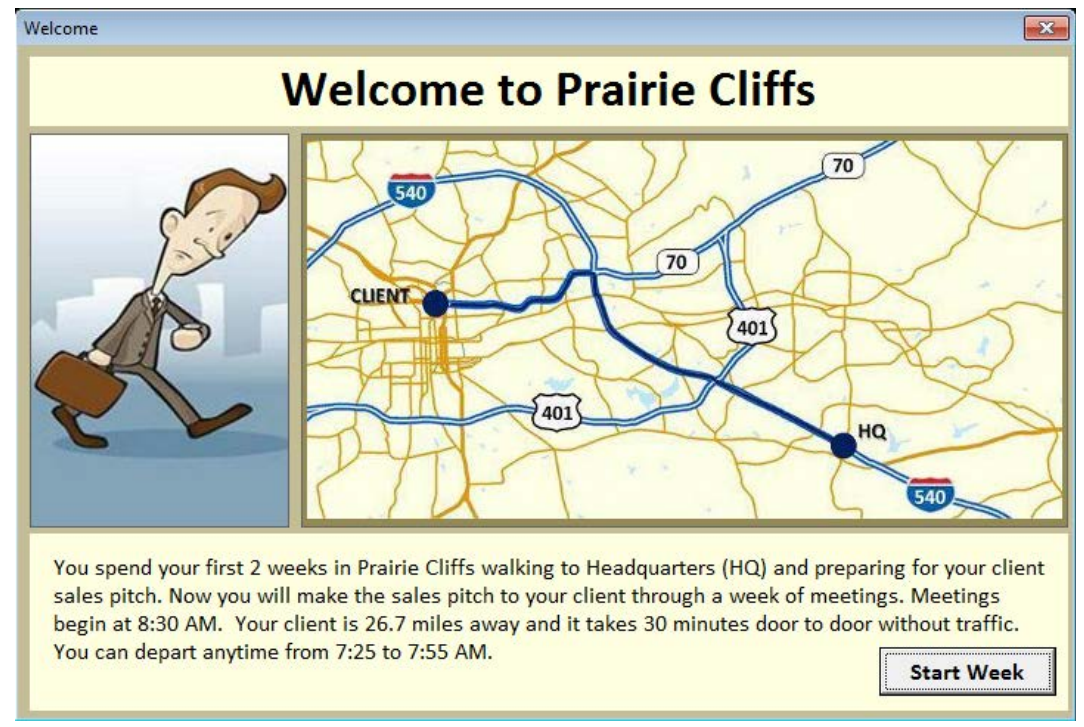

Figure H.17. City screenshot for Prairie Cliffs used for baseline travel information.

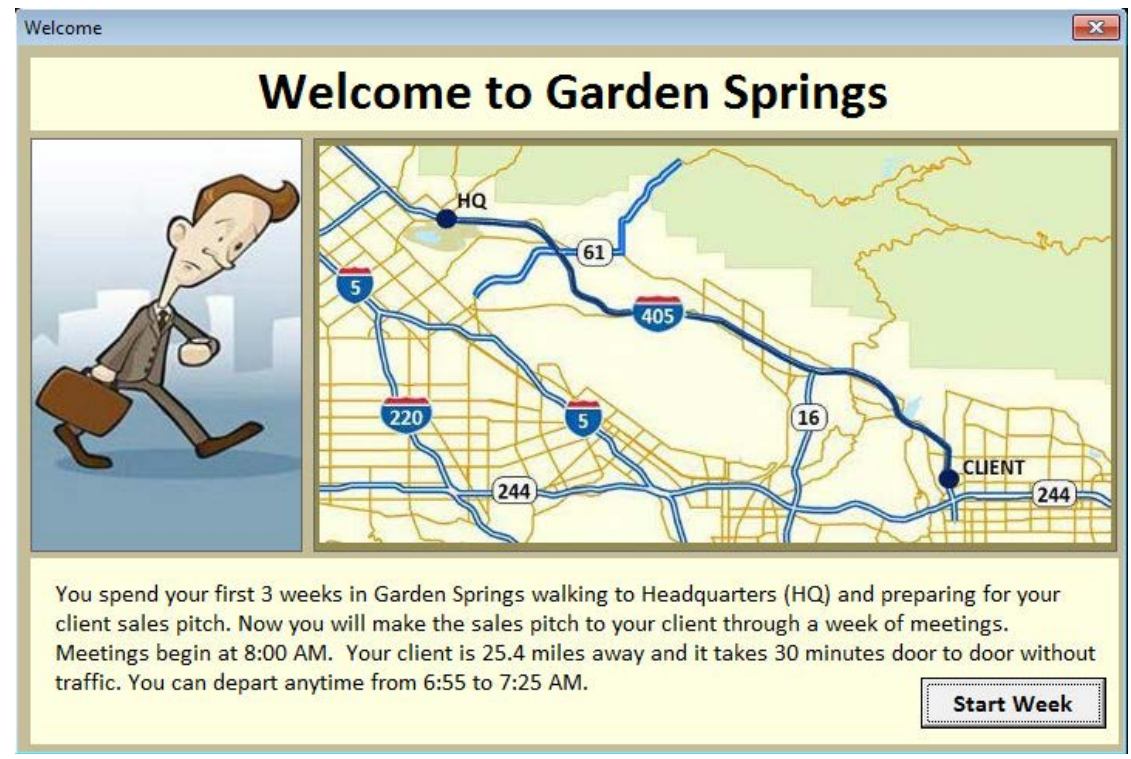

Figure H.18. City screenshot for Garden Springs used for textual 95th percentile and graphical good-, typical-, and bad-day reliability terms. 


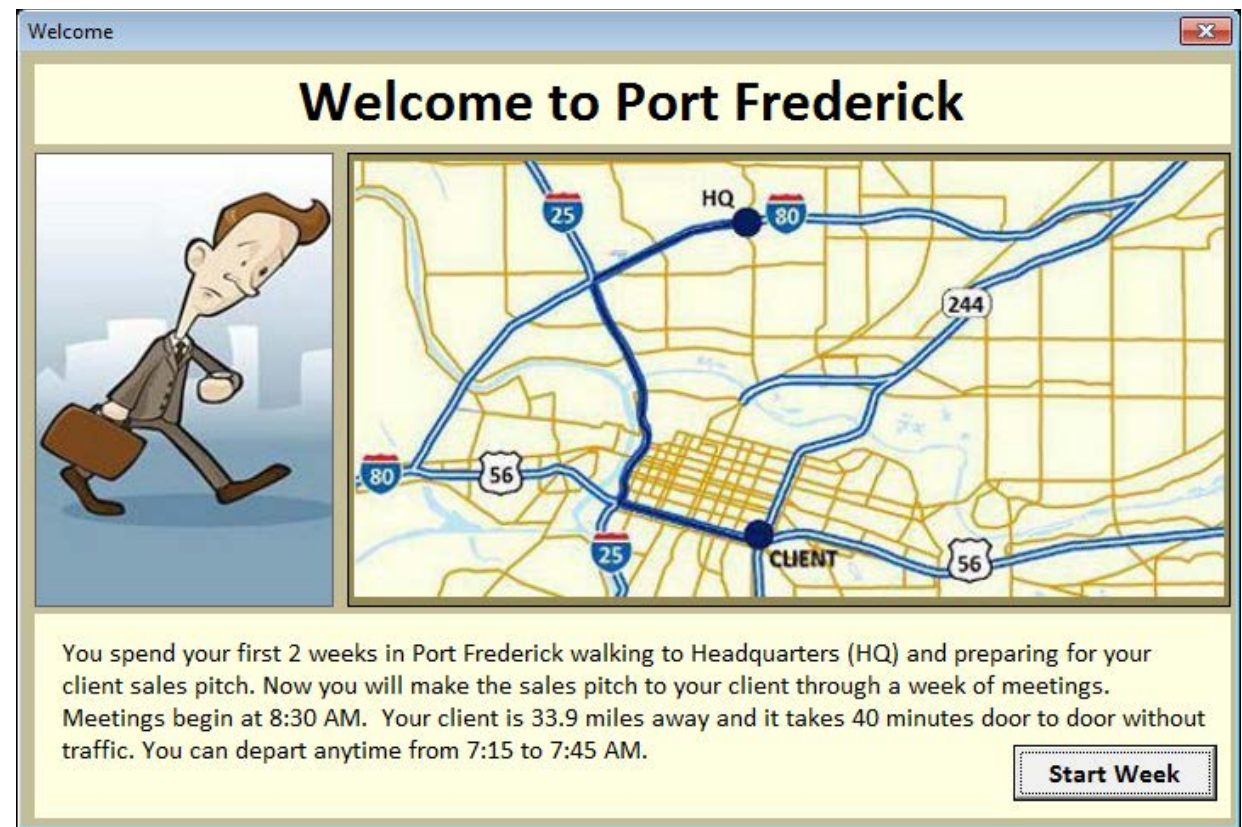

Figure H.19. City screenshot for Port Frederick used for textual average and 95th percentile reliability terms.

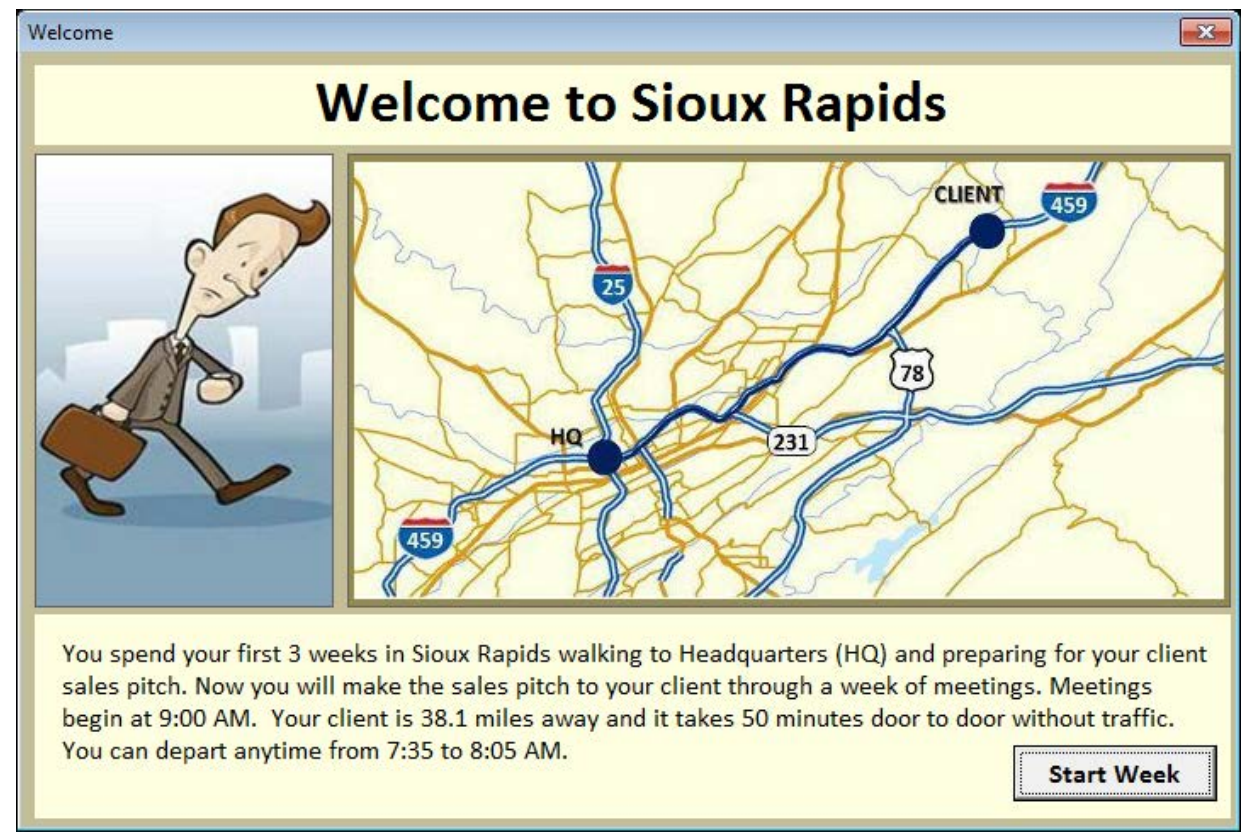

Figure H.20. City screenshot for Sioux Rapids used for textual good-, typical-, and bad-day reliability terms. 


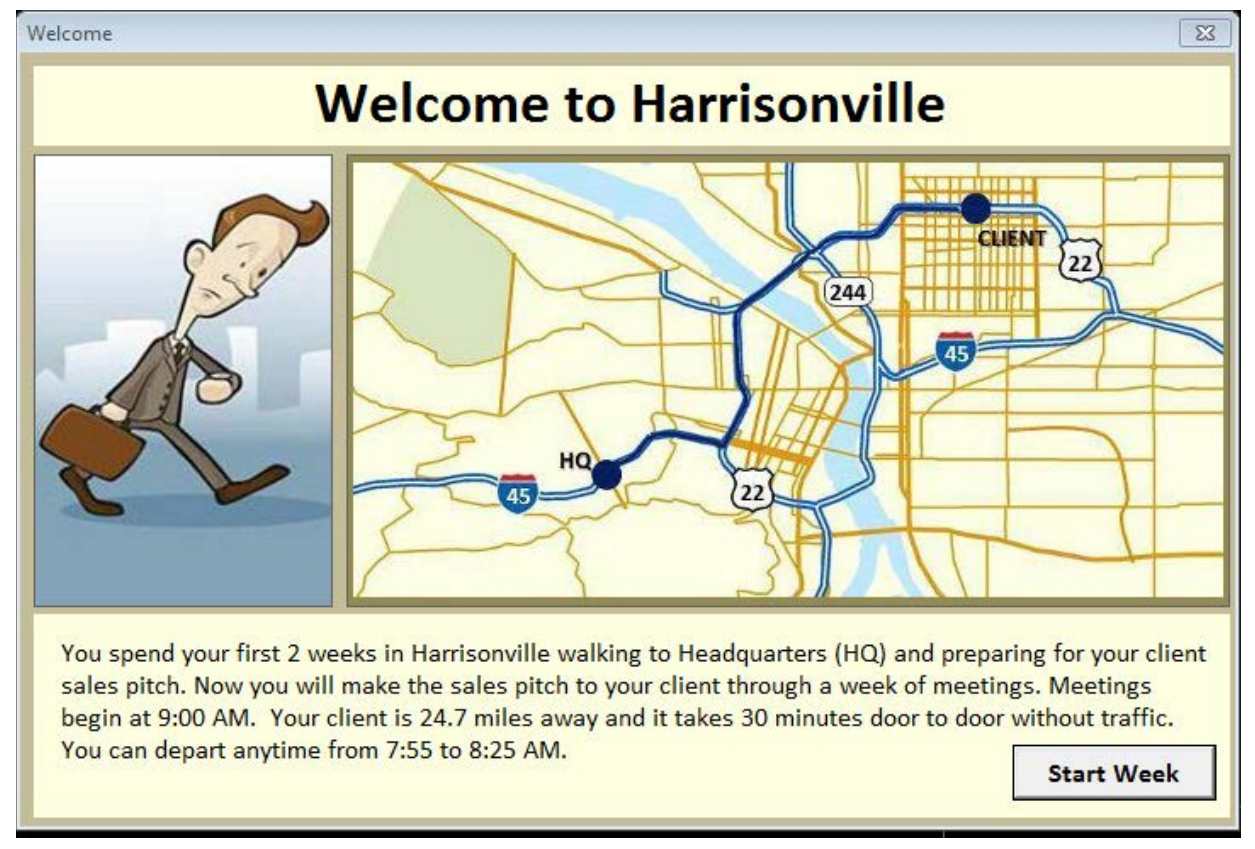

Figure H.21. City screenshot for Harrisonville used for reliability signposting and graphical average + 95th percentile reliability terms.

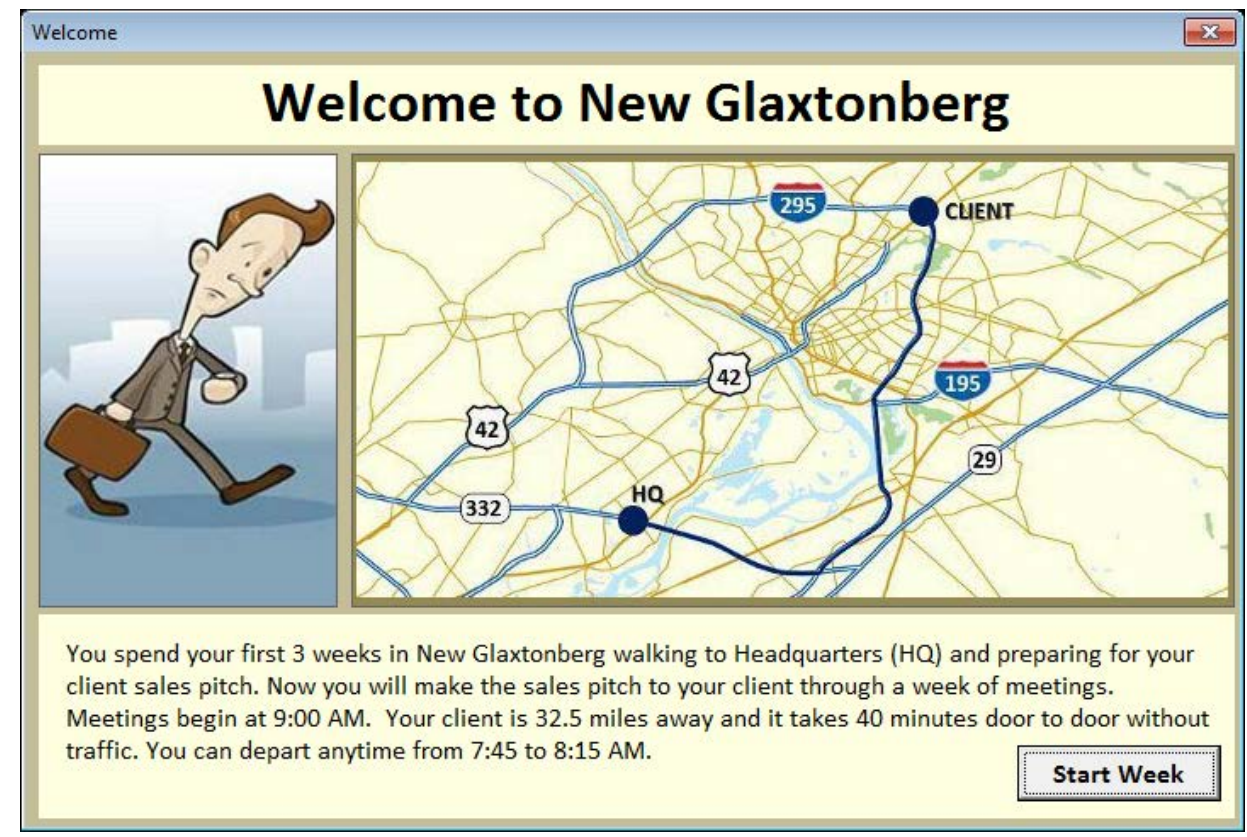

Figure H.22. City screenshot for New Glaxtonberg used for auditory 95th percentile reliability terms. 
Trip Time Data Tables for Experiment 1

\begin{tabular}{|c|c|c|c|c|}
\hline \multicolumn{5}{|c|}{$\begin{array}{l}\text { Data for Reliability Content A: } \\
\text { No Reliability Information }\end{array}$} \\
\hline $\begin{array}{l}\text { Info } \\
\text { Code }\end{array}$ & Day & $\begin{array}{l}\text { Dep } \\
\text { Time }\end{array}$ & $\begin{array}{c}\mathrm{TT} \\
(\mathrm{min})\end{array}$ & $\begin{array}{l}\text { Type of } \\
\text { Day }\end{array}$ \\
\hline A & 1 & 1 & 30 & Good \\
\hline A & 1 & 2 & 32 & Good \\
\hline$A$ & 1 & 3 & 34 & Good \\
\hline A & 1 & 4 & 34 & Good \\
\hline A & 1 & 5 & 37 & Good \\
\hline A & 1 & 6 & 38 & Good \\
\hline A & 1 & 7 & 39 & Good \\
\hline A & 2 & 1 & 44 & Moderate \\
\hline A & 2 & 2 & 51 & Moderate \\
\hline A & 2 & 3 & 56 & Moderate \\
\hline$A$ & 2 & 4 & 57 & Moderate \\
\hline$A$ & 2 & 5 & 59 & Moderate \\
\hline$A$ & 2 & 6 & 62 & Moderate \\
\hline A & 2 & 7 & 66 & Moderate \\
\hline$A$ & 3 & 1 & 31 & Typical \\
\hline$A$ & 3 & 2 & 37 & Typical \\
\hline A & 3 & 3 & 39 & Typical \\
\hline A & 3 & 4 & 40 & Typical \\
\hline A & 3 & 5 & 42 & Typical \\
\hline A & 3 & 6 & 49 & Typical \\
\hline A & 3 & 7 & 53 & Typical \\
\hline$A$ & 4 & 1 & 47 & Bad \\
\hline$A$ & 4 & 2 & 59 & Bad \\
\hline$A$ & 4 & 3 & 61 & $\mathrm{Bad}$ \\
\hline A & 4 & 4 & 55 & $\mathrm{Bad}$ \\
\hline$A$ & 4 & 5 & 53 & Bad \\
\hline A & 4 & 6 & 53 & Bad \\
\hline$A$ & 4 & 7 & 52 & Bad \\
\hline$A$ & 5 & 1 & 35 & Typical 2 \\
\hline$A$ & 5 & 2 & 42 & Typical 2 \\
\hline A & 5 & 3 & 43 & Typical 2 \\
\hline A & 5 & 4 & 46 & Typical 2 \\
\hline$A$ & 5 & 5 & 45 & Typical 2 \\
\hline$A$ & 5 & 6 & 47 & Typical 2 \\
\hline A & 5 & 7 & 50 & Typical 2 \\
\hline
\end{tabular}

\begin{tabular}{|c|c|c|c|c|}
\hline \multicolumn{5}{|c|}{$\begin{array}{l}\text { Data for Reliability Content B: } \\
\text { Textual 95th Percentile }\end{array}$} \\
\hline $\begin{array}{l}\text { Info } \\
\text { Code }\end{array}$ & Day & $\begin{array}{l}\text { Dep } \\
\text { Time }\end{array}$ & $\begin{array}{c}\mathrm{TT} \\
(\min )\end{array}$ & $\begin{array}{l}\text { Type of } \\
\text { Day }\end{array}$ \\
\hline$B$ & 1 & 1 & 44 & Moderate \\
\hline B & 1 & 2 & 51 & Moderate \\
\hline$B$ & 1 & 3 & 56 & Moderate \\
\hline B & 1 & 4 & 57 & Moderate \\
\hline$B$ & 1 & 5 & 59 & Moderate \\
\hline$B$ & 1 & 6 & 62 & Moderate \\
\hline$B$ & 1 & 7 & 66 & Moderate \\
\hline B & 2 & 1 & 31 & Typical \\
\hline$B$ & 2 & 2 & 37 & Typical \\
\hline$B$ & 2 & 3 & 39 & Typical \\
\hline B & 2 & 4 & 40 & Typical \\
\hline B & 2 & 5 & 42 & Typical \\
\hline$B$ & 2 & 6 & 49 & Typical \\
\hline$B$ & 2 & 7 & 53 & Typical \\
\hline$B$ & 3 & 1 & 47 & Bad \\
\hline$B$ & 3 & 2 & 59 & Bad \\
\hline$B$ & 3 & 3 & 61 & Bad \\
\hline$B$ & 3 & 4 & 55 & Bad \\
\hline B & 3 & 5 & 53 & Bad \\
\hline B & 3 & 6 & 53 & $\mathrm{Bad}$ \\
\hline B & 3 & 7 & 52 & Bad \\
\hline B & 4 & 1 & 35 & Good \\
\hline B & 4 & 2 & 42 & Good \\
\hline$B$ & 4 & 3 & 43 & Good \\
\hline$B$ & 4 & 4 & 46 & Good \\
\hline B & 4 & 5 & 45 & Good \\
\hline B & 4 & 6 & 47 & Good \\
\hline B & 4 & 7 & 50 & Good \\
\hline B & 5 & 1 & 30 & Typical 2 \\
\hline B & 5 & 2 & 32 & Typical 2 \\
\hline$B$ & 5 & 3 & 34 & Typical 2 \\
\hline B & 5 & 4 & 34 & Typical 2 \\
\hline B & 5 & 5 & 37 & Typical 2 \\
\hline B & 5 & 6 & 38 & Typical 2 \\
\hline B & 5 & 7 & 39 & Typical 2 \\
\hline
\end{tabular}

(continued on next page) 
Trip Time Data Tables for Experiment 1 (continued)

\begin{tabular}{|c|c|c|c|c|}
\hline \multicolumn{5}{|c|}{$\begin{array}{l}\text { Data for Reliability Content C: } \\
\text { Textual Average and 95th Percentile }\end{array}$} \\
\hline $\begin{array}{l}\text { Info } \\
\text { Code }\end{array}$ & Day & $\begin{array}{l}\text { Dep } \\
\text { Time }\end{array}$ & $\begin{array}{c}\mathrm{TT} \\
(\min )\end{array}$ & $\begin{array}{l}\text { Type of } \\
\text { Day }\end{array}$ \\
\hline C & 1 & 1 & 44 & Typical 2 \\
\hline C & 1 & 2 & 50 & Typical 2 \\
\hline C & 1 & 3 & 52 & Typical 2 \\
\hline C & 1 & 4 & 54 & Typical 2 \\
\hline C & 1 & 5 & 54 & Typical 2 \\
\hline C & 1 & 6 & 57 & Typical 2 \\
\hline C & 1 & 7 & 59 & Typical 2 \\
\hline C & 2 & 1 & 40 & Typical \\
\hline C & 2 & 2 & 46 & Typical \\
\hline C & 2 & 3 & 50 & Typical \\
\hline C & 2 & 4 & 51 & Typical \\
\hline C & 2 & 5 & 55 & Typical \\
\hline C & 2 & 6 & 55 & Typical \\
\hline C & 2 & 7 & 59 & Typical \\
\hline C & 3 & 1 & 55 & Bad \\
\hline C & 3 & 2 & 69 & Bad \\
\hline C & 3 & 3 & 70 & Bad \\
\hline C & 3 & 4 & 63 & Bad \\
\hline C & 3 & 5 & 62 & Bad \\
\hline C & 3 & 6 & 63 & Bad \\
\hline C & 3 & 7 & 62 & Bad \\
\hline C & 4 & 1 & 42 & Good \\
\hline C & 4 & 2 & 40 & Good \\
\hline C & 4 & 3 & 44 & Good \\
\hline C & 4 & 4 & 45 & Good \\
\hline C & 4 & 5 & 47 & Good \\
\hline C & 4 & 6 & 49 & Good \\
\hline C & 4 & 7 & 48 & Good \\
\hline $\mathrm{C}$ & 5 & 1 & 55 & Moderate \\
\hline C & 5 & 2 & 64 & Moderate \\
\hline C & 5 & 3 & 66 & Moderate \\
\hline C & 5 & 4 & 63 & Moderate \\
\hline C & 5 & 5 & 64 & Moderate \\
\hline C & 5 & 6 & 70 & Moderate \\
\hline C & 5 & 7 & 74 & Moderate \\
\hline
\end{tabular}

\begin{tabular}{|c|c|c|c|c|}
\hline \multicolumn{5}{|c|}{$\begin{array}{l}\text { Data for Reliability Content } D \text { : } \\
\text { Textual 20th, Average, and 95th Percentile }\end{array}$} \\
\hline $\begin{array}{l}\text { Info } \\
\text { Code }\end{array}$ & Day & $\begin{array}{l}\text { Dep } \\
\text { Time }\end{array}$ & $\begin{array}{c}\mathrm{TT} \\
(\mathrm{min})\end{array}$ & $\begin{array}{l}\text { Type of } \\
\text { Day }\end{array}$ \\
\hline D & 1 & 1 & 53 & Typical \\
\hline D & 1 & 2 & 57 & Typical \\
\hline D & 1 & 3 & 60 & Typical \\
\hline D & 1 & 4 & 58 & Typical \\
\hline D & 1 & 5 & 63 & Typical \\
\hline D & 1 & 6 & 68 & Typical \\
\hline D & 1 & 7 & 68 & Typical \\
\hline$D$ & 2 & 1 & 68 & Bad \\
\hline$D$ & 2 & 2 & 75 & $\mathrm{Bad}$ \\
\hline D & 2 & 3 & 82 & Bad \\
\hline$D$ & 2 & 4 & 74 & Bad \\
\hline D & 2 & 5 & 72 & $\mathrm{Bad}$ \\
\hline$D$ & 2 & 6 & 74 & Bad \\
\hline D & 2 & 7 & 74 & Bad \\
\hline D & 3 & 1 & 54 & Typical 2 \\
\hline D & 3 & 2 & 61 & Typical 2 \\
\hline$D$ & 3 & 3 & 62 & Typical 2 \\
\hline D & 3 & 4 & 63 & Typical 2 \\
\hline$D$ & 3 & 5 & 64 & Typical 2 \\
\hline D & 3 & 6 & 72 & Typical 2 \\
\hline D & 3 & 7 & 71 & Typical 2 \\
\hline D & 4 & 1 & 63 & Moderate \\
\hline$D$ & 4 & 2 & 70 & Moderate \\
\hline$D$ & 4 & 3 & 78 & Moderate \\
\hline$D$ & 4 & 4 & 73 & Moderate \\
\hline$D$ & 4 & 5 & 75 & Moderate \\
\hline$D$ & 4 & 6 & 79 & Moderate \\
\hline$D$ & 4 & 7 & 82 & Moderate \\
\hline$D$ & 5 & 1 & 51 & Good \\
\hline$D$ & 5 & 2 & 53 & Good \\
\hline$D$ & 5 & 3 & 52 & Good \\
\hline$D$ & 5 & 4 & 55 & Good \\
\hline$D$ & 5 & 5 & 58 & Good \\
\hline$D$ & 5 & 6 & 60 & Good \\
\hline$D$ & 5 & 7 & 57 & Good \\
\hline
\end{tabular}


Trip Time Data Tables for Experiment 1 (continued)

\begin{tabular}{|c|c|c|c|c|}
\hline \multicolumn{5}{|c|}{$\begin{array}{c}\text { Data for Reliability Content E: } \\
\text { Signpost Reliability }\end{array}$} \\
\hline $\begin{array}{l}\text { Info } \\
\text { Code }\end{array}$ & Day & $\begin{array}{l}\text { Dep } \\
\text { Time }\end{array}$ & $\begin{array}{l}\text { TT } \\
(\min )\end{array}$ & $\begin{array}{l}\text { Type of } \\
\text { Day }\end{array}$ \\
\hline E & 1 & 1 & 35 & Typical 2 \\
\hline E & 1 & 2 & 42 & Typical 2 \\
\hline E & 1 & 3 & 43 & Typical 2 \\
\hline E & 1 & 4 & 46 & Typical 2 \\
\hline E & 1 & 5 & 45 & Typical 2 \\
\hline E & 1 & 6 & 47 & Typical 2 \\
\hline E & 1 & 7 & 50 & Typical 2 \\
\hline E & 2 & 1 & 30 & Good \\
\hline$E$ & 2 & 2 & 32 & Good \\
\hline$E$ & 2 & 3 & 34 & Good \\
\hline E & 2 & 4 & 34 & Good \\
\hline$E$ & 2 & 5 & 37 & Good \\
\hline$E$ & 2 & 6 & 38 & Good \\
\hline$E$ & 2 & 7 & 39 & Good \\
\hline$E$ & 3 & 1 & 47 & Bad \\
\hline$E$ & 3 & 2 & 59 & Bad \\
\hline$E$ & 3 & 3 & 61 & Bad \\
\hline$E$ & 3 & 4 & 55 & Bad \\
\hline$E$ & 3 & 5 & 53 & Bad \\
\hline$E$ & 3 & 6 & 53 & Bad \\
\hline$E$ & 3 & 7 & 52 & Bad \\
\hline$E$ & 4 & 1 & 31 & Typical \\
\hline$E$ & 4 & 2 & 37 & Typical \\
\hline$E$ & 4 & 3 & 39 & Typical \\
\hline$E$ & 4 & 4 & 40 & Typical \\
\hline$E$ & 4 & 5 & 42 & Typical \\
\hline$E$ & 4 & 6 & 49 & Typical \\
\hline$E$ & 4 & 7 & 53 & Typical \\
\hline$E$ & 5 & 1 & 44 & Moderate \\
\hline$E$ & 5 & 2 & 51 & Moderate \\
\hline$E$ & 5 & 3 & 56 & Moderate \\
\hline $\mathrm{E}$ & 5 & 4 & 57 & Moderate \\
\hline$E$ & 5 & 5 & 59 & Moderate \\
\hline$E$ & 5 & 6 & 62 & Moderate \\
\hline$E$ & 5 & 7 & 66 & Moderate \\
\hline
\end{tabular}

\begin{tabular}{|c|c|c|c|c|}
\hline \multicolumn{5}{|c|}{$\begin{array}{l}\text { Data for Reliability Content F: } \\
\text { Graphical Average and 95th Percentile }\end{array}$} \\
\hline $\begin{array}{l}\text { Info } \\
\text { Code }\end{array}$ & Day & $\begin{array}{l}\text { Dep } \\
\text { Time }\end{array}$ & $\begin{array}{c}\mathrm{TT} \\
(\min )\end{array}$ & $\begin{array}{l}\text { Type of } \\
\text { Day }\end{array}$ \\
\hline $\mathrm{F}$ & 1 & 1 & 42 & Good \\
\hline $\mathrm{F}$ & 1 & 2 & 40 & Good \\
\hline $\mathrm{F}$ & 1 & 3 & 44 & Good \\
\hline $\mathrm{F}$ & 1 & 4 & 45 & Good \\
\hline $\mathrm{F}$ & 1 & 5 & 47 & Good \\
\hline $\mathrm{F}$ & 1 & 6 & 49 & Good \\
\hline $\mathrm{F}$ & 1 & 7 & 48 & Good \\
\hline $\mathrm{F}$ & 2 & 1 & 55 & Bad \\
\hline $\mathrm{F}$ & 2 & 2 & 69 & Bad \\
\hline $\mathrm{F}$ & 2 & 3 & 70 & Bad \\
\hline $\mathrm{F}$ & 2 & 4 & 63 & Bad \\
\hline $\mathrm{F}$ & 2 & 5 & 62 & $\mathrm{Bad}$ \\
\hline $\mathrm{F}$ & 2 & 6 & 63 & Bad \\
\hline $\mathrm{F}$ & 2 & 7 & 62 & Bad \\
\hline $\mathrm{F}$ & 3 & 1 & 44 & Typical 2 \\
\hline $\mathrm{F}$ & 3 & 2 & 50 & Typical 2 \\
\hline $\mathrm{F}$ & 3 & 3 & 52 & Typical 2 \\
\hline $\mathrm{F}$ & 3 & 4 & 54 & Typical 2 \\
\hline $\mathrm{F}$ & 3 & 5 & 54 & Typical 2 \\
\hline $\mathrm{F}$ & 3 & 6 & 57 & Typical 2 \\
\hline $\mathrm{F}$ & 3 & 7 & 59 & Typical 2 \\
\hline $\mathrm{F}$ & 4 & 1 & 55 & Moderate \\
\hline $\mathrm{F}$ & 4 & 2 & 64 & Moderate \\
\hline $\mathrm{F}$ & 4 & 3 & 66 & Moderate \\
\hline $\mathrm{F}$ & 4 & 4 & 63 & Moderate \\
\hline $\mathrm{F}$ & 4 & 5 & 64 & Moderate \\
\hline $\mathrm{F}$ & 4 & 6 & 70 & Moderate \\
\hline $\mathrm{F}$ & 4 & 7 & 74 & Moderate \\
\hline $\mathrm{F}$ & 5 & 1 & 40 & Typical \\
\hline $\mathrm{F}$ & 5 & 2 & 46 & Typical \\
\hline $\mathrm{F}$ & 5 & 3 & 50 & Typical \\
\hline $\mathrm{F}$ & 5 & 4 & 51 & Typical \\
\hline $\mathrm{F}$ & 5 & 5 & 55 & Typical \\
\hline $\mathrm{F}$ & 5 & 6 & 55 & Typical \\
\hline $\mathrm{F}$ & 5 & 7 & 59 & Typical \\
\hline
\end{tabular}


Trip Time Data Tables for Experiment 1 (continued)

\begin{tabular}{|c|c|c|c|c|}
\hline \multicolumn{5}{|c|}{$\begin{array}{l}\text { Data for Reliability Content G: } \\
\text { Graphical 20th, Average, } \\
\text { and 95th Percentile }\end{array}$} \\
\hline $\begin{array}{l}\text { Info } \\
\text { Code }\end{array}$ & Day & $\begin{array}{l}\text { Dep } \\
\text { Time }\end{array}$ & $\begin{array}{c}\text { TT } \\
(\min )\end{array}$ & $\begin{array}{c}\text { Type of } \\
\text { Day }\end{array}$ \\
\hline $\mathrm{G}$ & 1 & 1 & 63 & Good \\
\hline G & 1 & 2 & 70 & Good \\
\hline $\mathrm{G}$ & 1 & 3 & 78 & Good \\
\hline G & 1 & 4 & 73 & Good \\
\hline G & 1 & 5 & 75 & Good \\
\hline G & 1 & 6 & 79 & Good \\
\hline $\mathrm{G}$ & 1 & 7 & 82 & Good \\
\hline G & 2 & 1 & 54 & Bad \\
\hline G & 2 & 2 & 61 & Bad \\
\hline $\mathrm{G}$ & 2 & 3 & 62 & Bad \\
\hline $\mathrm{G}$ & 2 & 4 & 63 & $\mathrm{Bad}$ \\
\hline G & 2 & 5 & 64 & $\mathrm{Bad}$ \\
\hline $\mathrm{G}$ & 2 & 6 & 72 & $\mathrm{Bad}$ \\
\hline $\mathrm{G}$ & 2 & 7 & 71 & $\mathrm{Bad}$ \\
\hline $\mathrm{G}$ & 3 & 1 & 51 & Typical 2 \\
\hline $\mathrm{G}$ & 3 & 2 & 53 & Typical 2 \\
\hline G & 3 & 3 & 52 & Typical 2 \\
\hline G & 3 & 4 & 55 & Typical 2 \\
\hline G & 3 & 5 & 58 & Typical 2 \\
\hline G & 3 & 6 & 60 & Typical 2 \\
\hline $\mathrm{G}$ & 3 & 7 & 57 & Typical 2 \\
\hline $\mathrm{G}$ & 4 & 1 & 68 & Moderate \\
\hline $\mathrm{G}$ & 4 & 2 & 75 & Moderate \\
\hline $\mathrm{G}$ & 4 & 3 & 82 & Moderate \\
\hline $\mathrm{G}$ & 4 & 4 & 74 & Moderate \\
\hline $\mathrm{G}$ & 4 & 5 & 72 & Moderate \\
\hline $\mathrm{G}$ & 4 & 6 & 74 & Moderate \\
\hline G & 4 & 7 & 74 & Moderate \\
\hline $\mathrm{G}$ & 5 & 1 & 53 & Typical \\
\hline $\mathrm{G}$ & 5 & 2 & 57 & Typical \\
\hline $\mathrm{G}$ & 5 & 3 & 60 & Typical \\
\hline G & 5 & 4 & 58 & Typical \\
\hline G & 5 & 5 & 63 & Typical \\
\hline $\mathrm{G}$ & 5 & 6 & 68 & Typical \\
\hline G & 5 & 7 & 68 & Typical \\
\hline
\end{tabular}

\begin{tabular}{|c|c|c|c|c|}
\hline \multicolumn{5}{|c|}{$\begin{array}{l}\text { Data for Reliability Content H: } \\
\text { Auditory 95th Percentile }\end{array}$} \\
\hline $\begin{array}{l}\text { Info } \\
\text { Code }\end{array}$ & Day & $\begin{array}{l}\text { Dep } \\
\text { Time }\end{array}$ & $\begin{array}{c}\mathrm{TT} \\
(\mathrm{min})\end{array}$ & $\begin{array}{l}\text { Type of } \\
\text { Day }\end{array}$ \\
\hline $\mathrm{H}$ & 1 & 1 & 40 & Typical \\
\hline $\mathrm{H}$ & 1 & 2 & 46 & Typical \\
\hline $\mathrm{H}$ & 1 & 3 & 50 & Typical \\
\hline $\mathrm{H}$ & 1 & 4 & 51 & Typical \\
\hline $\mathrm{H}$ & 1 & 5 & 55 & Typical \\
\hline $\mathrm{H}$ & 1 & 6 & 55 & Typical \\
\hline $\mathrm{H}$ & 1 & 7 & 59 & Typical \\
\hline $\mathrm{H}$ & 2 & 1 & 55 & Moderate \\
\hline $\mathrm{H}$ & 2 & 2 & 64 & Moderate \\
\hline $\mathrm{H}$ & 2 & 3 & 66 & Moderate \\
\hline $\mathrm{H}$ & 2 & 4 & 63 & Moderate \\
\hline $\mathrm{H}$ & 2 & 5 & 64 & Moderate \\
\hline $\mathrm{H}$ & 2 & 6 & 70 & Moderate \\
\hline $\mathrm{H}$ & 2 & 7 & 74 & Moderate \\
\hline $\mathrm{H}$ & 3 & 1 & 42 & Good \\
\hline $\mathrm{H}$ & 3 & 2 & 40 & Good \\
\hline $\mathrm{H}$ & 3 & 3 & 44 & Good \\
\hline $\mathrm{H}$ & 3 & 4 & 45 & Good \\
\hline $\mathrm{H}$ & 3 & 5 & 47 & Good \\
\hline $\mathrm{H}$ & 3 & 6 & 49 & Good \\
\hline $\mathrm{H}$ & 3 & 7 & 48 & Good \\
\hline $\mathrm{H}$ & 4 & 1 & 44 & Typical 2 \\
\hline $\mathrm{H}$ & 4 & 2 & 50 & Typical 2 \\
\hline $\mathrm{H}$ & 4 & 3 & 52 & Typical 2 \\
\hline $\mathrm{H}$ & 4 & 4 & 54 & Typical 2 \\
\hline $\mathrm{H}$ & 4 & 5 & 54 & Typical 2 \\
\hline $\mathrm{H}$ & 4 & 6 & 57 & Typical 2 \\
\hline $\mathrm{H}$ & 4 & 7 & 59 & Typical 2 \\
\hline $\mathrm{H}$ & 5 & 1 & 55 & Bad \\
\hline $\mathrm{H}$ & 5 & 2 & 69 & Bad \\
\hline $\mathrm{H}$ & 5 & 3 & 70 & $\mathrm{Bad}$ \\
\hline $\mathrm{H}$ & 5 & 4 & 63 & Bad \\
\hline $\mathrm{H}$ & 5 & 5 & 62 & Bad \\
\hline $\mathrm{H}$ & 5 & 6 & 63 & Bad \\
\hline $\mathrm{H}$ & 5 & 7 & 62 & Bad \\
\hline
\end{tabular}

Note: Info Code = Information Code, Dep Time = Departure Time, and $\Pi=$ Travel Time. 
Experiment 2 Screenshot for Transitioning Participants from Week to Week

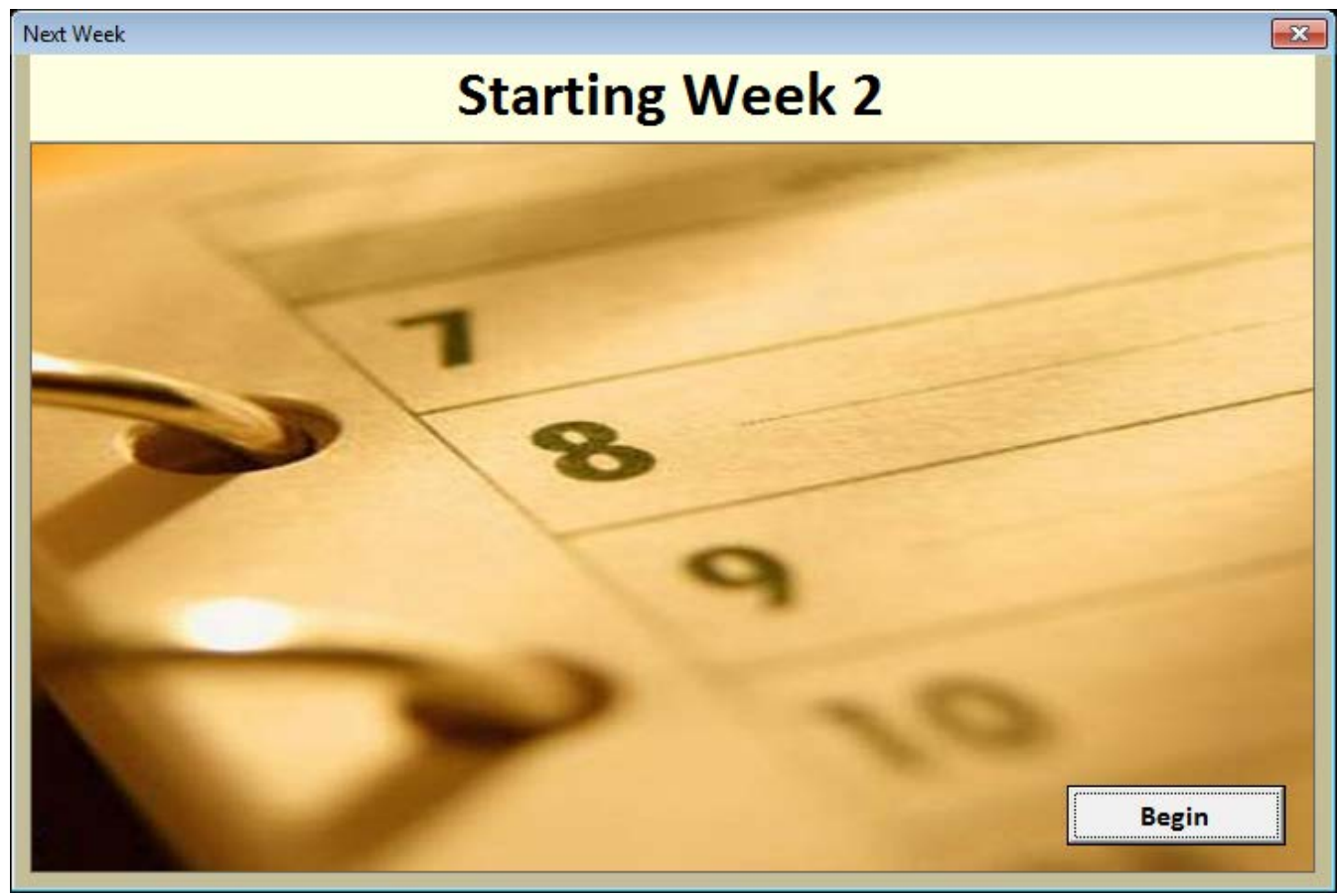




\section{Post-Experiment Paper Survey Conducted in Chicago and Houston}

\section{POST GAME SURVEY}

Thank you for completing the traffic game! Be low are a number of formats presenting different types of historic travel time inforcmation. Historic travel time information may be helpful in planning unfamiliar trips, particularly when on-tire arrival is critical. For each format below, please tell us three things:

(1) How easily are you are able to understand information presented?

(2) How useful would this information prove when planning unfamiliar trips?

(3) How complicated is the information that is presented?

\section{EORMAT 1: Historic Graph presentiog estimated travel time, and extra time for unexpected delavs}

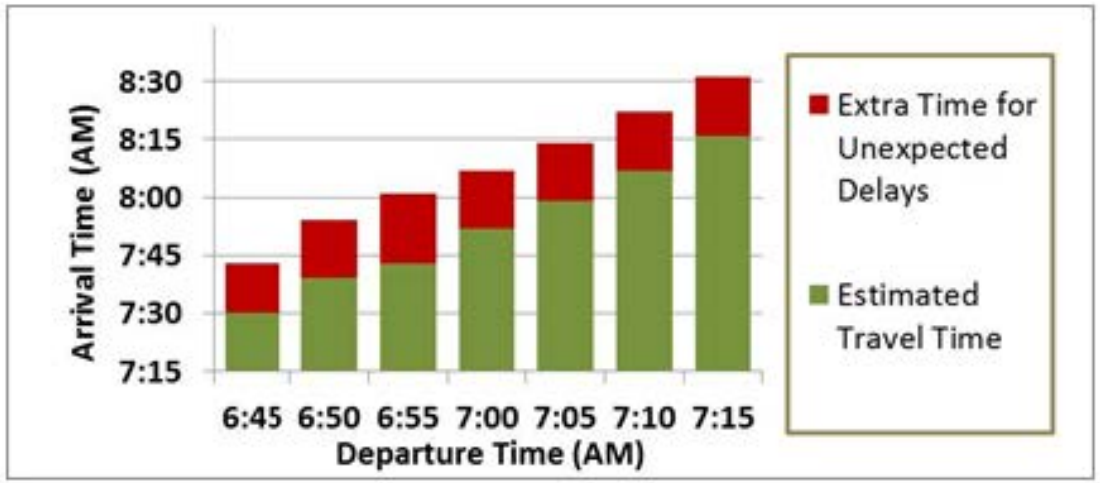

1a. How complicated is the infomation that is presented?

$\begin{array}{llllllll}1 & 2 & 3 & 4 & 5 & 6 & 7\end{array}$

( 1 = not complicated $4=$ neutral $\quad 7$ =very complicated)

1b. How easily are you able to understand information presented?

$\begin{array}{lllllll}1 & 2 & 3 & 4 & 5 & 6 & 7\end{array}$

$$
\text { (1 } 1 \text { very difficult } \quad 4=\text { neutral } \quad 7=\text { very easily) }
$$

1c. How useful would this infomation prove when planning unfamiliartrips? $\begin{array}{lllllll}1 & 2 & 3 & 4 & 5 & 6 & 7\end{array}$

$$
\text { (1 = not useful } \quad 4 \text { =neutral } \quad 7 \text { =very useful) }
$$

FORMAT 2: Historic Text with a slider bar for departure time presenting estimates for good, typical, and bad traffic days

Departing at 7:50am:

Good Day: 53 minutes or less

Typical Day: 63 minutes or less

Bad Day:
8:43 AM or earlier arrival

8:53 AM or earlier arrival

9:07 AM or earlier arrival

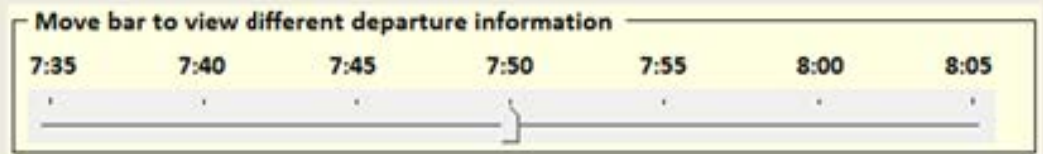

2a. How complicated is the infomation that is presented?

$\begin{array}{llllllll}1 & 2 & 3 & 4 & 5 & 6 & 7\end{array}$

$1=$ not complicated $4=$ neutra

$7=$ very complicated)

$2 b$. How easily are you able to understand information presented?

$$
\text { (1 = very difficult } \quad 4 \text { =neutral } \quad 7 \text { =very easily) }
$$

2c. How useful would this infomation prove when planning unfamiliar trips? $\begin{array}{lllllll}1 & 2 & 3 & 4 & 5 & 6 & 7\end{array}$

$$
\text { ( } 1 \text { = not useful } \quad 4 \text { =neutral } \quad 7 \text { =very useful })
$$


FORMAT 3: Historic Text with as slider bar for departure time presenting estimated and extra travel time information.

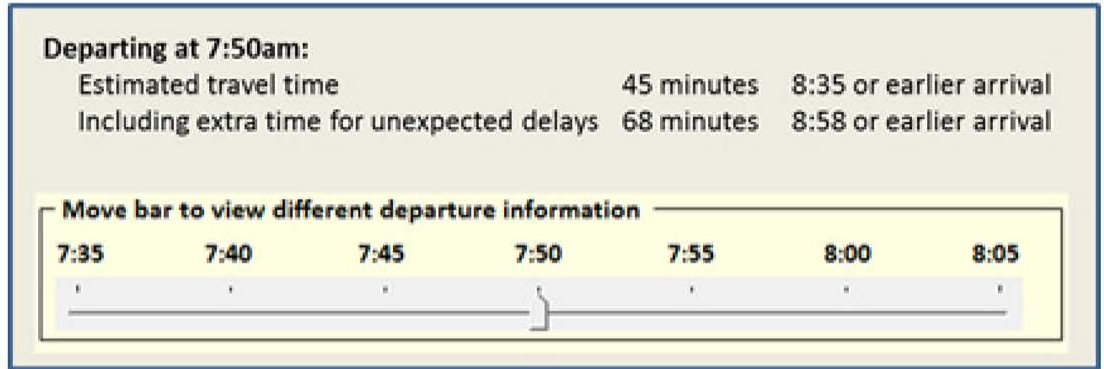

3a. How complicated is the information that is presented?

$\begin{array}{lllllll}1 & 2 & 3 & 4 & 5 & 6 & 7\end{array}$

(1 = not complicated $4=$ neutral $\quad 7=$ very complicated)

3b. How easily are you able to understand information presented?

(1 = very difficult $\quad 4=$ neutral $\quad 7=$ veryeasily)

3c. How useful would this information prove when planning unfamiliar trips? $\begin{array}{lllllll}2 & 3 & 4 & 5 & 6 & 7\end{array}$

( 1 = not useful $\quad 4$ =neutral $\quad 7$ =very useful)

FORMAT 4: Histeric Graph presenting arrival times for geod, tyaical, and bad daxs.

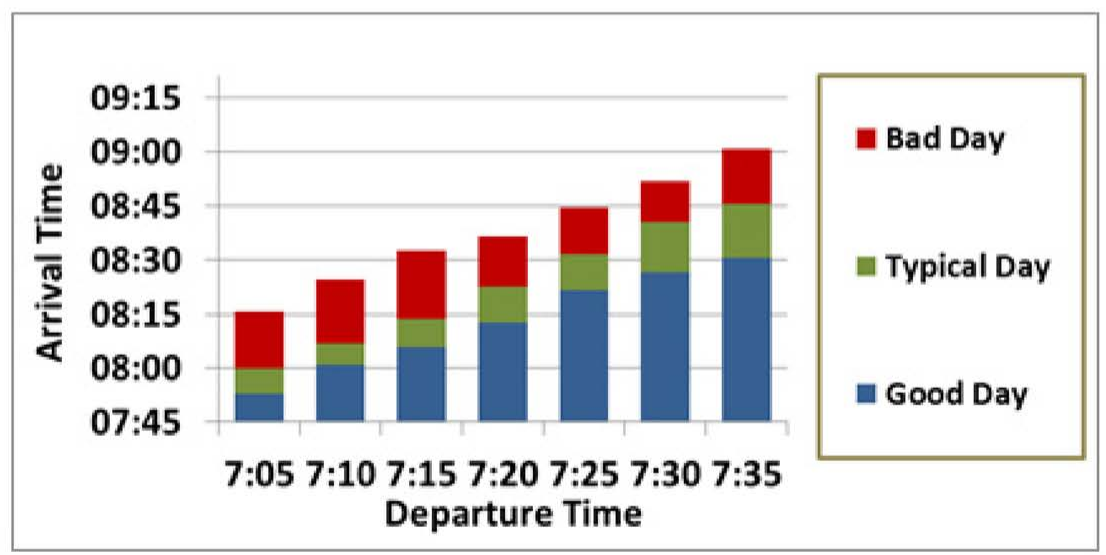

4a. How complicated is the information that is presented?

$\begin{array}{lllllll}1 & 2 & 3 & 4 & 5 & 6 & 7\end{array}$

(1 = not complicated $\quad 4=$ neutral $\quad 7=$ very complicated)

4b. How easily are you able to understand information presented?

( 1 = very difficult $\quad 4=$ neutral $\quad 7$ =veryeasily)

$\begin{array}{lllllll}1 & 2 & 3 & 4 & 5 & 6 & 7\end{array}$

4c. How useful would this information prove when planning unfamiliartrips? $\begin{array}{lllllll}2 & 3 & 4 & 5 & 6 & 7\end{array}$

(1 = not useful $\quad 4$ =neutral $\quad 7$ =very useful) 
FORMAT 5. Histaric Text with aslidec bar for de earture time.presenting travel time information.

Departing at 7:50am:

The majority of the time, this trip will take 68 minutes or less resulting in a

$8: 58$ or earlier arrival.

Move bar to view different departure information

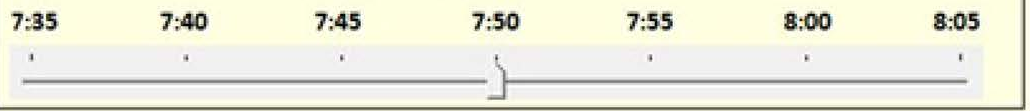

5a. How complicated is the information that is presented?

$\begin{array}{lllllll}1 & 2 & 3 & 4 & 5 & 6 & 7\end{array}$

( 1 = not complicated $4=$ neutral $\quad 7$ =very complicated)

5b. How easily are you able to understand information presented? $\quad 1234567$

(1) very difficult $\quad 4=$ neutral $\quad 7$ =veryeasily)

5c. How useful would this information prove when planningunfamiliartrips? $\begin{array}{lllllll}1 & 2 & 4 & 5 & 6 & 7\end{array}$

(1 = not useful $\quad 4$ =neutral $\quad 7$ =very useful)

FORMAT 6: REAL TIME color coded lines presenting current travel time ranges

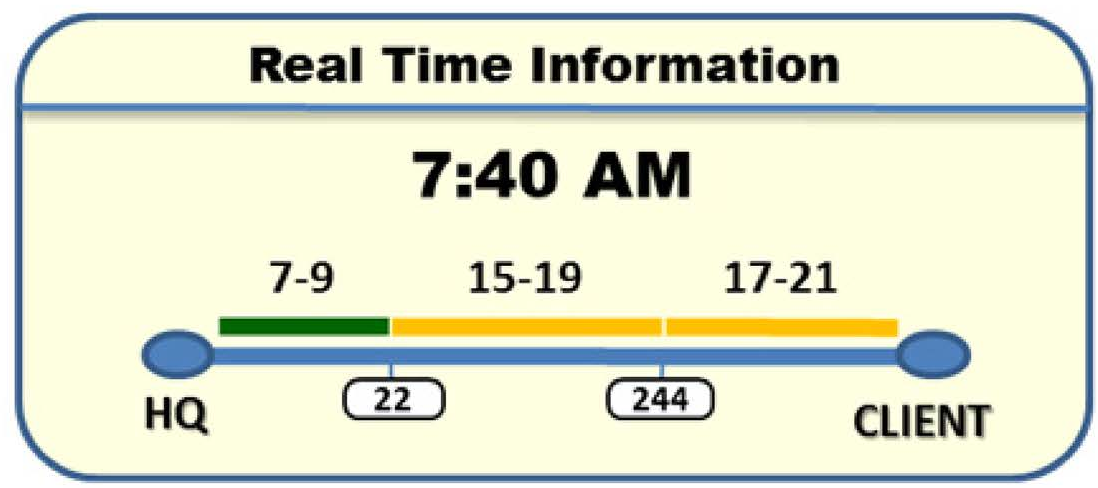

6a. How complicated is the information that is presented?

$\begin{array}{lllllll}1 & 2 & 3 & 4 & 5 & 6 & 7\end{array}$

( 1 = not complicated $4=$ neutral $\quad 7=$ very complicated)

6b. How easily are you able to understand information presented? $\quad \begin{array}{lllllll}2 & 3 & 4 & 5 & 6 & 7\end{array}$

(1 1 very difficult $\quad 4=$ neutral $\quad 7=$ veryeasily)

6c. How useful would this information prove when planningunfamiliartrips? $12 \begin{array}{llllll}1 & 4 & 4 & 6\end{array}$

(1 = not useful $\quad 4$ =neutral $\quad 7$ =very useful) 


\section{Supplemental Figures from Enhanced Laboratory Experiment Results}

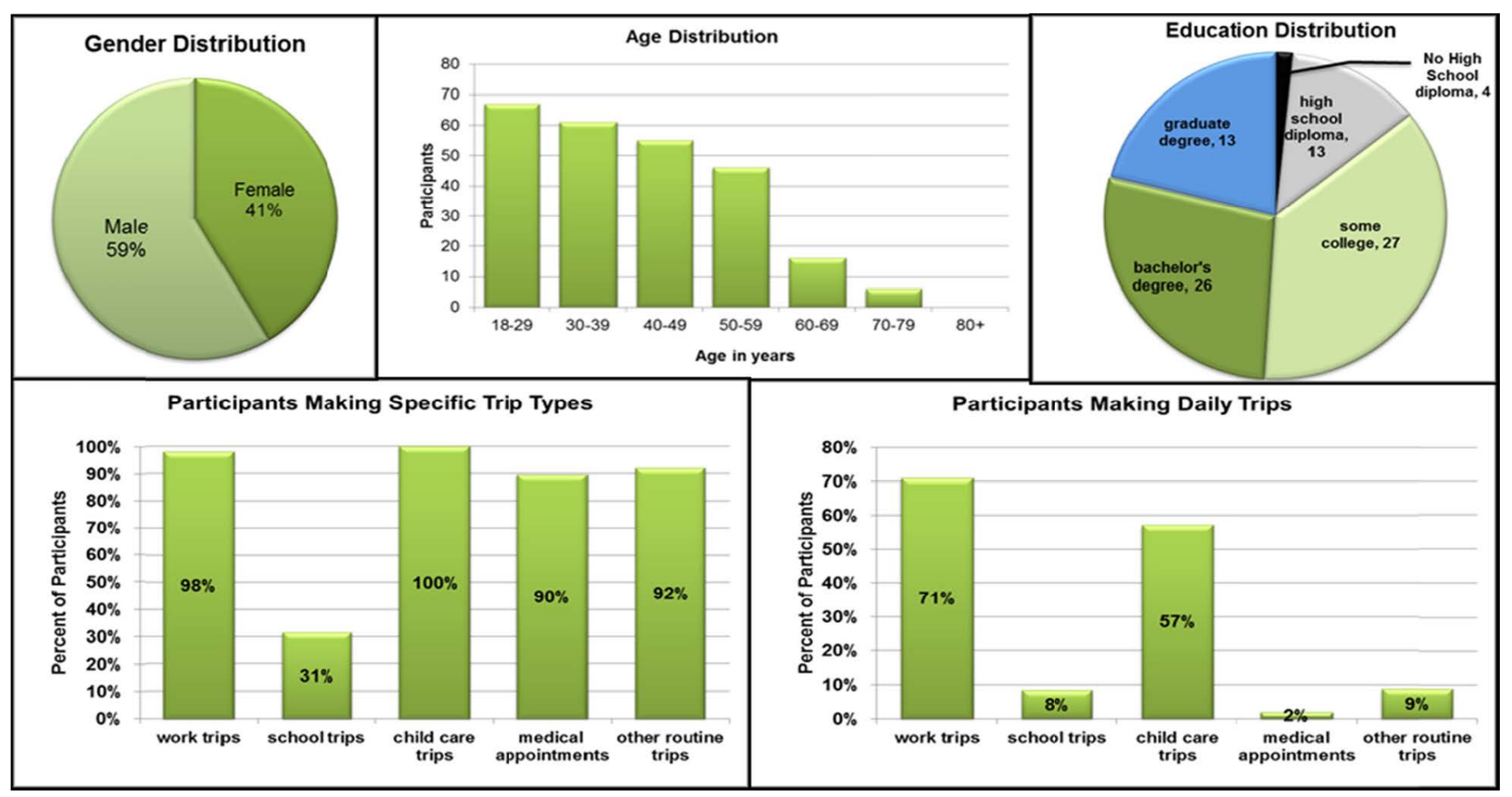

Figure I.1. Demographic and trip-making characteristics of experiment participants. 


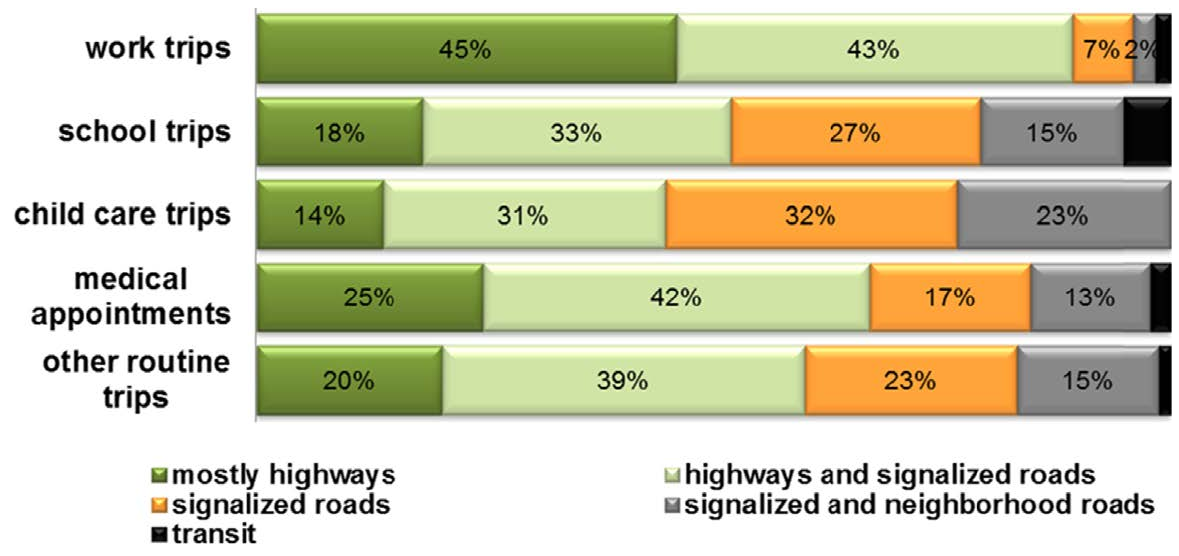

Figure I.2. Types of roads participants use for specific trip types.
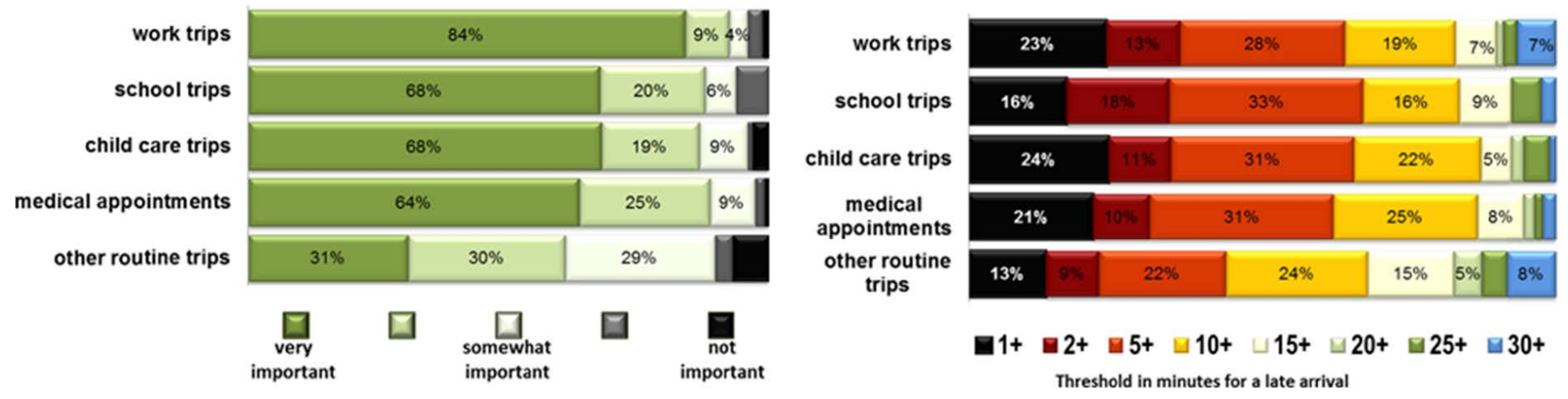

Figure I.3. Participants' rating of importance of on-time arrival (left) and definition of late arrival, by trip type (right).
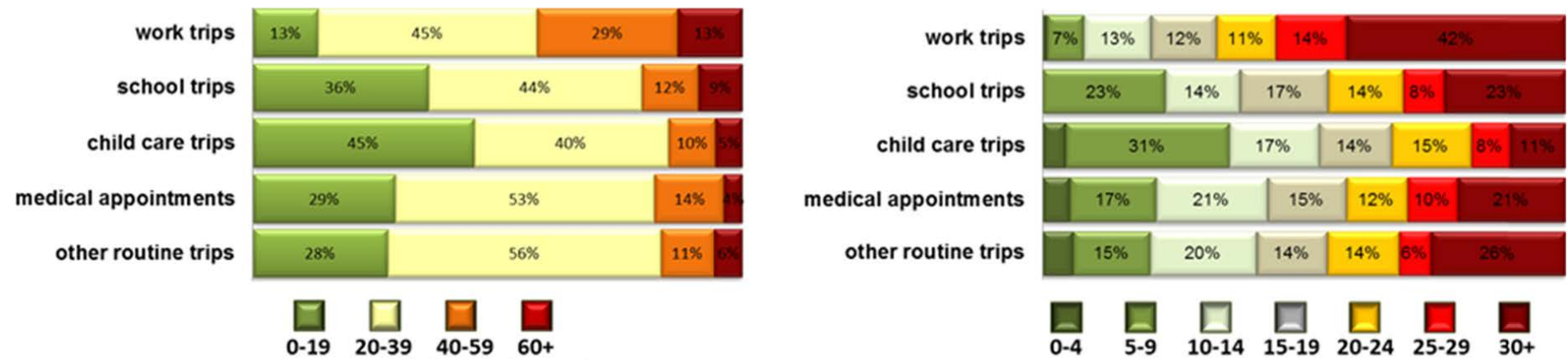

Figure I.4. Average (left) and additional (right) "bad day" trip duration, by trip type. 


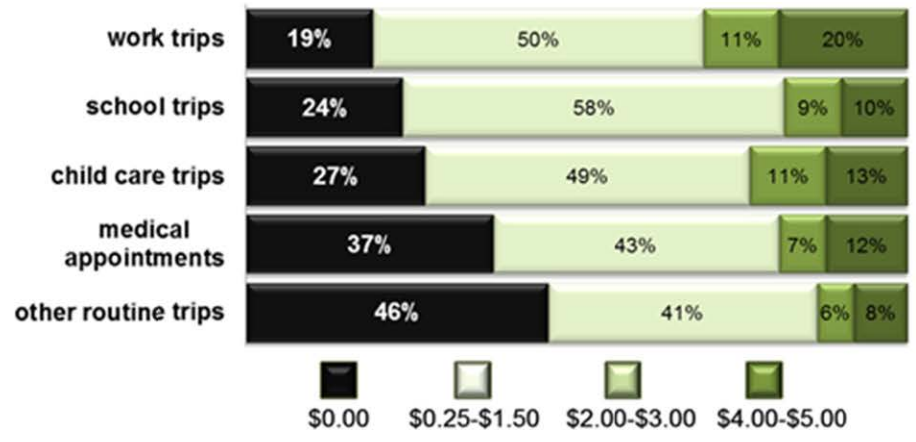

\begin{tabular}{|c|c|c|c|c|c|}
\hline work trips & $24 \%$ & $53 \%$ & $9 \%$ & & $3 \%$ \\
\hline school trips & $35 \%$ & $50 \%$ & & $8 \%$ & $8 \%$ \\
\hline child care trips & $38 \%$ & $41 \%$ & $7 \%$ & 15 & $5 \%$ \\
\hline $\begin{array}{c}\text { medical } \\
\text { appointments }\end{array}$ & $44 \%$ & $40 \%$ & & $9 \%$ & $7 \%$ \\
\hline other routine trips & & & $42 \%$ & & $0 \%$ \\
\hline
\end{tabular}

Figure I.5. Willingness to pay for guaranteed travel time (left) or late-arrival (right) knowledge. 


\section{TRB OVERSIGHT COMMITTEE FOR THE STRATEGIC HIGHWAY RESEARCH PROGRAM 2*}

CHAIR: Kirk T. Steudle, Director, Michigan Department of Transportation

\section{MEMBERS}

H. Norman Abramson, Executive Vice President (retired), Southwest Research Institute Alan C. Clark, MPO Director, Houston-Galveston Area Council

Frank L. Danchetz, Vice President, ARCADIS-US, Inc.

Malcolm Dougherty, Director, California Department of Transportation

Stanley Gee, Executive Deputy Commissioner, New York State Department of Transportation

Mary L. Klein, President and CEO, NatureServe

Michael P. Lewis, Director, Rhode Island Department of Transportation

John R. Njord, Executive Director (retired), Utah Department of Transportation

Charles F. Potts, Chief Executive Officer, Heritage Construction and Materials

Ananth K. Prasad, Secretary, Florida Department of Transportation

Gerald M. Ross, Chief Engineer (retired), Georgia Department of Transportation

George E. Schoener, Executive Director, I-95 Corridor Coalition

Kumares C. Sinha, Olson Distinguished Professor of Civil Engineering, Purdue University

Paul Trombino III, Director, Iowa Department of Transportation

\section{EX OFFICIO MEMBERS}

Victor M. Mendez, Administrator, Federal Highway Administration

David L. Strickland, Administrator, National Highway Transportation Safety Administration

Frederick "Bud" Wright, Executive Director, American Association of State Highway and Transportation Officials

\section{LIAISONS}

Ken Jacoby, Communications and Outreach Team Director, Office of Corporate Research, Technology, and Innovation Management, Federal Highway Administration

Tony Kane, Director, Engineering and Technical Services, American Association of State Highway and Transportation Officials Jeffrey F. Paniati, Executive Director, Federal Highway Administration John Pearson, Program Director, Council of Deputy Ministers Responsible for Transportation and Highway Safety, Canada Michael F. Trentacoste, Associate Administrator, Research, Development, and Technology, Federal Highway Administration

${ }^{\star}$ Membership as of July 2014 .

\section{RELIABILITY TECHNICAL COORDINATING COMMITTEE*}

CHAIR: Carlos Braceras, Deputy Director and Chief Engineer, Utah Department of Transportation

VICE CHAIR: John Corbin, Director, Bureau of Traffic Operations, Wisconsin Department of Transportation

VICE CHAIR: Mark F. Muriello, Assistant Director, Tunnels, Bridges, and Terminals, The Port Authority of New York and New Jersey

\section{MEMBERS}

Malcolm E. Baird, Consultant

Mike Bousliman, Chief Information Officer, Information Services Division, Montana Department of Transportation

Kevin W. Burch, President, Jet Express, Inc.

Leslie S. Fowler, ITS Program Manager, Intelligent Transportation Systems, Bureau of Transportation Safety and Technology, Kansas Department of Transportation

Steven Gayle, Consultant, Gayle Consult, LLC

Bruce R. Hellinga, Professor, Department of Civil and Environmental Engineering, University of Waterloo, Ontario, Canada

Sarath C. Joshua, ITS and Safety Program Manager, Maricopa Association of Governments

Sandra Q. Larson, Systems Operations Bureau Director, Iowa Department of Transportation

Dennis Motiani, Executive Director, Transportation Systems Management, New Jersey Department of Transportation

Richard J. Nelson, Nevada Department of Transportation

Richard Phillips, Director (retired), Administrative Services, Washington State Department of Transportation

Mark Plass, District Traffic Operations Engineer, Florida Department of Transportation

Constance S. Sorrell, Chief of Systems Operations, Virginia Department of Transportation

William Steffens, Vice President and Regional Manager, McMahon Associates

Jan van der Waard, Program Manager, Mobility and Accessibility, Netherlands Institute for Transport Policy Analysis

John P. Wolf, Assistant Division Chief, Traffic Operations, California Department of Transportation (Caltrans)

\section{FHWA LIAISONS}

Robert Arnold, Director, Transportation Management, Office of Operations, Federal Highway Administration Joe Conway, SHRP 2 Implementation Director, National Highway Institute

Jeffrey A. Lindley, Associate Administrator for Operations, Federal Highway Administration

\section{U.S. DEPARTMENT OF TRANSPORTATION LIAISON}

Patricia S. Hu, Director, Bureau of Transportation Statistics, U.S. Department of Transportation

\section{AASHTO LIAISON}

Gummada Murthy, Associate Program Director, Operations

\section{CANADA LIAISON}

Andrew Beal, Manager, Traffic Office, Highway Standards Branch, Ontario Ministry of Transportation

\footnotetext{
${ }^{\star}$ Membership as of July 2014.
} 


\section{Related SHRP 2 Research}

Forecasting and Delivery of Highway Travel Time Reliability Information (L15A) Establishing Monitoring Programs for Travel Time Reliability (LO2) 